
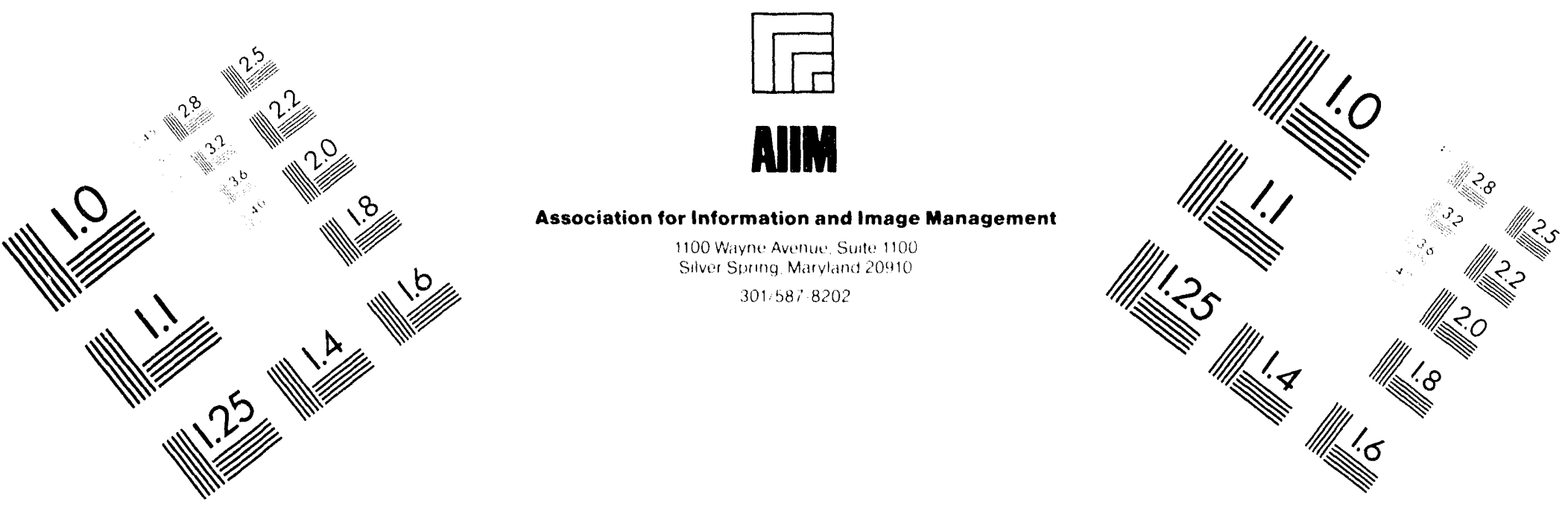

\title{
Centimeter
}

1mum

Inches
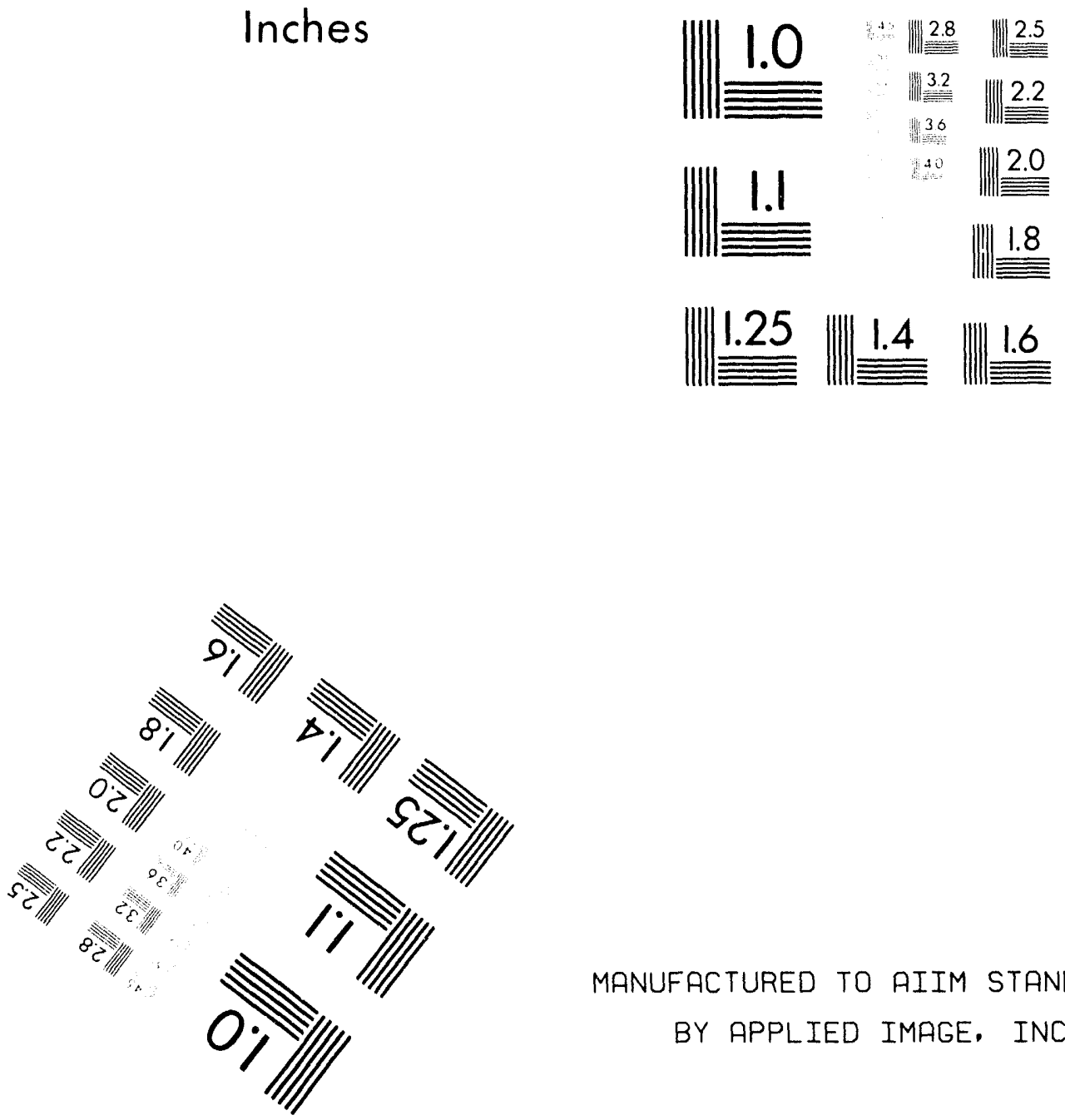

MANUFACTURED TO AIIM STANDARDS

BY APPLIED IMAGE, INC.

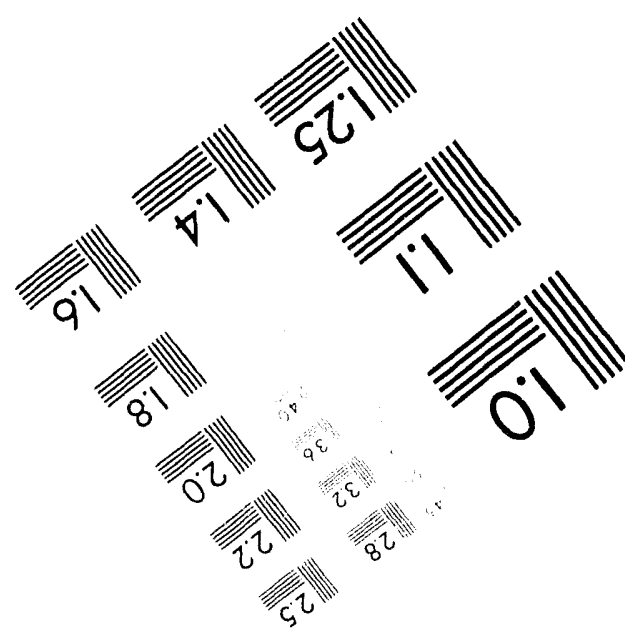



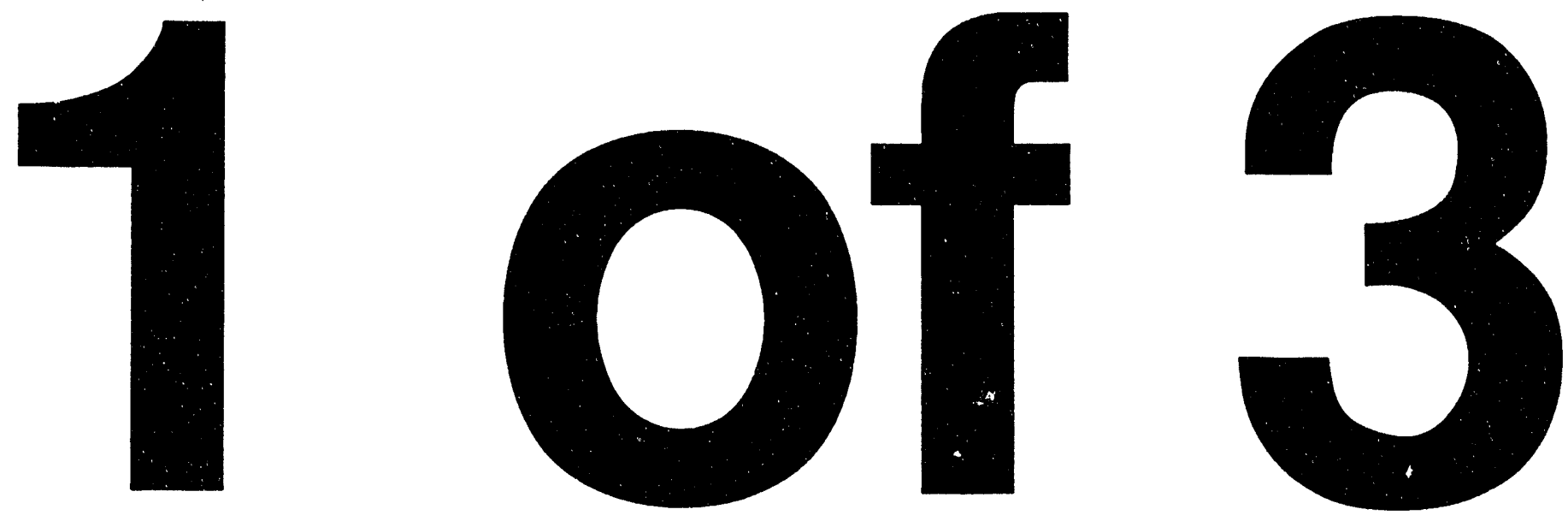


\title{
FINAL REPORT
}

\section{DEMONSTRATION TEST AND EVALUATION OF ULTRAVIOLET/ULTRAVIOLET CATALYZED PEROXIDE OXIDATION FOR GROUNDWATER REMEDIATION AT OAK RIDGE K-25 SITE}

MARCH 1994

\author{
Prepared 5y \\ W.J. SCHAFER ASSOCIATES \\ 321 Billerica Road \\ Chelmsford, Massachusetts 01824-4191 \\ 508-256-2070 \\ And \\ VULCAN PEROXIDATION SYSTEMS, INC. \\ a Wholly Owned Subsidiary of \\ Vulcan Materials Company \\ 5151 E. Broadway, Suite 600 \\ Tucson, Arizona 85711-3558
}

For

U.S. DEPARTMENT OF ENERGY

OAK RIDGE OPERATION

Under Contract DE-AC05-930R22000 
$1.0 \quad$ INTRODUCTION 3

2.0 REMEDIATION OF ORGANICS IN WATER

2.1 DOE Need 5

2.1.1 Regulatory Requirements for Water Remediation 5

2.1.2 Standard Water Remediation Technologies 5

2.2 Application to DOE of UV/UV Catalyzed Peroxide Oxidation Technology 6

2.3 Site Description 6

2.4 Demonstration Objectives $\quad 8$

3.0 DESCRIPTION OF UV/IJV CATALYZED PEROXIDE OXIDATION WATER REMEDIATION

3.1 Introduction 10

$\begin{array}{ll}3.2 & \text { Perox-Pure } \\ & \text { TM } \\ \text { Process } & 10\end{array}$

$\begin{array}{ll}\text { 3.3 Surface Discharge (SD) UV Lamp } & 12\end{array}$

3.4 SD UV Lamp System Upgrade 17

$\begin{array}{lll}3.4 .1 & \text { Summary } & 17\end{array}$

3.4.2 Water and Gas Recirculation Systems 19

3.4.3 Preliminary Integration with PSI Test Chamber 19

3.4.4 Laboratory Testing of UV Lamp System 19

3.5 Integration and Process Testing at PSI 21

$\begin{array}{lll}4.0 & \text { DEMONSTRATION TESTING } & 24\end{array}$

4.1 Summary 24

4.2 Test Objectives 25

4.3 Test Description 25

4.3.1 Test Setup and Site Description 25

4.3.2 Testing Procedures 25

4.3.3 Quality Assurance and Quality Control Test
Methods

4.4 Test Conditions 28

4.4.1 Phase I Tests with Perox-Pure ${ }^{\mathrm{TM}}$ System 28

4.4.2 Phase II Tests with SD-2 System 29 
TABLE OF CONTENTS (CONT.)

PAGE

4.5 Test Results 31

4.5.1 Water Characterization 31

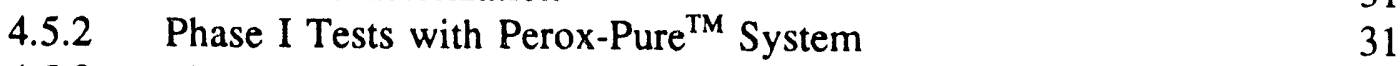

4.5.3 Phase II Tests with SD-2 System 32

4.6 Data Analysis 33

4.6.1 Perox-Pure ${ }^{\mathrm{TM}}$ System Data Analysis 36

4.6.2 SD-2 System Data Analysis 37

4.6.3 Continuous Water Monitoring Results $\quad 39$

4.6.4 Comparison of Treatment Systems 42

5.0 TECHNOLOGY EVALUATION

5.1 Progress and Status of SD Lamp Development 44

5.1.1 Background 44

5.1.2 Performance Improvements 44

5.1.3 Unique Characteristics of UV/UV Catalyzed Technology 45

5.2 Cost Savings and Benefits 45

5.2.1 Cost Comparison 45

5.2.2 Annual Savings Estimate 48

5.2.3 Return on Investment $\quad 50$

$\begin{array}{ll}5.3 \text { Other Benefits } & 50\end{array}$

5.3.1 Regulatory Advantages $\quad 50$

$\begin{array}{ll}\text { 5.3.2 Potential Acceptance Issues } & 50\end{array}$

$\begin{array}{lll}\text { 5.3.3 Risk Assessment } & 51\end{array}$

6.0 CONCLUSIONS AND RECOMMENDATIONS 52

SURFACE DISCHARGE LIGHT SOURCE REFERENCES 53

APPENDIX I WATER QUALITY DATA, SW-31 SPRING K-1070-C/D

BURIAL GROUND, K-25 SITE 54

APPENDIX II MAXIMUM CONTAMINATION LEVELS ALLOWED

FOR ORGANICS IN SW-31 SPRING WATER

$\begin{array}{lll}\text { APPENDIX III TERMINOLOGY AND DEFINITIONS } & 56\end{array}$

APPENDIX IV RESULTS OF PEROX-PURE ${ }^{\text {TM }}$ TESTING ON

SW-31 SPRING WATER AT DOE K-25 SITE 
TABLE OF CONTENTS (CONT.)

PAGE

APPENDIX $V$ RESULTS OF SD-2 TESTING ON SW-31

SPRING WATER AT DOE K-25 SITE

79

APPENDIX VI FIELD TEST OF DIRECT SAMPLING ION TRAP MASS SPECTROMETER FOR GROUNDWATER CLEANUP PROCESS MONITORING

APPENDIX VII ANALYTICAL REPORTS FOR PEROX-PURE ${ }^{\mathrm{TM}}$ AND SD-2 TESTS ON SW-31 SPRING

\section{LIST OF FIGURES}

FIGURE NO.

PAGE

2.1 Site of the Proposed Demonstration at the Oak Ridge K-25 Facility

3.1 Surface Discharge Schematic

3.2 Surface Discharge Cylindrical Lamp

3.3 SD UV Lamp Comparison with Commercial UV I amp

3.4 Surface Discharge Lamp System Block Diagram

3.5 Surface Discharge Lamp Layout

3.6 Gas Recirculation System Layout

3.7 SD Lamp System Integrated with PSI Demonstration Trailer

4.1 Run 17A DSITMS Continuous Water Monitoring of Treatment of K-25 Seep 


\section{LIST OF TABLES}

TABLE NO.

PAGE

2.1

SOW Success Criteria

3.1 Oxidation Rates of Ozone and Hydroxyl Radicals

Hydroxyl Radical Rate Constants

3.3 Surface Discharge Technology Development

4.1 Phase I Conditions for Perox-Pure ${ }^{\mathrm{TM}}$ Testing on SW-31 Spring Water

4.2 Phase II Conditions for SD UV Lamp Testing on SW-31 Spring Water

4.3 Characterization Results for SW-31 Spring Water

4.4 Summary Conclusions for Perox-Pure ${ }^{\mathrm{TM}}$ Testing

4.5 Summary Conclusions for SD Lamp Testing

4.6 Destruction Rates for SD-2 Testing on SW-31

Spring Water

4.7 DSITMS Analysis of Water for Run No. 12

4.8 DSITMS Analysis of Water for Run No. 17A

42

4.9 Summary Comparison of Perox-Pure ${ }^{T M}$ with SD-2 Test Results

5.1 Cost Estimate of Perox-Pure ${ }^{\mathrm{TM}}$ at SW-31 Spring

5.2 Cost Estimate of Perox-Pure ${ }^{\mathrm{TM}}$ SD UV Lamp-Based System at SW-31 Spring 


\section{EXECUTIVE SUMMARY}

In the Ultraviolet/Ultraviolet Catalyzed Groundwater Remediation program, W.J. Schafer Associates, Inc. (WJSA) demonstrated, tested and evaluated a new ultraviolet (UV) lamp integrated with an existing commercial technology employing UV catalyzed peroxide oxidation to destroy organics in groundwater at an Oak Ridge K-25 site. The existing commercial technology is the perox-pure ${ }^{\mathrm{TM}}$ process of Peroxidation Systems Incorporated (PSI) that employs standard UV lamp technology to catalyze $\mathrm{H}_{2} \mathrm{O}_{2}$ into $\mathrm{OH}$ radicals, which attack many organic molecules. In comparison to classical technologies for remediation of groundwater contaminated with organics, the perox-pure ${ }^{\mathrm{TM}}$ process not only is cost effective but also reduces contaminants to harmless by-products instead of transferring the contaminants from one medium to another (such as in activated carbon or air stripping). Although the perox-pure ${ }^{\mathrm{TM}}$ process is cost effective against many organics, it is not effective for some organic contaminants of interest to DOE such as TCA, which has the highest concentration of the organics at the K-25 test site. Contaminants such as TCA are treated more readily by direct photolysis using short wavelength UV light. WJSA has been developing a unique UV lamp which is very efficient in the short UV wavelength region. Consequently, combining this UV lamp with the perox-pure ${ }^{\mathrm{TM}}$ process results in a means for treating essentially all organic contaminants.

In the program reported here, the new UV lamp lifetime was improved and the lamp integrated into a PSI demonstration trailer. Even though this UV lamp operated at less than optimum power and UV efficiency, the destruction rate for the highest concentration organic (TCA) was more than double that of the commercial unit. An optimized UV lamp may double

again the destruction rate; i.e., a factor of four greater than the commercial system. The demonstration at K-25 included tests with (1) the commercial PSI system, (2) the new UV lampbased system and (3) the commercial PSI and new UV lamp systems in series. The evaluation of these tests indicates that:

1. The PSI system is cost-effective for treating water at the K-25 site. In comparison to the remediation approach imp'emented at K-25, the PSI system may save the Department of Energy additional cost.

2. The PSI system may have specific applicability at numerous DOE sites. 
3. The new UV system (alone and in tandem with the PSI system) can provide additional cost-reduction oi water treatment at many DOE sites for difficult organic contaminants like TCA.

4. Additional engineering development is necessary for full-scale operation with the new UV lamp.

Recommendations from the demonstration results include:

1. Use the perox-pure ${ }^{\mathrm{TM}}$ system results as a basis for considering further use at the SW31 Spring as well as at other DOE sites requiring water remediation.

2. Support further development of SD UV lamp technology to reduce the cost of water remediation. 


\subsection{INTRODUCTION}

This Ultraviolet/Ultraviolet Catalyzed Groundwater Remediation Demonstration Test and Evaluation program consisted of the integration of a special ultraviolet (UV) lamp with an existing commercial water treatment system to remediate groundwater contaminated with mixed organic compounds from the SW-31 Spring at the K-25 Plant in Oak Ridge, Tennessee. The commercially available perox-pure ${ }^{\mathrm{TM}}$ system of Peroxidation Systems Inc. (PSI) utilizes high intensity, medium pressure UV lamps along with hydrogen peroxide $\left(\mathrm{H}_{2} \mathrm{O}_{2}\right)$ to destroy organic contamination in water via photochemical oxidation. The destruction of the organic compounds occurs by a combination of mechanisms. $\mathrm{H}_{2} \mathrm{O}_{2}$ is catalyzed by $\mathrm{UV}$ light at a specific wavelength to form hydroxyl radicals, powerful chemical oxidants that break down organic molecules. At the same time, some chemical bonds are broken by UV light, aiding in the destruction of the organics. In comparison to classical water remediation technologies, the perox-pure ${ }^{\mathrm{TM}}$ process not only is cost effective but also reduces contaminants to harmless end-products instead of transferring the contaminants from one medium to another [such as in granulated active carbon (GAC) or air stripping]. Although the perox-pure ${ }^{\mathrm{TM}}$ process is cost effective against many organics, it is not as effective for some organic contaminants of interest to DOE such as TCA, which has the highest of the organic concentrations at SW-31. Such contaminants are treated more readily by direct photolysis using short wavelength UV light. W.J. Schafer Associates, Inc. (WJSA) has been developing a unique surface discharge (SD) UV lamp which is very efficient in the short UV wavelength region. Consequently, combining the SD UV lamp with the peroxpure $^{\mathrm{TM}}$ process results in a more effective means for treating a wide range of organics. Earlier

results of bench-scale testing indicated that the SD UV lamp can yield more rapid destruction of saturated volatile organic compounds (VOCs) than the commercially available system. The onsite demonstration at K-25 tested this concept under field conditions.

In the program reported here, the SD UV lamp was upgraded and integrated into a PSI demonstration trailer. The general project objectives were to:

1. Maximize the rate and extent of destruction of all organic contaminants in the influent waste stream by the existing and new advanced UV catalyzed oxidation systems, both separately and in series.

2. Evaluate the process economics of the UV catalyzed oxidation systems. 
The evaluation of the demonstration tests indicates that:

1. The PSI system is cost-effective for the K-25 site.

2. The PSI system has widespread applicability at DOE sites.

3. The SD UV system (alone and in series with the PSI system) can provide additional cost-reduction of water treatment at many DOE sites.

4. Additional development is necessary for practical water treatment with the SD UV lamp. 


\subsection{REMEDIATION OF ORGANICS IN WATER}

\subsection{DOE Need}

\subsubsection{Regulatory Requirements for Water Remediation}

Groundwater contaminated with a broad range of organic compounds is a common problem at many DOE sites. The organic contamination levels at the SW-31 Spring DT\&E site, summarized in Appendix I, are an example of the broad range of contaminants and concentrations which occur. Some of the DOE facilities at which groundwater contaminants have been identified are: the Feed Materials Production Center (Fernald, OH); the three Oak Ridge facilities (K-25, Y-12, and ORO); Mount Laboratory; the Kansas City Plant; and the Savannah River Plant.

The K-25 site, as well as other Oak Ridge sites, are on the National Priority List, and thus fall under the jurisdiction of both CERCLA and DOE/EPA Interagency Agreements. These regulations require consideration of alternatives for remediation. This demonstration project meets that requirement.

\subsubsection{Standard Water Remediation Technologies}

The Environmental Protection Agency (SPA) has accepted Packed Tower Aeration (PTA) "air stripping" and Granulated Activated Carbon (GAC) as the best demonstrated available technologies (BDAT). In air stripping, the most economical water treatment process, air is bubbled through the water, removes organic contaminants from the water and transfers them to the air. Stringent emission regulations have made this technique less acceptable. In GAC, organics are removed by absorption as water flows through the carbon medium. GAC is not effective for all organic contaminants, and results in contaminated carbon. The handling of a secondary contaminant is undesirable at many DOE sites. For both classical water remediation technologies, the contaminants are transferred from one medium to another resulting in a new contamination problem.

At the SW-31 Spring site, GAC is unacceptable due to the contaminated carbon byproduct. Consequently, the plan is to transport the water to the Y-12 site, which has a permit for air stripping. Alternative technologies are desirable. 


\subsection{Application to DOE of UV/UV Catalyzed Peroxide Oxidation Technology}

The use of Advanced Oxidation ( $\mathrm{AO}$ ) processes to destroy toxic and refractory organic compounds present in water has gained rapid acceptance since their emergence in the mid 1980's. Much of this acceptance is due to the ability of AO processes to reduce most organic contaminants in water to $\mathrm{CO}_{2}, \mathrm{H}_{2} \mathrm{O}$ and, if present, inorganic halides. The perox-pure ${ }^{\mathrm{TM}}$ process incorporates a UV lamp, hydrogen peroxide $\left(\mathrm{H}_{2} \mathrm{O}_{2}\right)$ and a patented reactor to provide total on-site destruction of the organics without by-products, solid wastes to dispose of, or gas emissions. This process has been demonstrated to be cost-effective for a wide variety of contaminated waters and wastewaters.

This technology, however, is not as cost-effective for some organics, which are better treated by direct UV photolysis. This group of organics includes 1,1,1 TCA, one of the major organic contaminants at the SW-31 Spring. Treating these organics cost-effectively requires the incorporation of a high intensity UV lamp with efficient light emission below $250 \mathrm{~nm}$. The surface discharge (SD) UV lamp has the desired UV light output. Thus, the combination of the UV peroxide process with UV photolysis allows remediation of essentially all organic water contamination at DOE sites.

\subsection{Site Description}

The K-25 site is located in Roane County between Oak Ridge and Kingston, Tennessee. The site was home to one of three U.S.-based uranium enrichment plants to support the nuclear fuel cycle. The plant, which is no longer in operation, is currently managed by Martin Marietta Energy Systems, Inc. (MMES) for DOE.

The SW-31 Spring influent to the UV system is located adjacent to the east side of Avenue $D$, about 110 feet south of the center of the intersection with 9th Street, on the eastern portion of the K-25 facility (Figure 2.1). The SW-31 Spring flows from a PVC pipe imbedded at the foot of a 6-foot high slope, and at the time of the test drained through an open concrete channel along the road into a storm drain (SD-180-04) approximately 80 feet north of the SW-31 Spring. The SW-31 Spring is located approximately 200 feet west of the boundary of the K-1070C/D Classified Burial Ground. 


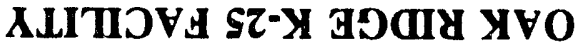

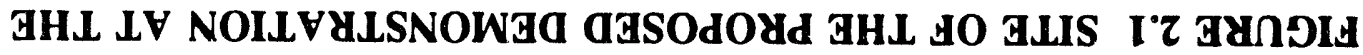

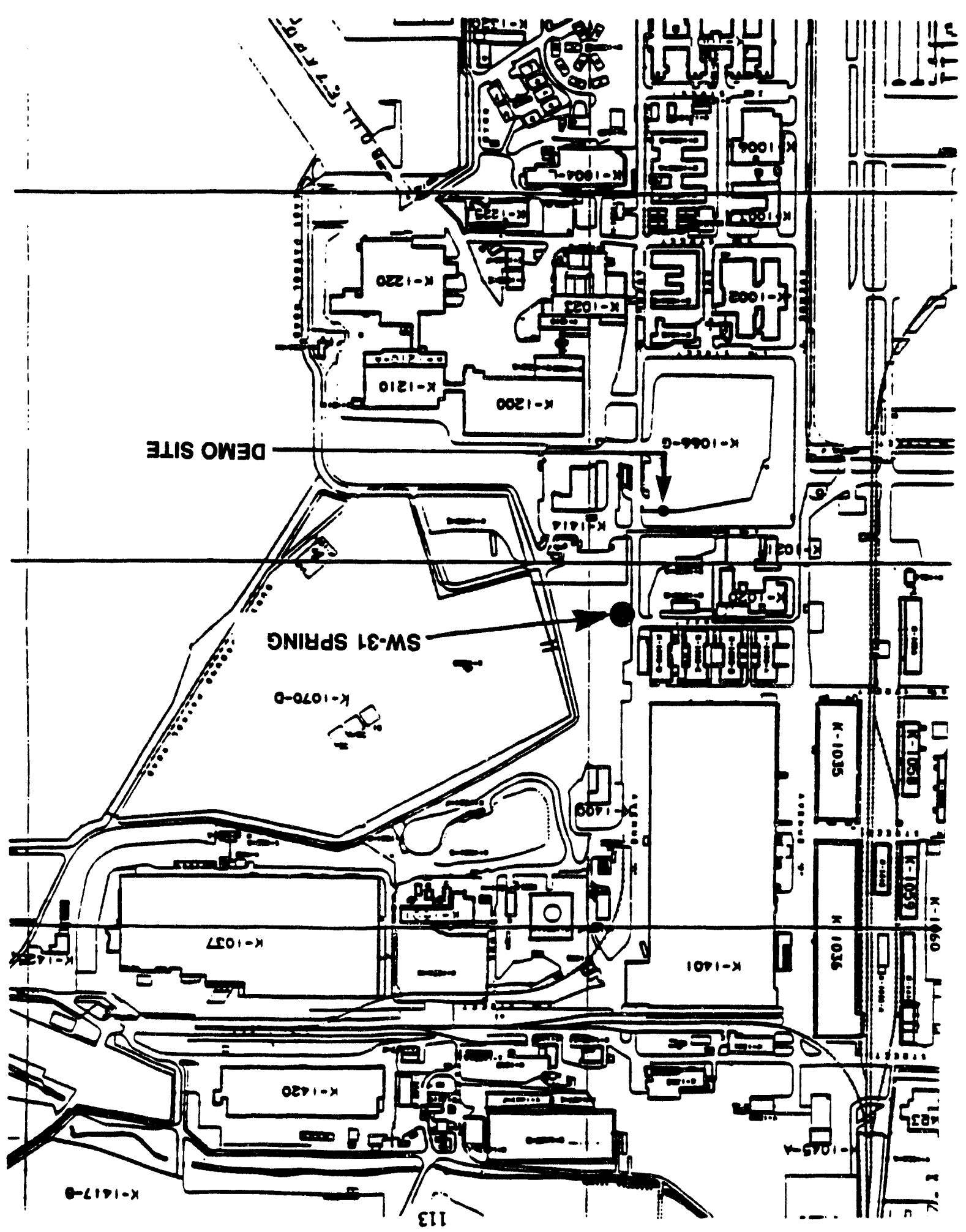


The base SW-31 Spring flow appears to be relatively constant at a few gal/min. Flow increases during and immediately following storms, and reportedly has stopped completely during extended dry periods.

Chemical characteristics of the SW-31 Spring have been determined and are summarized in Appendix I. This water, once it entered the storm drains, continued on to local streams.

Due to recently enacted treatment criteria for the spring water, an effort is currently underway to collect the 2 to $3 \mathrm{gpm}$ effluent. Two 12,500-gallon tanks have been placed at the Western base of the burial ground hill where the spring flows from the PVC pipe. Each day, the water in the collection tanks is hauled via tanker truck to the Groundwater Treatment Plant at the nearby Y-12 facility.

\subsection{Demonstration Objectives}

As stated in the Statement of Work (SOW), the objective of this DT\&E was "to demonstrate an integrated groundwater treatment system that oxidizes a wide variety of organic compounds." The specific "success criteria" from the SOW are listed in Table 2.1.

\section{TABLE 2.1 SOW SUCCESS CRITERIA}

1. All seep water target organic compounds must be reduced in the effluent to levels that meet Drinking Waste Standards.

2. Operational costs should be comparable to or less than existing groundwater remediation technologies.

3. Operational conditions should be optimized when the UV light source is integrated with the perox-pure ${ }^{\mathrm{TM}}$ system.

4. Secondary waster generation must be held to a minimum.

5. Environmental, safety and health documentation will be prepared and maintained.

6. Reporting of the demonstration activities, including observations, conclusions and recommendations, will be made to DOE for review and comment prior to finalization. 
The first is to reduce all organic compounds to concentration levels that meet drinking water standards while at the same time demonstrating that the water discharge requirements at SW-31 Spring are met. A comparison of the maximum contaminant levels allowed for drinking water (see Appendix II) with the effluent levels demonstrated (see Appendices IV and V) shows that the first success criteria was met. The DOE water discharge requirement at the SW-31 Spring, namely that the total toxic organics be below 2.13 milligrams per liter (see Appendix V, Table 2), is readily achievable and was met in many cases by the influent organic concentrations. The second primary objective is to demonstrate that the costs for the UV demonstration technologies are comparable or less than existing ground water remediation technologies. While precise cost comparisons are difficult in mixed waste waters such that exist at the SW-31 Spring unless demonstrations are carried out in parallel, the results of this program (see Section 5.2) demonstrate that the costs for the existing UV commercial technology is less than the currently identified alternative and is more acceptable from a regulatory standpoint than other classical alternatives. Furthermore, the test demonstrated that using the new UV light source, the destruction rate of the rate limiting organic (TCA) contaminant is increased by more than a factor of two (see Section 4.6.4) and, consequently, that once the new UV light source is commercialized, water remediation costs would be reduced. Also, the third objective was achieved since the fastest destruction rates occurred with the system in which the UV light source was integrated with the perox-pure ${ }^{\mathrm{TM}}$ system. Thie test also corroborated the fact that secondary waste is minimal with this UV-based water treatment system. Environmental Safety and Health documentation was successfully maintained, and with the completion of this report, al! required reporting activities under this effort are also complete. Consequently, all program objectives have been met. 


\subsection{DESCRIPTION OF UV/UV CATALYZED PEROXIDE OXIDATION WATER REMEDIATION}

\subsection{Introduction}

UV/UV catalyzed peroxide oxidation water remediation relies on two processes. In the first, UV light is absorbed by peroxide and produces $\mathrm{OH} \cdot$ radicals which are very strong oxidizers of organics. In the second (photolysis), organic molecules absorb UV light directly which breaks bonds and/or produces excited states which are chemically reactive. The commercial peroxpure $^{\mathrm{TM}}$ process relies primarily on the former, UV catalyzed peroxide oxidation, which is effective on many, but not all, organic contaminants. The UV light from the commercial lamp is weak in the short wavelength UV needed for the second process (photolysis). The surface discharge (SD) UV lamp is a strong source of short wavelength UV light and hence is suited for UV photolysis. Consequently, combining the SD UV lamp with the perox-pure ${ }^{\mathrm{TM}}$ process is an effective means for treating all organics.

Starting in 1989, WJSA and PSI collaborated in proof-of-concept experiments which lead to the successful demonstration of an SD UV lamp in an experimental PSI test chamber. Laboratory tests with synthetic water showed the advantages of the SD UV lamp over available commercial UV lamps. This proof-of-principle experiment led to the DT\&E program reported here.

Preparation for the field test in this program included engineering the SD UV lamp for improved reliability and integrating the improved SD lamp with a PSI field demonstration trailer. The following sections describe the technology, the improvements accomplished in this program and the integration with the demonstration trailer.

\subsection{Perox-Pure ${ }^{\mathrm{TM}}$ Process}

UV light catalyzes the chemical oxidation of organic contaminants in water by its combined effect upon the organic contaminant and its reaction with $\mathrm{H}_{2} \mathrm{O}_{2}$. Many organic contaminants absorb UV light and may be directly oxidized or form intermediates that are more reactive to chemical oxidants. More importantly, UV light at less than $400 \mathrm{~nm}$ wavelength reacts with $\mathrm{H}_{2} \mathrm{O}_{2}$ molecules to form hydroxyl radicals $(\mathrm{OH} \cdot)$. This process is described by: 


$$
\begin{gathered}
\mathrm{H}_{2} \mathrm{O}_{2}+\mathrm{UV} \rightarrow 2 \mathrm{OH} . \\
(<400 \mathrm{~nm})
\end{gathered}
$$

The $\mathrm{OH}$ - chemical oxidant then reacts with the organic contaminants in the water. Given sufficient $\mathrm{H}_{2} \mathrm{O}_{2}$ and $\mathrm{UV}$ dose the compounds are completely destroyed. The $\mathrm{H}_{2} \mathrm{O}_{2}$ concentration is not stoichiometric as other inorganic ions can consume hydroxyl radicals. Also, the UV light converts $\mathrm{H}_{2} \mathrm{O}_{2}$ to hydroxyl radicals regardless of the organic content. Rather, the required $\mathrm{H}_{2} \mathrm{O}_{2}$ dosage is dependent upon a number of parameters including contaminant type, water quality, oxidation time, and UV intensity.

Hydroxyl radicals are the strongest practical oxidant to use for water remediation. For example, oxidation rates for $\mathrm{OH} \cdot$ are $10^{6}$ to $10^{10}$ times faster than for ozone (see Table 3.1). Although many organic contaminants, such as TCE and benzene, are rapidly destroyed by hydroxyl radical attack, some compounds are not readily attacked by $\mathrm{OH}$. (see Table 3.2). Oxidation for these organics, such as chloroform, 1,1,1-TCA and carbon tetrachloride, depends on photolysis, i.e., direct reaction with UV light. Most of these organic compounds are absorptive only in the short UV region below $240 \mathrm{~nm}$ where standard UV lamps are inefficient.

TABLE 3.1 OXIDATION RATES OF OZONE AND HYDROXYL RADICALS

\begin{tabular}{|l|l|l|}
\hline Compound & \multicolumn{1}{|c|}{$\mathbf{O}_{\mathbf{3}}{ }^{*}$} & \multicolumn{1}{|c|}{$\mathbf{O H} \cdot *$} \\
\hline Olefins & $10^{3}-10^{5}$ & $10^{9}-10^{10}$ \\
Phenols & $10^{3}$ & $10^{9}$ \\
Aromatics & $10^{0}-10^{2}$ & $10^{8}-10^{10}$ \\
Aldehydes & 10 & $10^{9}$ \\
Ketones & 1 & $10^{9}-10^{10}$ \\
Alkanes & $10^{-2}$ & $10^{6}-10^{9}$ \\
\hline *K, in L mole ${ }^{-1} \mathrm{~S}^{-1}$ \\
\hline
\end{tabular}




\section{TABLE 3.2 HYDROXYL RADICAL RATE CONSTANTS}

\begin{tabular}{|l|c|}
\hline \multicolumn{1}{|c|}{ Compound } & $\begin{array}{c}\text { Rate Constant } \\
\left(\mathbf{K ~ x ~ 1 0} \mathbf{~ M}^{-1} \mathbf{S}^{-1)}\right.\end{array}$ \\
\hline Benzene & 7.8 \\
Vinyl Chloride & 7.1 \\
TCE & 4.0 \\
Dichloromethane & 0.058 \\
Chloroform & 0.005 \\
TCA & Very slow \\
Carbon Tetrachloride & Non-reactive \\
\hline
\end{tabular}

Since 1985, a number of improvements and advances have been made in the perox-pure ${ }^{\mathrm{TM}}$ technology to increase the oxidation rate of difficult to oxidize organic compounds. Many of these compounds are low molecular weight chlorinated alkanes such as chloroform $\left(\mathrm{CHCl}_{3}\right)$, carbon tetrachloride $\left(\mathrm{Ccl}_{4}\right), 1,1,1$-trichloroethane (1,1,1-TCA), methylene chloride $(\mathrm{MeCl})$, and 1,1-dichloroethane (1,1-DCA). Overall improvements in oxidation rates range from 200 to $400 \%$, depending on the compound.

Much of this advancement is due to UV lamp efficiency. The most recent innovation incorporates a novel UV lamp technology. Briefly this new lamp generates two to three times the UV energy with the same electrical input as conventional high intensity UV lamps. In addition, much of this UV light is generated below $250 \mathrm{~nm}$ wavelength where it has enough energy to oxidize chlorinated alkanes directly. Therefore, the combination of standard peroxpure $^{\mathrm{TM}}$ technology with the novel UV lamp results in an AO process that is effective for the destruction of a wide variety of organic compounds found in contaminated groundwater.

\subsection{Surface Discharge (SD) UV Lamp}

The SD is a pulsed plasma discharge along a dielectric surface between two electrodes. High UV efficiency is achieved by electrically driving the SD lamp at high intensity and producing UV light from the dielectric material that is generated in the plasma. 
An SD occurs when the electrical potential between the two electrodes is large enough to cause a gas breakdown. A schematic of a rectangular SD is shown in Figure 3.1. Capacitive coupling through the dielectric substrate with the conductor below the dielectric aids in generating the plasma along the surface. The breakdown occurs preferentially along the surface, resulting in a ribbon-like plasma which emits intense incoherent light. The light properties (i.e., peak intensity, spectrum, and pulselength) depend on several parameters including the electrical circuit components, gas composition and pressure, geometrical arrangement of the electrode/substrate conibination and the composition of the substrate material. For typical well designed systems, over $90 \%$ of the stored electrical energy is deposited in the plasma. Generally, over $70 \%$ of the energy deposited in the SD plasma is converted to light. Consequently, the SD is a very efficient source of light.

The radiant emission from the SD can be enhanced in a specific spectral region by choosing a substrate material with constituents that have emission lines in the desired spectral region. A summary of research and development of the SD light source conducted at the WJSA Laboratory is given in Table 3.3.

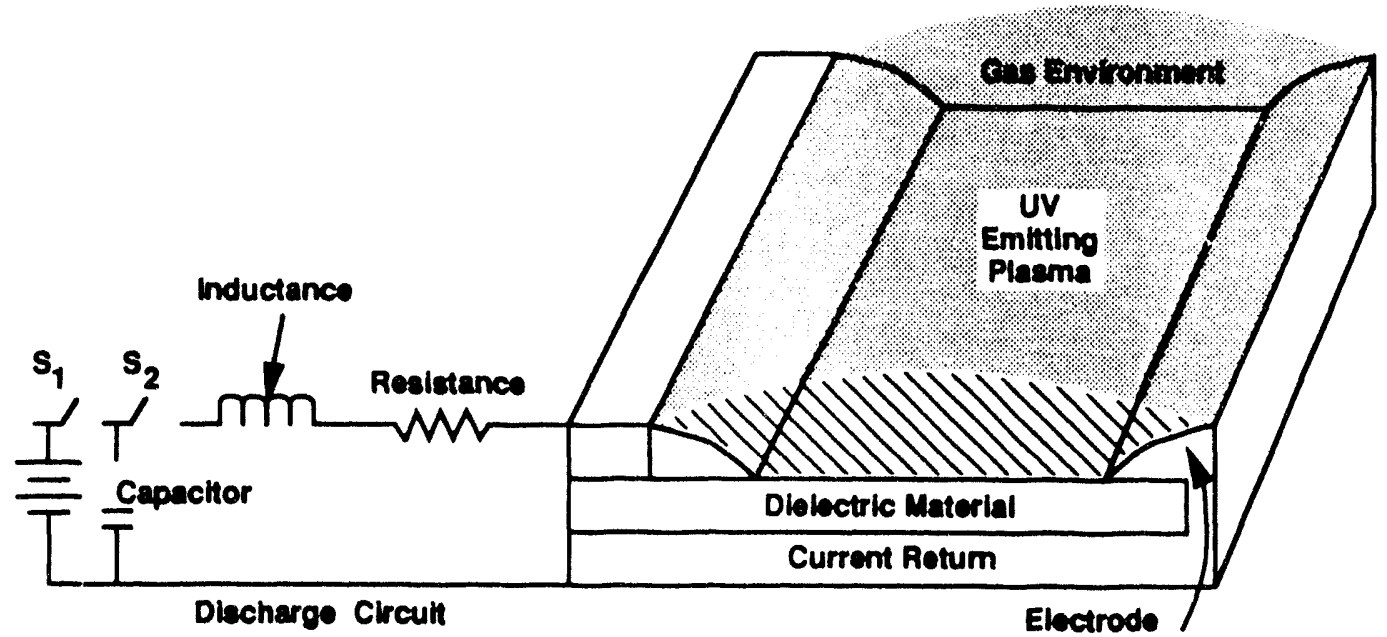

FIGURE 3.1 SURFACE DISCHARGE SCHEMATIC 
TABLE 3.3 SURFACE DISCHARGE TECHNOLOGY DEVELOPMENT ${ }^{(1-12)}$

\begin{tabular}{||l|l||}
\hline \hline High intensity radiant output $\left(\mathrm{W} / \mathrm{cm}^{2}\right)$ & $4 \times 10^{6}$ \\
\hline Large area emission $\left(\mathrm{cm}^{2}\right)$ & 750 \\
\hline Uniform radiant output & Yes \\
\hline Geometrical shaping & Lincar, rectangular, cylindrical, annular \\
\hline Spectral enhancement & Factor of 2 in $0.2-0.3 \mu \mathrm{m}$ band \\
\hline Repetitive pulse rate $(\mathrm{Hz})$ & 5000 \\
\hline Temporal pulsewidth $(\mathrm{s})$ & $7 \times 10^{-8}-10$ \\
\hline Lifetime (shots), fused silica substrate & $>10^{6}$ \\
\hline
\end{tabular}

The SD geometrical configuration is flexible. That is, the dielectric can be arranged in many different shapes, including rectangular (shown in Figure 3.1), annular, linear and cylindrical. We use a cylindrical SD lamp for in-water remediation applications, shown in Figure 3.2. In this configuration, the electrical discharge generates a plasma along a cylindrical rod between cylindrical electrodes. An outer envelope retains the rare gas used for the plasma discharge. Figure 3.2 also shows an open shutter photograph of the pulsed plasma discharge. Many streamers are generated along the circumference of the cathode, which together form a cylindrical light emitting plasma.

A comparison of the most efficient SD UV spectrum with a standard medium pressure mercury lamp (see Figure 2.3) shows the efficiency advantage of the SD lamp. In the wavelength region below $240 \mathrm{~nm}$ needed for photolysis, the SD UV efficiency shown is about five times that of the commercial lamp. This results in much faster organic destruction rates with the SD lamp.

Prior to this DT\&E program, WJSA and PSI collaborated in an internal research and development program using the SD cylindrical lamp to demonstrate the advantages of the short UV light. In order to achieve sufficient reliability for processing tests, the SD lamp was operated at: (1) one-fourth of the UV efficiency shown in Figure 3.3 and (2) at low power (200 W). In those tests, the destruction rates of organic compounds $1,1,1-\mathrm{TCA}$ and $\mathrm{CCL}_{4}$ (which are treated by UV photolysis with the SD lamp) were 1.5 times that with the standard lamp. This result established the advantage of the SD lamp and led to the DT\&E program reported here. 


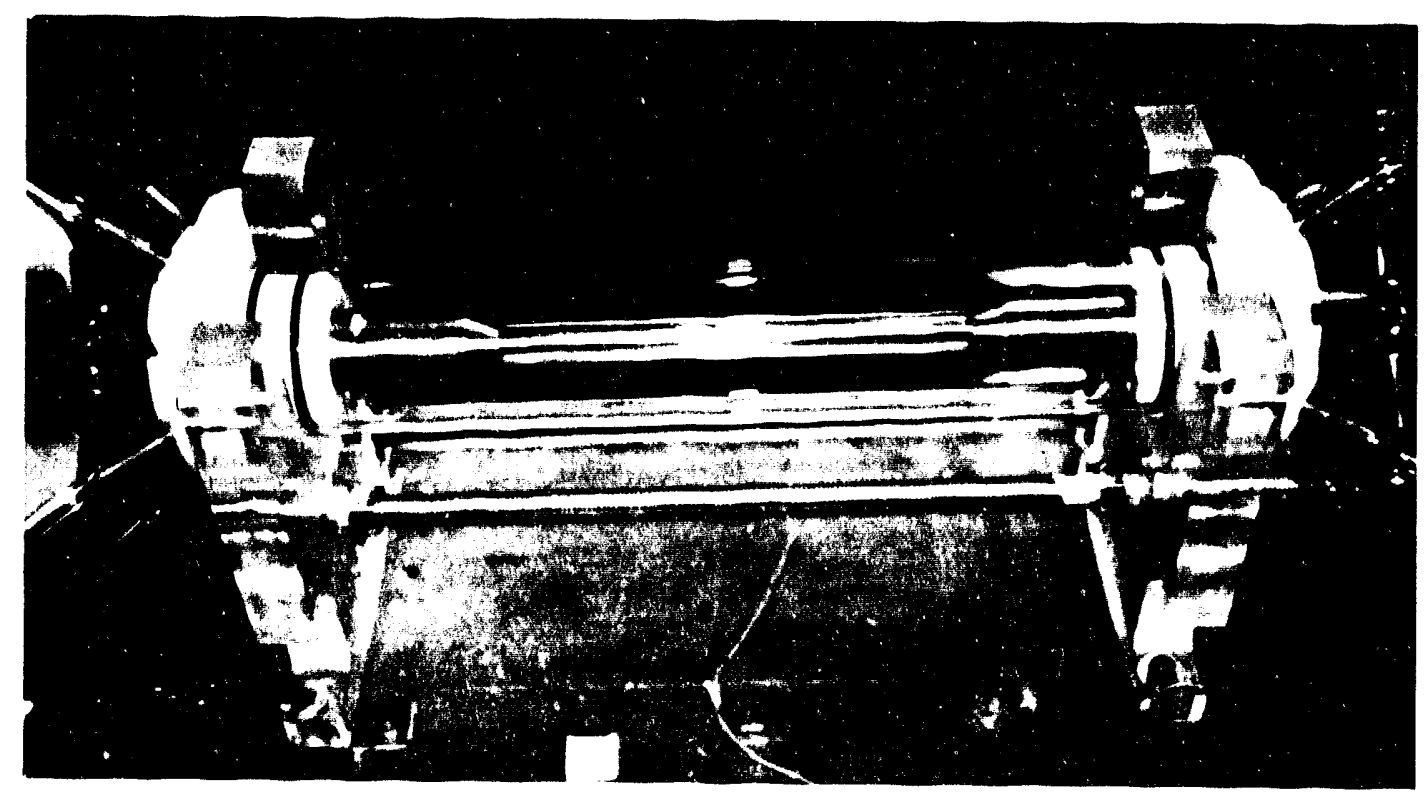

(a) Tare Shot of SD Lamp

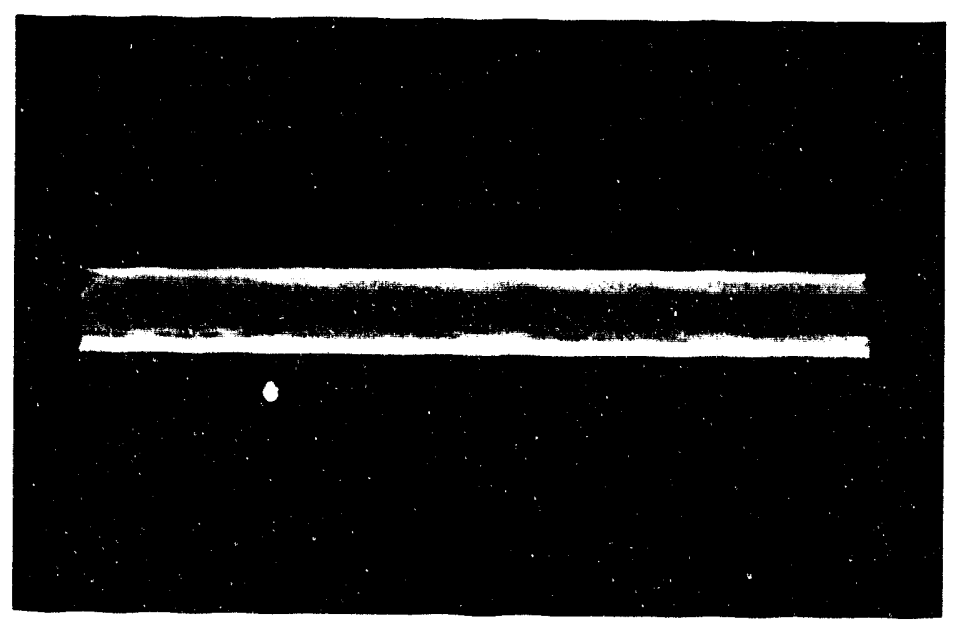

(b) Open Shutter Photo of SD Plasma

FIGURE 3.2 SURFACE DISCHARGE CYLINDRICAL LAMP 


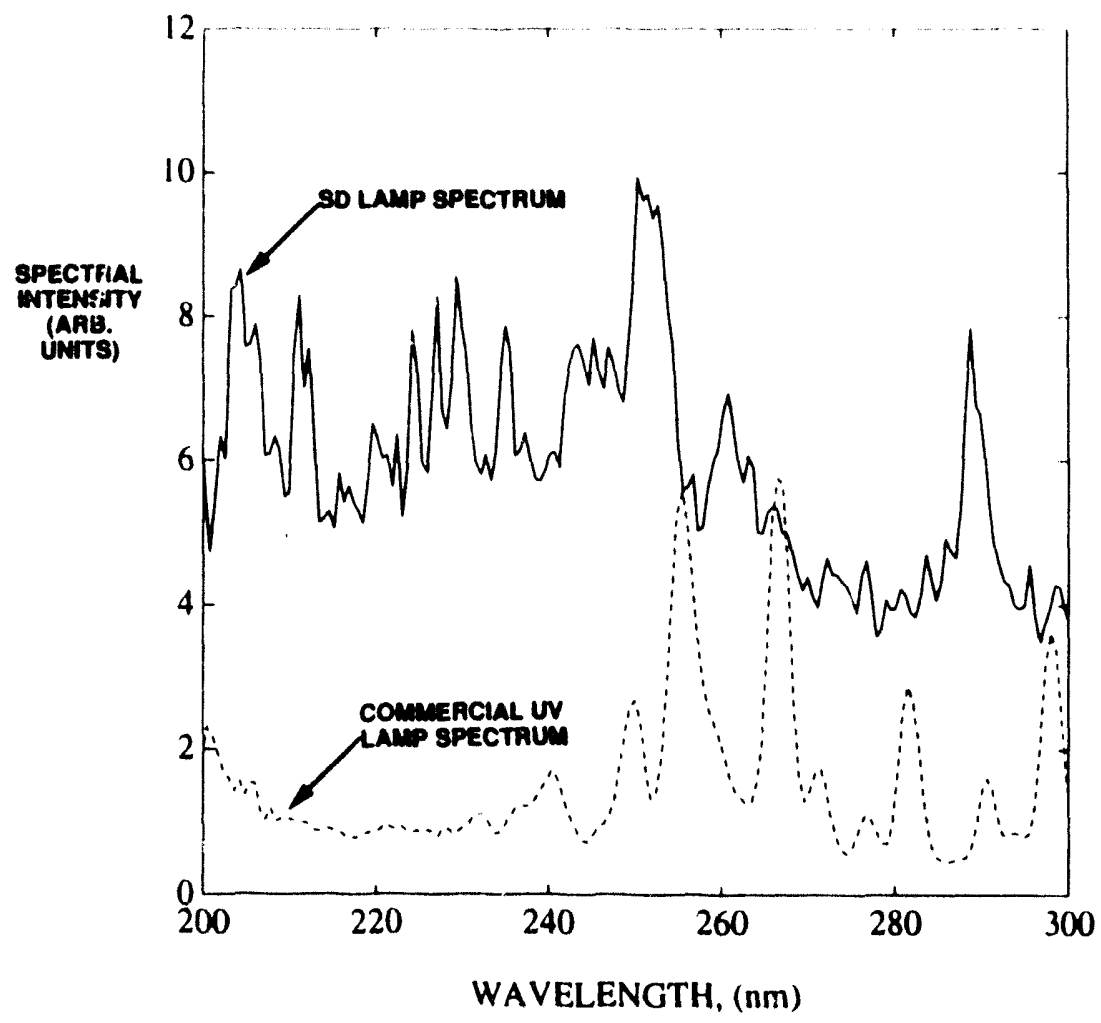

FIGURE 3.3 SD UV LAMP COMPARISON WITH COMMERCIAL UV LAMP 
The primary limitation in SD lamp operation in the laboratory tests was due to the accumulation of substrate species on the envelope, reducing UV transmission. In the laboratory tests, the SD lamp had to be disassembled and cleaned after approximately 15 minutes of operation. Consequently, the first step in this DT\&E program was to upgrade the lamp to operate at the higher power and longer operating times needed for a field test. This upgrade is described in the next section.

\subsection{SD UV Lamp System Upgrade}

\subsubsection{Summary}

At the start of this program, an upgraded SD lamp system, referred to as SD-2, was designed to improve performance for field tests and for integrating with the PSI demonstration system. Initial retrofit and upgrade design of SD-2 was followed by laboratory testing to establish the lifetime capabilities for the field test.

Both the laboratory and upgrade system designs have the same subcomponents shown in Figure 3.4. The SD lamp, which interfaces with a PSI test chamber, mounts against end flanges which orient the lamp axially in the center of a cylindrical processing chamber. Contaminated water flows in one end, is irradiated by the SD lamp (destroying the organic contaminants) and exits the other end of the chamber.

A gas recirculation system removes the gas from the discharge area, filters, cools and returns it to the lamp. The electrical driver stores and then discharges electrical energy into the lamp. A liquid cooling recirculation system cools the electrodes and substrate.

In the earlier laboratory prototype system, a powder from the SD substrate accumulated on the lamp envelope over time, which reduced the UV transmission. In that system, when operated at an average power of 200 watts, the UV transmission degraded after about fifteen minutes of operation. Insufficient electrode cooling and gas flow in the recirculation system were the key factors in that degradation.

SD-2 can operate as high as 1,500 watts, about a factor of 7 increase from the laboratory system. The liquid cooling flow rate is approximately a factor of 30 greater than the laboratory system. Also, the gas recirculation flow rate is larger, by over a factor of 100 , than the laboratory system and the gas filter system is greatly improved. 


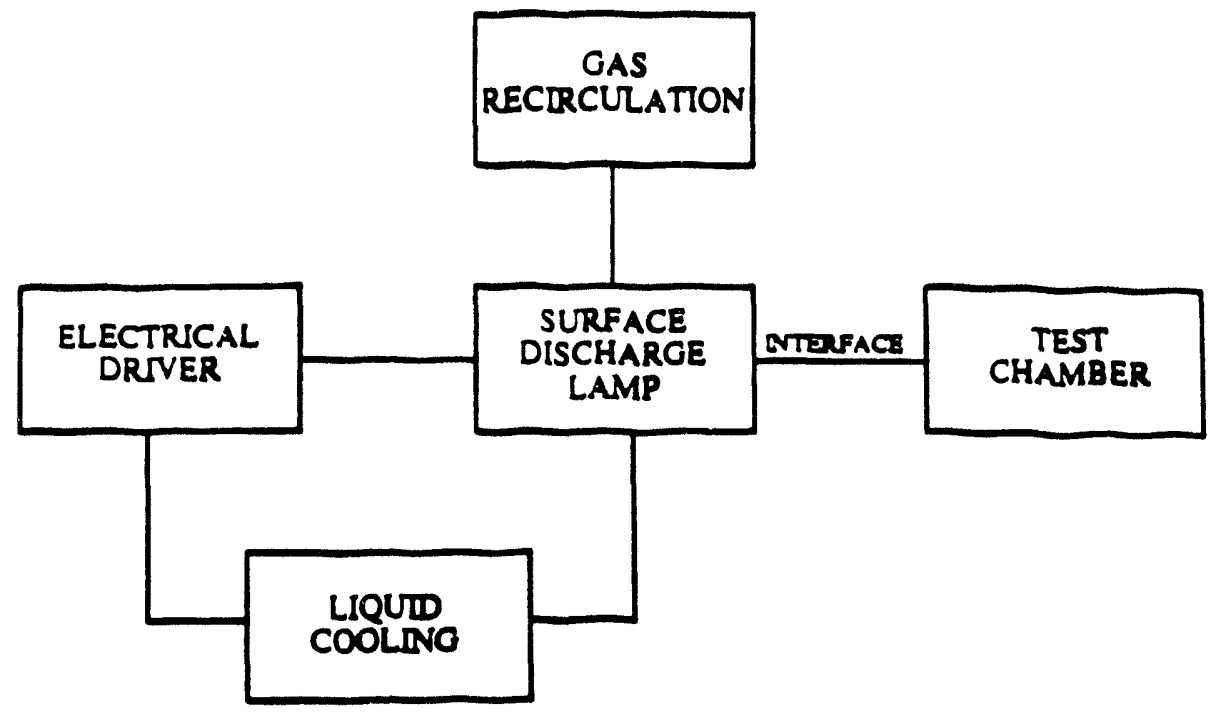

FIGURE 3.4 SURFACE DISCHARGE LAMP SYSTEM BLOCK DIAGRAM

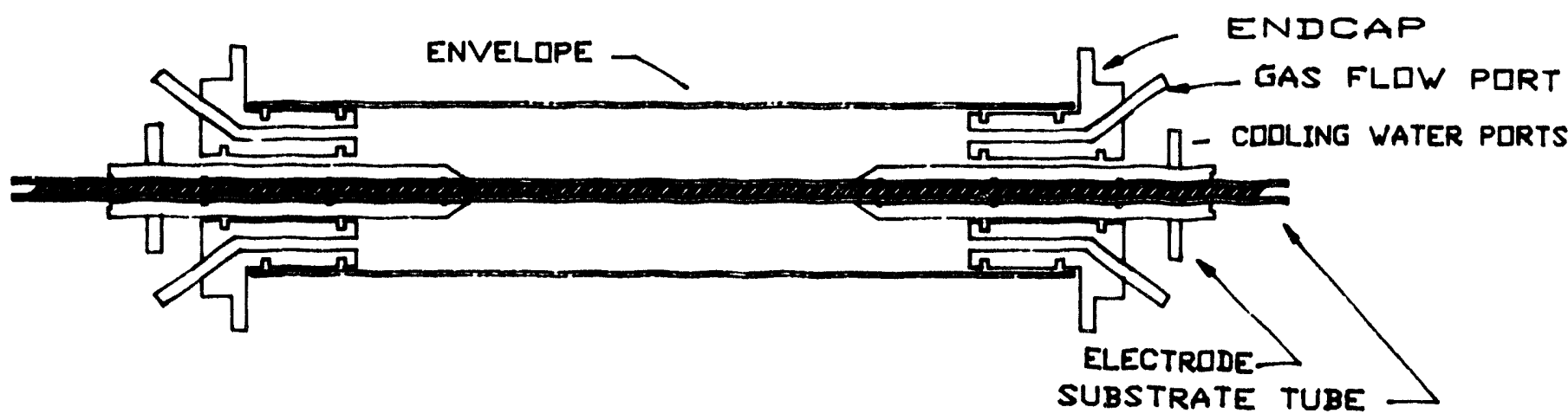

FIGURE 3.5 SURFACE DISCHARGE LAMP LAYOUT 


\subsubsection{Water and Gas Recirculation Systems}

The layout drawing for the new SD UV lamp in Figure 3.5 shows specific details for the gas flow, water ports, and electrodes. The number of cooling ports to the electrodes is larger by a factor of 3, and the flow rate by approximately a factor of 30 . The electrodes are approximately twice as massive, thereby providing better heat sinking.

The water circulation flow system cools the electrodes and the substrate of the SD lamp. The flow loop is arranged in series, with water flowing through the cathode first, then through the center of the lamp to the anode and back to a chiller that maintains the water at a selected temperature.

A system layout for the gas recirculation and filtering system is shown in Figure 3.6. The flow rate for this system removes the gas from the SD UV lamp between each shot for the maximum expected rep rate of five times per second. Expansion tanks allow the pressure from the discharge in the lamp to be relieved external to the lamp.

\subsubsection{Preliminary Integration with PSI Test Chamber}

The first step in integrating SD-2 with the PSI system was to integrate the lamp with the test chamber itself. Since the SD UV lamp has a different diameter than the standard lamp system used by PSI, custom end flanges were designed, fabricated and installed to mount the SD lamp to the test chamber.

\subsubsection{Laboratory Testing of UV Lamp System}

The fabrication of SD-2 led to laboratory testing. The SD UV lamp was assembled and the gas recirculation and liquid cooling systems checked out. Separately, the electrical driver was tested with a resistive load to verify design specifications. Next, the electrical driver was connected to the SD lamp mounted external to the test chamber. After observation of the plasma discharge visually for proper operation, the UV output was measured using an optical multichannel analyzer calibrated to an NBS traceable standard.

Next, the SD UV lamp was mounted in the test chamber and operated in a repetition rate mode. To simulate the field test, water was circulated through the laboratory reactor during SD operation. An optical fiber through the wall of the reactor served as a monitor of UV output from the lamp reaching the wall of the test chamber. The temperature of the gas and liquid flow 


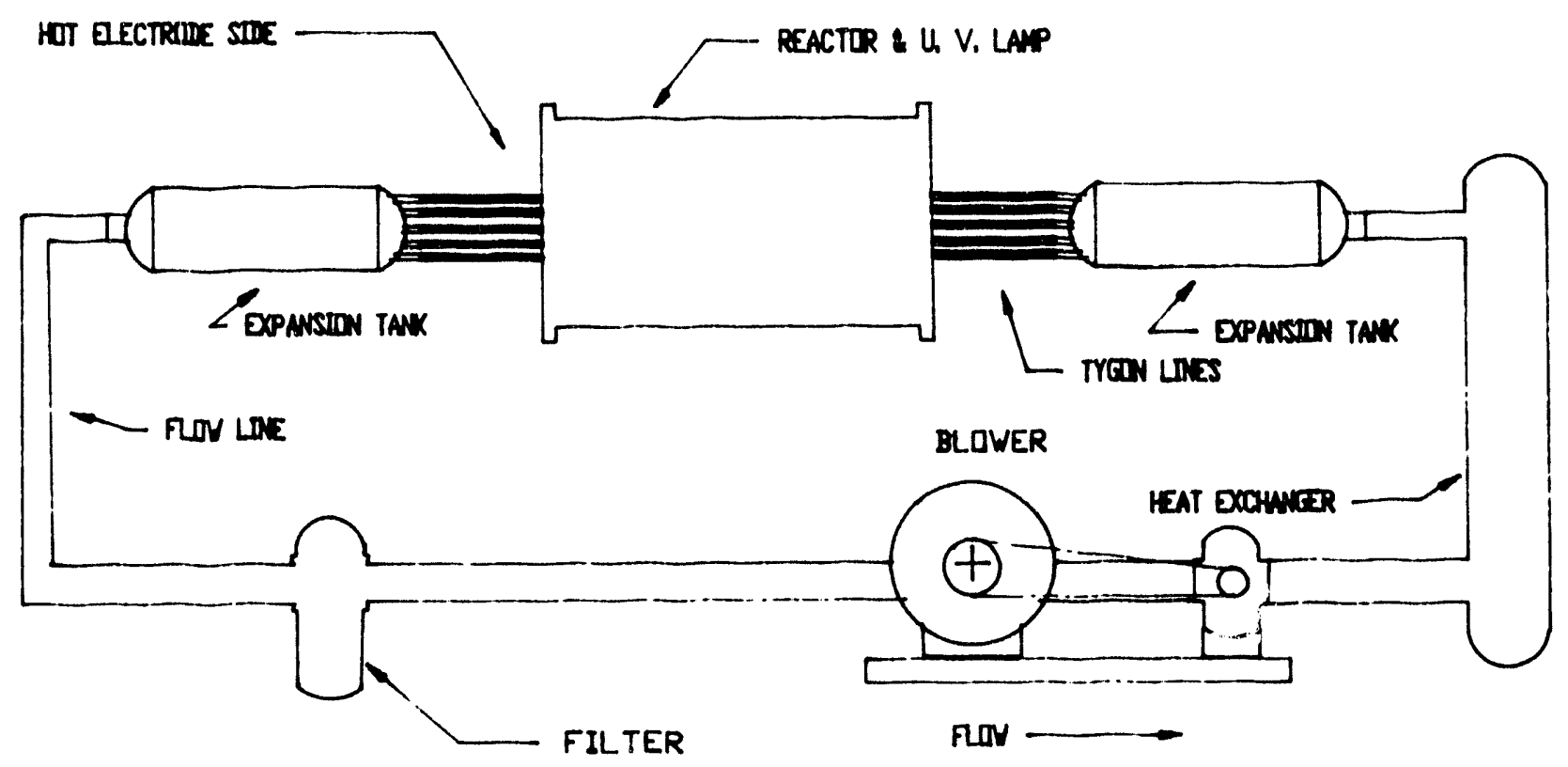

FIGURE 3.6 GAS RECIRCULATION SYSTEM LAYOUT 
was monitored at different locations and as a function of time. Initial tests were at low repetition rates and the repetition rate gradually increased to observe changes in thermal response and/or UV transmission. In the final laboratory test series, the SD lamp operated for three hours at an average power of 1,000 watts without degradation in UV output. Since the previous laboratory lamp operated at 200 watts for 15 minutes, the SD-2 lamp operated at five times higher power for twelve times longer, or an increase of a factor of sixty in total UV emitted. At the end of these tests, SD-2 was crated and transported to PSI for further integration and process testing.

\subsection{Integration and Process Testing at PSI}

SD-2 was integrated with a bench-scale perox-pure ${ }^{\mathrm{TM}}$ unit in PS!'s trailer field test demonstration unit. The closed-loop bench-scale unit consisted of a reactor, circulation pump, heat exchanger, recycle reservoir and $\mathrm{H}_{2} \mathrm{O}_{2}$ feed pump. The integration included installing the lamp and electrical driver pallets in the PSI demonstration trailer. Figure 3.7 shows the trailer, the electrical driver and lamp system pallets (located on the left-hand side of the trailer) as well as the perox-pure ${ }^{\mathrm{TM}}$ system (located on the right-hand side of the trailer). The perox-pure ${ }^{\mathrm{TM}}$ system in the trailer consisted of a perox-pure ${ }^{\mathrm{TM}}$ Model SSB-30R chemical oxidation unit and a Model PM-000 $\mathrm{X} \mathrm{H}_{2} \mathrm{O}_{2}$ Feed Module. Ancillary equipment used during the demonstration included a 300-gallon HDPE tank, a dual canister cartridge filter and a 50-gpm magnetic drive feed pump. Interconnecting piping and wiring was provided by PSI.

The perox-pure ${ }^{\mathrm{TM}}$ Model SSB-30R is a skid-mounted unit consisting of an oxidation chamber, a control panel, and an electrical enclosure. The oxidation chamber is made up of six horizontally-mounted sections. Each section consists of a cylindrical sub-chamber with a quartz tube mounted axially in the sub-chamber. One 5 kilowatt PSI UV lamp is positioned inside each quartz tube. Water to be treated enters the first subsection bottom of the oxidation chamber. The water is contained between the chamber wall and the quartz tube and does not contact the UV lamp. The water flows up through the chamber in a turbulent manner ensuring uniform mixing and contact with the UV light. The water exits the final subsection at the top of the oxidation chamber. The oxidation chamber is completely flooded during operation.

The control panel of the SSB-30R featured the process monitoring equipment, system control switches and alarms. The process monitoring equipment consisted of a flow meter, a flow accumulator, an hour meter, and ammeters for each UV lamp. The control switches 
included system startup, $\mathrm{H}_{2} \mathrm{O}_{2}$ feed pump startup, and individual UV lamp startup. A system power cutoff button was provided for emergency use. Alarm annunciators were provided in the control panel to inform the user of the cause of system shutdown, if one occurred.

Plumbing lines were hooked up and installed to prepare for batch recycle testing at DOE. Initial process testing at PSI showed that the system integration was complete. The trailer was then shipped to Oak Ridge, Tennessee. 


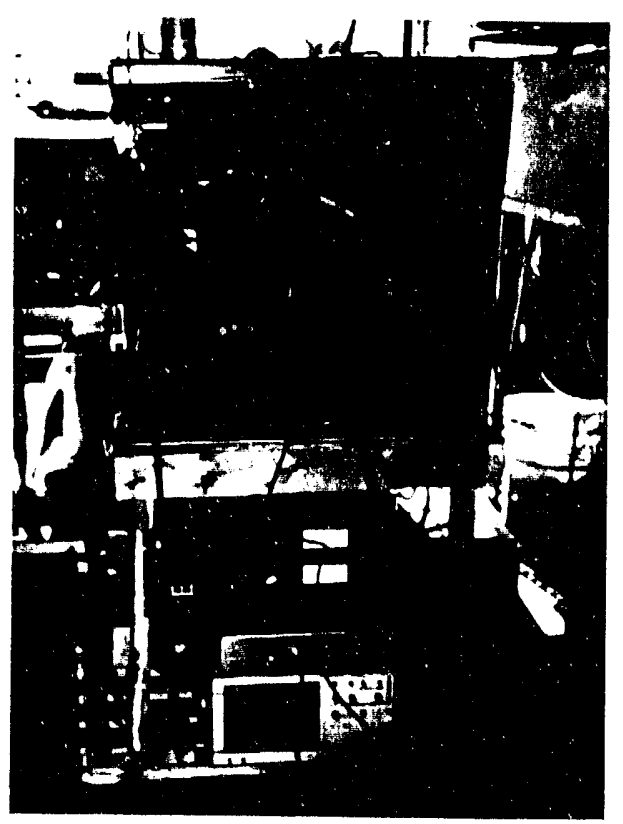

(a) View of SD System From Center of Trailer

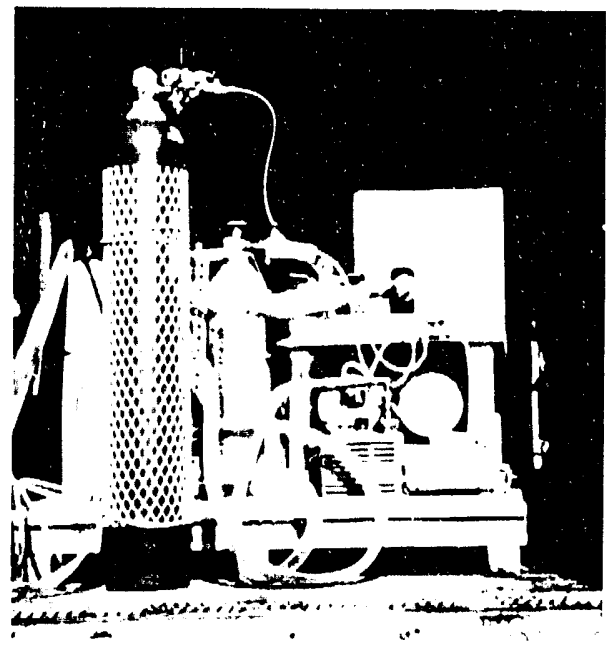

(b) View of SD System From End of Trailer

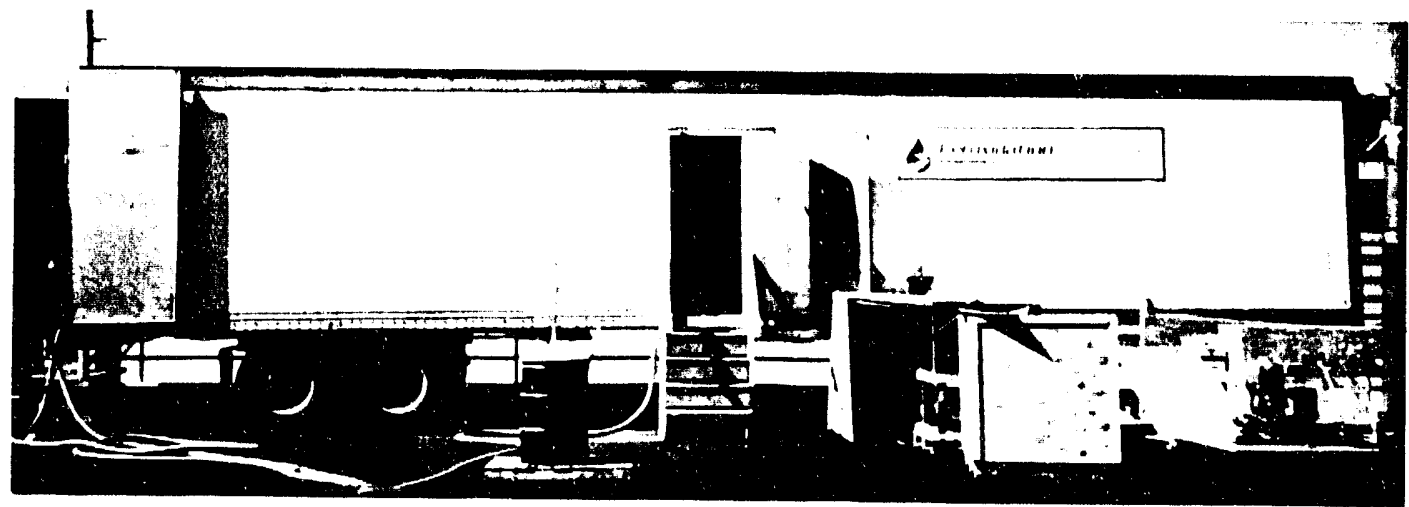

(c) Demonstration Trailer

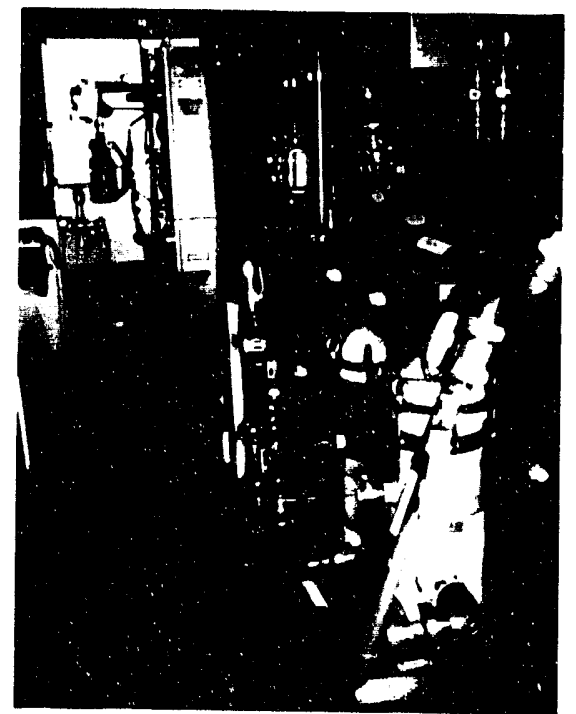

(d) View of Perox-Pure ${ }^{\mathrm{TM}}$ System From Center of Trailer

FIGURE 3.7 SD LAMP SYSTEM INTEGRATED WITH PSI DEMONSTRATION TRAILER 


\subsection{DEMONSTRATION TESTING}

\subsection{Summary}

The demonstration testing has three stages: setup, testing and data analysis. Martin Marietta Energy Systems (MMES) was responsible for the on-site setup which included permitting and other regulatory requirements, arranging for a pump system to transfer the water to the demonstration trailer site, arranging for electric power to be brought in and for handling the treated water. WJSA and PSI set up the test plan with the objective of demonstrating (1) the viability of the perox-pure ${ }^{\mathrm{TM}}$ water treatment system and (2) improvements in water remediation from using the SD UV lamp. The test plan includes variations in lamp operating conditions, $\mathrm{H}_{2} \mathrm{O}_{2}$ dosage, $\mathrm{pH}$ level, water pretreatment and catalyst dose. Phase I testing was with the perox-

pure $^{\mathrm{TM}}$ system alone whereas Phase II testing employed SD-2 alone and with the perox-pure ${ }^{\mathrm{TM}}$ system. The data analysis shows that the perox-pure ${ }^{\mathrm{TM}}$ system successfully treated the water from the SW-31 Spring and that for the major organic constituent, TCA, SD-2 had greater destruction rate.

\subsection{Test Objectives}

The general test objective was to evaluate the performance of UV/UV catalyzed peroxide water treatment remediation methods. Specifically, the objectives were to compare and contrast the existing commercial perox-pure ${ }^{\mathrm{TM}}$ system with SD-2. The test had two phases. The first was to optimize the operation of the perox-pure ${ }^{\mathrm{TM}}$ system by varying the peroxide concentration, $\mathrm{pH}$ level, catalyst dose and the pretreatment conditions. The second phase was to measure the water treatment capabilities of SD-2 over the range of lamp operating parameters available for these tests. This data both establishes the water remediation capabilities of SD-2 and provides a basis to extrapolate to water remediation capabilities with SD lamps after further engineering to increase lifetime and UV efficiency. 


\subsection{Test Description}

\subsubsection{Test Setup and Site Description}

The test site is on the eastern side of K-25. The SW-31 Spring is adjacent to the east side of Avenue D, approximately 200 feet west of the K-1070 classified burial ground. The SW-31 Spring flows from a PVC pipe which is embedded in the hillside below the classified burial ground. Flow rates vary depending on the weather, ranging from very little flow in dry weather up to six gallons per minute.

The location of the Spring is enclosed in an area requiring clearances and/or escorts. In order to facilitate our testing, the actual treatment site was located outside the fence. This allowed the workday to begin early and end late. The water from the Spring was pumped up the hillside approximately one block away to a holding tank at the demonstration site. Martin Marietta Energy Systems (MMES), in support of DOE, handled the site preparation as well as test support. MMES also arranged for power lines to be brought to the trailer site location to run the equipment. Almost all of the necessary equipment for running the demonstration was contained in the PSI demonstration trailer shown. In addition, tanker trucks were on-site to receive the treated water for transport over to the water treatment facility at the K-25 site for final discharge.

\subsubsection{Testing Procedures}

Testing with both the perox-pure ${ }^{\mathrm{TM}}$ and SD-2 systems was conducted with water collected from the SW-31 Spring. The SW-31 Spring emptied into a small sump. As needed, water spilling into the sump was pumped to the demonstration area approximately 100 yards away. The water was collected in a 330-gallon HDPE tote tank mounted inside a steel cage. The discharge from the water supply line was positioned inside the tank near the bottom to minimize volatilization losses of the VOCs. The tank was provided with a level control float to discontinue the water supply when 300 gallons had been collected. The water supply could also be manually controlled. In this way, water was collected in a sufficient volume on an as needed basis to insure that the untreated water was as fresh as possible for each day's testing.

The SW-31 Spring water contained 10 to $20 \mathrm{mg} / \mathrm{l}$ of ferrous iron in solution. Upon delivery to the demonstration collection tank, the iron began to oxidize due to contact with the atmosphere. The initially clear water turned turbid orange within a few hours of collection. 
Since iron removal was desired in most tests, the water was allowed to set for at least four hours before being used.

After oxidation of the iron was complete, water was pumped to the batch tank inside the PSI trailer as needed. When iron removal was desired, the water was circulated through the cartridge filter canister loaded with $3 \mu$ cartridges. In most instances, the iron concentration was reduced to less than $2 \mathrm{mg} / \mathrm{l}$ by running the water through the filters.

When desired, acid addition for $\mathrm{pH}$ adjustment was performed in the batch tank. Concentrated sulfuric acid was added to the batch in sufficient volume to produce the desired $\mathrm{pH}$ reduction. The water was circulated in the batch tank to facilitate mixing. Influent pH level controls the carbonate chemistry. Carbonate and bicarbonate ions scavenge hydroxyl radicals. Acid addition lowers the $\mathrm{pH}$ which shifts the carbonate equilibrium to carbonic acid, which reduces the completion for $\mathrm{OH}$. radical.

After completion of the desired pretreatment, the water was used in the perox-pure ${ }^{\mathrm{TM}}$ or SD-2 system tests. At the conclusion of the testing, the treated water was pumped to a 5,000gallon tanker truck for removal to a suitable location. Any untreated water was treated with the perox-pure $^{\mathrm{TM}}$ system for a time sufficient to significantly reduce the VOC concentrations before discharge to the tanker.

\subsubsection{Perox-Pure ${ }^{\mathrm{TM}}$ System}

The pretreated water was treated in batch mode with the perox-pure ${ }^{\mathrm{TM}}$ system to provide longer contact times than would be available in a flow-through mode of operation. The water was pumped at a rate of $40 \mathrm{gpm}$ from the batch tank into the oxidation chamber of the peroxpure $^{\mathrm{TM}}$ unit. $\mathrm{H}_{2} \mathrm{O}_{2}$ was added at a given rate into the water between the feed pump and the oxidation chamber. The effluent from the oxidation chamber was directed back into the batch tank, with a volume of about 75 gallons.

During each test, the UV lamps were warmed up for a period of 2 minutes to reach peak intensity. $\mathrm{H}_{2} \mathrm{O}_{2}$ addition was initiated immediately after the lamp warmup period. Water samples were collected from a sample valve downstream of the feed pump. The first sample, designated as 0 minutes of oxidation, was collected just before $\mathrm{H}_{2} \mathrm{O}_{2}$ addition was initiated. Subsequent samples were collected from the same point at specific time intervals. In certain tests, samples were collected at points across the oxidation chamber to provide a comparison of flow-through 
and batch mode VOC destruction rates. Throughout each test, the $\mathrm{H}_{2} \mathrm{O}_{2}$ addition rate was controlled to maintain the $\mathrm{H}_{2} \mathrm{O}_{2}$ concentration (see Section 3.2) at the influent of the oxidation chamber.

\subsubsection{SD-2 System}

Approximately 12 liters of pretreated water was transferred to the recycle reservoir of the SD-2 system before each test. The circulation pump was then turned on to fill the reactor and other components of the system. After presetting the SD-2 power supply to the desired setpoints, WJSA personnel initiated the discharge and slowly increased the discharge frequency. When the SD-2 UV source had been brought up to full power and frequency, a sample was collected from a valve upstream of the recycle reservoir. This sample was designated as 0 minutes of oxidation. Immediately, the desired dosage of $\mathrm{H}_{2} \mathrm{O}_{2}$ was added to the recycle reservoir, and the $\mathrm{H}_{2} \mathrm{O}_{2}$ feed pump was set at a rate to maintain the test $\mathrm{H}_{2} \mathrm{O}_{2}$ concentration at the influent to the reactor.

At predetermined time intervals, treated samples were collected via the sample valve. At the conclusion of each test, the UV source frequency was slowly decreased to "cool down" the system and prevent potential damage.

\subsubsection{Quality Assurance and Quality Control Test Methods}

During the demonstration period, samples of untreated and treated groundwater were analyzed for volatile organic contaminants (VOC's) by EPA Method 601 and EPA Method 602. These analyses provide data to determine the efficiency of the perox-pure ${ }^{\mathrm{TM}}$ and SD-based processes and their cost effectiveness. Additional samples were analyzed for non-volatile organics using EPA Method 625/8250 (Extractable Organics). The combination of EPA Methods 601,602 and 625 provide an accurate assessment of the success of the technology.

Peroxidation Systems, Inc. (PSI) regularly analyzes 300 to 500 samples per month for EPA Methods 601/602. Their laboratory in Tucson, Arizona has two complete gas chromatograph (GC) purge and trap systems using Hewlett-Packard 5890 chromatographs with O.I. analytical detectors and computerized data analysis. Also, some runs at the SW-31 Spring had a split

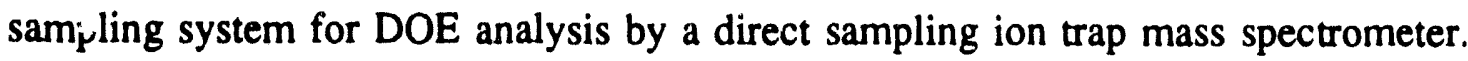




\subsection{Test Conditions}

\subsubsection{Phase I Tests with Perox-Pure ${ }^{\mathrm{TM}}$ System}

Sixteen (16) Phase I tests were performed on the SW-31 Spring water with the peroxpure $^{\mathrm{TM}}$ system. The test conditions are shown in Table 4.1. $\mathrm{H}_{2} \mathrm{O}_{2}$ concentration, influent pH,

\section{TABLE 4.1 PHASE I CONDITIONS FOR PEROX-PURE ${ }^{\mathrm{TM}}$ TESTING ON SW-31 SPRING WATER}

\begin{tabular}{|c|c|c|c|c|}
\hline Test & $\underset{\text { Maintained (mg/l) }}{\mathrm{H}_{2} \mathrm{O}_{2}{ }^{1}}$ & $\begin{array}{c}\text { Influent } \\
\text { pH }\end{array}$ & $\begin{array}{c}\text { Catalyst } \\
(\mathrm{mg} / \mathrm{I})\end{array}$ & $\begin{array}{c}\text { Water }^{2} \\
\text { Pretreated }\end{array}$ \\
\hline 1 & $20-100^{3}$ & 7 & 0 & Yes \\
\hline 2 & 20 & 7 & 0 & Yes \\
\hline 3 & 40 & 7 & 0 & Yes \\
\hline 4 & $100-400^{3}$ & 7 & 0 & Yes \\
\hline 5 & 50 & 6 & 0 & Yes \\
\hline 6 & 50 & 5 & 0 & Yes \\
\hline 7 & 50 & 3.5 & 0 & Yes \\
\hline 8 & 70 & 6.5 & 0 & Yes \\
\hline 9 & $200-600^{3}$ & 4 & 20 & Yes \\
\hline 10 & 50 & 3.5 & 20 & Yes \\
\hline 11 & 50 & 4 & 50 & Yes \\
\hline 12 & 50 & 4 & 100 & Yes \\
\hline 13 & 50 & 5 & 0 & No \\
\hline 14 & 50 & 6.5 & 0 & No \\
\hline 15 & 50 & 4.8 & 0 & Yes \\
\hline 16 & 50 & 3.2 & 20 & Yes \\
\hline
\end{tabular}


catalyst (proprietary to PSI) dosage, and water pretreatment (via filtration for iron remnval) were the process variables evaluated during the study. In Test 1,4 and 9 , the $\mathrm{H}_{2} \mathrm{O}_{2}$ concentration was not properly controlled. Hence, the conditions of these tests were repeated with the correct $\mathrm{H}_{2} \mathrm{O}_{2}$ concentrations in Tests 2,8 and 10 , respectively. Also, Tests 15 and 16 were performed to repeat the conditions of tests 6 and 10, respectively, in order to check the accuracy of the earlier tests across the oxidation chamber to provide flow-through treatment results.

\subsubsection{Phase II Tests with SD-2 System}

Nineteen (19) Phase II tests were performed on the SW-31 Spring water with the SD-2 system. The test conditions are shown in Table 4.2. $\mathrm{H}_{2} \mathrm{O}_{2}$ concentration, influent pH, catalyst dosage and water pretreatment were varied as in Phase I. Most of the tests were conducted with no $\mathrm{H}_{2} \mathrm{O}_{2}$ at a pH of 3 to 4 since these conditions were expected to be the most favorable. In addition, SD-2 lamp parameter variations included gas-type, charging voltage $\left(V_{c}\right)$, pulse repetition frequency (PRF), lamp envelope and substrate materials.

The tests included variations of all planned lamp system and water treatment parameters. However, technical problems caused some of the values of parameters to be changed. For instance, instead of an average power between 1,000 and 1,500 watts, the tests were between 500 and 1,000 watts due to glass envelope failure. The plan was to use Suprasil envelopes for most of the tests, but due to manufacturing flaws, Suprasil was used only for the first two tests. GE214 was used for the rest of the tests.

The failure of the high quality UV transmissive (Suprasil) lamp envelopes was unexpected. Minimum orders for Suprasil were expensive and had long delivery times, but since the manufacturer (Hareus-Amersil) located a small batch of Suprasil with acceptable dimensions, it was decided that, based on previous experience, ten Suprasil envelopes available should be more than sufficient. However, the purchased Suprasil material had greater variability in thickness and diameter than previous lots. Custom end caps were machined for each end of each envelope. Nevertheless, the variability in diameter and thickness resulted in breakage not expected at the beginning of the program. Consequently, once the available Suprasil material was consumed, the tests continued with standard quality UV lamp envelopes (GE-214). As discussed below, this reduced the organic destruction rate due to the increase in UV absorption in the standard quality envelope material. Thus, the analysis of the destruction rates in this program includes 
TABLE 4.2 PHASE II CONDITIONS FOR SD UV LAMP TESTING ON SW-31 SPRING WATER

\begin{tabular}{|c|c|c|c|c|c|c|c|c|}
\hline Test & $\begin{array}{c}\mathrm{H}_{2} \mathrm{O}_{2} \\
\mathrm{mg} /\end{array}$ & $\mathrm{pH}$ & $\begin{array}{c}\text { Pre- } \\
\text { treatment }\end{array}$ & $\begin{array}{c}\text { Catalyst } \\
\mathrm{mg} /\end{array}$ & $\begin{array}{c}\text { Power } \\
(\mathrm{W})\end{array}$ & $\mathrm{Gas}$ & $\begin{array}{c}\mathrm{V}_{\mathrm{c}} \\
(\mathrm{KV})\end{array}$ & $\begin{array}{c}\mathrm{PRF} \\
(\mathrm{Hz})\end{array}$ \\
\hline $1 \mathrm{~A}^{\mathrm{a}}$ & 0 & 3.8 & Yes & 0 & 1000 & $\mathrm{Kr}$ & 18.4 & 2.6 \\
\hline $2 \mathrm{~A}^{\mathrm{a}}$ & 0 & 3.3 & Yes & 0 & 1500 & $\mathrm{Kr}$ & 18.4 & 3.8 \\
\hline $3 \mathrm{~A}$ & 0 & 3.3 & Yes & 0 & 500 & $\mathrm{Argon}$ & 17.3 & 1.5 \\
\hline $4 \mathrm{~A}$ & 0 & 3.3 & Yes & 0 & 500 & $\mathrm{Kr}$ & 17.3 & 1.5 \\
\hline $5 \mathrm{~A}$ & 70 & 3.3 & Yes & 0 & 500 & $\mathrm{Kr}$ & 17.3 & 1.5 \\
\hline $6 \mathrm{~A}$ & 50 & 3.3 & Yes & 0 & 500 & $\mathrm{Kr}$ & 17.3 & 1.5 \\
\hline $7 \mathrm{~A}$ & 25 & 3.3 & Yes & 0 & 500 & $\mathrm{Kr}$ & 17.3 & 1.5 \\
\hline $8 \mathrm{~A}$ & 0 & 6.7 & Yes & 0 & 500 & $\mathrm{Kr}$ & 17.3 & 1.5 \\
\hline $9 \mathrm{~A}$ & 0 & 4.0 & Yes & 20 & 500 & $\mathrm{Kr}$ & 17.3 & 1.5 \\
\hline $10 \mathrm{~A}$ & 0 & 4.0 & Yes & 50 & 500 & $\mathrm{Kr}$ & 17.3 & 1.5 \\
\hline $11 \mathrm{~A}^{*}$ & 5 & 3.6 & No & 0 & 500 & $\mathrm{Kr}$ & 17.3 & 1.5 \\
\hline $12 \mathrm{~A}^{\Delta}$ & 0 & 3.0 & Yes & 0 & 1000 & $\mathrm{Kr}$ & 17.3 & 3.0 \\
\hline $13 \mathrm{~A}^{\Delta}$ & 0 & 3.0 & Yes & 0 & 500 & $\mathrm{Kr}$ & 17.3 & 1.5 \\
\hline $14 \mathrm{~A}$ & 0 & 6.3 & No & 0 & 500 & $\mathrm{Kr}$ & 17.3 & 1.5 \\
\hline $15 \mathrm{~A}$ & 0 & 3.0 & Yes & 0 & 500 & $\mathrm{Kr}$ & 17.3 & 1.5 \\
\hline $16 \mathrm{~A}$ & 0 & 3.0 & Yes & 0 & 500 & $\mathrm{Xe}$ & 17.3 & 1.5 \\
\hline $17 \mathrm{~A}$ & 0 & 3.0 & Yes & 0 & 1000 & $\mathrm{Xe}$ & 17.3 & 3.0 \\
\hline $18 \mathrm{~A}$ & 0 & 3.0 & Yes & 0 & 600 & $\mathrm{Xe}$ & 18.9 & 1.5 \\
\hline $19 \mathrm{~A}$ & 0 & 3.0 & Yes & 0 & 700 & $\mathrm{Xe}$ & 20.5 & 1.5 \\
\hline
\end{tabular}

Suprasil envelope

$\triangle$ GE214 substrate (replaces Suprasil substrate)

*In series, with PSI unit first

${ }^{O_{F}}$ Filter iron before treatment 
extrapolations based on comparison of tests with and without Suprasil to estimate the destruction rates of a system with Suprasil.

The UV emission efficiency increases with atomic weight of the gas. That is, the UV output from xenon is greater than krypton is greater than argon. Thus, the organic destruction rate is expected to be greatest with xenon, less with krypton and least with argon.

\subsection{Test Results}

The analytical reports for all tests are compiled in Appendix VII for completeness. Summary tables for review and analysis are in Appendices IV and V, and are discussed below. Discussion of an apparent anomaly in the vinyl chloride data is discussed in Appendix VII.

\subsubsection{Water Characterization}

On October 12,1993, during the initiation of the testing with the SD-2 system, 2 liters of SW-31 Spring water were obtained from the collection tank and shipped to the PSI Testing Laboratory in Tucson, Arizona. The water was characterized for a variety of water quality parameters of interest to the perox-pure ${ }^{\mathrm{TM}}$ and SD-2 processes. The characterization results are shown in Table 4.3. The iron and TSS settled rapidly leaving a clear, colorless solution with a residual iron (i.e., after oxidized iron settled) of $0.2 \mathrm{mg} /$.

\subsubsection{Phase I Tests with Perox-Pure ${ }^{\mathrm{TM}}$ System}

The perox-pure ${ }^{\mathrm{TM}}$ testing results are shown in Appendix IV. Chloroethane, 1,1dichloroethane and 1,1,1-trichloroethane were the primary contaminants detected. In some tests, trichlorofluoromethane was also detected at a significant concentration. Low $\mu \mathrm{g} / \mathrm{l}$ levels of several other halogenated and aromatic VOCs were also detected.

The variation in influent VOC concentration ( 0 minutes of oxidation) is attributed to the different holding times for each batch of spring water in the collection tank and the change in ambient temperature from day to day. Initial batches yielded a total VOC concentration of approximately $1 \mathrm{mg} /$. In one test, the total VOC concentration was more than $5 \mathrm{mg} /$. Typically, the total influent VOC concentration was between 1 and $2 \mathrm{mg} /$. 


\section{TABLE 4.3 CHARACTERIZATION RESULTS FOR SW-3I SPRING WATER}

\begin{tabular}{|l|c|}
\hline \multicolumn{1}{|c|}{ Parameter } & \multicolumn{1}{c|}{ Value } \\
\hline Visual Appearance & Turbid Orange \\
\hline $\mathrm{pH}$ & 6.9 \\
\hline TDS (mg/) & 350 \\
\hline Turbidity (FTU) & 21 \\
\hline Total Iron (mg/) & 16 \\
\hline Chloride (mg/) & 58 \\
\hline COD (mg/) & $13^{1}$ \\
\hline TOC (mg/) & 5 \\
\hline Alkalinity (mg/l) & 250 \\
\hline TSS (mg/) & 50 \\
\hline NO ${ }_{3}$ as N (mg/) & $<0.1$ \\
\hline Hardness (mg/) & 300 \\
\hline 'Valve obtained after solids allowed to settle \\
\hline
\end{tabular}

During Tests 3, 5 and 13, additional samples were collected for analysis of base neutral/acid semivolatile organic compounds and none was detected (see Appendices IV and VII). No semivolatile compounds were detected, either in the influent or treated samples.

\subsubsection{Phase II Tests With SD-2 System}

The treatment results obtained with the SD-2 system are shown in Appendix V. As was the case with the perox-pure ${ }^{\mathrm{TM}}$ system, 1,1-dichloroethane and 1,1,1-trichloroethane were two of the primary compounds detected. However, chloroethane was only detected in Test 1 and 2. Subsequent tests, conducted several weeks after Tests 1 and 2, contained methylene chloride instead. Low $\mu \mathrm{g} / \mathrm{l}$ levels of numerous other halogenated and aromatic VOCs were also detected.

The influent VOC concentration typically varied between 0.5 and $1 \mathrm{mg} / \mathrm{l}$. This is somewhat lower than the concentration range detected during the perox-pure ${ }^{\mathrm{TM}}$ testing. This may 
be attributed to the additional handling of the water in which water was transferred by bucket from the perox-pure ${ }^{\mathrm{TM}}$ feed to the SD batch tank required for the SD-2 tests.

\subsection{Data Analysis}

A performance analysis was conducted to correlate the organic destruction rates (see Appendix III for definition) with the test variables. As evident in the test results, TCA and DCA were the two organic contaminants with (by far) the highest initial concentrations. This was fortunate since it allows a comparative evaluation of the perox-pure ${ }^{\mathrm{TM}}$ and SD-2 systems. As discussed above, the perox-pure ${ }^{\mathrm{TM}}$ system is optimized for organic destruction via $\mathrm{OH}$ - oxidation (in terms of power, spectrum, processing chamber and $\mathrm{H}_{2} \mathrm{O}_{2}$ addition). Since DCA is responsive to $\mathrm{OH}$, its destruction rate is a valid benchmark for perox-pure ${ }^{\mathrm{TM}}$ processing. The SD-2 system, on the other hand, due to its short wavelength UV spectrum, should be advantageous for destroying organics which are more receptive to direct UV photolysis. TCA is relatively unresponsive to $\mathrm{OH} \cdot$ treatment, and is most responsive to UV photolysis. Thus, destruction rates of TCA should be sensitive to changes in SD-2 UV output and be processed relatively slowly with the perox-pure ${ }^{\mathrm{TM}}$ system. The ability of SD-2 to destroy TCA more quickly than the peroxpure $^{\mathrm{TM}}$ system, then, is the primary measure in these tests of the improvement afforded by SD-2. The summary conclusions for the perox-pure ${ }^{\mathrm{TM}}$ system, SD-2 system and a comparison are in Tables 4.4 and 4.5.

The results for the perox-pure ${ }^{\mathrm{TM}}$ system are straightforward and generally as expected. The VOC destruction rate increased with increasing peroxide concentration, with about 50 milligrams per liter being the nominal best value since further additions of peroxide to 70 milligrams per liter increased the destruction rate only slightly. A careful examination showed that the DCA destruction rate was strongly affected by the peroxide whereas the TCA destruction rate increased very slightly with increased peroxide concentration. This is to be expected since, as explained above, DCA is responsive to $\mathrm{OH}$. oxidation whereas TCA is not. The $\mathrm{pH}$ value had very little affect on the VOC destruction rates in general. A careful look at the data showed that the secondary constituents, such as Benzene and Xylene, did benefit greatly from reduced $\mathrm{pH}$. The pretreatment to filter out the iron significantly increased the VOC destruction rate by greater than a factor of 2. The catalyst (proprietary to PSI) provided no overall advantage. 
TABLE 4.4 SUMMARY CONCLUSIONS FOR PEROX-PURE ${ }^{\text {TM }}$ TESTING

\begin{tabular}{|l|l|}
\hline \multicolumn{1}{|c|}{ Variable } & \multicolumn{1}{c|}{ Conclusion } \\
\hline $\mathrm{H}_{2} \mathrm{O}_{2}$ Concentration & $\begin{array}{l}\text { Increasing } \mathrm{H}_{2} \mathrm{O}_{2} \text { concentration increased VOC } \\
\text { destruction rate, with } 50 \mathrm{mg} / \text {, the nominal "best" value }\end{array}$ \\
\hline $\mathrm{pH}$ & $\begin{array}{l}\text { A reduction in } \mathrm{pH} \text { from } 6 \text { to } 3 \text { slightly increased VOC } \\
\text { destruction rates }\end{array}$ \\
\hline Pretreatment & $\begin{array}{l}\text { Iron filtration significantly increases VOC destruction } \\
\text { rates }\end{array}$ \\
\hline Catalyst & The catalyst provided no overall advantage \\
\hline
\end{tabular}

TABLE 4.5 SUMMARY CONCLUSIONS FOR SD LAMP TESTING

\begin{tabular}{||l|l||}
\hline \multicolumn{1}{|c|}{ Variable } & \multicolumn{1}{c|}{ Conclusion } \\
\hline $\mathrm{H}_{2} \mathrm{O}_{2}$ Concentration & $\begin{array}{l}\text { The TCA destruction rate increased slightly with a } \\
\text { small dose of } \mathrm{H}_{2} \mathrm{O}_{2}\end{array}$ \\
\hline $\mathrm{pH}$ & $\begin{array}{l}\text { Lowering the } \mathrm{pH} \text { from greater than } 6 \text { to about } 3 \\
\text { increased the VOC destruction rate by greater than } \\
\text { a factor of 2 }\end{array}$ \\
\hline Pretreatment & $\begin{array}{l}\text { Iron filtration generally did not increase VOC } \\
\text { destruction rates }\end{array}$ \\
\hline Catalyst & The catalyst provided no benefit \\
\hline Envelope Material & $\begin{array}{l}\text { With Suprasil, TCA destruction rate was 2.7 times } \\
\text { greater than with GE-214 }\end{array}$ \\
\hline Xenon Versus Krypton & $\begin{array}{l}\text { The destruction rate of TCA was about 1.6 times } \\
\text { greater with Xenon than with Krypton }\end{array}$ \\
\hline Average Power & $\begin{array}{l}\text { The destruction rate of VOCs scales linearly with } \\
\text { power within experimental error }\end{array}$ \\
\hline
\end{tabular}


For SD-2 the increase in destruction rate with increase in $\mathrm{H}_{2} \mathrm{O}_{2}$ concentration was small with SD-2 since its spectrum is more attuned to photolysis. The TCA destruction rate did increase slightly (i.e., by about a factor of 1.17) with a small dose of $\mathrm{H}_{2} \mathrm{O}_{2}$. The effect on DCA, as expected, was much greater, with an increased destruction rate of 3.3 with the addition of 25 milligrams per liter of $\mathrm{H}_{2} \mathrm{O}_{2}$. Lowering the $\mathrm{pH}$ from greater than 6 to about 3 increased destruction rates by greater than a factor of 2 for both DCA and TCA in the SD-2 test. Unlike the perox-pure ${ }^{\mathrm{TM}}$ system, iron filtration did not increase the organic destruction rates. Also, the catalyst provided no benefit.

The most interesting SD-2 results were with the envelope material, the average power and the use of Xenon versus Krypton as a discharge gas. Although it was unfortunate that breakage of the Supersil resulted in most of the tests being conducted with a GE-214 envelope, the contrast between the two does corroborate the supposition that the SD-2 system operates primarily by photolysis. The difference between Suprasil and GE-214 is that Suprasil transmits UV light down to $180 \mathrm{~nm}$ whereas GE-214 begins to cut off at $240 \mathrm{~nm}$. The photolysis of TCA (and other similar organics) requires UV light below $240 \mathrm{~nm}$. Consequently, the destruction rate of TCA should be sensitive to whether Suprasil or GE-214 is used whereas DCA, which is not based primarily on UV photolysis, should be relatively insensitive to the envelope material. This is seen in the data where the change from GE-214 to Suprasil envelope increases the TCA destruction rate by a factor of 2.7 and the DCA destruction rate increase; by only 1.09. Also, within experimental error, the destruction rates of TCA and DCA are proportional to the SD-2 operating power. Finally, as expected, the replacement of Krypton with Xenon increased the destruction rate of both TCA and DCA by similar factors (1.6 for TCA and 1.9 for DCA).

A summary comparison of the two systems is as follows: As expected, the SD-2 system operated best with little or no peroxide whereas the perox-pure ${ }^{\mathrm{TM}}$ system optimized at about 50 milligrams per liter of $\mathrm{H}_{2} \mathrm{O}_{2}$. The reduction in $\mathrm{pH}$ benefitted $\mathrm{SD}$ destruction rates strongly (i.e., by in the order of a factor of 2 ) whereas the perox-pure ${ }^{\mathrm{TM}}$ system benefitted only slightly (except for secondary organic compounds). The catalyst had no net effect for either system. Pretreatment benefitted the perox-pure ${ }^{\mathrm{TM}}$ system, but not the SD-2 system. Perhaps the most important conclusion is in the comparison of the TCA destruction rates for the two systems. Due to the breakage problem with the Suprasil, the destruction rate for the combination of Suprasil and xenon, which would be best, had to be inferred from a combination of tests. This is 
explained in detail below. Based on this inference, the SD-2 destruction rate for TCA was more than double the rate for the perox-pure ${ }^{\mathrm{TM}}$ system. The actual best measured SD-2 destruction rate occurred in the first test with Suprasil in which the SD destruction rate was 1.35 times greater than the best perox-pure ${ }^{\mathrm{TM}}$ destruction rate. This test was run without the benefit of increased destruction rate from xenon and without the benefit of increased destruction rate of a small quantity of peroxide. In any case, the primary objective of the demonstration to demonstrate the improved destruction rate from the SD-2 system by means of photolysis was corroborated experimentally. Also since, as discussed above, the best UV efficiency demonstrated in the lab is about three times that of SD-2, further engineering improvements have the potential to increase organic destruction rates.

Finally, by operating the perox-pure ${ }^{\mathrm{TM}}$ and then SD-2 systems in series (in tests without pretreatment) both the TCA and DCA destruction rates benefitted with TCA destruction rate increasing by a 1.4 factor and the DCA destruction rate increasing by a 3.8 factor. This result indicates that a UV-based water treatment system optimized for a broad range of organics is likely to include test conditions which favor $\mathrm{OH}$ - oxidation in one portion of the treatment and photolytic oxidation at another point in the processing. Details of the test results are discussed further in the next paragraphs.

\subsubsection{Perox-Pure ${ }^{\mathrm{TM}}$ System Data Analysis}

The effects of variation in $\mathrm{H}_{2} \mathrm{O}_{2}$ dosage were demonstrated in Tests 2,3 and 8. The $\mathrm{H}_{2} \mathrm{O}_{2}$ concentration maintained in solution was varied from 20 to $70 \mathrm{mg} /$. All of these tests were conducted at a neutral $\mathrm{pH}$, without catalyst, using water that had been pretreated for iron removal. Increasing the $\mathrm{H}_{2} \mathrm{O}_{2}$ concentration from $20 \mathrm{mg} /$ (Test 2) to $40 \mathrm{mg} /$ (Test 3) resulted in substantial improvement in the rate of total VOC destruction. Only a moderate rate increase was achieved by increasing the $\mathrm{H}_{2} \mathrm{O}_{2}$ concentration to $70 \mathrm{mg} / \mathrm{l}$ (Test 8 ). The unsaturated and aromatic VOCs were all destroyed to near or below the analytical detection limit in the first treated sample.

The effects of influent $\mathrm{pH}$ were evaluated in Tests 3,5,6 and 7. The influent $\mathrm{pH}$ was varied between 3.5 and 7 in these tests. All of these tests were conducted with a $\mathrm{H}_{2} \mathrm{O}_{2}$ concentration of 40 to $50 \mathrm{mg} /$, without catalyst, using pretreated water. The treatment results were comparable in Tests $3(\mathrm{pH} 7), 6(\mathrm{pH} \mathrm{5)}$ and $7(\mathrm{pH} \mathrm{3.5)}$ indicating that $\mathrm{pH}$ reduction via acid 
addition does not significantly benefit the VOC destruction rates. While the results for Test 5 (pH 6) appear to be significantly better than the other tests, we believe this is an artifact of the higher influent VOC concentrations, particularly the unsaturated and aromatic VOCs, which are rapidly destroyed to below the detection limit at any $\mathrm{pH}$.

The benefits of catalyst addition were evaluated in Tests 7,11,12 and 16. The catalyst dosage was varied from 0 to $100 \mathrm{mg} /$. During each test, the $\mathrm{H}_{2} \mathrm{O}_{2}$ concentration was maintained at $50 \mathrm{mg} / 1$ with an influent $\mathrm{pH}$ of 3.5 to 4 using pretreated water. The addition of catalyst did not increase the total VOC destruction rate. At the higher dosages, catalyst addition negatively impacted the rate of VOC destruction.

The effects of pretreatment at high and low $\mathrm{pH}$ were demonstrated in Tests 3, 6, 13 and 14. These tests were conducted with a $\mathrm{H}_{2} \mathrm{O}_{2}$ concentration of 40 to $50 \mathrm{mg} / \mathrm{l}$ and no catalyst addition. Pretreatment of the groundwater significantly increased the rate of VOC destruction at either $\mathrm{pH}$. This is expected because the entrained iron interferes with UV penetration through the water. Lowering of the initial $\mathrm{pH}$ in Test 13 improved the total VOC destruction rate when the iron was not removed.

\subsubsection{SD-2 System Data Analysis}

Table 4.6 shows the destruction rates (see Appendix III for definition) for TCA and DCA in the SD-2 (Phase II) test series. These rates are "normalized" according to the power per unit volume in order to make a one-to-one comparison with perox-pure ${ }^{\mathrm{TM}}$ rates. This procedure is valid over the parameter range tested here based on PSI proprietary data showing that organic destruction rates are approximately proportional to the ratio of electrical power to processing chamber volume. The effects of $\mathrm{H}_{2} \mathrm{O}_{2}$ concentration on destruction rates were demonstrated in Tests 4A through 7A. $\mathrm{H}_{2} \mathrm{O}_{2}$ concentration was varied from 0 to $70 \mathrm{mg} / \mathrm{l}$ while all other parameters were held constant. The TCA and DCA destruction rates were increased by factors of 1.2 and 3.3, respectively, with the addition of $25 \mathrm{mg} / \mathrm{l}$ of $\mathrm{H}_{2} \mathrm{O}_{2}$ (Test 7A). Additional $\mathrm{H}_{2} \mathrm{O}_{2}$ did not provide further improvement, and in fact, decreased the TCA destruction rate. Note that the increase in DCA destruction rate $(\times 3.3)$ with the addition of $\mathrm{H}_{2} \mathrm{O}_{2}$ is much greater than for TCA ( $\times 1.2)$, supporting our earlier assertion that DCA is much more receptive to $\mathrm{OH} \cdot$ oxidation than TCA. 
TABLE 4.6 DESTRUCTION RATES FOR SD-2 TESTING ON SW-31 SPRING WATER

\begin{tabular}{|c|c|c|c|c|c|c|c|c|}
\hline Test & $\begin{array}{c}\mathrm{H}_{2} \mathrm{O}_{2} \\
\mathrm{mg} / \mathrm{l}\end{array}$ & $\mathrm{pH}$ & $\begin{array}{c}\text { Pre- } \\
\text { treatment }\end{array}$ & $\begin{array}{c}\text { Catalyst } \\
\mathrm{mg} / \mathrm{l}\end{array}$ & $\begin{array}{l}\text { Power } \\
\text { (W) }\end{array}$ & Gas & $\begin{array}{c}\text { DCA } \\
\text { Rate } \\
\left(\mathrm{min}^{-1}\right)\end{array}$ & $\begin{array}{c}\text { TCA } \\
\text { Rate } \\
\left(\mathrm{min}^{-1}\right)\end{array}$ \\
\hline $1 A^{a}$ & 0 & 3.8 & Yes & 0 & 1000 & $\mathrm{Kr}$ & 0.76 & 2.0 \\
\hline $2 A^{a}$ & 0 & 3.3 & Yes & 0 & 1500 & $\mathrm{Kr}$ & 0.54 & 1.8 \\
\hline $3 A$ & 0 & 3.3 & Yes & 0 & 500 & Argon & 0.59 & 0.77 \\
\hline $4 \mathrm{~A}$ & 0 & 3.3 & Yes & 0 & 500 & $\mathrm{Kr}$ & 0.70 & 0.75 \\
\hline $5 \mathrm{~A}$ & 70 & 3.3 & Yes & 0 & 500 & $\mathrm{Kr}$ & 2.4 & 0.76 \\
\hline $6 \mathrm{~A}$ & 50 & 3.3 & Yes & 0 & 500 & $\mathrm{Kr}$ & 2.3 & 0.78 \\
\hline $7 \mathrm{~A}$ & 25 & 3.3 & Yes & 0 & 500 & $\mathrm{Kr}$ & 2.3 & 0.89 \\
\hline $8 \mathrm{~A}$ & 0 & 6.7 & Yes & 0 & 500 & $\mathrm{Kr}$ & 0.16 & 0.25 \\
\hline $9 \mathrm{~A}$ & 0 & 4.0 & Yes & 20 & 500 & $\mathrm{Kr}$ & 0.34 & 0.45 \\
\hline $10 \mathrm{~A}$ & 0 & 4.0 & Yes & 50 & 500 & $\mathrm{Kr}$ & 0.39 & 0.47 \\
\hline $11 A^{*}$ & 5 & 3.6 & No & 0 & 500 & $\mathrm{Kr}$ & 0.98 & 0.50 \\
\hline $12 \mathrm{~A}^{\Delta}$ & 0 & 3.0 & Yes & 0 & 1000 & $\mathrm{Kr}$ & 0.38 & 0.31 \\
\hline $13 \mathrm{~A}^{\Delta}$ & 0 & 3.0 & Yes & 0 & 500 & $\mathrm{Kr}$ & 0.54 & 0.40 \\
\hline $14 \mathrm{~A}$ & 0 & 6.3 & No & 0 & 500 & $\mathrm{Kr}$ & 0.26 & 0.36 \\
\hline $15 \mathrm{~A}$ & 0 & 3.0 & Yes & 0 & 500 & $\mathrm{Kr}$ & 0.60 & 0.52 \\
\hline $16 \mathrm{~A}$ & 0 & 3.0 & Yes & 0 & 500 & $\mathrm{Xe}$ & 1.12 & 0.83 \\
\hline $17 \mathrm{~A}$ & 0 & 3.0 & Yes & 0 & 1000 & $\mathrm{Xe}$ & 0.66 & 0.62 \\
\hline $18 \mathrm{~A}$ & 0 & 3.0 & Yes & 0 & 600 & $\mathrm{Xe}$ & 0.81 & 0.65 \\
\hline $19 \mathrm{~A}$ & 0 & 3.0 & Yes & 0 & 700 & $\mathrm{Xe}$ & 0.71 & 0.58 \\
\hline
\end{tabular}

Suprasil envelope

${ }^{\Delta}$ GE214 substrate (replaces Suprasil substrate)

*In series, with PSI unit first

${ }^{\diamond}$ Filter iron before treatment 
The effects of $\mathrm{pH}$ adjustment were evaluated in Tests $4 \mathrm{~A}$ and $8 \mathrm{~A}$. The VOC destruction rates were significantly slower at neutral $\mathrm{pH}$ (Test $8 \mathrm{~A}$ ) than at a lower $\mathrm{pH}$ (Test $4 \mathrm{~A}$ ). Lowering the $\mathrm{pH}$ from 6.7 to 3.3 increased the TCA destruction rate by 3 and the DCA by greater than 4 .

Catalyst addition in Tests $9 \mathrm{~A}$ and $10 \mathrm{~A}, 20$ and $50 \mathrm{mg} /$, respectively, resulted in slower VOC destruction than treatment without catalyst (Test 4A).

The VOC destruction rate results were slightly better for Test $8 \mathrm{~A}$, conducted with iron removal, than for Test 14A, conducted without iron removal. Pretreatment increased TCA and DCA destruction rates by 1.5 and 1.4 , respectively.

The use of a Suprasil rather than a GE-214 envelope (tube) produced substantial improvement in the rate of VOC destruction because of the higher short UV transparency in Suprasil. The increase in TCA rate (2.7 factor) was much greater than for DCA (1.1) due to the sensitivity of TCA to UV light below $240 \mathrm{~nm}$, where GE-214 transmission decreases.

The variation of VOC destruction rates with power is complicated. As seen in the next section, SD-2 produced intermediates, and net (effective) destruction rates varied with time during a given test. This effect is expected to vary with the type and concentration of organic contaminants. Further testing would be needed to quantify these effects for the SW-31. For this data analysis, we use the average destruction rate over the entire run. With this assumption, the VOC destruction rates vary approximately linearly with SD-2 power.

\subsubsection{Continuous Water Monitoring Results}

For several of the individual tests, personnel from Oak Ridge National Laboratories (ORNL) incorporated a continuous water monitoring system. This is a direct sample ion trap mass spectrometer (DSITMS) in which the concentration levels of organic constituents are monitored in real time and continuously during the test. The data package provided by ORNL is included in Appendix VI. A data example of the organic concentration levels (in terms of ion abundance) versus time is given in Figure 4.1. The initial delay of over a minute in concentration decrease corresponds approximately to the recirculation time of the water in the treatment system. As expected, the destruction rate of 1,1,1-TCA is the slowest with most of the other organics, i.e., xylene, benzene and toluene, falling off very rapidly. Notice that the falloff in 1,1-DCA concentration doesn't begin until after $200 \mathrm{sec}$. The exact reason for this is uncertain -- one possibility is that photolysis of 1,1,1-TCA produces 1,1-DCA. The production of MEK 


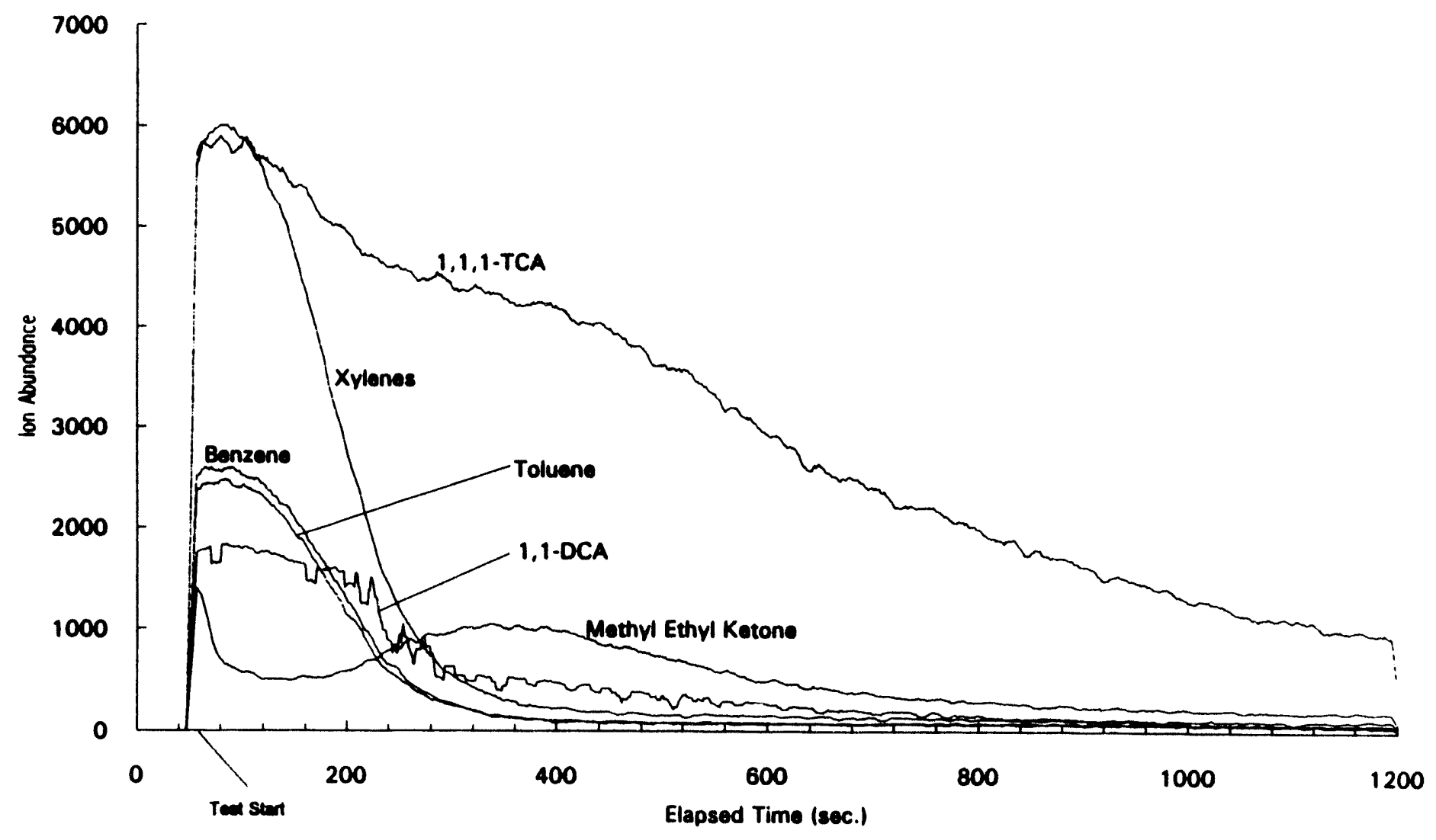

FIGURE 4.1 RUN 17A DSITMS CONTINUOUS WATER MONITORING OF TREATMENT OF K-25 SEEP 
as an intermediate early on in the process is evident as its concentration increases from about the 100-second to the 350-second mark. It also appears that the TCA destruction rate decreases, as the DCA destruction commences and the MEK concentration increases. This is a good example of a mixed organic contaminant problem in which the assumption of linear destruction rates for all organic contaminants is unfounded. Also, since the production of intermediates and their subsequent destruction can depend nonlinearly of the ultraviolet input power, extrapolations of the destruction rates as a function of system power must be made with caution. Destruction rate testing to determine actual requirements is necessary for accurate cost estimates.

An important motivation for including the DSITMS analysis was for a comparison of the concentration levels measured versus standard analytical techniques. Tables 4.7 and 4.8 exhibit two comparisons. Table 4.7 is for Run No. 12 with the perox-pure ${ }^{\mathrm{TM}}$ system and Table 4.8 is for Run No. 17A with SD-2. For 1,1-DCA, 1,1,1-TCA and benzene the agreement is generally within about a factor of two at the high end of measured concentration. At lower concentration, and for benzene, toluene and xylene, the quantitative agreement is less good.

It would have significantly enhanced the field tests if the DSITMS diagnostic had been used for all the tests. Real-time feedback would have been valuable in examining trends and revising the test plan during the test series. Consequently, we recommend that in future tests this diagnostic be included for the entire test series.

\section{TABLE 4.7 DSITMS ANALYSIS OF WATER FOR RUN NO. 12}

\begin{tabular}{||l|c|c|c|c|c|c||}
\hline & \multicolumn{2}{|c|}{$\begin{array}{c}\text { Concentration at } \\
\text { Test Start } \\
(\mathbf{n g} / \mathbf{m L})\end{array}$} & \multicolumn{2}{c|}{$\begin{array}{c}\text { Concentration at } \\
\text { 2 Min. } \\
(\mathbf{n g} / \mathbf{m L})\end{array}$} & \multicolumn{2}{c|}{$\begin{array}{c}\text { Concentration at } \\
\text { 3 Min. } \\
(\mathbf{n g} / \mathbf{m L})\end{array}$} \\
\hline Compound & ORNL* & PSI & ORNL* & PSI & ORNL* & PSI \\
\hline 1,1-DCA & 191 & 409 & 58 & 57 & 2 & 16 \\
\hline 1,1,1-TCA & 202 & 277 & 139 & 69 & 20 & 36 \\
\hline Benzene & 9 & 18 & 0 & 0 & 0 & 0 \\
\hline Toluene & 4 & 33 & 0 & 0 & 0 & 0 \\
\hline Xylene & 14 & 44 & 1 & 0 & 1 & 0 \\
\hline
\end{tabular}


TABLE 4.8 DSITMS ANALYSIS OF WATER FOR RUN NO. 17A

\begin{tabular}{|l|c|c|c|c||}
\hline & \multicolumn{2}{|c|}{$\begin{array}{c}\text { Concentration at } \\
\text { Test Start } \\
(\mathbf{n g} / \mathrm{mL})\end{array}$} & \multicolumn{2}{c|}{$\begin{array}{c}\text { Concentration at } \\
\text { Test Start } \\
(\mathbf{n g} / \mathbf{m L})\end{array}$} \\
\hline Compound & ORNL* & PSI & ORNL* & PSI \\
\hline 1,1-DCA & 624 & 407 & 36 & 67 \\
\hline 1,1,1-TCA & 552 & 366 & 81 & 67 \\
\hline Benzene & 46 & 18 & 2 & $<1$ \\
\hline Toluene & 48 & 15 & 3 & $<1$ \\
\hline Xylene & 218 & 11 & 3 & $<1$ \\
\hline \multicolumn{2}{|c|}{ *ORNL numbers are semi-quantitative field estimates. } \\
\hline
\end{tabular}

\subsubsection{Comparison of Treatment Systems}

The summary comparison of SD-2 with perox-pure ${ }^{\mathrm{TM}}$ in Table 4.9 emphasizes the TCA rate, which is representative of the photolysis-based oxidation rate (increase). The best peroxpure ${ }^{\mathrm{TM}}$ treatment results were achieved in Test 3 which was conducted with a $\mathrm{H}_{2} \mathrm{O}_{2}$ concentration of $40 \mathrm{mg} /$, neutral $\mathrm{pH}$, no catalyst, using pretreated water. These conditions are expected to be the optimum for perox-pure ${ }^{\mathrm{TM}}$ treatment of the SW-31 Spring water. At these conditions, the destruction rate constant for TCA was $1.5 \mathrm{~min}^{-1}$ (see Appendix III).

For comparison with SD-2, several different rates are examined since, due to Suprasil breakage problems, not all intended combinations of lamp variables should be tested. The first SD-2 treatment test (1A), performed on pretreated water without $\mathrm{H}_{2} \mathrm{O}_{2}$ or catalyst addition, at a $\mathrm{pH}$ of 3.8 and a discharge power equivalent to 1,000 watts, is the baseline for the analysis. The quartz tube was Suprasil and $\mathrm{Kr}$ gas was used. At these conditions, the first-order destruction rates for 1,1,1-trichloroethane was $2.0 \mathrm{~min}^{-1}$. This is a factor of 1.33 faster than the best peroxpure ${ }^{\mathrm{TM}}$ rate and was accomplished without the use of $\mathrm{H}_{2} \mathrm{O}_{2}$. 


\section{TABLE 4.9 SUMMARY COMPARISON OF PEROX-PURE ${ }^{\text {TM }}$ WITH}

SD.2 TEST RESULTS

\begin{tabular}{|c|c|}
\hline Variable & Conclusion \\
\hline $\mathrm{H}_{2} \mathrm{O}_{2}$ Concentration & $\begin{array}{l}\text { SD-2 was best with little }(\sim 25 \mathrm{mg} / \mathrm{l}) \text { or no } \mathrm{H}_{2} \mathrm{O}_{2} \\
\text { perox-pure }{ }^{\mathrm{TM}} \text { with larger }(\sim 50 \mathrm{mg} /) \mathrm{H}_{2} \mathrm{O}_{2} \\
\text { concentration }\end{array}$ \\
\hline pH & $\begin{array}{l}\text { SD-2 benefitted strongly but perox-pure }{ }^{\mathrm{TM}} \\
\text { benefitted only slightly }\end{array}$ \\
\hline Catalyst & No effect \\
\hline Pretreatment & $\begin{array}{l}\text { Pretreatment benefitted the perox-pure }{ }^{\mathrm{TM}} \text {, but } \\
\text { not the SD- } 2 \text { systems }\end{array}$ \\
\hline TCA Destruction Rate & $\begin{array}{l}\text { The best inferred SD- } 2 \text { destruction rate was } 2.5 \\
\text { times greater than perox-pure }{ }^{\mathrm{TM}} \text { rate }\end{array}$ \\
\hline Use of Both Systems & $\begin{array}{l}\text { The TCA destruction rates benefitted from } \\
\text { operating perox-pure }{ }^{T M} \text { and SD- } 2 \text { in series }\end{array}$ \\
\hline
\end{tabular}

To provide the best estimate possible with the available SD-2 test data, it is necessary to extrapolate the results of Test $1 \mathrm{~A}$ to account for the effects of using $\mathrm{Xe}$ gas rather than $\mathrm{Kr}$. In an internal research and development program, we (WJSA and PSI) showed that multiplication of different rates to give the net rate is a good approximation so long as each rate relates to UV efficiency, as in this case. By comparison of the results from Test $15 \mathrm{~A}$ ( $\mathrm{Kr}$ gas) and $16 \mathrm{~A}$ (Xe gas), the destruction rate increases by $\times 1.6$ for $1,1,1-$ trichloroethane. Applying this factor to the data from Test 1A, the projected destruction rates with the SD-2 system is $3.2 \mathrm{~min}^{-1}$ for 1,1,1,trichloroethane or 2.1 times faster than the best perox-pure ${ }^{\mathrm{TM}}$ rate.

Furthermore, the addition of a small quantity of $\mathrm{H}_{2} \mathrm{O}_{2}$, as shown in comparing Tests $7 \mathrm{~A}$ and $4 \mathrm{~A}$, increases the TCA destruction rate by about a factor of 1.2. Thus, an estimate of the results that might be expected with the SD-2 lamp system, including $\mathrm{Xe}$ and $\mathrm{H}_{2} \mathrm{O}_{2}$, is a TCA destruction rate of $3.8 \mathrm{~min}^{-1}$, or a factor of 2.5 times faster than the best rate with the peroxpure $^{\mathrm{TM}}$ system. Further development of SD lamp systems should include an experimental demonstration of these extrapolated destruction rates. 


\subsection{TECHNOLOGY EVALUATION}

\subsection{Progress and Status of SD Lamp Development}

\subsubsection{Background}

Prior to this program, the SD UV lamp was a laboratory system which had not been operated in the field. In single pulse measurements of UV efficiency, SD UV emission was up to five times greater efficiency (between 200 and $240 \mathrm{~nm}$ ) than the commercial high intensity UV lamps currently used. In that first SD system, inadequate gas flow resulted in the envelope UV transmission degradation in about 1,000 pulses when the lamp was operated at maximum efficiency. Consequently, to improve lifetime for the laboratory processing tests, the single pulse efficiency in the 200-240 $\mathrm{nm}$ region was approximately one-fourth of the best demonstrated in single pulse tests (i.e., about $25 \%$ higher than the medium pressure mercury lamp). At this single pulse efficiency, the average power was limited to 200-300 watts as compared to 2,000 watts for the commercial lamp. These SD UV lamp operating capabilities were inadequate for a field test. As described above, the SD-2 system designed and built in this program resulted in a power increase to 1,500 watts (from 300 watts) and demonstrated operating time of three hours (from 15 minutes).

\subsubsection{Performance Improvements}

For practical use the SD UV lamp requires performance improvements in the total energy delivery without degradation in UV transmission. Additional improvements are necessary to achieve commercially acceptable maintenance-free operating times. For instance, for eight weeks of maintenance-free operation for twenty-four hours a day, the degradation-free operating time must be increased by about a factor of 500 over the three hours demonstrated to date. We believe this goal is readily achievable with straightforward engineering. Commercial flashlamps, a similar technology to the SD lamp, have a demonstrated lifetime over 2,000 times greater than the three hours demonstrated with the SD lamp to date.

Also, the operational single pulse efficiency should be increased toward the maximum demonstrated single pulse efficiency. The single pulse efficiency of SD-2 was variable, but for the field tests was operated at close to the same efficiency as the laboratory unit (i.e., one-fourth 
the maximum demonstrated). Operating the lamp at increased UV efficiency will increase the organic destruction rate, which is highly desirable.

\subsubsection{Unique Characteristics of UV/UV Catalyzed Technology}

In comparing UV/UV catalyzed technology with classic water remediation technologies, such as air stripping and GAC, the biggest difference is that UV/UV catalyzed technology has no by-products requiring additional treatment. That is, the organic contaminants are destroyed by UV/UV catalyzed technology, whereas the contaminants are transferred to another medium with the classical technologies. Air stripping is used only where special permits have been granted, allowing air emission of organic contaminants at some maximum rate. This approach is not a long-term solution. The use of GAC on DOE sites is particularly difficult due to the production of a new contaminated waste that must be handled. The possibility of radio-active components in this contamination exacerbates the regulatory and permitting efforts that would be necessary if GAC were chosen for water remediation.

The use of a SD UV lamp in a UV-based water remediation system has the additional advantage of increasing the processing rate and reducing the water remediation cost for many mixed organic waste water remediation problems (see Section 5.2). The data from this field test (see Section 4.6) indicate that some combination of UV processing with and without peroxide will be optimum. Depending on the organics requiring destruction, in some cases, very little or no peroxide may be required using the SD UV lamp. This can reduce handling and materials cost.

\subsection{Cost Savings and Benefits}

\subsubsection{Cost Comparison}

Both general (DOE-wide) and specific (the SW-31 Spring) comparisons of the peroxpure $^{\mathrm{TM}}$ and SD UV-based water treatment systems with the classical air stripping and GAC techniques is difficult because of a combination of regulatory aspects and the variability in effectiveness of each technique with different organics. With a mixture of organics such as in the SW-31 Spring, the comparison is even more difficult. For the perox-pure ${ }^{\mathrm{TM}}$ process, as discussed above, hydroxyl destruction rate constants vary strongly with organic compound. Case studies have shown, for instance, that for TCE the perox-pure ${ }^{\mathrm{TM}}$ process cost ranges from 10 to 
30 cents per 1,000 gallons for $99.9 \%$ destruction. For a compound such as benzene the cost increases to 60 to 80 cents per 1,000 gallons and for compounds such as 1,1,1-TCA the cost increases further to about $\$ 1$ to $\$ 2$ per 1,000 gallons.

Air stripping is the least costly water treatment process, and in most cases would be the remediation process of choice if processing costs were the deciding factor. However, air emission regulations may exclude the use of air stripping. In addition, in many water treatment problems, additional pretreatment requirements (such as the requirement to remove iron from the water in the SW-31 Spring) are the dominant costs. Also, shown below, if the air stripper is not on location and transportation is required, then the cost can become relatively expensive.

For GAC, the cost quoted above for the perox-pure ${ }^{\mathrm{TM}}$ system to treat chemicals such as TCE and benzene the perox-pure ${ }^{\mathrm{TM}}$ process is competitive. For 1,1,1-TCA, however, GAC does not work well. Consequently, the perox-pure ${ }^{\mathrm{TM}}$ process is likely to be chosen over GAC. At many DOE sites, the use of GAC may be unacceptable because its removal from the site for further treatment would be a significant extra expense.

A cost estimate for using the perox-pure ${ }^{\mathrm{TM}}$ at the SW-31 Spring is summarized in Table 5.1 for two scenarios, the first without iron pretreatment and the second with iron pretreatment. The perox-pure ${ }^{\mathrm{TM}}$ capital cost is the price for engineering, equipment and instrumentation as a package and is not broken out. Testing and start-up services are extra and site/water specific. Operating labor is negligible since the systems operate automatically. Maintenance labor is typically estimated at $2 \%$ of the capital investment. Permitting and site preparation, if any, are site/water specific. The projected costs are compared to hauling to Y-12 because the peroxpure $^{\mathrm{TM}}$ system was proposed as an interim treatment measure until CNF at K-25 is completed. As shown in data above, iron pretreatment significantly increases the destruction rate; consequently, the cost for processing without iron pretreatment is approximately doubled. However, due to site disposal issues with the pretreatment filters, it may be preferable to operate the perox-pure ${ }^{\mathrm{TM}}$ system without iron pretreatment. In either case a 30 kilowatt full scale peroxpure $^{\mathrm{TM}}$ unit is sufficient for the SW-31 Spring. 
TABLE 5.1 COST ESTIMATE OF PEROX-PURE ${ }^{\text {TM }}$ AT SW-31 SPRING

\section{WITHOUT IRON PRETREATMENT}

CAN TREAT AT 30 GPM FOR 3.5 HR/DAY USING A $30 \mathrm{KW}$

FULL-SCALE PEROX-PURE ${ }^{\mathrm{TM}}$ SYSTEM

CAPITAL: APPROXIMATELY $\$ 100,000$ (COMPLETE SYSTEM)

MAINTENANCE: APPROXIMATELY $\$ 10,000$ (ONE YEAR)

CONSUMPTION: $\quad 17.3 \mathrm{KwH} / 1000 \mathrm{GAL}$

$1.2 \mathrm{LB} \mathrm{H}_{2} \mathrm{O}_{2} / 1000 \mathrm{GAL}$

OPERATING COSTS:

$\$ / 1000 \mathrm{GAL}$

ELECTRICAL ENERGY (@\$0.06/KWH)

$50 \% \mathrm{H}_{2} \mathrm{O}_{2}$ (@\$0.65/LB)

$\underline{0.78}$

TOTAL

\section{WITH IRON PRETREATMENT}

CAN TREAT AT 80 GPM FOR 1 HR/DAY USING A $30 \mathrm{KW}$ FULL-SCALE PEROX-PURE ${ }^{\text {TM }}$ SYSTEM

CAPITAL: APPROXIMATELY $\$ 100,000$ (COMPLETE SYSTEM)

MAINTENANCE: APPROXIMATELY $\$ 10,000$ (ONE YEAR)

CONSUMPTION: $\quad 7.0 \mathrm{KwH} / 1000 \mathrm{GAL}$

$0.7 \mathrm{LB} \mathrm{H}_{2} \mathrm{O}_{2} / 1000 \mathrm{GAL}$

OPERATING COSTS:

$\$ / 1000 \mathrm{GAL}$

ELECTRICAL ENERGY (@\$0.06/KWH)

0.42

$50 \% \mathrm{H}_{2} \mathrm{O}_{2}$ (@\$0.65/LB)

$\underline{0.45}$

TOTAL

0.89 
Since the SD UV lamp is not yet a commercial device, cost estimates are preliminary. Based on the field test conducted in this program, combined with laboratory demonstrations of UV efficiency with the SD lamp, we project that a commercial SD lamp system will increase the destruction rate by a factor of 3 of the most difficult organic, namely 1,1,1-TCA, at the SW-31 Spring. The capital cost per unit power of a SD UV lamp system is greater than a perox-pure ${ }^{\mathrm{TM}}$ system. We estimate that the capital cost for a SD UV lamp system-based water remediation system will be one-half of a perox-pure ${ }^{\mathrm{TM}}$ system (i.e., although the SD UV lamp is three times as efficient, it will cost half as much due to the higher capital cost per unit power). Also, the quantity of peroxide necessary would be reduced by approximately one half. The cost estimate for an SD UV lamp-based system, shown in Table 5.2, is based on these assumptions and indicates a processing cost reduction of greater than a factor of 2 . Furthermore, the test results indicate that the SD-2 system proceeds by a different destruction mechanism from the peroxpure $^{\mathrm{TM}}$ since the more highly chlorinated molecule was destroyed faster. It may be inferred from this result that the SD-2 cost efficiency should increase, compared to the perox-pure ${ }^{\mathrm{TM}}$ system, for more highly chlorinated VOCs. Thus, the cost savings with the SD-2 system should be much greater for contaminants like chloroform or carbon tetrachloride. This result was anticipated based upon the previous bench-scale testing with the SD-2 system. Thus, at DOE sites with highly chlorinated VOCs, the advantage of using an SD-2 system is greatest.

\subsubsection{Annual Savings Estimate}

The annual savings estimate provided here is specifically for the SW-31 Spring. The currently planned alternative is to haul the water to the DOE Y-12 water stripper system. The hauling cost alone is approximately $\$ 360 \mathrm{~K}$ per year according to MMES personnel. For the first year, the total cost of a perox-pure ${ }^{\mathrm{TM}}$ system is approximately $\$ 110 \mathrm{~K}$ (i.e., the capital equipment cost plus the maintenance cost). This does not include the cost of on-site personnel which is outside our cost estimate basis. Consequently, the savings in the first year is approximately $\$ 150 \mathrm{~K}$. Since the expected life of the perox-pure ${ }^{\mathrm{TM}}$ system is five years, for the additional four years, this cost savings per year would be approximately $\$ 250 \mathrm{~K}$ per year, for a total cost savings over a five-year period of $\$ 1.15 \mathrm{M}$. 
TABLE 5.2 COST ESTIMATE OF PEROX-PURE ${ }^{\text {TM }}$ SD UV LAMP-BASED SYSTEM AT SW-31 SPRING

\section{WITHOUT IRON PRETREATMENT}

CAN TREAT AT 30 GPM FOR 3.5 HR/DAY USING A $10 \mathrm{KW}$ FULL-SCALE PEROX-PURE ${ }^{\text {TM }}$ SYSTEM

CAPITAL: APPROXIMATELY $\$ 50,000$ (COMPLETE SYSTEM)

MAINTENANCE: APPROXIMATELY $\$ 10,000$ (ONE YEAR)

CONSUMPTION: $\quad 5.8 \mathrm{KwH} / 1000 \mathrm{GAL}$

$$
0.6 \mathrm{LB} \mathrm{H}_{2} \mathrm{O}_{2} / 1000 \mathrm{GAL}
$$

OPERATING COSTS:

ELECTRICAL ENERGY (@ $\$ 0.06 / \mathrm{KWH})$

$50 \% \mathrm{H}_{2} \mathrm{O}_{2}$ (@\$0.65/LB)

$\$ / 1000 \mathrm{GAL}$

TOTAL

$\underline{0.39}$

0.79

WITH IRON PRETREATMENT

CAN TREAT AT 80 GPM FOR 1 HR/DAY USING A $10 \mathrm{KW}$ FULL SCALE PEROX-PURE ${ }^{\text {TM }}$ SYSTEM

CAPITAL: APPROXIMATELY $\$ 50,000$ (COMPLETE SYSTEM)

MAINTENANCE: APPROXIMATELY $\$ 10,000$ (ONE YEAR)

CONSUMPTION: $\quad 2.5 \mathrm{KwH} / 1000 \mathrm{GAL}$

$$
0.35 \mathrm{LB} \mathrm{H}_{2} \mathrm{O}_{2} / 1000 \mathrm{GAL}
$$

OPERATING COSTS:

$\$ / 1000 \mathrm{GAL}$

ELECTRICAL ENERGY (@\$0.06/KWH)

0.15

$50 \% \mathrm{H}_{2} \mathrm{O}_{2}$ (@\$0.65/LB)

$\underline{0.23}$

TOTAL

0.38 


\subsubsection{Return on Investment}

This DT\&E program costs approximately $\$ 350 \mathrm{~K}$. If the perox-pure ${ }^{\mathrm{TM}}$ system were purchased and used for a period of five years, as discussed in the previous section, the total savings would be $\$ 800 \mathrm{~K}$, with a total return of investment of over $200 \%$ or yearly return of approximately $18 \%$ (compounded). If, as we hope, the perox-pure ${ }^{\mathrm{TM}}$ system will receive increased use at DOE sites with similar cost savings then the cost return on investment would increase greatly.

A calculation with a return on investment for a SD UV-based lamp system is inappropriate since further development is required for a full-scale system. Consequently, we provide here an example return on investment. Assume ten gallons per minute are treated for a period of five years (the expected lifetime of a commercial SD UV-based lamp system). If the processing cost with an SD system is cut in half from $\$ 1$ to 50 cents per 1,000 gallons, and if this scenario occurred at five DOE sites, then the total cost savings would be $\$ 6.5 \mathrm{M}$. We estimate the additional cost for the development of a full-scale commercial system to be about $\$ 1 \mathrm{M}$. The return on investment of this example warrants development of a SD UV-based water treatment technology.

\subsection{Other Benefits}

\subsubsection{Regulatory Advantages}

The primary regulatory advantage, mentioned above, is that the UV/UV catalyzed technology has no contaminated by-products. The most widely used water remediation technologies, air stripping and GAC require special permitting and/or special handling. GAC, once used, must either be incinerated or re-activated in a process which produces contaminants requiring disposal.

\subsubsection{Potential Acceptance Issues}

The existing perox-pure ${ }^{\mathrm{TM}}$ commercial system has received acceptance both at government facilities as well as in industry. The perceived issue (of some parties) of peroxide handling and storage has been resolved by having safe and proper equipment and handling techniques. Other potential acceptance issues with the SD UV lamp could be concerns with high intensity UV light and/or high voltage electrical equipment. 


\subsubsection{Risk Assessment}

The potential acceptance issues with the SD UV lamp system are similar to issues with other commercial hardware such as other UV lamps, lasers, and other systems with high voltage. The UV light is enclosed in the water processing reactor which can be designed to eliminate UV emission. Similarly, commercial high voltage systems routinely provide safe turn-key operation. Equipment would be labeled with cautions, interlocks and automatic turn-off switches incorporated into the system in case of unexpected failure. 


\subsection{CONCLUSIONS AND RECOMMENDATIONS}

The demonstration test and evaluation reported here successfully showed that ultraviolet/ultraviolet catalyzed peroxide oxidation groundwater remediation is practical for the SW-31 Spring tested here as well as at other DOE water remediation sites. Both the existing commercial perox-pure ${ }^{\mathrm{TM}}$ system and the new system incorporating an SD UV lamp were shown to be cost effective and to meet regulatory requirements intrinsically better than the current standard techniques.

For the SW-31 Spring, the perox-pure ${ }^{\mathrm{TM}}$ system would pay for itself in four months, and over a five-year period save more than $\$ 1 \mathrm{M}$ when compared to the alternative now planned at DOE. Also, based on tests conducted to date, a water remediation system based on the SD UV lamp would increase organic destruction rates of highly chlorinated VOCs by a factor of 3 or more and decrease processing costs by about a factor of 2 . Neither the perox-pure ${ }^{\mathrm{TM}}$ nor the SD UV lamp-based systems produce by-products requiring additional handling and/or treatment. This advantage is difficult to quantify in comparison to the current standard water remediation techniques.

Recommendations from the demonstration results include:

1. Use the perox-pure ${ }^{\mathrm{TM}}$ system results as a basis for considering further use at the SW-31 Spring as well as at other DOE sites requiring water remediation.

2. Support further development of SD UV lamp technology to reduce the cost of water remediation. 


\section{SURFACE DISCHARGE LIGHT SOURCE REFERENCES}

1. Schaefer, Raymond B., James P. Reilly, and M. John Yoder, "Pulsed Short Wavelength Laser Effects Simulator," Final Report, DNA-TR-84-222, Contract No. DNA-001-83-C0400 (1985).

2. Schaefer, Raymond B. and James P. Reilly, "Technology Development for High Irradiance and Large Impulse Surface Discharge Laser Effects Simulators," Technical Report, DNATR-104 (1986).

3. Schaefer, Raymond B., James P. Reilly, John M. Flaherty, and Glenn W. Zeiders, "Surface Discharge Laser Effects Simulator Development," Final Report, Contract No. DNA-001-850259 (1986).

4. Schaefer, Raymond B., Douglas G. Youmans, and Theodore W. Hilton, "Single Pulse Surface Discharge and Wavelength Conversion Experiments," Final Report, Contract No. DAAH01-83-D-A017, WJSA-FTR-84-03 (1983).

5. Schaefer, Raymond B., "Surface Discharges: Assessment of Soviet State-of-the-Art Capabilities," prepared for Army Foreign Science and Technology Center, Contract No. DAAK21-79-0109 (1979).

6. Schaefer, Raymond B. and Robert J. Grasso, "Development of Solid-State Lasers Pumped by the Surface Discharge Pulsed Incoherent Light Source," Contract No. DNA-001-PG-C0070 (1987).

7. Schaefer, Raymond B. and M. John Yoder, "Surface Discharge Preionized $\mathrm{CO}_{2}$ Laser Development," Lasers '87 Conference (1987).

8. Connolly, James J., Raymond B. Schaefer, and Michael S. Poirier, "Delivery and Development of a Surface Discharge Incoherent Pump Source," Contract No. 9-Y84-1686G (1988).

9. Schaefer, Raymond B., John Collins, Rodney A. Petr, and M. John Yoder, "Surface Discharge Pumped Ti: $\mathrm{Al}_{2} \mathrm{O}_{3}$ Laser," Final Report, Con No. DNA-001-87-C-0240 (1990).

10. Schaefer, Raymond B., et al., "Photolytic Detoxification Concept," Final Report prepared for the EPA under Contract No. 68-02-4159 (1986).

11. Schaefer, Raymond B., James P. Reilly, Alex Ballantyne, Douglas G. Youmans, and Michael Bernard, "The Surface Discharge Incoherent Light Source for Simulating Laser Effects and for Thermal Testing," Contract No. DNA-001-85-C-0259 (1990).

12. Schaefer, Raymond B., "Surface Discharge Photoinitiation of Pulsed Chemical Lasers," Prepared for Los Alamos Nat. Lab., Contract No. 9-X62-7319X-1 (1992). 
APPENDIX I. WATER QUALITY DATA, SW-31 SPRING

K-1070-C/D BURIAL GROUND, K-25 SITE

Data as of August 1993

(minimums and maximums collected from 1987 to 1993)

\begin{tabular}{|l|c|c|c|}
\hline \multicolumn{1}{|c|}{ Compound } & Minimum & Maximum & $6 / 25 / 93$ \\
\hline Volatiles ( $\mu$ g/L) & 150 \\
\hline Benzene & 190 & 1300 & $<100$ \\
\hline Chloroethane & $<10$ & 160 & $<50$ \\
\hline Chloroform & $<5$ & 5 & 90 \\
\hline Ethylbenzene & 190 & 430 & 920 \\
\hline 1,1-Dichloroethane & 460 & 3800 & 200 \\
\hline 1,1-Dichloroethane & 250 & 920 & $<50$ \\
\hline 1,2-Dichloroethane & $<5$ & 950 & $<50$ \\
\hline Methylene Chloride & 160 & 8000 & $<50$ \\
\hline Tetrachloroethane & $<5$ & 110 & 240 \\
\hline Toluene & 440 & 3100 & Not Detected \\
\hline Trans-1,2-Dichloroethane & 670 & 950 & 1300 \\
\hline 1,1,1-Trichloroethane & 4000 & 6800 & $<50$ \\
\hline 1,1,2-Trichloroethane & $<5$ & 170 & $39 \mathrm{~J}$ \\
\hline Trichloroethane & 140 & 430 & $<100$ \\
\hline Vinyl Chloride & $<10$ & 330 & 770 \\
\hline Xylene (total) & 1400 & 1900 & 160 \\
\hline 1,2-Dichloroethane (total) & No Data & No Data & \\
\hline
\end{tabular}




\section{APPENDIX II. MAXMUM CONTAMINATION LEVELS ALLOWED FOR ORGANICS IN SW-31 SPRING WATER(*)}

\begin{tabular}{||l|c||}
\hline \multicolumn{1}{|c|}{ Compound } & MCL $(\mu \mathrm{g} / \mathbf{l})$ \\
\hline Bromodichloromethane & 100 \\
\hline Bromoform & 100 \\
\hline Chloroethane & No Standard \\
\hline 1,1-Dichloroethane & No Standard \\
\hline 1,2-Dichloroethane & 5 \\
\hline 1,1-Dichloroethane & 7 \\
\hline 1,1,1-Trichloroethane & 200 \\
\hline Trichlorofluoromethane & No Standard \\
\hline Vinyl Chloride & 2 \\
\hline Benzene & 5 \\
\hline Toluene & 1,000 \\
\hline Ethylbenzene & 700 \\
\hline Total Xylene & 10,000 \\
\hline 1,3-Dichlorobenzene & 600 \\
\hline 1,4-Dichlorobenzene & 75 \\
\hline
\end{tabular}

*Drinking Water Regulations and Health Advisories, United States EPA, May (1993). 


\section{APPENDIX III. TERMINOLOGY AND DEFINITIONS}

\section{Reaction Rate (Oxidation or Destruction Rate)}

Reaction rate and oxidation rate are synonymous. The nomenclature used in the literature is pseudo-first order reaction rate constant, symbolized by $\mathrm{K}$ with units of inverse time. The reaction rate constant is calculated from the equation:

$$
\mathrm{K}=(1 / \mathrm{t}) \ln (\mathrm{Ci} / \mathrm{Ce})
$$

where $\mathrm{t}$ is the oxidation time in minutes, $\mathrm{Ci}$ is the influent contaminant concentration, and $\mathrm{Ce}$ is the effluent contaminant concentration after $t$ minutes of oxidation.

\section{Batch and Flow-Through Testing}

During flow-through testing, the water is pumped through the reactor only once. During batch testing, a fixed volume of water is recirculated through the reactor and back into the batch tank. Batch testing provides the opportunity to evaluate longer times while preserving a high flow rate to simulate full-scale operation.

\section{Oxidation Time}

The oxidation time for flow-through testing is calculated as follows:

$$
\mathrm{t}=\mathrm{V} / \mathrm{Q}
$$

where $t$ is the oxidation time in minutes, $\mathrm{V}$ is the reactor volume in gallons and $\mathrm{Q}$ is the flow rate in gallons per minute. The total contact volume in the S-30 is 15 gallons. More than one oxidation time can be obtained in each test by sampling at intermediate points (contact volumes) in the reactor.

For batch testing, the oxidation time is calculated as follows:

$$
t=t^{\prime}\left(V_{R} / V_{T}\right)
$$

where $t$ is the oxidation time in minutes, $t^{\prime}$ is the elapsed clock time in minutes, $V_{R}$ is the reactor volume in gallons and $V_{T}$ is the total batch volume. Batch volumes of 75 gallons are typically used during the perox-pure ${ }^{\mathrm{TM}}$ testing. 


\section{APPENDIX IV. RESULTS OF PEROX-PURE TM TESTING ON}

SW-J1 SPRING WATER AT DOE K-25 SITE

Table 1

Conditions for Perox-Pure ${ }^{\mathrm{TM}}$ Testing on SW-31 Spring Water at DOE K-25 Site

\begin{tabular}{|c|c|c|c|c||}
\hline Test & $\begin{array}{c}\mathbf{H}_{2} \mathbf{O}_{2}{ }^{1} \\
\text { Maintained }(\mathbf{m g} / \mathbf{l})\end{array}$ & $\begin{array}{c}\text { Influent } \\
\mathbf{p H}\end{array}$ & $\begin{array}{c}\text { Catalyst } \\
(\mathbf{m g} / \mathbf{)})\end{array}$ & $\begin{array}{c}\text { Water }^{2} \\
\text { Pretreated }\end{array}$ \\
\hline 1 & $20-100^{3}$ & 7 & 0 & Yes \\
\hline 2 & 20 & 7 & 0 & Yes \\
\hline 3 & 40 & 7 & 0 & Yes \\
\hline 4 & $100-400^{3}$ & 7 & 0 & Yes \\
\hline 5 & 50 & 6 & 0 & Yes \\
\hline 6 & 50 & 5 & 0 & Yes \\
\hline 7 & 50 & 3.5 & 0 & Yes \\
\hline 8 & 70 & 6.5 & 0 & Yes \\
\hline 9 & $200-600^{3}$ & 4 & 20 & Yes \\
\hline 10 & 50 & 3.5 & 20 & Yes \\
\hline 11 & 50 & 4 & 50 & Yes \\
\hline 12 & 50 & 4 & 100 & Yes \\
\hline 13 & 50 & 5 & 0 & No \\
\hline 14 & 50 & 6.5 & 0 & No \\
\hline 15 & 50 & 4.8 & 0 & Yes \\
\hline 16 & 50 & 3.2 & 20 & Yes \\
\hline
\end{tabular}

$1 \mathrm{H}_{2} \mathrm{O}_{2}$ concentration maintained during test via continuous addition of $50 \% \mathrm{H}_{2} \mathrm{O}_{2}$ solution.

2 Pretreatment for iron removal via filtration.

${ }^{3} \mathrm{H}_{2} \mathrm{O}_{2}$ concentration not effectively controlled. Conditions repeated in subsequent tests. 
Table 2

Conditions for WJSA SD Lamp Testing
on SW-31 Spring Water at DOE K-25 Site

\begin{tabular}{|c|c|c|c|c|c|c|}
\hline Test & $\begin{array}{l}\mathrm{H}_{2} \mathrm{O}^{1}{ }^{1} \\
\text { (mg/) }\end{array}$ & $\begin{array}{c}\text { Influent } \\
\text { pH }\end{array}$ & $\begin{array}{c}\text { Catalyst } \\
(\mathrm{mg} / \mathrm{l})\end{array}$ & $\begin{array}{c}\text { Water }^{2} \\
\text { Pretreated }\end{array}$ & $\begin{array}{c}\mathrm{SD}^{3} \\
\text { Conditions } \\
\end{array}$ & $\begin{array}{l}\text { Other } \\
\text { Notes }\end{array}$ \\
\hline $1 \mathrm{~A}$ & 0 & 3.8 & 0 & Yes & $\mathrm{A}$ & \\
\hline $2 \mathrm{~A}$ & 0 & 3.3 & 0 & Yes & B & \\
\hline $3 \mathrm{~A}$ & 0 & 3.3 & 0 & Yes & C & \\
\hline $4 \mathrm{~A}$ & 0 & 3.3 & 0 & Yes & D & \\
\hline $5 \mathrm{~A}$ & 70 & 3.3 & 0 & Yes & D & \\
\hline $6 \mathrm{~A}$ & 50 & 3.3 & 0 & Yes & D & \\
\hline $7 \mathrm{~A}$ & 25 & 3.3 & 0 & Yes & D & \\
\hline $8 \mathrm{~A}$ & 0 & 6.7 & 0 & Yes & D & \\
\hline $9 \mathrm{~A}$ & 0 & 4.0 & 20 & Yes & D & \\
\hline $10 \mathrm{~A}$ & 0 & 4.0 & 50 & Yes & D & \\
\hline $11 \mathrm{~A}$ & 5 & 3.6 & 0 & No & D & 4 \\
\hline $12 \mathrm{~A}$ & 0 & 3.0 & 0 & Yes & $\mathrm{E}$ & \\
\hline $13 \mathrm{~A}$ & 0 & 3.0 & 0 & Yes & F & \\
\hline $14 \mathrm{~A}$ & 0 & 6.3 & 0 & No & D & \\
\hline $15 \mathrm{~A}$ & 0 & 3.0 & 0 & Yes & D & \\
\hline $16 \mathrm{~A}$ & 0 & 3.0 & 0 & Yes & G & \\
\hline $17 \mathrm{~A}$ & 0 & 3.0 & 0 & Yes & $\mathrm{H}$ & \\
\hline $18 \mathrm{~A}$ & 0 & 3.0 & 0 & Yes & I & \\
\hline $19 \mathrm{~A}$ & 0 & 3.0 & 0 & Yes & $\mathbf{J}$ & \\
\hline
\end{tabular}

$\mathrm{H}_{2} \mathrm{O}_{2}$ concentration maintained in solution during test via continuous addition of $\mathrm{H}_{2} \mathrm{O}_{2}$ solution.

Pretreatment for iron removal via filtration.

SD conditions as follows:

A - $1000 \mathrm{~W}$ power, $\mathrm{Kr}$ gas, $65 \mathrm{~mm}$ tube, suprasil tube and substrate.

B - $1500 \mathrm{~W}$ power, $\mathrm{Kr}$ gas, $65 \mathrm{~mm}$ tube, suprasil tube and substrate.

C - $500 \mathrm{~W}$ power, Ar gas, $1.5 \mathrm{~Hz}, 65 \mathrm{~mm}$ tube, GE 214 tube, suprasil substrate.

D - $500 \mathrm{~W}$ power, $\mathrm{Kr}$ gas, $1.5 \mathrm{~Hz}, 65 \mathrm{~mm}$ tube, GE 214 tube, suprasil substrate.

$E$ - $1000 \mathrm{~W}$ power, $\mathrm{Kr}$ gas, $3 \mathrm{~Hz}, 65 \mathrm{~mm}$ tube, GE 214 tube and substrate.

$\mathrm{F}$ - $500 \mathrm{~W}$ power, $\mathrm{Kr}$ gas, $1.5 \mathrm{~Hz}, 65 \mathrm{~mm}$ tube, GE 214 lube and substrate.

G - $500 \mathrm{~W}$ power, Xe gas, $1.5 \mathrm{~Hz}, 65 \mathrm{~mm}$ tube, GE 614 tube, suprasil substrate.

$\mathrm{H}-1000 \mathrm{~W}$ power, Xe gas, $3 \mathrm{~Hz}, 65 \mathrm{~mm}$ tube, GE 214 tube, suprasil substrate.

I. $600 \mathrm{~W}$ power, (Higher Voltage), Xe gas, $1.5 \mathrm{~Hz}, 65 \mathrm{~mm}$ tube, GE 214 tube, suprasil substrate.

J - $700 \mathrm{~W}$ power (Highest Voltage), Xe gas, $1.5 \mathrm{~Hz}, 65 \mathrm{~mm}$ tube, GE 214, suprasil substrate.

4 Water treated in perox-pure ${ }^{\text {TM }}$ System before treatment with WJSA SD lamp. 
Table 3

Characterization Results for SW-31 Spring Water

\begin{tabular}{|l|c|}
\hline \multicolumn{1}{|c|}{ Parameter } & Value \\
\hline Visual Appearance & Turbid Orange \\
\hline pH & 6.9 \\
\hline TDS (mg/) & 350 \\
\hline Turbidity (FTU) & $2^{1}$ \\
\hline Total Iron (mg/) & 16 \\
\hline Chloride (mg/) & 58 \\
\hline COD (mg/) & $13^{1}$ \\
\hline TOC (mg/) & 5 \\
\hline Alkalinity (mg/) & 250 \\
\hline TSS (mg/) & 50 \\
\hline NO ${ }_{3}$ as N (mg/) & $<0.1$ \\
\hline Hardness $(\mathrm{mg} /)$ & 300 \\
\hline
\end{tabular}

'Value obtained after solids allowed to settle. 
'our! posdejo [enjor әцl אq

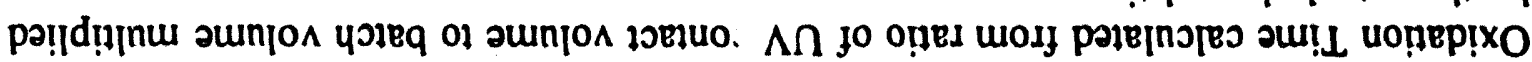

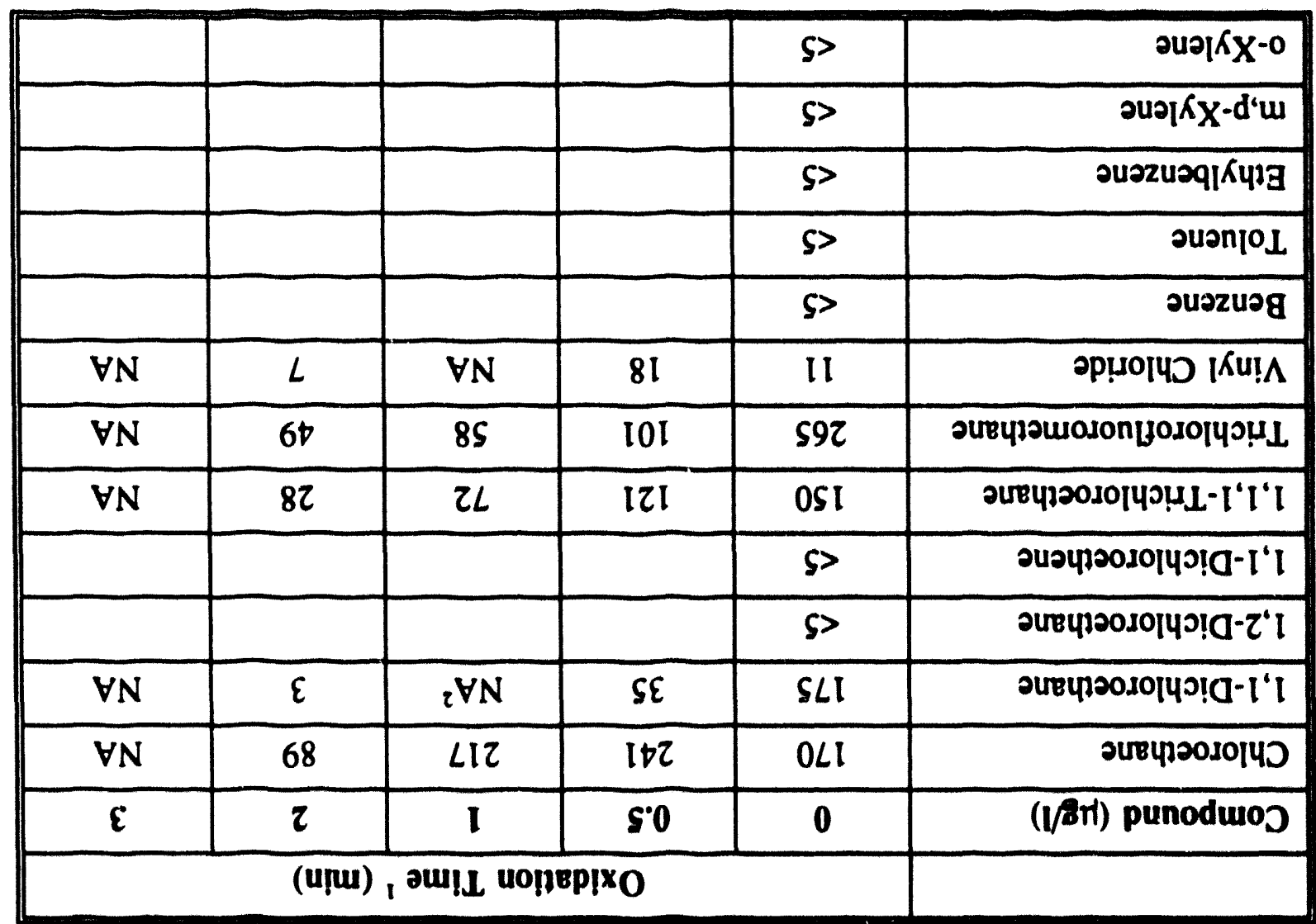

12501

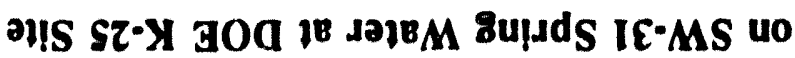

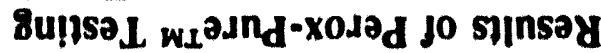

- गqEL 
Table 4 (cont.)

Results of Perox-Pure ${ }^{\mathrm{TM}}$ Testing

on SW-31 Spring Water at DOE K-25 Site

Test 2

\begin{tabular}{|l|c|c|c|c|c||}
\hline & \multicolumn{5}{|c|}{ Oxidation Time ${ }^{\mathbf{1}}(\mathrm{min})$} \\
\hline Compound $(\mu \mathrm{g} / \mathrm{l})$ & $\mathbf{0}$ & $\mathbf{0 . 5}$ & $\mathbf{1}$ & $\mathbf{2}$ & $\mathbf{3}$ \\
\hline Chloroethane & 286 & $\mathrm{NA}^{3}$ & 230 & $\mathrm{SSB}^{2}$ & 143 \\
\hline 1,1-Dichloroethane & 236 & 182 & 30 & & 5 \\
\hline 1,2-Dichloroethane & $<5$ & $<1$ & & & \\
\hline 1,1-Dichloroethene & 22 & 3 & $<1$ & & \\
\hline 1,1,1-Trichloroethane & 191 & 177 & 85 & & 19 \\
\hline Trichlorofluoromethane & 313 & 122 & 76 & & 71 \\
\hline Vinyl Chloride & 15 & 23 & 17 & & 11 \\
\hline Benzene & 8 & $<1$ & & & \\
\hline Toluene & 16 & 3 & 1 & & $<1$ \\
\hline Ethylbenzene & 9 & $<1$ & & & \\
\hline m,p-Xylene & 8 & $<1$ & & & \\
\hline o-Xylene & 10 & 2 & $<1$ & & \\
\hline
\end{tabular}

1 Oxidation Time calculated from ratio of UV contact volume to batch volume multiplied by the actual elapsed time.

2 Sample bottle broken in transit.

3 Result not available. 
Table 4 (cont.)

Results of Perox-Pure ${ }^{\mathrm{TM}}$ Testing

on SW-31 Spring Water at DOE K-25 Site

Test 3

\begin{tabular}{||l|c|c|c|c|c||}
\hline & \multicolumn{5}{|c|}{ Oxidation Time $^{\mathbf{1}}(\mathbf{m i n})$} \\
\hline Compound $(\mu \mathrm{g} / \mathrm{l})$ & $\mathbf{0}$ & $\mathbf{0 . 5}$ & $\mathbf{1}$ & $\mathbf{2}$ & $\mathbf{3}$ \\
\hline Chloroethane & 297 & 276 & 256 & 181 & 126 \\
\hline 1,1-Dichloroethane & $\mathrm{NA}^{3}$ & $\mathrm{NA}$ & $\mathrm{NA}$ & $\mathrm{NA}$ & $\mathrm{NA}$ \\
\hline 1,2-Dichloroethane & $<5$ & & & & \\
\hline 1,1-Dichloroethene & 14 & 2 & $<1$ & & \\
\hline 1,1,1-Trichloroethane & 237 & 133 & 79 & 30 & 13 \\
\hline Trichlorofluoromethane & 158 & 108 & 124 & 50 & 29 \\
\hline Vinyl Chloride & 20 & 21 & 19 & 14 & 9 \\
\hline Benzene & 5.8 & $<1$ & & & \\
\hline Toluene & 10 & 1 & $<1$ & & \\
\hline Ethylbenzene & $<5$ & & & & \\
\hline m,p-Xylene & $<5$ & & & & \\
\hline o-Xylene & 6.6 & $<1$ & & & \\
\hline
\end{tabular}

1 Oxidation Time calculated from ratio of UV contact volume to batch volume multiplied by the actual elapsed time.

$2 \quad$ Result not available. 
Table 4 (cont.)

Results of Perox-Pure ${ }^{\mathrm{TM}}$ Testing

on SW-31 Spring Water at DOE K-25 Site

Test 4

\begin{tabular}{||l|c|c|c|c|c||}
\hline & \multicolumn{5}{|c||}{ Oxidation Time $^{\mathbf{1}}(\mathbf{m i n})$} \\
\hline Compound $(\mu \mathrm{g} / \mathrm{l})$ & $\mathbf{0}$ & $\mathbf{0 . 5}$ & $\mathbf{1}$ & $\mathbf{2}$ & $\mathbf{3}$ \\
\hline Chloroethane & $\mathrm{NA}^{2}$ & 300 & 288 & 171 & 114 \\
\hline 1,1-Dichloroethane & $\mathrm{NA}$ & $\mathrm{NA}$ & $\mathrm{NA}$ & $\mathrm{NA}$ & NA \\
\hline 1,2-Dichloroethane & NA & & & & \\
\hline 1,1-Dichloroethene & NA & & & & \\
\hline 1,1,1-Trichloroethane & NA & 212 & 199 & 115 & 49 \\
\hline Trichlorofluoromethane & NA & NA & NA & NA & NA \\
\hline Vinyl Chloride & NA & 23 & 22 & 13 & 9 \\
\hline Benzene & NA & & & & \\
\hline Toluene & NA & & & & \\
\hline Ethylbenzene & NA & & & & \\
\hline m,p-Xylene & NA & & & & \\
\hline o-Xylene & NA & & & & \\
\hline
\end{tabular}

1 Oxidation Time calculated from ratio of UV contact volume to batch volume multiplied by the actual elapsed time.

$2 \quad$ Result not available. 
Table 4 (cont.)

Results of Perox-Pure ${ }^{\mathrm{TM}}$ Testing on SW-31 Spring Water at DOE K-25 Site

Test 5

\begin{tabular}{||l|c|c|c|c|c||}
\hline & \multicolumn{5}{|c||}{ Oxidation Time $^{\mathbf{1}}(\mathbf{m i n})$} \\
\hline Compound $(\mu \mathrm{g} / \mathrm{I})$ & $\mathbf{0}$ & $\mathbf{0 . 5}$ & $\mathbf{1}$ & $\mathbf{2}$ & $\mathbf{3}$ \\
\hline Chloroethane & 412 & $\mathrm{NA}^{2}$ & 361 & $\mathrm{NA}$ & $\mathrm{NA}$ \\
\hline 1,1-Dichloroethane & 298 & $\mathrm{NA}$ & 44 & 6 & $\mathrm{NA}$ \\
\hline 1,2-Dichloroethane & $<5$ & & & & \\
\hline 1,1-Dichloroethene & 57 & $<1$ & & & \\
\hline 1,1,1-Trichloroethane & 298 & $\mathrm{NA}$ & 139 & 45 & $\mathrm{NA}$ \\
\hline Trichlorofluoromethane & 206 & $\mathrm{NA}$ & 174 & 27 & $\mathrm{NA}$ \\
\hline Vinyl Chloride & $<5$ & & & & \\
\hline Benzene & $<5$ & & & & \\
\hline Toluene & 53 & $<1$ & & & \\
\hline Ethylbenzene & 9 & 1.3 & $<1$ & & \\
\hline m,p-Xylene & 14 & 1.4 & $<1$ & & \\
\hline o-Xylene & 34 & $<1$ & & & \\
\hline
\end{tabular}

1 Oxidation Time calculated from ratio of UV contact volume to batch volume multiplied by the actual elapsed time.

2 Result not available. 
Table 4 (cont.)

Results of Perox-Pure ${ }^{\mathrm{TM}}$ Testing

on SW-31 Spring Water at DOE K-25 Site

Test 6

\begin{tabular}{|l|c|c|c|c|c||}
\hline & \multicolumn{5}{|c|}{ Oxidation Time ${ }^{1}(\mathrm{~min})$} \\
\hline Compound $(\mu \mathrm{g} / \mathrm{l})$ & $\mathbf{0}$ & $\mathbf{0 . 5}$ & 1 & 2 & 3 \\
\hline Chloroethane & 316 & 281 & 236 & 149 & 88 \\
\hline 1,1-Dichloroethane & 501 & 139 & 29 & 5 & 3 \\
\hline 1,2-Dichloroethane & $<5$ & & & & \\
\hline 1,1-Dichloroethene & 54 & 2 & 1 & $<1$ & \\
\hline $1,1,1-$ Trichloroethane & 316 & 140 & 69 & 21 & 11 \\
\hline Trichlorofluoromethane & NA & NA & NA & NA & NA \\
\hline Vinyl Chloride & 30 & 21 & 18 & 10 & 6 \\
\hline Benzene & 29 & $<1$ & & & \\
\hline Toluene & 62 & 2 & $<1$ & & \\
\hline Ethylbenzene & 38 & 1 & $<1$ & & \\
\hline m,p-Xylene & 39 & 1 & $<1$ & & \\
\hline o-Xylene & 45 & 2 & $<1$ & & \\
\hline
\end{tabular}

1 Oxidation Time calculated from ratio of UV contact volume to batch volume multiplied by the actual elapsed time.

$2 \quad$ Result not available. 
Table 4 (cont.)

Results of Perox-Pure ${ }^{\mathrm{TM}}$ Testing

on SW-31 Spring Water at DOE K-25 Site

Test 7

\begin{tabular}{||l|c|c|c|c|c||}
\hline & \multicolumn{5}{|c||}{ Oxidation Time $^{\mathbf{1}}(\mathrm{min})$} \\
\hline Compound $(\mu \mathrm{g} / \mathrm{l})$ & $\mathbf{0}$ & $\mathbf{0 . 5}$ & $\mathbf{1}$ & $\mathbf{2}$ & $\mathbf{3}$ \\
\hline Chloroethane & 267 & 264 & 228 & 146 & 85 \\
\hline 1,1-Dichloroethane & 309 & 72 & 12 & 3 & 2 \\
\hline 1,2-Dichloroethane & $<5$ & & & & \\
\hline 1,1-Dichloroethene & $<5$ & & & & \\
\hline 1,1,1-Trichloroethane & 251 & 117 & 52 & 16 & 9 \\
\hline Trichlorofluoromethane & 300 & 140 & 115 & 72 & 61 \\
\hline Vinyl Chloride & 17 & 20 & 16 & 9 & 5 \\
\hline Benzene & $<5$ & & & & \\
\hline Toluene & $<5$ & & & & \\
\hline Ethylbenzene & $<5$ & & & & \\
\hline m,p-Xylene & $<5$ & & & & \\
\hline o-Xylene & $<5$ & & & & \\
\hline
\end{tabular}

1 Oxidation Time calculated from ratio of UV contact volume to batch volume multiplied by the actual elapsed time. 
Table 4 (cont.)

Results of Perox-Pure ${ }^{\mathrm{TM}}$ Testing

on SW-31 Spring Water at DOE K-25 Site

Test 8

\begin{tabular}{||l|c|c|c|c|c||}
\hline & \multicolumn{5}{|c||}{ Oxidation Time ${ }^{1}(\mathbf{m i n})$} \\
\hline Compound $(\mu \mathrm{g} / \mathrm{l})$ & $\mathbf{0}$ & $\mathbf{0 . 5}$ & $\mathbf{1}$ & $\mathbf{2}$ & $\mathbf{3}$ \\
\hline Chloroethane & 448 & 351 & 321 & 232 & 163 \\
\hline 1,1-Dichloroethane & 555 & 124 & 31 & 4 & 2 \\
\hline 1,2-Dichloroethane & 6 & 2 & $<1$ & & \\
\hline 1,1-Dichloroethene & 71 & 3 & $<1$ & & \\
\hline 1,1,1-Trichloroethane & 395 & 184 & 127 & 45 & 20 \\
\hline Trichlorofluoromethane & 215 & 173 & 148 & 67 & 30 \\
\hline Vinyl Chloride & 38 & 26 & 24 & 16 & 10 \\
\hline Benzene & 56 & 2 & $<1$ & & \\
\hline Toluene & 59 & 2 & $<1$ & & \\
\hline Ethylbenzene & 28 & 1 & $<1$ & & \\
\hline m,p-Xylene & 55 & 2 & $<1$ & & \\
\hline o-Xylene & 47 & 2 & $<1$ & & \\
\hline
\end{tabular}

1 Oxidation Time calculated from ratio of UV contact volume to batch volume multiplied by the actual elapsed time. 
Table 4 (cont.)

Results of Perox-Pure ${ }^{\mathrm{TM}}$ Testing on SW-31 Spring Water at DOE K-25 Site

Test 9

\begin{tabular}{||l|c|c|c|c|c||}
\hline & \multicolumn{5}{|c||}{ Oxidation Time $^{\mathbf{1}}$ (min) } \\
\hline Compound $(\mu \mathrm{g} / \mathrm{l})$ & $\mathbf{0}$ & $\mathbf{0 . 5}$ & $\mathbf{1}$ & $\mathbf{2}$ & $\mathbf{3}$ \\
\hline Chloroethane & 274 & 217 & 172 & 116 & 79 \\
\hline 1,1-Dichloroethane & 418 & 183 & 105 & 33 & 9 \\
\hline 1,2-Dichloroethane & 5 & 3 & 2 & $<1$ & \\
\hline 1,1-Dichloroethene & 15 & $<1$ & & & \\
\hline 1,1,1-Trichloroethane & 263 & 187 & 156 & 102 & 60 \\
\hline Trichlorofluoromethane & 134 & 52 & 32 & 18 & 16 \\
\hline Vinyl Chloride & 17 & 16 & 13 & 9 & 6 \\
\hline Benzene & 10 & $<1$ & & & \\
\hline Toluene & 9 & $<1$ & & & \\
\hline Ethylbenzene & $<5$ & & & & \\
\hline m,p-Xylene & 8 & $<1$ & & & \\
\hline o-Xylene & 13 & $<1$ & & & \\
\hline
\end{tabular}

1 Oxidation Time calculated from ratio of UV contact volume to batch volume multiplied by the actual elapsed time. 
Table 4 (cont.)

Results of Perox-Pure ${ }^{\mathrm{TM}}$ Testing

on SW-31 Spring Water at DOE K-25 Site

Test 10

\begin{tabular}{||l|l|c|c|c|c||}
\hline & \multicolumn{5}{|c||}{ Oxidation Time $^{\mathbf{1}}$ (min) } \\
\hline Compound $(\mu \mathrm{g} / \mathrm{l})$ & $\mathbf{0}$ & $\mathbf{0 . 5}$ & $\mathbf{1}$ & $\mathbf{2}$ & $\mathbf{3}$ \\
\hline Chloroethane & & & 306 & 221 & \\
\hline 1,1-Dichloroethane & & & 18 & 3 & \\
\hline 1,2-Dichloroethane & & & & & \\
\hline 1,1-Dichloroethene & & & & & \\
\hline 1,1,1-Trichloroethane & & & 83 & 19 & \\
\hline Trichlorofluoromethane & & & 151 & 98 & \\
\hline Vinyl Chloride & & & & & \\
\hline Benzene & & & & & \\
\hline Toluene & & & & & \\
\hline Ethylbenzene & & & & & \\
\hline m,p-Xylene & & & & & \\
\hline o-Xylene & & & & & \\
\hline
\end{tabular}

1 Oxidation Time calculated from ratio of UV contact volume to batch volume multiplied by the actual elapsed time. 
Table 4 (cont.)

Results of Perox-Pure ${ }^{\mathrm{TM}}$ Testing

on SW-31 Spring Water at DOE K-25 Site

Test 11: Batch

\begin{tabular}{||l|c|c|c|c|c||}
\hline & \multicolumn{5}{|c||}{ Oxidation Time $^{\mathbf{1}}(\mathbf{m i n})$} \\
\hline Compound $(\mu \mathrm{g} / \mathbf{l})$ & $\mathbf{0}$ & $\mathbf{0 . 5}$ & $\mathbf{1}$ & $\mathbf{2}$ & $\mathbf{3}$ \\
\hline Chloroethane & 535 & 385 & 352 & 286 & 197 \\
\hline 1,1-Dichloroethane & 640 & 231 & 126 & 10 & 3 \\
\hline 1,2-Dichloroethane & $<5$ & & & & \\
\hline 1,1-Dichloroethene & 48 & $<1$ & & & \\
\hline 1,1,1-Trichloroethane & 460 & 190 & 130 & 51 & 16 \\
\hline Trichlorofluoromethane & 473 & 191 & 167 & 140 & 100 \\
\hline Vinyl Chloride & 36 & 130 & 168 & 49 & 13 \\
\hline Benzene & 12 & 2 & $<1$ & & \\
\hline Toluene & 16 & $<1$ & & & \\
\hline Ethylbenzene & 6 & $<1$ & & & \\
\hline m,p-Xylene & 8 & $<1$ & & & \\
\hline o-Xylene & 12 & $<1$ & & & \\
\hline \hline
\end{tabular}

1 Oxidation Time calculated from ratio of UV contact volume to batch volume multiplied by the actual elapsed time. 
Table 4 (cont.)

Results of Perox-Pure ${ }^{\mathrm{TM}}$ Testing on SW-31 Spring Water at DOE K-25 Site

Test 11: Flow-Through

\begin{tabular}{||l|c|c||}
\hline & \multicolumn{2}{|c||}{ Oxidation Time ${ }^{\mathbf{1}}$ (min) } \\
\hline Compound $(\mu \mathrm{g} / \mathrm{l})$ & $\mathbf{0}$ (Influent) & $\mathbf{0 . 4}$ (Lamp 6) \\
\hline Chloroethane & 352 & 258 \\
\hline 1,1-Dichloroethane & 126 & 28 \\
\hline 1,2-Dichloroethane & & \\
\hline 1,1-Dichloroethene & & 66 \\
\hline 1,1,1-Trichloroethane & 130 & 127 \\
\hline Trichlorofluoromethane & NA $^{2}$ & \\
\hline Vinyl Chloride & & \\
\hline Benzene & & \\
\hline Toluene & & \\
\hline Ethylbenzene & & \\
\hline m,p-Xylene & & \\
\hline o-Xylene & & \\
\hline
\end{tabular}

1 Flow - through oxidation time.

2 Result no available. 
Table 4 (cont.)

Results of Perox-Pure ${ }^{\mathrm{TM}}$ Testing

on SW-31 Spring Water at DOE K-25 Site

Test 12

\begin{tabular}{||l|c|c|c|c|c||}
\hline & \multicolumn{5}{|c||}{ Oxidation Time $^{\mathbf{1}}(\mathbf{m i n})$} \\
\hline Compound $(\mu \mathrm{g} / \mathrm{l})$ & $\mathbf{0}$ & $\mathbf{0 . 5}$ & $\mathbf{1}$ & $\mathbf{2}$ & $\mathbf{3}$ \\
\hline Chloroethane & 308 & 279 & 266 & 227 & 184 \\
\hline 1,1-Dichloroethane & 409 & 233 & 73 & 57 & 16 \\
\hline 1,2-Dichloroethane & $<5$ & 3 & 3 & 1 & $<1$ \\
\hline 1,1-Dichloroethene & 34 & $<1$ & & & \\
\hline 1,1,1-Trichloroethane & 277 & 154 & 119 & 69 & 36 \\
\hline Trichlorofluoromethane & 175 & 113 & 103 & 78 & 57 \\
\hline Vinyl Chloride & 33 & $<1$ & & & \\
\hline Benzene & 18 & 6 & 3 & $<1$ & \\
\hline Toluene & 33 & $<1$ & & & \\
\hline Ethylbenzene & 15 & $<1$ & & & \\
\hline m,p-Xylene & 18 & $<1$ & & & \\
\hline o-Xylene & 26 & $<1$ & & & \\
\hline
\end{tabular}

Oxidation Time calculated from ratio of UV contact volume to batch volume multiplied by the actual elapsed time. 
Table 4 (cont.)

Results of Perox-Pure TM Testing

on SW-31 Spring Water at DOE K-25 Site

Test 13

\begin{tabular}{|l|c|c|c|c|c||}
\hline & \multicolumn{5}{|c|}{ Oxidation Time ${ }^{1}(\mathbf{m i n})$} \\
\hline Compound $(\mu \mathrm{g} / \mathrm{l})$ & $\mathbf{0}$ & $\mathbf{0 . 5}$ & $\mathbf{1}$ & $\mathbf{2}$ & $\mathbf{3}$ \\
\hline Chloroethane & 396 & 356 & 324 & 245 & 197 \\
\hline 1,1-Dichloroethane & 483 & 264 & 183 & 61 & 18 \\
\hline 1,2-Dichloroethane & $<5$ & 4 & 2 & $<1$ & \\
\hline 1,1-Dichloroethene & $<5$ & & & & \\
\hline 1,1,1-Trichloroethane & 354 & 215 & 189 & 120 & 71 \\
\hline Trichlorofluoromethane & 244 & 187 & 167 & 105 & 80 \\
\hline Vinyl Chloride & 49 & 47 & 40 & 27 & 19 \\
\hline Benzene & 7 & 2 & $<1$ & & \\
\hline Toluene & $<5$ & & & & \\
\hline Ethylbenzene & $<5$ & & & & \\
\hline m,p-Xylene & $<5$ & & & & \\
\hline o-Xylene & $<5$ & & & & \\
\hline
\end{tabular}

1 Oxidation Time calculated from ratio of UV contact volume to batch volume multiplied by the actual elapsed time. 
Table 4 (cont.)

Results of Perox-Pure ${ }^{\mathrm{TM}}$ Testing on SW.31 Spring Water at DOE K.25 Site

Test 14

\begin{tabular}{|l|c|c|c|c|c||}
\hline & \multicolumn{5}{|c|}{ Oxidation Time $^{\mathbf{1}}(\mathbf{m i n})$} \\
\hline Compound $(\mu \mathrm{g} / \mathrm{l})$ & $\mathbf{0}$ & $\mathbf{0 . 5}$ & $\mathbf{1}$ & $\mathbf{2}$ & 3 \\
\hline Chloroethane & 368 & 345 & 323 & 306 & 270 \\
\hline 1,1-Dichloroethane & 410 & 236 & 224 & 106 & 65 \\
\hline 1,2-Dichloroethane & $<5$ & 3 & 3 & 1 & $<1$ \\
\hline 1,1-Dichloroethene & $<5$ & & & & \\
\hline 1,1,1-Trichloroethane & 334 & 217 & 206 & 176 & 134 \\
\hline Trichlorofluoromethane & 221 & 200 & 178 & 149 & 119 \\
\hline Vinyl Chloride & $<5$ & & & & \\
\hline Benzene & $<5$ & & & & \\
\hline Toluene & $<5$ & & & & \\
\hline Ethylbenzene & $<5$ & & & & \\
\hline m,p-Xylene & $<5$ & & & & \\
\hline o-Xylene & $<5$ & & & & \\
\hline
\end{tabular}

1 Oxidation Time calculated from ratio of UV contact volume to batch volume multiplied by the actual elapsed time. 


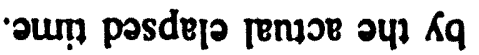

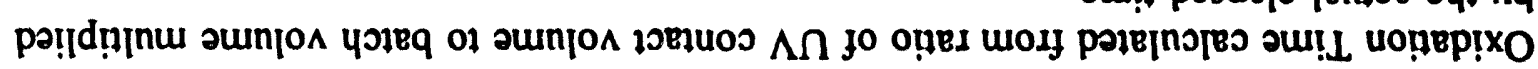

\begin{tabular}{|c|c|c|c|c|c|}
\hline$\tau$ & $\tau$ & & $\tau$ & $\boldsymbol{S}$ & จนข|KX-0 \\
\hline \multirow[t]{2}{*}{$b$} & b & & t & $L$ & วนข|אX-d'w \\
\hline & & & & I> & 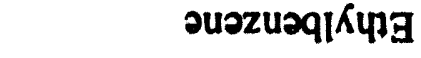 \\
\hline$\tau$ & $\tau$ & & $\tau$ & $\boldsymbol{S}$ & auən|0ل \\
\hline \multirow[t]{3}{*}{$\tau$} & $\tau$ & & $\tau$ & OI & ourzuog \\
\hline & & & & I> & 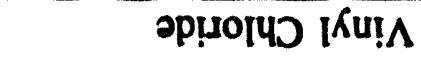 \\
\hline & & & & I> & 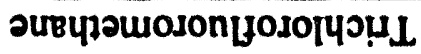 \\
\hline $9 I$ & 82 & & ILI & $6 E E$ & 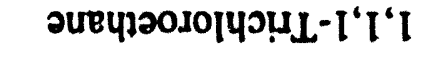 \\
\hline \multirow[t]{2}{*}{$\nabla I$} & $\nabla l$ & & $\nabla l$ & $\nabla l$ & әแәчрә0دо|Чग!ด-I'I \\
\hline & & & & I> & 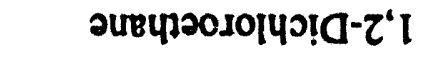 \\
\hline \multirow[t]{2}{*}{$\nabla$} & $\mathbf{S}$ & & 66 & $\varepsilon \nabla \varepsilon$ & 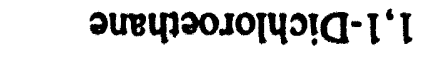 \\
\hline & & & & I> & 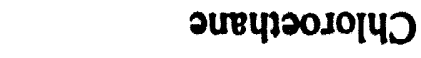 \\
\hline $\boldsymbol{\varepsilon}$ & $\boldsymbol{z}$ & $\boldsymbol{I}$ & S:O & $\mathbf{0}$ & (I/8rt) punoduros \\
\hline
\end{tabular}

40189:SI 150]

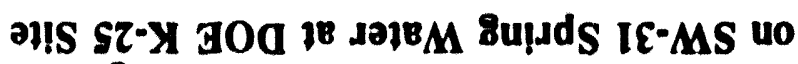

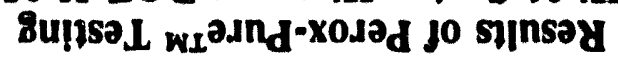

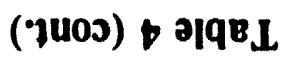


Table 4 (cont.)

Results of Perox-Pure ${ }^{\mathrm{TM}}$ Testing on SW-31 Spring Water at DOE K-25 Site

Test 15: Flow-Through

\begin{tabular}{|c|c|c|c|}
\hline & \multicolumn{3}{|c|}{ Oxidation Time ${ }^{1}$ (min) } \\
\hline Compound $(\mu \mathrm{g} / \mathrm{l})$ & $\begin{array}{c}0 \\
\text { (Influent) }\end{array}$ & $0.2($ Lamp 3) & 0.4 (Lamp 6) \\
\hline \multicolumn{4}{|l|}{ Chloroethane } \\
\hline 1,1-Dichloroethane & 13 & $<1$ & $<1$ \\
\hline \multicolumn{4}{|l|}{ 1,2-Dichloroethane } \\
\hline \multicolumn{4}{|l|}{ 1,1-Dichloroethene } \\
\hline 1,1,1-Trichloroethane & 97 & 50 & 53 \\
\hline \multicolumn{4}{|l|}{ Trichlorofluoromethane } \\
\hline \multicolumn{4}{|l|}{ Vinyl Chloride } \\
\hline Benzene & 2 & $<1$ & \\
\hline Toluene & 2 & $<1$ & \\
\hline \multicolumn{4}{|l|}{ Ethylbenzene } \\
\hline m,p-Xylene & 4 & $<1$ & \\
\hline o-Xylene & 2 & $<1$ & \\
\hline
\end{tabular}

1 Flow - through oxidation time. 
Table 4 (cont.)

Results of Perox-Pure ${ }^{\mathrm{TM}}$ Testing

on SW-31 Spring Water at DOE K-25 Site

Test 16: Batch

\begin{tabular}{||l|c|c|c|c|c||}
\hline & \multicolumn{5}{|c||}{ Oxidation Time ${ }^{\mathbf{1}}(\mathbf{m i n})$} \\
\hline Compound $(\mu \mathrm{g} / \mathrm{l})$ & $\mathbf{0}$ & $\mathbf{0 . 5}$ & $\mathbf{1}$ & $\mathbf{2}$ & $\mathbf{3}$ \\
\hline Chloroethane & $<1$ & & $\mathrm{~N}$ & & \\
\hline 1,1-Dichloroethane & 343 & 99 & & 5 & 4 \\
\hline 1,2-Dichloroethane & $<1$ & & & & \\
\hline 1,1-Dichloroethene & 14 & $<1$ & & & \\
\hline 1,1,1-Trichloroethane & 340 & 171 & & 28 & 16 \\
\hline Trichlorofluoromethane & 439 & 355 & & 229 & 161 \\
\hline Vinyl Chloride & 30 & 22 & & 12 & 7 \\
\hline Benzene & 109 & 2 & & 2 & $<1$ \\
\hline Toluene & 6 & 2 & & 2 & $<1$ \\
\hline Ethylbenzene & $<1$ & 2 & & 2 & $<1$ \\
\hline m,p-Xylene & 8 & 2 & & 2 & 5 \\
\hline o-Xylene & 5 & 2 & & 2 & $<1$ \\
\hline
\end{tabular}

1 Oxidation Time calculated from ratio of UV contact volume to batch volume multiplied by the actual elapsed time. 
Table 4 (cont.)

Results of Perox-Pure ${ }^{\mathrm{TM}}$ Testing

on SW-31 Spring Water at DOE K-25 Site

Test 16: Flow-Through

\begin{tabular}{|c|c|c|c|}
\hline \multirow[b]{2}{*}{ Compound $(\mu \mathrm{g} / \mathrm{l})$} & \multicolumn{3}{|c|}{ Oxidation Time ${ }^{1}$ (min) } \\
\hline & $\begin{array}{c}0 \\
\text { (Influent) }\end{array}$ & 0.2 (Lamps) & 0.4 (Lamp 6) \\
\hline Chloroethane & $<1$ & & \\
\hline 1,1-Dichloroethane & 13 & $<1$ & \\
\hline 1,2-Dichloroethane & $<1$ & & \\
\hline 1,1-Dichloroethene & $<1$ & & \\
\hline 1,1,1-Trichloroethane & 97 & 50 & 53 \\
\hline Trichlorofluoromethane & 339 & 273 & 317 \\
\hline Vinyl Chloride & 20 & 15 & 18 \\
\hline Benzene & 2 & $<1$ & \\
\hline Toluene & 2 & $<1$ & \\
\hline Ethylbenzene & 2 & $<1$ & \\
\hline m,p-Xylene & 4 & $<1$ & \\
\hline 0-Xylene & 2 & $<1$ & \\
\hline
\end{tabular}

1 Flow - through oxidation time. 


\title{
APPENDIX V. RESULTS OF SD-2 TESTING ON SW-31 SPRING WATER AT DOE K-25 SITE
}

Table 1

\author{
Results of SD-2 Testing \\ on SW-31 Spring Water at DOE K-25 Site
}

Test 1A

\begin{tabular}{||l|c|c|c|c||}
\hline & \multicolumn{4}{|c||}{ Oxidation Time ${ }^{1}(\mathbf{m i n})$} \\
\hline Compound $(\mu \mathrm{g} / \mathrm{l})$ & $\mathbf{0}$ & $\mathbf{2}$ & $\mathbf{4}$ & $\mathbf{6}$ \\
\hline Chloroethane & 290 & 176 & 161 & 145 \\
\hline Chloroform & $<5$ & $<1$ & & \\
\hline 1,1-Dichloroethane & 322 & 152 & 113 & 82 \\
\hline 1,2-Dichloroethane & $<5$ & $<1$ & & \\
\hline 1,2-Dichloroethene & $<5$ & $<1$ & & \\
\hline Methylene Chloride & $<5$ & $<1$ & & \\
\hline Tetrachloroethene & $<5$ & $<1$ & & \\
\hline 1,1,1-Trichloroethane & 186 & 57 & 20 & 5 \\
\hline 1,1,2-Trichloroethane & $<5$ & $<1$ & & \\
\hline Trichloroethene & $<5$ & $<1$ & & \\
\hline Benzene & 6 & $<1$ & & \\
\hline Toluene & 7 & $<1$ & & \\
\hline Ethylbenzene & $<5$ & $<1$ & & \\
\hline m,p-Xylene & $<5$ & $<1$ & & \\
\hline o-Xylene & $<5$ & $<1$ & & \\
\hline 1,2-Dichlorobenzene & $<5$ & $<1$ & & \\
\hline 1,3-Dichlorobenzene & $<5$ & $<1$ & & \\
\hline
\end{tabular}

1 Oxidation time calculated from ratio of UV contact voluine to batch volume multiplied by the elapsed time. 
Table 1 (cont.)

Results of SD-2 Testing

on SW-31 Spring Water at DOE K-25 Site

Test 2A

\begin{tabular}{||l|c|c|c|c||}
\hline & \multicolumn{4}{|c|}{ Oxidation Time $^{1}(\mathbf{m i n})$} \\
\hline Compound $(\mu \mathrm{g} / \mathrm{l})$ & $\mathbf{0}$ & $\mathbf{2}$ & $\mathbf{4}$ & $\mathbf{6}$ \\
\hline Chloroethane & 243 & 215 & 203 & 163 \\
\hline Chloroform & $<5$ & $<1$ & & \\
\hline 1,1-Dichloroethane & 258 & 161 & 109 & 59 \\
\hline 1,2-Dichloroethane & $<5$ & 3 & 3 & 3 \\
\hline 1,1-Dichloroethene & 7 & $<1$ & & \\
\hline Methylene Chloride & $<5$ & $<1$ & & \\
\hline Tetrachloroethene & $<5$ & $<1$ & & \\
\hline 1,1,1-Trichloroethane & 131 & 34 & 5 & 1 \\
\hline 1,1,2-Trichloroethane & $<5$ & 2 & 2 & 1 \\
\hline Trichloroethene & $<5$ & $<1$ & & \\
\hline Benzene & $<5$ & $<1$ & & \\
\hline Toluene & 6 & $<1$ & & \\
\hline Ethylbenzene & $<5$ & $<1$ & & \\
\hline m,p-Xylene & $<5$ & $<1$ & & \\
\hline o-Xylene & $<5$ & $<1$ & & \\
\hline Trichlorofluoromethane & 115 & 69 & 46 & 26 \\
\hline Vinyl Chloride & 17 & 63 & $<1$ & \\
\hline
\end{tabular}

Oxidation time calculated from ratio of UV contact volume to batch volume multiplied by the elapsed time. 
Table 1 (cont.)

Results of SD-2 Testing

on SW-31 Spring Water at DOE K-25 Site

Test 3A

\begin{tabular}{|c|c|c|c|c|}
\hline \multirow[b]{2}{*}{ Compound $(\mu \mathrm{g} / \mathrm{l})$} & \multicolumn{4}{|c|}{ Oxidation Time ${ }^{1}$ (min) } \\
\hline & $\mathbf{0}$ & 3 & 6 & 9 \\
\hline Chloroethane & 11 & 7 & 3 & $<1$ \\
\hline Chloroform & 1 & $<1$ & & \\
\hline 1,1-Dichloroethane & 239 & 195 & 145 & 107 \\
\hline 1,2-Dichloroethane & 2 & 2 & 1 & $<1$ \\
\hline 1,1-Dichloroethene & $<1$ & $<1$ & & \\
\hline Methylene Chloride & 78 & 72 & 60 & 54 \\
\hline Tetrachloroethene & $<1$ & & & \\
\hline 1,1,1-Trichloroethane & 199 & 148 & 103 & 70 \\
\hline 1,1,2-Trichloroethane & 3 & 2 & 2 & 2 \\
\hline Trichloroethene & $<1$ & & & \\
\hline Trichlorofluoromethane & 139 & 132 & 117 & 105 \\
\hline Benzene & 2 & $<1$ & & \\
\hline Toluene & $<1$ & & & \\
\hline Ethylbenzene & $<1$ & & & \\
\hline m,p-Xylene & $<1$ & & & \\
\hline 0-Xylene & $<1$ & & & \\
\hline
\end{tabular}

Oxidation time calculated from ratio of UV contact volume to batch volume multiplied by the elapsed time. 
Table 1 (cont.)

Results of SD-2 Testing

on SW-31 Spring Water at DOE K-25 Site

Test 4A

\begin{tabular}{|c|c|c|c|c|}
\hline \multirow[b]{2}{*}{ Compound $(\mu \mathrm{g} / \mathrm{l})$} & \multicolumn{4}{|c|}{ Oxidation Time ${ }^{1}(\mathrm{~min})$} \\
\hline & $\mathbf{0}$ & 3 & 6 & 9 \\
\hline Chloroethane & 17 & 4 & 4 & $<1$ \\
\hline Chloroform & $<1$ & & & \\
\hline 1,1-Dichloroethane & 274 & 215 & 149 & 106 \\
\hline 1,2-Dichloroethane & 2 & 2 & 1 & $<1$ \\
\hline 1,1-Dichloroethene & $<1$ & & & \\
\hline Methylene Chloride & 101 & 81 & 66 & 56 \\
\hline Tetrachloroethene & $<1$ & & & \\
\hline 1,1,1-Trichloroethane & 232 & 175 & 117 & 85 \\
\hline 1,1,2-Trichloroethane & 3 & 2 & 2 & 2 \\
\hline Trichloroethene & $<1$ & & & \\
\hline Trichlorofluoromethane & 195 & 142 & 147 & 107 \\
\hline Benzene & 3 & $<1$ & & \\
\hline Toluene & 2 & $<1$ & & \\
\hline Ethylbenzene & $<1$ & & & \\
\hline m,p-Xylene & $<1$ & & & \\
\hline o-Xylene & $<1$ & & & \\
\hline
\end{tabular}

1 Oxidation time calculated from ratio of UV contact volume to batch volume multiplied by the elapsed time. 
Table 1 (cont.)

Results of SD-2 Testing

on SW-31 Spring Water at DOE K-25 Site

Test 5A

\begin{tabular}{|c|c|c|c|c|}
\hline \multirow[b]{2}{*}{ Compound $(\mu \mathrm{g} / \mathrm{l})$} & \multicolumn{4}{|c|}{ Oxidation Time ${ }^{1}$ (min) } \\
\hline & $\mathbf{0}$ & 3 & 6 & 9 \\
\hline Chloroethane & 13 & 1 & $<1$ & \\
\hline Chloroform & $<1$ & & & \\
\hline 1,1-Dichloroethane & 265 & 139 & 42 & 10 \\
\hline 1,2-Dichloroethane & 2 & $<1$ & & \\
\hline 1,1-Dichloroethene & $<1$ & & & \\
\hline Methylene Chloride & 101 & 91 & 72 & 56 \\
\hline Tetrachloroethene & $<1$ & & & \\
\hline 1,1,1-Trichloroethane & 242 & 196 & 132 & 86 \\
\hline 1,1,2-Trichloroethane & 3 & 2 & $<1$ & \\
\hline Trichloroethene & $<1$ & & & \\
\hline Benzene & 4 & $<1$ & & \\
\hline Toluene & 2 & $<1$ & & \\
\hline Ethylbenzene & $<1$ & & & \\
\hline m,p-Xylene & $<1$ & & & \\
\hline o-Xylene & $<1$ & & & \\
\hline
\end{tabular}

1 Oxidation time calculated from ratio of UV contact volume to batch volume multiplied by the elapsed time. 
Table 1 (cont.)

Results of SD-2 Testing on SW-31 Spring Water at DOE K-25 Site

Test 6A

\begin{tabular}{||l|c|c|c|c||}
\hline & \multicolumn{4}{|c|}{ Oxidation Time $^{\mathbf{1}}$ (min) } \\
\hline Compound $(\mu \mathrm{g} / \mathrm{l})$ & $\mathbf{0}$ & $\mathbf{3}$ & $\mathbf{6}$ & $\mathbf{9}$ \\
\hline Chloroethane & 15 & 2 & $<1$ & \\
\hline Chloroform & $<1$ & & & \\
\hline 1,1-Dichloroethane & 277 & 139 & 40 & 12 \\
\hline 1,2-Dichloroethane & 2 & $<1$ & & \\
\hline 1,2-Dichloroethene & $<1$ & & & \\
\hline Methylene Chloride & 107 & 89 & 68 & 59 \\
\hline Tetrachloroethene & $<1$ & & & \\
\hline 1,1,1-Trichloroethane & 242 & 180 & 123 & 84 \\
\hline 1,1,2-Trichloroethane & $<1$ & & & \\
\hline Trichloroethene & $<1$ & & & \\
\hline Trichlorofluoromethane & 187 & 160 & 128 & 115 \\
\hline Benzene & 2 & $<1$ & & \\
\hline Toluene & $<1$ & & & \\
\hline Ethylbenzene & $<1$ & & & \\
\hline m,p-Xylene & $<1$ & & & \\
\hline o-Xylene & $<1$ & & & \\
\hline
\end{tabular}

Oxidation time calculated from ratio of UV contact volume to batch volume multiplied by the elapsed time. 
Table 1 (cont.)

Results of SD-2 Testing

on SW-31 Spring Water at DOE K-25 Site

Test 7A

\begin{tabular}{|c|c|c|c|c|}
\hline \multirow[b]{2}{*}{ Compound $(\mu \mathrm{g} / \mathrm{l})$} & \multicolumn{4}{|c|}{ Oxidation 1.me $\left.\mathrm{m}^{\mathbf{1}} \mathrm{min}\right)$} \\
\hline & 0 & 3 & 6 & 9 \\
\hline Chloroethane & 18 & 2 & $<1$ & \\
\hline Chloroform & $<1$ & & & \\
\hline 1,1-Dichloroethane & 240 & 119 & 32 & 10 \\
\hline 1,2-Dichloroethane & 2 & $<1$ & & \\
\hline 1,1-Dichloroethene & $<1$ & $<1$ & & \\
\hline Methylene Chloride & 111 & 89 & 61 & 63 \\
\hline Tetrachloroethene & $<1$ & & & \\
\hline 1,1,1-Trichloroethane & 232 & 173 & 102 & 69 \\
\hline 1,1,2-Trichloroethane & 3 & 1 & $<1$ & \\
\hline Trichloroethene & $<1$ & & & \\
\hline Trichlorofluoromethane & 264 & 226 & 112 & 163 \\
\hline Benzene & 2 & $<1$ & & \\
\hline Toluene & $<1$ & & & \\
\hline Ethylbenzene & $<1$ & & & \\
\hline m,p-Xylene & $<1$ & & & \\
\hline o-Xylene & $<1$ & & & \\
\hline
\end{tabular}

Oxidation time calculated from ratio of UV contact volume to batch volume multiplied by the elapsed time. 
Table 1 (cont.)

Results of SD-2 Testing

on SW-31 Spring Water at DOE K-25 Site

Test 8A

\begin{tabular}{|c|c|c|c|c|}
\hline \multirow[b]{2}{*}{ Compound $(\mu \mathrm{g} / \mathrm{l})$} & \multicolumn{4}{|c|}{ Oxidation Time ${ }^{1}$ (min) } \\
\hline & $\mathbf{0}$ & 3 & 6 & 9 \\
\hline Chloroethane & 35 & 33 & 27 & 25 \\
\hline Chloroform & 3 & 1 & $<1$ & \\
\hline 1,1-Dichloroethane & 400 & 323 & 331 & 321 \\
\hline 1,2-Dichloroethane & 3 & 3 & 3 & 3 \\
\hline 1,2-Dichloroethene & 2 & 5 & 4 & 3 \\
\hline Methylene Chloride & 169 & 149 & 128 & 117 \\
\hline Tetrachloroethene & 4 & $<1$ & & \\
\hline 1,1,1-Trichloroethane & 326 & 298 & 257 & 232 \\
\hline 1,1,2-Trichloroethane & 4 & 4 & 3 & 3 \\
\hline Trichloroethene & 8 & 4 & $<1$ & \\
\hline Trichlorofluormethane & 354 & 337 & 275 & 263 \\
\hline Benzene & 54 & 38 & 26 & 17 \\
\hline Toluene & 52 & 32 & 18 & 11 \\
\hline Ethylbenzene & 23 & 16 & 9 & 6 \\
\hline m,p-Xylene & 80 & 44 & 18 & 10 \\
\hline o-Xylene & 39 & 22 & 10 & 6 \\
\hline
\end{tabular}

Oxidation time calculated from ratio of UV contact volume to batch volume multiplied by the elapsed time. 
Table 1 (cont.)

Results of SD-2 Testing

on SW-31 Spring Water at DOE K-25 Site

Test 9A

\begin{tabular}{||l|c|c|c|c||}
\hline & \multicolumn{4}{|c|}{ Oxidation Time ${ }^{1}(\mathbf{m i n})$} \\
\hline Compound $(\mu \mathrm{g} / \mathrm{l})$ & $\mathbf{0}$ & $\mathbf{3}$ & $\mathbf{6}$ & $\mathbf{9}$ \\
\hline Chloroethane & 18 & $\mathrm{NA}^{2}$ & 11 & 13 \\
\hline Chloroform & 2 & $<1$ & & \\
\hline 1,1-Dichloroethane & 291 & 238 & 218 & 183 \\
\hline 1,2-Dichloroethane & 3 & 3 & 2 & 2 \\
\hline 1,2-Dichloroethene & 1 & $<1$ & & \\
\hline Methylene Chloride & 89 & 79 & 70 & 57 \\
\hline Tetrachloroethene & 1 & $<1$ & & \\
\hline 1,1,1-Trichloroethane & 214 & 194 & 155 & 119 \\
\hline $1,1,2$-Trichloroethane & 4 & 4 & 4 & 5 \\
\hline Trichloroethene & 3 & $<1$ & & \\
\hline Trichlorofluoromethane & 190 & 172 & 145 & 130 \\
\hline Carbon Tetrachloride & $<1$ & 2 & 4 & 4 \\
\hline Benzene & 20 & $<1$ & & \\
\hline Toluene & 17 & $<1$ & & \\
\hline Ethylbenzene & 8 & $<1$ & & \\
\hline m,p-Xylene & 22 & $<1$ & & \\
\hline o-Xylene & 11 & $<1$ & & \\
\hline
\end{tabular}

1 Oxidation time calculated from ratio of UV contact volume to batch volume multiplied by the elapsed time.

$2 \quad$ Result not available. 
Table 1 (cont.)

Results of SD-2 Testing

on SW-31 Spring Water at DOE K-25 Site

Test 10A

\begin{tabular}{||l|c|c|c|c||}
\hline & \multicolumn{4}{|c|}{${\text { Oxidation } \text { Time }^{1}(\mathbf{m i n})}$} \\
\hline Compound $(\mu \mathrm{g} / \mathrm{l})$ & 0 & 3 & 6 & 9 \\
\hline Chloroethane & 27 & 22 & 19 & 19 \\
\hline Chloroform & 1 & 3 & 3 & 2 \\
\hline 1,1-Dichloroethane & 394 & 339 & 278 & 231 \\
\hline 1,2-Dichloroethane & 3 & 3 & 2 & 2 \\
\hline 1,2-Dichloroethene & 3 & 1 & $<1$ & \\
\hline Methylene Chloride & 133 & 118 & 105 & 86 \\
\hline Tetrachloroethene & 2 & $<1$ & & \\
\hline 1,1,1-Trichloroethane & 313 & 263 & 206 & 164 \\
\hline $1,1,2-$ Trichloroethane & 4 & 4 & 5 & 6 \\
\hline Trichloroethene & 6 & $<1$ & & \\
\hline Trichlorofluoromethane & 276 & 260 & 220 & 188 \\
\hline Carbon Tetrachloride & 6 & 6 & 5 & 6 \\
\hline Benzene & 39 & 4 & 1 & 1 \\
\hline Toluene & 34 & 2 & $<1$ & \\
\hline Ethylbenzene & 16 & 2 & $<1$ & \\
\hline m,p-Xylene & 46 & 2 & $<1$ & \\
\hline o-Xylene & 24 & 2 & $<1$ & \\
\hline
\end{tabular}

1 Oxidation time calculated from ratio of UV contact volume to batch volume multiplied by the elapsed time. 
Table 1 (cont.)

Results of SD.2 Testing

on SW-31 Spring Water at DOE K-25 Site

Test 11A

\begin{tabular}{|c|c|c|c|}
\hline \multirow[b]{2}{*}{ Compound $(\mu \mathrm{g} / \mathrm{l})$} & \multicolumn{3}{|c|}{ Oxidation Time ${ }^{1}$ (min) } \\
\hline & $0^{2}$ & 3 & 6 \\
\hline Chloroethane & $<1$ & & \\
\hline Chloroform & $<1$ & & \\
\hline 1,1-Dichloroethane & 83 & 55 & 34 \\
\hline 1,2-Dichloroethane & $<1$ & & \\
\hline 1,2-Dichloroethene & $<1$ & & \\
\hline Methylene Chloride & 107 & 90 & 73 \\
\hline Tetrachloroethene & $<1$ & & \\
\hline 1,1,1-Trichloroethane & 178 & 144 & 113 \\
\hline 1,1,2-Trichloroethane & 1 & $<1$ & \\
\hline Trichloroethene & $<1$ & & \\
\hline Trichlorofluoromethane & 242 & 205 & 173 \\
\hline Benzene & $<1$ & & \\
\hline Toluene & $<1$ & & \\
\hline Ethylbenzene & $<1$ & & \\
\hline m,p-Xylene & $<1$ & & \\
\hline o-Xylene & $<1$ & 5 & $<1$ \\
\hline
\end{tabular}

1 Oxidation time calculated from ratio of UV contact volume to batch volume multiplied by the elapsed time.

2 After perox-pure ${ }^{\mathrm{TM}}$ treatment for 0.5 minutes. 
Table 1 (cont.)

Results of SD-2 Testing

on SW-31 Spring Water at DOE K-25 Site

Test 12A

\begin{tabular}{|c|c|c|c|c|}
\hline \multirow[b]{2}{*}{ Compound $(\mu \mathrm{g} / \mathrm{l})$} & \multicolumn{4}{|c|}{ Oxidation Time ${ }^{1}(\mathrm{~min})$} \\
\hline & $\mathbf{0}$ & 3 & 6 & 9 \\
\hline Chloroethane & 26 & 13 & 3 & 1 \\
\hline Chloroform & 1 & 1 & $<1$ & \\
\hline 1,1-Dichloroethane & 376 & 279 & 181 & 133 \\
\hline 1,2-Dichloroethane & 3 & 2 & 1 & 1 \\
\hline 1,2-Dichloroethene & 2 & $<1$ & & \\
\hline Methylene Chloride & 146 & 120 & 94 & 86 \\
\hline Tetrachloroethene & $<1$ & & & \\
\hline 1,1,1-Trichloroethane & 322 & 248 & 180 & 137 \\
\hline 1,1,2-Trichloroethane & 4 & 3 & 3 & 3 \\
\hline Trichloroethene & 7 & $<1$ & & \\
\hline Benzene & 36 & $<1$ & & \\
\hline Toluene & 37 & $<1$ & & \\
\hline Ethylbenzene & 27 & $<1$ & & \\
\hline m,p-Xylene & 59 & $<1$ & & \\
\hline 0-Xylene & 28 & $<1$ & & \\
\hline
\end{tabular}

1 Oxidation time calculated from ratio of UV contact volume to batch volume multiplied by the elapsed time. 

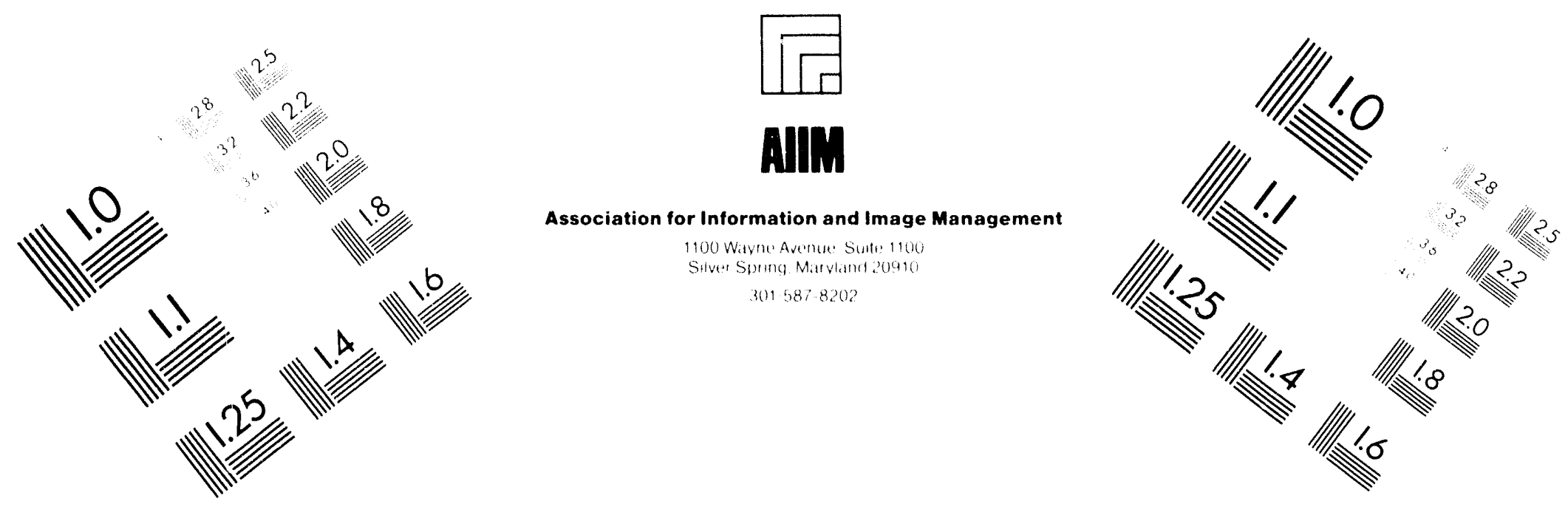

\section{Centimeter}

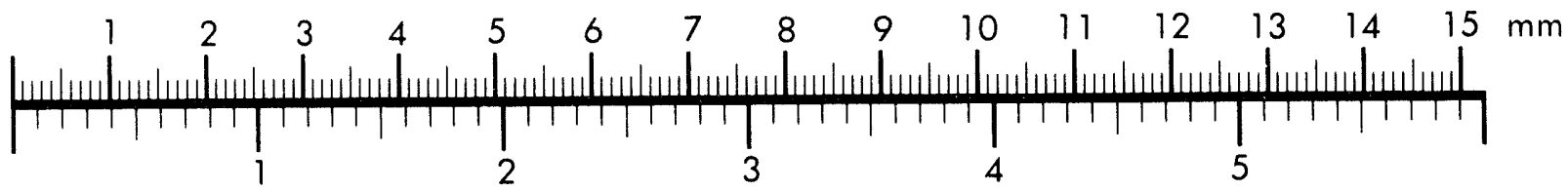

Inches
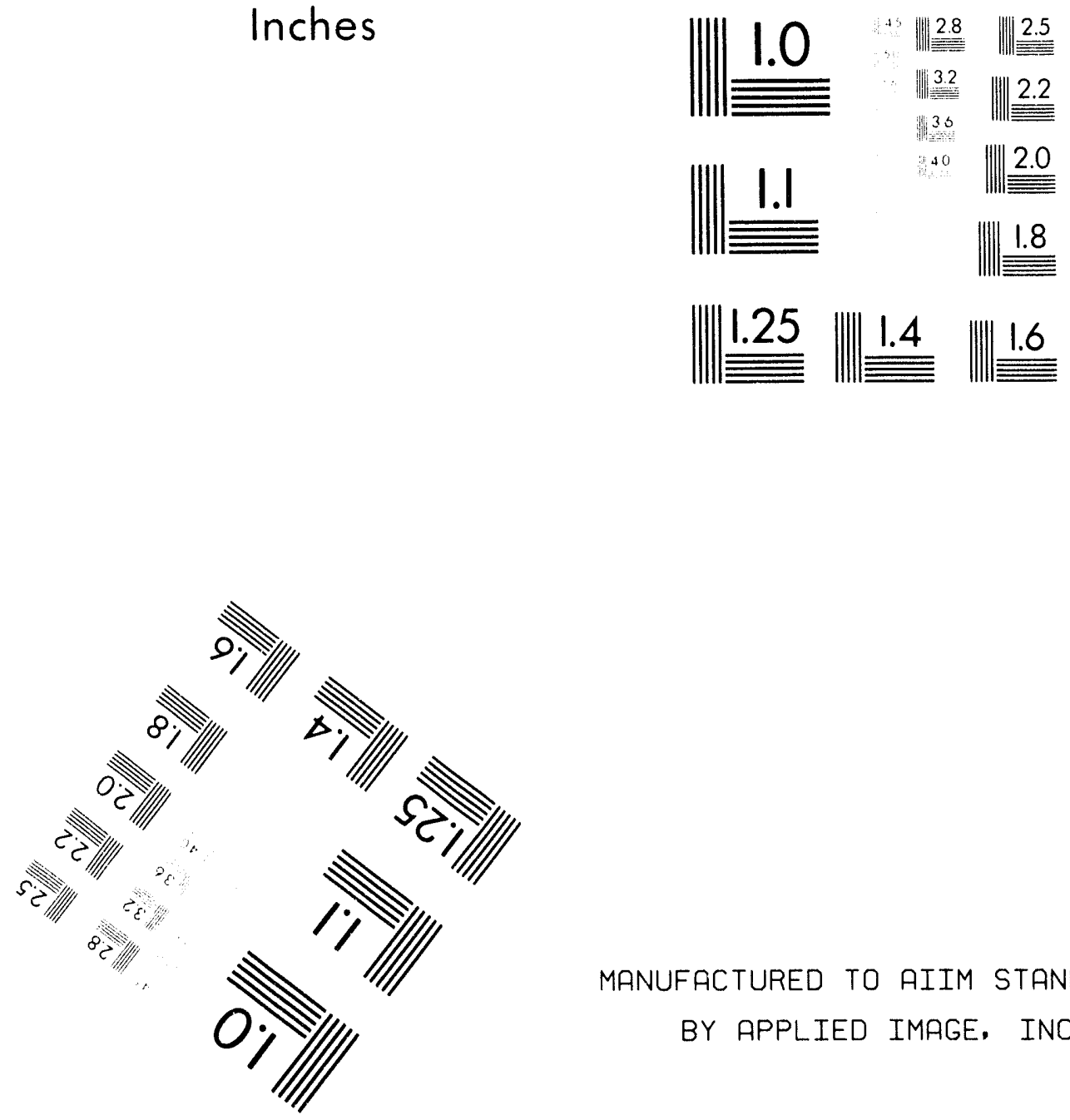

MANUFACTURED TO AIIM STANDARDS

BY APPLIED IMAGE, INC.

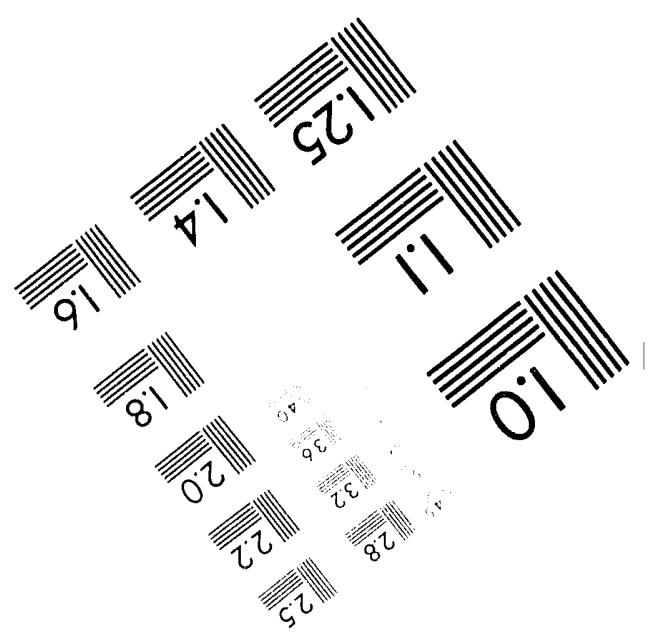



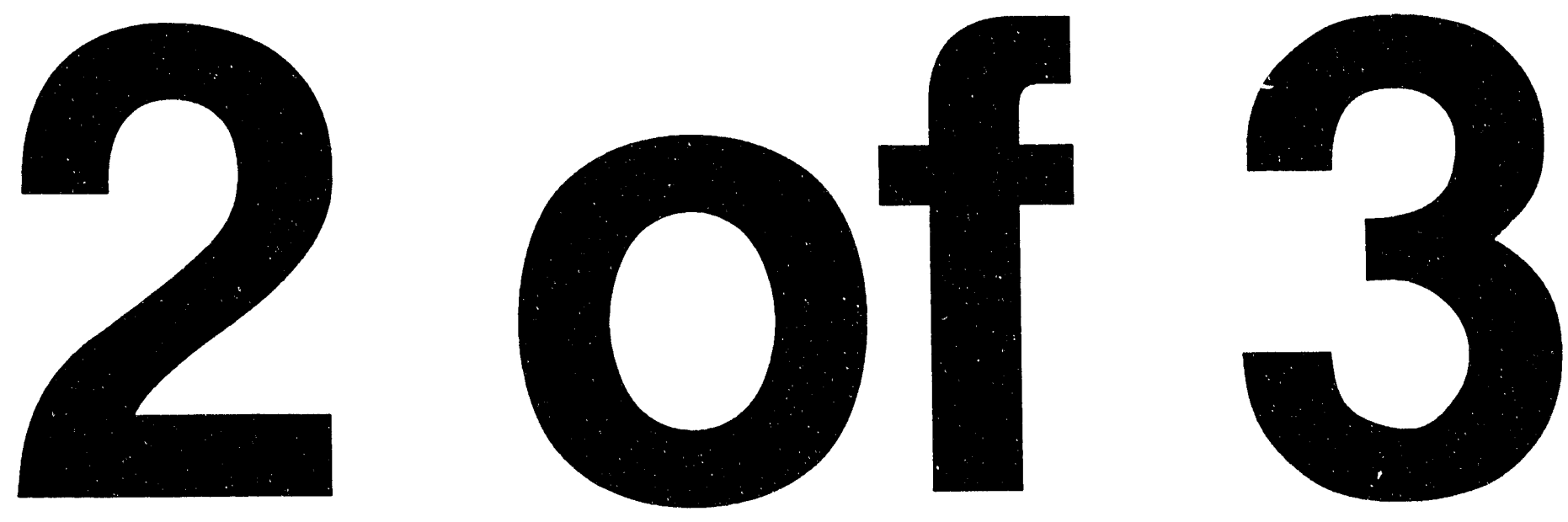
Table 1 (cont.)

Results of SD-2 Testing

on SW-31 Spring Water at DOE K-25 Site

Test 13A

\begin{tabular}{||l|c|c|c|c||}
\hline & \multicolumn{4}{|c|}{ Oxidation Time $^{\mathbf{1}}(\mathbf{m i n})$} \\
\hline Compound $(\mu \mathrm{g} / \mathrm{l})$ & $\mathbf{0}$ & $\mathbf{3}$ & $\mathbf{6}$ & $\mathbf{9}$ \\
\hline Chloroethane & 27 & 18 & 9 & 5 \\
\hline Chloroform & 1 & 1 & $<1$ & \\
\hline 1,1-Dichloroethane & 368 & 300 & 232 & 177 \\
\hline 1,2-Dichloroethane & 3 & 2 & 2 & 1 \\
\hline 1,2-Dichloroethene & $<1$ & 2 & $<1$ & \\
\hline Methylene Chloride & 144 & 124 & 105 & 91 \\
\hline Tetrachloroethene & 3 & $<1$ & & \\
\hline 1,1,1-Trichloroethane & 322 & 257 & 221 & 186 \\
\hline 1,1,2-Trichloroethane & 4 & 3 & 3 & 2 \\
\hline Trichloroethene & 5 & $<1$ & & \\
\hline Trichlorofluoromethane & 279 & 252 & 220 & 191 \\
\hline Benzene & 24 & $<1$ & & \\
\hline Toluene & 24 & $<1$ & & \\
\hline Ethylbenzene & 18 & $<1$ & & \\
\hline m,p-Xylene & 39 & $<1$ & & \\
\hline o-Xylene & 19 & $<1$ & & \\
\hline
\end{tabular}

Oxidation time calculated from ratio of UV contact volume to batch volume multiplied by the elapsed time. 
Table 1 (cont.)

Results of SD-2 Testing

on SW-31 Spring Water at DOE K-25 Site

Test 14A

\begin{tabular}{||l|c|c|c|c||}
\hline & \multicolumn{4}{|c|}{${\text { Oxidation } \text { Time }^{1}(\mathbf{m i n})}$} \\
\hline Compound $(\mu \mathrm{g} / \mathrm{l})$ & $\mathbf{0}$ & $\mathbf{3}$ & $\mathbf{6}$ & $\mathbf{9}$ \\
\hline Chloroethane & 28 & 24 & $\mathrm{SBB}^{2}$ & 18 \\
\hline Chloroform & 1 & 1 & & \\
\hline 1,1-Dichloroethane & 385 & 315 & & 271 \\
\hline 1,2-Dichloroethane & 3 & 3 & & 2 \\
\hline 1,2-Dichloroethene & 3 & 5 & & 3 \\
\hline Methylene Chloride & 175 & 142 & & 114 \\
\hline Tetrachloroethene & 4 & 1 & & $<1$ \\
\hline 1,1,1-Trichloroethane & 348 & 278 & & 214 \\
\hline 1,1,2-Trichloroethane & 4 & 3 & & 3 \\
\hline Trichloroethene & 7 & 3 & & $<1$ \\
\hline Trichlorofluoromethane & 309 & 352 & & 198 \\
\hline Carbon Tetrachloride & $<1$ & $<1$ & & 2 \\
\hline Benzene & 54 & 40 & & 19 \\
\hline Toluene & 56 & 36 & & 15 \\
\hline Ethylbenzene & 38 & 27 & & 12 \\
\hline m,p-Xylene & 101 & 61 & & 20 \\
\hline o-Xylene & 45 & 31 & & 9 \\
\hline
\end{tabular}

Oxidation time calculated from ratio of UV contact volume to batch volume multiplied by the elapsed time.

$2 \quad$ Sample bottle broken in transit. 
Table 1 (cont.)

Results of SD-2 Testing

on SW-31 Spring Water at DOE K-25 Site

Test 15A

\begin{tabular}{||l|c|c|c|c||}
\hline & \multicolumn{4}{|c|}{ Oxidation Time $^{1}(\mathbf{m i n})$} \\
\hline Compound $(\mu \mathrm{g} / \mathrm{l})$ & $\mathbf{0}$ & $\mathbf{3}$ & $\mathbf{6}$ & $\mathbf{9}$ \\
\hline Chloroethane & $27<1$ & 17 & 9 & 2 \\
\hline Chloroform & 1 & 1 & $<1$ & \\
\hline 1,1-Dichloroethane & 374 & 298 & 229 & 165 \\
\hline 1,2-Dichloroethane & 3 & 2 & 2 & 1 \\
\hline 1,2-Dichloroethene & 2 & $<1$ & & \\
\hline Methylene Chloride & 139 & 119 & 97 & 85 \\
\hline Tetrachloroethene & 3 & $<1$ & & 157 \\
\hline 1,1,1-Trichloroethane & 318 & 252 & 207 & 3 \\
\hline $1,1,2-$ Trichloroethane & 4 & 3 & 3 & 170 \\
\hline Trichloroethene & 6 & $<1$ & & \\
\hline Trichlorofluoromethane & 278 & 237 & 201 & \\
\hline Benzene & 37 & $<1$ & & \\
\hline Toluene & 38 & $<1$ & & $<1$ \\
\hline Ethylbenzene & 26 & $<1$ & & \\
\hline m,p-Xylene & 59 & 1 & $<1$ & $<1$ \\
\hline o-Xylene & 29 & 1 & & \\
\hline
\end{tabular}

1 Oxidation time calculated from ratio of UV contact volume to batch volume multiplied by the elapsed time. 
Table 1 (cont.)

Results of SD-2 Testing

on SW-31 Spring Water at DOE K-25 Site

Test 16A

\begin{tabular}{||l|c|c|c|c||}
\hline & \multicolumn{4}{|c|}{ Oxidation Time $^{\mathbf{1}}(\mathbf{m i n})$} \\
\hline Compound $(\mu \mathrm{g} / \mathrm{l})$ & $\mathbf{0}$ & $\mathbf{3}$ & $\mathbf{6}$ & $\mathbf{9}$ \\
\hline Chloroethane & 35 & 18 & 2 & $<1$ \\
\hline Cinloroform & 2 & $<1$ & & \\
\hline 1,1-Dichloroethane & 476 & 323 & 203 & 103 \\
\hline 1,2-Dichloroethane & 4 & 3 & 1 & $<1$ \\
\hline 1,2-Dichloroethene & 2 & $<1$ & & \\
\hline Methylene Chloride & 187 & 134 & 77 & 43 \\
\hline Tetrachloroethene & 4 & $<1$ & & \\
\hline 1,1,1-Trichloroethane & 415 & 288 & 207 & 133 \\
\hline 1,1,2-Trichloroethane & 6 & 4 & 3 & 2 \\
\hline Trichloroethene & 8 & $<1$ & & \\
\hline Trichlorofluoromethane & 243 & 291 & 221 & 160 \\
\hline Benzene & 30 & $<1$ & & \\
\hline Toluene & 30 & $<1$ & & \\
\hline Ethylbenzene & 21 & $<1$ & & \\
\hline m,p-Xylene & 45 & $<1$ & & \\
\hline o-Xylene & 20 & $<1$ & & \\
\hline
\end{tabular}

1 Oxidation time calculated from ratio of UV contact volume to batch volume multiplied by the elapsed time. 
Table 1 (cont.)

\section{Results of SD-2 Testing \\ on SW-31 Spring Water at DOE K-25 Site}

Test 17A

\begin{tabular}{||l|c|c|c|c||}
\hline & \multicolumn{4}{|c|}{ Oxidation Time $^{1}(\mathbf{m i n})$} \\
\hline Compound $(\mu \mathrm{g} / \mathrm{l})$ & $\mathbf{0}$ & $\mathbf{3}$ & $\mathbf{6}$ & $\mathbf{9}$ \\
\hline Chloroethane & 17 & 4 & 1 & $<1$ \\
\hline Chloroform & 3 & $<1$ & & \\
\hline 1,1-Dichloroethane & 407 & 237 & 135 & 67 \\
\hline 1,2-Dichloroethane & $<1$ & & & \\
\hline 1,2-Dichloroethene & 5 & $<1$ & & \\
\hline Methylene Chloride & $<1$ & & & \\
\hline Tetrachloroethene & 3 & 3 & $<1$ & \\
\hline 1,1,1-Trichloroethane & 366 & 217 & 118 & 67 \\
\hline $1,1,2-$ Trichloroethane & 1 & $<1$ & & \\
\hline Trichloroethene & 4 & $<1$ & & \\
\hline Trichlorofluoromethane & 399 & 333 & 254 & 203 \\
\hline Carbon Tetrachloride & 6 & 2 & 2 & $<1$ \\
\hline Benzene & 18 & $<1$ & & \\
\hline Toluene & 15 & $<1$ & & \\
\hline Ethylbenzene & $<1$ & & & \\
\hline m,p-Xylene & 23 & $<1$ & & \\
\hline o-Xylene & 11 & $<1$ & & \\
\hline
\end{tabular}

1 Oxidation time calculated from ratio of UV contact volume to batch volume multiplied by the elapsed time. 
Table 1 (cont.)

Results of SD-2 Testing

on SW-31 Spring Water at DOE K-25 Site

Test 18A

\begin{tabular}{|c|c|c|c|c|}
\hline & \multicolumn{4}{|c|}{ Oxidation Time ${ }^{1}$ (min) } \\
\hline Compound $(\mu \mathrm{g} / \mathrm{l})$ & $\mathbf{0}$ & 3 & 6 & 9 \\
\hline Chloroethane & 19 & 8 & 2 & $<1$ \\
\hline Chloroform & $<1$ & & & \\
\hline 1,1-Dichloroethane & 426 & 305 & 185 & 113 \\
\hline 1,2-Dichloroethane & $<1$ & & & \\
\hline 1,2-Dichloroethene & 7 & $<1$ & & \\
\hline Methylene Chloride & $<1$ & & & \\
\hline Tetrachloroethene & 4 & $<1$ & & \\
\hline 1,1,1-Trichloroethane & 371 & 276 & 186 & 128 \\
\hline 1,1,2-Trichloroethane & $<1$ & & & \\
\hline Trichloroethene & 7 & $<1$ & & \\
\hline Trichlorofluoromethane & 423 & 364 & 283 & 228 \\
\hline Carbon Tetrachloride & 5 & 3 & 1 & $<1$ \\
\hline Benzene & 24 & $<1$ & & \\
\hline Toluene & 23 & $<1$ & & \\
\hline Ethylbenzene & $<1$ & & & \\
\hline m,p-Xylene & 33 & $<1$ & & \\
\hline o-Xylene & 16 & $<1$ & & \\
\hline
\end{tabular}

1 Oxidation time calculated from ratio of UV contact volume to batch volume multiplied by the elapsed time. 
Table 1 (cont.)

Results of SD-2 Testing

on SW-31 Spring Water at DOE K-25 Site

Test 19A

\begin{tabular}{||l|c|c|c|c||}
\hline & \multicolumn{4}{|c|}{ Oxidation Time ${ }^{1}(\mathbf{m i n})$} \\
\hline Compound $(\mu \mathrm{g} / \mathrm{l})$ & $\mathbf{0}$ & $\mathbf{3}$ & $\mathbf{6}$ & $\mathbf{9}$ \\
\hline Chloroethane & $<1$ & & & \\
\hline Chloroform & $<1$ & & & \\
\hline 1,1-Dichloroethane & 394 & 269 & 168 & 102 \\
\hline 1,2-Dichloroethane & $<1$ & & & \\
\hline 1,2-Dichloroethene & 6 & $<1$ & & \\
\hline Methylene Chloride & $<1$ & & & \\
\hline Tetrachloroethene & 4 & $<1$ & & \\
\hline 1,1,1-Trichloroethane & 354 & 255 & 170 & 116 \\
\hline 1,1,2-Trichloroethane & $<1$ & & & \\
\hline Trichloroethene & 4 & $<1$ & & \\
\hline Benzene & 21 & 2 & & \\
\hline Toluene & 17 & $<1$ & & \\
\hline Ethylbenzene & $<1$ & & & \\
\hline m,p-Xylene & 26 & 4 & $<1$ & \\
\hline o-Xylene & 13 & $<1$ & & \\
\hline
\end{tabular}

1 Oxidation time calculated from ratio of UV contact volume to batch volume multiplied by the elapsed time. 
Table 2

Primary Organic Treatment Criteria for SW-31 Spring Water

\begin{tabular}{||l|c|c||}
\hline Contaminant & Influent $(\mathbf{m g} / \mathbf{l})$ & Effluent Objective (mg/) \\
\hline Benzene & 0.15 & \\
\hline Chloroethane & $<0.10$ & \\
\hline Chloroform & $<0.05$ & \\
\hline Ethylbenzene & 0.09 & \\
\hline 1,1-Dichloroethane & 0.92 & \\
\hline 1,1-Dichloroethene & 0.20 & \\
\hline 1,2-Dichloroethane & $<0.05$ & \\
\hline Methylene Chloride & $<0.05$ & \\
\hline Tetrachloroethane & $<0.05$ & \\
\hline Toluene & 0.24 & \\
\hline 1,1,1-Trichloroethane & 1.30 & \\
\hline 1,1,2-Trichloro:thane & $<0.05$ & \\
\hline Vinyl Chloride & $<0.10$ & \\
\hline Xylene (Total) & 0.77 & \\
\hline 1,2-Dichloroethene (Total) & 0.16 & \\
\hline Total Toxic Organics & $3.83-4.28$ & \\
\hline
\end{tabular}




\title{
APPENDIX VI. FIELD TEST OF DIRECT SAMPLING ION TRAP MASS SPECTROMETER FOR GROUNDWATER CLEANUP PROCESS MONITORING*
}

\author{
C.V. Thompson, M.B. Wise and M.R. Guerin \\ Chemical and Analytical Sciences Division \\ Oak Ridge National Laboratory
}

February 21, 1994

\section{Introduction}

A field-transportable Direct Sampling Ion Trap Mass Spectrometer (DSITMS) developed by ORNL for the Department of Energy Office of Technology Development (EM-50) was evaluated for its utility as a process monitoring aid. DSITMS allows the very rapid ( 3 minutes or less) quantification of volatile organic chemicals in water so it was thought the multiple samples could be taken, analyzed on-site, and results reported to the operators in more-nearly real-time.

Two field tests were carried out. The first test was designed to determine whether DSITMS was applicable to the analysis of the multiple contaminar! water being processed. This involved field analyses of samples by DSITMS for comparison with results generated using standard EPA methods performed by the process support laboratory. The second test resulted from an observation by the ORNL field team that it might be possible to monitor the process in a truly continuous, on-line, real-time manner.

Both tests were very successful. Results suggest that it would be possible and worthwhile to develop a continuous monitoring version of the instrument. The current instrument is already applicable if manual operation and quantification are acceptable. Summaries of data and observations follow.

"Modified version of "DSITMS Real-Time Monitoring of VOC's in Peroxidation Systems Water Treatment System," Summary Report Draft, C. V. Thompson and M. B. Wise, 1/4/94. Modifications (by M. R. Guerin) remove brief descriptions of the process and of observations which are treated more completely by the main document. 


\section{First Field Test}

In the first test, conducted on September 3,1993, $40 \mathrm{~mL}$ grab samples of water from the treatment process, and water standards of the pertinent VOCs, were analyzed at the site by DSITMS. Results from these analyses were calculated the next work day. Qualitative analysis of the samples was possible in real-time by monitoring the spectral display of the analyses (Figure 3). Table 1 is a comparison of data from the DSITMS analysis with data generated by a standard GC/MS method.

Table 1. Analysis of Water Samples from Treatment of K-25 Seep

\begin{tabular}{|c|r|l|r|l|r|l|}
\hline \multirow{2}{*}{} & \multicolumn{2}{|c|}{ Test Start } & \multicolumn{2}{c|}{ 2 Min. Elapsed Time } & \multicolumn{2}{c|}{ 3 Min. Elapsed Time } \\
\cline { 2 - 7 } & \multicolumn{2}{|c|}{$\mathrm{ng} / \mathrm{mL}$} & \multicolumn{2}{c|}{$\mathrm{ng} / \mathrm{mL}$} & \multicolumn{2}{c|}{$\mathrm{ng} / \mathrm{mL}$} \\
\hline Compound & ORNL $^{\mathrm{a}}$ & Per-Ox $^{\mathrm{b}}$ & ORNL & Per-Ox & ORNL & Per-Ox \\
\hline 1,1,-DCA & 191 & 409 & 56 & 57 & 2 & 16 \\
\hline $1,1,1-\mathrm{TCA}$ & 202 & 277 & 139 & 69 & 20 & 36 \\
\hline Benzene & 9 & 18 & 0 & 0 & 0 & 0 \\
\hline Toluene & 4 & 33 & 0 & 0 & 0 & 0 \\
\hline Xylenes & 14 & 22 & 1 & 0 & 1 & 0 \\
\hline
\end{tabular}

(' DSITMS $\left.{ }^{\mathrm{b}} \mathrm{GCMS}\right)$

The data generated by DSITMS are based on crude field standards and represent semi-quantitative results at best. The results for both methods show the same trends of decaying concentrations for each of the analytes. In addition to the analytes listed in Table 1 , acetone and $\mathrm{C}_{3}$ - and $\mathrm{C}_{4}$ - benzenes were also present in the samples but were not quantitated due to a lack of appropriate standards. These three analytes were not mentioned in the GCMS analytical report and apparently were not looked for in the analysis.

\section{Second Field Test}

For the second field test which was conducted on October 18 and 19, 1993, a continuous water monitor developed here at the laboratory, was deployed to provide realtime analysis of VOCs in the water from the treatment process. The continuous water monitor employs a new Venturi-based technology which is presently under patent consideration. The delay time between processing of the sample in the continuous water monitor and display of the spectrum on screen was about 7 seconds. 
The continuous water monitor was used in conjunction with five tests of the experimental water treatment system and with two tests of the commercial system. Figures 4-10 are graphs of the temporal responses for analytes of interest for each of these tests. The data for each analyte has been subjected to a running ten-point smoothing routine to reduce random noise. It should be noted that relative ion abundances for the analytes do not correlate to relative concentrations. Although xylenes and 1,1,1-TCA have comparable ion abundances, they differ in concentrations by a factor of 2 to 3. Test start times are marked on each of the graphs. Tables 2-7 contain starting and ending concentrations derived from standards run for the tests. In each of the tests, analytes expected to be in the seep water in significant concentrations included 1,1,1trichloroethane, 1,1-dichloroethane. benzene, toluene, and xylenes. Another analyte, possibly methyl ethyl ketone (MEK), appears to have been an intermediate formed during the treatment process and eventually consumed by it. Its presence was unexpected and had not been reported prior to the use of the continuous water monitor with the treatment processes. Standards used with the continuous water monitor did not include MEK, so no quantitation of it is possible in the tests.

The first experimental test which was monitored had no corresponding standards for calibration; therefore, it has not been quantified. The fourth experimental test had no standards for calibration, but the standards from the third and fifth tests have been averaged and used to provide estimates of the analyte concentrations in this test. The second commercial test included the introduction of acetone into the water undergoing treatment at a concentration of $20 \mathrm{mg} / \mathrm{L}$ at an elapsed time of about 6.5 minutes from the start of the test. This addition appeared to accelerate the rate of degradation of the organics. However, it is also possible that the large concentration of acetone ions in the ion trap depressed the ions from other analytes through space charging. One other effect of the acetone addition was an immediate increase in the concentration of MEK.

One benefit of a system like the continuous water monitor is the ability to provide data in real time of the effects of treatment processes such as the ones used at K-25. When operating parameters for the treatment systems are changed, their effects on the rates of degradation of various compounds can be immediately observed, allowing for optimization of the process in a much shorter time than if standard analyses had to be performed using grab samples. With a temporal resolution of one data point per second for the continuous water monitor, rate constants for degradation of individual compounds by the treatment process can be easily determined from the generated data. This represents a significant benefit of the continuous water monitor over the relatively small number of grab samples which can be acquired during a test.

Analytical results are listed from laboratory analyses of grab samples from commercial tests 16 and 17 are listed in Tables 8 and 9 , respectively. Concentrations as determined by the continuous water monitor are given for comparison. The results from the DSITMS analyses are significantly lower than the concentrations as determined by the continuous water monitor. Results from the standard GC/MS analysis of split 
samples performed at ORNL are even lower. One possible reason for these differences is that the peroxide and catalysts added to the samples continued to degrade the organics in the samples, albeit at much slower rates, leading to the progressively lower numbers as the samples aged.

Table 2. Continuous Water Monitor - Semi-quantitative Concentration Estimates Second Field Test, 10/18/93, Run \#12A Experimental Treatment System

\begin{tabular}{|c|c|c|}
\hline Compound & $\begin{array}{c}\text { Test Start } \\
(\mathrm{ng} / \mathrm{mL})\end{array}$ & $\begin{array}{c}\text { Test End } \\
(\mathrm{ng} / \mathrm{mL})\end{array}$ \\
\hline $1,1-\mathrm{DCA}$ & 605 & 115 \\
\hline $1,1,1-\mathrm{TCA}$ & 662 & 299 \\
\hline Benzene & 33 & 3 \\
\hline Toluene & 26 & 5 \\
\hline Xylene & 108 & 6 \\
\hline
\end{tabular}

Table 3. Continuous Water Monitor - Semi-quantitative Concentration Estimates Second Field Test, 10/18/93, Run \#14A Experimental Treatment System

\begin{tabular}{|c|c|c|}
\hline Compound & $\begin{array}{c}\text { Test Start } \\
(\mathrm{ng} / \mathrm{mL})\end{array}$ & $\begin{array}{c}\text { Test End } \\
(\mathrm{ng} / \mathrm{mL})\end{array}$ \\
\hline $1,1-\mathrm{DCA}$ & 1431 & 461 \\
\hline $1,1,1-\mathrm{TCA}$ & 939 & 579 \\
\hline Benzene & 51 & 22 \\
\hline Toluene & 44 & 15 \\
\hline Xylene & 209 & 49 \\
\hline
\end{tabular}


Table 4. Continuous Water Monitor - Semi-quantitative Concentration Estimates* Second Field Test, 10/18/93, Run \#15A Experimental Treatment System

\begin{tabular}{|c|c|c|}
\hline Compound & $\begin{array}{c}\text { Test Start } \\
(\mathrm{ng} / \mathrm{mL})\end{array}$ & $\begin{array}{c}\text { Test End } \\
(\mathrm{ng} / \mathrm{mL})\end{array}$ \\
\hline $1,1-\mathrm{DCA}$ & 373 & 131 \\
\hline $1,1,1-\mathrm{TCA}$ & 368 & 273 \\
\hline Benzene & 32 & 2 \\
\hline Toluene & 32 & 3 \\
\hline Xylene & 136 & 2 \\
\hline
\end{tabular}

"These estimates were generated by averaging the standards for the runs preceding and following this one.

Table 5. Continuous Water Monitor - Semi-quantitative Concentration Estimates Second Field Test, 10/18/93, Run \#17A Experimental Treatment System

\begin{tabular}{|c|c|c|}
\hline Compound & $\begin{array}{c}\text { Test Start } \\
(\mathrm{ng} / \mathrm{mL})\end{array}$ & $\begin{array}{c}\text { Test End } \\
(\mathrm{ng} / \mathrm{mL})\end{array}$ \\
\hline $1,1-\mathrm{DCA}$ & 624 & 36 \\
\hline $1,1,1-\mathrm{TCA}$ & 552 & 81 \\
\hline Benzene & 46 & 2 \\
\hline Toluene & 48 & 3 \\
\hline Xylene & 218 & 3 \\
\hline
\end{tabular}


Table 6. Continuous Water Monitor - Semi-quantitative Concentration Estimates Second Field Test, 10/19/93, Run \#16 Commercial Treatment System

\begin{tabular}{|c|c|c|}
\hline Compound & $\begin{array}{c}\text { Test Start } \\
(\mathrm{ng} / \mathrm{mL})\end{array}$ & $\begin{array}{c}\text { Test End } \\
(\mathrm{ng} / \mathrm{mL})\end{array}$ \\
\hline $1,1-\mathrm{DCA}$ & 259 & 8 \\
\hline $1,1,1-\mathrm{TCA}$ & 460 & 10 \\
\hline Benzene & 32 & 2 \\
\hline Toluene & 24 & 3 \\
\hline Xylene & 107 & 4 \\
\hline
\end{tabular}

Table 7. Continuous Water Monitor - Semi-quantitative Concentration Estimates Second Field Test, 10/19/93, Run \#17 Commercial Treatment System

\begin{tabular}{|c|c|c|}
\hline Compound & $\begin{array}{c}\text { Test Start } \\
(\mathrm{ng} / \mathrm{mL})\end{array}$ & $\begin{array}{c}\text { Test End } \\
(\mathrm{ng} / \mathrm{mL})\end{array}$ \\
\hline $1,1-\mathrm{DCA}$ & 114 & 10 \\
\hline $1,1,1-\mathrm{TCA}$ & 338 & 14 \\
\hline Benzene & 29 & 2 \\
\hline Toluene & 27 & 3 \\
\hline Xylene & 124 & 3 \\
\hline
\end{tabular}


Table 8. Analysis of Water Samples from Treatment of K-25 Seep Second Field Test, 10/19/93, Run \#16, Commercial Treatment

\begin{tabular}{|c|r|r|l|r|r|r|}
\hline & \multicolumn{3}{|c|}{ Test Start } & \multicolumn{3}{c|}{ Test End } \\
\cline { 2 - 8 } & \multicolumn{3}{|c|}{$\mathrm{ng} / \mathrm{mL}$} & \multicolumn{3}{c|}{$\mathrm{ng} / \mathrm{mL}$} \\
\cline { 2 - 8 } & $\begin{array}{c}\text { Cont. } \\
\text { Monitor }\end{array}$ & DSITMS & GC/MS & $\begin{array}{c}\text { Cont. } \\
\text { Monitor }\end{array}$ & DSITMS & GC/MS \\
\hline Date Analyzed & $10 / 19 / 93$ & $10 / 20 / 93$ & $10 / 29 / 93$ & $10 / 19 / 93$ & $10 / 20 / 93$ & $10 / 29 / 93$ \\
\hline Compound & \multicolumn{3}{|c|}{} & & \multicolumn{3}{c|}{} \\
\hline $1,1,-$ DCA & 259 & 129 & 7 & 8 & 8 & 2 \\
\hline $1,1,1-T C A$ & 460 & 294 & 60 & 10 & 20 & 9 \\
\hline Benzene & 32 & 5 & ND & 2 & 2 & ND \\
\hline Toluene & 24 & 5 & ND & 3 & 4 & ND \\
\hline Xylenes & 107 & 32 & ND & 4 & 28 & ND \\
\hline
\end{tabular}

Table 9. Analysis of Water Samples from Treatment of K-25 Seep Second Field Test, 10/19/93, Run \#17, Commercial Treatment

\begin{tabular}{|c|r|r|l|r|r|r|}
\hline & \multicolumn{3}{|c|}{ Test Start } & \multicolumn{3}{c|}{ Test End } \\
\cline { 2 - 8 } & \multicolumn{3}{|c|}{$\mathrm{ng} / \mathrm{mL}$} & \multicolumn{3}{c|}{$\mathrm{ng} / \mathrm{mL}$} \\
\cline { 2 - 8 } & $\begin{array}{c}\text { Cont. } \\
\text { Monitor }\end{array}$ & DSITMS & GC/MS & $\begin{array}{r}\text { Cont. } \\
\text { Monitor }\end{array}$ & DSITMS & GC/MS \\
\hline Date Analyzed & $10 / 19 / 93$ & $10 / 20 / 93$ & $10 / 29 / 93$ & $10 / 19 / 93$ & $10 / 20 / 93$ & $10 / 29 / 93$ \\
\hline Compound & & & & 10 & 5 & 1 \\
\hline $1,1,-$-DCA & 114 & 109 & 4 & 14 & 20 & 7 \\
\hline $1,1,1-$ TCA & 338 & 265 & 54 & 2 & 1 & ND \\
\hline Benzene & 29 & 3 & ND & 3 & 2 & ND \\
\hline Toluene & 27 & 3 & ND & 3 & 16 & ND \\
\hline Xylenes & 124 & 22 & ND & & & \\
\hline
\end{tabular}




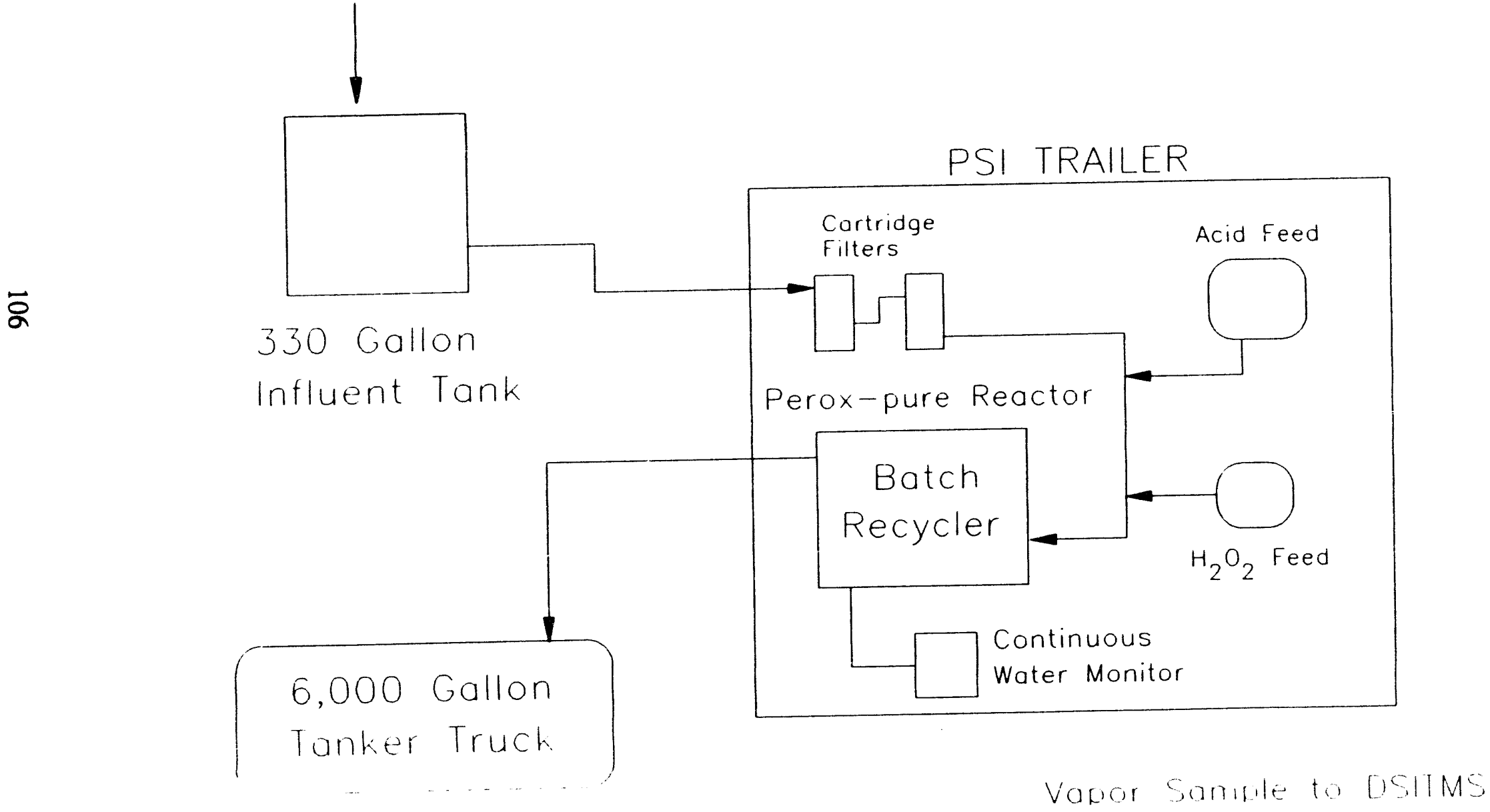




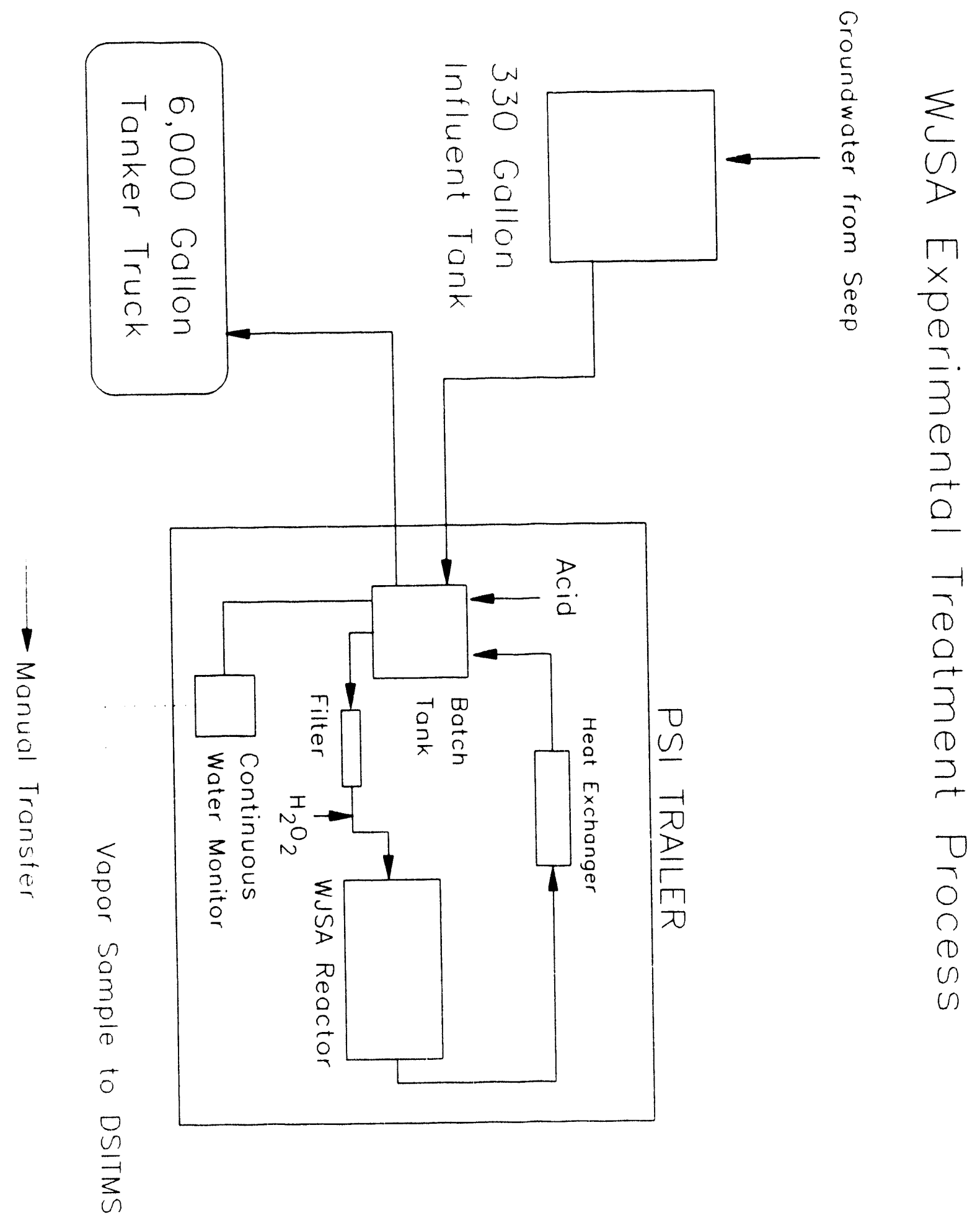


File: E:MAG 1018ct5 Date: Oct-18-1993 16:11:13

Chro Analysis Coment: -25 Seep Sample, with stds after, blank, low, high, h2oci,m1600 Coment: K-25 Seep Sample, with sts arter, RIC: 32899 Metention Time: 3:07 187 Range: 47-150 Plotted: 1 to 1273

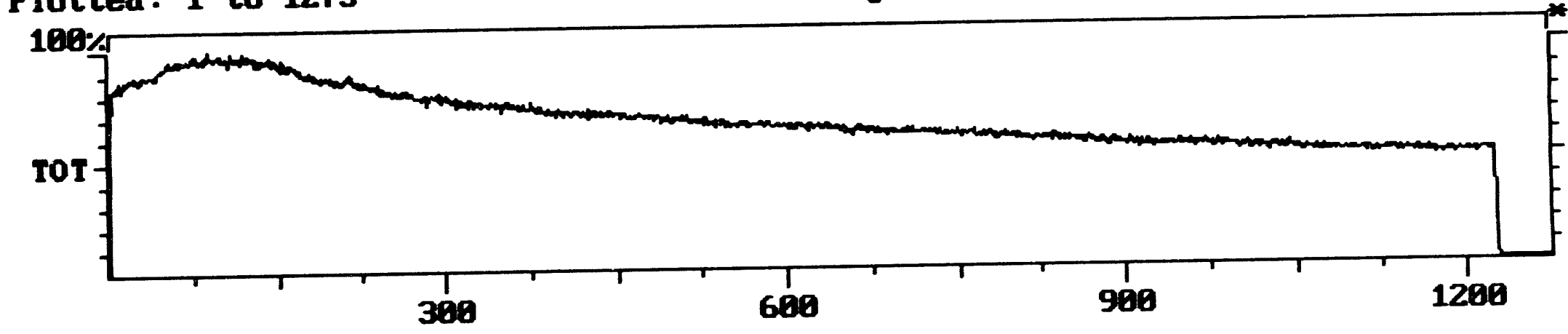

$\bar{\infty}$
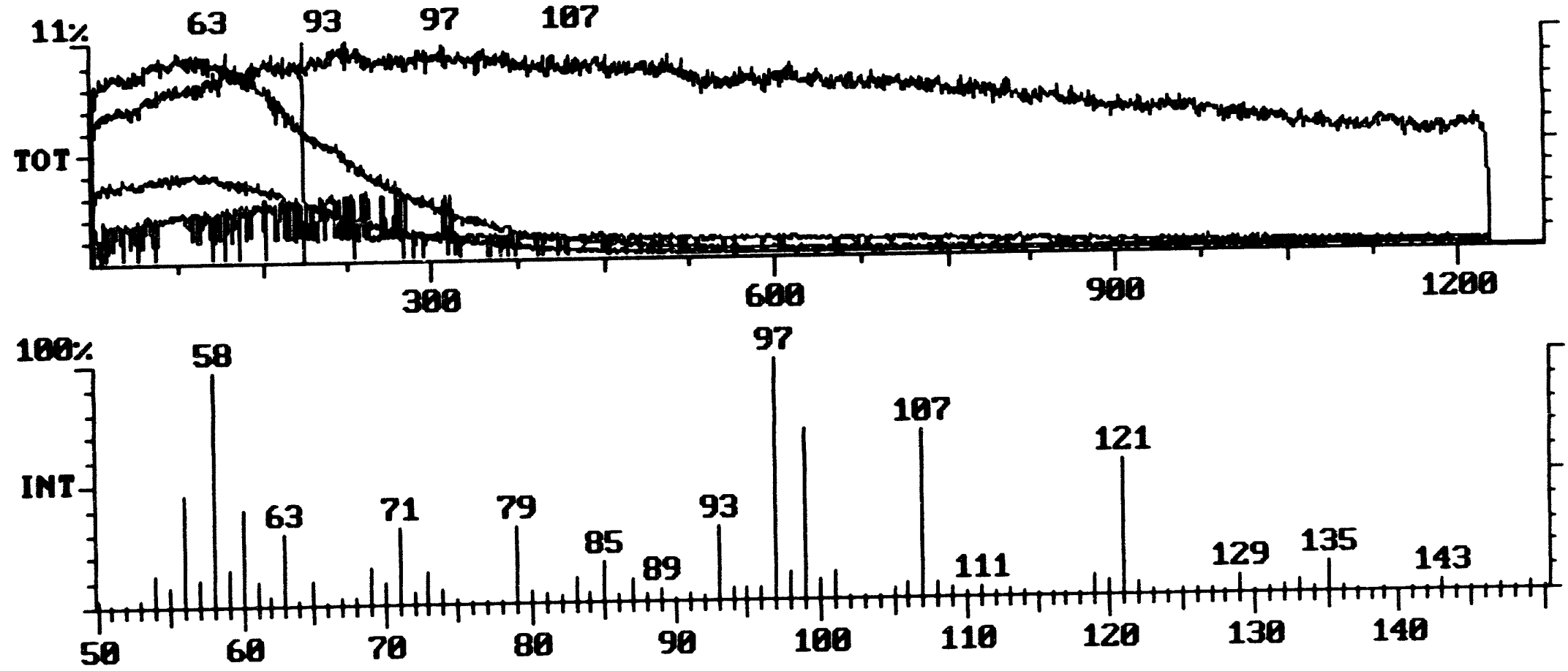


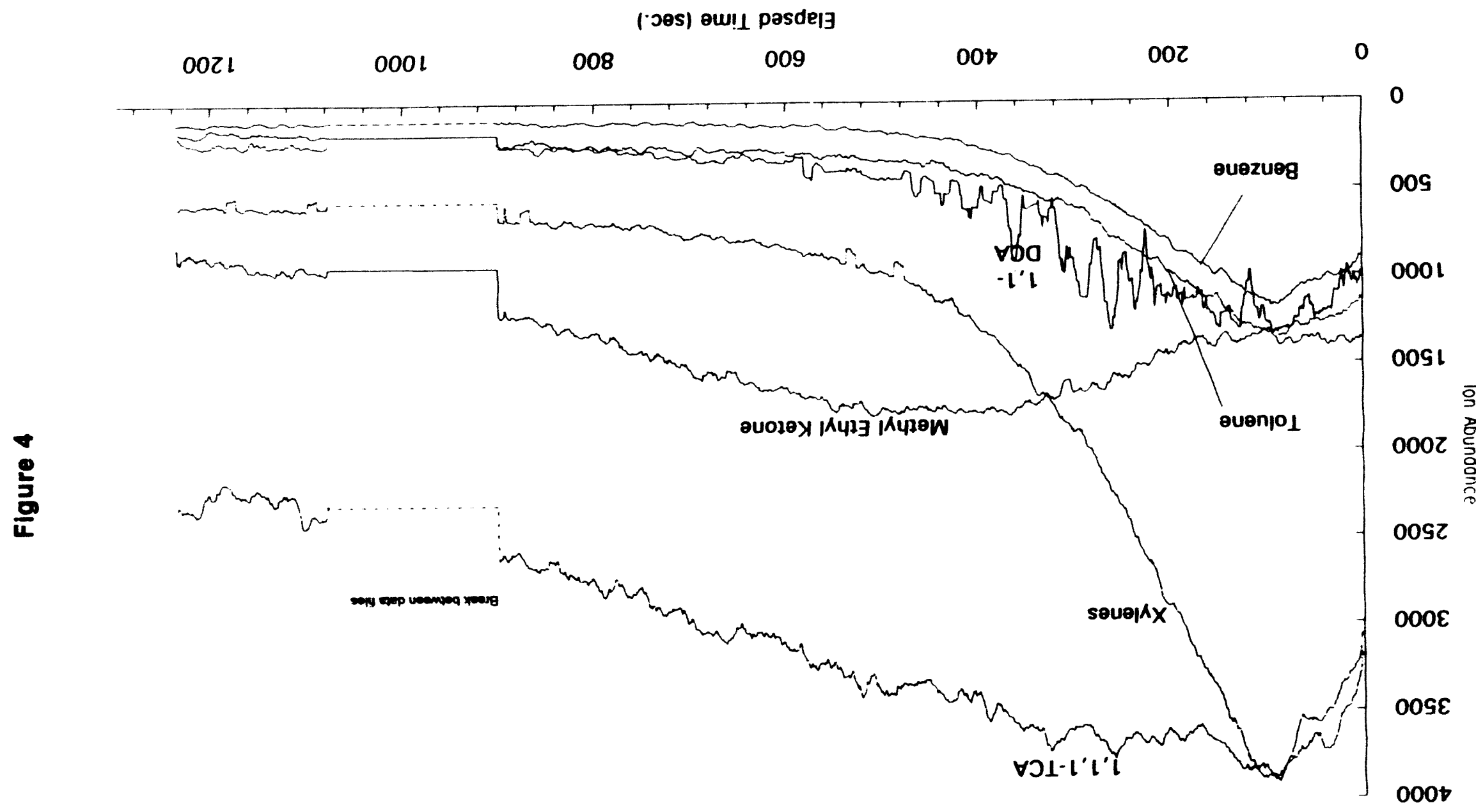

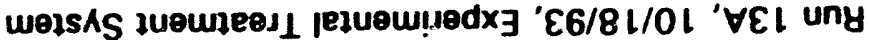

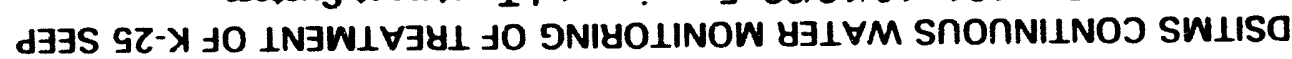


DSITMS CONTINUOUS WATER MONITORING OF TREATMENT OF K-2ऽ SEEP

Run 12A, 10/18/93, Experimental Treatment System

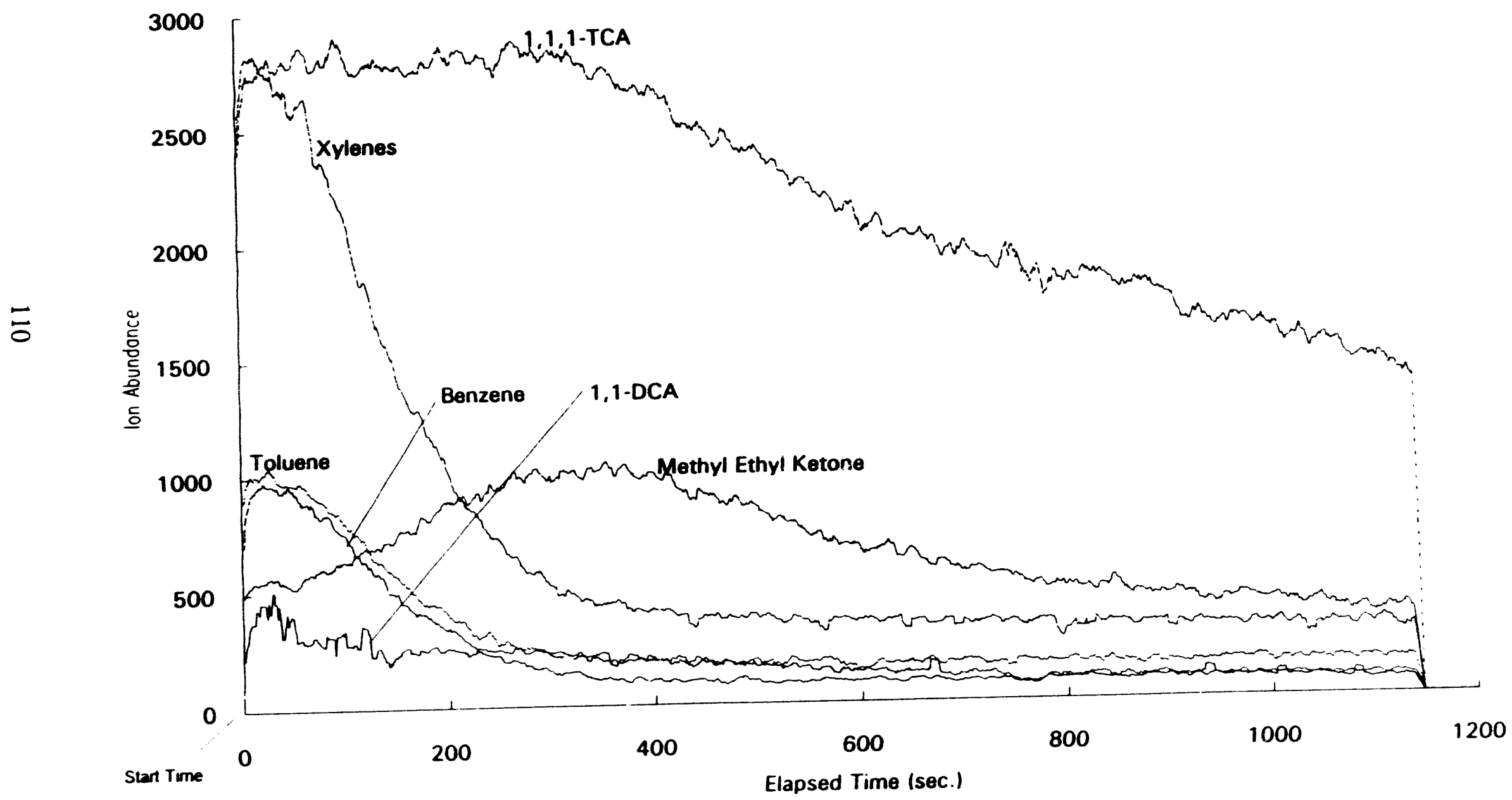


DSITMS CONTINUOUS WATER MONITORING OF TREATMENT OF K-25 SEEP

Run 14A - Iron left in seep water, 10/18/93, Experimental Treatment System

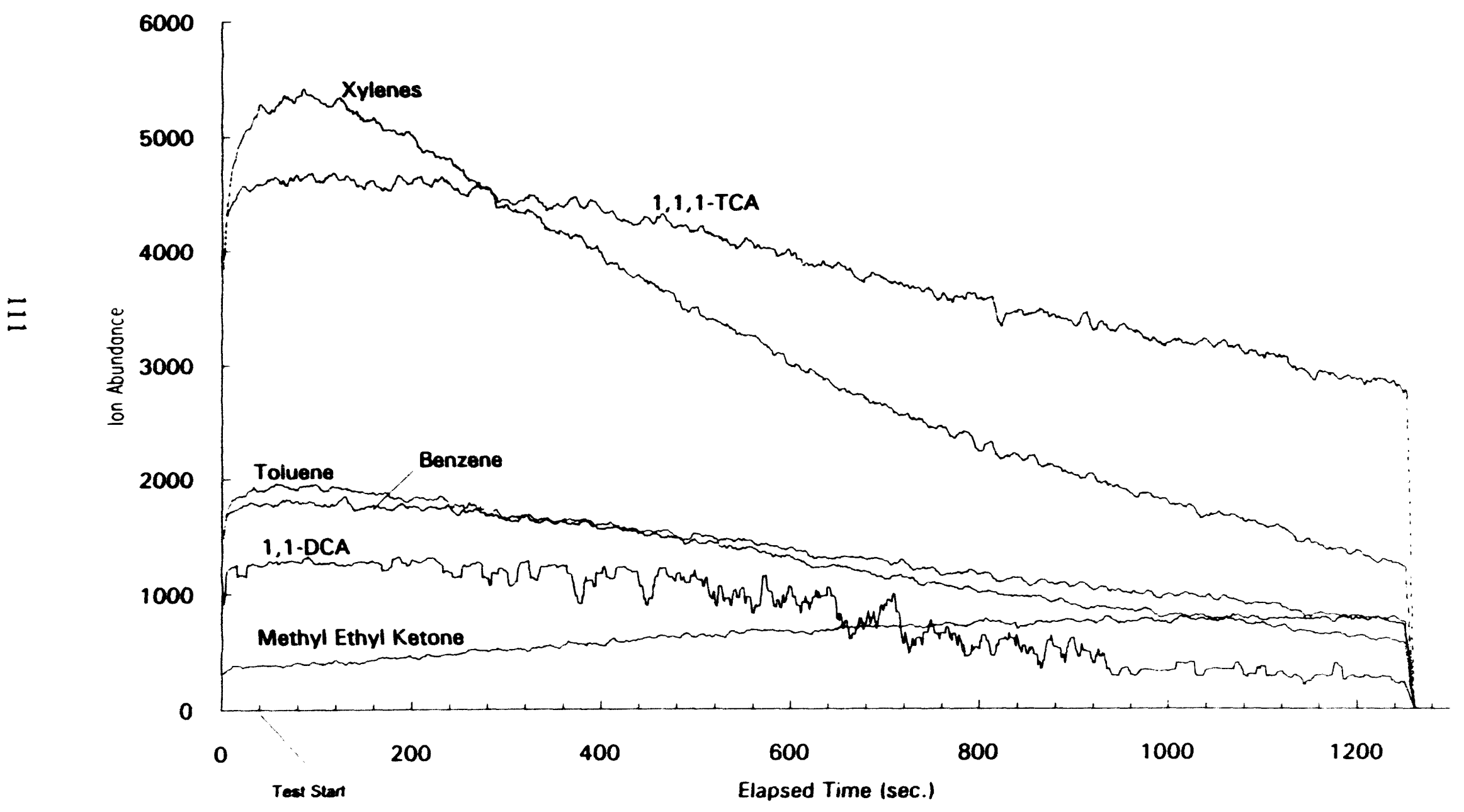


DSITMS CONTINUOUS WATER MONITORING OF TREATMENT OF K-25 SEEP

Run 15A, 10/18/93, Experimental Treatment System

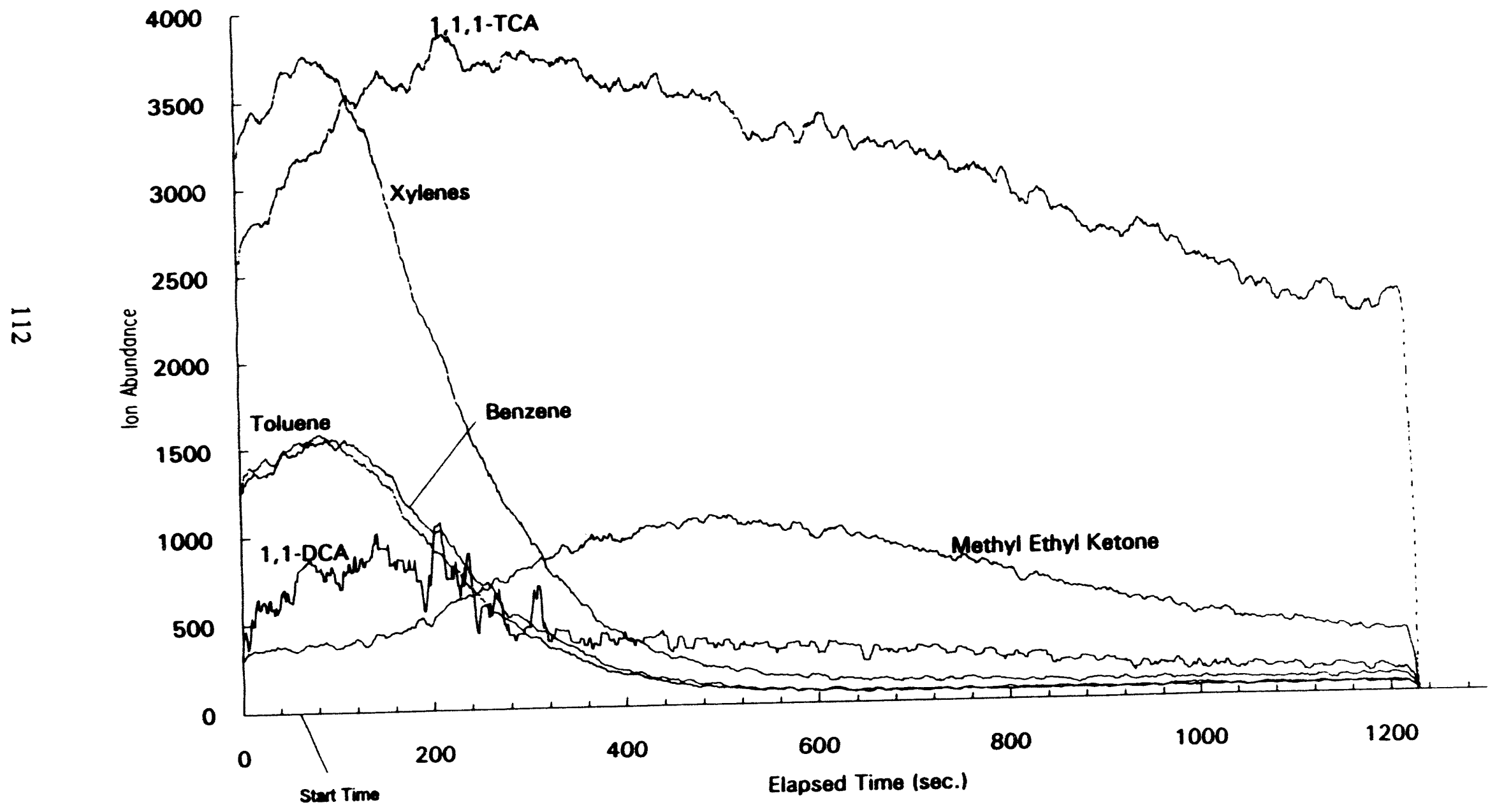


DSITMS CONTINUOUS WATER MONITORING OF TREATMENT OF K-25 SEEP

Run 17A, 10/18/93, Experimental Treatment System, Higher Energy UV

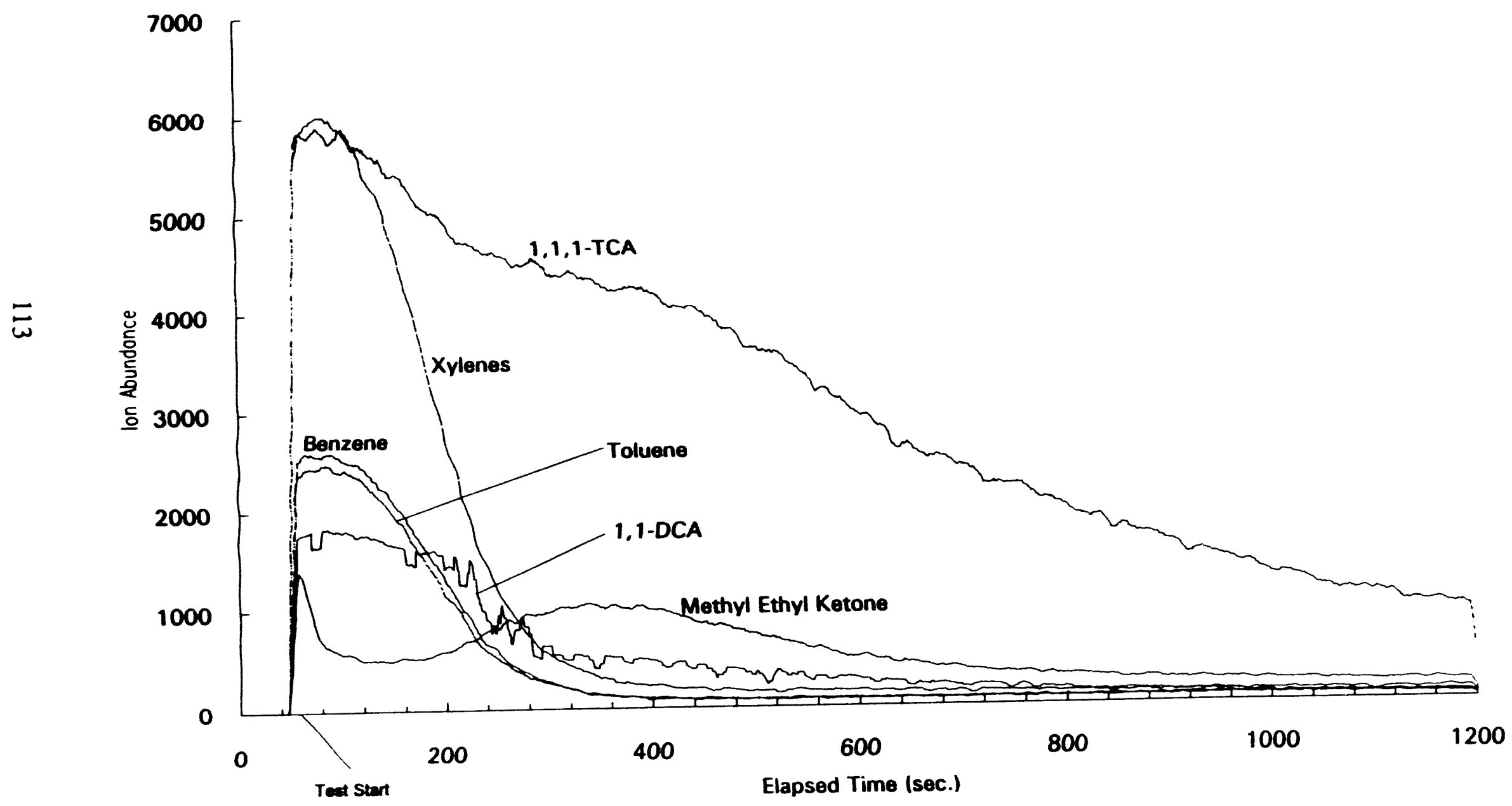


DSITMS CONTINUOUS WATER MONITORING OF TREATMENT OF K-25 SEEP

Run 16, 10/19/93, Commercial Treatment System

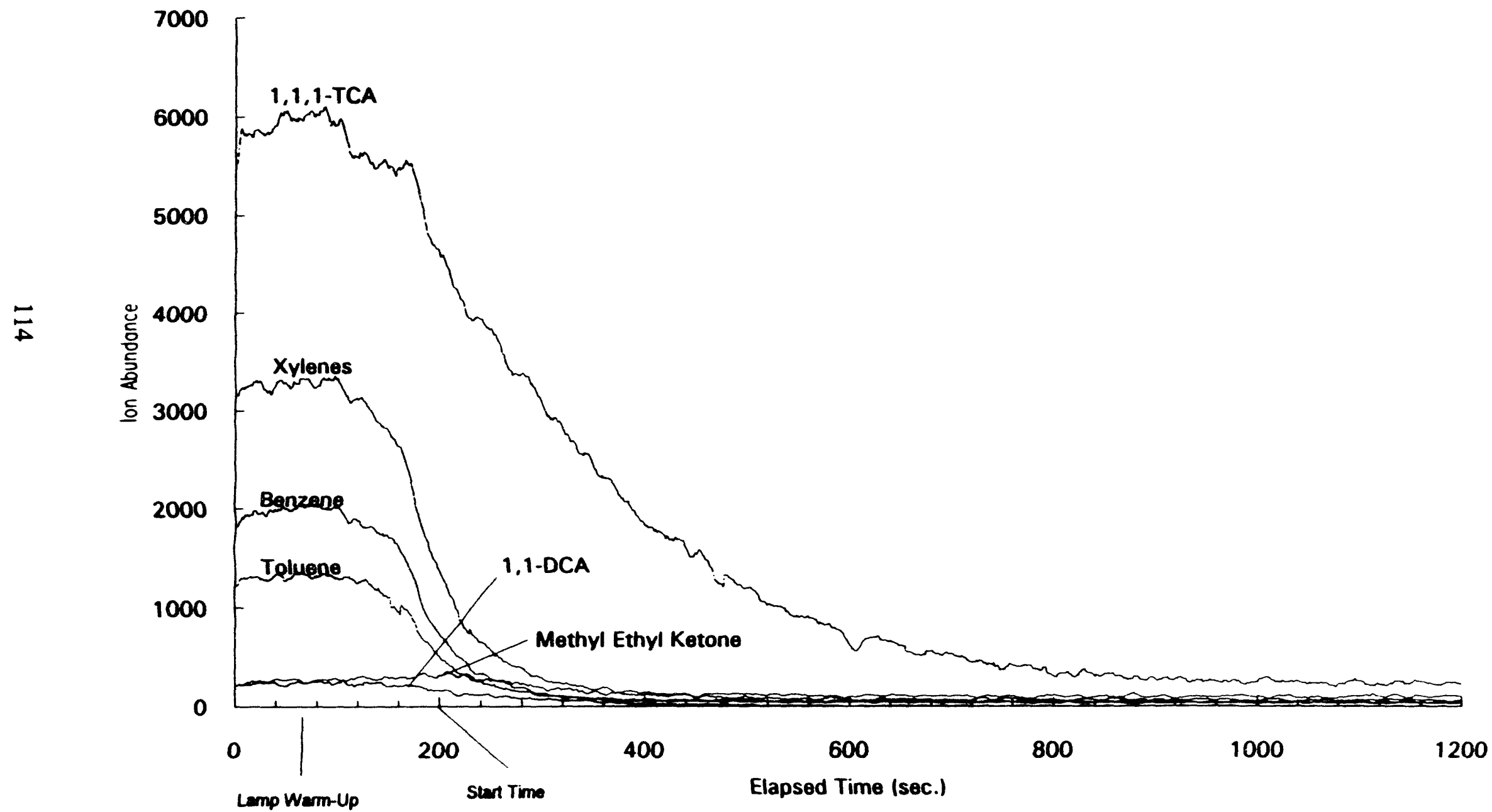


DSITMS CONTINUOUS WATER MONITORING OF TREATMENT OF K-25 SEEP Run 17, 10/19/93, Commercial Treatment System

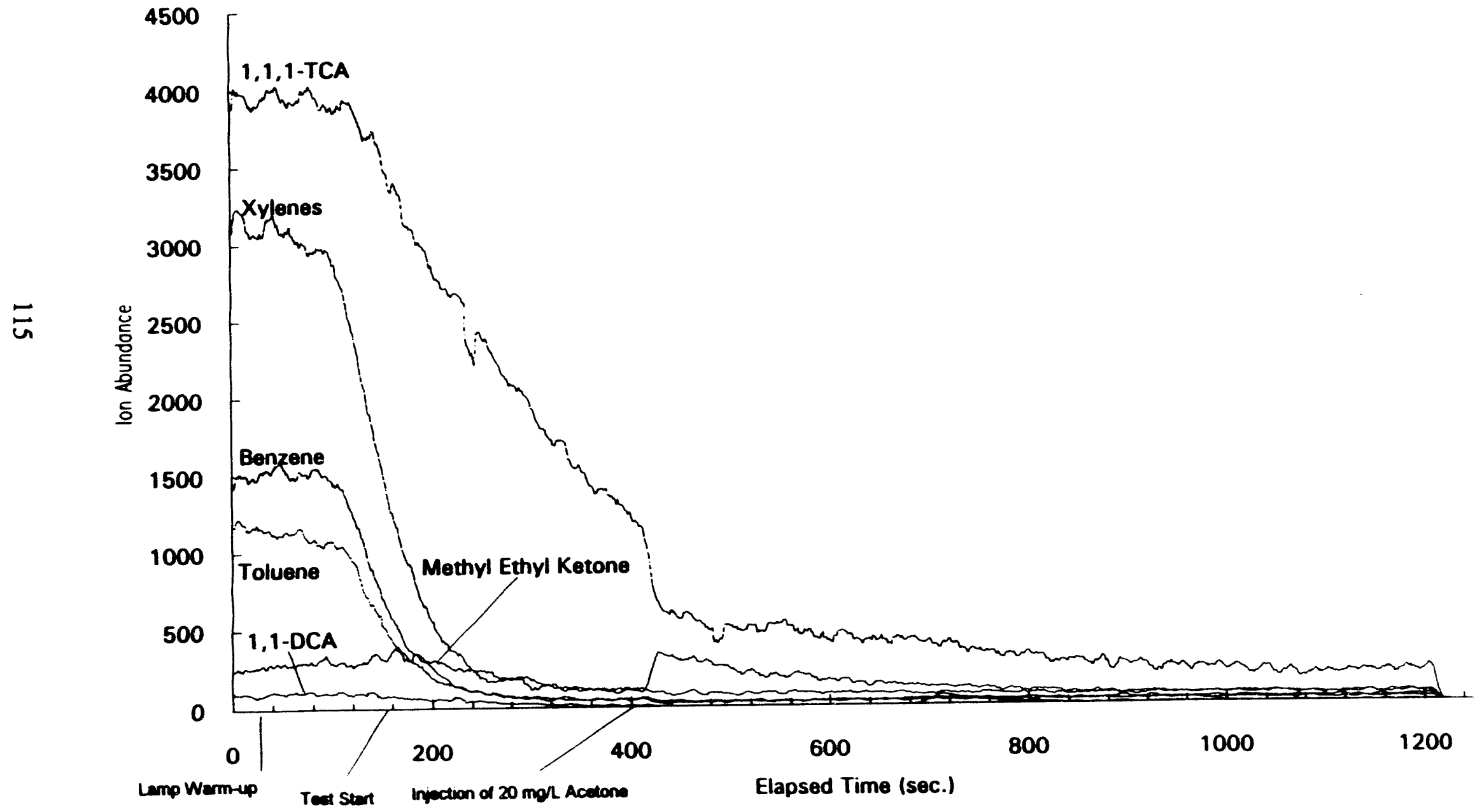




\section{APPENDIX VII. ANALYTICAL REPORTS FOR PEROX-PURE ${ }^{\mathrm{TM}}$ AND SD-2 TESTS ON SW-31 SPRING}

\section{Comment on Vinyl Chloride Measurements}

Based on the analytical analyses performed in this study, vinyl chloride was "detected" as being present in the SW-31 Spring water. However, the destruction rates for vinyl chloride demonstrated by the analytical results are significantly lower in magnitude than historically demonstrated destruction rates for the perox-pure ${ }^{\mathrm{TM}}$ process, and are less than expected for the SD-2 system based on the destruction rates achieyed in this study for other chlorinated ethylene compounds such as TCE. Therefore, an anomaly exists between the vinyl chloride analytical results and the expected/demonstrated destruction rates. This anomaly is even further supported by the fact that the destruction rates for the other more difficult to treat VOC's in the SW-31 water (such as TCA and DCA) were close to expected results. Because the analytical methodology utilized in this study for VOC analysis (i.e., EPA Method 601/602) was not of a confirmatory nature [because it did not employ gas chromatograph/mass spectroscopy (GC/MS) per EPA Method 624]; and the level of vinyl chloride detected was very low, it is uncertain that the compound "detected" as vinyl chloride was actually vinyl chloride.

In future studies at the SW-31 Spring site, it is recommended that GC/MS analysis be performed for VOC's using EPA Method 624 or B240 to confirm the presence of vinyl chloride (or not) and its destruction rates. 


\section{CHAIN OF CUSTODY FORM}

Aapen Environmental Laboratory

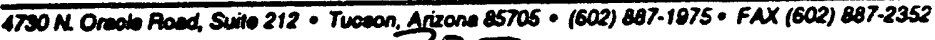

COMPANY PSI

ADDRESS

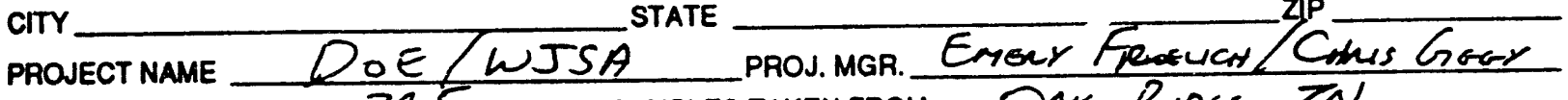
PROJECT NUMBER 345 SAMPLES TAKEN FROM: DAK ROG, TN

ON \& 24 HOUR RUSH (__ \% Surcharg) $\square$ 48 HOUR RUSH ( $\%$ Surchargo)

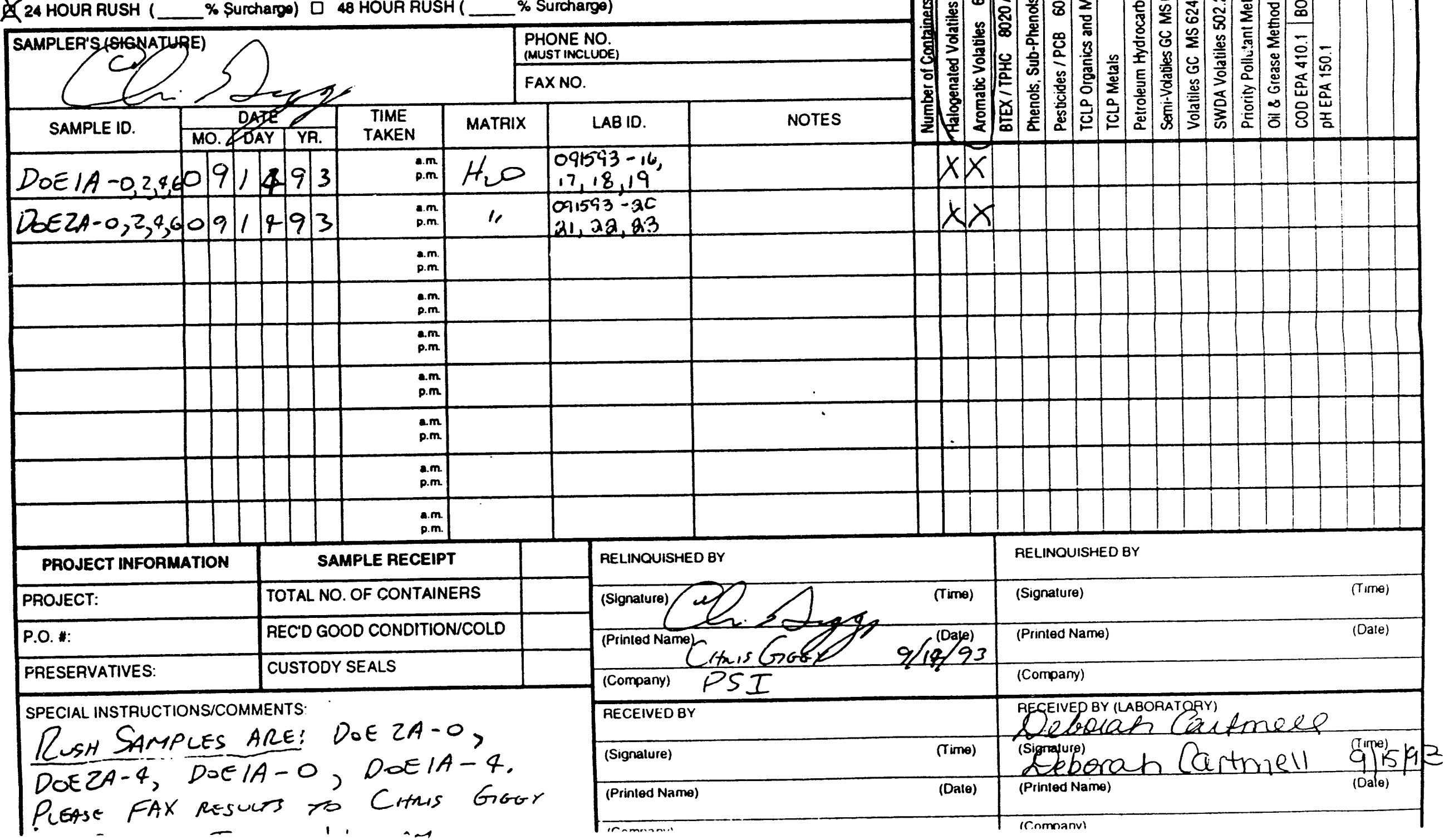




\section{CHAIN OF CUSTODY FORM \\ Arizona Certified Laboratory}

Page 1 of 1

Aspon Environmonted Laboratory

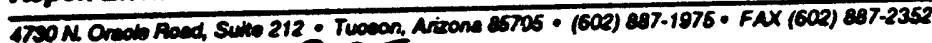

COMPANY PSI

ADDRESS Tesen Orfice

CITY

PROJECT NAME WJSA/POE

PROJECT NUMBER STATE ZIP

2. 24 HOUR RUSH ( SAMPLES TAKEN FROM: VOE

SAMPLER'S (SIGNATURE)

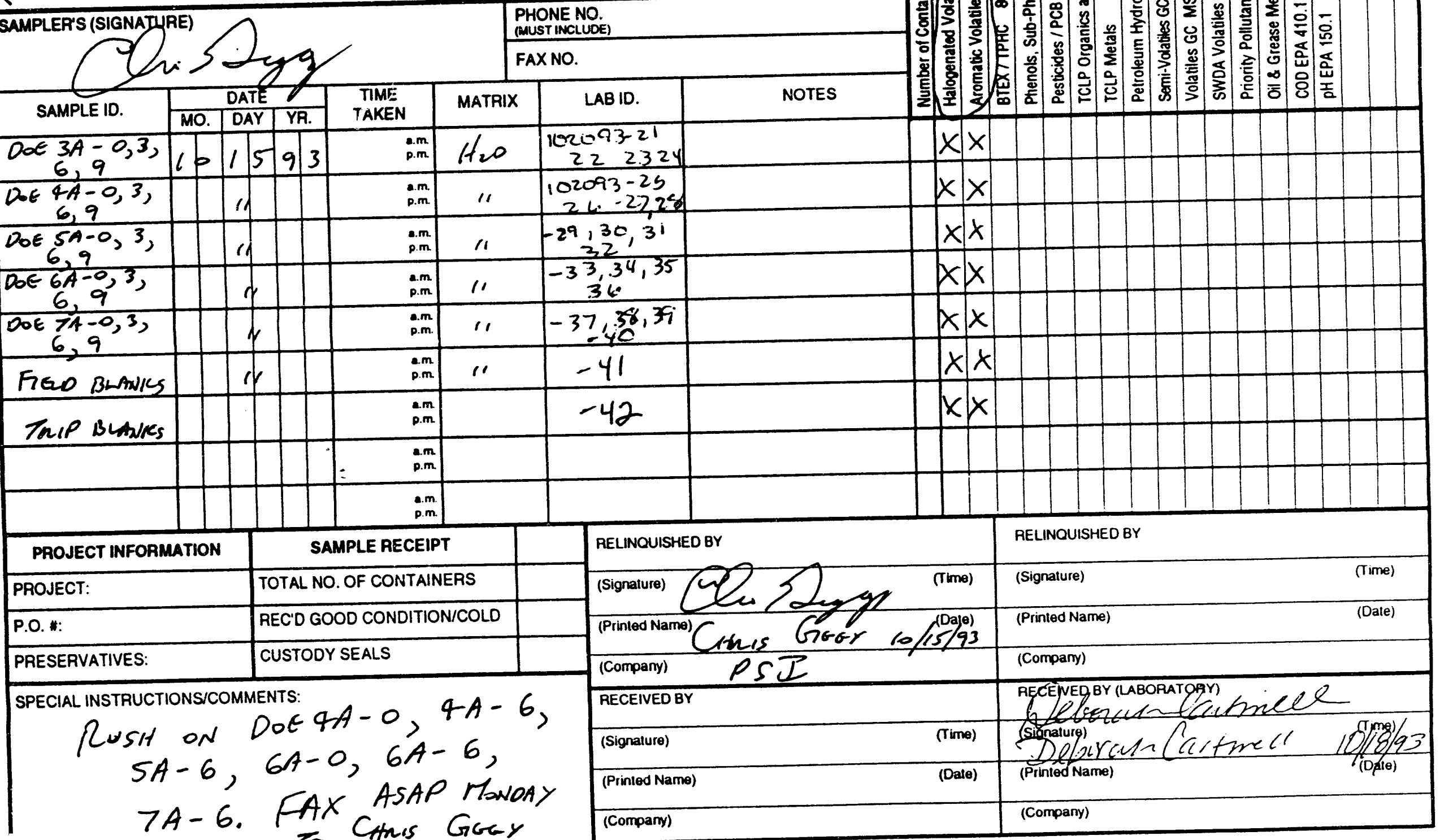




\section{CHAIN OF CUSTODY FORM}

Page 1 of 1

Aspon Environmentwl Laboratory

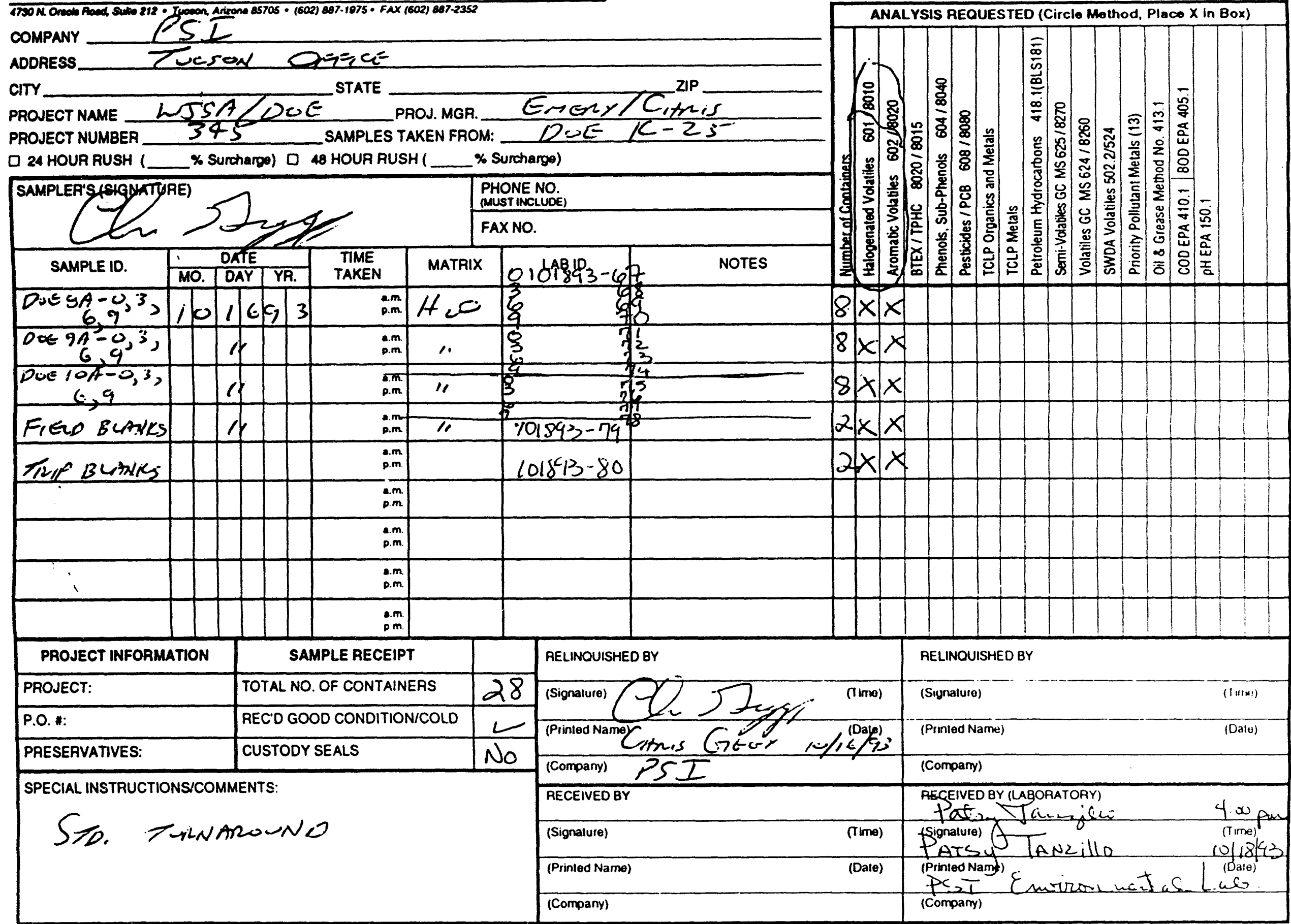




\section{CHAIN OF CUSTODY FORM \\ Arizona Certifled Laboratory}

Aspen Environmentel Laboratory

Page 1 of 1

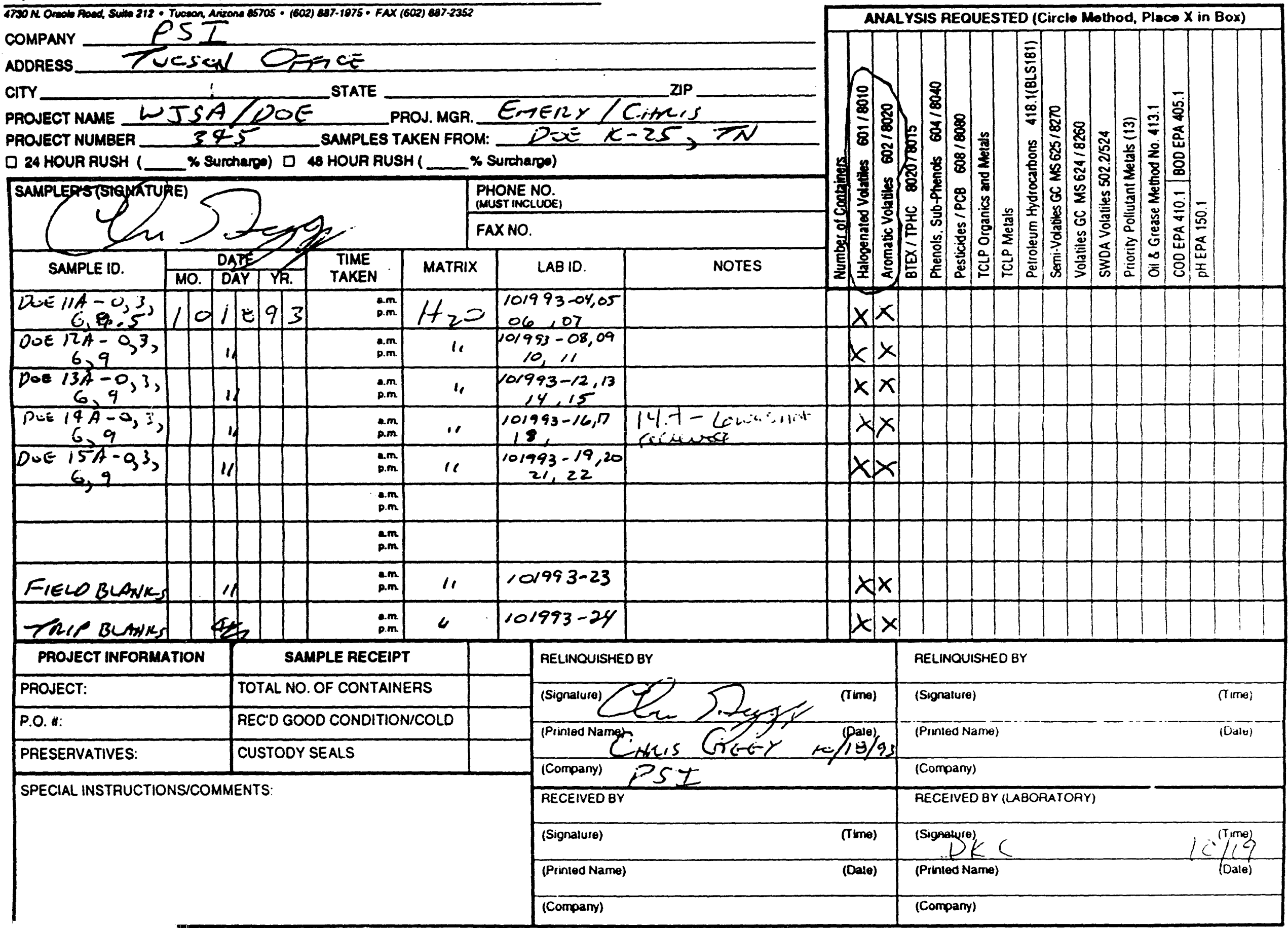




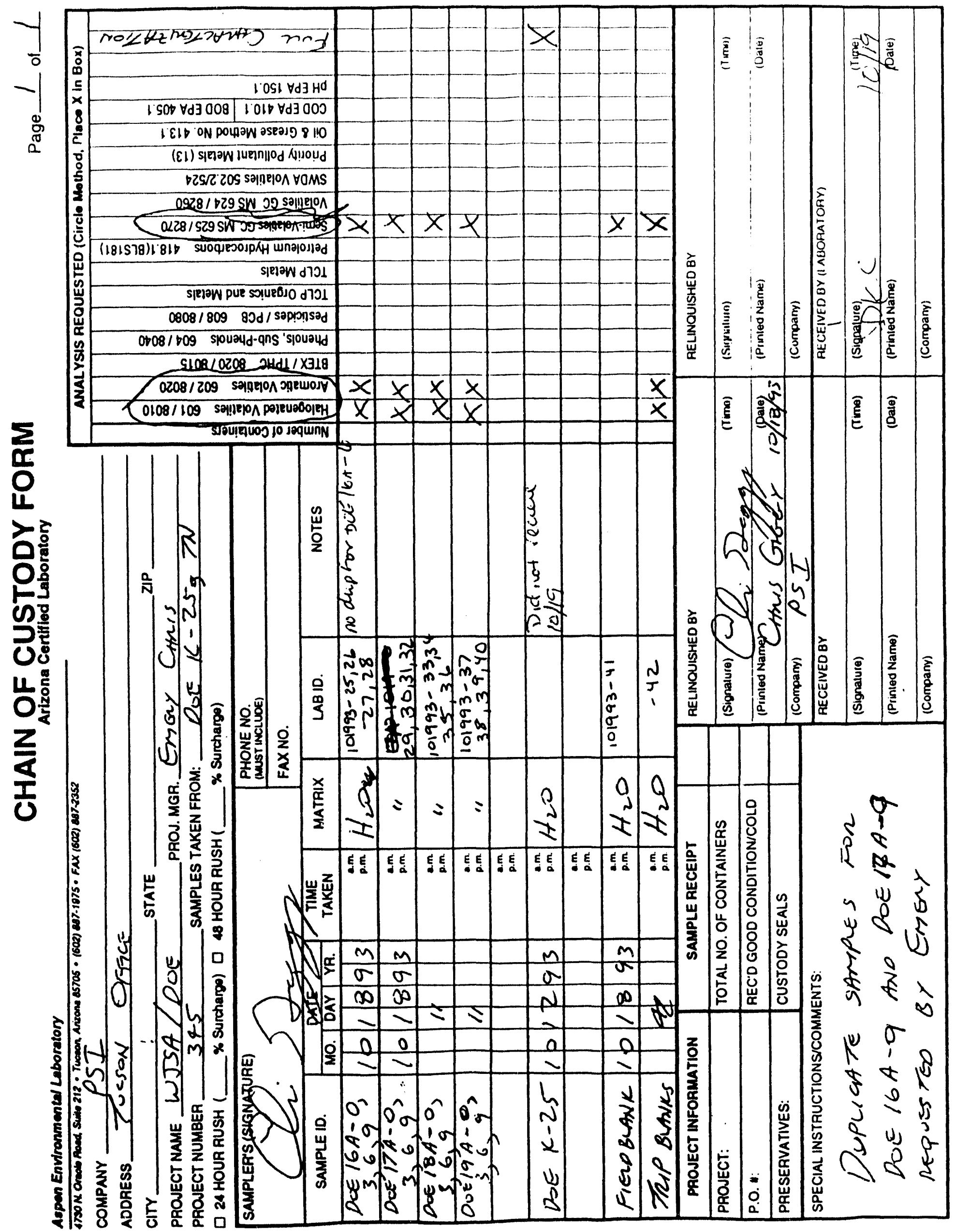


ANALYTICAL REPORT

State of Arizona License $\# 0009$

Client:

Project Name:

Project Number:

Sample ID:
Peroxidation Systems, Inc.

5151 E. Broadway, Suite 600

Tucson, Arizona 85711

WJSA/DOE

345

$091593-16,18,20,22$
Sample Type: Groundwater.

Date Sampled: $9 / 14 / 93$

Date Received: $9 / 15 / 93$

Date Analyzed: 9/15/93

Analyzed By: Deb Cartmell

EPA Method 601 - Purgeable Halocarbons

Reported in $\mu \mathrm{g} / \mathrm{L}$ (ppb)

\begin{tabular}{|c|c|c|c|c|c|}
\hline Compounds & $\begin{array}{l}\text { Limit of } \\
\text { Detection }\end{array}$ & $1 A-0^{\circ}$ & $1 A-4$ & $2 A-0^{\circ}$ & $2 A-4$ \\
\hline Bromodicloromethane & 1.0 & $<5.0$ & $<1.0$ & $<5.0$ & $<1.0$ \\
\hline Bromoform & 1.0 & $<5.0$ & $<1.0$ & $<5.0$ & $<1.0$ \\
\hline Bromomethane & 1.0 & $<5.0$ & $<1.0$ & $<5.0$ & $<1.0$ \\
\hline Carbon Tetrachloride & 1.0 & $<5.0$ & $<1.0$ & $<5.0$ & $<1.0$ \\
\hline Chlorobenzene & 1.0 & $<5.0$ & $<1.0$ & $<5.0$ & $<1.0$ \\
\hline Chloroethane & 1.0 & 290 & 161 & 243 & 203 \\
\hline Chloroform & 1.0 & $<5.0$ & $<1.0$ & $<5.0$ & $<1.0$ \\
\hline Chloromethane & 1.0 & $<5.0$ & $<1.0$ & $<5.0$ & $<1.0$ \\
\hline 2-Chloroethyl vinyl ether & 1.0 & $<5.0$ & $<1.0$ & $<5.0$ & $<1.0$ \\
\hline Dibromochloromethane & 1.0 & $<5.0$ & $<1.0$ & $<5.0$ & $<1.0$ \\
\hline 1,2-Dichlorobenzene & 1.0 & $<5.0$ & $<1.0$ & $<5.0$ & $<1.0$ \\
\hline 1,3-Dichlorobenzene & 1.0 & $<5.0$ & $<1.0$ & $<5.0$ & $<1.0$ \\
\hline 1,4-Dichlorobenzene & 1.0 & $<5.0$ & $<1.0$ & $<5.0$ & $<1.0$ \\
\hline 1,1-Dichloroethane & 1.0 & 322 & 113 & 258 & 109 \\
\hline 1,2-Dichloroethane & 1.0 & $<5.0$ & 1.7 & $<5.0$ & 3.4 \\
\hline 1,1-Dichloroethene & 1.0 & 10 & $<1.0$ & 7.0 & $<1.0$ \\
\hline Trans-1,2-Dichloroethene & 1.0 & $<5.0$ & $<1.0$ & $<5.0$ & $<1.0$ \\
\hline 1,2-Dichloropropane & 1.0 & $<5.0$ & $<1.0$ & $<5.0$ & $<1.0$ \\
\hline Cis-1,3-Dichloropropene & 1.0 & $<5.0$ & $<1.0$ & $<5.0$ & $<1.0$ \\
\hline Trans-1,3-Dichloropropene & 1.0 & $<5.0$ & $<1.0$ & $<5.0$ & $<1.0$ \\
\hline Methylene Chloride & 1.0 & $<5.0$ & $<1.0$ & $<5.0$ & $<1.0$ \\
\hline 1,1,2,2-Tetrachloroethane & 1.0 & $<5.0$ & $<1.0$ & $<5.0$ & $<1.0$ \\
\hline Tetrachloroethene & 1.0 & $<5.0$ & $<1.0$ & $<5.0$ & $<1.0$ \\
\hline 1,1,1-Trichloroethane & 1.0 & 186 & 20 & 131 & 4.9 \\
\hline 1,1,2-Trichloroethane & 1.0 & $<5.0$ & 1.5 & $<5.0$ & 1.9 \\
\hline Trichloroethene & 1.0 & $<5.0$ & $<1.0$ & $<5.0$ & $<1.0$ \\
\hline Trichlorofluoromethane & 1.0 & 134 & 48 & 115 & 46 \\
\hline Vinyl Chloride & 1.0 & 16 & 57 & 17 & $<1.0$ \\
\hline
\end{tabular}

Comments:

5:1 Dilution made on this sample.

Reviewed By:

Tarelli 


\section{ANALYTICAL REPORT}

State of Arizona License $\# 0009$

$\begin{array}{llll}\text { Client: } & \begin{array}{l}\text { Peroxidation Systems, Inc. } \\ \text { 5151 E. Broadway, Suite } 600\end{array} & \text { Sample Type: } & \text { Groundwater } \\ & \text { Tucson, Arizona 85711 } & \text { Date Sampled: } & 9 / 14 / 93 \\ \text { Project Name: } & \text { WJSA/DOE } & \text { Date Received: } & 9 / 15 / 93 \\ \text { Project Number: } & 345 & \text { Date Analyzed: } & 9 / 15 / 93 \\ \text { Sample ID: } & 091593-16,18,20,22 & \text { Analyzed By: } & \text { Deb Cartmell }\end{array}$

EPA Method 602 - Purgeable Aromatics (G.C. - PID)

Reported in $\mu \mathrm{g} / \mathrm{L}(\mathrm{ppb})$

\begin{tabular}{|l|c|c|c|c|c|c|}
\hline \multicolumn{1}{|c|}{ Compounds } & $\begin{array}{c}\text { Limit of } \\
\text { Detection }\end{array}$ & $1 \mathrm{~A}-0^{\circ}$ & $1 \mathrm{~A}-4$ & $2 \mathrm{~A}-0^{\circ}$ & $2 \mathrm{~A}-4$ & \\
\hline Benzene & 1.0 & 6.0 & $<1.0$ & $<5.0$ & $<1.0$ & $<1.0$ \\
Toluene & 1.0 & 7.3 & $<1.0$ & 5.6 & $<5.0$ & $<1.0$ \\
Chlorobenzene & 1.0 & $<5.0$ & $<1.0$ & $<5.0$ & $<1.0$ & $<1.0$ \\
Ethyl Benzene & 1.0 & $<5.0$ & $<1.0$ & $<5.0$ & $<5.0$ & $<1.0$ \\
m,p-Xylene & 1.0 & $<5.0$ & $<1.0$ & $<5.0$ & $<1.0$ \\
0-Xylene & 1.0 & $<5.0$ & $<1.0$ & $<5.0$ & $<1.0$ & $<1.0$ \\
1,2-Dichlorobenzene & 1.0 & $<5.0$ & $<1.0$ & $<5.0$ & $<5.0$ & $<1.0$ \\
1,3-Dichlorobenzene & 1.0 & $<5.0$ & $<1.0$ & $<5.0$ & $<1.0$ \\
1,4-Dichlorobenzene & 1.0 & $<5.0$ & $<1.0$ & $<5.0$ & \\
\hline
\end{tabular}

Comments:

5:1 Dilution made on this sample.

Reviewed By:

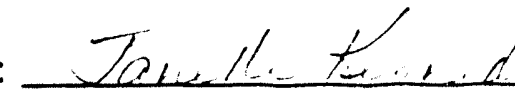

Date 


\section{PSI Environmental Laboratories}

2595 E. Airport Drive

Tucwon. Arizana 85706

$602.573-6565$

FAX 602.573-6550

1.600-P51-1.ABS

\section{ANALYTICAL REPORT}

State of Arizona License $\# 0009$

Client:

Peroxidation Systems, Inc.

5151 E. Broadway, Suite 600

Tucson, Arizona 85711

Project Name: WJSA/DOE

Project Number: $\quad 345$

Sample ID:
$091593-17,19,21,23$
Sample Type: Groundwater

Date Sampled: $\quad$ 9/14/93

Date Received: $\quad 9 / 15 / 93$

Date Analyzed: 9/15/93

Analyzed By: Deb Cartmell

EPA Method 601 - Purgeable Halocarbons

Reported in $\mu \mathrm{g} / \mathrm{L}$ (ppb)

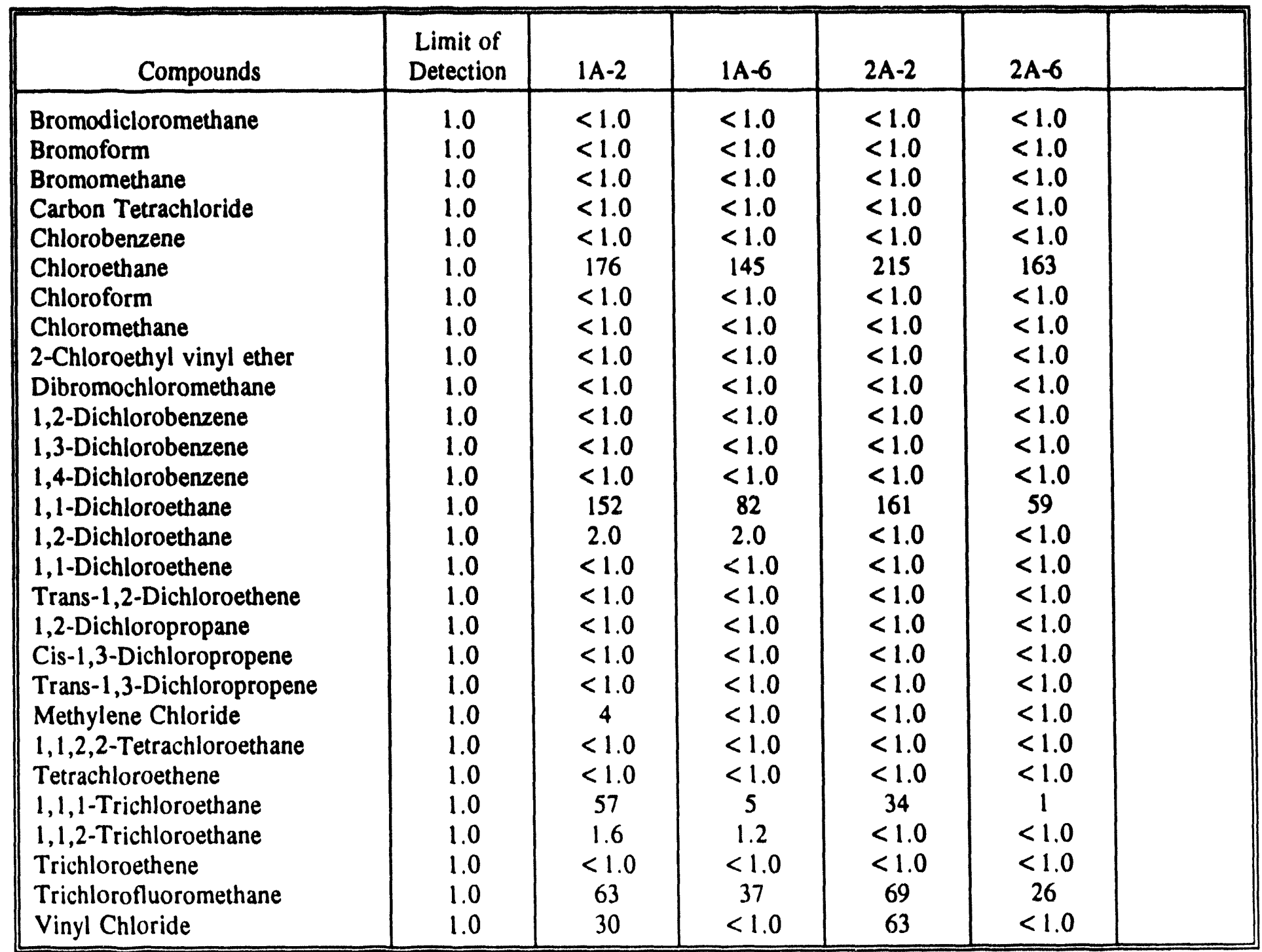

Comments:

Reviewed By: 
Client:

Peroxidation Systems, Inc.

5151 E. Broadway, Suite 600

Tucson, Arizona 85711

Projoct Name:

WJSA/DOE

Project Number:

345

Sample ID:

$091593-17,19,21,23$
Sample Type: Groundwater

Date Sampled: $\quad 9 / 14 / 93$

Date Received: $\quad 9 / 15 / 93$

Date Analyzed: $\quad 9 / 15 / 93$

Analyzed By: Deb Cartmell

EPA Method 602 - Purgeable Aromatics (G.C. - PID)

Reported in $\mu \mathrm{g} / \mathrm{L}$ (ppb)

\begin{tabular}{|c|c|c|c|c|c|}
\hline Compounds & $\begin{array}{l}\text { Limit of } \\
\text { Detection }\end{array}$ & $1 A-2$ & $1 A-6$ & $2 A-2$ & $2 A-6$ \\
\hline Benzene & 1.0 & $<1.0$ & $<1.0$ & $<1.0$ & $<1.0$ \\
\hline Toluene & 1.0 & $<1.0$ & $<1.0$ & $<1.0$ & $<1.0$ \\
\hline Chlorobenzene & 1.0 & $<1.0$ & $<1.0$ & $<1.0$ & $<1.0$ \\
\hline Ethyl Benzene & 1.0 & $<1.0$ & $<1.0$ & $<1.0$ & $<1.0$ \\
\hline m,p-Xylene & 1.0 & $<1.0$ & $<1.0$ & $<1.0$ & $<1.0$ \\
\hline $0-X y l e n e$ & 1.0 & $<1.0$ & $<1.0$ & $<1.0$ & $<1.0$ \\
\hline 1,2-Dichlorobenzene & 1.0 & $<1.0$ & $<1.0$ & $<1.0$ & $<1.0$ \\
\hline 1,3-Dichlorobenzene & 1.0 & $<1.0$ & $<1.0$ & $<1.0$ & $<1.0$ \\
\hline 1,4-Dichlorobenzene & 1.0 & $<1.0$ & $<1.0$ & $<1.0$ & $<1.0$ \\
\hline
\end{tabular}

Comments:

Reviewed By:

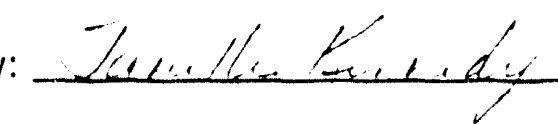

Date 


\section{PSI Environmental Laboratories}

Client:

Peroxidation Systems, Inc. 5151 E. Broadway, Suite 600 Tucson, Arizona 85711

$\begin{array}{ll}\text { Project Name: } & \text { WJSA/DOE } \\ \text { Project Number: } & 345 \\ \text { Sample ID: } & 102093-21-24\end{array}$

Sample Type: Groundwater.

Date Sampled: $\quad 10 / 15 / 93$

Date Received: $\quad 10 / 18 / 93$

Date Analyzed: 10/18/93

Analyzed By: Deb Cartmell

EPA Method 601 - Purgeable Halocarbons

Reported in $\mu \mathrm{g} / \mathrm{L}(\mathrm{ppb})$

\begin{tabular}{|c|c|c|c|c|c|c|}
\hline Compounds & $\begin{array}{l}\text { Limit of } \\
\text { Detection }\end{array}$ & $3 A-0$ & $3 A-3$ & $3 A-6$ & $3 A-9$ & \\
\hline Bromodicloromethane & 1.0 & $<1.0$ & $<1.0$ & $<1.0$ & $<1.0$ & \\
\hline Bromoform & 1.0 & $<1.0$ & $<1.0$ & $<1.0$ & $<1.0$ & \\
\hline Bromomethane & 1.0 & $<1.0$ & $<1.0$ & $<1.0$ & $<1.0$ & \\
\hline Carbon Tetrachloride & 1.0 & $<1.0$ & $<1.0$ & $<1.0$ & $<1.0$ & \\
\hline Chlorobenzene & 1.0 & $<1.0$ & $<1.0$ & $<1.0$ & $<1.0$ & \\
\hline Chloroethane & 1.0 & 11 & 7 & 3 & $<1.0$ & \\
\hline Chloroform & 1.0 & 1.0 & $<1.0$ & $<1.0$ & $<1.0$ & \\
\hline Chloromethane & 1.0 & $<1.0$ & $<1.0$ & $<1.0$ & $<1.0$ & \\
\hline 2-Chloroethyl vinyl ether & 1.0 & $<1.0$ & $<1.0$ & $<1.0$ & $<1.0$ & \\
\hline Dibromochloromethane & 1.0 & $<1.0$ & $<1.0$ & $<1.0$ & $<1.0$ & \\
\hline 1,2-Dichlorobenzene & 1.0 & $<1.0$ & $<1.0$ & $<1.0$ & $<1.0$ & \\
\hline 1,3-Dichlorobenzene & 1.0 & $<1.0$ & $<1.0$ & $<1.0$ & $<1.0$ & \\
\hline 1,4-Dichlorobenzene & 1.0 & $<1.0$ & $<1.0$ & $<1.0$ & $<1.0$ & \\
\hline 1,1-Dichloroethane & 1.0 & 239 & 195 & 145 & 107 & \\
\hline 1,2-Dichloroethane & 1.0 & $<1.0$ & $<1.0$ & $<1.0$ & $<1.0$ & \\
\hline 1,1-Dichloroethene & 1.0 & $<1.0$ & $<1.0$ & $<1.0$ & $<1.0$ & \\
\hline Trans-1,2-Dichloroethene & 1.0 & $<1.0$ & $<1.0$ & $<1.0$ & $<1.0$ & \\
\hline 1,2-Dichloropropane & 1.0 & $<1.0$ & $<1.0$ & $<1.0$ & $<1.0$ & \\
\hline Cis-1,3-Dichloropropene & 1.0 & $<1.0$ & $<1.0$ & $<1.0$ & $<1.0$ & \\
\hline Trans-1,3-Dichloropropene & 1.0 & $<1.0$ & $<1.0$ & $<1.0$ & $<1.0$ & \\
\hline Methylene Chloride & 1.0 & 78 & 72 & 60 & 54 & \\
\hline 1,1,2,2-Tetrachloroethane & 1.0 & $<1.0$ & $<1.0$ & $<1.0$ & $<1.0$ & \\
\hline Tetrachloroethene & 1.0 & $<1.0$ & $<1.0$ & $<1.0$ & $<1.0$ & \\
\hline 1,1,1-Trichloroethane & 1.0 & 199 & 148 & 103 & 70 & \\
\hline 1,1,2-Trichloroethane & 1.0 & 3.0 & 2.0 & 2.0 & 2.0 & \\
\hline Trichloroethene & 1.0 & $<1.0$ & $<1.0$ & $<1.0$ & $<1.0$ & \\
\hline Trichlorofluoromethane & 1.0 & 139 & 132 & 117 & 105 & \\
\hline Vinyl Chloride & 1.0 & $<1.0$ & $<1.0$ & $<1.0$ & $<1.0$ & \\
\hline
\end{tabular}

Comments:

Reviewed By: 


\section{PSI Environmental Laboratories}

2595 E. Alnuort Drive Tucson, Arlzono 85706 602.573.6565 FAX 602.573.6550 1.800.P5I-LAB5

\section{ANALYTICAL REPORT}

State of Arizona License $\$ 0009$

$\begin{array}{llll}\text { Client: } & \begin{array}{l}\text { Peroxidation Systems, Inc. } \\ \text { 5151 E. Broadway, Suite 600 } \\ \text { Tucson, Arizona 85711 }\end{array} & \text { Sample Type: } & \text { Groundwater } \\ & \text { WJSA/DOE } & \text { Date Sampled: } & 10 / 15 / 93 \\ \text { Project Name: } & \text { Date Received: } & \text { 10/18/93 } \\ \text { Project Number: } & 345 & \text { Date Analyzed: } & \text { 10/18/93 } \\ \text { Sample ID: } & 102093-21-24 & \text { Analyzed By: } & \text { Deb Cartmell }\end{array}$

EPA Method 602 - Purgeable Aromatics (G.C. - PID)

Reported in $\mu \mathrm{g} / \mathrm{L}$ (ppb)

\begin{tabular}{|l|c|c|c|c|c|c|}
\hline \multicolumn{1}{|c|}{ Compounds } & $\begin{array}{c}\text { Limit of } \\
\text { Detection }\end{array}$ & $3 \mathrm{~A}-0$ & $3 \mathrm{~A}-3$ & $3 \mathrm{~A}-6$ & $3 \mathrm{~A}-9$ & \\
\hline Benzene & 1.0 & 1.7 & $<1.0$ & $<1.0$ & $<1.0$ & $<1.0$ \\
Toluene & 1.0 & $<1.0$ & $<1.0$ & $<1.0$ & $<1.0$ & $<1.0$ \\
Chlorobenzene & 1.0 & $<1.0$ & $<1.0$ & $<1.0$ & $<1.0$ & $<1.0$ \\
Ethyl Benzene & 1.0 & $<1.0$ & $<1.0$ & $<1.0$ & $<1.0$ & $<1.0$ \\
m,p-Xylene & 1.0 & $<1.0$ & $<1.0$ & $<1.0$ & $<1.0$ \\
0-Xylene & 1.0 & $<1.0$ & $<1.0$ & $<1.0$ & $<1.0$ & $<1.0$ \\
1,2-Dichlorobenzene & 1.0 & $<1.0$ & $<1.0$ & $<1.0$ & $<1.0$ & $<1.0$ \\
1,3-Dichlorobenzene & 1.0 & $<1.0$ & $<1.0$ & $<1.0$ & $<1.0$ & $<1.0$ \\
1,4-Dichlorobenzene & 1.0 & $<1.0$ & $<1.0$ & $<1.0$ & \\
\hline
\end{tabular}

Comments:

Reviewed By: Ted, lle terendy

Date $1,15 / 93$ 


\section{PSI Environmental Laboratories}

Client:

Peroxidation Systems, Inc. 5151 E. Broadway, Suite 600 Tucson, Arizona 85711

Project Name:

WJSA/DOE

Project Number: 345

Sample ID:
$102093-25,27,31,33,35$
Sample Type: Groundwater

Date Sampled: $\quad 10 / 15 / 93$

Date Received: 10/18/93

Date Analyzed: $10 / 18 / 93$

Analyzed By: Deb Cartmell

\section{EPA Method 601 - Purgeable Halocarbons \\ Reported in $\mu \mathrm{g} / \mathrm{L}$ (ppb)}

\begin{tabular}{|c|c|c|c|c|c|c|}
\hline Compounds & $\begin{array}{l}\text { Limit of } \\
\text { Detection }\end{array}$ & $4 A-0$ & $4 A-6$ & $5 A-6$ & $6 \mathrm{~A}-0$ & $6 A-6$ \\
\hline Bromodicloromethane & 1.0 & $<1.0$ & $<1.0$ & $<1.0$ & $<1.0$ & $<1.0$ \\
\hline Bromoform & 1.0 & $<1.0$ & $<1.0$ & $<1.0$ & $<1.0$ & $<1.0$ \\
\hline Bromomethane & 1.0 & $<1.0$ & $<1.0$ & $<1.0$ & $<1.0$ & $<1.0$ \\
\hline Carbon Tetrachloride & 1.0 & $<1.0$ & $<1.0$ & $<1.0$ & $<1.0$ & $<1.0$ \\
\hline Chlorobenzene & 1.0 & $<1.0$ & $<1.0$ & $<1.0$ & $<1.0$ & $<1.0$ \\
\hline Chloroethane & 1.0 & 17 & 4.0 & $<1.0$ & 15 & $<1.0$ \\
\hline Chloroform & 1.0 & $<1.0$ & $<1.0$ & $<1.0$ & $<1.0$ & $<1.0$ \\
\hline Chloromethane & 1.0 & $<1.0$ & $<1.0$ & $<1.0$ & $<1.0$ & $<1.0$ \\
\hline 2-Chloroethyl vinyl ether & 1.0 & $<1.0$ & $<1.0$ & $<1.0$ & $<1.0$ & $<1.0$ \\
\hline Dibromochloromethane & 1.0 & $<1.0$ & $<1.0$ & $<1.0$ & $<1.0$ & $<1.0$ \\
\hline 1,2-Dichlorobenzene & 1.0 & $<1.0$ & $<1.0$ & $<1.0$ & $<1.0$ & $<1.0$ \\
\hline 1,3-Dichlorobenzene & 1.0 & $<1.0$ & $<1.0$ & $<1.0$ & $<1.0$ & $<1.0$ \\
\hline 1,4-Dichlorobenzene & 1.0 & $<1.0$ & $<1.0$ & $<1.0$ & $<1.0$ & $<1.0$ \\
\hline 1,1-Dichloroethane & 1.0 & 274 & 149 & 42 & 277 & 40 \\
\hline 1,2-Dichloroethane & 1.0 & $<1.0$ & $<1.0$ & $<1.0$ & $<1.0$ & $<1.0$ \\
\hline 1,1-Dichloroethene & 1.0 & $<1.0$ & $<1.0$ & $<1.0$ & $<1.0$ & $<1.0$ \\
\hline Trans-1,2-Dichloroethene & 1.0 & $<1.0$ & $<1.0$ & $<1.0$ & $<1.0$ & $<1.0$ \\
\hline 1,2-Dichloropropane & 1.0 & $<1.0$ & $<1.0$ & $<1.0$ & $<1.0$ & $<1.0$ \\
\hline Cis-1,3-Dichloropropene & 1.0 & $<1.0$ & $<1.0$ & $<1.0$ & $<1.0$ & $<1.0$ \\
\hline Trans-1,3-Dichloropropene & 1.0 & $<1.0$ & $<1.0$ & $<1.0$ & $<1.0$ & $<1.0$ \\
\hline Methylene Chloride & 1.0 & 101 & 66 & 72 & 107 & 68 \\
\hline 1,1,2,2-Tetrachloroethane & 1.0 & $<1.0$ & $<1.0$ & $<1.0$ & $<1.0$ & $<1.0$ \\
\hline Tetrachloroethene & 1.0 & $<1.0$ & $<1.0$ & $<1.0$ & $<1.0$ & $<1.0$ \\
\hline 1,1,1-Trichloroethane & 1.0 & 232 & 117 & 132 & 242 & 123 \\
\hline 1,1,2-Trichloroethane & 1.0 & 3.0 & 2.0 & $<1.0$ & $<1.0$ & $<1.0$ \\
\hline Trichloroethene & 1.0 & $<1.0$ & $<1.0$ & $<1.0$ & $<1.0$ & $<1.0$ \\
\hline Trichlorofluoromethane & 1.0 & 195 & 147 & NA & 187 & 128 \\
\hline Vinyl Chloride & 1.0 & $<1.0$ & $<1.0$ & $<1.0$ & $<1.0$ & $<1.0$ \\
\hline
\end{tabular}

Comments: NA - Data not available, Pending QA/OC.

Reviewed By: 


\section{PSI Environmental Laboratories}

ANALYTICAL REPORT

State of Arizona License $\# 0009$

Client:

Peroxidation Systems, Inc. 5151 E. Broadway, Suite 600 Tucson, Arizona 85711

Project Name:

WJSA/DOE

Project Number: 345

Sample ID:
Sample Type: Groundwater

Date Sampled: $\quad 10 / 15 / 93$

Date Received: $\quad 10 / 18 / 93$

Date Analyzed: $10 / 18 / 93$

Analyzed By: Deb Cartmell

EPA Method 602 - Purgeable Aromatics (G.C. - PID)

Reported in $\mu \mathrm{g} / \mathrm{L}(\mathrm{ppb})$

\begin{tabular}{|l|c|c|c|c|c|c|}
\hline \multicolumn{1}{|c|}{ Compounds } & $\begin{array}{c}\text { Limit of } \\
\text { Detection }\end{array}$ & $4 \mathrm{~A}-0$ & $4 \mathrm{~A}-6$ & $5 \mathrm{~A}-6$ & $6 \mathrm{~A}-0$ & $6 \mathrm{~A}-6$ \\
\hline Benzene & 1.0 & 3.3 & $<1.0$ & $<1.0$ & 2.0 & $<1.0$ \\
Toluene & 1.0 & 1.8 & $<1.0$ & $<1.0$ & $<1.0$ & $<1.0$ \\
Chlorobenzene & 1.0 & $<1.0$ & $<1.0$ & $<1.0$ & $<1.0$ & $<1.0$ \\
Ethyl Benzene & 1.0 & $<1.0$ & $<1.0$ & $<1.0$ & $<1.0$ & $<1.0$ \\
m,p-Xylene & 1.0 & $<1.0$ & $<1.0$ & $<1.0$ & $<1.0$ & $<1.0$ \\
0-Xylene & 1.0 & $<1.0$ & $<1.0$ & $<1.0$ & $<1.0$ & $<1.0$ \\
1,2-Dichlorobenzene & 1.0 & $<1.0$ & $<1.0$ & $<1.0$ & $<1.0$ & $<1.0$ \\
1,3-Dichlorobenzene & 1.0 & $<1.0$ & $<1.0$ & $<1.0$ & $<1.0$ & $<1.0$ \\
1,4-Dichlorobenzene & 1.0 & $<1.0$ & $<1.0$ & $<1.0$ & $<1.0$ & $<1.0$ \\
\hline
\end{tabular}

Comments:

Reviewed By:

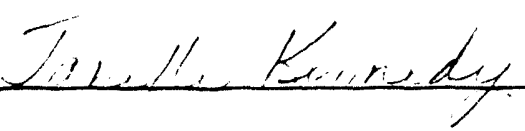

Date

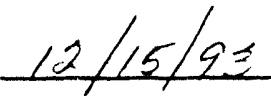


2595 E. Alport Drive Tucson. Arizana 85706 602-573-6565 FAX 602-573-6550 I-800-P5I-LAB5 ANALYTICAL REPORT

State of Arizona License $\# 0009$

Client:

Peroxidation Systems, Inc. 5151 E. Broadway, Suite 600

Tucson, Arizona 85711

Project Name: WJSA/DOE

Project Number: 345

Sample ID:

$102093-26,28,29,30,32$
Sample Type: Groundwater

Date Sampled: $\quad 10 / 15 / 93$

Date Received: $\quad 10 / 18 / 93$

Date Analyzed: 10/18/93

Analyzed By: Deb Cartmell

EPA Method 601 - Purgeable Halocarbons

Reported in $\mu \mathrm{g} / \mathrm{L}$ (ppb)

\begin{tabular}{|c|c|c|c|c|c|c|}
\hline Compounds & $\begin{array}{l}\text { Limit of } \\
\text { Detection }\end{array}$ & $4 A-3$ & $4 A-9$ & $5 A-0$ & $5 A-3$ & $5 A-9$ \\
\hline Bromodicloromethane & 1.0 & $<1.0$ & $<1.0$ & $<1.0$ & $<1.0$ & $<1.0$ \\
\hline Bromoform & 1.0 & $<1.0$ & $<1.0$ & $<1.0$ & $<1.0$ & $<1.0$ \\
\hline Bromomethane & 1.0 & $<1.0$ & $<1.0$ & $<1.0$ & $<1.0$ & $<1.0$ \\
\hline Carbon Tetrachloride & 1.0 & $<1.0$ & $<1.0$ & $<1.0$ & $<1.0$ & $<1.0$ \\
\hline Chlorobenzene & 1.0 & $<1.0$ & $<1.0$ & $<1.0$ & $<1.0$ & $<1.0$ \\
\hline Chloroethane & 1.0 & 7.0 & $<1.0$ & 13 & 1.0 & $<1.0$ \\
\hline Chloroform & 1.0 & $<1.0$ & $<1.0$ & $<1.0$ & $<1.0$ & $<1.0$ \\
\hline Chloromethane & 1.0 & $<1.0$ & $<1.0$ & $<1.0$ & $<1.0$ & $<1.0$ \\
\hline 2-Chloroethyl vinyl ether & 1.0 & $<1.0$ & $<1.0$ & $<1.0$ & $<1.0$ & $<1.0$ \\
\hline Dibromochloromethane & 1.0 & $<1.0$ & $<1.0$ & $<1.0$ & $<1.0$ & $<1.0$ \\
\hline 1,2-Dichlorobenzene & 1.0 & $<1.0$ & $<1.0$ & $<1.0$ & $<1.0$ & $<1.0$ \\
\hline 1,3-Dichlorobenzene & 1.0 & $<1.0$ & $<1.0$ & $<1.0$ & $<1.0$ & $<1.0$ \\
\hline 1,4-Dichlorobenzene & 1.0 & $<1.0$ & $<1.0$ & $<1.0$ & $<1.0$ & $<1.0$ \\
\hline 1,1-Dichloroethane & 1.0 & 215 & 106 & 265 & 139 & 10 \\
\hline 1,2-Dichloroethane & 1.0 & 2.0 & $<1.0$ & 2.0 & $<1.0$ & $<1.0$ \\
\hline 1,1-Dichloroethene & 1.0 & $<1.0$ & $<1.0$ & $<1.0$ & $<1.0$ & $<1.0$ \\
\hline Trans-1,2-Dichloroethene & 1.0 & $<1.0$ & $<1.0$ & $<1.0$ & $<1.0$ & $<1.0$ \\
\hline 1,2-Dichloropropane & 1.0 & $<1.0$ & $<1.0$ & $<1.0$ & $<1.0$ & $<1.0$ \\
\hline Cis-1,3-Dichloropropene & 1.0 & $<1.0$ & $<1.0$ & $<1.0$ & $<1.0$ & $<1.0$ \\
\hline Trans-1,3-Dichloropropene & 1.0 & $<1.0$ & $<1.0$ & $<1.0$ & $<1.0$ & $<1.0$ \\
\hline Methylene Chloride & 1.0 & 81 & 56 & 101 & 91 & 56 \\
\hline 1,1,2,2-Tetrachloroethane & 1.0 & $<1.0$ & $<1.0$ & $<1.0$ & $<1.0$ & $<1.0$ \\
\hline Tetrachloroethene & 1.0 & $<1.0$ & $<1.0$ & $<1.0$ & $<1.0$ & $<1.0$ \\
\hline 1,1,1-Trichloroethane & 1.0 & 175 & 85 & 242 & 196 & 86 \\
\hline 1,1,2-Trichloroethane & 1.0 & $<1.0$ & $<1.0$ & 3.0 & 2.0 & $<1.0$ \\
\hline Trichloroethene & 1.0 & $<1.0$ & $<1.0$ & $<1.0$ & $<1.0$ & $<1.0$ \\
\hline Trichlorofluoromethane & 1.0 & 142 & 107 & NA & NA & NA \\
\hline Vinyl Chloride & 1.0 & $<1.0$ & $<1.0$ & $<1.0$ & $<1.0$ & $<1.0$ \\
\hline
\end{tabular}

Comments: NA - Data not availahle, Pending OA/OC. 
ANALYTICAL REPORT

State of Arizona License \#0009

$\begin{array}{llll}\text { Client: } & \begin{array}{l}\text { Peroxidation Systems, Inc. } \\ \text { 5151 E. Broadway, Suite } 600\end{array} & \text { Sample Type: } & \text { Groundwater } \\ & \text { Tucson, Arizona 85711 } & \text { Date Sampled: } & 10 / 15 / 93 \\ \text { Project Name: } & \text { WJSA/DOE } & \text { Date Received: } & 10 / 18 / 93 \\ \text { Project Number: } & 345 & \text { Date Analyzed: } & 10 / 18 / 93 \\ \text { Sample ID: } & 102093-26,28,29,30,32 & \text { Analyzed By: } & \text { Deb Cartmell }\end{array}$

EPA Method 602 - Purgeable Aromatics (G.C. - PID)

Reported in $\mu \mathrm{g} / \mathrm{L}(\mathrm{ppb})$

\begin{tabular}{||l|c|c|c|c|c|c||}
\hline \multicolumn{1}{|c|}{ Compounds } & $\begin{array}{c}\text { Limit of } \\
\text { Detection }\end{array}$ & $4 \mathrm{~A}-3$ & $4 \mathrm{~A}-9$ & $5 \mathrm{~A}-0$ & $5 \mathrm{~A}-3$ & $5 \mathrm{~A}-9$ \\
\hline Benzene & 1.0 & $<1.0$ & $<1.0$ & 3.6 & $<1.0$ & $<1.0$ \\
Toluene & 1.0 & $<1.0$ & $<1.0$ & 2.0 & $<1.0$ & $<1.0$ \\
Chlorobenzene & 1.0 & $<1.0$ & $<1.0$ & $<1.0$ & $<1.0$ & $<1.0$ \\
Ethyl Benzene & 1.0 & $<1.0$ & $<1.0$ & $<1.0$ & $<1.0$ & $<1.0$ \\
m,p-Xylene & 1.0 & $<1.0$ & $<1.0$ & $<1.0$ & $<1.0$ & $<1.0$ \\
o-Xylene & 1.0 & $<1.0$ & $<1.0$ & $<1.0$ & $<1.0$ & $<1.0$ \\
1,2-Dichlorobenzene & 1.0 & $<1.0$ & $<1.0$ & $<1.0$ & $<1.0$ & $<1.0$ \\
1,3-Dichlorobenzene & 1.0 & $<1.0$ & $<1.0$ & $<1.0$ & $<1.0$ & $<1.0$ \\
1,4-Dichlorobenzene & 1.0 & $<1.0$ & $<1.0$ & $<1.0$ & $<1.0$ & $<1.0$ \\
\hline
\end{tabular}

Comments:

Reviewed By:

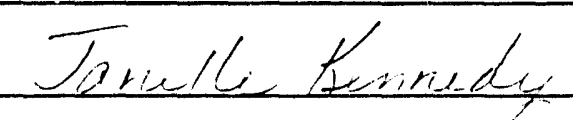

Date

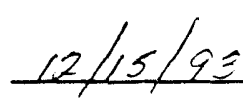


ANALYTICAL REPORT

State of Arizona License $\# 0009$

Client: $\quad$ Peroxidation Systems, Inc. 5151 E. Broadway, Suite 600 Tucson, Arizona 85711

Project Name: $\quad$ WJSA/DOE

Project Number: $\quad 345$

Sample ID: $\quad$ 102093-34, 36, 39
Sample Type: Groundwater

Date Sampled: $\quad 10 / 15 / 93$

Date Received: $\quad$ 10/18/93

Date Analyzed: 10/18/93

Analyzed By: Deb Cartmell

EPA Method 601 - Purgeable Halocarbons

Reported in $\mu \mathrm{g} / \mathrm{L}$ (ppb)

\begin{tabular}{|c|c|c|c|c|}
\hline Compounds & $\begin{array}{l}\text { Limit of } \\
\text { Detection }\end{array}$ & $6 A-3$ & $6 A-9$ & $7 A-6$ \\
\hline Bromodicloromethane & 1.0 & $<1.0$ & $<1.0$ & $<1.0$ \\
\hline Bromoform & 1.0 & $<1.0$ & $<1.0$ & $<1.0$ \\
\hline Bromomethane & 1.0 & $<1.0$ & $<1.0$ & $<1.0$ \\
\hline Carbon Tetrachloride & 1.0 & $<1.0$ & $<1.0$ & $<1.0$ \\
\hline Chlorobenzene & 1.0 & $<1.0$ & $<1.0$ & $<1.0$ \\
\hline Chloroethane & 1.0 & 2.0 & $<1.0$ & $<1.0$ \\
\hline Chloroform & 1.0 & $<1.0$ & $<1.0$ & $<1.0$ \\
\hline Chloromethane & 1.0 & $<1.0$ & $<1.0$ & $<1.0$ \\
\hline 2-Chloroethyl vinyl ether & 1.0 & $<1.0$ & $<1.0$ & $<1.0$ \\
\hline Dibromochloromethane & 1.0 & $<1.0$ & $<1.0$ & $<1.0$ \\
\hline 1,2-Dichlorobenzene & 1.0 & $<1.0$ & $<1.0$ & $<1.0$ \\
\hline 1,3-Dichlorobenzene & 1.0 & $<1.0$ & $<1.0$ & $<1.0$ \\
\hline 1,4-Dichlorobenzene & 1.0 & $<1.0$ & $<1.0$ & $<1.0$ \\
\hline 1,1-Dichloroethane & 1.0 & 139 & 12 & 32 \\
\hline 1,2-Dichloroethane & 1.0 & $<1.0$ & $<1.0$ & $<1.0$ \\
\hline 1,1-Dichloroethene & 1.0 & $<1.0$ & $<1.0$ & $<1.0$ \\
\hline Trans-1,2-Dichloroethene & 1.0 & $<1.0$ & $<1.0$ & $<1.0$ \\
\hline 1,2-Dichloropropane & 1.0 & $<1.0$ & $<1.0$ & $<1.0$ \\
\hline Cis-1,3-Dichloropropene & 1.0 & $<1.0$ & $<1.0$ & $<1.0$ \\
\hline Trans-1,3-Dichloropropene & 1.0 & $<1.0$ & $<1.0$ & $<1.0$ \\
\hline Methylene Chloride & 1.0 & 89 & 59 & 61 \\
\hline 1,1,2,2-Tetrachloroethane & 1.0 & $<1.0$ & $<1.0$ & $<1.0$ \\
\hline Tetrachloroethene & 1.0 & $<1.0$ & $<1.0$ & $<1.0$ \\
\hline 1,1,1-Trichloroethane & 1.0 & 180 & 84 & 102 \\
\hline 1,1,2-Trichloroethane & 1.0 & $<1.0$ & $<1.0$ & $<1.0$ \\
\hline Trichloroethene & 1.0 & $<1.0$ & $<1.0$ & $<1.0$ \\
\hline Trichlorofluoromethane & 1.0 & 160 & 115 & 112 \\
\hline Vinyl Chloride & 1.0 & $<1.0$ & $<1.0$ & $<1.0$ \\
\hline
\end{tabular}

Comments:

Reviewed By: 


\section{A PI Environmental Laboratories}

2595 E. Alrpart Drive Tucson. Arizona 85706 602-57.3-6565 FAX 602-573-6550 1-800-P5I-LAB5

\section{ANALYTICAL REPORT}

State of Arizona License \#0009

Client:

Peroxidation Systems, Inc. 5151 E. Broadway, Suite 600 Tucson, Arizona 85711

Project Name:

WJSA/DOE

Project Number: $\quad 345$

Sample ID:

102093-34, 36, 39
Sample Type: Groundwater

Date Sampled: $\quad 10 / 15 / 93$

Date Received: $\quad 10 / 18 / 93$

Date Analyzed: $10 / 18 / 93$

Analyzed By: Deb Cartmell

EPA Method 602 - Purgeable Aromatics (G.C. - PID)

Reported in $\mu \mathrm{g} / \mathrm{L}(\mathrm{ppb})$

\begin{tabular}{||l|c|c|c|c|c|c||}
\hline \multicolumn{1}{|c|}{ Compounds } & $\begin{array}{c}\text { Limit of } \\
\text { Detection }\end{array}$ & $6 \mathrm{~A}-3$ & $6 \mathrm{~A}-9$ & $7 \mathrm{~A}-6$ & & \\
\hline Benzene & 1.0 & $<1.0$ & $<1.0$ & $<1.0$ & & \\
Toluene & 1.0 & $<1.0$ & $<1.0$ & $<1.0$ & & \\
Chlorobenzene & 1.0 & $<1.0$ & $<1.0$ & $<1.0$ & & \\
Ethyl Benzene & 1.0 & $<1.0$ & $<1.0$ & $<1.0$ & & \\
m,p-Xylene & 1.0 & $<1.0$ & $<1.0$ & $<1.0$ & & \\
0-Xylene & 1.0 & $<1.0$ & $<1.0$ & $<1.0$ & & \\
1,2-Dichlorobenzene & 1.0 & $<1.0$ & $<1.0$ & $<1.0$ & & \\
1,3-Dichlorobenzene & 1.0 & $<1.0$ & $<1.0$ & $<1.0$ & & \\
1,4-Dichlorobenzene & 1.0 & $<1.0$ & $<1.0$ & $<1.0$ & & \\
\hline
\end{tabular}

Comments:

Reviewed By:

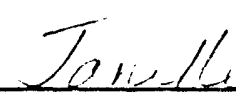

Date

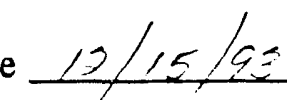


2595 E. Almport Drive Tucson. Arlzona 85706 602-573-6565 FAX 602-573-6550 1-800-P5I-LA85 ANALYTICAL REPORT

State of Arizona License $\# 0009$

Client:

5151 E. Broadway, Suite 600
Tucson, Arizona 85711

Project Name: $\quad$ WJSA/DOE

Project Number: $\quad 345$

Sample ID: $\quad$ 102093-37, 38, 40
Sample Type: Groundwater

Date Sampled: $\quad 10 / 15 / 93$

Date Received: $\quad 10 / 18 / 93$

Date Analyzed: 10/18/93

Analyzed By: Deb Cartmell

\section{EPA Method 601 - Purgeable Halocarbons}

Reported in $\mu \mathrm{g} / \mathrm{L}(\mathrm{ppb})$

\begin{tabular}{|c|c|c|c|c|c|}
\hline Compounds & $\begin{array}{l}\text { Limit of } \\
\text { Detection }\end{array}$ & $7 \mathrm{~A}-0$ & $7 A-3$ & 7A-9 & \\
\hline Bromodicloromethane & 1.0 & $<1.0$ & $<1.0$ & $<1.0$ & \\
\hline Bromoform & 1.0 & $<1.0$ & $<1.0$ & $<1.0$ & \\
\hline Bromomethane & 1.0 & $<1.0$ & $<1.0$ & $<1.0$ & \\
\hline Carbon Tetrachloride & 1.0 & $<1.0$ & $<1.0$ & $<1.0$ & \\
\hline Chlorobenzene & 1.0 & $<1.0$ & $<1.0$ & $<1.0$ & \\
\hline Chloroethane & 1.0 & 18 & 2.0 & $<1.0$ & \\
\hline Chloroform & 1.0 & $<1.0$ & $<1.0$ & $<1.0$ & \\
\hline Chloromethane & 1.0 & $<1.0$ & $<1.0$ & $<1.0$ & \\
\hline 2-Chloroethyl vinyl ether & 1.0 & $<1.0$ & $<1.0$ & $<1.0$ & \\
\hline Dibromochloromethane & 1.0 & $<1.0$ & $<1.0$ & $<1.0$ & \\
\hline 1,2-Dichlorobenzene & 1.0 & $<1.0$ & $<1.0$ & $<1.0$ & \\
\hline 1,3-Dichlorobenzene & 1.0 & $<1.0$ & $<1.0$ & $<1.0$ & \\
\hline 1,4-Dichlorobenzene & 1.0 & $<1.0$ & $<1.0$ & $<1.0$ & \\
\hline 1,1-Dichloroethane & 1.0 & 240 & 119 & 10 & \\
\hline 1,2-Dichloroethane & 1.0 & 2 & $<1.0$ & $<1.0$ & \\
\hline 1,1-Dichloroethene & 1.0 & $<1.0$ & $<1.0$ & $<1.0$ & \\
\hline Trans-1,2-Dichloroethene & 1.0 & $<1.0$ & $<1.0$ & $<1.0$ & \\
\hline 1,2-Dichloropropane & 1.0 & $<1.0$ & $<1.0$ & $<1.0$ & \\
\hline Cis-1,3-Dichloropropene & 1.0 & $<1.0$ & $<1.0$ & $<1.0$ & \\
\hline Trans-1,3-Dichloropropene & 1.0 & $<1.0$ & $<1.0$ & $<1.0$ & \\
\hline Methylene Chloride & 1.0 & 111 & 89 & 63 & \\
\hline 1,1,2,2-Tetrachloroethane & 1.0 & $<1.0$ & $<1.0$ & $<1.0$ & \\
\hline Tetrachloroethene & 1.0 & $<1.0$ & $<1.0$ & $<1.0$ & \\
\hline 1,1,1-Trichloroethane & 1.0 & 232 & 173 & 69 & \\
\hline 1,1,2-Trichloroethane & 1.0 & 3 & 1 & $<1.0$ & \\
\hline Trichloroethene & 1.0 & $<1.0$ & $<1.0$ & $<1.0$ & \\
\hline Trichlorofluoromethane & 1.0 & 264 & 226 & 163 & \\
\hline Vinyl Chloride & 1.0 & $<1.0$ & $<1.0$ & $<1.0$ & \\
\hline
\end{tabular}

Comments:

Reviewed By:

Date 


\section{ANALYTICAL REPORT}

State of Arizona License $\# 0009$

$\begin{array}{llll}\text { Client: } & \begin{array}{l}\text { Peroxidation Systems, Inc. } \\ \text { 5151 E. Broadway, Suite } 600\end{array} & \text { Sample Type: } & \text { Groundwater } \\ & \text { Tucson, Arizona 85711 } & \text { Date Sampled: } & 10 / 15 / 93 \\ \text { Project Name: } & \text { WJSA/DOE } & \text { Date Received: } & 10 / 18 / 93 \\ \text { Project Number: } & 345 & \text { Date Analyzed: } & 10 / 18 / 93 \\ \text { Sample ID: } & 102093-37,38,40 & \text { Analyzed By: } & \text { Deb Cartmell }\end{array}$

EPA Method 602 - Purgeable Aromatics (G.C. - PID)

Reported in $\mu \mathrm{g} / \mathrm{L}$ (ppb)

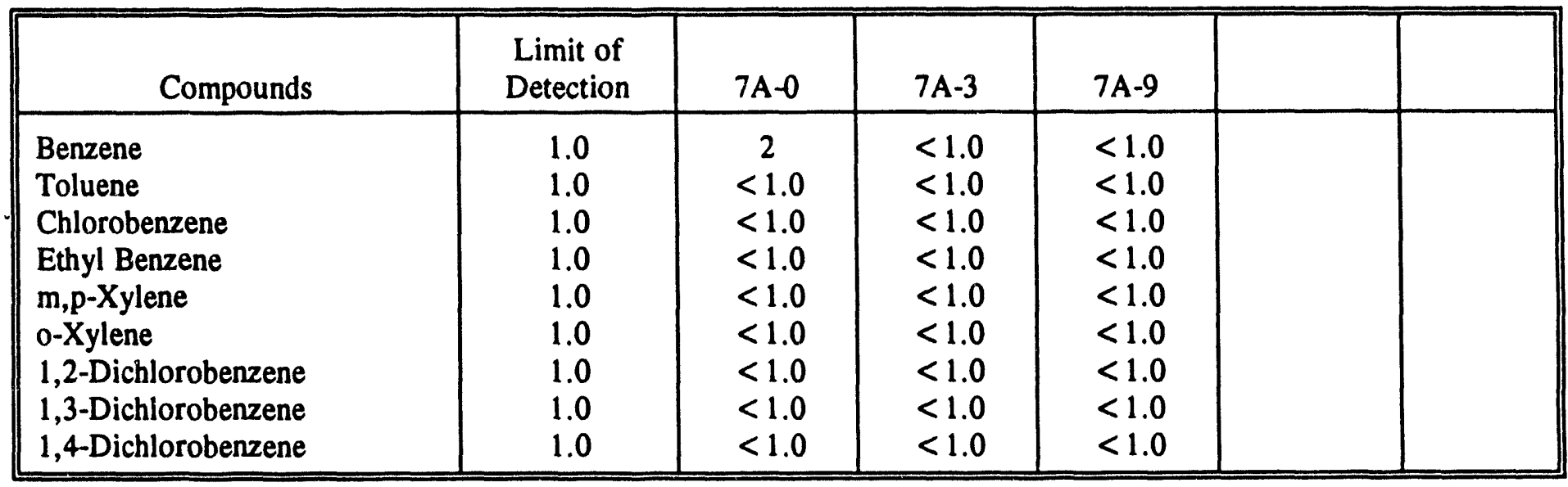

Comments:

Reviewed By: Tumille friridy

Date 
FAX 602-573-6550

I-BOO-PSI-LAES ANALYTICAL REPORT

State of Arizona License $\sharp 0009$

Client:

\section{Project Name:}

Project Number:

Sample ID:
Peroxidation Systems, Inc. 5151 E. Broadway, Suite 600 Tucson, Arizona 85711

WJSA/DOE

345

101893-67 - 70
Sample Type: Groundwater

Date Sampled: $\quad 10 / 16 / 93$

Date Received: $\quad$ 10/18/93

Date Analyzed: 10/26/93

Analyzed By: Deb Cartmell

EPA Method 601 - Purgeable Halocarbons

Reported in $\mu \mathrm{g} / \mathrm{L}$ (ppb)

\begin{tabular}{|c|c|c|c|c|c|}
\hline Compounds & $\begin{array}{l}\text { Limit of } \\
\text { Detection }\end{array}$ & $8 \mathrm{~A}-0$ & $8 A-3$ & $8 A-6$ & $8 A-9$ \\
\hline Bromodicloromethane & 1.0 & $<1.0$ & $<1.0$ & $<1.0$ & $<1.0$ \\
\hline Bromoform & 1.0 & $<1.0$ & $<1.0$ & $<1.0$ & $<1.0$ \\
\hline Bromomethane & 1.0 & $<1.0$ & $<1.0$ & $<1.0$ & $<1.0$ \\
\hline Carbon Tetrachloride & 1.0 & $<1.0$ & $<1.0$ & $<1.0$ & $<1.0$ \\
\hline Chlorobenzene & 1.0 & $<1.0$ & $<1.0$ & $<1.0$ & $<1.0$ \\
\hline Chloroethane & 1.0 & 35 & 33 & 27 & 25 \\
\hline Chloroform & 1.0 & 3 & 1.0 & $<1.0$ & $<1.0$ \\
\hline Chloromethane & 1.0 & $<1.0$ & $<1.0$ & $<1.0$ & $<1.0$ \\
\hline 2-Chloroethyl vinyl ether & 1.0 & $<1.0$ & $<1.0$ & $<1.0$ & $<1.0$ \\
\hline Dibromochloromethane & 1.0 & $<1.0$ & $<1.0$ & $<1.0$ & $<1.0$ \\
\hline 1,2-Dichlorobenzene & 1.0 & $<1.0$ & $<1.0$ & $<1.0$ & $<1.0$ \\
\hline 1,3-Dichlorobenzene & 1.0 & $<1.0$ & $<1.0$ & $<1.0$ & $<1.0$ \\
\hline 1,4-Dichlorobenzene & 1.0 & $<1.0$ & $<1.0$ & $<1.0$ & $<1.0$ \\
\hline 1,1-Dichloroethane & 1.0 & 400 & 323 & 331 & 321 \\
\hline 1,2-Dichloroethane & 1.0 & 3 & 3 & 3 & 3 \\
\hline 1,1-Dichloroethene & 1.0 & $<1.0$ & $<1.0$ & $<1.0$ & $<1.0$ \\
\hline Trans-1,2-Dichloroethene & 1.0 & 2 & 5 & 4 & 3 \\
\hline 1,2-Dichloropropane & 1.0 & $<1.0$ & $<1.0$ & $<1.0$ & $<1.0$ \\
\hline Cis-1,3-Dichloropropene & 1.0 & $<1.0$ & $<1.0$ & $<1.0$ & $<1.0$ \\
\hline Trans-1,3-Dichloropropene & 1.0 & $<1.0$ & $<1.0$ & $<1.0$ & $<1.0$ \\
\hline Methylene Chloride & 1.0 & 169 & 149 & 128 & 117 \\
\hline 1,1,2,2-Tetrachloroethane & 1.0 & $<1.0$ & $<1.0$ & $<1.0$ & $<1.0$ \\
\hline Tetrachloroethene & 1.0 & 4.0 & $<1.0$ & $<1.0$ & $<1.0$ \\
\hline 1,1,1-Trichloroethane & 1.0 & 326 & 298 & 257 & 232 \\
\hline 1,1,2-Trichloroethane & 1.0 & 4.0 & 4.0 & 3.0 & 3.0 \\
\hline Trichloroethene & 1.0 & 8 & 4.0 & $<1.0$ & $<1.0$ \\
\hline Trichlorofluoromethane & 1.0 & 354 & 337 & 275 & 263 \\
\hline Vinyl Chloride & 1.0 & $<1.0$ & $<1.0$ & $<1.0$ & $<1.0$ \\
\hline
\end{tabular}

Comments:

Reviewed By: 
ANALYTICAL REPORT

State of Arizona License \#0009

Client:

Peroxidation Systems, Inc.

5151 E. Broadway, Suite 600

Tucson, Arizona 85711

Project Name: WJSA/DOE

Project Number: $\quad 345$

Sample ID: $\quad$ 101893-67 - 70
Sample Type: Groundwater

Date Sampled: $\quad 10 / 16 / 93$

Date Received: 10/18/93

Date Analyzed: 10/26/93

Analyzed By: Deb Cartmell

EPA Method 602 - Purgeable Aromatics (G.C. - PID)

Reported in $\mu \mathrm{g} / \mathrm{L}$ (ppb)

\begin{tabular}{|c|c|c|c|c|c|}
\hline Compounds & $\begin{array}{l}\text { Limit of } \\
\text { Detection }\end{array}$ & $8 A-0$ & $8 A-3$ & $8 A-6$ & $8 A-9$ \\
\hline $\begin{array}{l}\text { Benzene } \\
\text { Toluene } \\
\text { Chlorobenzene } \\
\text { Ethyl Benzene } \\
\text { m,p-Xylene } \\
\text { 0-Xylene } \\
\text { 1,2-Dichlorobenzene } \\
\text { 1,3-Dichlorobenzene } \\
\text { 1,4-Dichlorobenzene }\end{array}$ & $\begin{array}{l}1.0 \\
1.0 \\
1.0 \\
1.0 \\
1.0 \\
1.0 \\
1.0 \\
1.0 \\
1.0\end{array}$ & $\begin{array}{l}54 \\
52 \\
<1.0 \\
23 \\
80 \\
39 \\
<1.0 \\
<1.0 \\
<1.0\end{array}$ & $\begin{array}{c}38 \\
32 \\
<1.0 \\
16 \\
44 \\
22 \\
<1.0 \\
<1.0 \\
<1.0\end{array}$ & $\begin{array}{c}26 \\
18 \\
<1.0 \\
9 \\
18 \\
10 \\
<1.0 \\
<1.0 \\
<1.0\end{array}$ & $\begin{array}{c}17 \\
11 \\
<1.0 \\
6 \\
10 \\
6 \\
<1.0 \\
<1.0 \\
<1.0\end{array}$ \\
\hline
\end{tabular}

Comments:

Reviewed By:

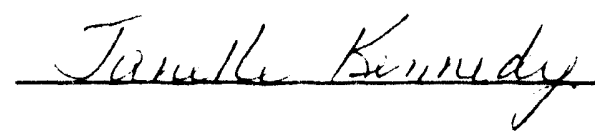

Date

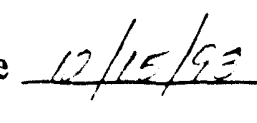




\section{ANALYTICAL REPORT \\ State of Arizona License $\# 0009$}

Client:

Peroxidation Systems, Inc.

5151 E. Broadway, Suite 600

Tucson, Arizona 85711

Project Name: WJSA/DOE

Project Number: 345

Sample ID:

101893-71- 74
Sample Type: Groundwater

Date Sampled: $\quad 10 / 16 / 93$

Date Received: $\quad 10 / 18 / 93$

Date Analyzed: 10/26/93

Analyzed By: Deb Cartmell

\section{EPA Method 601 - Purgeable Halocarbons \\ Reported in $\mu \mathrm{g} / \mathrm{L}$ (ppb)}

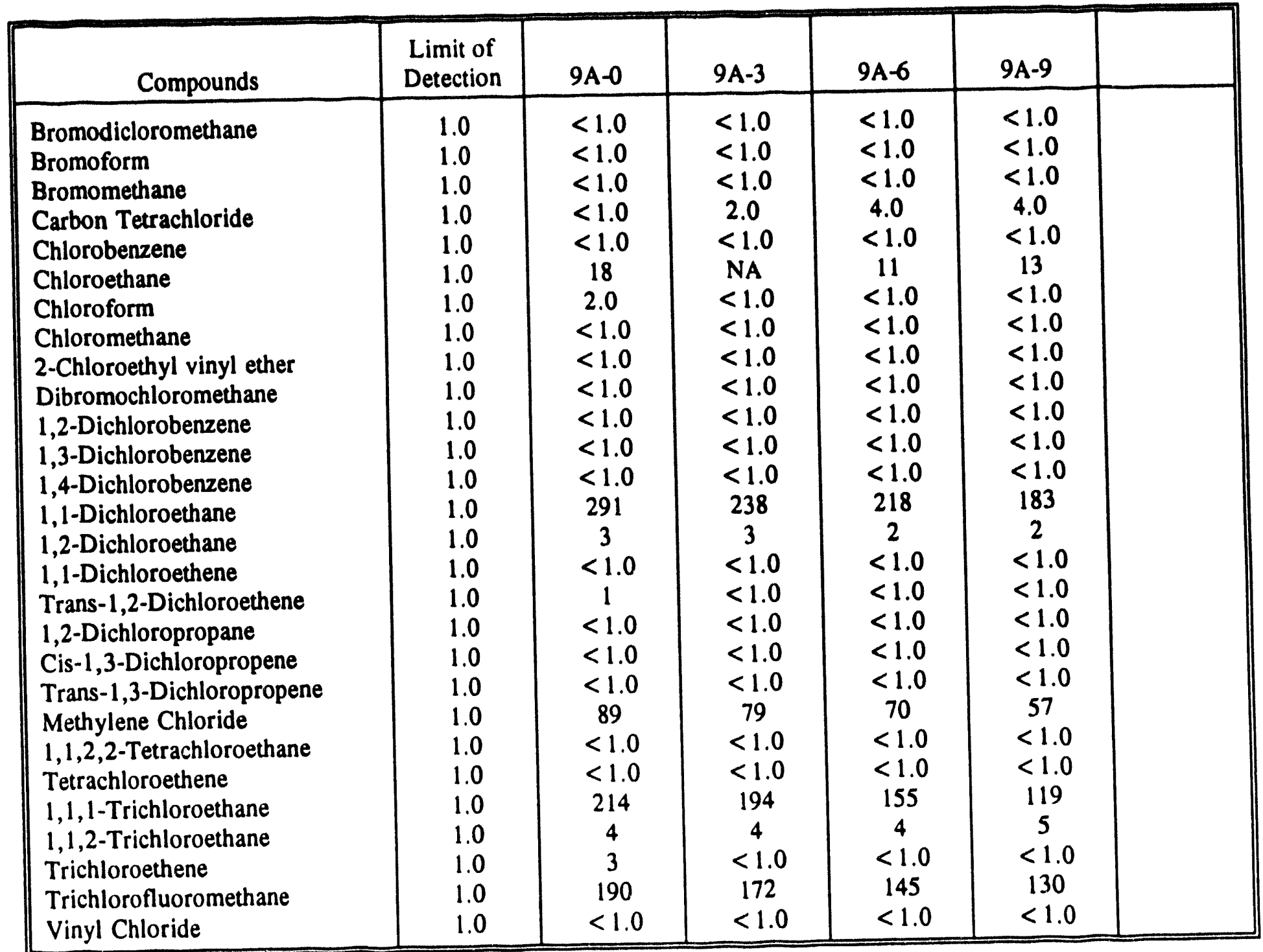

Comments: NA - Data not available, Pending QA/OC.

Reviewed By: 


\section{ANALYTICAL REPORT}

State of Arizona License "

$\begin{array}{llll}\text { Client: } & \begin{array}{l}\text { Peroxidation Systems, Inc. } \\ \text { 5151 E. Broadway, Suite 600 }\end{array} & \text { Sample Type: } & \text { Groundwater } \\ & \text { Tucson, Arizona 85711 } & \text { Date Sampled: } & 10 / 16 / 93 \\ \text { Project Name: } & \text { WJSA/DOE } & \text { Date Received: } & 10 / 18 / 93 \\ \text { Project Number: } & 345 & \text { Date Analyzed: } & 10 / 26 / 93 \\ \text { Sample DD: } & 101893-71-74 & \text { Analyzed By: } & \text { Deb Cartmell }\end{array}$

EPA Method 602 - Purgeable Aromatics (G.C. - PID)

Reported in $\mu \mathrm{g} / \mathrm{L}(\mathrm{ppb})$

\begin{tabular}{|c|c|c|c|c|c|}
\hline Compounds & $\begin{array}{l}\text { Limit of } \\
\text { Detection }\end{array}$ & $9 A-0$ & $9 A-3$ & $9 A-6$ & $9 A-9$ \\
\hline $\begin{array}{l}\text { Benzene } \\
\text { Toluene } \\
\text { Chlorobenzene } \\
\text { Ethyl Benzene } \\
\text { m,p-Xylene } \\
\text { o-Xylene } \\
\text { 1,2-Dichlorobenzene } \\
\text { 1,3-Dichlorobenzene } \\
\text { 1,4-Dichlorobenzene }\end{array}$ & $\begin{array}{l}1.0 \\
1.0 \\
1.0 \\
1.0 \\
1.0 \\
1.0 \\
1.0 \\
1.0 \\
1.0\end{array}$ & $\begin{array}{c}20 \\
17 \\
<1.0 \\
8 \\
22 \\
11 \\
<1.0 \\
<1.0 \\
<1.0\end{array}$ & $\begin{array}{l}<1.0 \\
<1.0 \\
<1.0 \\
<1.0 \\
<1.0 \\
<1.0 \\
<1.0 \\
<1.0 \\
<1.0\end{array}$ & $\begin{array}{l}<1.0 \\
<1.0 \\
<1.0 \\
<1.0 \\
<1.0 \\
<1.0 \\
<1.0 \\
<1.0 \\
<1.0\end{array}$ & $\begin{array}{l}<1.0 \\
<1.0 \\
<1.0 \\
<1.0 \\
<1.0 \\
<1.0 \\
<1.0 \\
<1.0 \\
<1.0\end{array}$ \\
\hline
\end{tabular}

Comments:

Reviewed By: 
Client:

$\begin{array}{ll}\text { Project Name: } & \text { WJSA/DOE } \\ \text { Project Number: } & 345 \\ \text { Sample 1D: } & 101893-75-78\end{array}$

Sample Type: Groundwater.

Date Sampled: $\quad 10 / 16 / 93$

Date Received: $\quad 10 / 18 / 93$

Date Analyzed: 10/26/93

Analyzed By: Deb Cartmell

EPA Method 601 - Purgeable Halocarbons

Reported in $\mu \mathrm{g} / \mathrm{L}$ (ppb)

\begin{tabular}{|c|c|c|c|c|c|c|}
\hline Compounds & $\begin{array}{l}\text { Limit of } \\
\text { Detection }\end{array}$ & $10 A-0$ & $10 A-3$ & $10 A-6$ & $10 A-9$ & \\
\hline Bromodicloromethane & 1.0 & $<1.0$ & $<1.0$ & $<1.0$ & $<1.0$ & \\
\hline Bromoform & 1.0 & $<1.0$ & $<1.0$ & $<1.0$ & $<1.0$ & \\
\hline Bromomethane & 1.0 & $<1.0$ & $<1.0$ & $<1.0$ & $<1.0$ & \\
\hline Carbon Tetrachloride & 1.0 & 6 & 6 & 5 & 6 & \\
\hline Chlorobenzene & 1.0 & $<1.0$ & $<1.0$ & $<1.0$ & $<1.0$ & \\
\hline Chloroethane & 1.0 & 27 & 22 & 19 & 19 & \\
\hline Chloroform & 1.0 & 1 & 3 & 3 & 2 & \\
\hline Chloromethane & 1.0 & $<1.0$ & $<1.0$ & $<1.0$ & $<1.0$ & \\
\hline 2-Chloroethyl vinyl ether & 1.0 & $<1.0$ & $<1.0$ & $<1.0$ & $<1.0$ & \\
\hline Dibromochloromethane & 1.0 & $<1.0$ & $<1.0$ & $<1.0$ & $<1.0$ & \\
\hline 1,2-Dichlorobenzene & 1.0 & $<1.0$ & $<1.0$ & $<1.0$ & $<1.0$ & \\
\hline 1,3-Dichlorobenzene & 1.0 & $<1.0$ & $<1.0$ & $<1.0$ & $<1.0$ & \\
\hline 1,4-Dichlorobenzene & 1.0 & $<1.0$ & $<1.0$ & $<1.0$ & $<1.0$ & \\
\hline 1,1-Dichloroethane & 1.0 & 394 & 339 & 278 & 231 & \\
\hline 1,2-Dichloroethane & 1.0 & 3 & 3 & 2 & 2 & \\
\hline 1,1-Dichloroethene & 1.0 & $<1.0$ & $<1.0$ & $<1.0$ & $<1.0$ & \\
\hline Trans-1,2-Dichloroethene & 1.0 & 3 & 1 & $<1.0$ & $<1.0$ & \\
\hline 1,2-Dichloropropane & 1.0 & $<1.0$ & $<1.0$ & $<1.0$ & $<1.0$ & \\
\hline Cis-1,3-Dichloropropene & 1.0 & $<1.0$ & $<1.0$ & $<1.0$ & $<1.0$ & \\
\hline Trans-1,3-Dichloropropene & 1.0 & $<1.0$ & $<1.0$ & $<1.0$ & $<1.0$ & \\
\hline Methylene Chloride & 1.0 & 133 & 118 & 105 & 86 & \\
\hline 1,1,2,2-Tetrachloroethane & 1.0 & $<1.0$ & $<1.0$ & $<1.0$ & $<1.0$ & \\
\hline Tetrachloroethene & 1.0 & 2 & $<1.0$ & $<1.0$ & $<1.0$ & \\
\hline 1,1,1-Trichloroethane & 1.0 & 313 & 263 & 206 & 164 & \\
\hline 1,1,2-Trichloroethane & 1.0 & 4 & 4 & 5 & 6 & \\
\hline Trichloroethene & 1.0 & 6 & $<1.0$ & $<1.0$ & $<1.0$ & \\
\hline Trichlorofluoromethane & 1.0 & 276 & 260 & 220 & 188 & \\
\hline Vinyl Chloride & 1.0 & $<1.0$ & $<1.0$ & $<1.0$ & $<1.0$ & \\
\hline
\end{tabular}

Comments:

Reviewed By: 
ANALYTICAL REPORT

State of Arizona License $\# 0009$
Client:
Peroxidation Systems, Inc.
5151 E. Broadway, Suite 600
Sample Type: Groundwater
Tucson, Arizona 85711
Date Sampled: $\quad 10 / 16 / 93$
Project Name:
WJSA/DOE
Date Received: 10/18/93
Project Number: $\quad 345$
Sample ID:
$101893.75-78$
Date Analyzed: 10/26/93
Analyzed By: Deb Cartmell
EPA Method 602 - Purgeable Aromatics (G.C. - PID)
Reported in $\mu \mathrm{g} / \mathrm{L}$ (ppb)

\begin{tabular}{|l|c|c|c|c|c|c|}
\hline \multicolumn{1}{|c|}{ Compounds } & $\begin{array}{c}\text { Limit of } \\
\text { Detection }\end{array}$ & $10 \mathrm{~A}-0$ & $10 \mathrm{~A}-3$ & $10 \mathrm{~A}-6$ & $10 \mathrm{~A}-9$ & \\
\hline Benzene & 1.0 & 39 & 4 & 1 & 1 & \\
Toluene & 1.0 & 34 & 2 & $<1.0$ & $<1.0$ & \\
Chlorobenzene & 1.0 & $<1.0$ & $<1.0$ & $<1.0$ & $<1.0$ & \\
Ethyl Benzene & 1.0 & 16 & 2 & $<1.0$ & $<1.0$ & \\
m,p-Xylene & 1.0 & 46 & 2 & $<1.0$ & $<1.0$ & $<1.0$ \\
o-Xylene & 1.0 & 24 & 2 & $<1.0$ & $<1.0$ & \\
1,2-Dichlorobenzene & 1.0 & $<1.0$ & $<1.0$ & $<1.0$ & $<1.0$ & \\
1,3-Dichlorobenzene & 1.0 & $<1.0$ & $<1.0$ & $<1.0$ & $<1.0$ & \\
1,4-Dichlorobenzene & 1.0 & $<1.0$ & $<1.0$ & $<1.0$ & $<1.0$ & \\
\hline
\end{tabular}

Comments:

Reviewed By:

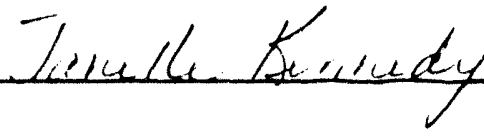

Date

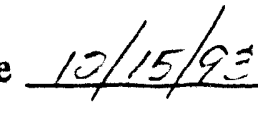


2595 E. Alport Drlve Tuceon, Arlzana 85706 602.573.6565 FAX 602-573-6550 1-800-P5I-LABS ANALYTICAL REPORT

State of Arizona License $\# 0009$

$\begin{array}{llll}\text { Client: } & \begin{array}{l}\text { Peroxidation Systems, Inc. } \\ \text { 5151 E. Broadway, Suite 600 } \\ \text { Tucson, Arizona 85711 }\end{array} & \text { Sample Type: } & \text { Groundwater } \\ & & \text { Date Sampled: } & 10 / 18 / 93 \\ \text { Project Name: } & \text { WJSA/DOE } & \text { Date Received: } & 10 / 19 / 93 \\ \text { Project Number: } & 345 & \text { Date Analyzed: } & 10 / 26 / 93 \\ \text { Sample ID: } & 101993-4-7 & \text { Analyzed By: } & \text { Deb Cartmell }\end{array}$

EPA Method 601 - Purgeable Halocarbons

Reported in $\mu \mathrm{g} / \mathrm{L}$ (ppb)

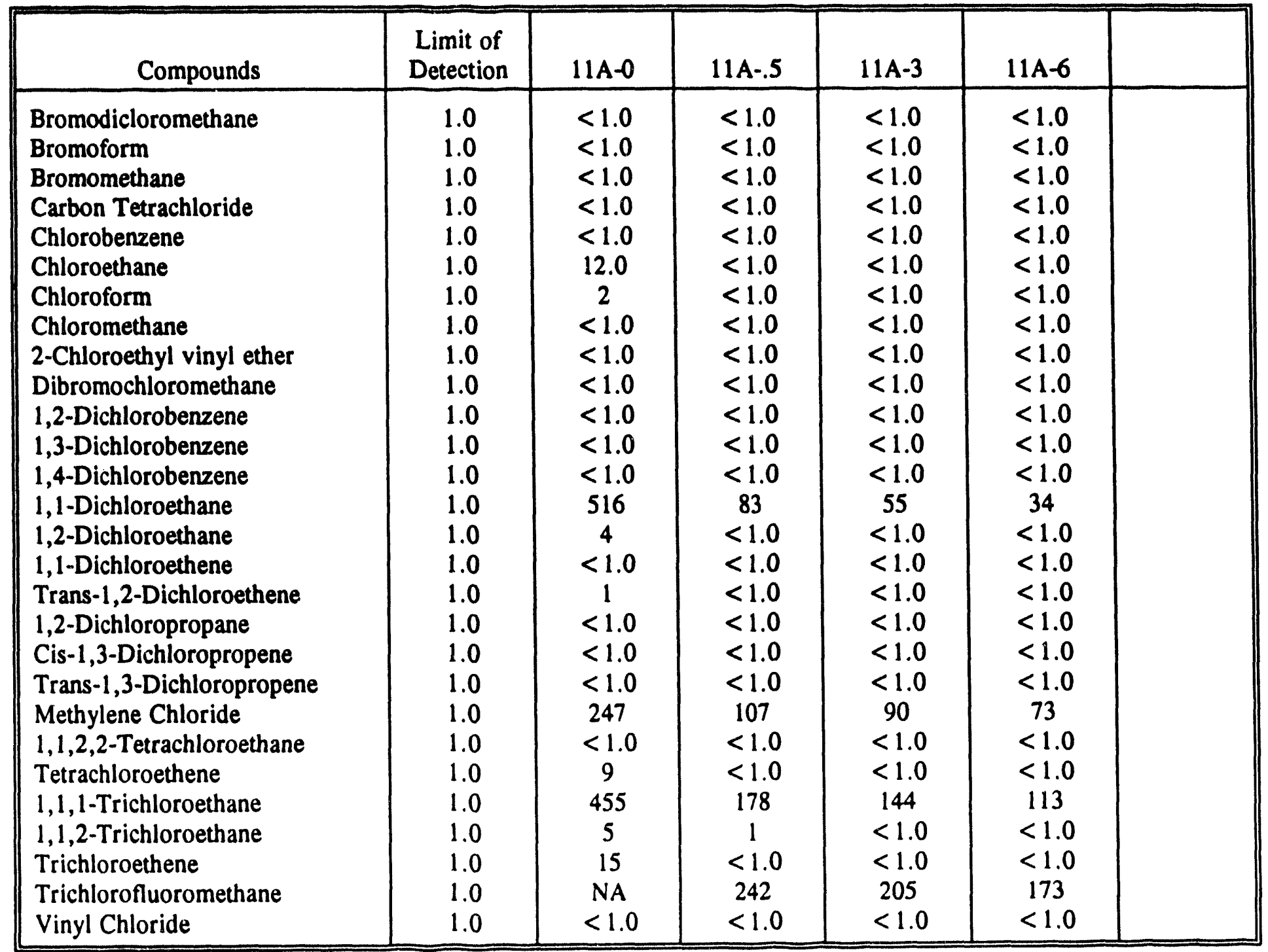

Comments: NA - Data not available, Pending $\mathrm{OA} / \mathrm{OC}$

Reviewed By: Tarulle funnedy 


\section{PSI Environmental Laboratories}

ANALYTICAL REPORT

State of Arizona License \#0009

Client:

Peroxidation Systems, Inc. 5151 E. Broadway, Suite 600 Tucson, Arizona 85711

Project Name: $\quad$ WJSA/DOE

Project Number: $\quad 345$

Sample ID:

$101993-4-7$
Sample Type: Groundwater

Date Sampled: $\quad 10 / 18 / 93$

Date Received: $\quad 10 / 19 / 93$

Date Analyzed: $10 / 26 / 93$

Analyzed By: Deb Cartmell

EPA Method 602 - Purgeable Aromatics (G.C. - PID)

Reported in $\mu \mathrm{g} / \mathrm{L}(\mathrm{ppb})$

\begin{tabular}{||l|c|c|c|c|c|c||}
\hline \multicolumn{1}{|c|}{ Compounds } & $\begin{array}{c}\text { Limit of } \\
\text { Detection }\end{array}$ & $11 \mathrm{~A}-0$ & $11 \mathrm{~A}-.5$ & $11 \mathrm{~A}-3$ & $11 \mathrm{~A}-6$ & \\
\hline Benzene & 1.0 & 85 & $<1.0$ & $<1.0$ & $<1.0$ & $<1.0$ \\
Toluene & 1.0 & 90 & $<1.0$ & $<1.0$ & $<1.0$ & $<1.0$ \\
Chlorobenzene & 1.0 & $<1.0$ & $<1.0$ & $<1.0$ & $<1.0$ & $<1.0$ \\
Ethyl Benzene & 1.0 & 66 & $<1.0$ & $<1.0$ & $<1.0$ & $<1.0$ \\
m,p-Xylene & 1.0 & 164 & $<1.0$ & $<$ & $<1.0$ \\
0-Xylene & 1.0 & 72 & $<1.0$ & 5 & $<1.0$ \\
1,2-Dichlorobenzene & 1.0 & $<1.0$ & $<1.0$ & $<1.0$ & $<1.0$ & $<1.0$ \\
1,3-Dichlorobenzene & 1.0 & $<1.0$ & $<1.0$ & $<1.0$ & $<1.0$ \\
1,4-Dichlorobenzene & 1.0 & 1 & $<1.0$ & $<1.0$ & \\
\hline
\end{tabular}

Comments:

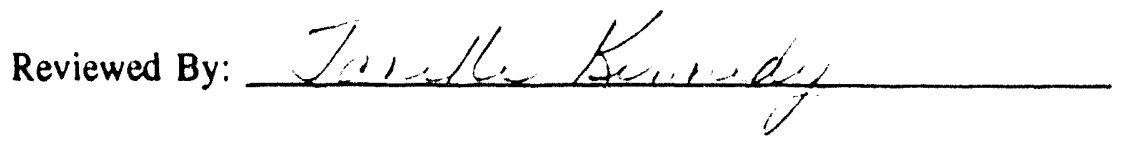

Date:

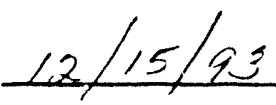


Client:

Project Name:

WJSA/DOE

Project Number:

345

Sample ID:
Sample Type: Groundwater

Date Sampled: $\quad 10 / 18 / 93$

Date Received: $\quad 10 / 19 / 93$

Date Analyzed: 11/19/93

Analyzed By: Deb Cartmell

EPA Method 601 - Purgeable Halocarbons

Reported in $\mu \mathrm{g} / \mathrm{L}(\mathrm{ppb})$

\begin{tabular}{|c|c|c|c|c|c|}
\hline Compounds & $\begin{array}{l}\text { Limit of } \\
\text { Detection }\end{array}$ & $12 \mathrm{~A}-0$ & $12 \mathrm{~A}-3$ & $12 A-6$ & $12 A-9$ \\
\hline Bromodicloromethane & 1.0 & $<1.0$ & $<1.0$ & $<1.0$ & $<1.0$ \\
\hline Bromoform & 1.0 & $<1.0$ & $<1.0$ & $<1.0$ & $<1.0$ \\
\hline Bromomethane & 1.0 & $<1.0$ & $<1.0$ & $<1.0$ & $<1.0$ \\
\hline Carbon Tetrachloride & 1.0 & $<1.0$ & $<1.0$ & $<1.0$ & $<1.0$ \\
\hline Chlorobenzene & 1.0 & $<1.0$ & $<1.0$ & $<1.0$ & $<1.0$ \\
\hline Chloroethane & 1.0 & 26 & 13 & 3 & $<1.0$ \\
\hline Chloroform & 1.0 & 1 & 1 & $<1.0$ & $<1.0$ \\
\hline Chloromethane & 1.0 & $<1.0$ & $<1.0$ & $<1.0$ & $<1.0$ \\
\hline 2-Chloroethyl vinyl ether & 1.0 & $<1.0$ & $<1.0$ & $<1.0$ & $<1.0$ \\
\hline Dibromochloromethane & 1.0 & $<1.0$ & $<1.0$ & $<1.0$ & $<1.0$ \\
\hline 1,2-Dichlorobenzene & 1.0 & $<1.0$ & $<1.0$ & $<1.0$ & $<1.0$ \\
\hline 1,3-Dichlorobenzene & 1.0 & $<1.0$ & $<1.0$ & $<1.0$ & $<1.0$ \\
\hline 1,4-Dichlorobenzene & 1.0 & $<1.0$ & $<1.0$ & $<1.0$ & $<1.0$ \\
\hline 1,1-Dichloroethane & 1.0 & 376 & 279 & 181 & 133 \\
\hline 1,2-Dichloroethane & 1.0 & 3 & 2 & 1 & 1 \\
\hline 1,1-Dichloroethene & 1.0 & $<1.0$ & $<1.0$ & $<1.0$ & $<1.0$ \\
\hline Trans-1,2-Dichloroethene & 1.0 & 2 & $<1.0$ & $<1.0$ & $<1.0$ \\
\hline 1,2-Dichloropropane & 1.0 & $<1.0$ & $<1.0$ & $<1.0$ & $<1.0$ \\
\hline Cis-1,3-Dichloropropene & 1.0 & $<1.0$ & $<1.0$ & $<1.0$ & $<1.0$ \\
\hline Trans-1,3-Dichloropropene & 1.0 & $<1.0$ & $<1.0$ & $<1.0$ & $<1.0$ \\
\hline Methylene Chloride & 1.0 & 146 & 120 & 94 & 86 \\
\hline 1,1,2,2-Tetrachloroethane & 1.0 & $<1.0$ & $<1.0$ & $<1.0$ & $<1.0$ \\
\hline Tetrachloroethene & 1.0 & $<1.0$ & $<1.0$ & $<1.0$ & $<1.0$ \\
\hline 1,1,1-Trichloroethane & 1.0 & 322 & 248 & 180 & $137^{\circ}$ \\
\hline 1,1,2-Trichloroethane & 1.0 & 4 & 3 & 3 & 3 \\
\hline Trichloroethene & 1.0 & 7 & $<1.0$ & $<1.0$ & $<1.0$ \\
\hline Trichlorofluoromethane & 1.0 & NA & NA & NA & NA \\
\hline Vinyl Chloride & 1.0 & $<1.0$ & $<1.0$ & $<1.0$ & $<1.0$ \\
\hline
\end{tabular}

Comments: NA - Data not available, Pending QA/OC.

Reviewed By:

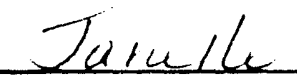


ANALYTICAL REPORT

State of Arizona License $\# 0009$
Client: $\quad$ Peroxidation Systems, Inc. 5151 E. Broadway, Suite 600 Tucson, Arizona 85711

Project Name: WJSA/DOE

Project Number: 345

Sample ID: $\quad$ 101993-8 - 11
Sample Type: Groundwater

Date Sampled: $\quad 10 / 18 / 93$

Date Received: $10 / 19 / 93$

Date Analyzed: 11/19/93

Analyzed By: Deb Cartmell

EPA Method 602 - Purgeable Aromatics (G.C. - PID)

Reported in $\mu \mathrm{g} / \mathrm{L}$ (ppb)

\begin{tabular}{|l|c|c|c|c|c|c|}
\hline \multicolumn{1}{|c|}{ Compounds } & $\begin{array}{c}\text { Limit of } \\
\text { Detection }\end{array}$ & $12 \mathrm{~A}-0$ & $12 \mathrm{~A}-3$ & $12 \mathrm{~A}-6$ & $12 \mathrm{~A}-9$ & \\
\hline Benzene & 1.0 & 36 & $<1.0$ & $<1.0$ & $<1.0$ & $<1.0$ \\
Toluene & 1.0 & 37 & $<1.0$ & $<1.0$ & $<1.0$ & $<1.0$ \\
Chlorobenzene & 1.0 & $<1.0$ & $<1.0$ & $<1.0$ \\
Ethyl Benzene & 1.0 & 27 & $<1.0$ & $<1.0$ & $<1.0$ & $<1.0$ \\
m,p-Xylene & 1.0 & 59 & $<1.0$ & $<1.0$ & $<1.0$ & $<1.0$ \\
o-Xylene & 1.0 & 28 & $<1.0$ & $<1.0$ \\
1,2-Dichlorobenzene & 1.0 & $<1.0$ & $<1.0$ & $<1.0$ & $<1.0$ & $<1.0$ \\
1,3-Dichlorobenzene & 1.0 & $<1.0$ & $<1.0$ & $<1.0$ & $<1.0$ \\
1,4-Dichlorobenzene & 1.0 & $<1.0$ & $<1.0$ & $<1.0$ & $<1.0$ \\
\hline
\end{tabular}

Comments:

Reviewed By: Terulle fuered 
Client:

Peroxidation Systems, Inc. 5151 E. Broadway, Suite 600

Tucson, Arizona 85711

Project Name: WJSA/DOE

Project Number: 345

Sample ID:
101993-12 - 15
Sample Type: Groundwater

Date Sampled: $\quad 10 / 18 / 93$

Date Received: 10/19/93

Date Analyzed: 10/26/93

Analyzed By: Deb Cartmell

EPA Method 601 - Purgeable Halocarbons

Reported in $\mu \mathrm{g} / \mathrm{L}$ (ppb)

\begin{tabular}{|c|c|c|c|c|c|}
\hline Compounds & $\begin{array}{l}\text { Limit of } \\
\text { Detection }\end{array}$ & $13 A-0$ & $13 A-3$ & $13 A-6$ & $13 A-9$ \\
\hline Bromodicloromethane & 1.0 & $<1.0$ & $<1.0$ & $<1.0$ & $<1.0$ \\
\hline Bromoform & 1.0 & $<1.0$ & $<1.0$ & $<1.0$ & $<1.0$ \\
\hline Bromomethane & 1.0 & $<1.0$ & $<1.0$ & $<1.0$ & $<1.0$ \\
\hline Carbon Tetrachloride & 1.0 & $<1.0$ & $<1.0$ & $<1.0$ & $<1.0$ \\
\hline Chlorobenzene & 1.0 & $<1.0$ & $<1.0$ & $<1.0$ & $<1.0$ \\
\hline Chloroethane & 1.0 & 27 & 18 & 9 & 5 \\
\hline Chloroform & 1.0 & 1 & 1 & $<1.0$ & $<1.0$ \\
\hline Chloromethane & 1.0 & $<1.0$ & $<1.0$ & $<1.0$ & $<1.0$ \\
\hline 2-Chloroethyl vinyl ether & 1.0 & $<1.0$ & $<1.0$ & $<1.0$ & $<1.0$ \\
\hline Dibromochloromethane & 1.0 & $<1.0$ & $<1.0$ & $<1.0$ & $<1.0$ \\
\hline 1,2-Dichlorobenzene & 1.0 & $<1.0$ & $<1.0$ & $<1.0$ & $<1.0$ \\
\hline 1,3-Dichlorobenzene & 1.0 & $<1.0$ & $<1.0$ & $<1.0$ & $<1.0$ \\
\hline 1,4-Dichlorobenzene & 1.0 & $<1.0$ & $<1.0$ & $<1.0$ & $<1.0$ \\
\hline 1,1-Dichloroethane & 1.0 & 368 & 300 & 232 & 177 \\
\hline 1,2-Dichloroethane & 1.0 & 3 & 2 & 2 & 1 \\
\hline 1,1-Dichloroethene & 1.0 & $<1.0$ & $<1.0$ & $<1.0$ & $<1.0$ \\
\hline Trans-1,2-Dichloroethene & 1.0 & $<1.0$ & $<1.0$ & $<1.0$ & $<1.0$ \\
\hline 1,2-Dichloropropane & 1.0 & $<1.0$ & $<1.0$ & $<1.0$ & $<1.0$ \\
\hline Cis-1,3-Dichloropropene & 1.0 & $<1.0$ & $<1.0$ & $<1.0$ & $<1.0$ \\
\hline Trans-1,3-Dichloropropene & 1.0 & $<1.0$ & $<1.0$ & $<1.0$ & $<1.0$ \\
\hline Methylene Chloride & 1.0 & 144 & 124 & 105 & 91 \\
\hline 1,1,2,2-Tetrachloroethane & 1.0 & $<1.0$ & $<1.0$ & $<1.0$ & $<1.0$ \\
\hline Tetrachloroethene & 1.0 & 3 & $<1.0$ & $<1.0$ & $<1.0$ \\
\hline 1,1,1-Trichloroethane & 1.0 & 322 & 257 & 221 & 186 \\
\hline 1,1,2-Trichloroethane & 1.0 & 4 & 3 & 3 & 2 \\
\hline Trichloroethene & 1.0 & 5 & $<1.0$ & $<1.0$ & $<1.0$ \\
\hline Trichlorofluoromethane & 1.0 & 279 & 252 & 220 & 191 \\
\hline Vinyl Chloride & 1.0 & $<1.0$ & $<1.0$ & $<1.0$ & $<1.0$ \\
\hline
\end{tabular}

Comments:

Reviewed By: 


\section{ANALYTICAL REPORT}

State of Arizona License $\# 0009$

Client: $\quad$ Peroxidation Systems, Inc. 5151 E. Broadway, Suite 600 Tucson, Arizona 85711

Project Name:

WJSA/DOE

Project Number: $\quad 345$

Sample ID:

$101993-12-15$
Sample Type: Groundwater

Date Sampled: $\quad 10 / 18 / 93$

Date Received: 10/19/93

Date Analyzed: 10/26/93

Analyzed By: Deb Cartmell

EPA Method 602 - Purgeable Aromatics (G.C. - PID)

Reported in $\mu \mathrm{g} / \mathrm{L}(\mathrm{ppb})$

\begin{tabular}{|c|c|c|c|c|c|}
\hline Compounds & $\begin{array}{l}\text { Limit of } \\
\text { Detection }\end{array}$ & $13 A-0$ & $13 A-3$ & $13 A-6$ & $13 A-9$ \\
\hline Benzene & 1.0 & 24 & $<1.0$ & $<1.0$ & $<1.0$ \\
\hline Toluene & 1.0 & 24 & $<1.0$ & $<1.0$ & $<1.0$ \\
\hline Chlorobenzene & 1.0 & $<1.0$ & $<1.0$ & $<1.0$ & $<1.0$ \\
\hline Ethyl Benzene & 1.0 & 18 & $<1.0$ & $<1.0$ & $<1.0$ \\
\hline m,p-Xylene & 1.0 & 39 & $<1.0$ & $<1.0$ & $<1.0$ \\
\hline $0-$ Xylene & 1.0 & 19 & $<1.0$ & $<1.0$ & $<1.0$ \\
\hline 1,2-Dichlorobenzene & 1.0 & $<1.0$ & $<1.0$ & $<1.0$ & $<1.0$ \\
\hline 1,3-Dichlorobenzene & 1.0 & $<1.0$ & $<1.0$ & $<1.0$ & $<1.0$ \\
\hline 1,4-Dichlorobenzene & 1.0 & $<1.0$ & $<1.0$ & $<1.0$ & $<1.0$ \\
\hline
\end{tabular}

Comments:

Reviewed By:

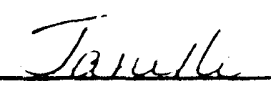

Date 


\section{PSI Environmental Laboratories}

Tucson. Arizond 85706 602-573-6565 FAX 602-573-6550

I-8O0-PSI-LAQS

ANALYTICAL REPORT

State of Arizona License $\# 0009$

$\begin{array}{llll}\text { Client: } & \begin{array}{l}\text { Peroxidation Systems, Inc. } \\ \text { 5151 E. Broadway, Suite 600 }\end{array} & \text { Sample Type: } & \text { Groundwater } \\ & \text { Tucson, Arizona 85711 } & \text { Date Sampled: } & 10 / 18 / 93 \\ \text { Project Name: } & \text { WJSA/DOE } & \text { Date Received: } & 10 / 19 / 93 \\ \text { Project Number: } & 345 & \text { Date Analyzed: } & 11 / 19 / 93 \\ \text { Sample ID: } & 101993-16-18 & \text { Analyzed By: } & \text { Deb Cartmell }\end{array}$

EPA Method 601 - Purgeable Halocarbons

Reported in $\mu \mathrm{g} / \mathrm{L}$ (ppb)

\begin{tabular}{|c|c|c|c|c|c|}
\hline Compounds & $\begin{array}{l}\text { Limit of } \\
\text { Detection }\end{array}$ & $14 A-0$ & $14 A-3$ & $14 A-9$ & \\
\hline Bromodicloromethane & 1.0 & $<1.0$ & $<1.0$ & $<1.0$ & \\
\hline Bromoform & 1.0 & $<1.0$ & $<1.0$ & $<1.0$ & \\
\hline Bromomethane & 1.0 & $<1.0$ & $<1.0$ & $<1.0$ & \\
\hline Carbon Tetrachloride & 1.0 & $<1.0$ & $<1.0$ & 2 & \\
\hline Chlorobenzene & 1.0 & $<1.0$ & $<1.0$ & $<1.0$ & \\
\hline Chloroethane & 1.0 & 28 & 24 & 18 & \\
\hline Chloroform & 1.0 & 1 & 1 & $<1.0$ & \\
\hline Chloromethane & 1.0 & $<1.0$ & $<1.0$ & $<1.0$ & \\
\hline 2-Chloroethyl vinyl ether & 1.0 & $<1.0$ & $<1.0$ & $<1.0$ & \\
\hline Dibromochloromethane & 1.0 & $<1.0$ & $<1.0$ & $<1.0$ & \\
\hline 1,2-Dichlorobenzere & 1.0 & $<1.0$ & $<1.0$ & $<1.0$ & \\
\hline 1,3-Dichlorobenzene & 1.0 & $<1.0$ & $<1.0$ & $<1.0$ & \\
\hline 1,4-Dichlorobenzene & 1.0 & $<1.0$ & $<1.0$ & $<1.0$ & \\
\hline 1,1-Dichloroethane & 1.0 & 385 & 315 & 271 & \\
\hline 1,2-Dichloroethane & 1.0 & 3 & 3 & 2 & \\
\hline 1,1-Dichloroethene & 1.0 & $<1.0$ & $<1.0$ & $<1.0$ & \\
\hline Trans-1,2-Dichloroethene & 1.0 & 3 & 5 & 3 & \\
\hline 1,2-Dichloropropane & 1.0 & $<1.0$ & $<1.0$ & $<1.0$ & \\
\hline Cis-1,3-Dichloropropene & 1.0 & $<1.0$ & $<1.0$ & $<1.0$ & \\
\hline Trans-1,3-Dichloropropene & 1.0 & $<1.0$ & $<1.0$ & $<1.0$ & \\
\hline Methylene Chloride & 1.0 & 175 & 142 & 114 & \\
\hline $1,1,2,2$-Tetrachloroethane & 1.0 & $<1.0$ & $<1.0$ & $<1.0$ & \\
\hline Tetrachloroethene & 1.0 & 4 & 1 & $<1.0$ & \\
\hline 1,1,1-Trichloroethane & 1.0 & 348 & 278 & 214 & \\
\hline 1,1,2-Trichloroethane & 1.0 & 3.5 & 3.4 & 2.7 & \\
\hline Trichloroethene & 1.0 & 7 & 3 & $<1.0$ & \\
\hline Trichlorofluoromethane & 1.0 & 309 & 252 & 198 & \\
\hline Vinyl Chloride & 1.0 & $<1.0$ & $<1.0$ & $<1.0$ & \\
\hline
\end{tabular}

Comments:

Reviewed By: 


\section{PSI Environmental Laboratories}

2595 E. Airport Ortve Twcson. Arizona 85706 602-573-6565 FAX 602-573-6550 1-800-P5I-LABS

ANALYTICAL REPORT

State of Arizona License $\# 0009$
Client:

Peroxidation Systems, Inc.

5151 E. Broadway, Suite 600

Tucson, Arizona 85711

Project Name: $\quad$ WJSA/DOE

Project Number: $\quad 345$

Sample ID: $\quad$ 101993-16-18
Sample Type: Groundwater

Date Sampled: $\quad 10 / 18 / 93$

Date Received: 10/19/93

Date Analyzed: 11/19/93

Analyzed By: Deb Cartmell

EPA Method 602 - Purgeable Aromatics (G.C. - PID)

Reported in $\mu \mathrm{g} / \mathrm{L}$ (ppb)

\begin{tabular}{||l|c|c|c|c|c|c|}
\hline \multicolumn{1}{|c|}{ Compounds } & $\begin{array}{c}\text { Limit of } \\
\text { Detection }\end{array}$ & $14 \mathrm{~A}-0$ & $14 \mathrm{~A}-3$ & $14-9$ & & \\
\hline Benzene & 1.0 & 54 & 40 & 19 & & \\
Toluene & 1.0 & 56 & 36 & 15 & & \\
Chlorobenzene & 1.0 & $<1.0$ & $<1.0$ & $<1.0$ & & \\
Ethyl Benzene & 1.0 & 38 & 27 & 12 & & \\
m,p-Xylene & 1.0 & 101 & 61 & 20 & & \\
0-Xylene & 1.0 & 45 & 31 & $<$ & & \\
1,2-Dichlorobenzene & 1.0 & $<1.0$ & $<1.0$ & $<1.0$ & & \\
1,3-Dichlorobenzene & 1.0 & $<1.0$ & $<1.0$ & $<1.0$ & & \\
1,4-Dichlorobenzene & 1.0 & $<1.0$ & $<1.0$ & $<1.0$ & & \\
\hline
\end{tabular}

Comments:

Reviewed By: Tamelleteredy

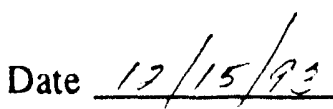


FAX 602.573-6550

Client:

Peroxidation Systems, Inc.

5151 E. Broadway, Suite 600

Tucson, Arizona 85711

Project Name: WJSA/DOE

Project Number: $\quad 345$

Sample ID:

101993-19- 22
Sample Type: Groundwater

Date Sampled: $10 / 18 / 93$

Date Received: $\quad$ 10/19/93

Date Analyzed: 11/19/93

Analyzed By: Deb Cartmell

EPA Method 601 - Purgeable Halocarbons

Reported in $\mu \mathrm{g} / \mathrm{L}$ (ppb)

\begin{tabular}{|c|c|c|c|c|c|}
\hline Compounds & $\begin{array}{l}\text { Limit of } \\
\text { Detection }\end{array}$ & $15 A-0$ & $15 A-3$ & $15 A-6$ & $15 A-9$ \\
\hline Bromodicloromethane & 1.0 & $<1.0$ & $<1.0$ & $<1.0$ & $<1.0$ \\
\hline Bromoform & 1.0 & $<1.0$ & $<1.0$ & $<1.0$ & $<1.0$ \\
\hline Bromomethane & 1.0 & $<1.0$ & $<1.0$ & $<1.0$ & $<1.0$ \\
\hline Carbon Tetrachloride & 1.0 & $<1.0$ & $<1.0$ & $<1.0$ & $<1.0$ \\
\hline Chlorobenzene & 1.0 & $<1.0$ & $<1.0$ & $<1.0$ & $<1.0$ \\
\hline Chloroethane & 1.0 & 27 & 17 & 9 & 2 \\
\hline Chloroform & 1.0 & 1 & 1 & $<1.0$ & $<1.0$ \\
\hline Chloromethane & 1.0 & $<1.0$ & $<1.0$ & $<1.0$ & $<1.0$ \\
\hline 2-Chloroethyl vinyl ether & 1.0 & $<1.0$ & $<1.0$ & $<1.0$ & $<1.0$ \\
\hline Dibromochloromethane & 1.0 & $<1.0$ & $<1.0$ & $<1.0$ & $<1.0$ \\
\hline 1,2-Dichlorobenzene & 1.0 & $<1.0$ & $<1.0$ & $<1.0$ & $<1.0$ \\
\hline 1,3-Dichlorobenzene & 1.0 & $<1.0$ & $<1.0$ & $<1.0$ & $<1.0$ \\
\hline 1,4-Dichlorobenzene & 1.0 & $<1.0$ & 2 & 1 & 1 \\
\hline 1,1-Dichloroethane & 1.0 & 374 & 298 & 229 & 165 \\
\hline 1,2-Dichloroethane & 1.0 & 3 & 2 & 2 & 1 \\
\hline 1,1-Dichloroethene & 1.0 & $<1.0$ & $<1.0$ & $<1.0$ & $<1.0$ \\
\hline Trans-1,2-Dichloroethene & 1.0 & 2 & $<1.0$ & $<1.0$ & $<1.0$ \\
\hline 1,2-Dichloropropane & 1.0 & $<1.0$ & $<1.0$ & $<1.0$ & $<1.0$ \\
\hline Cis-1,3-Dichloropropene & 1.0 & $<1.0$ & $<1.0$ & $<1.0$ & $<1.0$ \\
\hline Trans-1,3-Dichloropropene & 1.0 & $<1.0$ & $<1.0$ & $<1.0$ & $<1.0$ \\
\hline Methylene Chloride & 1.0 & 139 & 119 & 97 & 85 \\
\hline $1,1,2,2$-Tetrachloroethane & 1.0 & $<1.0$ & $<1.0$ & $<1.0$ & $<1.0$ \\
\hline Tetrachloroethene & 1.0 & 3 & $<1.0$ & $<1.0$ & $<1.0$ \\
\hline 1,1,1-Trichloroethane & 1.0 & 318 & 252 & 207 & 157 \\
\hline 1,1,2-Trichloroethane & 1.0 & 4 & 3 & 3 & 3 \\
\hline Trichloroethene & 1.0 & 6 & $<1.0$ & $<1.0$ & $<1.0$ \\
\hline Trichlorofluoromethane & 1.0 & 278 & 237 & 201 & 170 \\
\hline Vinyl Chloride & 1.0 & $<1.0$ & $<1.0$ & $<1.0$ & $<1.0$ \\
\hline
\end{tabular}

Comments:

Reviewed By: 


\section{ANALYTICAL REPORT}

State of Arizona License $\# 0009$

Client:

Peroxidation Systems, Inc.

5151 E. Broadway, Suite 600

Tucson, Arizona 85711

Project Name: WJSA/DOE

Project Number: $\quad 345$

Sample ID: $\quad$ 101993-19-22
Sample Type: Groundwater

Date Sampled: $\quad 10 / 18 / 93$

Date Received: 10/19/93

Date Analyzed: 11/19/93

Analyzed By: Deb Cartmell

EPA Method 602 - Purgeable Aromatics (G.C. - PID)

Reported in $\mu \mathrm{g} / \mathrm{L}$ (ppb)

\begin{tabular}{|c|c|c|c|c|c|}
\hline Compounds & $\begin{array}{l}\text { Limit of } \\
\text { Detection }\end{array}$ & $15 A-0$ & $15 A-3$ & $15 A-6$ & $15 A-9$ \\
\hline $\begin{array}{l}\text { Benzene } \\
\text { Toluene } \\
\text { Chlorobenzene } \\
\text { Ethyl Benzene } \\
\text { m,p-Xylene } \\
\text { 0-Xylene } \\
\text { 1,2-Dichlorobenzene } \\
\text { 1,3-Dichlorobenzene } \\
\text { 1,4-Dichlorobenzene }\end{array}$ & $\begin{array}{l}1.0 \\
1.0 \\
1.0 \\
1.0 \\
1.0 \\
1.0 \\
1.0 \\
1.0 \\
1.0\end{array}$ & $\begin{array}{c}37 \\
38 \\
<1.0 \\
26 \\
59 \\
29 \\
<1.0 \\
<1.0 \\
<1.0\end{array}$ & $\begin{array}{l}<1.0 \\
<1.0 \\
<1.0 \\
<1.0 \\
<1.0 \\
<1.0 \\
<1.0 \\
<1.0 \\
<1.0\end{array}$ & $\begin{array}{l}<1.0 \\
<1.0 \\
<1.0 \\
<1.0 \\
<1.0 \\
<1.0 \\
<1.0 \\
<1.0 \\
<1.0\end{array}$ & $\begin{array}{l}<1.0 \\
<1.0 \\
<1.0 \\
<1.0 \\
<1.0 \\
<1.0 \\
<1.0 \\
<1.0 \\
<1.0\end{array}$ \\
\hline
\end{tabular}

Comments:

Reviewed By:

Date $19 / 1,5 / 9=$ 
Client:

Peroxidation Systems, Inc.

5151 E. Broadway, Suite 600

Tucson, Arizona 85711

Project Name: WJSA/DOE

Project Number: $\quad 345$

Sample ID: $\quad$ 101993-25 - 28
Sample Type: Groundwater

Date Sampled: $\quad 10 / 18 / 93$

Date Received: 10/19/93

Date Analyzed: 11/2/93

Analyzed By: Deb Cartmell

EPA Method 601 - Purgeable Halocarbons

Reported in $\mu \mathrm{g} / \mathrm{L}(\mathrm{ppb})$

\begin{tabular}{|c|c|c|c|c|c|}
\hline Compounds & $\begin{array}{l}\text { Limit of } \\
\text { Detection }\end{array}$ & $16 A-0$ & $16 A-3$ & $16 A-6$ & $16 A-9$ \\
\hline Bromodicloromethane & 1.0 & $<1.0$ & $<1.0$ & $<1.0$ & $<1.0$ \\
\hline Bromoform & 1.0 & $<1.0$ & $<1.0$ & $<1.0$ & $<1.0$ \\
\hline Bromomethane & 1.0 & $<1.0$ & $<1.0$ & $<1.0$ & $<1.0$ \\
\hline Carbon Tetrachloride & 1.0 & $<1.0$ & $<1.0$ & $<1.0$ & $<1.0$ \\
\hline Chlorobenzene & 1.0 & $<1.0$ & $<1.0$ & $<1.0$ & $<1.0$ \\
\hline Chloroethane & 1.0 & 35 & 18 & 2 & $<1.0$ \\
\hline Chloroform & 1.0 & 2 & $<1.0$ & $<1.0$ & $<1.0$ \\
\hline Chloromethane & 1.0 & $<1.0$ & $<1.0$ & $<1.0$ & $<1.0$ \\
\hline 2-Chloroethyl vinyl ether & 1.0 & $<1.0$ & $<1.0$ & $<1.0$ & $<1.0$ \\
\hline Dibromochloromethane & 1.0 & $<1.0$ & $<1.0$ & $<1.0$ & $<1.0$ \\
\hline 1,2-Dichlorobenzene & 1.0 & $<1.0$ & $<1.0$ & $<1.0$ & $<1.0$ \\
\hline 1,3-Dichlorobenzene & 1.0 & $<1.0$ & $<1.0$ & $<1.0$ & $<1.0$ \\
\hline 1,4-Dichlorobenzene & 1.0 & $<1.0$ & $<1.0$ & $<1.0$ & $<1.0$ \\
\hline 1,1-Dichloroethane & 1.0 & 476 & 323 & 203 & 103 \\
\hline 1,2-Dichloroethane & 1.0 & 4 & 3 & 1 & $<1.0$ \\
\hline 1,1-Dichloroethene & 1.0 & $<1.0$ & 1.0 & $<1.0$ & $<1.0$ \\
\hline Trans-1,2-Dichloroethene & 1.0 & 3 & $<1.0$ & $<1.0$ & $<1.0$ \\
\hline 1,2-Dichloropropane & 1.0 & $<1.0$ & $<1.0$ & $<1.0$ & $<1.0$ \\
\hline Cis-1,3-Dichloropropene & 1.0 & $<1.0$ & $<1.0$ & $<1.0$ & $<1.0$ \\
\hline Trans-1,3-Dichloropropene & 1.0 & $<1.0$ & $<1.0$ & $<1.0$ & $<1.0$ \\
\hline Methylene Chloride & 1.0 & 187 & 134 & 77 & 43 \\
\hline 1,1,2,2-Tetrachloroethane & 1.0 & $<1.0$ & $<1.0$ & $<1.0$ & $<1.0$ \\
\hline Tetrachloroethene & 1.0 & 4 & $<1.0$ & $<1.0$ & $<1.0$ \\
\hline 1,1,1-Trichloroethane & 1.0 & 415 & 288 & 207 & 133 \\
\hline 1,1,2-Trichloroethane & 1.0 & 6 & 4 & 3 & 2 \\
\hline Trichloroethene & 1.0 & 8 & $<1.0$ & $<1.0$ & $<1.0$ \\
\hline Trichlorofluoromethane & 1.0 & 342 & 291 & 221 & 163 \\
\hline Vinyl Chloride & 1.0 & $<1.0$ & $<1.0$ & $<1.0$ & $<1.0$ \\
\hline
\end{tabular}

Comments:

Reviewed By: 


\section{PSI Environmental Laboratories}

\section{ANALYTICAL REPORT}

State of Arizona License " 0009

Client:

Peroxidation Systems, Inc. 5151 E. Broadway, Suite 600 Tucson, Arizona 85711

Project Name: $\quad$ WJSA/DOE

Project Number: $\quad 345$

Sample ID:
101993-25 - 28
Sample Type: Groundwater

Date Sampled: $\quad 10 / 18 / 93$

Date Received: 10/19/93

Date Analyzed: 11/2/93

Analyzed By: Deb Cartmell

EPA Method 602 - Purgeable Aromatics (G.C. - PID)

Reported in $\mu \mathrm{g} / \mathrm{L}$ (ppb)

\begin{tabular}{|c|c|c|c|c|c|}
\hline Compounds & $\begin{array}{l}\text { Limit of } \\
\text { Detection }\end{array}$ & $16 A-0$ & $16 A-3$ & $16 A-6$ & $16 A-9$ \\
\hline $\begin{array}{l}\text { Benzene } \\
\text { Toluene } \\
\text { Chlorobenzene } \\
\text { Ethyl Benzene } \\
\text { m,p-Xylene } \\
\text { 0-Xylene } \\
\text { 1,2-Dichlorobenzene } \\
\text { 1,3-Dichlorobenzene } \\
\text { 1,4-Dichlorobenzene }\end{array}$ & $\begin{array}{l}1.0 \\
1.0 \\
1.0 \\
1.0 \\
1.0 \\
1.0 \\
1.0 \\
1.0 \\
1.0\end{array}$ & $\begin{array}{c}30 \\
30 \\
<1.0 \\
21 \\
45 \\
20 \\
<1.0 \\
<1.0 \\
<1.0\end{array}$ & $\begin{array}{l}<1.0 \\
<1.0 \\
<1.0 \\
<1.0 \\
<1.0 \\
<1.0 \\
<1.0 \\
<1.0 \\
<1.0\end{array}$ & $\begin{array}{l}<1.0 \\
<1.0 \\
<1.0 \\
<1.0 \\
<1.0 \\
<1.0 \\
<1.0 \\
<1.0 \\
<1.0\end{array}$ & $\begin{aligned}< & 1.0 \\
< & 1.0 \\
< & 1.0 \\
< & 1.0 \\
< & 1.0 \\
& 2 \\
< & 1.0 \\
< & <1.0 \\
< & 1.0\end{aligned}$ \\
\hline
\end{tabular}

Comments:

Reviewed By: 


\section{PSI Enviranmental Laboratorles}

FAX 602.573-6550

1-BOO-PST-LAGS

ANALYTICAL REPORT

State of Arizona License 10009

Client:

Project Number:

Sample ID:
Peroxidation Systems, Inc. 5151 E. Broadway, Suite 600 Tucson, Arizona 85711

WJSA/DOE

345

101993-29- 32
Sample Type: Groundwater

Date Sampled: $\quad 10 / 18 / 93$

Date Received: $\quad 10 / 19 / 93$

Date Analyzed: 11/2/93

Analyzed By: Deb Cartmell

\section{EPA Method 601 - Purgeable Halocarbons \\ Reported in $\mu \mathrm{g} / \mathrm{L}$ (ppb)}

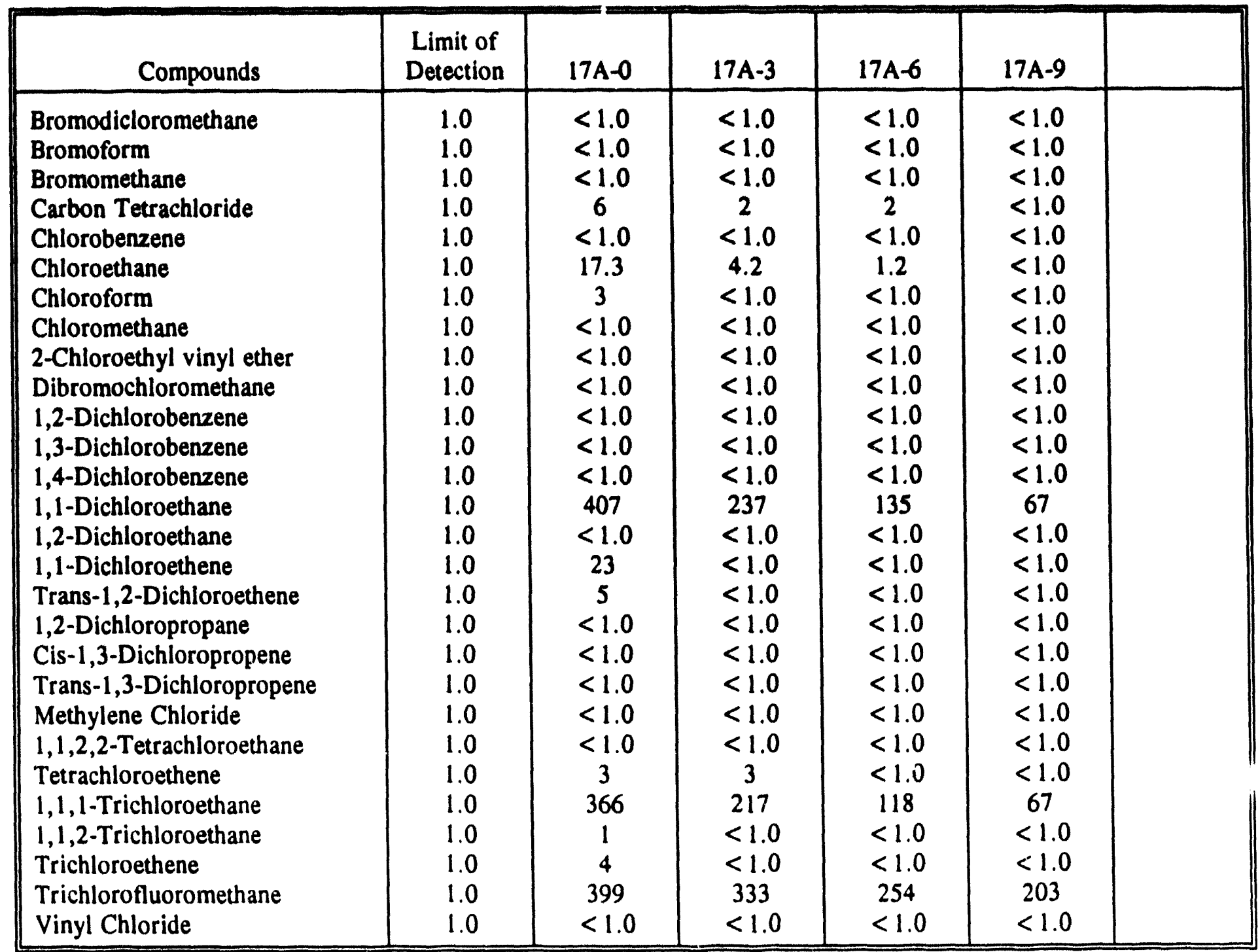

Comments: 
ANALYTICAL REPORT

State of Arizona License "

Client: $\quad$ Peroxidation Systems, Inc. 5151 E. Broadway, Suite 600

Tucson, Arizona 85711

Project Name: $\quad$ WJSA/DOE

Project Number: $\quad 345$

Sample ID:
101993-29 - 32
Sample Type: Groundwater

Date Sampled: $\quad 10 / 18 / 93$

Date Received: $\quad 10 / 19 / 93$

Date Analyzed: 11/2/93

Analyzed By: Deb Cartmell

EPA Method 602 - Purgeable Aromatics (G.C. - PID)

Reported in $\mu \mathrm{g} / \mathrm{L}(\mathrm{ppb})$

\begin{tabular}{|c|c|c|c|c|c|}
\hline Compounds & $\begin{array}{l}\text { Limit of } \\
\text { Detection }\end{array}$ & $17 A-0$ & $17 A-3$ & $17 A-6$ & $17 A-9$ \\
\hline $\begin{array}{l}\text { Benzene } \\
\text { Toluene } \\
\text { Chlorobenzene } \\
\text { Ethyl Benzene } \\
\text { m,p-Xylene } \\
\text { o-Xylene } \\
\text { 1,2-Dichlorobenzene } \\
\text { 1,3-Dichlorobenzene } \\
\text { 1,4-Dichlorobenzene }\end{array}$ & $\begin{array}{l}1.0 \\
1.0 \\
1.0 \\
1.0 \\
1.0 \\
1.0 \\
1.0 \\
1.0 \\
1.0\end{array}$ & $\begin{array}{l}18 \\
15 \\
<1.0 \\
<1.0 \\
23 \\
11 \\
<1.0 \\
<1.0 \\
<1.0\end{array}$ & $\begin{array}{l}<1.0 \\
<1.0 \\
<1.0 \\
<1.0 \\
<1.0 \\
<1.0 \\
<1.0 \\
<1.0 \\
<1.0\end{array}$ & $\begin{array}{l}<1.0 \\
<1.0 \\
<1.0 \\
<1.0 \\
<1.0 \\
<1.0 \\
<1.0 \\
<1.0 \\
<1.0\end{array}$ & $\begin{array}{l}<1.0 \\
<1.0 \\
<1.0 \\
<1.0 \\
<1.0 \\
<1.0 \\
<1.0 \\
<1.0 \\
<1.0\end{array}$ \\
\hline
\end{tabular}

Comments:

Reviewed By:

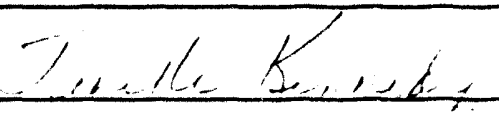

Date $12 / 1,1 / 3$ 
Client:

Project Name:

Project Number:

Sample ID:
Peroxidation Systems, Inc.

5151 E. Broadway, Suite 600

Tucson, Arizona 85711

WJSA/DOE

345

101993-33 - 36
Sample Type: Groundwater

Date Sampled: 10/18/93

Date Received: 10/19/93

Date Analyzed: 11/2/93

Analyzed By: Deb Cartmell

EPA Method 601 - Purgeable Halocarbons

Reported in $\mu \mathrm{g} / \mathrm{L}(\mathrm{ppb})$

\begin{tabular}{|c|c|c|c|c|c|}
\hline Compounds & $\begin{array}{l}\text { Limit of } \\
\text { Detection }\end{array}$ & $18 \mathrm{~A}-0$ & $18 A-3$ & $18 A-6$ & $18 A-9$ \\
\hline Bromodicloromethane & 1.0 & $<1.0$ & $<1.0$ & $<1.0$ & $<1.0$ \\
\hline Bromoform & 1.0 & $<1.0$ & $<1.0$ & $<1.0$ & $<1.0$ \\
\hline Bromomethane & 1.0 & $<1.0$ & $<1.0$ & $<1.0$ & $<1.0$ \\
\hline Carbon Tetrachloride & 1.0 & 5 & 3 & 1 & $<1.0$ \\
\hline Chlorobenzene & 1.0 & $<1.0$ & $<1.0$ & $<1.0$ & $<1.0$ \\
\hline Chloroethane & 1.0 & 19 & 8 & 2 & $<1.0$ \\
\hline Chloroform & 1.0 & $<1.0$ & $<1.0$ & $<1.0$ & $<1.0$ \\
\hline Chloromethane & 1.0 & $<1.0$ & $<1.0$ & $<1.0$ & $<1.0$ \\
\hline 2-Chloroethyl vinyl ether & 1.0 & $<1.0$ & $<1.0$ & $<1.0$ & $<1.0$ \\
\hline Dibromochloromethane & 1.0 & $<1.0$ & $<1.0$ & $<1.0$ & $<1.0$ \\
\hline 1,2-Dichlorobenzene & 1.0 & $<1.0$ & $<1.0$ & $<1.0$ & $<1.0$ \\
\hline 1,3-Dichlorobenzene & 1.0 & $<1.0$ & $<1.0$ & $<1.0$ & $<1.0$ \\
\hline 1,4-Dichlorobenzene & 1.0 & $<1.0$ & $<1.0$ & $<1.0$ & $<1.0$ \\
\hline 1,1-Dichloroethane & 1.0 & 426 & 305 & 185 & 113 \\
\hline 1,2-Dichloroethane & 1.0 & $<1.0$ & $<1.0$ & $<1.0$ & $<1.0$ \\
\hline 1,1-Dichloroethene & 1.0 & 28 & $<1.0$ & $<1.0$ & $<1.0$ \\
\hline Trans-1,2-Dichloroethene & 1.0 & 7 & $<1.0$ & $<1.0$ & $<1.0$ \\
\hline 1,2-Dichloropropane & 1.0 & $<1.0$ & $<1.0$ & $<1.0$ & $<1.0$ \\
\hline Cis-1,3-Dichloropropene & 1.0 & $<1.0$ & $<1.0$ & $<1.0$ & $<1.0$ \\
\hline Trans-1,3-Dichloropropene & 1.0 & $<1.0$ & $<1.0$ & $<1.0$ & $<1.0$ \\
\hline Methylene Chloride & 1.0 & $<1.0$ & $<1.0$ & $<1.0$ & $<1.0$ \\
\hline 1,1,2,2-Tetrachloroethane & 1.0 & $<1.0$ & $<1.0$ & $<1.0$ & $<1.0$ \\
\hline Tetrachloroethene & 1.0 & 4 & $<1.0$ & $<1.0$ & $<1.0$ \\
\hline 1,1,1-Trichloroethane & 1.0 & 371 & 276 & 186 & 128 \\
\hline 1,1,2-Trichloroethane & 1.0 & $<1.0$ & $<1.0$ & $<1.0$ & $<1.0$ \\
\hline Trichloroethene & 1.0 & 7 & $<1.0$ & $<1.0$ & $<1.0$ \\
\hline Trichlorofluoromethane & 1.0 & 423 & 364 & 263 & 228 \\
\hline Vinyl Chloride & 1.0 & $<1.0$ & $<1.0$ & $<1.0$ & $<1.0$ \\
\hline
\end{tabular}

Comments:

Reviewed By: 


\section{ANALYTICAL REPORT}

State of Arizona License

$\begin{array}{llll}\text { Client: } & \begin{array}{l}\text { Peroxidation Systems, Inc. } \\ \text { 5151 E. Broadway, Suite 600 }\end{array} & \text { Sample Type: } & \text { Groundwater } \\ & \text { Tucson, Arizona 85711 } & \text { Date Sampled: } & 10 / 18 / 93 \\ & \text { WJSA/DOE } & \text { Date Received: } & 10 / 19 / 93 \\ \text { Project Name: } & 345 & \text { Date Analyzed: } & 11 / 2 / 93 \\ \text { Project Number: } & 101993-33-36 & \text { Analyzed By: } & \text { Deb Cartmell } \\ \text { Sample ID: } & 10193 & & \end{array}$

EPA Method 602 - Purgeable Aromatics (G.C. - PID)

Reported in $\mu \mathrm{g} / \mathrm{L}(\mathrm{ppb})$

\begin{tabular}{|c|c|c|c|c|c|}
\hline Compounds & $\begin{array}{l}\text { Limit of } \\
\text { Detection }\end{array}$ & $18 \mathrm{~A}-0$ & $18 A-3$ & $18 A-6$ & $18 \mathrm{~A}-9$ \\
\hline Benzene & 1.0 & 24 & $<1.0$ & $<1.0$ & $<1.0$ \\
\hline Toluene & 1.0 & 23 & $<1.0$ & $<1.0$ & $<1.0$ \\
\hline Chlorobenzene & 1.0 & $<1.0$ & $<1.0$ & $<1.0$ & $<1.0$ \\
\hline Ethyl Benzene & 1.0 & $<1.0$ & $<1.0$ & $<1.0$ & $<1.0$ \\
\hline m,p-Xylene & 1.0 & 33 & $<1.0$ & $<1.0$ & $<1.0$ \\
\hline $0-$ Xylene & 1.0 & 16 & $<1.0$ & $<1.0$ & $<1.0$ \\
\hline 1,2-Dichlorobenzene & 1.0 & $<1.0$ & $<1.0$ & $<1.0$ & $<1.0$ \\
\hline 1,3-Dichlorobenzene & 1.0 & $<1.0$ & $<1.0$ & $<1.0$ & $<1.0$ \\
\hline 1,4-Dichlorobenzene & 1.0 & $<1.0$ & $<1.0$ & $<1.0$ & $<1.0$ \\
\hline
\end{tabular}

Comments:

Reviewed By:

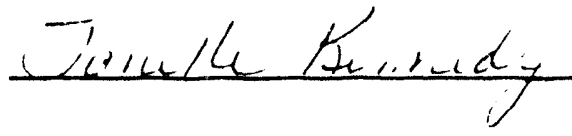

Date Li, $/ 1,5 / 0 ?$ 


\section{PSI Environmental Laboratories}

2595 E. Airport Drive Tucson. Arizana 85706 602-573-6565 FAX 602-573-6550 1-800-P5I-LA日5 ANALYTICAL REPORT

State of Arizona License $\# 0009$

Client:

Project Number: $\quad 345$

Sample ID: $\quad 101993-37-40$
Sample Type: Groundwater

Date Sampled: $\quad 10 / 18 / 93$

Date Received: $\quad 10 / 19 / 93$

Date Analyzed: 10/26/93

Analyzed By: Deb Cartmell

EPA Method 601 - Purgeable Halocarbons

Reported in $\mu \mathrm{g} / \mathrm{L}$ (ppb)

\begin{tabular}{|c|c|c|c|c|c|}
\hline Compounds & $\begin{array}{l}\text { Limit of } \\
\text { Detection }\end{array}$ & $19 \mathrm{~A}-0$ & $19 A-3$ & $19 A-6$ & $19 A-9$ \\
\hline Bromodicloromethane & 1.0 & $<1.0$ & $<1.0$ & $<1.0$ & $<1.0$ \\
\hline Bromoform & 1.0 & $<1.0$ & $<1.0$ & $<1.0$ & $<1.0$ \\
\hline Bromomethane & 1.0 & $<1.0$ & $<1.0$ & $<1.0$ & $<1.0$ \\
\hline Carbon Tetrachloride & 1.0 & $<1.0$ & $<1.0$ & $<1.0$ & $<1.0$ \\
\hline Chlorobenzene & 1.0 & $<1.0$ & $<1.0$ & $<1.0$ & $<1.0$ \\
\hline Chloroethane & 1.0 & NA & NA & NA & NA \\
\hline Chloroform & 1.0 & $<1.0$ & $<1.0$ & $<1.0$ & $<1.0$ \\
\hline Chloromethane & 1.0 & $<1.0$ & $<1.0$ & $<1.0$ & $<1.0$ \\
\hline 2-Chloroethyl vinyl ether & 1.0 & $<1.0$ & $<1.0$ & $<1.0$ & $<1.0$ \\
\hline Dibromochloromethane & 1.0 & $<1.0$ & $<1.0$ & $<1.0$ & $<1.0$ \\
\hline 1,2-Dichlorobenzene & 1.0 & $<1.0$ & $<1.0$ & $<1.0$ & $<1.0$ \\
\hline 1,3-Dichlorobenzene & 1.0 & $<1.0$ & $<1.0$ & $<1.0$ & $<1.0$ \\
\hline 1,4-Dichlorobenzene & 1.0 & $<1.0$ & $<1.0$ & $<1.0$ & $<1.0$ \\
\hline 1,1-Dichloroethane & 1.0 & 394 & 269 & 168 & 102 \\
\hline 1,2-Dichloroethane & 1.0 & $<1.0$ & $<1.0$ & $<1.0$ & $<1.0$ \\
\hline 1,1-Dichloroethene & 1.0 & 24 & $<1.0$ & $<1.0$ & $<1.0$ \\
\hline Trans-1,2-Dichloroethene & 1.0 & 6 & $<1.0$ & $<1.0$ & $<1.0$ \\
\hline 1,2-Dichloropropane & 1.0 & $<1.0$ & $<1.0$ & $<1.0$ & $<1.0$ \\
\hline Cis-1,3-Dichloropropene & 1.0 & $<1.0$ & $<1.0$ & $<1.0$ & $<1.0$ \\
\hline Trans-1,3-Dichloropropene & 1.0 & $<1.0$ & $<1.0$ & $<1.0$ & $<1.0$ \\
\hline Methylene Chloride & 1.0 & $<1.0$ & $<1.0$ & $<1.0$ & $<1.0$ \\
\hline $1,1,2,2$-Tetrachloroethane & 1.0 & $<1.0$ & $<1.0$ & $<1.0$ & $<1.0$ \\
\hline Tetrachloroethene & 1.0 & 4 & $<1.0$ & $<1.0$ & $<1.0$ \\
\hline $1,1,1$-Trichloroethane & 1.0 & 354 & 255 & 170 & 116 \\
\hline 1,1,2-Trichloroethane & 1.0 & $<1.0$ & $<1.0$ & $<1.0$ & $<1.0$ \\
\hline Trichloroethene & 1.0 & 4 & $<1.0$ & $<1.0$ & $<1.0$ \\
\hline Trichlorofluoromethane & 1.0 & NA & NA & NA & NA \\
\hline Vinyl Chloride & 1.0 & $<1.0$ & $<1.0$ & $<1.0$ & $<1.0$ \\
\hline
\end{tabular}

Comments: NA - Data not available, Pending QA/OC.

Reviewed By:

Turulle Fernedy

Date 


\section{PSI Environmental Laboratories}

2595 E. Airpart Drive Tucson. Arizona 85706 602-573-6565 FAX 602-573-6550 1-800-P5I-LABS

\section{ANALYTICAL REPORT}

State of Arizona License \#0009

$\begin{array}{llll}\text { Client: } & \begin{array}{l}\text { Peroxidation Systems, Inc. } \\ \text { 5151 E. Broadway, Suite 600 }\end{array} & \text { Sample Type: } & \text { Groundwater } \\ & \text { Tucson, Arizona 85711 } & \text { Date Sampled: } & 10 / 18 / 93 \\ \text { Project Name: } & \text { WJSA/DOE } & \text { Date Received: } & \text { 10/19/93 } \\ \text { Project Number: } & 345 & \text { Date Analyzed: } & \text { 10/26/93 } \\ \text { Sample ID: } & 101993-37-40 & \text { Analyzed By: } & \text { Deb Cartmell }\end{array}$

EPA Method 602 - Purgeable Aromatics (G.C. - PID)

Reported in $\mu \mathrm{g} / \mathrm{L}(\mathrm{ppb})$

\begin{tabular}{|c|c|c|c|c|c|}
\hline Compounds & $\begin{array}{l}\text { Limit of } \\
\text { Detection }\end{array}$ & $19 A-0$ & $19 A-3$ & $19 A-6$ & $19 A-9$ \\
\hline Benzene & 1.0 & 21 & 2 & $<1.0$ & $<1.0$ \\
\hline Toluene & 1.0 & 17 & $<1.0$ & $<1.0$ & $<1.0$ \\
\hline Chlorobenzene & 1.0 & $<1.0$ & $<1.0$ & $<1.0$ & $<1.0$ \\
\hline Ethyl Benzene & 1.0 & $<1.0$ & $<1.0$ & $<1.0$ & $<1.0$ \\
\hline m,p-Xylene & 1.0 & 26 & 4 & $<1.0$ & $<1.0$ \\
\hline 0 -Xylene & 1.0 & 13 & $<1.0$ & $<1.0$ & $<1.0$ \\
\hline 1,2-Dichlorobenzene & 1.0 & $<1.0$ & $<1.0$ & $<1.0$ & $<1.0$ \\
\hline 1,3-Dichlorobenzene & 1.0 & $<1.0$ & $<1.0$ & $<1.0$ & $<1.0$ \\
\hline 1,4-Dichlorobenzene & 1.0 & $<1.0$ & $<1.0$ & $<1.0$ & $<1.0$ \\
\hline
\end{tabular}

Comments:

Reviewed By: 
COMPANY PSI

ADDRESS 5151 E. Breadway

cirr Tucson:

STATE Az

ZPP

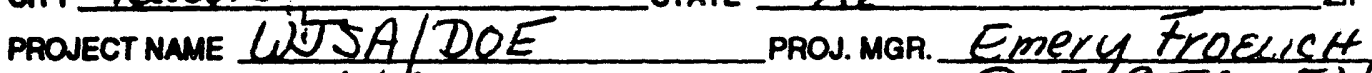

PRONECT NUMBER 345 SAMPLES TAKEN FROM: DOE SITE, TN

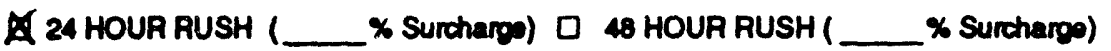

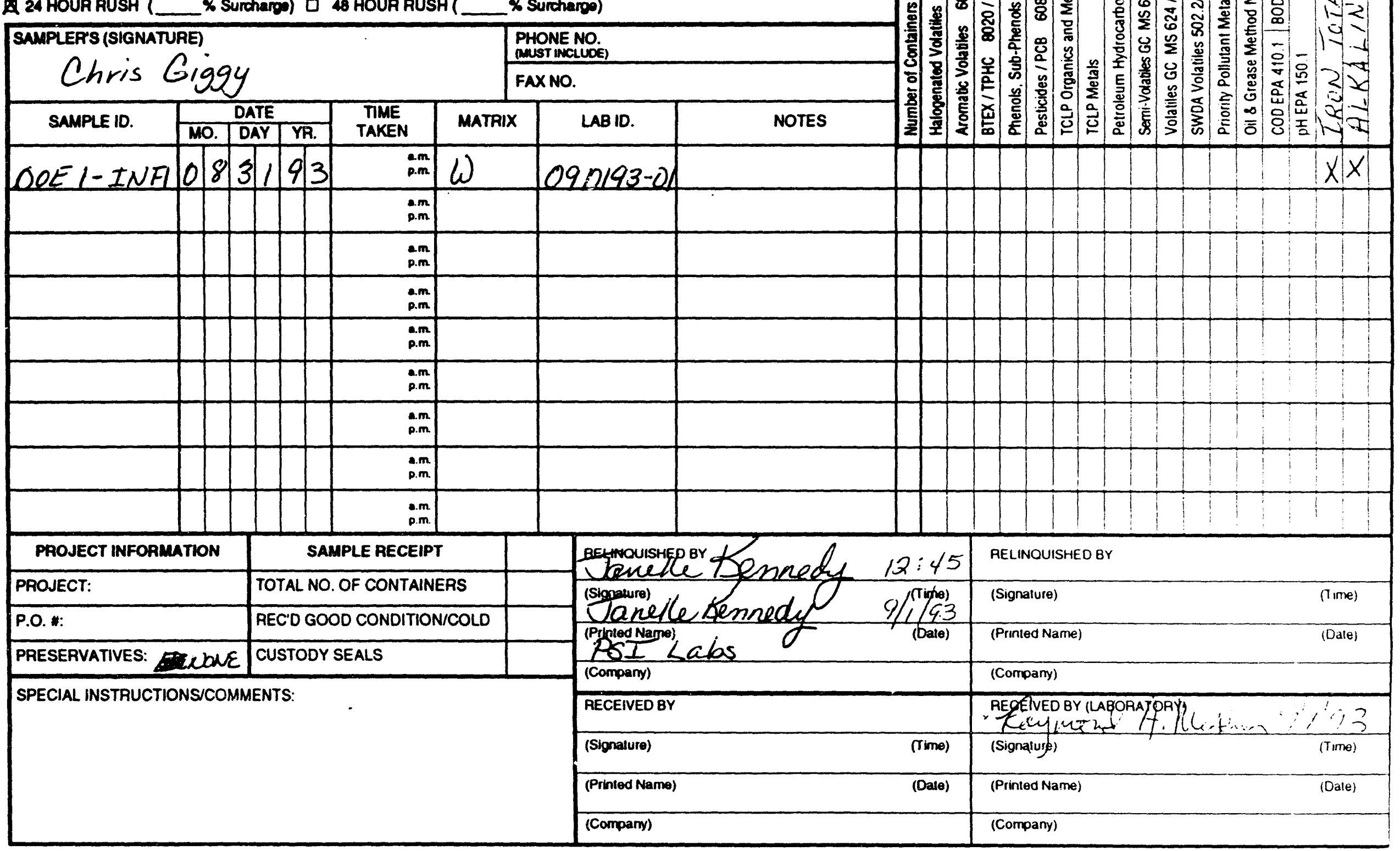




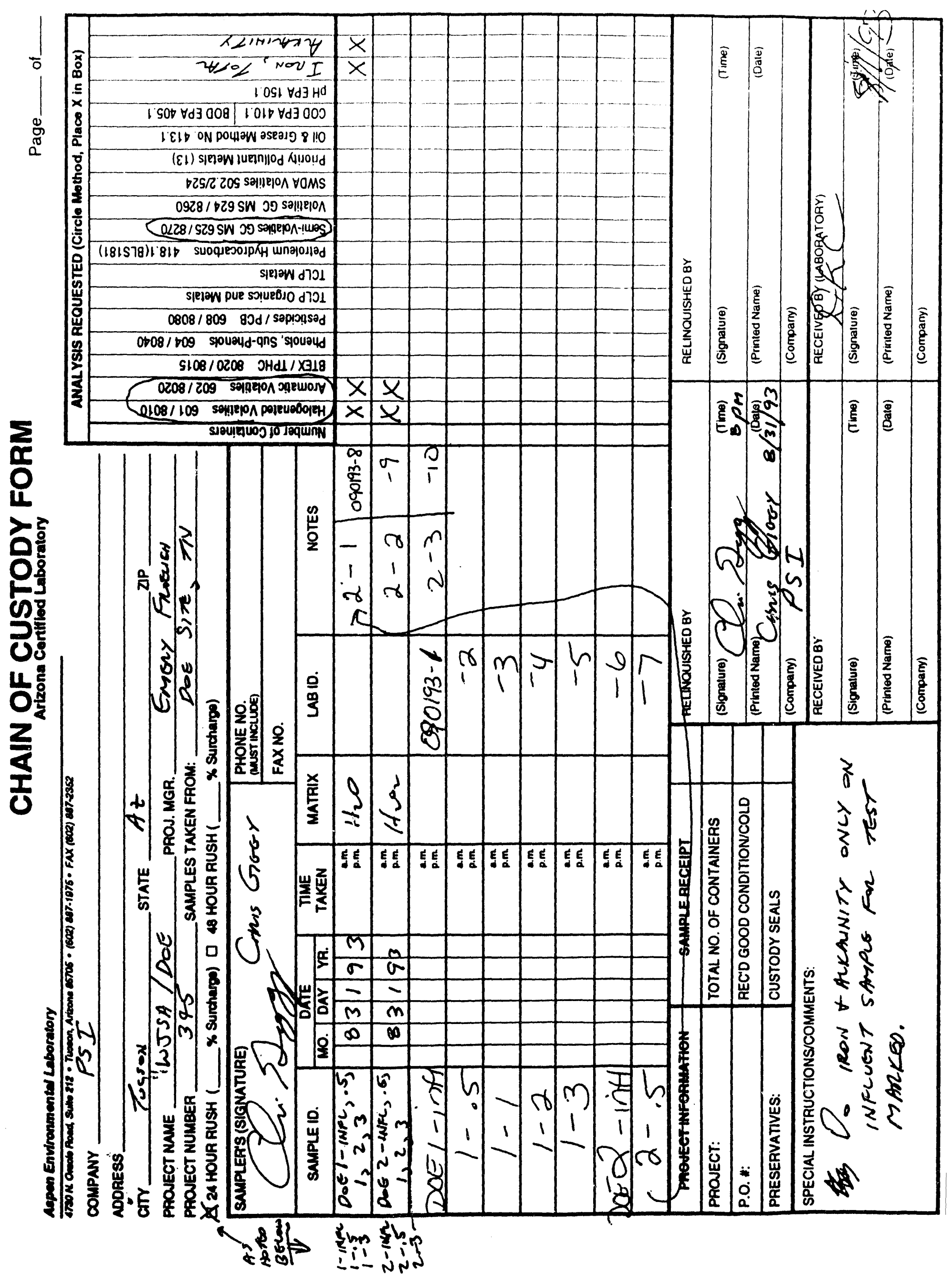


UחAII Ur YuO IU I runivi

Arizona Certified Laboratory

Page ___ of

Anvironmented Laboratory

is

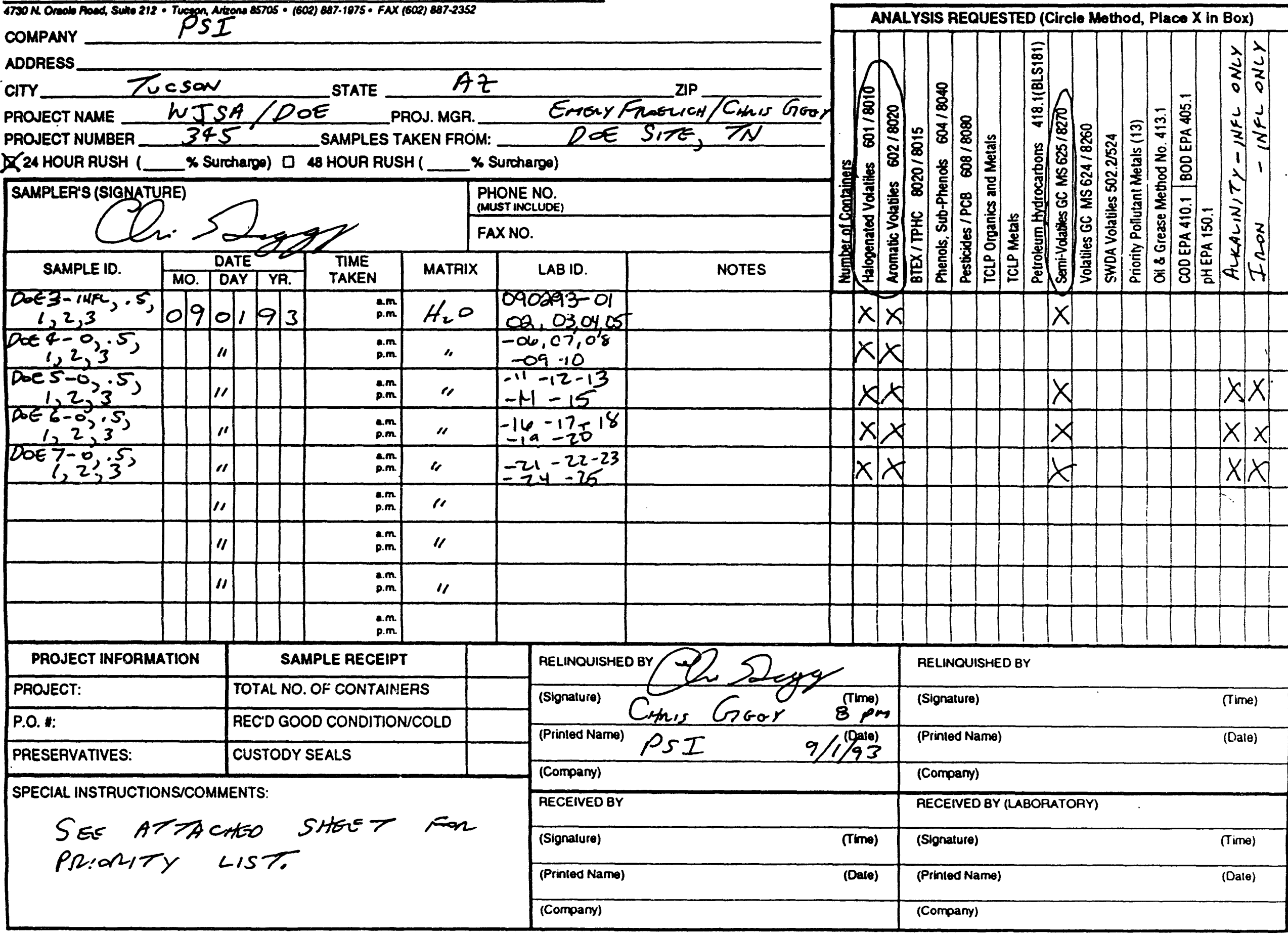


CHAIN OF CUSTODY FORM

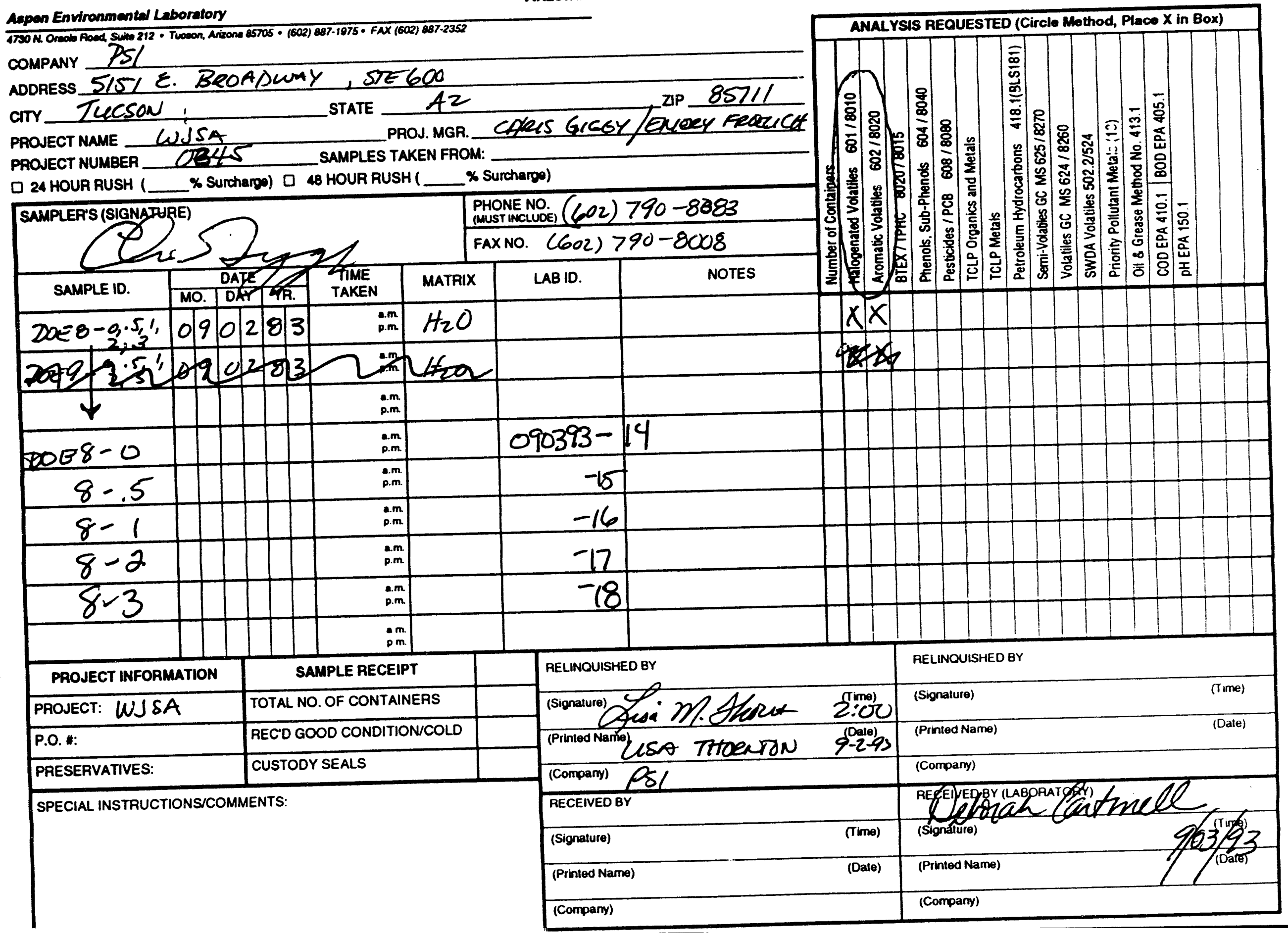


ANALYSIS REQUESTED (Circlo Mothod, Placo $X$ in Box) COMPANY $\rho S I$

ADDRESS

CITY TUESON STATE AZ Az PROJECT NAME WJSA/OOE PROJECT NUMBER 345 SAMPLES TAKEN FROM: lOE SITo, TN 口 24 HOUR RUSH (__ \% Surchargo) ם 48 HOUR RUSH (__ \% Surchargo)

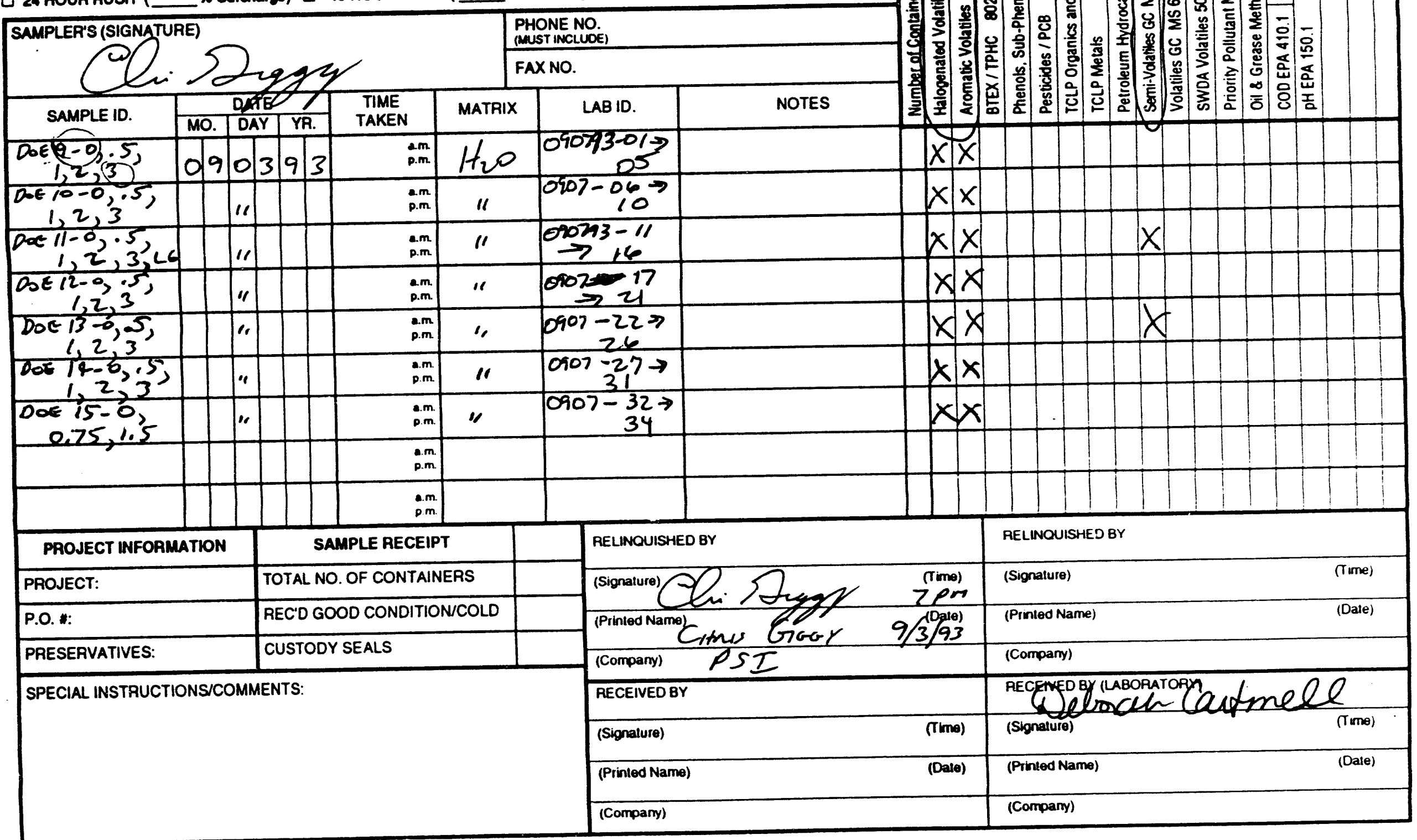


CHAIN OF CUSTODY FORM

Page 1 of 1

Aapan Environmental Laboratory

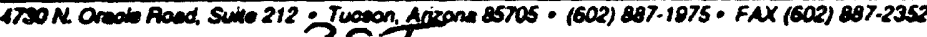

COMPANY

ADDRESS

$\cos 2$

dasac

CITY

project name wJSA/DOE

PAOJECT NUMBER 345

PROJ. MGR.

口 24 HOUR RUSH (__ \% Surchargo) $\square$ 48 HOUR RUSH (

\% Surchargo)

SAMPLER'S (SIGNATURE)

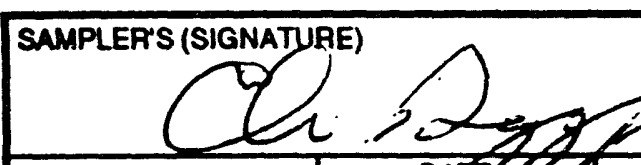

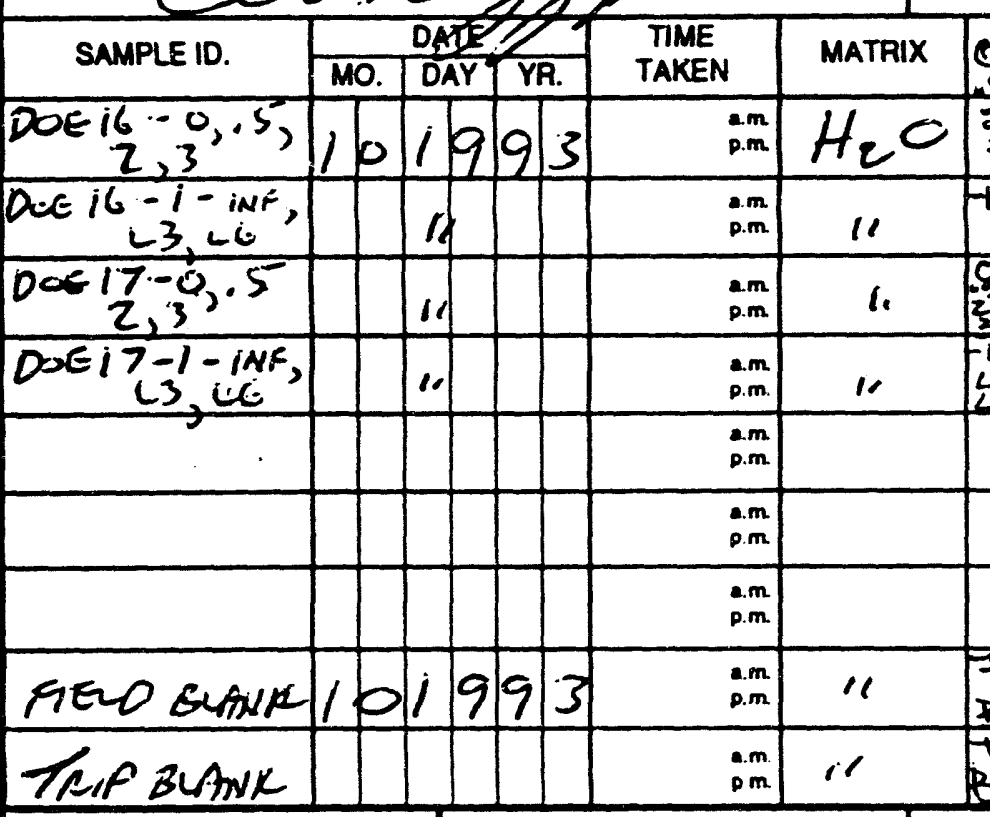

PHONE NO.

FAX NO.

\begin{tabular}{l|l|l|}
\hline \multicolumn{1}{|c|}{ PROJECT INFORMATION } & \multicolumn{1}{|c|}{ SAMPLE RECEIPT } & \\
\hline PROJECT: & TOTAL NO. OF CONTAINERS & 32 \\
\hline P.O. *: & RECD GOOD CONDITION/COLD & \\
\hline PRESERVATIVES: & CUSTODY SEALS & \\
\hline
\end{tabular}

SPECIAL INSTRUCTIONS/COMMENTS

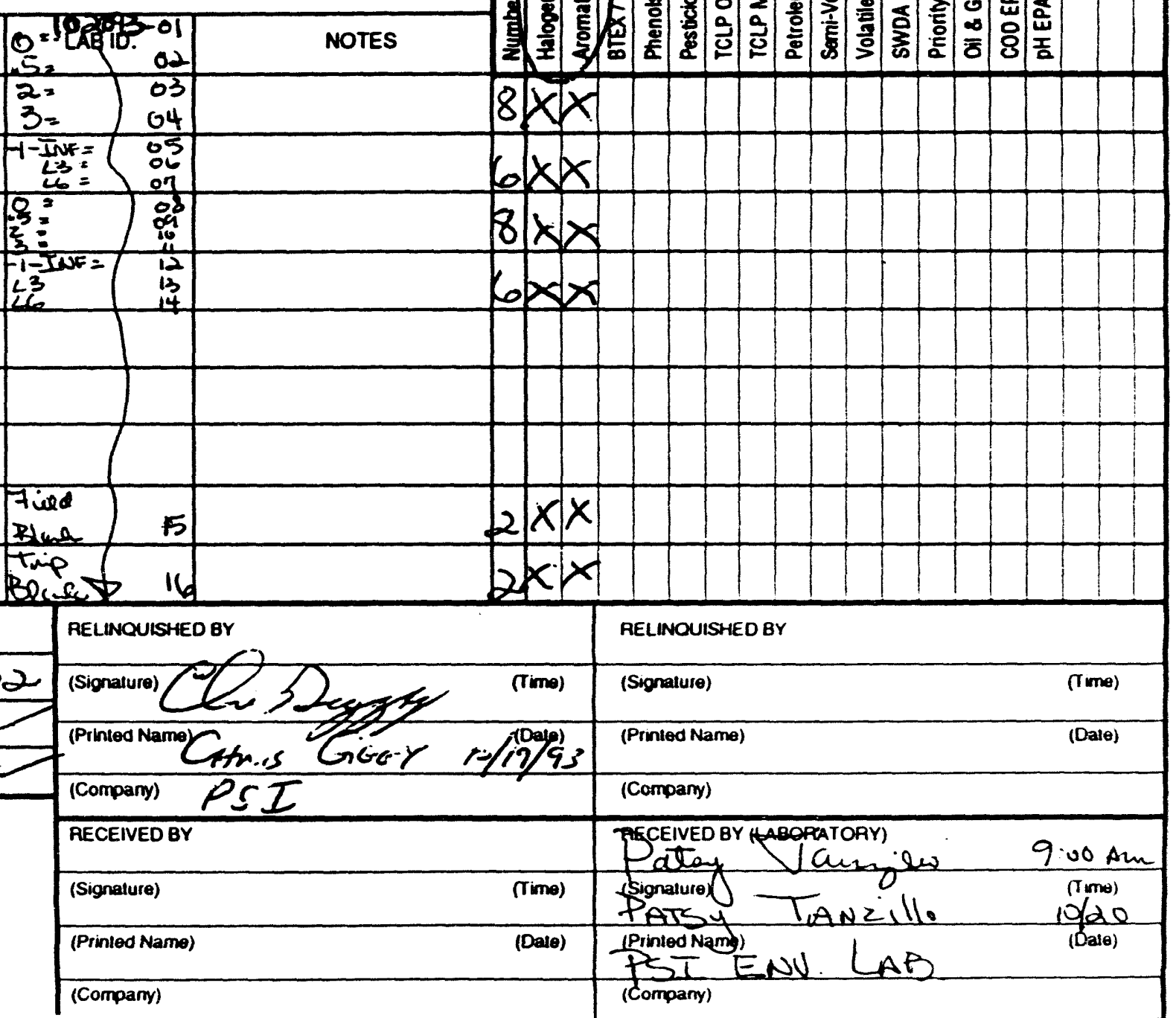


Client:

Peroxidation Systems, Inc.

5151 E. Broadway, Suite 600

Tucson, Arizona 85711

Project Name: $\quad$ WJSA/DOE

Project Number: $\quad 345$

Sample ID:

$090193-01-05$
Sample Type: Groundwater

Date Sampled: $\quad 8 / 31 / 93$

Date Received: $9 / 1 / 93$

Date Analyzed: 9/13/93

Analyzed By: Deb Cartmell

EPA Method 601 - Purgeable Halocarbons

Reported in $\mu \mathrm{g} / \mathrm{L}$ (ppb)

\begin{tabular}{|c|c|c|c|c|c|c|}
\hline Compounds & $\begin{array}{l}\text { Limit of } \\
\text { Detection }\end{array}$ & $1-\operatorname{Infl} 1^{\circ}$ & $1-.5$ & $1-1$ & $1-2$ & $1-3$ \\
\hline Bromodicloromethane & 1.0 & $<5.0$ & $<1.0$ & $<1.0$ & $<1.0$ & $<1.0$ \\
\hline Bromoform & 1.0 & 16 & $<1.0$ & $<1.0$ & $<1.0$ & $<1.0$ \\
\hline Bromomethane & 1.0 & $<5.0$ & $<1.0$ & $<1.0$ & $<1.0$ & $<1.0$ \\
\hline Carbon Tcrachloride & 1.0 & $<5.0$ & $<1.0$ & $<1.0$ & $<1.0$ & $<1.0$ \\
\hline Chlorobenzene & 1.0 & $<5.0$ & $<1.0$ & $<1.0$ & $<1.0$ & $<1.0$ \\
\hline Chloroethane & 1.0 & 170 & 241 & 217 & 89 & NA \\
\hline Chloroform & 1.0 & $<5.0$ & $<1.0$ & $<1.0$ & $<1.0$ & $<1.0$ \\
\hline Chloromethane & 1.0 & $<5.0$ & 1.0 & $<1.0$ & $<1.0$ & $<1.0$ \\
\hline 2-Chloroethyl vinyl ether & 1.0 & $<5.0$ & $<1.0$ & $<1.0$ & $<1.0$ & $<1.0$ \\
\hline Dibromochloromethane & 1.0 & $<5.0$ & $<1.0$ & $<1.0$ & $<1.0$ & $<1.0$ \\
\hline 1,2-Dichlorobenzene & 1.0 & $<5.0$ & $<1.0$ & $<1.0$ & $<1.0$ & $<1.0$ \\
\hline 1,3-Dichlorobenzene & 1.0 & $<5.0$ & $<1.0$ & $<1.0$ & $<1.0$ & $<1.0$ \\
\hline 1,4-Dichlorobenzene & 1.0 & $<5.0$ & $<1.0$ & $<1.0$ & $<1.0$ & $<1.0$ \\
\hline 1,1-Dichloroethane & 1.0 & 175 & 35 & NA & 3 & NA \\
\hline 1,2-Dichloroethane & 1.0 & $<5.0$ & $<1.0$ & $<1.0$ & $<1.0$ & $<1.0$ \\
\hline 1,1-Dichloroethene & 1.0 & $<5.0$ & $<1.0$ & 1.3 & $<1.0$ & $<1.0$ \\
\hline Trans-1,2-Dichloroethene & 1.0 & $<5.0$ & $<1.0$ & $<1.0$ & $<1.0$ & $<1.0$ \\
\hline 1,2-Dichloropropane & 1.0 & $<5.0$ & $<1.0$ & $<1.0$ & $<1.0$ & $<1.0$ \\
\hline Cis-1,3-Dichloropropene & 1.0 & $<5.0$ & $<1.0$ & $<1.0$ & $<1.0$ & $<1.0$ \\
\hline Trans-1,3-Dichloropropene & 1.0 & $<5.0$ & $<1.0$ & $<1.0$ & $<1.0$ & $<1.0$ \\
\hline Methylene Chloride & 1.0 & $<5.0$ & $<1.0$ & $<1.0$ & $<1.0$ & $<1.0$ \\
\hline $1,1,2,2$-Tetrachloroethane & 1.0 & $<5.0$ & $<1.0$ & $<1.0$ & $<1.0$ & $<1.0$ \\
\hline Tetrachloroethene & 1.0 & $<5.0$ & $<1.0$ & $<1.0$ & $<1.0$ & $<1.0$ \\
\hline 1,1,1-Trichloroethane & 1.0 & 150 & 121 & 72 & 23 & NA \\
\hline 1,1,2-Trichloroethane & 1.0 & $<5.0$ & $<1.0$ & $<1.0$ & $<1.0$ & $<1.0$ \\
\hline Trichloroethene & 1.0 & $<5.0$ & $<1.0$ & $<1.0$ & $<1.0$ & $<1.0$ \\
\hline Trichlorofluoromethane & 1.0 & 265 & 101 & 58 & 49 & NA \\
\hline Vinyl Chloride & 1.0 & 11 & 18 & NA & 7.0 & NA \\
\hline
\end{tabular}

Comments: NA - Data not available, Pending QA/QC

\section{5:1 Dilution was made on 1-Infl}

Reviewed By: 
ANALYTICAL REPORT

State of Arizona License \#0009

\begin{tabular}{|c|c|c|c|c|c|c|}
\hline Client: & $\begin{array}{l}\text { Peroxidation Systems, In } \\
5151 \text { E. Broadway, Suite } \\
\text { Tucson, Arizona } 85711\end{array}$ & & $\begin{array}{l}\text { Sample Type: } \\
\text { Date Sampled: }\end{array}$ & $\begin{array}{l}\text { Ground } \\
8 / 31 / 93\end{array}$ & & \\
\hline Project Name: & WJSA/DOE & & Date Received: & $9 / 1 / 93$ & & \\
\hline Project Number: & 345 & & Date Analyzed: & $9 / 2 / 93$ & & \\
\hline Sample ID: & $090193-01-05$ & & Analyzed By: & Deb C & & \\
\hline & 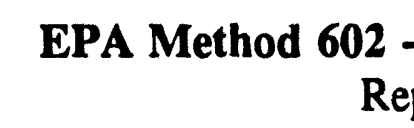 & $\begin{array}{l}\text { geabl } \\
d \text { in } \mu\end{array}$ & $\begin{array}{l}\text { Aromatics (C } \\
\mathrm{g} / \mathrm{L}(\mathrm{ppb})\end{array}$ & C. $-\mathbf{P}$ & & \\
\hline Compounds & $\begin{array}{l}\text { Limit of } \\
\text { Detection }\end{array}$ & $1-\operatorname{Infl}^{*}$ & $1-.5$ & $1-1$ & $1-2$ & $1-3$ \\
\hline $\begin{array}{l}\text { Benzene } \\
\text { Toluene } \\
\text { Chlorobenzene } \\
\text { Ethyl Benzene } \\
\text { m,p-Xylene } \\
\text { 0-Xylene } \\
\text { 1,2-Dichlorobenzene } \\
\text { 1,3-Dichlorobenzene } \\
\text { 1,4-Dichlorobenzene }\end{array}$ & $\begin{array}{l}1.0 \\
1.0 \\
1.0 \\
1.0 \\
1.0 \\
1.0 \\
1.0 \\
1.0 \\
1.0\end{array}$ & $\begin{array}{l}<5.0 \\
<5.0 \\
<5.0 \\
<5.0 \\
<5.0 \\
<5.0 \\
<5.0 \\
<5.0 \\
<5.0\end{array}$ & $\begin{array}{l}<1.0 \\
<1.0 \\
<1.0 \\
<1.0 \\
<1.0 \\
<1.0 \\
<1.0 \\
<1.0 \\
<1.0\end{array}$ & $\begin{array}{l}<1.0 \\
<1.0 \\
<1.0 \\
<1.0 \\
<1.0 \\
<1.0 \\
<1.0 \\
<1.0 \\
<1.0\end{array}$ & $\begin{array}{l}<1.0 \\
<1.0 \\
<1.0 \\
<1.0 \\
<1.0 \\
<1.0 \\
<1.0 \\
<1.0 \\
<1.0\end{array}$ & $\begin{array}{l}<1.0 \\
<1.0 \\
<1.0 \\
<1.0 \\
<1.0 \\
<1.0 \\
<1.0 \\
<1.0 \\
<1.0\end{array}$ \\
\hline
\end{tabular}

Comments:

5:1 Dilution was made on this sample.

Reviewed By: 
State of Arizona License 10009

Client:

Peroxidation Systems, Inc. 5151 E. Broadway, Suite 600

Tucson, Arizona 85711

Project Name: WJSA/DOE

Project Number: $\quad 345$

Sample ID: $\quad$ 090193-06-10
Sample Type: Groundwater

Date Sampled: $\quad 8 / 31 / 93$

Date Received: $9 / 1 / 93$

Date Analyzed: 9/13/93

Analyzed By: Deb Cartmell

EPA Method 601 - Purgeable Halocarbons

Reported in $\mu \mathrm{g} / \mathrm{L}(\mathrm{ppb})$

\begin{tabular}{|c|c|c|c|c|c|c|}
\hline Compounds & $\begin{array}{l}\text { Limit of } \\
\text { Detection }\end{array}$ & $2-\ln f^{*}$ & $2-.5$ & $2-1$ & $2-2^{\infty}$ & $2-3$ \\
\hline Bromodicloromethane & 1.0 & $<5.0$ & $<1.0$ & $<1.0$ & & $<1.0$ \\
\hline Bromoform & 1.0 & $<5.0$ & $<1.0$ & $<1.0$ & & $<1.0$ \\
\hline Bromomethane & 1.0 & $<5.0$ & $<1.0$ & $<1.0$ & & $<1.0$ \\
\hline Carbon Tetrachloride & 1.0 & $<5.0$ & $<1.0$ & $<1.0$ & & $<1.0$ \\
\hline Chlorobenzene & 1.0 & $<5.0$ & $<1.0$ & $<1.0$ & & $<1.0$ \\
\hline Chloroethane & 1.0 & 193 & 299 & 230 & & 143 \\
\hline Chloroform & 1.0 & $<5.0$ & $<1.0$ & $<1.0$ & & $<1.0$ \\
\hline Chloromethane & 1.0 & $<5.0$ & $<1.0$ & $<1.0$ & & $<1.0$ \\
\hline 2-Chloroethyl vinyl ether & 1.0 & $<5.0$ & $<1.0$ & $<1.0$ & & $<1.0$ \\
\hline Dibromochloromethane & 1.0 & $<5.0$ & $<1.0$ & $<1.0$ & & $<1.0$ \\
\hline 1,2-Dichlorobenzene & 1.0 & $<5.0$ & $<1.0$ & $<1.0$ & & $<1.0$ \\
\hline 1,3-Dichlorobenzene & 1.0 & $<5.0$ & $<1.0$ & $<1.0$ & & $<1.0$ \\
\hline 1,4-Dichlorobenzene & 1.0 & $<5.0$ & $<1.0$ & $<1.0$ & & $<1.0$ \\
\hline 1,1-Dichloroethane & 1.0 & 286 & NA & 30 & & 5 \\
\hline 1,2-Dichloroethane & 1.0 & $<5.0$ & $<1.0$ & $<1.0$ & & $<1.0$ \\
\hline 1,1-Dichloroethene & 1.0 & 22 & 3 & $<1.0$ & & $<1.0$ \\
\hline Trans-1,2-Dichloroethene & 1.0 & $<5.0$ & $<1.0$ & $<1.0$ & & $<1.0$ \\
\hline 1,2-Dichloropropane & 1.0 & $<5.0$ & $<1.0$ & $<1.0$ & & $<1.0$ \\
\hline Cis-1,3-Dichloropropene & 1.0 & $<5.0$ & $<1.0$ & $<1.0$ & & $<1.0$ \\
\hline Trans-1,3-Dichloropropene & 1.0 & $<5.0$ & $<1.0$ & $<1.0$ & & $<1.0$ \\
\hline Methylene Chloride & 1.0 & $<5.0$ & $<1.0$ & $<1.0$ & & $<1.0$ \\
\hline 1,1,2,2-Tetrachloroethane & 1.0 & $<5.0$ & $<1.0$ & $<1.0$ & & $<1.0$ \\
\hline Tetrachloroethene & 1.0 & $<5.0$ & $<1.0$ & $<1.0$ & & $<1.0$ \\
\hline 1,1,1-Trichloroethane & 1.0 & 191 & 177 & 85 & & 19 \\
\hline 1,1,2-Trichloroethane & 1.0 & $<5.0$ & $<1.0$ & $<1.0$ & & $<1.0$ \\
\hline Trichloroethene & 1.0 & $<5.0$ & $<1.0$ & $<1.0$ & & $<1.0$ \\
\hline Trichlorofluoromethane & 1.0 & 313 & 122 & 76 & & 71 \\
\hline Vinyl Chloride & 1.0 & 15 & 23 & 17 & & 11 \\
\hline
\end{tabular}

Comments: NA - Data not available, Pending OA/OC

Reviewed By:

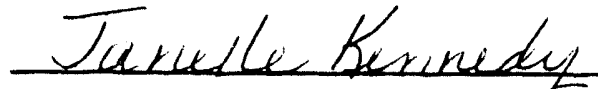

Date

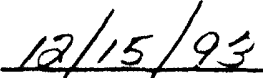




\section{ANALYTICAL REPORT \\ State of Arizona License $" 0009$}

Client: $\quad$ Peroxidation Systems, Inc. 5151 E. Broadway, Suite 600 Tucson, Arizona 85711

WJSA/DOE

345

$090193-06 \cdot 10$
Sample Type: Groundwater

Date Sampled: $\quad 8 / 31 / 93$

Date Received: $9 / 1 / 93$

Date Analyzed: $9 / 13 / 93$

Analyzed By: Deb Cartmell

EPA Method 602 - Purgeable Aromatics (G.C. - PID)

Reported in $\mu \mathrm{g} / \mathrm{L}$ (ppb)

\begin{tabular}{|c|c|c|c|c|c|c|}
\hline Compounds & $\begin{array}{l}\text { Limit of } \\
\text { Detection }\end{array}$ & $2-\operatorname{Infl}^{\circ}$ & $2-.5$ & $2-1$ & $2-2^{-\infty}$ & $2-3$ \\
\hline $\begin{array}{l}\text { Benzene } \\
\text { Toluene } \\
\text { Chlorobenzene } \\
\text { Ethyl Benzene } \\
\text { m,p-Xylene } \\
\text { 0-Xylene } \\
\text { 1,2-Dichlorobenzene } \\
\text { 1,3-Dichlorobenzene } \\
\text { 1,4-Dichlorobenzene }\end{array}$ & $\begin{array}{l}1.0 \\
1.0 \\
1.0 \\
1.0 \\
1.0 \\
1.0 \\
1.0 \\
1.0 \\
1.0\end{array}$ & $\begin{array}{c}8 \\
16 \\
<5.0 \\
9 \\
8 \\
10 \\
<5.0 \\
<5.0 \\
<5.0\end{array}$ & $\begin{array}{l}<1.0 \\
3.0 \\
<1.0 \\
<1.0 \\
<1.0 \\
2.0 \\
<1.0 \\
<1.0 \\
<1.0\end{array}$ & $\begin{array}{l}<1.0 \\
1.0 \\
<1.0 \\
<1.0 \\
<1.0 \\
<1.0 \\
<1.0 \\
<1.0 \\
<1.0\end{array}$ & & $\begin{array}{l}<1.0 \\
<1.0 \\
<1.0 \\
<1.0 \\
<1.0 \\
<1.0 \\
<1.0 \\
<1.0 \\
<1.0\end{array}$ \\
\hline
\end{tabular}

Comments:

5:1 Dilution was made on 2-Infl "Sample bottle broken

Reviewed By: 
FAX 602-573-6550

1.8DO-PSI-LABS ANALYTICAL REPORT

State of Arizona License "0009

Client:

Peroxidation Systems, Inc.

5151 E. Broadway, Suite 600

Tucson, Arizona 85711

Project Name: WJSA/DOE

Project Number: $\quad 345$

Sample ID:

$090293-01-05$
Sample Type: Groundwater

Date Sampled: $\quad 9 / 1 / 93$

Date Received: $\quad 9 / 2 / 93$

Date Analyzed: 9/13/93

Analyzed By: Deb Cartmell

EPA Method 601 - Purgeable Halocarbons

Reported in $\mu \mathrm{g} / \mathrm{L}$ (ppb)

\begin{tabular}{|c|c|c|c|c|c|c|}
\hline Compounds & $\begin{array}{l}\text { Limit of } \\
\text { Detection }\end{array}$ & 3-Infl & $3-.5$ & $3-2$ & $3-3$ & $3-1$ \\
\hline Bromodicloromethane & 1.0 & $<5.0$ & $<1.0$ & $<1.0$ & $<1.0$ & $<1.0$ \\
\hline Bromoform & 1.0 & $<5.0$ & $<1.0$ & $<1.0$ & $<1.0$ & $<1.0$ \\
\hline Bromomethane & 1.0 & $<5.0$ & $<1.0$ & $<1.0$ & $<1.0$ & $<1.0$ \\
\hline Carbon Tetrachloride & 1.0 & $<5.0$ & $<1.0$ & $<1.0$ & $<1.0$ & $<1.0$ \\
\hline Chlorobenzene & 1.0 & $<5.0$ & $<1.0$ & $<1.0$ & $<1.0$ & $<1.0$ \\
\hline Chloroethane & 1.0 & 297 & 276 & 181 & 126 & 256 \\
\hline Chloroform & 1.0 & $<5.0$ & $<1.0$ & $<1.0$ & $<1.0$ & $<1.0$ \\
\hline Chloromethane & 1.0 & $<5.0$ & $<1.0$ & $<1.0$ & $<1.0$ & $<1.0$ \\
\hline 2-Chloroethyl vinyl ether & 1.0 & $<5.0$ & $<1.0$ & $<1.0$ & $<1.0$ & $<1.0$ \\
\hline Dibromochloromethane & 1.0 & $<5.0$ & $<1.0$ & $<1.0$ & $<1.0$ & $<1.0$ \\
\hline 1,2-Dichlorobenzene & 1.0 & $<5.0$ & $<1.0$ & $<1.0$ & $<1.0$ & $<1.0$ \\
\hline 1,3-Dichlorobenzene & 1.0 & $<5.0$ & $<1.0$ & $<1.0$ & $<1.0$ & $<1.0$ \\
\hline 1,4-Dichlorobenzene & 1.0 & $<5.0$ & $<1.0$ & $<1.0$ & $<1.0$ & $<1.0$ \\
\hline 1,1-Dichloroethane & 1.0 & 387 & 125 & 5.4 & 3 & 32 \\
\hline 1,2-Dichloroethane & 1.0 & $<5.0$ & $<1.0$ & $<1.0$ & $<1.0$ & $<1.0$ \\
\hline 1,1-Dichloroethene & 1.0 & 14 & 2 & $<1.0$ & $<1.0$ & $<1.0$ \\
\hline Trans-1,2-Dichloroethene & 1.0 & $<5.0$ & $<1.0$ & $<1.0$ & $<1.0$ & $<1.0$ \\
\hline 1,2-Dichloropropane & 1.0 & $<5.0$ & $<1.0$ & $<1.0$ & $<1.0$ & $<1.0$ \\
\hline Cis-1,3-Dichloropropene & 1.0 & $<5.0$ & $<1.0$ & $<1.0$ & $<1.0$ & $<1.0$ \\
\hline Trans-1,3-Dichloropropene & 1.0 & $<5.0$ & $<1.0$ & $<1.0$ & $<1.0$ & $<1.0$ \\
\hline Methylene Chloride & 1.0 & $<5.0$ & $<1.0$ & $<1.0$ & $<1.0$ & $<1.0$ \\
\hline 1,1,2,2-Tetrachloroethane & 1.0 & $<5.0$ & $<1.0$ & $<1.0$ & $<1.0$ & $<1.0$ \\
\hline Tetrachloroethene & 1.0 & $<5.0$ & $<1.0$ & $<1.0$ & $<1.0$ & $<1.0$ \\
\hline 1,1,1-Trichloroethane & 1.0 & 237 & 133 & 30 & 13 & 79 \\
\hline 1,1,2-Trichloroethane & 1.0 & $<5.0$ & $<1.0$ & $<1.0$ & $<1.0$ & $<1.0$ \\
\hline Trichloroethene & 1.0 & $<5.0$ & $<1.0$ & $<1.0$ & $<1.0$ & $<1.0$ \\
\hline Trichlorofluoromethane & 1.0 & 158 & 108 & 50 & 29 & 124 \\
\hline Vinyl Chloride & 1.0 & 20 & 21 & 14 & 9 & 19 \\
\hline
\end{tabular}

Comments:

5:1 Dilution was made on this sample.

Reviewed By:

Turelle ferrendy

Date $12 / 15 / 93$ 


\section{PSI Environmental Laboratories}

2595 E. Alport Drive Tuceon. Arizana 85706 602.573-6565 FAX 602.573-6550 1-600-p5I-LABS

\section{ANALYTICAL REPORT}

State of Arizona License 10009

Client:

Peroxidation Systems, Inc.

5151 E. Broadway, Suite 600

Tucson, Arizona 85711

Project Name: $\quad$ WJSA/DOE

Project Number: $\quad 345$

Sample ID:
090293-01-05
Sample Type: Groundwater

Date Sampled: $\quad 9 / 1 / 93$

Date Received: $\quad 9 / 2 / 93$

Date Analyzed: $\quad 9 / 13 / 93$

Analyzed By: Deb Cartmell

EPA Method 602 - Purgeable Aromatics (G.C. - PID)

Reported in $\mu \mathrm{g} / \mathrm{L}(\mathrm{ppb})$

\begin{tabular}{|l|c|c|c|c|c|c|}
\hline \multicolumn{1}{|c|}{ Compounds } & $\begin{array}{c}\text { Limit of } \\
\text { Detection }\end{array}$ & $3-$ Infl $^{\circ}$ & $3-.5$ & $3-1$ & $3-2$ & $3-3$ \\
\hline Benzene & 1.0 & 5.8 & $<1.0$ & $<1.0$ & $<1.0$ & $<1.0$ \\
Toluene & 1.0 & 10.4 & 1.0 & $<1.0$ & $<1.0$ & $<1.0$ \\
Chlorobenzene & 1.0 & $<5.0$ & $<1.0$ & $<1.0$ & $<1.0$ & $<1.0$ \\
Ethyl Benzene & 1.0 & $<5.0$ & $<1.0$ & $<1.0$ & $<1.0$ & $<1.0$ \\
m,p-Xylene & 1.0 & $<5.0$ & $<1.0$ & $<1.0$ & $<1.0$ & $<1.0$ \\
0-Xylene & 1.0 & 6.60 & $<1.0$ & $<1.0$ & $<1.0$ & $<1.0$ \\
1,2-Dichlorobenzene & 1.0 & $<5.0$ & $<1.0$ & $<1.0$ & $<1.0$ & $<1.0$ \\
1,3-Dichlorobenzene & 1.0 & $<5.0$ & $<1.0$ & $<1.0$ & $<1.0$ & $<1.0$ \\
1,4-Dichlorobenzene & 1.0 & $<5.0$ & $<1.0$ & $<1.0$ & $<1.0$ & $<1.0$ \\
\hline
\end{tabular}

Comments:

"5il Dilution was made on this sample.

Reviewed By:

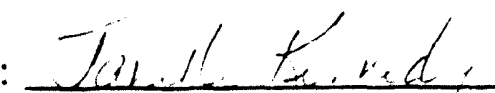


Client:

Project Name:

WJSA/DOE

Project Number: $\quad 345$

Sample ID: $\quad$ 090293-06-10
Sample Type: Groundwater

Date Sampled: $\quad 9 / 1 / 93$

Date Received: $\quad 9 / 2 / 93$

Date Analyzed: 9/7/93

Analyzed By: Deb Cartmell

EPA Method 601 - Purgeable Halocarbons

Reported in $\mu \mathrm{g} / \mathrm{L}(\mathrm{ppb})$

\begin{tabular}{|c|c|c|c|c|c|c|}
\hline Compounds & $\begin{array}{c}\text { Limit of } \\
\text { Detection }\end{array}$ & $4-0^{*}$ & $4-.5$ & $4-2$ & $4-3$ & 4-1 \\
\hline Bromodicloromethane & 1.0 & NA & $<1.0$ & $<1.0$ & $<1.0$ & $<1.0$ \\
\hline Bromoform & 1.0 & NA & $<1.0$ & $<1.0$ & $<1.0$ & $<1.0$ \\
\hline Bromomethane & 1.0 & NA & $<1.0$ & $<1.0$ & $<1.0$ & $<1.0$ \\
\hline Carbon Tetrachloride & 1.0 & NA & $<1.0$ & $<1.0$ & $<1.0$ & $<1.0$ \\
\hline Chlorobenzene & 1.0 & NA & $<1.0$ & $<1.0$ & $<1.0$ & $<1.0$ \\
\hline Chloroethane & 1.0 & NA & 300 & 171 & 114 & 288 \\
\hline Chloroform & 1.0 & NA & $<1.0$ & $<1.0$ & $<1.0$ & $<1.0$ \\
\hline Chloromethane & 1.0 & NA & $<1.0$ & $<1.0$ & $<1.0$ & $<1.0$ \\
\hline 2-Chloroethyl vinyl ether & 1.0 & NA & $<1.0$ & $<1.0$ & $<1.0$ & $<1.0$ \\
\hline Dibromochloromethane & 1.0 & NA & $<1.0$ & $<1.0$ & $<1.0$ & $<1.0$ \\
\hline 1,2-Dichlorobenzene & 1.0 & NA & $<1.0$ & $<1.0$ & $<1.0$ & $<1.0$ \\
\hline 1,3-Dichlorobenzene & 1.0 & NA & $<1.0$ & $<1.0$ & $<1.0$ & $<1.0$ \\
\hline 1,4-Dichlorobenzene & 1.0 & NA & $<1.0$ & $<1.0$ & $<1.0$ & $<1.0$ \\
\hline 1,1-Dichloroethane & 1.0 & NA & NA & NA & NA & 123 \\
\hline 1,2-Dichloroethane & 1.0 & NA & $<1.0$ & $<1.0$ & $<1.0$ & $<1.0$ \\
\hline 1,1-Dichloroethene & 1.0 & NA & $<1.0$ & $<1.0$ & $<1.0$ & $<1.0$ \\
\hline Trans-1,2-Dichloroethene & 1.0 & NA & $<1.0$ & $<1.0$ & $<1.0$ & $<1.0$ \\
\hline 1,2-Dichloropropane & 1.0 & NA & $<1.0$ & $<1.0$ & $<1.0$ & $<1.0$ \\
\hline Cis-1,3-Dichloropropene & 1.0 & NA & $<1.0$ & $<1.0$ & $<1.0$ & $<1.0$ \\
\hline Trans-1,3-Dichloropropene & 1.0 & NA & $<1.0$ & $<1.0$ & $<1.0$ & $<1.0$ \\
\hline Methylene Chloride & 1.0 & NA & $<1.0$ & $<1.0$ & $<1.0$ & $<1.0$ \\
\hline 1,1,2,2-Tetrachloroethane & 1.0 & NA & $<1.0$ & $<1.0$ & $<1.0$ & $<1.0$ \\
\hline Tetrachloroethene & 1.0 & NA & $<1.0$ & $<1.0$ & $<1.0$ & $<1.0$ \\
\hline 1,1,1-Trichloroethane & 1.0 & NA & 212 & 115 & 49 & 199 \\
\hline 1,1,2-Trichloroethane & 1.0 & NA & $<1.0$ & $<1.0$ & $<1.0$ & $<1.0$ \\
\hline Trichloroethene & 1.0 & NA & $<1.0$ & $<1.0$ & $<1.0$ & $<1.0$ \\
\hline Trichlorofluoromethane & 1.0 & NA & 98 & 27 & 18 & 97 \\
\hline Vinyl Chloride & 1.0 & NA & 23 & 13 & 9.0 & 22 \\
\hline
\end{tabular}

Comments: NA - Data not available - Pending QA/OC

"5:1 Dilution was made on this sample.

Reviev'red By:

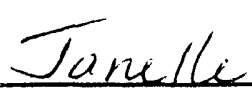


ANALYTICAL REPORT

State of Arizona License $\# 0009$

$\begin{array}{llll}\text { Client: } & \begin{array}{l}\text { Peroxidation Systems, Inc. } \\ \text { 5151 E. Broadway, Suite 600 } \\ \text { Tucson, Arizona 85711 }\end{array} & \text { Sample Type: } & \text { Groundwater } \\ & & \text { Date Sampled: } & 9 / 1 / 93 \\ \text { Project Name: } & \text { WJSA/DOE } & \text { Date Received: } & 9 / 2 / 93 \\ \text { Project Number: } & 345 & \text { Date Analyzed: } & 9 / 7 / 93 \\ \text { Sample ID: } & 090293-06-10 & \text { Analyzed By: } & \text { Deb Cartmell }\end{array}$

EPA Method 602 - Purgeable Aromatics (G.C. - PID)

Reported in $\mu \mathrm{g} / \mathrm{L}(\mathrm{ppb})$

\begin{tabular}{||l|c|c|c|c|c|c||}
\hline \multicolumn{1}{|c|}{ Compounds } & $\begin{array}{c}\text { Limit of } \\
\text { Detection }\end{array}$ & $4-0$ & $4-.5$ & $4-2$ & $4-3$ & $4-1$ \\
\hline Benzene & 1.0 & NA & $<1.0$ & $<1.0$ & $<1.0$ & $<1.0$ \\
Toluene & 1.0 & NA & $<1.0$ & $<1.0$ & $<1.0$ & $<1.0$ \\
Chlorobenzene & 1.0 & NA & $<1.0$ & $<1.0$ & $<1.0$ & $<1.0$ \\
Ethyl Benzene & 1.0 & NA & $<1.0$ & $<1.0$ & $<1.0$ & $<1.0$ \\
m,p-Xylene & 1.0 & NA & $<1.0$ & $<1.0$ & $<1.0$ & $<1.0$ \\
o-Xylene & 1.0 & NA & $<1.0$ & $<1.0$ & $<1.0$ & $<1.0$ \\
1,2-Dichlorobenzene & 1.0 & NA & $<1.0$ & $<1.0$ & $<1.0$ & $<1.0$ \\
1,3-Dichlorobenzene & 1.0 & NA & $<1.0$ & $<1.0$ & $<1.0$ & $<1.0$ \\
1,4-Dichlorobenzene & 1.0 & NA & $<1.0$ & $<1.0$ & $<1.0$ & $<1.0$ \\
\hline
\end{tabular}

Comments: NA - Data not available, Pending QA/QC.

Reviewed By: Tarulc Krinety

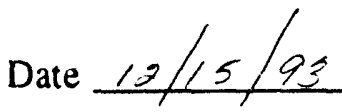




\section{PSI Environmental Laboratories}

2595 E. Airport Drive Tucson. Arizona 85706 G02-573-6565 FAX 602-573-6550 1-800-P5I-LAB5 ANALYTICAL REPORT

State of Arizona License $\# 0009$

Client:

\section{Project Name:}

Project Number:

Sample ID:
Peroxidation Systems, Inc. 5151 E. Broadway, Suite 600

Tucson, Arizona 85711

WJSA/DOE

345

090293-11-15
Sample Type: Groundwater.

Date Sampled: $\quad$ 9/1/93

Date Received: 9/2/93

Date Analyzed: 9/7/93

Analyzed By: Deb Cartmell

EPA Method 601 - Purgeable Halocarbons

Reported in $\mu \mathrm{g} / \mathrm{L}$ (ppb)

\begin{tabular}{|c|c|c|c|c|c|c|}
\hline Compounds & $\begin{array}{l}\text { Limit of } \\
\text { Detection }\end{array}$ & $5-.5^{*}$ & $5-1$ & $5-2$ & $5-3$ & $5-0^{-\infty}$ \\
\hline Bromodicloromethane & 1.0 & NA & $<1.0$ & NA & NA & $<5.0$ \\
\hline Bromoform & 1.0 & NA & $<1.0$ & NA & NA & $<5.0$ \\
\hline Bromomethane & 1.0 & NA & $<1.0$ & NA & NA & $<5.0$ \\
\hline Carbon Tetrachloride & 1.0 & NA & $<1.0$ & NA & NA & $<5.0$ \\
\hline Chlorobenzene & 1.0 & NA & $<1.0$ & NA & NA & $<5.0$ \\
\hline Chloroethane & 1.0 & NA & 361 & NA & NA & 412 \\
\hline Chloroform & 1.0 & NA & $<1.0$ & NA & NA & $<5.0$ \\
\hline Chloromethane & 1.0 & NA & $<1.0$ & NA & NA & $<5.0$ \\
\hline 2-Chloroethyl vinyl ether & 1.0 & NA & $<1.0$ & NA & NA & $<5.0$ \\
\hline Dibromochloromethane & 1.0 & NA & $<1.0$ & NA & NA & $<5.0$ \\
\hline 1,2-Dichlorobenzene & 1.0 & NA & $<1.0$ & NA & NA & $<5.0$ \\
\hline 1,3-Dichlorobenzene & 1.0 & NA & $<1.0$ & NA & NA & $<5.0$ \\
\hline 1,4-Dichlorobenzene & 1.0 & NA & $<1.0$ & NA & NA & $<5.0$ \\
\hline 1,1-Dichloroethane & 1.0 & NA & 44 & NA & NA & 298 \\
\hline 1,2-Dichloroethane & 1.0 & NA & $<1.0$ & NA & NA & $<5.0$ \\
\hline 1,1-Dichloroethene & 1.0 & NA & $<1.0$ & NA & NA & 57 \\
\hline Trans-1,2-Dichloroethene & 1.0 & NA & $<1.0$ & NA & NA & $<5.0$ \\
\hline 1,2-Dichloropropane & 1.0 & NA & $<1.0$ & NA & NA & $<5.0$ \\
\hline Cis-1,3-Dichloropropene & 1.0 & NA & $<1.0$ & NA & NA & $<5.0$ \\
\hline Trans-1,3-Dichloropropene & 1.0 & NA & $<1.0$ & NA & NA & $<5.0$ \\
\hline Methylene Chloride & 1.0 & NA & $<1.0$ & NA & NA & $<5.0$ \\
\hline 1,1,2,2-Tetrachloroethane & 1.0 & NA & $<1.0$ & NA & NA & $<5.0$ \\
\hline Tetrachloroethene & 1.0 & NA & $<1.0$ & NA & NA & $<5.0$ \\
\hline 1,1,1-Trichloroethane & 1.0 & NA & 139 & NA & NA & 248 \\
\hline 1,1,2-Trichloroethane & 1.0 & NA & $<1.0$ & NA & NA & $<5.0$ \\
\hline Trichloroethene & 1.0 & NA & $<1.0$ & NA & NA & $<5.0$ \\
\hline Trichlorofluoromethane & 1.0 & NA & 174 & NA & NA & 206 \\
\hline Vinyl Chloride & 1.0 & NA & $<1.0$ & NA & NA & $<5.0$ \\
\hline
\end{tabular}

Comments: NA - Data not available. Pending QA/OC

'Sample run with headspace.

"5:1 Dilution was made on this sample.

Reviewed By:

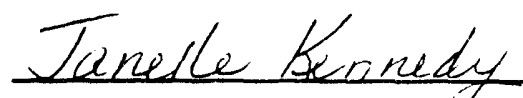

Date $12 / 15 / 93$ 


\section{P5I Environmental Laboratories}

ANALYTICAL REPORT

State of Arizona License \#0009

Client:

Peroxidation Systems, Inc.

5151 E. Broadway, Suite 600

Tucson, Arizona 85711

Project Name:

WJSA/DOE

Project Number: $\quad 345$

Sample ID:
090293-11 - 15
Sample Type: Groundwater

Date Sampled: $\quad 9 / 1 / 93$

Date Received: $\quad 9 / 2 / 93$

Date Analyzed: $\quad 9 / 7 / 93$

Analyzed By: Deb Cartmell

\section{EPA Method 602 - Purgeable Aromatics (G.C. - PID) \\ Reported in $\mu \mathrm{g} / \mathrm{L}$ (ppb)}

\begin{tabular}{|l|c|c|c|c|c|c|}
\hline \multicolumn{1}{|c|}{ Compounds } & $\begin{array}{c}\text { Limit of } \\
\text { Detection }\end{array}$ & $5-.5^{\circ}$ & $5-1$ & $5-2$ & $5-3$ & $5-0$ \\
\hline Benzene & 1.0 & $\mathrm{NA}$ & $<1.0$ & $<1.0$ & $<1.0$ & $<5^{\circ}$ \\
Toluene & 1.0 & $\mathrm{NA}$ & $<1.0$ & $<1.0$ & $<1.0$ & 53 \\
Chlorobenzene & 1.0 & $<1.0$ & $<1.0$ & $<1.0$ & $<1.0$ & $<5^{\circ}$ \\
Ethyl Benzene & 1.0 & $\mathrm{NA}$ & $<1.0$ & $<1.0$ & $<1.0$ & $<5^{\circ}$ \\
m,p-Xylene & 1.0 & $\mathrm{NA}$ & 1.1 & $\mathrm{NA}$ & $\mathrm{NA}$ & 14 \\
o-Xylene & 1.0 & $<1.0$ & $<1.0$ & $<1.0$ & $<1.0$ & 34 \\
1,2-Dichlorobenzene & 1.0 & $<1.0$ & $<1.0$ & $<1.0$ & $<1.0$ & $<5^{\circ}$ \\
1,3-Dichlorobenzene & 1.0 & $<1.0$ & $<1.0$ & $<1.0$ & $<1.0$ & NA \\
1,4-Dichlorobenzene & 1.0 & $<1.0$ & $<1.0$ & $<1.0$ & $<1.0$ & NA \\
\hline
\end{tabular}

Comments: NA - Data not Available, Pending QA/OC

5:1 Dilution was necessary due to the levels of contaminants in the sample.

Reviewed By:

7

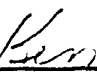

Date $12 / 15 / 93$ 
Client:

Peroxidation Systems, Inc.

5151 E. Broadway, Suite 600

Tucson, Arizona 85711

Project Name: $\quad$ WJSA/DOE

Project Number: $\quad 345$

Sample ID:
090293-16-20
Sample Type: Groundwater

Date Sampled: $\quad 9 / 1 / 93$

Date Received: 9/2/93

Date Analyzed: $9 / 13 / 93$

Analyzed By: Deb Cartmell

EPA Method 601 - Purgeable Halocarbons

Reported in $\mu \mathrm{g} / \mathrm{L}$ (ppb)

\begin{tabular}{|c|c|c|c|c|c|c|}
\hline Compounds & $\begin{array}{l}\text { Limit of } \\
\text { Detection }\end{array}$ & $6-0^{*}$ & $6-.5$ & $6-1$ & $6-2$ & $6-3$ \\
\hline Bromodicloromethane & 1.0 & $<5.0$ & $<1.0$ & $<1.0$ & $<1.0$ & $<1.0$ \\
\hline Bromoform & 1.0 & $<5.0$ & $<1.0$ & $<1.0$ & $<1.0$ & $<1.0$ \\
\hline Bromomethane & 1.0 & $<5.0$ & $<1.0$ & $<1.0$ & $<1.0$ & $<1.0$ \\
\hline Carbon Tetrachloride & 1.0 & $<5.0$ & $<1.0$ & $<1.0$ & $<1.0$ & $<1.0$ \\
\hline Chlorobenzene & 1.0 & $<5.0$ & $<1.0$ & $<1.0$ & $<1.0$ & $<1.0$ \\
\hline Chloroethane & 1.0 & 316 & 281 & 236 & 149 & 88 \\
\hline Chloroform & 1.0 & $<5.0$ & $<1.0$ & $<1.0$ & $<1.0$ & $<1.0$ \\
\hline Chloromethane & 1.0 & $<5.0$ & $<1.0$ & $<1.0$ & $<1.0$ & $<1.0$ \\
\hline 2-Chloroethyl vinyl ether & 1.0 & $<5.0$ & $<1.0$ & $<1.0$ & $<1.0$ & $<1.0$ \\
\hline Dibromochloromethane & 1.0 & $<5.0$ & $<1.0$ & $<1.0$ & $<1.0$ & $<1.0$ \\
\hline 1,2-Dichlorobenzene & 1.0 & $<5.0$ & $<1.0$ & $<1.0$ & $<1.0$ & $<1.0$ \\
\hline 1,3-Dichlorobenzene & 1.0 & $<5.0$ & $<1.0$ & $<1.0$ & $<1.0$ & $<1.0$ \\
\hline 1,4-Dichlorobenzene & 1.0 & $<5.0$ & $<1.0$ & $<1.0$ & $<1.0$ & $<1.0$ \\
\hline 1,1-Dichloroethane & 1.0 & 501 & 139 & 29 & 5 & 3 \\
\hline 1,2-Dichloroethane & 1.0 & $<5.0$ & $<1.0$ & $<1.0$ & $<1.0$ & $<1.0$ \\
\hline 1,1-Dichloroethene & 1.0 & 54 & 2 & 1 & $<1.0$ & $<1.0$ \\
\hline Trans-1,2-Dichloroethene & 1.0 & $<5.0$ & $<1.0$ & $<1.0$ & $<1.0$ & $<1.0$ \\
\hline 1,2-Dichloropropane & 1.0 & $<5.0$ & $<1.0$ & $<1.0$ & $<1.0$ & $<1.0$ \\
\hline Cis-1,3-Dichloropropene & 1.0 & $<5.0$ & $<1.0$ & $<1.0$ & $<1.0$ & $<1.0$ \\
\hline Trans-1,3-Dichloropropene & 1.0 & $<5.0$ & $<1.0$ & $<1.0$ & $<1.0$ & $<1.0$ \\
\hline Methylene Chloride & 1.0 & $<5.0$ & $<1.0$ & $<1.0$ & $<1.0$ & $<1.0$ \\
\hline 1,1,2,2-Tetrachloroethane & 1.0 & $<5.0$ & $<1.0$ & $<1.0$ & $<1.0$ & $<1.0$ \\
\hline Tetrachloroethene & 1.0 & $<5.0$ & $<1.0$ & $<1.0$ & $<1.0$ & $<1.0$ \\
\hline 1,1,1-Trichloroethane & 1.0 & 316 & 140 & 69 & 21 & 11 \\
\hline 1,1,2-Trichloroethane & 1.0 & $<5.0$ & $<1.0$ & $<1.0$ & $<1.0$ & $<1.0$ \\
\hline Trichloroethene & 1.0 & $<5.0$ & $<1.0$ & $<1.0$ & $<1.0$ & $<1.0$ \\
\hline Trichlorofluoromethane & 1.0 & 326 & 141 & 118 & 77 & 59 \\
\hline Vinyl Chloride & 1.0 & 30 & 21 & 18 & 10 & 6 \\
\hline
\end{tabular}

Comments:

5:1 Dilution was made on this sample.

Reviewed By: 


\section{ANALYTICAL REPORT}

State of Arizona License $\# 0009$

$\begin{array}{llll}\text { Client: } & \begin{array}{l}\text { Peroxidation Systems, Inc. } \\ \text { 5151 E. Broadway, Suite 600 }\end{array} & \text { Sample Type: } & \text { Groundwater } \\ & \text { Tucson, Arizona 85711 } & \text { Date Sampled: } & 9 / 1 / 93 \\ \text { Project Name: } & \text { WJSA/DOE } & \text { Date Received: } & 9 / 2 / 93 \\ \text { Project Number: } & 345 & \text { Date Analyzed: } & 9 / 13 / 93 \\ \text { Sample ID: } & 090293-16-20 & \text { Analyzed By: } & \text { Deb Cartmell }\end{array}$

EPA Method 602 - Purgeable Aromatics (G.C. - PID)

Reported in $\mu \mathrm{g} / \mathrm{L}(\mathrm{ppb})$

\begin{tabular}{||l|c|c|c|c|c|c||}
\hline \multicolumn{1}{|c|}{ Compounds } & $\begin{array}{c}\text { Limit of } \\
\text { Detection }\end{array}$ & $6-0^{\circ}$ & $6-.5$ & $6-1$ & $6-2$ & $6-3$ \\
\hline Benzene & 1.0 & 29 & $<1.0$ & $<1.0$ & $<1.0$ & $<1.0$ \\
Toluene & 1.0 & 62 & 2.0 & 1.0 & 1.0 & $<1.0$ \\
Chlorobenzene & 1.0 & $<5.0$ & $<1.0$ & $<1.0$ & $<1.0$ & $<1.0$ \\
Ethyl Benzene & 1.0 & 38 & 1.0 & $<1.0$ & 1.0 & $<1.0$ \\
m,p-Xylene & 1.0 & 39 & 1.0 & $<1.0$ & 1.0 & $<1.0$ \\
0-Xylene & 1.0 & 45 & 2.0 & $<1.0$ & 1.0 & $<1.0$ \\
1,2-Dichlorobenzene & 1.0 & $<5.0$ & $<1.0$ & $<1.0$ & $<1.0$ & $<1.0$ \\
1,3-Dichlorobenzene & 1.0 & $<1.0$ & $<1.0$ & $<1.0$ & $<1.0$ & $<1.0$ \\
1,4-Dichlorobenzene & 1.0 & $<1.0$ & $<1.0$ & $<1.0$ & $<1.0$ & $<1.0$ \\
\hline
\end{tabular}

Comments:

5:1 Dilution was made on this sample.

Reviewed By:

Tamlleterindy

Date

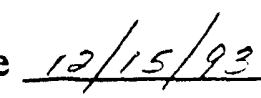


2595 E. Alport Drlve Tucson. Arizano 85706 602-573-6565 FAx 602-573-6550 1-800-P5I-LABS ANALYTICAL REPORT

State of Arizona License $\# 0009$

Client:

Project Name: $\quad$ WJSA/DOE

Project Number: $\quad 345$

Sample ID:
Peroxidation Systems, Inc.

5151 E. Broadway, Suite 600

Tucson, Arizona 85711

090293-21- 25
Sample Type: Groundwater

Date Sampled: $\quad 9 / 1 / 93$

Date Received: $\quad 9 / 2 / 93$

Date Analyzed: 9/13/93

Analyzed By: Deb Cartmell

EPA Method 601 - Purgeable Halocarbons

Reported in $\mu \mathrm{g} / \mathrm{L}(\mathrm{ppb})$

\begin{tabular}{|c|c|c|c|c|c|c|}
\hline Compounds & $\begin{array}{l}\text { Limit of } \\
\text { Detection }\end{array}$ & $7-0^{\circ}$ & $7-.5$ & $7-1$ & $7-2$ & $7-3$ \\
\hline Bromodicloromethane & 1.0 & $<5.0$ & $<1.0$ & $<1.0$ & $<1.0$ & $<1.0$ \\
\hline Bromoform & 1.0 & $<5.0$ & $<1.0$ & $<1.0$ & $<1.0$ & $<1.0$ \\
\hline Bromomethane & 1.0 & $<5.0$ & $<1.0$ & $<1.0$ & $<1.0$ & $<1.0$ \\
\hline Carbon Tetrachloride & 1.0 & $<5.0$ & $<1.0$ & $<1.0$ & $<1.0$ & $<1.0$ \\
\hline Chlorobenzene & 1.0 & $<5.0$ & $<1.0$ & $<1.0$ & $<1.0$ & $<1.0$ \\
\hline Chloroethane & 1.0 & 267 & 264 & 228 & 146 & 85 \\
\hline Chloroform & 1.0 & $<5.0$ & $<1.0$ & $<1.0$ & $<1.0$ & $<1.0$ \\
\hline Chloromethane & 1.0 & $<5.0$ & $<1.0$ & $<1.0$ & $<1.0$ & $<1.0$ \\
\hline 2-Chloroethyl vinyl ether & 1.0 & $<5.0$ & $<1.0$ & $<1.0$ & $<1.0$ & $<1.0$ \\
\hline Dibromochloromethane & 1.0 & $<5.0$ & $<1.0$ & $<1.0$ & $<1.0$ & $<1.0$ \\
\hline 1,2-Dichlorobenzene & 1.0 & $<5.0$ & $<1.0$ & $<1.0$ & $<1.0$ & $<1.0$ \\
\hline 1,3-Dichlorobenzene & 1.0 & $<5.0$ & $<1.0$ & $<1.0$ & $<1.0$ & $<1.0$ \\
\hline 1,4-Dichlorobenzene & 1.0 & $<5.0$ & $<1.0$ & $<1.0$ & $<1.0$ & $<1.0$ \\
\hline 1,1-Dichloroethane & 1.0 & 309 & 72 & 12 & 3.0 & 2.0 \\
\hline 1,2-Dichloroethane & 1.0 & $<5.0$ & $<1.0$ & $<1.0$ & $<1.0$ & $<1.0$ \\
\hline 1,1-Dichloroethene & 1.0 & $<5.0$ & 1.0 & $<1.0$ & $<1.0$ & $<1.0$ \\
\hline Trans-1,2-Dichloroethene & 1.0 & $<5.0$ & $<1.0$ & $<1.0$ & $<1.0$ & $<1.0$ \\
\hline 1,2-Dichloropropane & 1.0 & $<5.0$ & $<1.0$ & $<1.0$ & $<1.0$ & $<1.0$ \\
\hline Cis-1,3-Dichloropropene & 1.0 & $<5.0$ & $<1.0$ & $<1.0$ & $<1.0$ & $<1.0$ \\
\hline Trans-1,3-Dichloropropene & 1.0 & $<5.0$ & $<1.0$ & $<1.0$ & $<1.0$ & $<1.0$ \\
\hline Methylene Chloride & 1.0 & $<5.0$ & $<1.0$ & $<1.0$ & $<1.0$ & $<1.0$ \\
\hline 1,1,2,2-Tetrachloroethane & 1.0 & $<5.0$ & $<1.0$ & $<1.0$ & $<1.0$ & $<1.0$ \\
\hline Tetrachloroethene & 1.0 & $<5.0$ & $<1.0$ & $<1.0$ & $<1.0$ & $<1.0$. \\
\hline 1,1,1-Trichloroethane & 1.0 & 251 & 117 & 52 & 16 & 9 \\
\hline 1,1,2-Trichloroethane & 1.0 & $<5.0$ & $<1.0$ & $<1.0$ & $<1.0$ & $<1.0$ \\
\hline Trichloroethene & 1.0 & $<5.0$ & $<1.0$ & $<1.0$ & $<1.0$ & $<1.0$ \\
\hline Trichlorofluoromethane & 1.0 & 300 & 140 & 115 & 72 & 61 \\
\hline Vinyl Chloride & 1.0 & 17 & 20 & 16 & 9 & 5 \\
\hline
\end{tabular}

Comments:

5:1 Dilution was made on influent sample.

Reviewed By: Tarelle Ferinedy

Date 10/15/93 
ANALYTICAL REPORT

State of Arizona License $\$ 0009$

$\begin{array}{llll}\text { Client: } & \begin{array}{l}\text { Peroxidation Systems, Inc. } \\ \text { 5151 E. Broadway, Suite 600 } \\ \text { Tucson, Arizona 85711 }\end{array} & \text { Sample Type: } & \text { Groundwater } \\ & & \text { Date Sampled: } & 9 / 1 / 93 \\ \text { Project Name: } & \text { WJSA/DOE } & \text { Date Received: } & 9 / 2 / 93 \\ \text { Project Number: } & 345 & \text { Date Analyzed: } & 9 / 13 / 93 \\ \text { Sample ID: } & 090293-21-25 & \text { Analyzed By: } & \text { Deb Cartmell }\end{array}$

EPA Method 602 - Purgeable Aromatics (G.C. - PID)

Reported in $\mu \mathrm{g} / \mathrm{L}$ (ppb)

\begin{tabular}{||l|c|c|c|c|c|c||}
\hline \multicolumn{1}{|c|}{ Compounds } & $\begin{array}{c}\text { Limit of } \\
\text { Detection }\end{array}$ & $7-0^{*}$ & $7-.5$ & $7-1$ & $7-2$ & $7-3$ \\
\hline Benzene & 1.0 & $<5.0$ & $<1.0$ & $<1.0$ & $<1.0$ & $<1.0$ \\
Toluene & 1.0 & $<5.0$ & 1.0 & 1.0 & 1.0 & 1.0 \\
Chlorobenzene & 1.0 & $<5.0$ & $<1.0$ & $<1.0$ & $<1.0$ & $<1.0$ \\
Ethyl Benzene & 1.0 & $<5.0$ & 1.0 & $<1.0$ & 1.0 & 1.0 \\
m,p-Xylene & 1.0 & $<5.0$ & $<1.0$ & $<1.0$ & $<1.0$ & $<1.0$ \\
o-Xylene & 1.0 & $<5.0$ & 1.0 & $<1.0$ & 1.0 & 1.0 \\
1,2-Dichlorobenzene & 1.0 & $<5.0$ & $<1.0$ & $<1.0$ & $<1.0$ & $<1.0$ \\
1,3-Dichlorobenzene & 1.0 & $<5.0$ & $<1.0$ & $<1.0$ & $<1.0$ & $<1.0$ \\
1,4-Dichlorobenzene & 1.0 & $<5.0$ & $<1.0$ & $<1.0$ & $<1.0$ & $<1.0$ \\
\hline
\end{tabular}

Comments:

"5:1 Dilution was made on influent sample.

Reviewed By: Therlle ferindy 


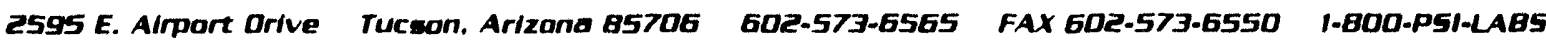
ANALYTICAL REPORT

State of Arizona License \#0009

Client: $\quad$ Peroxidation Systems, Inc. 5151 E. Broadway, Suite 600

Tucson, Arizona 85711

Project Name: $\quad$ WJSA/DOE

Project Number: 345

Sample ID: NA
Sample Type: Groundwater

Date Sampled: $\quad 9 / 02 / 93$

Date Received: 9/07/93

Date Ans.lyzed: 9/23/93

Analyzed By: Deb Cartmell

EPA Method 601 - Purgeable Halocarbons

Reported in $\mu \mathrm{g} / \mathrm{L}$ (ppb)

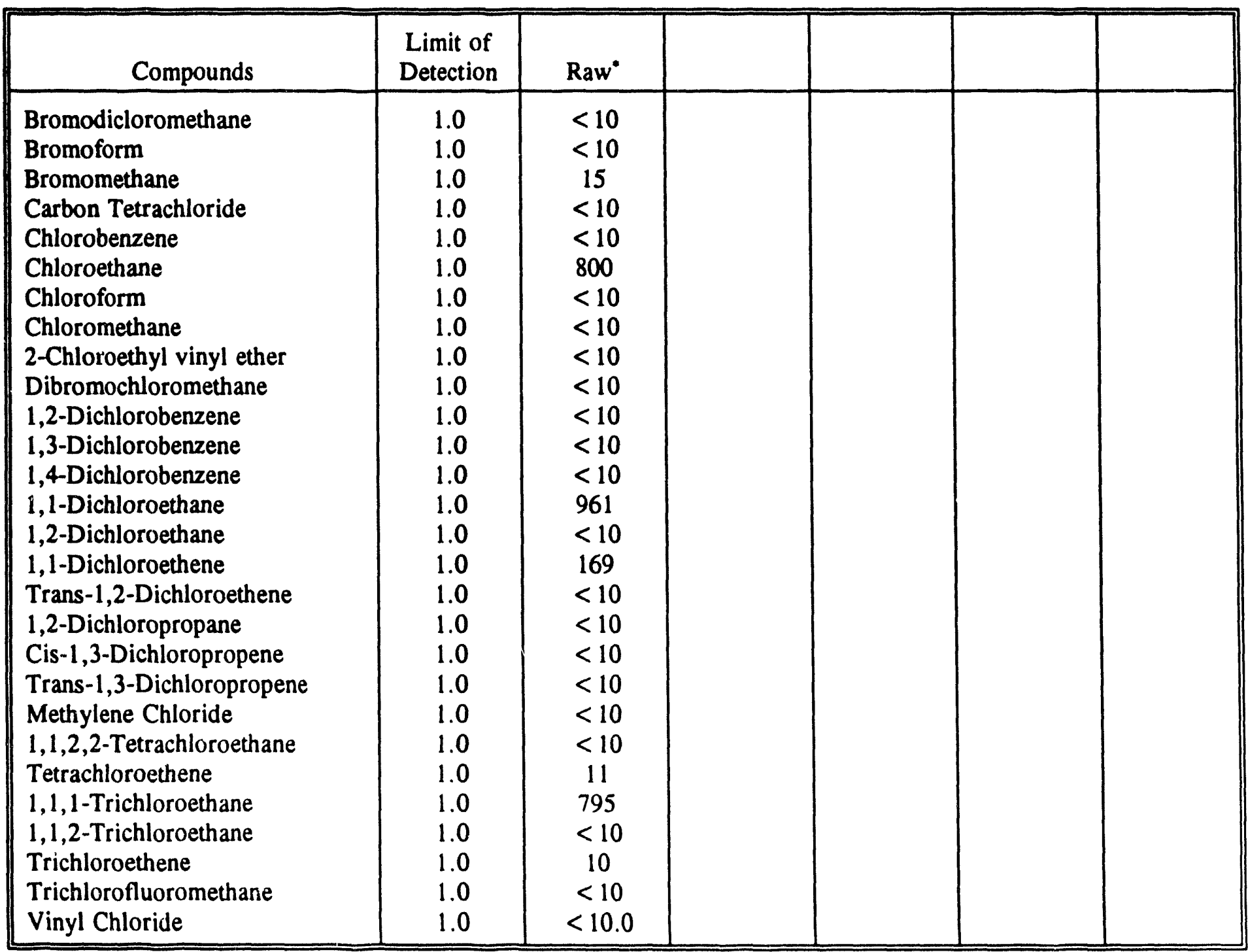

Ci 1 ments:

'0:1 Dilution was made on this sample.

Reviewed By: Tarcile Serinedy 


\section{ANALYTICAL REPORT}

State of Arizona License $\# 0009$

Client:

Peroxidation Systems, Inc. 5151 E. Broadway, Suite 600

Tucson, Arizona 85711

Project Name: WJSA/DOE

Project Number: $\quad 345$

Sample ID: NA
Sample Type: Groundwater

Date Sampled: $\quad 9 / 02 / 93$

Date Received: 9/07/93

Date Analyzed: 9/23/93

Analyzed By: Deb Cartmell

EPA Method 602 - Purgeable Aromatics (G.C. - PID)

Reported in $\mu \mathrm{g} / \mathrm{L}$ (ppb)

\begin{tabular}{||l|c|c|c|c|c|c|}
\hline \multicolumn{1}{|c|}{ Compounds } & $\begin{array}{c}\text { Limit of } \\
\text { Detection }\end{array}$ & Raw & & & & \\
\hline Benzene & 1.0 & 137 & & & & \\
Toluene & 1.0 & 129 & & & & \\
Chlorobenzene & 1.0 & $<10.0$ & & & & \\
Ethyl Benzene & 1.0 & $<10.0$ & & & & \\
m,p-Xylene & 1.0 & 344 & & & & \\
0-Xylene & 1.0 & 145 & & & & \\
1,2-Dichlorobenzene & 1.0 & $<10.0$ & & & & \\
1,3-Dichlorobenzene & 1.0 & $<10.0$ & & & & \\
1,4-Dichlorobenzene & 1.0 & 55 & & & \\
\hline
\end{tabular}

Comments:

10:1 Dilution was made on this sample.

Reviewed By:

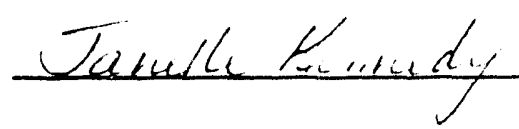

Date

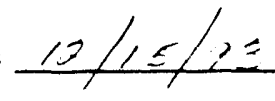


Client:

Project Name:

WJSA/DOE

Project Number: $\quad 345$

Sample ID: $\quad$ 090393-14-18
Sample Type: Groundwater.

Date Sampled: $\quad 9 / 2 / 93$

Date Received: $\quad 9 / 3 / 93$

Date Analyzed: 9/13/93

Analyzed By: Deb Cartmell

\section{EPA Method 601 - Purgeable Halocarbons \\ Reported in $\mu \mathrm{g} / \mathrm{L}$ (ppb)}

\begin{tabular}{|c|c|c|c|c|c|c|}
\hline Compounds & $\begin{array}{l}\text { Limit of } \\
\text { Detection }\end{array}$ & $8-0^{\circ}$ & $8-.5$ & $8-1$ & $8-2$ & $8-3$ \\
\hline Bromodicloromethane & 1.0 & $<5.0$ & $<1.0$ & $<1.0$ & $<1.0$ & $<1.0$ \\
\hline Bromoform & 1.0 & $<5.0$ & $<1.0$ & $<1.0$ & $<1.0$ & $<1.0$ \\
\hline Bromomethane & 1.0 & $<5.0$ & $<1.0$ & $<1.0$ & $<1.0$ & $<1.0$ \\
\hline Carbon Tetrachloride & 1.0 & $<5.0$ & $<1.0$ & $<1.0$ & $<1.0$ & $<1.0$ \\
\hline Chlorobenzene & 1.0 & $<5.0$ & $<1.0$ & $<1.0$ & $<1.0$ & $<1.0$ \\
\hline Chloroethane & 1.0 & 448 & 351 & 321 & 232 & 163 \\
\hline Chloroform & 1.0 & $<5.0$ & $<1.0$ & $<1.0$ & $<1.0$ & $<1.0$ \\
\hline Chloromethane & 1.0 & $<5.0$ & $<1.0$ & $<1.0$ & $<1.0$ & $<1.0$ \\
\hline 2-Chloroethyl vinyl ether & 1.0 & $<5.0$ & $<1.0$ & $<1.0$ & $<1.0$ & $<1.0$ \\
\hline Dibromochloromethane & 1.0 & $<5.0$ & $<1.0$ & $<1.0$ & $<1.0$ & $<1.0$ \\
\hline 1,2-Dichlorobenzene & 1.0 & $<5.0$ & $<1.0$ & $<1.0$ & $<1.0$ & $<1.0$ \\
\hline 1,3-Dichlorobenzene & 1.0 & $<5.0$ & $<1.0$ & $<1.0$ & $<1.0$ & $<1.0$ \\
\hline 1,4-Dichlorobenzene & 1.0 & $<5.0$ & $<1.0$ & $<1.0$ & $<1.0$ & $<1.0$ \\
\hline 1,1-Dichloroethane & 1.0 & 555 & 124 & 31 & 4 & 2 \\
\hline 1,2-Dichloroethane & 1.0 & $<5.0$ & $<1.0$ & $<1.0$ & $<1.0$ & $<1.0$ \\
\hline 1,1-Dichloroethene & 1.0 & 70 & 2 & 1 & $<1.0$ & $<1.0$ \\
\hline Trans-1,2-Dichloroethene & 1.0 & $<5.0$ & $<1.0$ & $<1.0$ & $<1.0$ & $<1.0$ \\
\hline 1,2-Dichloropropane & 1.0 & $<5.0$ & $<1.0$ & $<1.0$ & $<1.0$ & $<1.0$ \\
\hline Cis-1,3-Dichloropropene & 1.0 & $<5.0$ & $<1.0$ & $<1.0$ & $<1.0$ & $<1.0$ \\
\hline Trans-1,3-Dichloropropene & 1.0 & $<5.0$ & $<1.0$ & $<1.0$ & $<1.0$ & $<1.0$ \\
\hline Methylene Chloride & 1.0 & $<5.0$ & $<1.0$ & $<1.0$ & $<1.0$ & $<1.0$ \\
\hline 1,1,2,2-Tetrachloroethane & 1.0 & $<5.0$ & $<1.0$ & $<1.0$ & $<1.0$ & $<1.0$ \\
\hline Tetrachloroethene & 1.0 & $<5.0$ & $<1.0$ & $<1.0$ & $<1.0$ & $<1.0$ \\
\hline 1,1,1-Trichloroethane & 1.0 & 395 & 184 & 127 & 45 & 20 \\
\hline 1,1,2-Trichloroethane & 1.0 & $<5.0$ & $<1.0$ & $<1.0$ & $<1.0$ & $<1.0$ \\
\hline Trichloroethene & 1.0 & $<5.0$ & $<1.0$ & $<1.0$ & $<1.0$ & $<1.2$ \\
\hline Trichlorofluoromethane & 1.0 & 215 & 173 & 148 & 67 & 30 \\
\hline Vinyl Chloride & 1.0 & 38 & 26 & 24 & 16 & 10 \\
\hline
\end{tabular}

\section{Comments:}


ANALYTICAL REPORT

State of Arizona License "0009

Client: $\quad$ Peroxidation Systems, Inc.

5151 E. Broadway, Suite 600

Tucson, Arizona 85711

Project Name: $\quad$ WJSA/DOE

Project Number: $\quad 345$

Sample ID:
$090393-14-18$
Sample Type: Groundwater

Date Sampled: $\quad 9 / 2 / 93$

Date Received: $\quad 9 / 3 / 93$

Date Analyzed: $\quad 9 / 13 / 93$

Analyzed By: Deb Cartmell

EPA Method 602 - Purgeable Aromatics (G.C. - PID)

Reported in $\mu \mathrm{g} / \mathrm{L}$ (ppb)

\begin{tabular}{|l|c|c|c|c|c|c|}
\hline \multicolumn{1}{|c|}{ Compounds } & $\begin{array}{c}\text { Limit of } \\
\text { Detection }\end{array}$ & $8.0^{\circ}$ & $8-.5$ & $8-1$ & $8-2$ & $8-3$ \\
\hline Benzene & 1.0 & 56 & 2 & $<1.0$ & $<1.0$ & $<1.0$ \\
Toluene & 1.0 & 59 & 2 & $<1.0$ & $<1.0$ & $<1.0$ \\
Chlorobenzene & 1.0 & $<5.0$ & 1 & $<1.0$ & $<1.0$ & $<1.0$ \\
Ethyl Benzene & 1.0 & 28 & 1 & $<1.0$ & $<1.0$ & $<1.0$ \\
m,p-Xylene & 1.0 & 55 & 2 & $<1.0$ & $<1.0$ & $<1.0$ \\
0-Xylene & 1.0 & 47 & 2 & $<1.0$ & $<1.0$ & $<1.0$ \\
1,2-Dichlorobenzene & 1.0 & $<5.0$ & $<1.0$ & $<1.0$ & $<1.0$ & $<1.0$ \\
1,3-Dichlorobenzene & 1.0 & $<5.0$ & $<1.0$ & $<1.0$ & $<1.0$ & $<1.0$ \\
1,4-Dichlorobenzene & 1.0 & $<5.0$ & $<1.0$ & $<1.0$ & $<1.0$ & $<1.0$ \\
\hline
\end{tabular}

Comments:

-5:1 Dilution was made on sample.

Reviewed By:

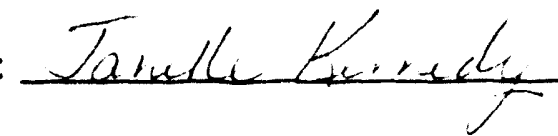

Date

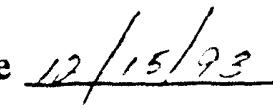


Client:

Peroxidation Systems, Inc.

5151 E. Broadway, Suite 600

Tucson, Arizona 85711

Project Name: WJSA/DOE

Project Number: $\quad 345$

Sample ID: $\quad$ 090793-01-05
Sample Type: Groundwater.

Date Sampled: $\quad 9 / 3 / 93$

Date Received: 9/4/93

Date Analyzed: 9/13/93

Analyzed By: Deb Cartmell

EPA Method 601 - Purgeable Halocarbons

Reported in $\mu \mathrm{g} / \mathrm{L}(\mathrm{ppb})$

\begin{tabular}{|c|c|c|c|c|c|c|}
\hline Compounds & $\begin{array}{l}\text { Limit of } \\
\text { Detection }\end{array}$ & $9-0^{\circ}$ & $9-.5$ & $9-1$ & $9-2$ & $9-3$ \\
\hline Bromodicloromethane & 1.0 & $<5.0$ & $<1.0$ & $<1.0$ & $<1.0$ & $<1.0$ \\
\hline Bromoform & 1.0 & $<5.0$ & $<1.0$ & $<1.0$ & $<1.0$ & $<1.0$ \\
\hline Bromomethane & 1.0 & $<5.0$ & $<1.0$ & $<1.0$ & $<1.0$ & $<1.0$ \\
\hline Carbon Tetrachloride & 1.0 & $<5.0$ & $<1.0$ & $<1.0$ & $<1.0$ & $<1.0$ \\
\hline Chlorobenzene & 1.0 & $<5.0$ & $<1.0$ & $<1.0$ & $<1.0$ & $<1.0$ \\
\hline Chloroethane & 1.0 & 274 & 217 & 172 & 116 & 79 \\
\hline ChInroform & 1.0 & $<5.0$ & $<1.0$ & $<1.0$ & $<1.0$ & $<1.0$ \\
\hline Chloromethane & 1.0 & $<5.0$ & $<1.0$ & $<1.0$ & $<1.0$ & $<1.0$ \\
\hline 2-Chloroethyl vinyl ether & 1.0 & $<5.0$ & $<1.0$ & $<1.0$ & $<1.0$ & $<1.0$ \\
\hline Dibromochloromethane & 1.0 & $<5.0$ & $<1.0$ & $<1.0$ & $<1.0$ & $<1.0$ \\
\hline 1,2-Dichlorobenzene & 1.0 & $<5.0$ & $<1.0$ & $<1.0$ & $<1.0$ & $<1.0$ \\
\hline 1,3-Dichlorobenzene & 1.0 & $<5.0$ & $<1.0$ & $<1.0$ & $<1.0$ & $<1.0$ \\
\hline 1,4-Dichlorobenzene & 1.0 & $<5.0$ & $<1.0$ & $<1.0$ & $<1.0$ & $<1.0$ \\
\hline 1,1-Dichloroethane & 1.0 & 418 & 183 & 105 & 33 & 9 \\
\hline 1,2-Dichloroethane & 1.0 & 5 & 3 & $=$ & $<1.0$ & $<1.0$ \\
\hline 1,1-Dichloroethene & 1.0 & 15 & $<1.0$ & $<1.0$ & $<1.0$ & $<1.0$ \\
\hline Trans-1,2-Dichloroethene & 1.0 & $<5.0$ & $<1.0$ & $<1.0$ & $<1.0$ & $<1.0$ \\
\hline 1,2-Dichloropropane & 1.0 & $<5.0$ & $<1.0$ & $<1.0$ & $<1.0$ & $<1.0$ \\
\hline Cis-1,3-Dichloropropene & 1.0 & $<5.0$ & $<1.0$ & $<1.0$ & $<1.0$ & $<1.0$ \\
\hline Trans-1,3-Dichloropropene & 1.0 & $<5.0$ & $<1.0$ & $<1.0$ & $<1.0$ & $<1.0$ \\
\hline Methylene Chloride & 1.0 & $<5.0$ & $<1.0$ & $<1.0$ & $<1.0$ & $<1.0$ \\
\hline 1,1,2,2-Tetrachloroethane & 1.0 & $<5.0$ & $<1.0$ & $<1.0$ & $<1.0$ & $<1.0$ \\
\hline Tetrachloroethene & 1.0 & $<5.0$ & $<1.0$ & $<1.0$ & $<1.0$ & $<1.0$ \\
\hline 1,1,1-Trichloroethane & 1.0 & 263 & 187 & 156 & 102 & 60 \\
\hline 1,1,2-Trichloroethane & 1.0 & $<5.0$ & $<1.0$ & $<1.0$ & $<1.0$ & $<1.0$ \\
\hline Trichloroethene & 1.0 & $<5.0$ & $<1.0$ & $<1.0$ & $<1.0$ & $<1.0$ \\
\hline Trichlorofluoromethane & 1.0 & 134 & 52 & 32 & 18 & 16 \\
\hline Vinyl Chloride & 1.0 & 17 & 16 & 13 & 9 & 6 \\
\hline
\end{tabular}

Comments:

5:1 Dilution was made on influent sample due to the high levels of contaminants.

Reviewed By: Turulle fernedy Date $12 / 15 / 93$ 
ANALYTICAL REPORT

State of Arizona License $\# 0009$

$\begin{array}{llll}\text { Client: } & \begin{array}{l}\text { Peroxidation Systems, Inc. } \\ \text { 5151 E. Broadway, Suite 600 }\end{array} & \text { Sample Type: } & \text { Groundwater } \\ & \text { Tucson, Arizona 85711 } & \text { Date Sampled: } & 9 / 3 / 93 \\ \text { Project Name: } & \text { WJSA/DOE } & \text { Date Received: } & 9 / 4 / 93 \\ \text { Project N. mber: } & 345 & \text { Date Analyzed: } & 9 / 13 / 93 \\ \text { Sample ID: } & 090793-01-05 & \text { Analyzed By: } & \text { Deb Cartmell }\end{array}$

EPA Method 602 - Purgeable Aromatics (G.C. - PID)

Reported in $\mu \mathrm{g} / \mathrm{L}(\mathrm{ppb})$

\begin{tabular}{|l|c|c|c|c|c|c||}
\hline \multicolumn{1}{|c|}{ Compounds } & $\begin{array}{c}\text { Limit of } \\
\text { Detection }\end{array}$ & $9-0^{*}$ & $9-.5$ & $9-1$ & $9-2$ & $9-3$ \\
\hline Benzene & 1.0 & 10 & $<1.0$ & $<1.0$ & $<1.0$ & $<1.0$ \\
Toluene & 1.0 & 9 & $<1.0$ & $<1.0$ & $<1.0$ & $<1.0$ \\
Chlorobenzene & 1.0 & $<5.0$ & $<1.0$ & $<1.0$ & $<1.0$ & $<1.0$ \\
Ethyl Benzene & 1.0 & $<5.0$ & $<1.0$ & $<1.0$ & $<1.0$ & $<1.0$ \\
m,p-Xylene & 1.0 & 8 & $<1.0$ & $<1.0$ & $<1.0$ & $<1.0$ \\
o-Xylene & 1.0 & 13 & $<1.0$ & $<1.0$ & $<1.0$ & $<1.0$ \\
1,2-Dichlorobenzene & 1.0 & $<5.0$ & $<1.0$ & $<1.0$ & $<1.0$ & $<1.0$ \\
1,3-Dichlorobenzene & 1.0 & $<5.0$ & $<1.0$ & $<1.0$ & $<1.0$ & $<1.0$ \\
1,4-Dichlorobenzene & 1.0 & $<5.0$ & $<1.0$ & $<1.0$ & $<1.0$ & $<1.0$ \\
\hline
\end{tabular}

Comments:

5:1 Dilution was made on influent sample.

Reviewed By:

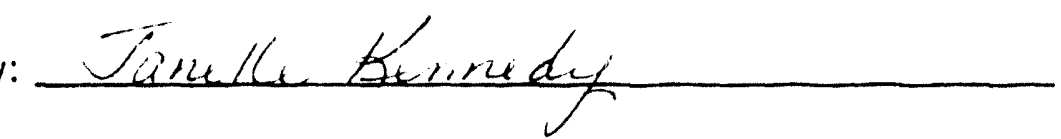

Date 

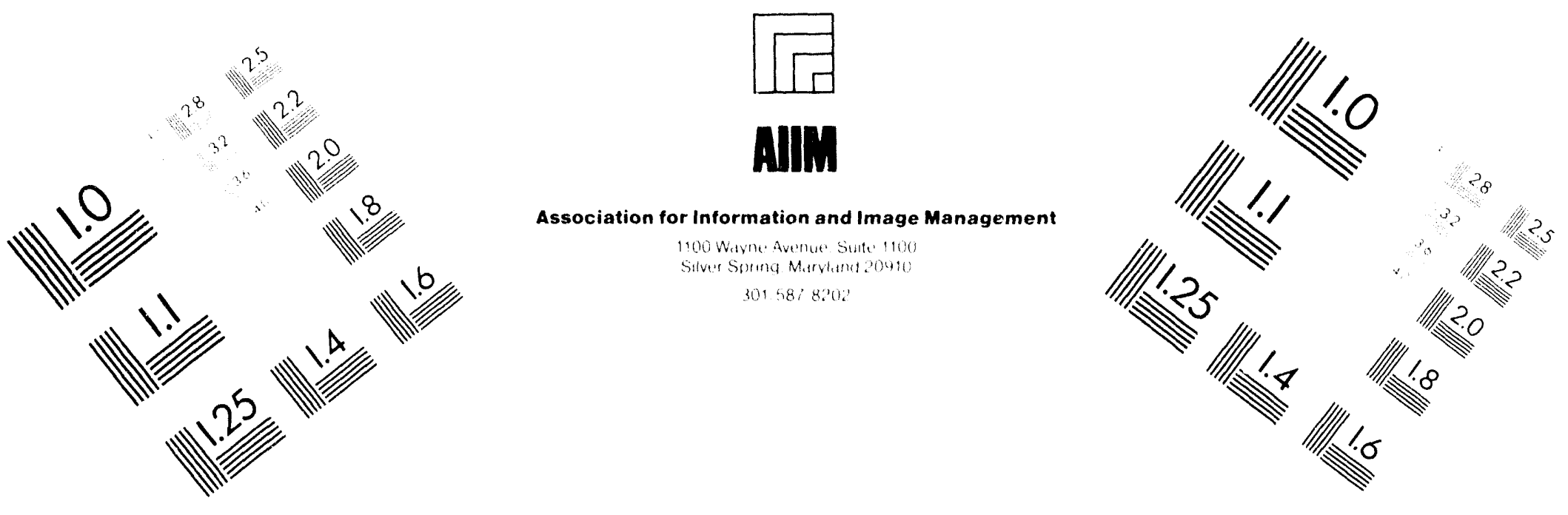

Centimeter

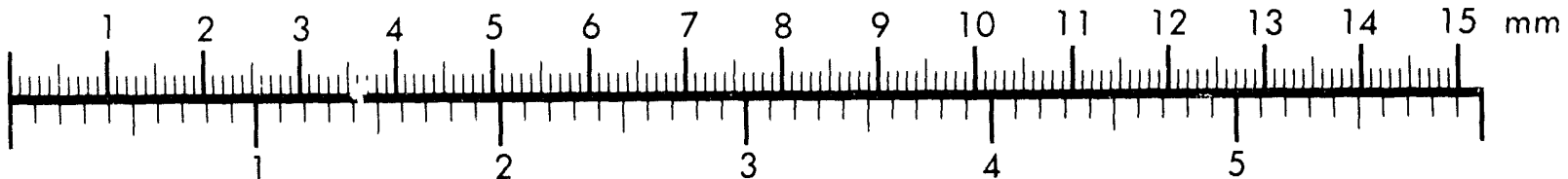

Inches
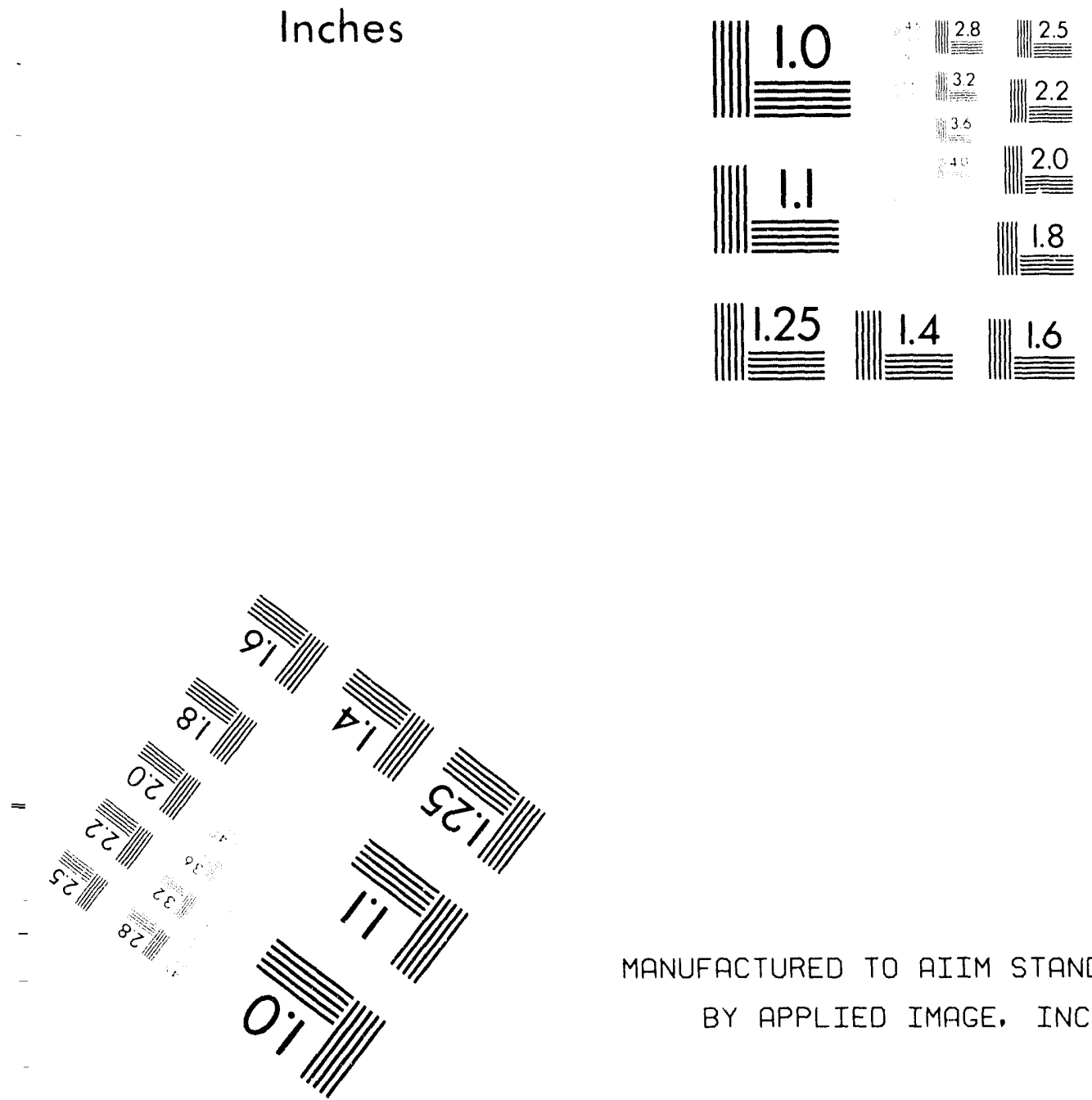

MANUFACTURED TO AIIM STANDARDS

BY APPLIED IMAGE. INC.

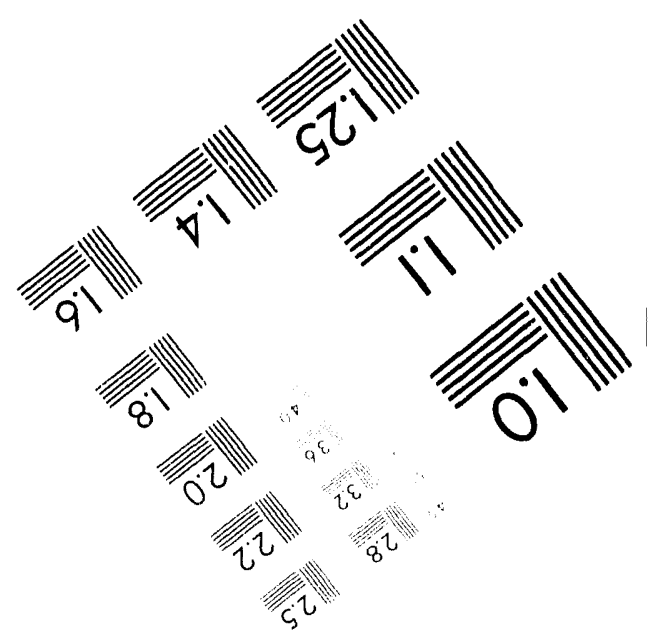



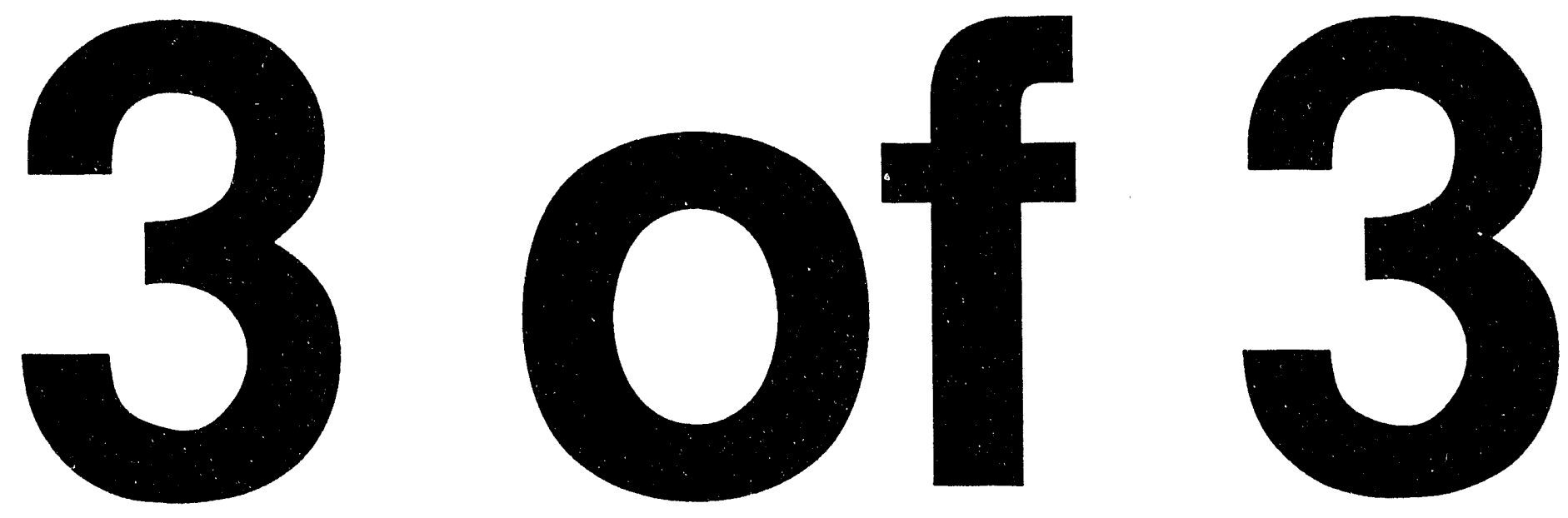


\section{PSI Enviranmental Laboratories}

2595 E. Airport Drive Tucson. Arizona B5706 602-573-6565 FAX 602-573-6550 1-800-P5I-LABS

ANALYTICAL REPORT

State of Arizona License $\# 0009$

Client: $\quad$ Peroxidation Systems, Inc.

5151 E. Broadway, Suite 600

Tucson, Arizona 85711

Project Name: $\quad$ WJSA/DOE

Project Number: $\quad 345$

Sample ID: $\quad$ 090793-16, 8, 9
Sample Type: Groundwater

Date Sampled: $\quad 9 / 3 / 93$

Date Received: $\quad 9 / 4 / 93$

Date Analyzed: 9/14/93

Analyzed By: Deb Cartmell

EPA Method 601 - Purgeable Halocarbons

Reported in $\mu \mathrm{g} / \mathrm{L}$ (ppb)

\begin{tabular}{|c|c|c|c|c|}
\hline Compounds & $\begin{array}{l}\text { Limit of } \\
\text { Detection }\end{array}$ & 11-L6 & $10-1$ & $10-2$ \\
\hline Bromodicloromethane & 1.0 & $<1.0$ & $<1.0$ & $<1.0$ \\
\hline Bromoform & 1.0 & $<1.0$ & $<1.0$ & $<1.0$ \\
\hline Bromomethane & 1.0 & $<1.0$ & $<1.0$ & $<1.0$ \\
\hline Carbon Tetrachloride & 1.0 & $<1.0$ & $<1.0$ & $<1.0$ \\
\hline Chlorobenzene & 1.0 & $<1.0$ & $<1.0$ & $<1.0$ \\
\hline Chloroethane & 1.0 & 258 & 306 & 221 \\
\hline Chloroform & 1.0 & $<1.0$ & $<1.0$ & $<1.0$ \\
\hline Chloromethane & 1.0 & $<1.0$ & $<1.0$ & $<1.0$ \\
\hline 2-Chloroethyl vinyl ether & 1.0 & $<1.0$ & $<1.0$ & $<1.0$ \\
\hline Dibromochloromethane & 1.0 & $<1.0$ & $<1.0$ & $<1.0$ \\
\hline 1,2-Dichlorobenzene & 1.0 & $<1.0$ & $<1.0$ & $<1.0$ \\
\hline 1,3-Dichlorobenzene & 1.0 & $<1.0$ & $<1.0$ & $<1.0$ \\
\hline 1,4-Dichlorobenzene & 1.0 & $<1.0$ & $<1.0$ & $<1.0$ \\
\hline 1,1-Dichloroethane & 1.0 & 28 & 18 & 3 \\
\hline 1,2-Dichloroethane & 1.0 & $<1.0$ & $<1.0$ & $<1.0$ \\
\hline 1,1-Dichloroethene & 1.0 & $<1.0$ & $<1.0$ & $<1.0$ \\
\hline Trans-1,2-Dichloroethene & 1.0 & $<1.0$ & $<1.0$ & $<1.0$ \\
\hline 1,2-Dichloropropane & 1.0 & $<1.0$ & $<1.0$ & $<1.0$ \\
\hline Cis-1,3-Dichloropropene & 1.0 & $<1.0$ & $<1.0$ & $<1.0$ \\
\hline Trans-1,3-Dichloropropene & 1.0 & $<1.0$ & $<1.0$ & $<1.0$ \\
\hline Methylene Chloride & 1.0 & $<1.0$ & $<1.0$ & $<1.0$ \\
\hline 1,1,2,2-Tetrachloroethane & 1.0 & $<1.0$ & $<1.0$ & $<1.0$ \\
\hline Tetrachloroethene & 1.0 & $<1.0$ & $<1.0$ & $<1.0$ \\
\hline 1,1,1-Trichloroethane & 1.0 & 66 & 83 & 19 \\
\hline 1,1,2-Trichloroethane & 1.0 & $<1.0$ & $<1.0$ & $<1.0$ \\
\hline Trichloroethene & 1.0 & $<1.0$ & $<1.0$ & $<1.0$ \\
\hline Trichlorofluoromethane & 1.0 & 127 & 151 & 98 \\
\hline Vinyl Chloride & 1.0 & $<1.0$ & $<1.0$ & $<1.0$ \\
\hline
\end{tabular}

Comments:

Reviewed By: 


\section{PSI Environmental Laboratories}

2595 E. Airpart Drive Tucson. Arizand 85706 602-573-6565 FAX 602-573-6550 I-800-PSI-LABS

ANALYTICAL REPORT

State of Arizona License \#0009
Client: $\quad$ Peroxidation Systems, Inc. 5151 E. Broadway, Suite 600 Tucson, Arizona 85711

Project Name: WJSA/DOE

Project Number: $\quad 345$

Sample ID: $\quad$ 090793-16, 8, 9
Sample Type: Groundwater

Date Sampled: $\quad 9 / 3 / 93$

Date Received: $\quad 9 / 4 / 93$

Date Analyzed: $9 / 14 / 93$

Analyzed By: Deb Cartmell

EPA Method 602 - Purgeable Aromatics (G.C. - PID)

Reported in $\mu \mathrm{g} / \mathrm{L}(\mathrm{ppb})$

\begin{tabular}{||l|c|c|c|c|c|c|}
\hline \multicolumn{1}{|c|}{ Compounds } & $\begin{array}{c}\text { Limit of } \\
\text { Detection }\end{array}$ & $11-\mathrm{L} 6$ & $10-1$ & $10-2$ & - & \\
\hline Benzene & 1.0 & $<1.0$ & $<1.0$ & $<1.0$ & & \\
Toluene & 1.0 & $<1.0$ & $<1.0$ & $<1.0$ & & \\
Chlorobenzene & 1.0 & $<1.0$ & $<1.0$ & $<1.0$ & & \\
Ethyl Benzene & 1.0 & $<1.0$ & $<1.0$ & $<1.0$ & & \\
m,p-Xylene & 1.0 & $<1.0$ & $<1.0$ & $<1.0$ & & \\
o-Xylene & 1.0 & $<1.0$ & $<1.0$ & $<1.0$ & & \\
1,2-Dichlorobenzene & 1.0 & $<1.0$ & $<1.0$ & $<1.0$ & & \\
1,3-Dichlorobenzene & 1.0 & $<1.0$ & $<1.0$ & $<1.0$ & & \\
1,4-Dichlorobenzene & 1.0 & $<1.0$ & $<1.0$ & $<1.0$ & & \\
\hline
\end{tabular}

Comments:

- Rest of Test 10 vials were broken.

Reviewed By: Larulle Fnnedy

Date

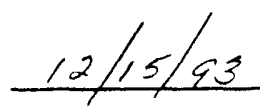


2595 E. Airpart Drive Tucsan. Arizana 85706 602-573-6565 FAX 602-573-6550 1-800-P51-LAB5

\section{ANALYTICAL REPORT}

State of Arizona License $\# 0009$

Client: $\quad$ Peroxidation Systems, Inc. 5151 E. Broadway, Suite 600 Tucson, Arizona 85711

Project Name: WJSA/DOE

Project Number: $\quad 345$

Sample ID:

$090793-11-16$
Sample Type: Groundwater

Date Sampled: $\quad 9 / 3 / 93$

Date Received: 9/4/93

Date Analyzed: $9 / 14 / 93$

Analyzed By: Deb Cartmell

EPA Method 601 - Purgeable Halocarbons

Reported in $\mu \mathrm{g} / \mathrm{L}$ (ppb)

\begin{tabular}{|c|c|c|c|c|c|c|}
\hline Compounds & $\begin{array}{l}\text { Limit of } \\
\text { Detection }\end{array}$ & $11-0^{*}$ & $11-.5$ & $11-1$ & $11-2$ & $11-3$ \\
\hline Bromodicloromethane & 1.0 & $<5.0$ & $<1.0$ & $<1.0$ & $<1.0$ & $<1.0$ \\
\hline Bromoform & 1.0 & $<5.0$ & $<1.0$ & $<1.0$ & $<1.0$ & $<1.0$ \\
\hline Bromomethane & 1.0 & $<5.0$ & $<1.0$ & $<1.0$ & $<1.0$ & $<1.0$ \\
\hline Carbon Tetrachloride & 1.0 & $<5.0$ & $<1.0$ & $<1.0$ & $<1.0$ & $<1.0$ \\
\hline Chlorobenzene & 1.0 & $<5.0$ & $<1.0$ & $<1.0$ & $<1.0$ & $<1.0$ \\
\hline Chloroethane & 1.0 & 535 & 385 & 352 & 286 & 197 \\
\hline Chloroform & 1.0 & $<5.0$ & $<1.0$ & $<1.0$ & $<1.0$ & $<1.0$ \\
\hline Chloromethane & 1.0 & $<5.0$ & $<1.0$ & $<1.0$ & $<1.0$ & $<1.0$ \\
\hline 2-Chloroethyl vinyl ether & 1.0 & $<5.0$ & $<1.0$ & $<1.0$ & $<1.0$ & $<1.0$ \\
\hline Dibromochloromethane & 1.0 & $<5.0$ & $<1.0$ & $<1.0$ & $<1.0$ & $<1.0$ \\
\hline 1,2-Dichlorobenzene & 1.0 & $<5.0$ & $<1.0$ & $<1.0$ & $<1.0$ & $<1.0$ \\
\hline 1,3-Dichlorobenzene & 1.0 & $<5.0$ & $<1.0$ & $<1.0$ & $<1.0$ & $<1.0$ \\
\hline 1,4-Dichlorobenzene & 1.0 & $<5.0$ & $<1.0$ & $<1.0$ & $<1.0$ & $<1.0$ \\
\hline 1,1-Dichloroethane & 1.0 & 640 & 231 & 126 & 10 & 3 \\
\hline 1,2-Dichloroethane & 1.0 & $<5.0$ & $<1.0$ & $<1.0$ & $<1.0$ & $<1.0$ \\
\hline 1,1-Dichloroethene & 1.0 & 48 & $<1.0$ & $<1.0$ & $<1.0$ & $<1.0$ \\
\hline Trans-1,2-Dichloroethene & 1.0 & $<5.0$ & $<1.0$ & $<1.0$ & $<1.0$ & $<1.0$ \\
\hline 1,2-Dichloropropane & 1.0 & $<5.0$ & $<1.0$ & $<1.0$ & $<1.0$ & $<1.0$ \\
\hline Cis-1,3-Dichloropropene & 1.0 & $<5.0$ & $<1.0$ & $<1.0$ & $<1.0$ & $<1.0$ \\
\hline Trans-1,3-Dichloropropene & 1.0 & $<5.0$ & $<1.0$ & $<1.0$ & $<1.0$ & $<1.0$ \\
\hline Methylene Chloride & 1.0 & $<5.0$ & $<1.0$ & $<1.0$ & $<1.0$ & $<1.0$ \\
\hline 1,1,2,2-Tetrachloroethane & 1.0 & $<5.0$ & $<1.0$ & $<1.0$ & $<1.0$ & $<1.0$ \\
\hline Tetrachloroethene & 1.0 & $<5.0$ & $<1.0$ & $<1.0$ & $<1.0$ & $<1.0$ \\
\hline 1,1,1-Trichloroethane & 1.0 & 460 & 190 & 130 & 51 & 16 \\
\hline 1,1,2-Trichloroethane & 1.0 & $<5.0$ & $<1.0$ & $<1.0$ & $<1.0$ & $<1.0$ \\
\hline Trichloroethene & 1.0 & $<5.0$ & $<1.0$ & $<1.0$ & $<1.0$ & $<1.0$ \\
\hline Trichlorofluoromethane & 1.0 & 473 & 191 & 167 & 140 & 100 \\
\hline Vinyl Chloride & 1.0 & 36 & 130 & 168 & 49 & 13 \\
\hline
\end{tabular}

Comments:

5:1 Dilution was made on this influent sample

Reviewed By: 


\section{ANALYTICAL REPORT}

State of Arizona License $\$ 0009$

$\begin{array}{llll}\text { Client: } & \begin{array}{l}\text { Peroxidation Systems, Inc. } \\ \text { 5151 E. Broadway, Suite } 600\end{array} & \text { Sample Type: } & \text { Groundwater } \\ & \text { Tucson, Arizona 85711 } & \text { Date Sampled: } & 9 / 3 / 93 \\ & \text { WJSA/DOE } & \text { Date Received: } & 9 / 4 / 93 \\ \text { Project Name: } & 345 & \text { Date Analyzed: } & 9 / 14 / 93 \\ \text { Project Number: } & 090793-11-16 & \text { Analyzed By: } & \text { Deb Cartmell } \\ \text { Sample ID: } & & & \end{array}$

EPA Method 602 - Purgeable Aromatics (G.C. - PID)

Reported in $\mu \mathrm{g} / \mathrm{L}(\mathrm{ppb})$

\begin{tabular}{||l|c|c|c|c|c|c|}
\hline \multicolumn{1}{|c|}{ Compounds } & $\begin{array}{c}\text { Limit of } \\
\text { Detection }\end{array}$ & $11-0^{\circ}$ & $11-.5$ & $11-1$ & $11-2$ & $11-3$ \\
\hline Benzene & 1.0 & 12 & 2.0 & $<1.0$ & $<1.0$ & $<1.0$ \\
Toluene & 1.0 & 16 & $<1.0$ & $<1.0$ & $<1.0$ & $<1.0$ \\
Chlorobenzene & 1.0 & $<5.0$ & $<1.0$ & $<1.0$ & $<1.0$ & $<1.0$ \\
Ethyl Benzene & 1.0 & 6 & $<1.0$ & $<1.0$ & $<1.0$ & $<1.0$ \\
m,p-Xylene & 1.0 & 8 & $<1.0$ & $<1.0$ & $<1.0$ & $<1.0$ \\
0-Xylene & 1.0 & 12 & $<1.0$ & $<1.0$ & $<1.0$ & $<1.0$ \\
1,2-Dichlorobenzene & 1.0 & $<5.0$ & $<i .0$ & $<1.0$ & $<1.0$ & $<1.0$ \\
1,3-Dichlorobenzene & 1.0 & $<5.0$ & $<1.0$ & $<1.0$ & $<1.0$ & $<1.0$ \\
1,4-Dichlorobenzene & 1.0 & $<5.0$ & $<1.0$ & $<1.0$ & $<1.0$ & $<1.0$ \\
\hline
\end{tabular}

Comments:

5:1 Dilution was made on this sample.

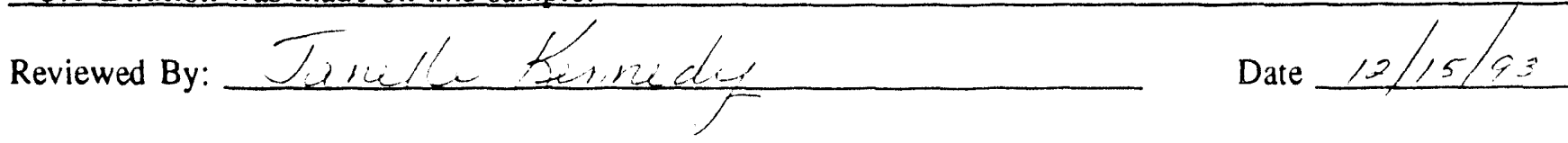


2595 E. Airport Drive Tucson. Arizona 85706 602-573-6565 FAX 602-573-6550 1-8U0-P5I-LABS

\section{ANALYTICAL REPORT}

State of Arizona License $\# 0009$

$\begin{array}{llll}\text { Client: } & \begin{array}{l}\text { Peroxidation Systems, Inc. } \\ \text { 5151 E. Broadway, Suite } 600\end{array} & \text { Sample Type: } & \text { Groundwater } \\ & \text { Tucson, Arizona 85711 } & \text { Date Sampled: } & 9 / 3 / 93 \\ & & & \\ \text { Project Name: } & \text { WJSA/DOE } & \text { Date Received: } & 9 / 4 / 93 \\ \text { Project Number: } & 345 & \text { Date Analyzed: } & 9 / 15 / 93 \\ \text { Sample ID: } & 090793-17-21 & \text { Analyzed By: } & \text { Deb Cartmell }\end{array}$

EPA Method 601 - Purgeable Halocarbons

Reported in $\mu \mathrm{g} / \mathrm{L}$ (ppb)

\begin{tabular}{|c|c|c|c|c|c|c|}
\hline Compounds & $\begin{array}{l}\text { Limit of } \\
\text { Detection }\end{array}$ & $12-0^{\circ}$ & $12-.5$ & $12-1$ & $12-2$ & $12-3$ \\
\hline Bromodicloromethane & 1.0 & $<5.0$ & $<1.0$ & $<1.0$ & $<1.0$ & $<1.0$ \\
\hline Bromoform & 1.0 & $<5.0$ & $<1.0$ & $<1.0$ & $<1.0$ & $<1.0$ \\
\hline Bromomethane & 1.0 & $<5.0$ & $<1.0$ & $<1.0$ & $<1.0$ & $<1.0$ \\
\hline Carbon Tetrachloride & 1.0 & $<5.0$ & $<1.0$ & $<1.0$ & $<1.0$ & $<1.0$ \\
\hline Chlorobenzene & 1.0 & $<5.0$ & $<1.0$ & $<1.0$ & $<1.0$ & $<1.0$ \\
\hline Chloroethane & 1.0 & 308 & 279 & 266 & 227 & 184 \\
\hline Chloroform & 1.0 & $<5.0$ & $<1.0$ & $<1.0$ & $<1.0$ & $<1.0$ \\
\hline Chloromethane & 1.0 & $<5.0$ & $<1.0$ & $<1.0$ & $<1.0$ & $<1.0$ \\
\hline 2-Chloroethyl vinyl ether & 1.0 & $<5.0$ & $<1.0$ & $<1.0$ & $<1.0$ & $<1.0$ \\
\hline Dibromochloromethane & 1.0 & $<5.0$ & $<1.0$ & $<1.0$ & $<1.0$ & $<1.0$ \\
\hline 1,2-Dichlorobenzene & 1.0 & $<5.0$ & $<1.0$ & $<1.0$ & $<1.0$ & $<1.0$ \\
\hline 1,3-Dichlorobenzene & 1.0 & $<5.0$ & $<1.0$ & $<1.0$ & $<1.0$ & $<1.0$ \\
\hline 1,4-Dichlorobenzene & 1.0 & $<5.0$ & $<1.0$ & $<1.0$ & $<1.0$ & $<1.0$ \\
\hline 1,1-Dichloroethane & 1.0 & 409 & 233 & 73 & 57 & 16 \\
\hline 1,2-Dichloroethane & 1.0 & $<5.0$ & 3.0 & 3.0 & 1.0 & $<1.0$ \\
\hline 1,1-Dichloroethene & 1.0 & 34 & $<1.0$ & $<1.0$ & $<1.0$ & $<1.0$ \\
\hline Trans-1,2-Dichloroethene & 1.0 & $<5.0$ & $<1.0$ & $<1.0$ & $<1.0$ & $<1.0$ \\
\hline 1,2-Dichloropropane & 1.0 & $<5.0$ & $<1.0$ & $<1.0$ & $<1.0$ & $<1.0$ \\
\hline Cis-1,3-Dichloropropene & 1.0 & $<5.0$ & $<1.0$ & $<1.0$ & $<1.0$ & $<1.0$ \\
\hline Trans-1,3-Dichloropropene & 1.0 & $<5.0$ & $<1.0$ & $<1.0$ & $<1.0$ & $<1.0$ \\
\hline Methylene Chloride & 1.0 & $<5.0$ & $<1.0$ & $<1.0$ & $<1.0$ & $<1.0$ \\
\hline $1,1,2,2$-Tetrachloroethane & 1.0 & $<5.0$ & $<1.0$ & $<1.0$ & $<1.0$ & $<1.0$ \\
\hline Tetrachloroethene & 1.0 & $<5.0$ & $<1.0$ & $<1.0$ & $<1.0$ & $<1.0$ \\
\hline 1,1,1-Trichloroethane & 1.0 & 277 & 154 & 119 & 69 & 36 \\
\hline 1,1,2-Trichloroethane & 1.0 & $<5.0$ & $<1.0$ & $<1.0$ & $<1.0$ & $<1.0$ \\
\hline Trichloroethene & 1.0 & $<5.0$ & $<1.0$ & $<1.0$ & $<1.0$ & $<1.0$ \\
\hline Trichlorofluoromethane & 1.0 & 175 & 113 & 103 & 78 & 57 \\
\hline Vinyl Chloride & 1.0 & 33 & $<1.0$ & $<1.0$ & $<1.0$ & $<1.0$ \\
\hline
\end{tabular}

Comments:

5:1 Dilution was made on this sample.

Reviewed By: 
ANALYTICAL REPORT

State of Arizona License $\# 0009$

$\begin{array}{llll}\text { Client: } & \begin{array}{l}\text { Peroxidation Systems, Inc. } \\ \text { 5151 E. Broadway, Suite } 600\end{array} & \text { Sample Type: } & \text { Groundwater } \\ & \text { Tucson, Arizona 85711 } & \text { Date Sampled: } & 9 / 3 / 93 \\ & & \text { Date Received: } & 9 / 4 / 93 \\ \text { Project Name: } & \text { WJSA/DOE } & \text { Date Analyzed: } & 9 / 15 / 93 \\ \text { Project Number: } & 345 & \text { Analyzed By: } & \text { Deb Cartmell } \\ \text { Sample ID: } & 090793-17-21 & & \end{array}$

EPA Method 602 - Purgeable Aromatics (G.C. - PID)

Reported in $\mu \mathrm{g} / \mathrm{L}(\mathrm{ppb})$

\begin{tabular}{||l|c|c|c|c|c|c||}
\hline \multicolumn{1}{|c|}{ Compounds } & $\begin{array}{c}\text { Limit of } \\
\text { Detection }\end{array}$ & $12-0^{*}$ & $12-.5$ & $12-1$ & $12-2$ & $12-3$ \\
\hline Benzene & 1.0 & 18 & 6 & 3 & $<1.0$ & $<1.0$ \\
Toluene & 1.0 & 33 & $<1.0$ & $<1.0$ & $<1.0$ & $<1.0$ \\
Chlorobenzene & 1.0 & $<5.0$ & $<1.0$ & $<1.0$ & $<1.0$ & $<1.0$ \\
Ethyl Benzene & 1.0 & 15 & $<1.0$ & $<1.0$ & $<1.0$ & $<1.0$ \\
m,p-Xylene & 1.0 & 18 & $<1.0$ & $<1.0$ & $<1.0$ & $<1.0$ \\
0-Xylene & 1.0 & 26 & $<1.0$ & $<1.0$ & $<1.0$ & $<1.0$ \\
1,2-Dichlorobenzene & 1.0 & $<5.0$ & $<1.0$ & $<1.0$ & $<1.0$ & $<1.0$ \\
1,3-Dichlorobenzene & 1.0 & $<5.0$ & $<1.0$ & $<1.0$ & $<1.0$ & $<1.0$ \\
1,4-Dichlorobenzene & 1.0 & $<5.0$ & $<1.0$ & $<1.0$ & $<1.0$ & $<1.0$ \\
\hline
\end{tabular}

Comments:

5:1 Dilution was made on this sample

Reviewed By: Zurulle ferind

Date

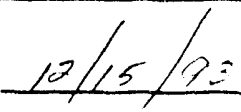


State of Arizona License $\# 0009$

$\begin{array}{llll}\text { Client: } & \text { Peroxidation Systems, Inc. } & \text { Sample Type: } & \text { Groundwater } \\ & \begin{array}{l}\text { 5151 E. Broadway, Suite } 600 \\ \text { Tucson, Arizona 85711 }\end{array} & \text { Date Sampled: } & 9 / 3 / 93 \\ & & & \\ \text { Project Name: } & \text { WJSA/DOE } & \text { Date Received: } & 9 / 4 / 93 \\ \text { Project Number: } & 345 & \text { Date Analyzed: } & 9 / 15 / 93 \\ \text { Sample ID: } & 090793-22-26 & \text { Analyzed By: } & \text { Deb Cartmell }\end{array}$

EPA Method 601 - Purgeable Halocarbons

Reported in $\mu \mathrm{g} / \mathrm{L}$ (ppb)

\begin{tabular}{|c|c|c|c|c|c|c|}
\hline Compounds & $\begin{array}{l}\text { Limit of } \\
\text { Detection }\end{array}$ & $13-0^{*}$ & $13-.5$ & $13-1$ & $13-2$ & $13-3$ \\
\hline Bromodicloromethane & 1.0 & $<5.0$ & $<1.0$ & $<1.0$ & $<1.0$ & $<1.0$ \\
\hline Bromoform & 1.0 & $<5.0$ & $<1.0$ & $<1.0$ & $<1.0$ & $<1.0$ \\
\hline Bromomethane & 1.0 & $<5.0$ & $<1.0$ & $<1.0$ & $<1.0$ & $<1.0$ \\
\hline Carbon Tetrachloride & 1.0 & $<5.0$ & $<1.0$ & $<1.0$ & $<1.0$ & $<1.0$ \\
\hline Chlorobenzene & 1.0 & $<5.0$ & $<1.0$ & $<1.0$ & $<1.0$ & $<1.0$ \\
\hline Chloroethane & 1.0 & 396 & 356 & 324 & 245 & 197 \\
\hline Chloroform & 1.0 & $<5.0$ & $<1.0$ & $<1.0$ & $<1.0$ & $<1.0$ \\
\hline Chloromethane & 1.0 & $<5.0$ & $<1.0$ & $<1.0$ & $<1.0$ & $<1.0$ \\
\hline 2-Chloroethyl vinyl ether & 1.0 & $<5.0$ & $<1.0$ & $<1.0$ & $<1.0$ & $<1.0$ \\
\hline Dibromochloromethane & 1.0 & $<5.0$ & $<1.0$ & $<1.0$ & $<1.0$ & $<1.0$ \\
\hline 1,2-Dichlorobenzene & 1.0 & $<5.0$ & 3 & 2 & $<1.0$ & $<1.0$ \\
\hline 1,3-Dichlorobenzene & 1.0 & $<5.0$ & $<1.0$ & $<1.0$ & $<1.0$ & $<1.0$ \\
\hline 1,4-Dichlorobenzene & 1.0 & $<5.0$ & $<1.0$ & $<1.0$ & $<1.0$ & $<1.0$ \\
\hline 1,1-Dichloroethane & 1.0 & 483 & 264 & 183 & 61 & 18 \\
\hline 1,2-Dichloroethane & 1.0 & $<5.0$ & 4 & 2 & $<1.0$ & $<1.0$ \\
\hline 1,1-Dichloroethene & 1.0 & $<5.0$ & $<1.0$ & $<1.0$ & $<1.0$ & $<1.0$ \\
\hline Trans-1,2-Dichloroethene & 1.0 & $<5.0$ & $<1.0$ & $<1.0$ & $<1.0$ & $<1.0$ \\
\hline 1,2-Dichloropropane & 1.0 & $<5.0$ & $<1.0$ & $<1.0$ & $<1.0$ & $<1.0$ \\
\hline Cis-1,3-Dichloropropene & 1.0 & $<5.0$ & $<1.0$ & $<1.0$ & $<1.0$ & $<1.0$ \\
\hline Trans-1,3-Dichloropropene & 1.0 & $<5.0$ & $<1.0$ & $<1.0$ & $<1.0$ & $<1.0$ \\
\hline Methylene Chloride & 1.0 & $<5.0$ & $<1.0$ & $<1.0$ & $<1.0$ & $<1.0$ \\
\hline 1,1,2,2-Tetrachloroethane & 1.0 & $<5.0$ & $<1.0$ & $<1.0$ & $<1.0$ & $<1.0$ \\
\hline Tetrachloroethene & 1.0 & $<5.0$ & $<1.0$ & $<1.0$ & $<1.0$ & $<1.0$ \\
\hline 1,1,1-Trichloroethane & 1.0 & 354 & 215 & 189 & 120 & 71 \\
\hline 1,1,2-Trichloroethane & 1.0 & $<5.0$ & $<1.0$ & $<1.0$ & $<1.0$ & $<1.0$ \\
\hline Trichloroethene & 1.0 & $<5.0$ & $<1.0$ & $<1.0$ & $<1.0$ & $<1.0$ \\
\hline Trichlorofluoromethane & 1.0 & 244 & 187 & 167 & 105 & 80 \\
\hline Vinyl Chloride & 1.0 & 49 & 47 & 40 & 27 & 19 \\
\hline
\end{tabular}

Comments:

5:1 Dilution made on this sample

Reviewed By: 


\section{PSI Environmental Laboratories}

ANALYTICAL REPORT

State of Arizona License $\$ 0009$

$\begin{array}{llll}\text { Client: } & \begin{array}{l}\text { Peroxidation Systems, Inc. } \\ \text { 5151 E. Broadway, Suite 600 }\end{array} & \text { Sample Type: } & \text { Groundwater } \\ & \text { Tucson, Arizona 85711 } & \text { Date Sampled: } & 9 / 3 / 93 \\ & \text { WJSA/DOE } & \text { Date Received: } & 9 / 4 / 93 \\ \text { Project Name: } & 345 & \text { Date Analyzed: } & 9 / 15 / 93 \\ \text { Project Number: } & 090793-22-26 & \text { Analyzed By: } & \text { Deb Cartmell } \\ \text { Sample ID: } & & & \end{array}$

EPA Method 602 - Purgeable Aromatics (G.C. - PID)

Reported in $\mu \mathrm{g} / \mathrm{L}$ (ppb)

\begin{tabular}{|l|c|c|c|c|c|c|}
\hline \multicolumn{1}{|c|}{ Compounds } & $\begin{array}{c}\text { Limit of } \\
\text { Detection }\end{array}$ & $13-0^{\circ}$ & $13-.5$ & $13-1$ & $13-2$ & $13-3$ \\
\hline Benzene & 1.0 & 7.0 & 2 & $<1.0$ & $<1.0$ & $<1.0$ \\
Tol:iene & 1.0 & $<5.0$ & $<1.0$ & $<1.0$ & $<1.0$ & $<1.0$ \\
Chlorobenzene & 1.0 & $<5.0$ & $<1.0$ & $<1.0$ & $<1.0$ & $<1.0$ \\
Ethyl Benzene & 1.0 & $<5.0$ & $<1.0$ & $<1.0$ & $<1.0$ & $<1.0$ \\
m,p-Xylene & 1.0 & $<5.0$ & $<1.0$ & $<1.0$ & $<1.0$ & $<1.0$ \\
0-Xylene & 1.0 & $<5.0$ & $<1.0$ & $<1.0$ & $<1.0$ & $<1.0$ \\
1,2-Dichlorobenzene & 1.0 & $<5.0$ & $<1.0$ & $<1.0$ & $<1.0$ & $<1.0$ \\
1,3-Dichlorobenzene & 1.0 & $<5.0$ & $<1.0$ & $<1.0$ & $<1.0$ & $<1.0$ \\
1,4-Dichlorobenzene & 1.0 & $<5.0$ & $<1.0$ & $<1.0$ & $<1.0$ & $<1.0$ \\
\hline
\end{tabular}

Comments:

5:1 Dilution was made on this sample.

Reviewed By:

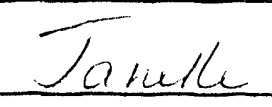


2595 E. Airport Drive Tucson, Arizona 85706 602-573-6565 FAX 602.573-6550 1.800-p51-LAB5

ANALYTICAL REPORT

State of Arizona License $\# 0 C 09$

Client:

Peroxidation Systems, Inc.

5151 E. Broadway, Suite 600

Tucson, Arizona 85711

Project Name: $\quad$ WJSA/DOE

Project Number: $\quad 345$

Sample ID:

090793-27- 31
Sample Type: Groundwater

Date Sampled: $\quad 9 / 3 / 93$

Date Received: $\quad 9 / 4 / 93$

Date Analyzed: $9 / 17 / 93$

Analyzed By: Deb Cartmell

EPA Method 601 - Purgeable Halocarbons

Reported in $\mu \mathrm{g} / \mathrm{L}$ (ppb)

\begin{tabular}{|c|c|c|c|c|c|c|}
\hline Compounds & $\begin{array}{l}\text { Limit of } \\
\text { Detection }\end{array}$ & $14-0^{*}$ & $14-.5$ & $14-1$ & $14-2$ & $14-3$ \\
\hline Bromodicloromethane & 1.0 & $<5.0$ & $<1.0$ & $<1.0$ & $<1.0$ & $<1.0$ \\
\hline Bromoform & 1.0 & $<5.0$ & $<1.0$ & $<1.0$ & $<1.0$ & $<1.0$ \\
\hline Bromomethane & 1.0 & $<5.0$ & $<1.0$ & $<1.0$ & $<1.0$ & $<1.0$ \\
\hline Carbon Tetrachloride & 1.0 & $<5.0$ & $<1.0$ & $<1.0$ & $<1.0$ & $<1.0$ \\
\hline Chlorobenzene & 1.0 & $<5.0$ & $<1.0$ & $<1.0$ & $<1.0$ & $<1.0$ \\
\hline Chloroethane & 1.0 & 368 & 345 & 323 & 306 & 270 \\
\hline Chloroform & 1.0 & $<5.0$ & $<1.0$ & $<1.0$ & $<1.0$ & $<1.0$ \\
\hline Chloromethane & 1.0 & $<5.0$ & $<1.0$ & $<1.0$ & $<1.0$ & $<1.0$ \\
\hline 2-Chloroethyl vinyl ether & 1.0 & $<5.0$ & $<1.0$ & $<1.0$ & $<1.0$ & $<1.0$ \\
\hline Dibromochloromethane & 1.0 & $<5.0$ & $<1.0$ & $<1.0$ & $<1.0$ & $<1.0$ \\
\hline 1,2-Dichlorobenzene & 1.0 & $<5.0$ & $<1.0$ & $<1.0$ & $<1.0$ & $<1.0$ \\
\hline 1,3-Dichlorobenzene & 1.0 & $<5.0$ & $<1.0$ & $<1.0$ & $<1.0$ & $<1.0$ \\
\hline 1,4-Dichlorobenzene & 1.0 & $<5.0$ & $<1.0$ & $<1.0$ & $<1.0$ & $<1.0$ \\
\hline 1,1-Dichloroethane & 1.0 & 410 & 236 & 224 & 106 & 65 \\
\hline 1,2-Dichloroethane & 1.0 & $<5.0$ & 3 & 3 & 1 & $<1.0$ \\
\hline 1,1-Dichloroethene & 1.0 & $<5.0$ & $<1.0$ & $<1.0$ & $<1.0$ & $<1.0$ \\
\hline Trans-1,2-Dichloroethene & 1.0 & $<5.0$ & $<1.0$ & $<1.0$ & $<1.0$ & $<1.0$ \\
\hline 1,2-Dichloropropane & 1.0 & $<5.0$ & $<1.0$ & $<1.0$ & $<1.0$ & $<1.0$ \\
\hline Cis-1,3-Dichloropropene & 1.0 & $<5.0$ & $<1.0$ & $<1.0$ & $<1.0$ & $<1.0$ \\
\hline Trans-1,3-Dichloropropene & 1.0 & $<5.0$ & $<1.0$ & $<1.0$ & $<1.0$ & $<1.0$ \\
\hline Methylene Chloride & 1.0 & $<5.0$ & $<1.0$ & $<1.0$ & $<1.0$ & $<1.0$ \\
\hline $1,1,2,2-$ Tetrachloroethane & 1.0 & $<5.0$ & $<1.0$ & $<1.0$ & $<1.0$ & $<1.0$ \\
\hline Tetrachloroethene & 1.0 & $<5.0$ & $<1.0$ & $<1.0$ & $<1.0$ & $<1.0$ \\
\hline 1,1,1-Trichloroethane & 1.0 & 334 & 217 & 206 & 176 & 134 \\
\hline 1,1,2-Trichloroethane & 1.0 & $<5.0$ & 2 & 2 & $<1.0$ & $<1.0$ \\
\hline Trichloroethene & 1.0 & $<5.0$ & $<1.0$ & $<1.0$ & $<1.0$ & $<1.0$ \\
\hline Trichlorofluoromethane & 1.0 & 221 & 200 & 178 & 149 & 119 \\
\hline Vinyl Chloride & 1.0 & $<5.0$ & $<1.0$ & $<1.0$ & $<1.0$ & $<1.0$ \\
\hline
\end{tabular}

Comments:

5:1 Dilution was made on this sample

Reviewed By: 
ANALYTICAL REPORT

State of Arizona License \#0009
Client: $\quad$ Peroxidation Systems, Inc. 5151 E. Broadway, Suite 600 Tucson, Arizona 85711

WJSA/DOE

345

090793-27- 31
Sample Type: Groundwater

Date Sampled: $\quad 9 / 3 / 93$

Date Received: $\quad 9 / 4 / 93$

Date Analyzed: $\quad 9 / 17 / 93$

Analyzed By: Deb Cartmell

EPA Method 602 - Purgeable Aromatics (G.C. - PID)

Reported in $\mu \mathrm{g} / \mathrm{L}(\mathrm{ppb})$

\begin{tabular}{|l|c|c|c|c|c|c|}
\hline \multicolumn{1}{|c|}{ Compounds } & $\begin{array}{c}\text { Limit of } \\
\text { Detection }\end{array}$ & $14-0^{\circ}$ & $14-.5$ & $14-1$ & $14-2$ & $14-3$ \\
\hline Benzene & 1.0 & $<5.0$ & $<1.0$ & $<1.0$ & $<1.0$ & $<1.0$ \\
Toluene & 1.0 & $<5.0$ & $<1.0$ & $<1.0$ & $<1.0$ & $<1.0$ \\
Chlorobenzene & 1.0 & $<5.0$ & $<1.0$ & $<1.0$ & $<1.0$ & $<1.0$ \\
Ethyl Benzene & 1.0 & $<5.0$ & $<1.0$ & $<1.0$ & $<1.0$ & $<1.0$ \\
m,p-Xylene & 1.0 & $<5.0$ & $<1.0$ & $<1.0$ & $<1.0$ & $<1.0$ \\
o-Xylerie & 1.0 & $<5.0$ & $<1.0$ & $<1.0$ & $<1.0$ & $<1.0$ \\
1,2-Dichlorobenzene & 1.0 & $<5.0$ & $<1.0$ & $<1.0$ & $<1.0$ & $<1.0$ \\
1,3-Dichlorobenzene & 1.0 & $<5.0$ & $<1.0$ & $<1.0$ & $<1.0$ & $<1.0$ \\
1,4-Dichlorobenzene & 1.0 & $<5.0$ & $<1.0$ & $<1.0$ & $<1.0$ & $<1.0$ \\
\hline
\end{tabular}

Comments:

5:1 Dilution was made on this sample.

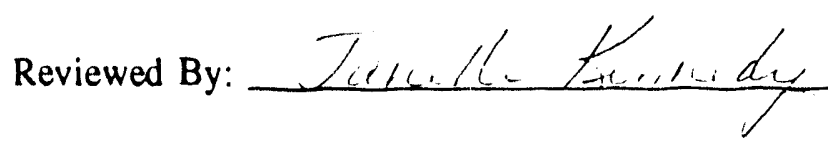

Date 


\section{PSI Environmental Laboratories}

Tucson. Arizona 85706

602-573-6565

FAX 602-573-6550

1-BOO-PS/-LABS ANALYTICAL REPORT

State of Arizona License $\# 0009$

Client:

\section{Project Name:}

Project Number:

Sample ID:
Peroxidation Systems, Inc. 5151 E. Broadway, Suite 600 Tucson, Arizona 85711

WJSA/DOE

345

090793-32 - 34
Sample Type: Groundwater

Date Sampled: $\quad 9 / 3 / 93$

Date Received: 9/4/93

Date Analyzed: 11/19/93

Analyzed By: Deb Cartmell

EPA Method 601 - Purgeable Halocarbons

Reported in $\mu \mathrm{g} / \mathrm{L}$ (ppb)

\begin{tabular}{|c|c|c|c|c|}
\hline Compounds & $\begin{array}{l}\text { Limit of } \\
\text { Detection }\end{array}$ & $15-0^{*}$ & $15-.75$ & $15-1.5$ \\
\hline Bromodicloromethane & 1.0 & $<5.0$ & $<1.0$ & $<1.0$ \\
\hline Bromoform & 1.0 & $<5.0$ & $<1.0$ & $<1.0$ \\
\hline Bromomethane & 1.0 & $<5.0$ & $<1.0$ & $<1.0$ \\
\hline Carbon Tetrachloride & 1.0 & $<5.0$ & $<1.0$ & $<1.0$ \\
\hline Chlorobenzene & 1.0 & $<5.0$ & $<1.0$ & $<1.0$ \\
\hline Chloroethane & 1.0 & 491 & 482 & 467 \\
\hline Chloroform & 1.0 & $<5.0$ & $<1.0$ & $<1.0$ \\
\hline Chloromethane & 1.0 & $<5.0$ & $<1.0$ & $<1.0$ \\
\hline 2-Chloroethyl vinyl ether & 1.0 & $<5.0$ & $<1.0$ & $<1.0$ \\
\hline Dibromochloromethane & 1.0 & $<5.0$ & $<1.0$ & $<1.0$ \\
\hline 1,2-Dichlorobenzene & 1.0 & $<5.0$ & $<1.0$ & $<1.0$ \\
\hline 1,3-Dichlorobenzene & 1.0 & $<5.0$ & $<1.0$ & $<1.0$ \\
\hline 1,4-Dichlorobenzene & 1.0 & $<5.0$ & $<1.0$ & $<1.0$ \\
\hline 1,1-Dichloroethane & 1.0 & 579 & 291 & 274 \\
\hline 1,2-Dichloroethane & 1.0 & 6.0 & 5 & 4 \\
\hline 1,1-Dichloroethene & 1.0 & $<5.0$ & $<1.0$ & $<1.0$ \\
\hline Trans-1,2-Dichloroethene & 1.0 & $<5.0$ & $<1.0$ & $<1.0$ \\
\hline 1,2-Dichloropropane & 1.0 & $<5.0$ & $<1.0$ & $<1.0$ \\
\hline Cis-1,3-Dichloropropene & 1.0 & $<5.0$ & $<1.0$ & $<1.0$ \\
\hline Trans-1,3-Dichloropropene & 1.0 & $<5.0$ & $<1.0$ & $<1.0$ \\
\hline Methylene Chloride & 1.0 & $<5.0$ & $<1.0$ & $<1.0$ \\
\hline 1,1,2,2-Tetrachloroethane & 1.0 & $<5.0$ & $<1.0$ & $<1.0$ \\
\hline Tetrachloroethene & 1.0 & $<5.0$ & $<1.0$ & $<1.0$ \\
\hline 1,1,1-Trichloroethane & 1.0 & 444 & 255 & 247 \\
\hline 1,1,2-Trichloroethane & 1.0 & $<5.0$ & 4 & 3 \\
\hline Trichloroethene & 1.0 & $<5.0$ & $<1.0$ & $<1.0$ \\
\hline Trichlorofluoromethane & 1.0 & NA & NA & NA \\
\hline Vinyl Chloride & 1.0 & $<5.0$ & $<1.0$ & $<1.0$ \\
\hline
\end{tabular}

Comments: NA - Data not Available, Pending QA/QC.

\section{5:1 Dilution was made on this sample.}

Reviewed By: 
ANALYTICAL REPORT

State of Arizona License $\# 0009$

Client:

Peroxidation Systems, Inc.

5151 E. Broadway, Suite 600

Tucson, Arizona 85711

Project Name: WJSA/DOE

Project Number: $\quad 345$

Sample ID:
Sample Type: Groundwater

Date Sampled: $\quad 9 / 3 / 93$

Date Received: $\quad 9 / 4 / 93$

Date Analyzed: $\quad 11 / 19 / 93$

Analyzed By: Deb Cartmell

EPA Method 602 - Purgeable Aromatics (G.C. - PID)

Reported in $\mu \mathrm{g} / \mathrm{L}(\mathrm{ppb})$

\begin{tabular}{|l|c|c|c|c|c|c|}
\hline \multicolumn{1}{|c|}{ Compounds } & $\begin{array}{c}\text { Limit of } \\
\text { Detection }\end{array}$ & $15-0^{\circ}$ & $15-.75$ & $15-1.5$ & & \\
\hline Benzene & 1.0 & 50 & 1 & $<1.0$ & & \\
Toluene & 1.0 & 128 & $<1.0$ & $<1.0$ & & \\
Chlorobenzene & 1.0 & $<5.0$ & $<1.0$ & $<1.0$ & & \\
Ethyl Benzene & 1.0 & 82 & $<1.0$ & $<1.0$ & & \\
m,p-Xylene & 1.0 & 111 & $<1.0$ & $<1.0$ & & \\
o-Xylene & 1.0 & 116 & $<1.0$ & $<1.0$ & & \\
1,2-Dichlorobenzene & 1.0 & $<5.0$ & $<1.0$ & $<1.0$ & & \\
1,3-Dichlorobenzene & 1.0 & $<5.0$ & $<1.0$ & $<1.0$ & & \\
1,4-Dichlorobenzene & 1.0 & $<5.0$ & $<1.0$ & $<1.0$ & & \\
\hline
\end{tabular}

Comments:

"5:1 Dilution was made on this sample.

Reviewed By:

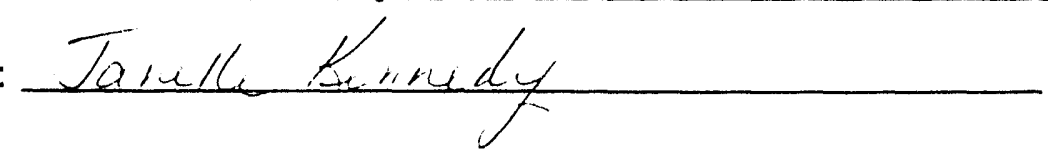

Date

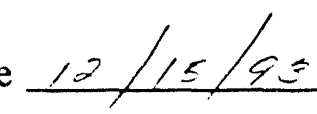


Client:

Peroxidation Systems, Inc. 5151 E. Broadway, Suite 600

Tucson, Arizona 85711

Project Name: WJSA/DOE

Project Number: $\quad 345$

Sample ID:

102093-1 - 4
Sample Type: Groundwater

Date Sampled: $\quad 10 / 17 / 93$

Date Received: $\quad 10 / 20 / 93$

Date Analyzed: 11/2/93

Analyzed By: Deb Cartmell

EPA Method 601 - Purgeable Halocarbons

Reported in $\mu \mathrm{g} / \mathrm{L}(\mathrm{ppb})$

\begin{tabular}{|c|c|c|c|c|c|}
\hline Compounds & $\begin{array}{l}\text { Limit of } \\
\text { Detection }\end{array}$ & $16-0$ & $16-.5$ & $16-2$ & $16-3$ \\
\hline Bromodicloromethane & 1.0 & $<1.0$ & $<1.0$ & $<1.0$ & $<1.0$ \\
\hline Bromoform & 1.0 & $<1.0$ & $<1.0$ & $<1.0$ & $<1.0$ \\
\hline Bromomethane & 1.0 & $<1.0$ & $<1.0$ & $<1.0$ & $<1.0$ \\
\hline Carbon Tetrachloride & 1.0 & $<1.0$ & $<1.0$ & $<1.0$ & $<1.0$ \\
\hline Chlorobenzene & 1.0 & $<1.0$ & 1.0 & 1.0 & 1.0 \\
\hline Chloroethane & 1.0 & NA & NA & NA & NA \\
\hline Chloroform & 1.0 & $<1.0$ & $<1.0$ & $<1.0$ & $<1.0$ \\
\hline Chloromethane & 1.0 & $<1.0$ & $<1.0$ & $<1.0$ & $<1.0$ \\
\hline 2-Chloroethyl vinyl ether & 1.0 & $<1.0$ & $<1.0$ & $<1.0$ & $<1.0$ \\
\hline Dibromochloromethane & 1.0 & $<1.0$ & $<1.0$ & $<1.0$ & $<1.0$ \\
\hline 1,2-Dichlorobenzene & 1.0 & $<1.0$ & $<1.0$ & $<1.0$ & $<1.0$ \\
\hline 1,3-Dichlorobenzene & 1.0 & $<1.0$ & $<1.0$ & $<1.0$ & $<1.0$ \\
\hline 1,4-Dichlorobenzene & 1.0 & $<1.0$ & $<1.0$ & $<1.0$ & $<1.0$ \\
\hline 1,1-Dichloroethane & 1.0 & 343 & 99 & 5 & 4 \\
\hline 1,2-Dichloroethane & 1.0 & $<1.0$ & $<1.0$ & $<1.0$ & $<1.0$ \\
\hline 1,1-Dichloroethene & 1.0 & 14.0 & $<1.0$ & $<1.0$ & $<1.0$ \\
\hline Trans-1,2-Dichloroethene & 1.0 & $<1.0$ & $<1.0$ & $<1.0$ & $<1.0$ \\
\hline 1,2-Dichloropropane & 1.0 & $<1.0$ & $<1.0$ & $<1.0$ & $<1.0$ \\
\hline Cis-1,3-Dichloropropene & 1.0 & $<1.0$ & $<1.0$ & $<1.0$ & $<1.0$ \\
\hline Trans-1,3-Dichloropropene & 1.0 & $<1.0$ & $<1.0$ & $<1.0$ & $<1.0$ \\
\hline Methylene Chloride & 1.0 & $<1.0$ & $<1.0$ & $<1.0$ & $<1.0$ \\
\hline 1,1,2,2-Tetrachloroethane & 1.0 & $<1.0$ & $<1.0$ & $<1.0$ & $<1.0$ \\
\hline Tetrachloroethene & 1.0 & $<1.0$ & $<1.0$ & $<1.0$ & $<1.0$ \\
\hline 1,1,1-Trichloroethane & 1.0 & 339 & 171 & 28 & 16 \\
\hline 1,1,2-Trichloroethane & 1.0 & $<1.0$ & $<1.0$ & $<1.0$ & $<1.0$ \\
\hline Trichloroethene & 1.0 & $<1.0$ & $<1.0$ & $<1.0$ & $<1.0$ \\
\hline Trichlorofluoromethane & 1.0 & 439 & 355 & 229 & 161 \\
\hline Vinyl Chloride & 1.0 & 30 & 22 & 12 & 7 \\
\hline
\end{tabular}

Comments: NA - Data not available, Pending QA/OC.

Reviewed By: 


\section{ANALYTICAL REPORT}

State of Arizona License $\$ 0009$

$\begin{array}{llll}\text { Client: } & \begin{array}{l}\text { Peroxidation Systems, Inc. } \\ \text { 5151 E. Broadway, Suite 600 } \\ \text { Tucson, Arizona 85711 }\end{array} & \text { Sample Type: } & \text { Groundwater } \\ & \text { Date Sampled: } & 10 / 17 / 93 \\ \text { Project Name: } & \text { WSA/DOE } & \text { Date Received: } & 10 / 20 / 93 \\ \text { Project Number: } & 345 & \text { Date Analyzed: } & 11 / 2 / 93 \\ \text { Sample ID: } & 102093-1-4 & \text { Analyzed By: } & \text { Deb Cartmell }\end{array}$

EPA Method 602 - Purgeable Aromatics (G.C. - PID)

Reported in $\mu \mathrm{g} / \mathrm{L}(\mathrm{ppb})$

\begin{tabular}{|c|c|c|c|c|c|}
\hline Compounds & $\begin{array}{l}\text { Limit of } \\
\text { Detection }\end{array}$ & $16-0$ & $16-.5$ & $16-2$ & $16-3$ \\
\hline Benzene & 1.0 & 10 & 2 & 2 & $<1.0$ \\
\hline Toluene & 1.0 & 5 & 2 & 2 & $<1.0$ \\
\hline Chlorobenzene & 1.0 & $<1.0$ & $<1.0$ & $<1.0$ & $<1.0$ \\
\hline Ethyl Benzene & 1.0 & $<1.0$ & $<1.0$ & $<1.0$ & $<1.0$ \\
\hline m,p-Xylene & 1.0 & 8 & 2 & 2 & 5 \\
\hline $0-X y l e n e$ & 1.0 & 5 & 2 & 2 & $<1.0$ \\
\hline 1,2-Dichlorobenzene & 1.0 & $<1.0$ & $<1.0$ & $<1.0$ & $<1.0$ \\
\hline 1,3-Dichlorobenzene & 1.0 & $<1.0$ & $<1.0$ & $<1.0$ & $<1.0$ \\
\hline 1,4-Dichlorobenzene & 1.0 & $<1.0$ & $<1.0$ & $<1.0$ & $<1.0$ \\
\hline
\end{tabular}

Comments:

Reviewed By:

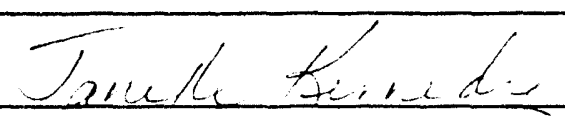

Date 


\section{PSI Environmental Laboratories}

2595 E. Alrport Drive Tucson. Arizona 85706 602-573-6565 FAX 602-573-6550 I-800-P5I-LABS ANALYTICAL REPORT

State of Arizona License $\# 0009$

Client: $\quad$ Peroxidation Systems, Inc.

5151 E. Broadway, Suite 600

Tucson, Arizona 85711

Project Name: $\quad$ WJSA/DOE

Project Number: 345

Sample ID: $\quad$ 102093-5 - 7, Blank
Sample Type: Groundwater-

Date Sampled: $\quad 10 / 17 / 93$

Date Received: $\quad 10 / 20 / 93$

Date Analyzed: 11/2/93

Analyzed By: Deb Cartmell

EPA Method 601 - Purgeable Halocarbons

Reported in $\mu \mathrm{g} / \mathrm{L}$ (ppb)

\begin{tabular}{|c|c|c|c|c|c|c|}
\hline Compounds & $\begin{array}{l}\text { Limit of } \\
\text { Detection }\end{array}$ & 16-1-Infl & 16-1-L3 & 16-1-L6 & Field 10/16 & \\
\hline Bromodicloromethane & 1.0 & $<1.0$ & $<1.0$ & $<1.0$ & $<1.0$ & \\
\hline Bromoform & 1.0 & $<1.0$ & $<1.0$ & $<1.0$ & $<1.0$ & \\
\hline Bromomethane & 1.0 & $<1.0$ & $<1.0$ & $<1.0$ & $<1.0$ & \\
\hline Carbon Tetrachloride & 1.0 & $<1.0$ & $<1.0$ & $<1.0$ & $<1.0$ & \\
\hline Chlorobenzene & 1.0 & $<1.0$ & $<1.0$ & $<1.0$ & $<1.0$ & \\
\hline Chloroethane & 1.0 & $<1.0$ & $<1.0$ & $<1.0$ & $<1.0$ & \\
\hline Chloroform & 1.0 & $<1.0$ & $<1.0$ & $<1.0$ & $<1.0$ & \\
\hline Chloromethane & 1.0 & $<1.0$ & $<1.0$ & $<1.0$ & $<1.0$ & \\
\hline 2-Chloroethyl vinyl ether & 1.0 & $<1.0$ & $<1.0$ & $<1.0$ & $<1.0$ & \\
\hline Dibromochloromethane & 1.0 & $<1.0$ & $<1.0$ & $<1.0$ & $<1.0$ & \\
\hline 1,2-Dichlorobenzene & 1.0 & $<1.0$ & $<1.0$ & $<1.0$ & $<1.0$ & \\
\hline 1,3-Dichlorobenzene & 1.0 & $<1.0$ & $<1.0$ & $<1.0$ & $<1.0$ & \\
\hline 1,4-Dichlorobenzene & 1.0 & $<1.0$ & $<1.0$ & $<1.0$ & $<1.0$ & \\
\hline 1,1-Dichloroethane & 1.0 & 13 & $<1.0$ & $<1.0$ & $<1.0$ & \\
\hline 1,2-Dichloroethane & 1.0 & $<1.0$ & $<1.0$ & $<1.0$ & $<1.0$ & \\
\hline 1,1-Dichloroethene & 1.0 & $<1.0$ & $<1.0$ & $<1.0$ & $<1.0$ & \\
\hline Trans-1,2-Dichloroethene & 1.0 & $<1.0$ & $<1.0$ & $<1.0$ & $<1.0$ & \\
\hline 1,2-Dichloropropane & 1.0 & $<1.0$ & $<1.0$ & $<1.0$ & $<1.0$ & \\
\hline Cis-1,3-Dichloropropene & 1.0 & $<1.0$ & $<1.0$ & $<1.0$ & $<1.0$ & \\
\hline Trans-1,3-Dichloropropene & 1.0 & $<1.0$ & $<1.0$ & $<1.0$ & $<1.0$ & \\
\hline Methylene Chloride & 1.0 & $<1.0$ & $<1.0$ & $<1.0$ & $<1.0$ & \\
\hline 1,1,2,2-Tetrachloroethane & 1.0 & $<1.0$ & $<1.0$ & $<1.0$ & $<1.0$ & \\
\hline Tetrachloroethene & 1.0 & $<1.0$ & $<1.0$ & $<1.0$ & $<1.0$ & \\
\hline 1,1,1-Trichloroethane & 1.0 & 97 & 50 & 53 & $<1.0$ & \\
\hline 1,1,2-Trichloroethane & 1.0 & $<1.0$ & $<1.0$ & $<1.0$ & $<1 . n$ & \\
\hline Trichloroethene & 1.0 & $<1.0$ & $<1.0$ & $<1.0$ & $<1.0$ & \\
\hline Trichlorofluoromethane & 1.0 & 339 & 273 & 317 & $<1.0$ & \\
\hline Vinyl Chloride & 1.0 & 20 & 15 & 18 & $<1.0$ & \\
\hline
\end{tabular}

Comments:

Reviewed By: Tarelle fermed

Date $12 / 15 / 93$ 
ANALYTICAL REPORT

State of Arizona License $\# 0009$

Client: $\quad$ Peroxidation Systems, Inc. 5151 E. Broadway, Suite 600

Tucson, Arizona 85711

Project Name: WJSA/DOE

Project Number: 345

Sample ID: $\quad$ 102093-5 - 7, Blank
Sample Type: Groundwater

Date Sampled: $\quad 10 / 17 / 93$

Date Received: $\quad 10 / 20 / 93$

Date Analyzed: 11/2/93

Analyzed By: Deb Cartmell

EPA Method 602 - Purgeable Aromatics (G.C. - PID)

Reported in $\mu \mathrm{g} / \mathrm{L}(\mathrm{ppb})$

\begin{tabular}{|c|c|c|c|c|c|}
\hline Compounds & $\begin{array}{l}\text { Limit of } \\
\text { Detection }\end{array}$ & $16-1-\operatorname{Infl}$ & $16-1-\mathrm{L} 3$ & 16-1-L6 & Field $10 / 16$ \\
\hline Benzene & 1.0 & 2 & $<1.0$ & $<1.0$ & $<1.0$ \\
\hline Toluene & 1.0 & 2 & $<1.0$ & $<1.0$ & 2 \\
\hline Chlorobenzene & 1.0 & $<1.0$ & $<1.0$ & $<1.0$ & $<1.0$ \\
\hline Ethyl Benzene & 1.0 & $<1.0$ & $<1.0$ & $<1.0$ & $<1.0$ \\
\hline m,p-Xylene & 1.0 & 4 & $<1.0$ & $<1.0$ & $<1.0$ \\
\hline $0-$ Xylene & 1.0 & 2 & $<1.0$ & $<1.0$ & $<1.0$ \\
\hline 1,2-Dichlorobenzene & 1.0 & $<1.0$ & $<1.0$ & $<1.0$ & $<1.0$ \\
\hline 1,3-Dichlorobenzene & 1.0 & $<1.0$ & $<1.0$ & $<1.0$ & $<1.0$ \\
\hline 1,4-Dichlorobenzene & 1.0 & $<1.0$ & $<1.0$ & $<1.0$ & $<1.0$ \\
\hline
\end{tabular}

Comments:

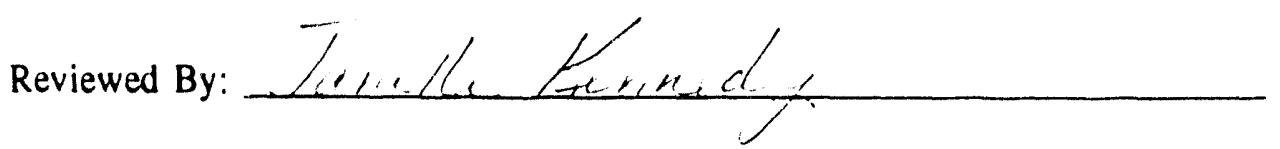


2595 E. Aipport Drive Tucson. Arizona 85706 602.573-6565 FAX 602.573-6550 1-800-p5I-LA85

ANALYTICAL REPORT

State of Arizona License $\# 0009$

Client:

Peroxidation Systems, Inc.

5151 E. Broadway, Suite 600

Tucson, Arizona 85711

Project Name: WJSA/DOE

Project Number: $\quad 345$

Sample ID: $\quad$ 102093-8 - 11
Sample Type: Groundwater.

Date Sampled: $\quad 10 / 17 / 93$

Date Received: $\quad 10 / 20 / 93$

Date Analyzed: $11 / 5 / 93$

Analyzed By: Deb Cartmell

EPA Method 601 - Purgeable Halocarbons

Reported in $\mu \mathrm{g} / \mathrm{L}(\mathrm{ppb})$

\begin{tabular}{|c|c|c|c|c|c|}
\hline Compounds & $\begin{array}{l}\text { Limit of } \\
\text { Detection }\end{array}$ & $17-0$ & $17-.5$ & $17-2$ & $17-3$ \\
\hline Bromodicloromethane & 1.0 & $<1.0$ & $<1.0$ & $<1.0$ & $<1.0$ \\
\hline Bromoform & 1.0 & $<1.0$ & $<1.0$ & $<1.0$ & $<1.0$ \\
\hline Bromomethane & 1.0 & $<1.0$ & $<1.0$ & $<1.0$ & $<1.0$ \\
\hline Carbon Tetrachloride & 1.0 & $<1.0$ & $<1.0$ & $<1.0$ & $<1.0$ \\
\hline Chlorobenzene & 1.0 & $<1.0$ & $<1.0$ & $<1.0$ & $<1.0$ \\
\hline Chloroethane & 1.0 & $<1.0$ & $<1.0$ & $<1.0$ & $<1.0$ \\
\hline Chloroform & 1.0 & $<1.0$ & $<1.0$ & $<1.0$ & $<1.0$ \\
\hline Chloromethane & 1.0 & $<1.0$ & $<1.0$ & $<1.0$ & $<1.0$ \\
\hline 2-Chloroethyl vinyl ether & 1.0 & $<1.0$ & $<1.0$ & $<1.0$ & $<1.0$ \\
\hline Dibromochloromethane & 1.0 & $<1.0$ & $<1.0$ & $<1.0$ & $<1.0$ \\
\hline 1,2-Dichlorobenzene & 1.0 & $<1.0$ & $<1.0$ & $<1.0$ & $<1.0$ \\
\hline 1,3-Dichlorobenzene & 1.0 & $<1.0$ & $<1.0$ & $<1.0$ & $<1.0$ \\
\hline 1,4-Dichlorobenzene & 1.0 & $<1.0$ & $<1.0$ & $<1.0$ & $<1.0$ \\
\hline 1,1-Dichloroethane & 1.0 & 283 & 48 & 4 & $<1.0$ \\
\hline 1,2-Dichloroethane & 1.0 & $<1.0$ & $<1.0$ & $<1.0$ & $<1.0$ \\
\hline 1,1-Dichloroethene & 1.0 & 8 & $<1.0$ & $<1.0$ & $<1.0$ \\
\hline Trans-1,2-Dichloroethene & 1.0 & $<1.0$ & $<1.0$ & $<1.0$ & $<1.0$ \\
\hline 1,2-Dichloropropane & 1.0 & $<1.0$ & $<1.0$ & $<1.0$ & $<1.0$ \\
\hline Cis-1,3-Dichloropropene & 1.0 & $<1.0$ & $<1.0$ & $<1.0$ & $<1.0$ \\
\hline Trans-1,3-Dichloropropene & 1.0 & $<1.0$ & $<1.0$ & $<1.0$ & $<1.0$ \\
\hline Methylene Chloride & 1.0 & $<1.0$ & $<1.0$ & $<1.0$ & $<1.0$ \\
\hline 1,1,2,2-Tetrachloroethane & 1.0 & $<1.0$ & $<1.0$ & $<1.0$ & $<1.0$ \\
\hline Tetrachloroethene & 1.0 & $<1.0$ & $<1.0$ & $<1.0$ & $<1.0$ \\
\hline 1,1,1-Trichloroethane & 1.0 & 306 & 162 & 28 & 14 \\
\hline 1,1,2-Trichloroethane & 1.0 & $<1.0$ & $<1.0$ & $<1.0$ & $<1.0$ \\
\hline Trichloroethene & 1.0 & 3 & 3 & 3 & 3 \\
\hline Trichlorofluoromethane & 1.0 & $<1.0$ & $<1.0$ & $<1.0$ & $<1.0$ \\
\hline Vinyl Chloride & 1.0 & $<1.0$ & $<1.0$ & $<1.0$ & $<1.0$ \\
\hline
\end{tabular}

Comments:

Reviewed By: 


\section{PSI Environmental Laboratories}

2595 E. Alrport Drive Tucson. Arizona 85706 602-573-6565 FAX 602.573-6550 1-800-PSI-LABS

ANALYTICAL REPORT

State of Arizona License $\# 0009$

Client:

Peroxidation Systems, Inc. 5151 E. Broadway, Suite 600

Tucson, Arizona 85711

Project Name: $\quad$ WJSA/DOE

Project Number: $\quad 345$

Sample ID: $\quad$ 102093-8 - $\quad 11$
Sample Type: Groundwater

Date Sampled: $\quad 10 / 17 / 93$

Date Received: 10/20/93

Date Analyzed: 11/5/93

Analyzed By: Deb Cartmell

EPA Method 602 - Purgeable Aromatics (G.C. - PID)

Reported in $\mu \mathrm{g} / \mathrm{L}(\mathrm{ppb})$

\begin{tabular}{|c|c|c|c|c|c|}
\hline Compounds & $\begin{array}{l}\text { Limit of } \\
\text { Detection }\end{array}$ & $17-0$ & $17-.5$ & $17-2$ & $17-3$ \\
\hline Benzene & 1.0 & 7 & 1 & 1 & $<1.0$ \\
\hline Toluene & 1.0 & 3 & $<1.0$ & $<1.0$ & $<1.0$ \\
\hline Chlorobenzene & 1.0 & $<1.0$ & $<1.0$ & $<1.0$ & $<1.0$ \\
\hline Ethyl Benzene & 1.0 & 2 & $<1.0$ & $<1.0$ & $<1.0$ \\
\hline $\mathrm{m}, \mathrm{p}-\mathrm{X}$ ylene & 1.0 & 4 & $<1.0$ & $<1.0$ & $<1.0$ \\
\hline $0-X y l e n e$ & 1.0 & 2 & $<1.0$ & $<1.0$ & $<1.0$ \\
\hline 1,2-Dichlorobenzene & 1.0 & $<1.0$ & $<1.0$ & $<1.0$ & $<1.0$ \\
\hline 1,3-Dichlorobenzene & 1.0 & $<1.0$ & $<1.0$ & $<1.0$ & $<1.0$ \\
\hline 1,4-Dichlorobenzene & 1.0 & $<1.0$ & $<1.0$ & $<1.0$ & $<1.0$ \\
\hline
\end{tabular}

Comments:

Reviewed By:

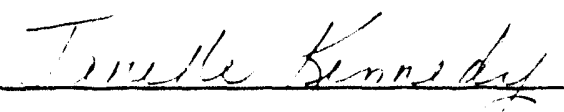

Date $12 / 15 / 93$ 
Client:

Project Name:

Project Number: $\quad 345$

Sample ID:
WJSA/DOE

Peroxidation Systems, Inc.

5151 E. Broadway, Suite 600

Tucson, Arizona 85711

$102093-12-16$
Sample Type: Groundwater

Date Sampled: $\quad 10 / 17 / 93$

Date Received: 10/20/93

Date Analyzed: 11/5/93

Analyzed By: Deb Cartmell

EPA Method 601 - Purgeable Halocarbons

Reported in $\mu \mathrm{g} / \mathrm{L}(\mathrm{ppb})$

\begin{tabular}{|c|c|c|c|c|c|c|}
\hline Compounds & $\begin{array}{l}\text { Limit of } \\
\text { Detection }\end{array}$ & 17-1-Infl & 17-1-L3 & 17-1-L6 & Field $10 / 18$ & Field 10/19 \\
\hline Bromodicloromethane & 1.0 & $<1.0$ & $<1.0$ & $<1.0$ & $<1.0$ & $<1.0$ \\
\hline Bromoform & 1.0 & $<1.0$ & $<1.0$ & $<1.0$ & $<1.0$ & $<1.0$ \\
\hline Bromomethane & 1.0 & $<1.0$ & $<1.0$ & $<1.0$ & $<1.0$ & $<1.0$ \\
\hline Carbon Tetrachloride & 1.0 & $<1.0$ & $<1.0$ & $<1.0$ & $<1.0$ & $<1.0$ \\
\hline Chlorobenzene & 1.0 & $<1.0$ & $<1.0$ & $<1.0$ & $<1.0$ & $<1.0$ \\
\hline Chloroethane & 1.0 & $<1.0$ & $<1.0$ & $<1.0$ & $<1.0$ & $<1.0$ \\
\hline Chloroform & 1.0 & $<1.0$ & $<1.0$ & $<1.0$ & $<1.0$ & $<1.0$ \\
\hline Chloromethane & 1.0 & $<1.0$ & $<1.0$ & $<1.0$ & $<1.0$ & $<1.0$ \\
\hline 2-Chloroethyl vinyl ether & 1.0 & $<1.0$ & $<1.0$ & $<1.0$ & $<1.0$ & $<1.0$ \\
\hline Dibromochloromethane & 1.0 & $<1.0$ & $<1.0$ & $<1.0$ & $<1.0$ & $<1.0$ \\
\hline 1,2-Dichlorobenzene & 1.0 & $<1.0$ & $<1.0$ & $<1.0$ & $<1.0$ & $<1.0$ \\
\hline 1,3-Dichlorobenzene & 1.0 & $<1.0$ & $<1.0$ & $<1.0$ & $<1.0$ & $<1.0$ \\
\hline 1,4-Dichlorobenzene & 1.0 & $<1.0$ & $<1.0$ & $<1.0$ & $<1.0$ & $<1.0$ \\
\hline 1,1-Dichloroethane & 1.0 & 8 & 3 & $<1.0$ & $<1.0$ & $<1.0$ \\
\hline 1,2-Dichloroethane & 1.0 & $<1.0$ & $<1.0$ & $<1.0$ & $<1.0$ & $<1.0$ \\
\hline 1,1-Dichloroethene & 1.0 & $<1.0$ & $<1.0$ & $<1.0$ & $<1.0$ & $<1.0$ \\
\hline Trans-1,2-Dichloroethene & 1.0 & $<1.0$ & $<1.0$ & $<1.0$ & $<1.0$ & $<1.0$ \\
\hline 1,2-Dichloropropane & 1.0 & $<1.0$ & $<1.0$ & $<1.0$ & $<1.0$ & $<1.0$ \\
\hline Cis-1,3-Dichloropropene & 1.0 & $<1.0$ & $<1.0$ & $<1.0$ & $<1.0$ & $<1.0$ \\
\hline Trans-1,3-Dichloropropene & 1.0 & $<1.0$ & $<1.0$ & $<1.0$ & $<1.0$ & $<1.0$ \\
\hline Methylene Chloride & 1.0 & $<1.0$ & $<1.0$ & $<1.0$ & $<1.0$ & $<1.0$ \\
\hline 1,1,2,2-Tetrachloroethane & 1.0 & $<1.0$ & $<1.0$ & $<1.0$ & $<1.0$ & $<1.0$ \\
\hline Tetrachloroethene & 1.0 & $<1.0$ & $<1.0$ & $<1.0$ & $<1.0$ & $<1.0$ \\
\hline 1,1,1-Trichloroethane & 1.0 & 78 & 61 & 42 & $<1.0$ & $<1.0$ \\
\hline 1,1,2-Trichloroethane & 1.0 & $<1.0$ & $<1.0$ & $<1.0$ & $<1.0$ & $<1.0$ \\
\hline Trichloroethene & 1.0 & $<1.0$ & $<1.0$ & $<1.0$ & $<1.0$ & $<1.0$ \\
\hline Trichlorofluorumethane & 1.0 & $<1.0$ & $<1.0$ & $<1.0$ & $<1.0$ & $<1.0$ \\
\hline Vinyl Chloride & 1.0 & $<1.0$ & $<1.0$ & $<1.0$ & $<1.0$ & $<1.0$ \\
\hline
\end{tabular}

Comments:

Reviewed By: 


\section{PSI Enviranmental Laboratories}

2595 E. Airport Drive Tucson, Arizona 85706 602.573-6565 FAX 602-573-6550 1-800.P51-LABS

ANALYTICAL REPORT

State of Arizona License $\# 0009$

\begin{tabular}{|c|c|c|c|}
\hline Client: & $\begin{array}{l}\text { Peroxidation Systems, Inc. } \\
5151 \text { E. Broadway, Suite } 600 \\
\text { Tucson, Arizona } 85711\end{array}$ & $\begin{array}{l}\text { Sample Type: } \\
\text { Date Sampled: }\end{array}$ & $\begin{array}{l}\text { Groundwater } \\
10 / 17 / 93\end{array}$ \\
\hline Project Name: & WJSA/DOE & Date Received: & $10 / 20 / 93$ \\
\hline Project Number: & 345 & Date Analyzed: & $11 / 5 / 93$ \\
\hline Sample ID: & $102093-12-16$ & Analyzed By: & Deb Cartmell \\
\hline
\end{tabular}

EPA Method 602 - Purgeable Aromatics (G.C. - PID)

Reported in $\mu \mathrm{g} / \mathrm{L}(\mathrm{ppb})$

\begin{tabular}{|l|c|c|c|c|c|c||}
\hline \multicolumn{1}{|c|}{ Compounds } & $\begin{array}{c}\text { Limit of } \\
\text { Detection }\end{array}$ & $17-1$-Infl & $17-1-\mathrm{L} 3$ & $17-1-$ L6 & Field 10/18 & Field 10/19 \\
\hline Benzene & 1.0 & 2 & $<1.0$ & $<1.0$ & $<1.0$ & $<1.0$ \\
Toluene & 1.0 & $<1.0$ & $<1.0$ & $<1.0$ & 2 & 2 \\
Chlorobenzene & 1.0 & $<1.0$ & $<1.0$ & $<1.0$ & $<1.0$ & $<1.0$ \\
Ethyl Benzene & 1.0 & $<1.0$ & $<1.0$ & $<1.0$ & $<1.0$ & $<1.0$ \\
m,p-Xylene & 1.0 & $<1.0$ & $<1.0$ & $<1.0$ & $<1.0$ & $<1.0$ \\
0-Xylene & 1.0 & $<1.0$ & $<1.0$ & $<1.0$ & $<1.0$ & $<1.0$ \\
1,2-Dichlorobenzene & 1.0 & $<1.0$ & $<1.0$ & $<1.0$ & $<1.0$ & $<1.0$ \\
1,3-Dichlorobenzene & 1.0 & $<1.0$ & $<1.0$ & $<1.0$ & $<1.0$ & $<1.0$ \\
1,4-Dichlorobenzene & 1.0 & $<1.0$ & $<1.0$ & $<1.0$ & $<1.0$ & $<1.0$ \\
\hline
\end{tabular}

Comments:

Reviewed By:

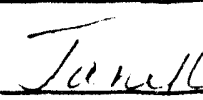


2595 E. Alrpart Drive Tucson, Arizona 85706 602-573-6565 FAX 602-573-6550 I-800-P5I-LA85 ANALYTICAL REPORT

State of Arizona License $\# 0009$

Client:

Project Name: WJSA/DOE

Project Number: $\quad 345$

Sample ID: NA
Sample Type: Groundwater

Date Sampled: See Table

Date Received: 8/20/93 - 9/15/93

Date Analyzed: 9/24/93

Analyzed By: Deb Cartmell

EPA Method 601 - Purgeable Halocarbons

Reported in $\mu \mathrm{g} / \mathrm{L}$ (ppb)

\begin{tabular}{|c|c|c|c|c|c|c|}
\hline Compounds & $\begin{array}{l}\text { Limit of } \\
\text { Detection }\end{array}$ & Trip 8/19 & Field $8 / 31$ & Field $9 / 1$ & Field $9 / 3$ & Field $9 / 14$ \\
\hline Bromodicloromethane & 1.0 & $<1.0$ & $<1.0$ & $<1.0$ & $<1.0$ & $<1.0$ \\
\hline Bromoform & 1.0 & $<1.0$ & $<1.0$ & $<1.0$ & $<1.0$ & $<1.0$ \\
\hline Bromomethane & 1.0 & $<1.0$ & $<1.0$ & $<1.0$ & $<1.0$ & $<1.0$ \\
\hline Carbon Tetrachloride & 1.0 & $<1.0$ & $<1.0$ & $<1.0$ & $<1.0$ & $<1.0$ \\
\hline Chlorobenzene & 1.0 & $<1.0$ & $<1.0$ & $<1.0$ & $<1.0$ & $<1.0$ \\
\hline Chloroethane & 1.0 & $<1.0$ & $<1.0$ & $<1.0$ & $<1.0$ & $<1.0$ \\
\hline Chloroform & 1.0 & $<1.0$ & $<1.0$ & $<1.0$ & $<1.0$ & $<1.0$ \\
\hline Chloromethane & 1.0 & $<1.0$ & $<1.0$ & $<1.0$ & $<1.0$ & $<1.0$ \\
\hline 2-Chloroethyl vinyl ether & 1.0 & $<1.0$ & $<1.0$ & $<1.0$ & $<1.0$ & $<1.0$ \\
\hline Dibromochloromethane & 1.0 & $<1.0$ & $<1.0$ & $<1.0$ & $<1.0$ & $<1.0$ \\
\hline 1,2-Dichlorobenzene & 1.0 & $<1.0$ & $<1.0$ & $<1.0$ & $<1.0$ & $<1.0$ \\
\hline 1,3-Dichlorobenzene & 1.0 & $<1.0$ & $<1.0$ & $<1.0$ & $<1.0$ & $<1.0$ \\
\hline 1,4-Dichlorobenzene & 1.0 & $<1.0$ & $<1.0$ & $<1.0$ & $<1.0$ & $<1.0$ \\
\hline 1,1-Dichloroethane & 1.0 & $<1.0$ & $<1.0$ & $<1.0$ & $<1.0$ & $<1.0$ \\
\hline 1,2-Dichloroethane & 1.0 & $<1.0$ & $<1.0$ & $<1.0$ & $<1.0$ & $<1.0$ \\
\hline 1,1-Dichloroethtse & 1.0 & $<1.0$ & $<1.0$ & $<1.0$ & $<1.0$ & $<1.0$ \\
\hline Trans-1,2-Dichloroethene & 1.0 & $<1.0$ & $<1.0$ & $<1.0$ & $<1.0$ & $<1.0$ \\
\hline 1,2-Dichloropropane & 1.0 & $<1.0$ & $<1.0$ & $<1.0$ & $<1.0$ & $<1.0$ \\
\hline Cis-1,3-Dichloropropene & 1.0 & $<1.0$ & $<1.0$ & $<1.0$ & $<1.0$ & $<1.0$ \\
\hline Trans-1,3-Dichloropropene & 1.0 & $<1.0$ & $<1.0$ & $<1.0$ & $<1.0$ & $<1.0$ \\
\hline Methylene Chloride & 1.0 & $<1.0$ & $<1.0$ & $<1.0$ & $<1.0$ & $<1.0$ \\
\hline 1,1,2,2-Tetrachloroethane & 1.0 & $<1.0$ & $<1.0$ & $<1.0$ & $<1.0$ & $<1.0$ \\
\hline Tetrachloroethene & 1.0 & $<1.0$ & $<1.0$ & $<1.0$ & $<1.0$ & $<1.0$ \\
\hline 1,1,1-Trichloroethane & 1.0 & $<1.0$ & $<1.0$ & $<1.0$ & $<1.0$ & $<1.0$ \\
\hline 1,1,2-Trichloroethane & 1.0 & $<1.0$ & $<1.0$ & $<1.0$ & $<1.0$ & $<1.0$ \\
\hline Trichloroethene & 1.0 & $<1.0$ & $<1.0$ & $<1.0$ & $<1.0$ & $<1.0$ \\
\hline Trichlorofluoromethane & 1.0 & $<1.0$ & $<1.0$ & $<1.0$ & $<1.0$ & $<1.0$ \\
\hline Vinyl Chloride & 1.0 & $<1.0$ & $<1.0$ & $<1.0$ & $<1.0$ & $<1.0$ \\
\hline
\end{tabular}

Comments:

Reviewed By:

Ta1, 11

Date 17/15/7s 


\section{ANALYTICAL REPORT}

State of Arizona License \#0009

\begin{tabular}{|c|c|c|c|c|c|c|}
\hline Client: & \multicolumn{2}{|c|}{$\begin{array}{l}\text { Peroxidation Systems, Inc. } \\
5151 \text { E. Broadway, Suite } 600 \\
\text { Tucson, Arizona } 85711\end{array}$} & $\begin{array}{l}\text { mple Type: } \\
\text { te Sampled: }\end{array}$ & $\begin{array}{l}\text { Groundv } \\
\text { See Tabl }\end{array}$ & & \\
\hline Project Name: & \multicolumn{2}{|l|}{ WJSA/DOE } & Date Received: & \multicolumn{2}{|c|}{$8 / 20 / 93-9 / 15 / 93$} & \\
\hline Project Number: & \multicolumn{2}{|l|}{345} & te Analyzed & \multicolumn{2}{|l|}{$9 / 24 / 93$} & \\
\hline Sample ID: & NA & & alyzed By: & \multicolumn{2}{|c|}{ Deb Cartmell } & \\
\hline \multicolumn{7}{|c|}{$\begin{array}{c}\text { EPA Method } 602 \text { - Purgeable Aromatics (G.C. - PID) } \\
\text { Reported in } \mu \mathrm{g} / \mathrm{L}(\mathrm{ppb})\end{array}$} \\
\hline Compounds & $\begin{array}{l}\text { Limit of } \\
\text { Detection }\end{array}$ & Trip 8/19 & Field $8 / 31$ & Field $9 / 1$ & Field 9/3 & Field $9 / 14$ \\
\hline $\begin{array}{l}\text { Benzene } \\
\text { Toluene } \\
\text { Chlorobenzene } \\
\text { Ethyl Benzene } \\
\text { m,p-Xylene } \\
\text { 0-Xylene } \\
\text { 1,2-Dichlorobenzene } \\
\text { 1,3-Dichlorobenzene } \\
\text { 1,4-Dichlorobenzene }\end{array}$ & $\begin{array}{l}1.0 \\
1.0 \\
1.0 \\
1.0 \\
1.0 \\
1.0 \\
1.0 \\
1.0 \\
1.0\end{array}$ & $\begin{array}{l}1.0 \\
<1.0 \\
<1.0 \\
<1.0 \\
<1.0 \\
<1.0 \\
<1.0 \\
<1.0 \\
<1.0\end{array}$ & $\begin{array}{l}<1.0 \\
<1.0 \\
<1.0 \\
<1.0 \\
<1.0 \\
<1.0 \\
<1.0 \\
<1.0 \\
<1.0\end{array}$ & $\begin{array}{l}<1.0 \\
<1.0 \\
<1.0 \\
<1.0 \\
<1.0 \\
<1.0 \\
<1.0 \\
<1.0 \\
<1.0\end{array}$ & $\begin{array}{l}<1.0 \\
<1.0 \\
<1.0 \\
<1.0 \\
<1.0 \\
<1.0 \\
<1.0 \\
<1.0 \\
<1.0\end{array}$ & $\begin{array}{l}<1.0 \\
<1.0 \\
<1.0 \\
<1.0 \\
<1.0 \\
1.6 \\
<1.0 \\
<1.0 \\
<1.0\end{array}$ \\
\hline
\end{tabular}

Comments:

Reviewed By:

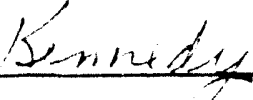

Date $12 / 15 / 9=$ 
CHAIN OF CUSTODY FORM

Page 1 of 2

Aspen Environmental Laboratory

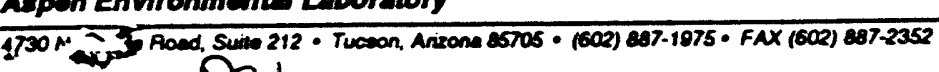

COMPANY PSI

ADDRESS

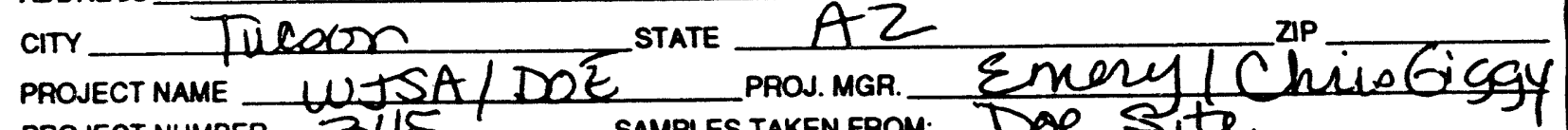

PROJECT NUMBER 345

$\square 24$ HOUR RUSH (

SAMPLER'S (SIGNATURE) S Surcharge) D 48 HOUR RUSH (

* Surcharge)

$$
\text { }
$$

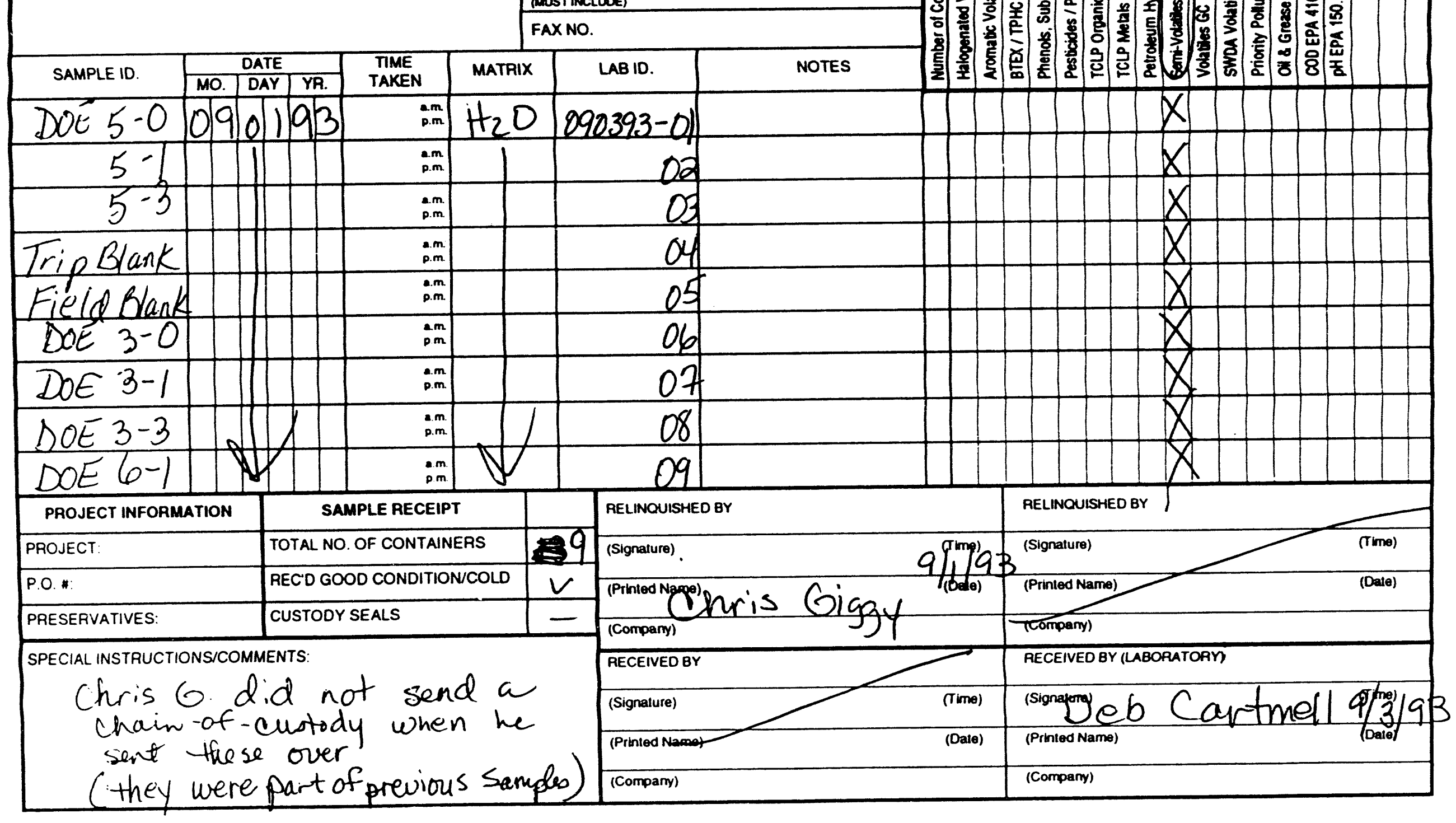




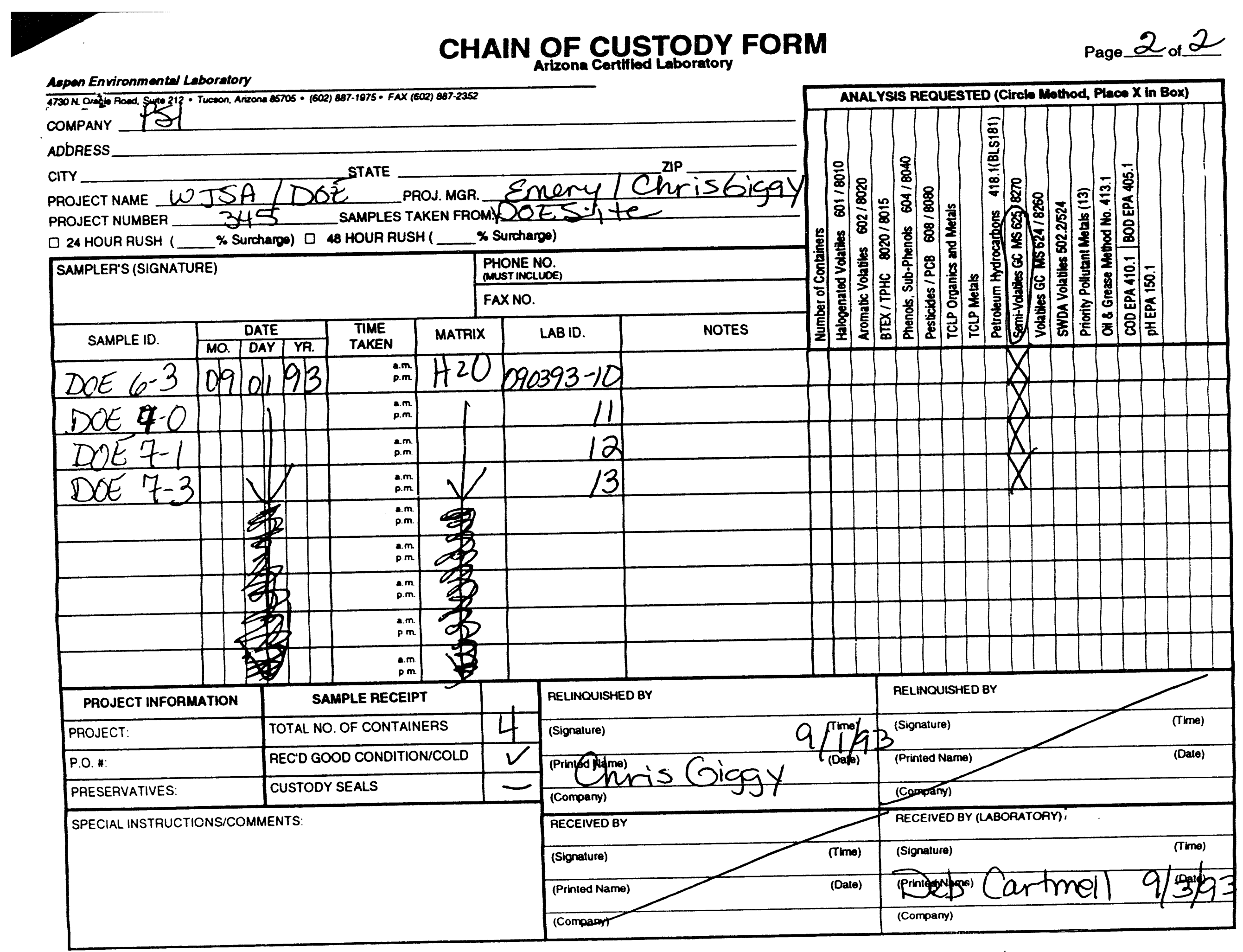




\section{PSI Environmental Laboratories}

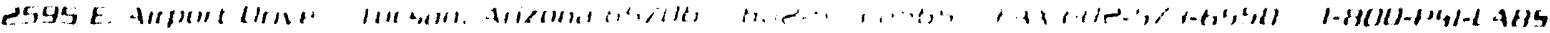

ANALYTICAL REPORT

State of Arizona License $\$ 0009$

Client:

Peroxidation Systems Inc.

5151 E. Rroadway, Suite 600

Tucson, AZ 85711

Project Number: 0345

P.O. Number: None
Sample I.D.: $\quad 5-0$

Laboratory I.D.: (090393-01

Sample Type: Water

Date Sampled: $\quad 09 / 01 / 1993$

Date Received: 09/03/1993

Date Analyzed: $\quad 10 / 12 / 1993$

Analyzed By: $\quad$ See Comments

Base Neutral/Acid Semivolatile Oroanic Compounds

EPA Methods 3510/8270

Base Neutral Analyte

$\mathrm{N}$-Nitrosodimethylamine

Bis(2-chloroethyl) Ether

1,2-Dichlorobenzene

1,3-Dichlorobenzene

1,4-Dichlorobenzene

Bis(2-chloroisopropyl) Ether

$N$-Nitrosodi-n-propylamine

Hexachloroethane

Nitrobenzene

Isophorone

Bis(2-chloroethoxy) methane

1,2,4-Trichlorobenzene

Naphthalene

4-Chloroaniline

Hexachlorobutadiene

2-Methylnaphthalene

Hexachlorocyclopentadiene

2-Chloronaphthalene

2-Nitroaniline

Dimethyl Phthalate

Acenaphthylene

3-Nitroaniline

Acenaphthene

Dibenzofuran

2,4-Dinitrotoluene

Acid Analyte

Phenol

2-Chiorophenol

Benzyl Alcohol

2-Methyiphenol

3- and 4-Methylphenol'

2-Nitrophenol

2,4-Dimethylphend

Benzoic Acid

MRL
10
10
10
10
10
10
10
10
10
10
10
10
10
10
10
10
20
10
40
10
10
40
10
10
10

$\mu \mathrm{O} \Omega(\mathrm{ppb})$

Result

ND

ND

ND

ND

ND

ND

ND

ND

ND

ND

ND

ND

ND

ND

ND

ND

ND

ND

ND

ND

ND

ND

ND

ND

ND

MRL**

Result

ND

ND

ND

ND

ND

ND

ND

ND
Base Neutral Analrte

2,6-Dinitrotoluene

Diethyl Phthalate

4-Chlorophenyl Phenyl Ether

Fluorene

4-Nitroaniline

$\mathrm{N}$-Nitrosodiphenylamine

4-Bromophenyl Phenyl Ether

Hexachlorobenzene

Phenanthrene

Anthracene

Di-n-butyl Phthalate

Fluoranthene

Pyrene

Butylbenzyl Phthalate

3,3'-Dichlorobenzidine

Benz(a)anthracene

Bis(2-ethylhexyl) Phthalate

Chrysene

Di-n-octyl Phthalate

Benzo(b)fluoranthene

Benzo(k)fluoranthene

Benzola)pyrene

Indeno(1,2,3-c,d)pyrene

Dibenz(a,h)anthracene

Benzolo, $h, i)$ perylene

Acid Analyte

2,4-Dichlorophenol

4-Chloro-3-methylpheno!

2,4,6-Trichlorophenol

2,4,5-Trichlorophenol

2,4-Dinitrophenol

4-Nitrophenol

2-Methyt-4,6-dinitrophenol

Pentachlorophenol

$\begin{array}{cc}\text { MRL.* } & \text { Result } \\ 10 & \text { ND } \\ 10 & \text { ND } \\ 10 & \text { ND } \\ 10 & \text { ND } \\ 100 & \text { ND } \\ 10 & \text { ND } \\ 10 & \text { ND } \\ 10 & \text { ND } \\ 10 & \text { ND } \\ 10 & \text { ND } \\ 10 & \text { ND } \\ 10 & \text { ND } \\ 10 & \text { ND } \\ 10 & \text { ND } \\ 100 & \text { ND } \\ 10 & \text { ND } \\ 10 & 28 \\ 10 & \text { ND } \\ 10 & \text { ND } \\ 10 & \text { ND } \\ 10 & \text { ND } \\ 10 & \text { ND } \\ 10 & \text { ND } \\ 10 & \text { ND } \\ 10 & \text { ND }\end{array}$

MRL“- Result

$\begin{array}{rr}10 & \text { ND } \\ 10 & \text { ND } \\ 10 & \text { ND } \\ 10 & \text { ND } \\ 100 & \text { ND } \\ 100 & \text { ND } \\ 40 & \text { ND } \\ 60 & \text { ND }\end{array}$

- Extraction performed by PSI Environmental Laboratories.

MRLMethod Reporting Limit

- MRL is elevated. Sample extract final volume was twice the value normally obtained by Golden State/CAS Laboratories, Inc.

ND None Detected at or above the method reporting limit

- Quantified as 4-methylphenol.

Comments:

Golden State/CAS Laboratories, Inc.
Reviewed By:

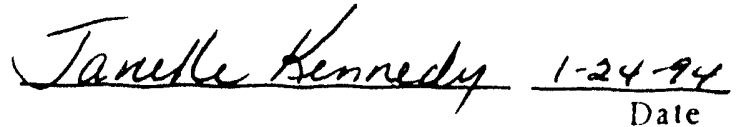




\section{PSI Enviranmental Láuarataries}

2595 E. Airport Orive

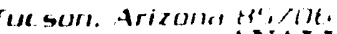

ANALYTICAL REPORT

State of Arizona License $\$ 0009$

Client:

Peroxidation Systems Inc.

5151 E. Broadway, Suite 600

Tucson, AZ 85711

Project Name: DOE/WJSA

Project Number: 0345

P.O. Number: None

\author{
Sample I.D.: $\quad 5-1$ \\ Laboratory I.D.: 090393-02 \\ Sample Type: Water \\ Date Sampled: 09/01/1993 \\ Date Received: 09/03/1993 \\ Date Analyzed: $\quad 10 / 12 / 1993$
}

Analyzed By: See Comments

Base Neutral/Acid Semivolatile Organic Compounds EPA Methods 3510/8270 $\mu \mathrm{g} \Omega(\mathrm{ppb})$

Base Neutral Analyte

N-Nitrosodimethylamine

Bis(2-chloroethyl) Ether

1,2-Dichlorobenzene

1,3-Dichlorobenzene

1,4-Dichlorobenzene

Bis(2-chloroisopropyl) Ether

$N$-Nitrosodi-n-propylamine

Hexachloroethane

Nitrobenzene

Isophorone

Bis(2-chloroethoxy) methane

1,2,4-Trichlorobenzene

Naphthalene

4-Chloroaniline

Hexachlorobutadierie

2-Miethylnaphthalene

Hexachlorocyclopentadiene

2-Chloronaphthalene

2-Nitroaniline

Dimethyl Phthalate

Acenaphthylene

3-Nitroaniline

Acenaphthene

Dibenzofuran

2,4-Dinitrotoluene

Result

ND

ND

ND

ND

ND

ND

ND

ND

ND

ND

ND

ND

ND

ND

ND

ND

ND

ND

ND

ND

ND

ND

ND

ND

ND

Acid Analyte

Phenol

2-Chlorophenol

Benayl Alcohol

2-Methylphend

3- and 4-Methylphenol"

2-Nitrophenol

2,4-Dimethylphenol

Benzoic Acid
Base Neutral Analyte

2,6-Dinitrotoluene

Diethyl Phthalate

4-Chlorophenyl Phenyl Ether

Flúorene

4-Nitroaniline

N-Nitrosodiphenylamine

4-Bromophenyl Phenyl Ether

Hexachlorobenzene

Phenanthrene

Anthracene

Di-n-butyl Phthalate

Fluoranthene

Pyrene

Butylbenzyl Phthalate

3,3'-Dichlorobenzidine

Benz(a)anthracene

Bis(2-ethythexy) Phthalate

Chrysene

Di-n-octyl Phthalate

Benzolblfluoranthene

Benzo/kJfluoranthene

Benzola)pyrene

Indenol1,2,3-c,dlpyrene

Dibenz(a,h)anthracene

Benzolo, $h, i)$ perylene

Acid Analyte

2.4-Dichlorophenol

4-Chioro-3-methyiphenol

2,4,6-Trichlorophenol

2,4,5-Trithlorophenol

2,4-Dinitrophenol

4-Nitrophenol

2-Mothy-4,6-dinitrophenol

Pentachlorophenol

$\begin{array}{cc}\text { MRL } & \text { Result } \\ 10 & \text { ND } \\ 10 & \text { ND } \\ 10 & \text { ND } \\ 10 & \text { ND } \\ 100 & \text { ND } \\ 10 & \text { ND } \\ 10 & \text { ND } \\ 10 & \text { ND } \\ 10 & \text { ND } \\ 10 & \text { ND } \\ 10 & \text { ND } \\ 10 & \text { ND } \\ 10 & \text { ND } \\ 10 & \text { ND } \\ 100 & \text { ND } \\ 10 & \text { ND } \\ 10 & \text { ND } \\ 10 & \text { ND } \\ 10 & \text { ND } \\ 10 & \text { ND } \\ 10 & \text { ND } \\ 10 & \text { ND } \\ 10 & \text { ND } \\ 10 & \text { ND } \\ 10 & \text { ND }\end{array}$

MRL • Result

$\begin{array}{rr}10 & \text { ND } \\ 10 & \text { ND } \\ 10 & \text { ND } \\ 10 & \text { ND } \\ 100 & \text { ND } \\ 100 & \text { ND } \\ 40 & \text { ND } \\ 60 & \text { ND }\end{array}$

- Exträction performed by PSI Environmental Laboratories.

MRLMothod Reporting Limit

- MRL is elevated. Sample extract final volume was twice the value normally obtained by Golden State/CAS Laboratories, Inc.

ND None Detected at or above the method reporting limit

- Quantified as 4-methytphenol.

Comments:

Golden State/CAS Laboratories, In 6925 Canoga Avenue

Canoga Park, CA 91303
Reviewed By:

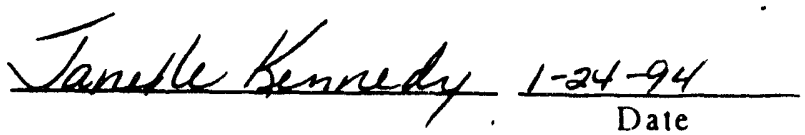




\section{PSI Environmental Laboratories}

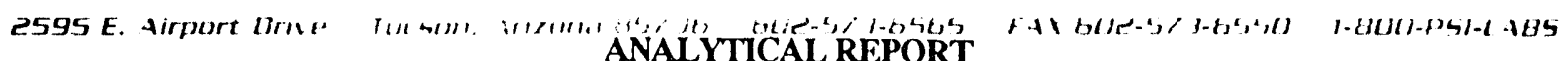

State of Arizona License $\$ 0009$

Client:

Peroxidation Systems Inc.

5151 E. Broadway, Suite 600

Tucson, AZ 85711

Sample I.D.: $\quad 5-3$

Laboratory I.D.: 090393-03

Sample Type: Water

Date Sampled: $\quad 09 / 01 / 1993$

Project Name: DOE/WJSA

Date Received: $\quad 09 / 03 / 1993$

Project Number: 0345

Date Analyzed: $\quad 10 / 12 / 1993$

P.O. Number: None

Analyzed By: See Comments Base Neutral/Acid Semivolatile Organic Compounds

EPA Methods 3510/8270 $\mu \mathrm{O} \Omega(\mathrm{ppb})$

Base Neutral Analyte

$\mathrm{N}$-Nitrosodimethylamine

Bis(2-chloroethyl). Ether

1,2-Dichlorobenzene

1,3-Dichlorobenzene

1,4-Dichlorobenzene

Bis(2-chloroisopropyl) Ether

$\mathrm{N}$-Nitrosodi-n-propylamine

Hexachloroethane

Nitrobenzene

Isophorone

Bis(2-chloroethoxy) methane

1,2,4-Trichl robenzene

Naphthalene

4-Chloroaniline

Hexachlorobutadiene

2-Methyinaphthalene

Hexachlorocyclopentadiene

2-Chloronaphthalene

2-Nitroaniline

Dimethyt Phthalate

Acenaphthylene

3-Nitroaniline

Acenaphthene

Dibenzofuran

2,4-Dinitrotoluene

Acid Analyto

Phenol

2-Chlorophenol

Benzyl Alcohol

2-Methylphenol

3- and 4-Methylphenol"

2-Nitrophenol

2,4-Dimethylphenal

Benzoic Acid

$\begin{array}{cc}\text { MRL** } & \text { Result } \\ 10 & \text { ND } \\ 10 & \text { ND } \\ 10 & \text { ND } \\ 10 & \text { ND } \\ 10 & \text { ND } \\ 10 & \text { ND } \\ 10 & \text { ND } \\ 10 & \text { ND } \\ 10 & \text { ND } \\ 10 & \text { ND } \\ 10 & \text { ND } \\ 10 & \text { ND } \\ 10 & \text { ND } \\ 10 & \text { ND } \\ 10 & \text { ND } \\ 10 & \text { ND } \\ 20 & \text { ND } \\ 10 & \text { ND } \\ 40 & \text { ND } \\ 10 & \text { ND } \\ 10 & \text { ND } \\ 40 & \text { ND } \\ 10 & \text { ND } \\ 10 & \text { ND } \\ 10 & \text { ND } \\ 10 & \end{array}$

MRL* Resuit-

$\begin{array}{rr}10 & \text { ND } \\ 10 & \text { ND } \\ 20 & \text { ND } \\ 10 & \text { ND } \\ 10 & \text { ND } \\ 10 & \text { ND } \\ 10 & \text { ND } \\ 100 & \text { ND }\end{array}$

Base Neutral Analrte

2,6-Dinitrotoluene

Diethyl Phthalate

4-Chlorophenyl Phenyl Ether

Fluorene

4-Nitroaniline

$\mathrm{N}$-Nitrosodiphenylamine

4-Bromophenyl Phenyl Ether

Hexachlorobenzene

Phenanthrene

Anthracene

Di-n-butyl Phthalate

Fluoranthene

Pyrene

Butylbenzyl Phthalate

3,3'-Dichlorobenzidine

Benz(a)anthracene

Bis(2-ethylhexyl) Phthalate

Chrysene

Di-n-octyl Phthalate

Benzolbifluoranthene

Benzo(k)fluoranthene

Benzolalpyrene

Indeno(1,2,3-c,d)pyrene

Dibenz (a,h)anthracene

Benzo(g,h,i)perylene

Acid Analyto

2,4-Dichlorophenol

4-Chloro-3-methylphenol

2,4,6-Trichlorophenol

2,4,5-Trichlorophenol

2,4-Dinitrophenol

4-Nitrophenol

2-Methyt-4,6-dinitrophenol

Pentachlorophenol

$\begin{array}{cc}\text { MRL* } & \text { Resul } \\ 10 & \text { ND } \\ 10 & \text { ND } \\ 10 & \text { ND } \\ 10 & \text { ND } \\ 100 & \text { ND } \\ 10 & \text { ND } \\ 10 & \text { ND } \\ 10 & \text { ND } \\ 10 & \text { ND } \\ 10 & \text { ND } \\ 10 & \text { ND } \\ 10 & \text { ND } \\ 10 & \text { ND } \\ 10 & \text { ND } \\ 100 & \text { ND } \\ 10 & \text { ND } \\ 10 & \text { ND } \\ 10 & \text { ND } \\ 10 & \text { ND } \\ 10 & \text { ND } \\ 10 & \text { ND } \\ 10 & \text { ND } \\ 10 & \text { ND } \\ 10 & \text { ND } \\ 10 & \text { ND } \\ 10 & \end{array}$

MRL * Result

$\begin{array}{rr}10 & \text { ND } \\ 10 & \text { ND } \\ 10 & \text { ND } \\ 10 & \text { ND } \\ 100 & \text { ND } \\ 100 & \text { ND } \\ 40 & \text { ND } \\ 60 & \text { ND }\end{array}$

- Extraction performed by PSI Environmental Laboratories.

MRLMethod Reporting Limit

- MRL is elevated. Sample extract final volume was twice the value normally obtained by Golden State/CAS Laboratories, Inc.

ND None Detected at or above the method reporting limit

- Quantified as 4-methylphenol.

Reviewed By:

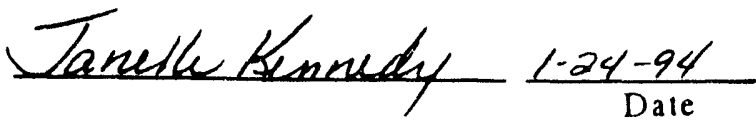




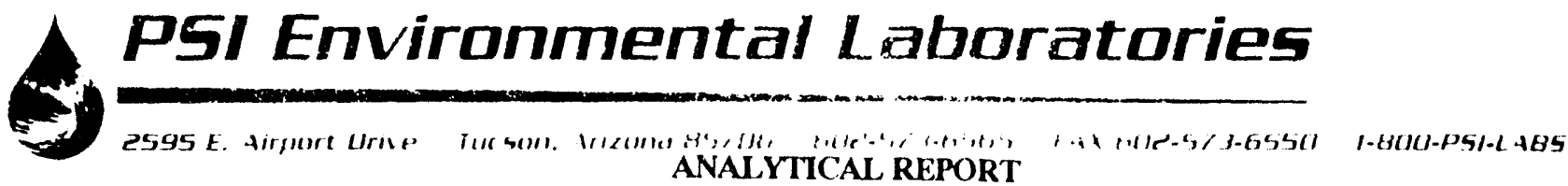

State of Arizona License $\# 0009$

Client:

Peroxidation Systems Inc.

Sample I.D.: $\quad 3-0$

5151 E. Broadway, Suite 600

Tucson, AZ 85711

Laboratory I.D.: 090393.06

Sample Type: Water

Date Sampled: $\quad 09 / 01 / 1993$

Project Name: DOE/WJSA

Date Received: 09/03/1993

Project Number: 0345

Date Analyzed: $\quad 10 / 12 / 1993$

P.O. Number: None

Analyzed By: See Comments

Base Neutral/Acid Semivolatile Organic Compounds EPA Methods 3510/8270

Base Neutral Analyte

$\mathrm{N}$-Nitrosodimethylamine

Bis(2-chloroethyl) Ether

1.2-Dichlorobenzene

1,3-Dichlorobenzene

1,4-Dichlorobenzene

Bis(2-chloroisopropyl) Ether

$\mathrm{N}$-Nitrosodi-n-propylamine

Hexachloroethane

Nitrobenzene

Isophorone

Bis(2-chloroethoxy) methane

1,2,4-Trichlorobenzene

Naphthalene

4-Chloroaniline

Hexachlorobutadiene

2-Methyinaphthalene

Hexachlorocyclopentadiene

2-Chloronaphthalene

2-Nitroaniline

Dimethyl Phthalate

Acenaphthylene

3-Nitroaniline

Acenaphthene

Dibenzofuran

2,4-Dinitrotoluene

Acid Analrte

Phenol

2-Chlorophenol

Benzyl Alcohol

2-Methyiphenol

3- and 4-Methyiphenol"

2-Nitrophenol

2,4-Dimethylphenol

MRL $\bullet$

10

10

10

10

10

10

10

10

10

10

10

10

10

10

10

10

20

10

40

10

10

40

10

10

10

Result $\mu \mathrm{g} / \mathrm{ppb})$

RDes

ND

ND

ND

ND

ND

ND

ND

ND

ND

ND

ND

ND

ND

ND

ND

ND

ND

ND

ND

ND

ND

ND

ND

ND

MRL * Result

10

10

20

10

10

10

10

100

ND

ND

ND

ND

ND

ND

ND

NO
Base Neutral Analyte

2,6-Dinitrotoluene

Diethyl Phthalate

4-Chlorophenyl Phenyl Ether

Fluorene

4-Nitroaniline

N-Nitrosodiphenylamine

4-Bromophenyl Phenyl Ether

Hexachlorobenzene

Phenanthrene

Anthracene

Di-n-butyl Phthalate

Fluoranthene

Pyrene

Butylbenzyl Phthalate

3,3'-Dichlorobenzidine

Benz(a)anthracene

Bis(2-ethylhexyl) Phthalate

Chrysene

Di-n-octyl Phthalate

Benzo(b)fluoranthene

Benzo(k)fluoranthene

Benzo(a)pyrene

Indeno(1,2,3-c,d)pyrene

Dibenz(a,h)anthracene

Benzo(g,h,i)perylene

Acid Analyte

2,4-Dichlorophenol

4-Chloro-3-methylphenol

2,4,6-Trichlorophenol

2,4,5-Trichlorophenol

2,4-Dinitrophenol

4-Nitrophenol

2-Methyl-4,6-dinitrophenol

Pentachlorophenol

$\begin{array}{rc}\text { MRL・ } & \text { Result } \\ 10 & \text { ND } \\ 10 & \text { ND } \\ 10 & \text { ND } \\ 10 & \text { ND } \\ 100 & \text { ND } \\ 10 & \text { ND } \\ 10 & \text { ND } \\ 10 & \text { ND } \\ 10 & \text { ND } \\ 10 & \text { ND } \\ 10 & \text { ND } \\ 10 & \text { ND } \\ 10 & \text { ND } \\ 10 & \text { ND } \\ 100 & \text { ND } \\ 10 & \text { ND } \\ 10 & \text { ND } \\ 10 & \text { ND } \\ 10 & \text { ND } \\ 10 & \text { ND } \\ 10 & \text { ND } \\ 10 & \text { ND } \\ 10 & \text { ND } \\ 10 & \text { ND } \\ 10 & \text { ND }\end{array}$

MRL * Result

$\begin{array}{rr}10 & \text { ND } \\ 10 & \text { ND } \\ 10 & \text { ND } \\ 10 & \text { ND } \\ 100 & \text { ND } \\ 100 & \text { ND } \\ 40 & \text { ND } \\ 60 & \text { ND }\end{array}$

- Extractión performed by PSI Environmental Laboratories.

MRLMethod Reporting Limit

- MRL is elevated. Sample extract final volume was twice the value normally obtained by Golden State/CAS Laboratories, Inc.

ND None Detected at or above the method reporting limit

- Quantified as 4-methylphenol. 


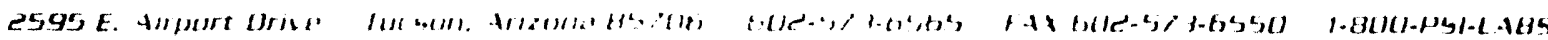

ANALYTICAL REPORT

State of Arizona License $\$ 0009$

Client:

Peroxidation Systems Inc.

Sample I.D.: $\quad 3-3$

5151 E. Broadway, Suite 600

Tucson, AZ 85711

Laboratory I.D.: 090393-08

$\begin{array}{llll} & & \text { Sample Type: } & \text { Water } \\ \text { Project Name: } & \text { DOE/WJSA } & \text { Date Sampled: } & 09 / 01 / 1993 \\ \text { Project Number: } & 0345 & \text { Date Received: } & 09 / 03 / 1993 \\ \text { P.O. Number: } & \text { None } & \text { Date Analyzed: } & \text { 10/13/1993 }\end{array}$

Base Neutral/Acid Semivolatile Organic Compounds EPA Methods $3510 / 8270$

$\mu \mathrm{g} \Omega(\mathrm{ppb})$

Base Neutral Analyte
N-Nitrosodimethylamine
Bis(2-chloroethyl) Ether
1,2-Dichlorobenzene
1,3-Dichlorobenzene
1,4-Dichlorobenzene
Bis(2-chloroisopropyl) Ether
N-Nitrosodi-n-propylamine
Hexachloroethane
Nitrobenzene
Isophorone
Bis(2-chloroethoxy) methane
1,2,4-Trichlorobenzene
Naphthalene
4-Chloroaniline
Hexachlorobutadiene
2-Methylnaphthalene
Hexachlorocyclopentadiene
2-Chloronaphthalene
2-Nitroaniline
Dimethyl Phthalate
Acenaphthylene
3-Nitroaniline
Acenaphthene
Dibenzofuran
2,4-Dinitrotoluene
Acid Analyte
Phenol
2-Chlorophenol
Benzyl Alcohol
2-Methylphenol
3- and 4-Methylphenol
2-Nitrophenol
2,4-Dimethylphenol
Benzoic Acid
(n)

\begin{tabular}{|c|c|c|}
\hline $\begin{array}{r}\text { MRL } \\
5 \\
5 \\
5 \\
5 \\
5 \\
5 \\
5 \\
5 \\
5 \\
5 \\
5 \\
5 \\
5 \\
5 \\
5 \\
5 \\
10 \\
5 \\
20 \\
5 \\
5 \\
20 \\
5 \\
5 \\
5\end{array}$ & $\begin{array}{l}\text { Result } \\
\text { ND } \\
\text { ND } \\
\text { ND } \\
\text { ND } \\
\text { ND } \\
\text { ND } \\
\text { ND } \\
\text { ND } \\
\text { ND } \\
\text { ND } \\
\text { ND } \\
\text { ND } \\
\text { ND } \\
\text { ND } \\
\text { ND } \\
\text { ND } \\
\text { ND } \\
\text { ND } \\
\text { ND } \\
\text { ND } \\
\text { ND } \\
\text { ND } \\
\text { ND } \\
\text { ND } \\
\text { ND }\end{array}$ & $\begin{array}{l}\text { Base Neutral Analyte } \\
\text { 2,6-Dinitrotoluene } \\
\text { Diethyl Phthalate } \\
\text { 4-Chlorophenyl Phenyl Ether } \\
\text { Fluorene } \\
\text { 4-Nitroaniline } \\
\text { N-Nitrosodiphenylamine } \\
\text { 4-Bromophenyl Phenyl Ether } \\
\text { Hexachlorobenzene } \\
\text { Phenanthrene } \\
\text { Anthracene } \\
\text { Di-n-butyl Phthalate } \\
\text { Fluoranthene } \\
\text { Pyrene } \\
\text { Butylbenzyl Phthalate } \\
\text { 3,3'-Dichlorobenzidine } \\
\text { Benz(a)anthracene } \\
\text { Bis(2-ethylhexyl) Phthalate } \\
\text { Chrysene } \\
\text { Di-n-octyl Phthalate } \\
\text { Benzolb)fluoranthene } \\
\text { Benzolk)fluoranthene } \\
\text { Benzola)pyrene } \\
\text { Indenol1,2,3-c,d)pyrene } \\
\text { Dibenz(a,hlanthracene } \\
\text { Benzolo,h,i)perylene }\end{array}$ \\
\hline MRL & Result & Acid Analyte \\
\hline $\begin{array}{r}5 \\
5 \\
10 \\
5 \\
5 \\
5 \\
5 \\
50\end{array}$ & $\begin{array}{l}\text { ND } \\
\text { ND } \\
\text { ND } \\
\text { ND } \\
\text { ND } \\
\text { ND } \\
\text { ND } \\
\text { ND }\end{array}$ & $\begin{array}{l}\text { 2,4-Dichlorophenol } \\
\text { 4-Chloro-3-methylphenol } \\
\text { 2,4,6-Trichlorophenol } \\
\text { 2,4,5-Trichlorophenol } \\
\text { 2,4-Dinitrophenol } \\
\text { 4-Nitrophenol } \\
\text { 2-Methyl-4,6-dinitrophenol } \\
\text { Pentachlorophenol }\end{array}$ \\
\hline
\end{tabular}

$\begin{array}{rc}\text { MRL } & \text { Result } \\ \mathbf{5} & \text { ND } \\ \mathbf{5} & \text { ND } \\ \mathbf{5} & \text { ND } \\ \mathbf{5} & \text { ND } \\ 20 & \text { ND } \\ \mathbf{5} & \text { ND } \\ \mathbf{5} & \text { ND } \\ \mathbf{5} & \text { ND } \\ \mathbf{5} & \text { ND } \\ \mathbf{5} & \text { ND } \\ \mathbf{5} & \text { ND } \\ \mathbf{5} & \text { ND } \\ \mathbf{5} & \text { ND } \\ \mathbf{5} & \text { ND } \\ \mathbf{2 0} & \text { ND } \\ \mathbf{5} & \text { ND } \\ \mathbf{5} & \text { ND } \\ \mathbf{5} & \text { ND } \\ \mathbf{5} & \text { ND } \\ \mathbf{5} & \text { ND } \\ \mathbf{5} & \text { ND } \\ \mathbf{5} & \text { ND } \\ \mathbf{5} & \text { ND } \\ \mathbf{5} & \text { ND } \\ \mathbf{5} & \text { ND } \\ & \\ \mathbf{M R L} & \text { Result } \\ \mathbf{5} & \text { ND } \\ \mathbf{5} & \text { ND } \\ \mathbf{5} & \text { ND } \\ \mathbf{5} & \text { ND } \\ \mathbf{5 0} & \text { ND } \\ \mathbf{5 0} & \text { ND } \\ \mathbf{2 0} & \text { ND } \\ \mathbf{3 0} & \text { ND }\end{array}$

MRLMethod Reporting Limit

ND None Detected at or above the method reporting limit

- Quantified as 4-methylphenol.

Reviewed By:

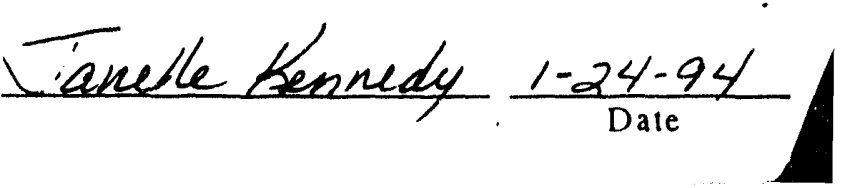




\section{PSI Environmental Laboratories}

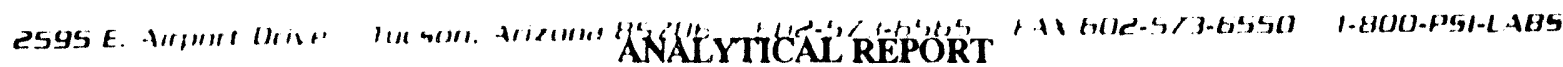

State of Arizona License $\$ 0009$

Client:

Peroxidation Systems Inc.

5151 E. Broadway, Suite 600

Tucson, AZ 85711

Project Name: DOE/WJSA

Project Number: 0345

P.O. Number: None
Sample I.D.: Method Blank

I aboratory I.D.: Method Blank

Sample Type: Water

Date Sampled: $\quad 09 / 01 / 1993$

Date Received: $\quad 09 / 03 / 1993$

Date Analyzed: $\quad 10 / 12 / 1993$

Analyzed By: See Comments Base Neutral/Acid Semivolatile Organic Compounds EPA Methods $3510 / 8270$

Base Neutral Analyte
N-Nitrosodimethylamine
Bis(2-chloroethyl) Ether
1,2-Dichlorobenzene
1,3-Dichlorobenzene
1,4-Dichlorobenzene
Bis(2-chloroisopropyl) Ether
N-Nitrosodi-n-propylamine
Hexachloroethane
Nitrobenzene
Isophorone
Bis(2-chloroethoxy) methane
1,2,4-Trichlorobenzene
Naphthalene
4-Chloroaniline
Hexachlorobutadiene
2-Methylnaphthalene
Hexachlorocyclopentadiene
2-Chloronaphthalene
2-Nitroaniline
Dimethyl Phthalate
Acenaphthylene
3-Nitroaniline
Acenaphthene
Dibenzofuran
2,4-Dinitrotoluene
Acid Analyte
Phenol
2-Chlorophenol
Benzyl Alcohol
2-Methylphenol
3- and 4-Methylphenol"
2-Nitrophenol
2,4-Dimethylphenol
Benzoic Acid
a

$\begin{array}{cc}\text { MRL } & \text { Result } \\ 10 & \text { ND } \\ 10 & \text { ND } \\ 10 & \text { ND } \\ 10 & \text { ND } \\ 10 & \text { ND } \\ 10 & \text { ND } \\ 10 & \text { ND } \\ 10 & \text { ND } \\ 10 & \text { ND } \\ 10 & \text { ND } \\ 10 & \text { ND } \\ 10 & \text { ND } \\ 10 & \text { ND } \\ 10 & \text { ND } \\ 10 & \text { ND } \\ 10 & \text { ND } \\ 20 & \text { ND } \\ 10 & \text { ND } \\ 40 & \text { ND } \\ 10 & \text { ND } \\ 10 & \text { ND } \\ 40 & \text { ND } \\ 10 & \text { ND } \\ 10 & \text { ND } \\ 10 & \text { ND } \\ & \end{array}$

Base Neutral Analrte

2,6-Dinitrotoluene

Diethyl Phthalate

4-Chlorophenyl Phenyl Ether

Fluorene

4-Nitroaniline

N-Nitrosodiphenylamine

4-Bromophenyl Phenyl Ether

Hexachlorobenzene

Phenanthrene

Anthracene

Di-n-butyl Phthalate

Fluoranthene

Pyrene

Butylbenzyl Phthalate

3,3'-Dichlorobenzidine

Benz(a)anthracene

Bis(2-ethylhexyl) Phthalate

Chrysene

Di-n-octyl Phthalate

Benzo(b)fluoranthene

Benzo(k)fluoranthene

Benzolalpyrene

Indeno(1,2,3-c,d)pyrene

Dibenz(a,h)anthracene

Benzo(g,h,i)perylene

$\begin{array}{rr}\text { MRL* } & \text { Result } \\ 10 & \text { ND } \\ 10 & \text { ND } \\ 20 & \text { ND } \\ 10 & \text { ND } \\ 10 & \text { ND } \\ 10 & \text { ND } \\ 10 & \text { ND } \\ 100 & \text { ND }\end{array}$

Acid Analrte

2,4-Dichlorophenol

4-Chloro-3-methylphenol

2,4,6-Trichlorophenol

2,4,5-Trichlorophenol

2,4-Dinitrophenol

4-Nitrophenol

2-Methyt-4,6-dinitrophenol

Pentachlorophenol

$\begin{array}{cc}\text { MRL* } & \text { Result } \\ 10 & \text { ND } \\ 10 & \text { ND } \\ 10 & \text { ND } \\ 10 & \text { ND } \\ 100 & \text { ND } \\ 10 & \text { ND } \\ 10 & \text { ND } \\ 10 & \text { ND } \\ 10 & \text { ND } \\ 10 & \text { ND } \\ 10 & \text { ND } \\ 10 & \text { ND } \\ 10 & \text { ND } \\ 10 & \text { ND } \\ 100 & \text { ND } \\ 10 & \text { ND } \\ 10 & 86 \\ 10 & \text { ND } \\ 10 & \text { ND } \\ 10 & \text { ND } \\ 10 & \text { ND } \\ 10 & \text { ND } \\ 10 & \text { ND } \\ 10 & \text { ND } \\ 10 & \text { ND } \\ \text { MRL } & \text { Result } \\ 10 & \text { ND } \\ 10 & \text { ND } \\ 10 & \text { ND } \\ 10 & \text { ND } \\ 100 & \text { ND } \\ 100 & \text { ND } \\ 40 & \text { ND } \\ 60 & \text { ND }\end{array}$

- Extraction performed by PSI Environmental Laboratories.

MRLMethod Reporting Limit

- MRL is elevated. Sample extract final volume was twice the value normally obtained by Golden State/CAS Laboratories, Inc.

ND None Detected at or above the method reporting limit

- Quantified as 4-methylphenol.

Comments:

Golden State/CAS Laboratories, Inc.

6925 Canoga Avenue

Canoga Park. CA 91303
Reviewed By:

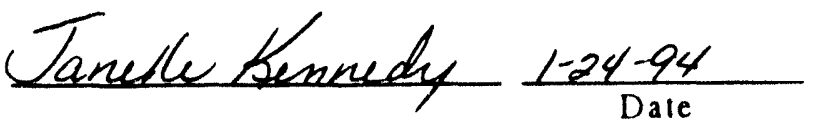




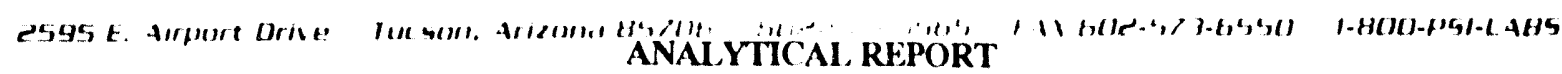

State of Arizona License $\$ 0009$

Client:

Peroxidation Systems Inc. 5151 E. Broadway, Suite 600

Tucson, AZ 85711

Project Name: DOE/WJSA

Project Number: 0345

P.O. Number: None
Sample I.D.: See Below

Laboratory I.D.: See Below

Sample Type: Water

Date Sampled: $\quad 09 / 01 / 1993$

Date Received: $\quad 09 / 03 / 1993$

Date Analyzed: $\quad 10 / 12 / 1993$

Analyzed By: See Comments

Surrogate Recovery Summary

Base Neutral/Acid Semivolatile Organic Compounds EPA Methods $3510 / 8270$

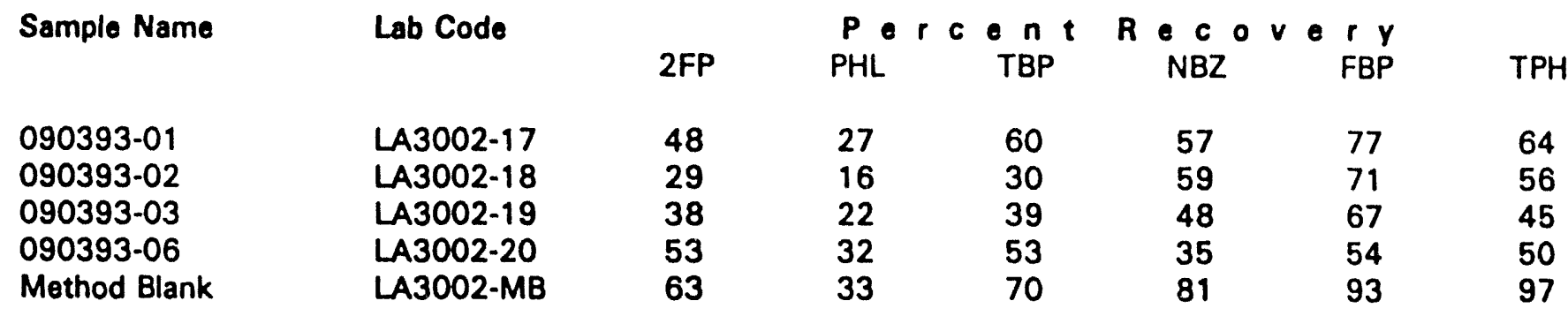

EPA Acceptance Criteria

$21-100 \quad 10-94 \quad 10-123 \quad 35-114 \quad 43-116$

33-141

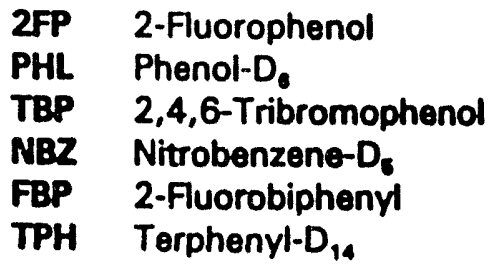




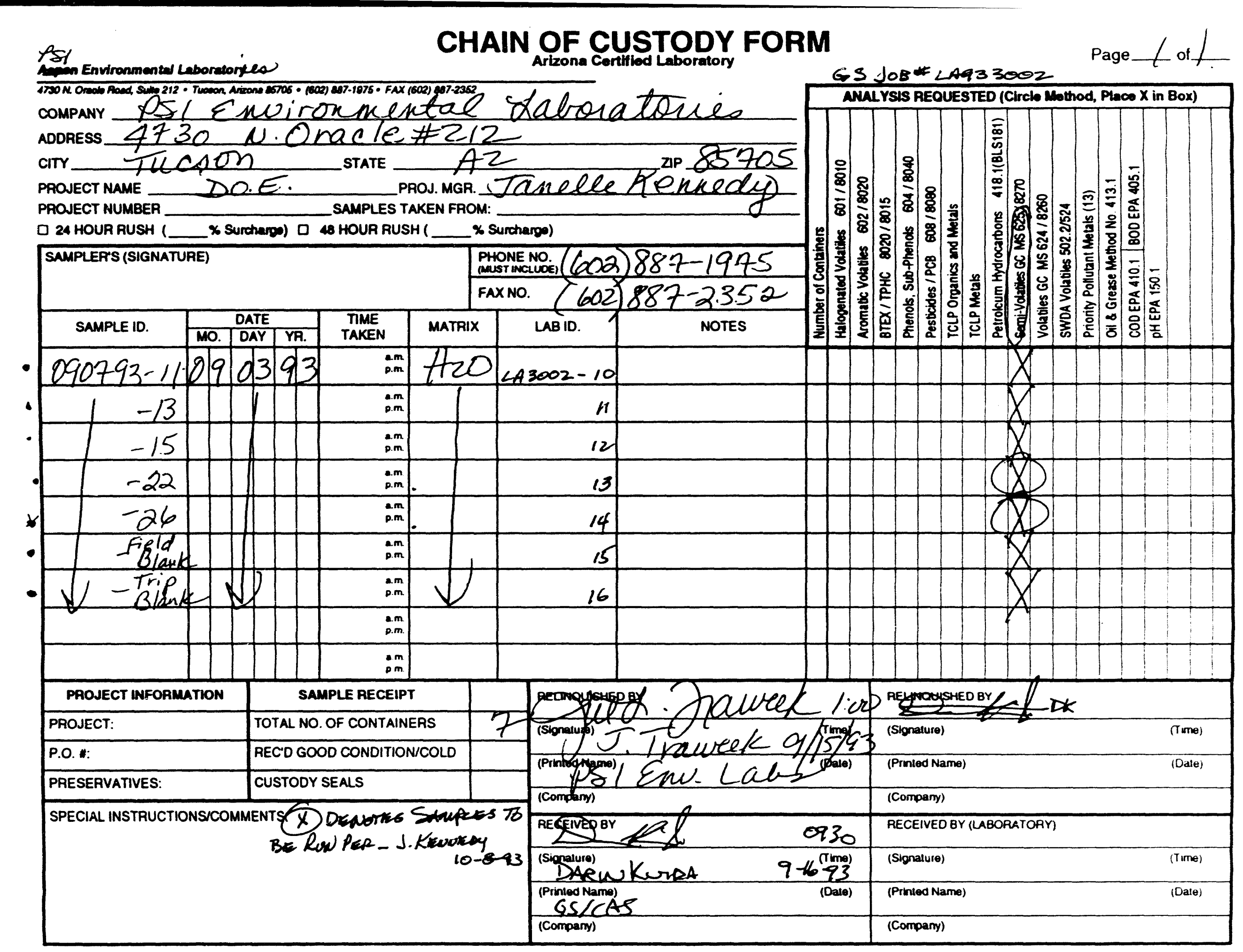




\section{PSI Environmental Laboratories}

2595 E. Alrport Drive Tucson. Arizona 85706 602.573-6565 FAX 602.573-6550 1.800.P5/-LA85

ANALYTCAL REPORT

State of Arizona License $\$ 0009$

Client:

Peroxidation Systems Inc.

5151 E. Broadway, Suite 600

Tucson, AZ 85711

Sample 1.D.:

$13-0$

Laboratory I.D.: 090793.22

$\begin{array}{llll} & & \text { Sample Type: } & \text { Water } \\ \text { Project Name: } & \text { DOE } & \text { Date Sampled: } & 09 / 03 / 1993 \\ \text { Project Number: } & 0345 & \text { Date Received: } & 09 / 07 / 1993 \\ \text { P.O. Number: } & \text { None } & \text { Date Analyzed: } & 10 / 13 / 1993 \\ & & \text { Analyzed By: } & \text { See Comments }\end{array}$

Base Neutral/Acid Semivolatile Organic Compounds

EPA Methods $3510 / 8270$

$\mu \mathrm{g} / \mathrm{(ppb)}$

Base Neutral Analyte

N-Nitrosodimethylamine

Bis (2-chloroethyl) Ether

1,2-Dichlorobenzene

1,3-Dichlorobenzene

1,4-Dichlorobenzene

Bis(2-chloroisopropyl) Ether

$\mathrm{N}$-Nitrosodi-n-propylamine

Hexachloroethane

Nitrobenzene

Isophorone

Bis(2-chloroethoxy) methane

1,2,4-Trichlorobenzene

Naphthalene

4-Chloroaniline

Hexachlorobutadiene

2-Methyinaphthalene

Hexachlorocyclopentadiene

2-Chloronaphthalene

2-Nitroaniline

Dimethyl Phthalate

Acenaphthylene

3-Nitroaniline

Acenaphthene

Dibenzofuran

2,4-Dinitrotoluene

Acid Analyto

Phenol

2-Chlorophenol

Benzyl Alcohol

2-Methylphenol

3- and 4-Methylphenol"

2-Nitrophenol

2,4-Dimethylphenol

Benzoic Acid

MRLMethod Reporting Limit

ND None Detected at or above the method reporting limit

- Quantified as 4-methylphenol.

$\begin{array}{rc}\text { MRL } & \text { Result } \\ \mathbf{5} & \text { ND } \\ \mathbf{5} & \text { ND } \\ \mathbf{5} & \text { ND } \\ \mathbf{5} & \text { ND } \\ \mathbf{5} & \text { ND } \\ \mathbf{5} & \text { ND } \\ \mathbf{5} & \text { ND } \\ \mathbf{5} & \text { ND } \\ \mathbf{5} & \text { ND } \\ \mathbf{5} & \text { ND } \\ \mathbf{5} & \text { ND } \\ \mathbf{5} & \text { ND } \\ \mathbf{5} & \text { ND } \\ \mathbf{5} & \text { ND } \\ \mathbf{5} & \text { ND } \\ \mathbf{5} & \text { ND } \\ \mathbf{1 0} & \text { ND } \\ \mathbf{5} & \text { ND } \\ \mathbf{2 0} & \text { ND } \\ \mathbf{5} & \text { ND } \\ \mathbf{5} & \text { ND } \\ \mathbf{2 0} & \text { ND } \\ \mathbf{5} & \text { ND } \\ \mathbf{5} & \text { ND } \\ \mathbf{5} & \text { ND } \\ & \end{array}$

MRL Result

$\begin{aligned} 5 & \text { ND } \\ 5 & \text { ND } \\ 10 & \text { ND } \\ 5 & \text { ND } \\ 5 & \text { ND } \\ 5 & \text { ND } \\ 5 & \text { ND } \\ 50 & \text { ND }\end{aligned}$

Base Neutrd Analrte

2,6-Dinitrotoluene

Diethyl Phthalate

4-Chlorophenyl Phenyl Ether

Fluorene

4-Nitroaniline

N-Nitrosodiphenylamine

4-Bromophenyl Phenyl Ether

Hexachlorobenzene

Phenanthrene

Anthracene

Di-n-butyl Phthalate

Fuoranthene

Pyreno

Butylbenzyl Phthalate

3,3'-Dichlorobenzidine

Benzla)anthracene

Bis(2-othylhexyl) Phthalate

Chrysene

Di-n-octy Phthalate

Benzolb)fluoranthene

Benzo(k)fluoranthene

Benzola)pyrene

Indeno(1,2,3-c,d)pyrene

Dibenz(a,h)anthracene

Benzolo,h,il)perylene

Acid Analyto

2,4-Dichlorophenol

4-Chloro-3-methylphenol

2,4,6-Trichlorophenol

2,4,5-Trichlorophenol

2,4-Dinitrophenol

4-Nitrophenol

2-Methyl-4,6-dinitrophenol

Pentachlorophenol

$\begin{array}{rr}\text { MRL } & \text { Result } \\ \mathbf{5} & \text { ND } \\ \mathbf{5} & \text { ND } \\ \mathbf{5} & \text { ND } \\ \mathbf{5} & \text { ND } \\ \mathbf{2 0} & \text { ND } \\ \mathbf{5} & \text { ND } \\ \mathbf{5} & \text { ND } \\ \mathbf{5} & \text { ND } \\ \mathbf{5} & \text { ND } \\ \mathbf{5} & \text { ND } \\ \mathbf{5} & \text { ND } \\ \mathbf{5} & \text { ND } \\ \mathbf{5} & \text { ND } \\ \mathbf{5} & \text { ND } \\ \mathbf{2 0} & \text { ND } \\ \mathbf{5} & \text { ND } \\ \mathbf{5} & \text { ND } \\ \mathbf{5} & \text { ND } \\ \mathbf{5} & \text { ND } \\ \mathbf{5} & \text { ND } \\ \mathbf{5} & \text { ND } \\ \mathbf{5} & \text { ND } \\ \mathbf{5} & \text { ND } \\ \mathbf{5} & \text { ND } \\ \mathbf{5} & \text { ND }\end{array}$

MRL Result

$\begin{aligned} \mathbf{5} & \text { ND } \\ \mathbf{5} & \text { ND } \\ \mathbf{5} & \text { ND } \\ \mathbf{5} & \text { ND } \\ 50 & \text { ND } \\ 50 & \text { ND } \\ 20 & \text { ND } \\ 30 & \text { ND }\end{aligned}$




\section{PSI Environmental Laboratories}

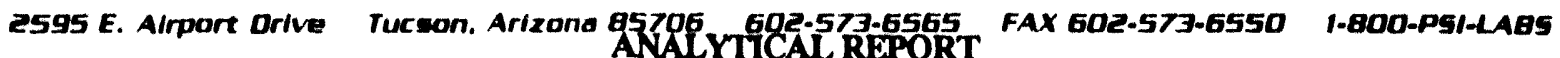
State of Arizona License $\$ 0009$

Client:

Peroxidation Systems Inc. 5151 E. Broadway, Suite 600 Tucson, AZ 85711

Project Name: DOE

Project Number: 0345

P.O. Number: None
Sample 1.D.:

13-3

Laboratory I.D.: 090793-26

Sample Type: Water

Date Sampled: $\quad 09 / 03 / 1993$

Date Received: $\quad 09 / 07 / 1993$

Date Analyzed: $\quad 10 / 13 / 1993$

Analyze I By: See Comments

Base Neutral/Acid Semivolatile Organic Compounds EPA Methods 3510/8270

$\mu \mathrm{p} \Omega(p p b)$

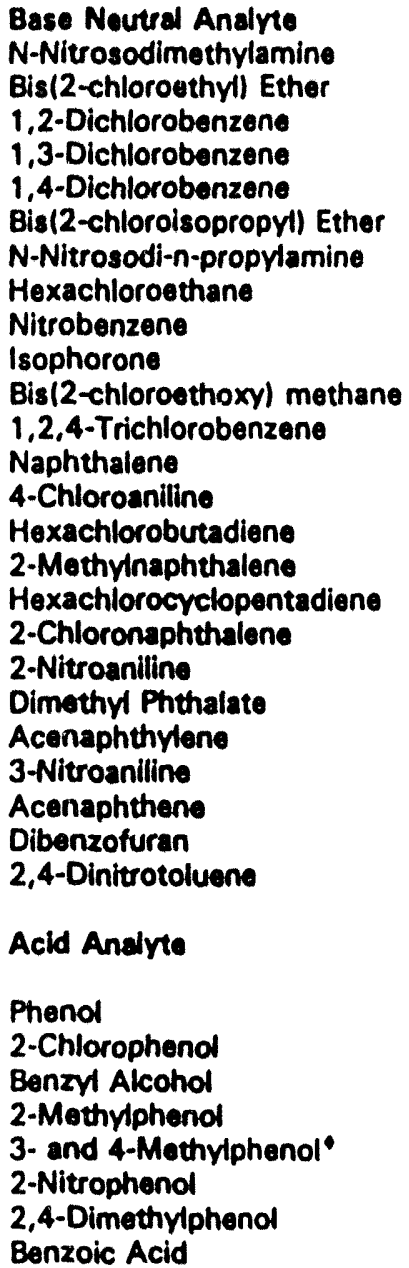

Base Neutral Analrte

N-Nitrosodimethylamine

1,2-Dichlorobenzene

1,3-Dichlorobenzene

Bis(2-chloroisopropyl) Ether

N-Nitrosodi-n-propylamin

Bis (2-chloroethoxy) methane

Hexachlorobutadiene

2-Methyinaphthalene

Hexachlorocyclopentadiene

ronaphthalene

Dimethyl Phthalate

$\begin{array}{rc}\text { MRL } & \text { Result } \\ \mathbf{5} & \text { ND } \\ \mathbf{5} & \text { ND } \\ \mathbf{5} & \text { ND } \\ \mathbf{5} & \text { ND } \\ \mathbf{5} & \text { ND } \\ \mathbf{5} & \text { ND } \\ \mathbf{5} & \text { ND } \\ \mathbf{5} & \text { ND } \\ \mathbf{5} & \text { ND } \\ \mathbf{5} & \text { ND } \\ \mathbf{5} & \text { ND } \\ \mathbf{5} & \text { ND } \\ \mathbf{5} & \text { ND } \\ \mathbf{5} & \text { ND } \\ \mathbf{5} & \text { ND } \\ \mathbf{5} & \text { ND } \\ 10 & \text { ND } \\ \mathbf{5} & \text { ND } \\ 20 & \text { ND } \\ \mathbf{5} & \text { ND } \\ \mathbf{5} & \text { ND } \\ 20 & \text { ND } \\ \mathbf{5} & \text { ND } \\ \mathbf{5} & \text { ND } \\ \mathbf{5} & \text { ND } \\ & \end{array}$

MRL Result

$\begin{array}{rr}\mathbf{5} & \text { ND } \\ \mathbf{5} & \text { ND } \\ 10 & \text { ND } \\ \mathbf{5} & \text { ND } \\ \mathbf{5} & \text { ND } \\ \mathbf{5} & \text { ND } \\ \mathbf{5} & \text { ND } \\ \mathbf{5 0} & \text { ND }\end{array}$

MRLMethod Reporting Limit

ND None Detected at or above the method reporting limit

- Quantified as 4-methylphenol.
Base Neutrel Analyte

2,6-Dinitrotoluene

Diethyl Phthalate

4-Chlorophenyl Phenyl Ether

Fluorene

4-Nitroaniline

N-Nitrosodiphenylamine

4-Bromophenyl Phenyl Ether

Hexachlorobenzene

Phenanthrene

Anthracene

Di-n-butyl Phthalate

Fluoranthene

Pyrene

Butylbenzy Phthalate

3,3'-Dichlorobenzidine

Benz(a)anthracene

Bis (2-othylhexyl) Phthalate

Chrysene

Di-n-octyl Phthalate

Benzo(b)filuoranthene

Benzo(k)fluoranthene

Benzolalpyrene

Indeno(1,2,3-c,d)pyrene

Dibenzla,hlanthracene

Benzolg,h,ilperylene

Acid Anarto

2,4-Dichlorophenol

4-Chloro-3-mothylphenol

2,4,6-Trichlorophenol

2,4,5-Trichlorophenol

2,4-Dinitrophenol

4-Nitrophenol

2-Methy-4,6-dinitrophenol

Pentachlorophenol

$\begin{array}{rc}\text { MRL } & \text { Result } \\ \mathbf{5} & \text { ND } \\ \mathbf{5} & \text { ND } \\ \mathbf{5} & \text { ND } \\ \mathbf{5} & \text { ND } \\ \mathbf{2 0} & \text { ND } \\ \mathbf{5} & \text { ND } \\ \mathbf{5} & \text { ND } \\ \mathbf{5} & \text { ND } \\ \mathbf{5} & \text { ND } \\ \mathbf{5} & \text { ND } \\ \mathbf{5} & \text { ND } \\ \mathbf{5} & \text { ND } \\ \mathbf{5} & \text { ND } \\ \mathbf{5} & \text { ND } \\ \mathbf{2 0} & \text { ND } \\ \mathbf{5} & \text { ND } \\ \mathbf{5} & \text { ND } \\ \mathbf{5} & \text { ND } \\ \mathbf{5} & \text { ND } \\ \mathbf{5} & \text { ND } \\ \mathbf{5} & \text { ND } \\ \mathbf{5} & \text { ND } \\ \mathbf{5} & \text { ND } \\ \mathbf{5} & \text { ND } \\ \mathbf{5} & \text { ND } \\ & \end{array}$

MRL Result

$\begin{array}{rr}\mathbf{5} & \text { ND } \\ \mathbf{5} & \text { ND } \\ \mathbf{5} & \text { ND } \\ \mathbf{5} & \text { ND } \\ \mathbf{5 0} & \text { ND } \\ \mathbf{5 0} & \text { ND } \\ \mathbf{2 0} & \text { ND } \\ 30 & \text { ND }\end{array}$




\section{ASI Environmental Laboratories}

Client:

Peroxidation Systems lnc.

5151 E. Broadway, Suite 600

Tucson, AZ 85711
Sample I.D.: Blank

Laboratory I.D.: Method Blank

Sample Type: Waler

Date Sampled: $\quad 09 / 03 / 1993$

Date Received: $\quad 09 / 07 / 1993$

Date Analyzed: $\quad 10 / 13 / 1993$

Analyzed By: See Comments

P.O. Number: None

Base Neutral/Acid Semivolatile Organic Compounds

EPA Methods 3510/8270 uph (ppb)

Base Neurtrd Analyte
N-Nitrosodimethylamine
Bis(2-chloroethyl) Ether
1,2-Dichlorobenzene
1.3-Dichlorobenzene
1,4-Dichlorobenzene
Bis(2-chloroisopropyl) Ether
N-Nitrosodi-n-propylamine
Hexachloroethane
Nitrobenzene
Isophorone
Bis/2-chlorosthoxy) methane
1,2,4-Trichlorobenzene
Naphthalene
4-Chloroaniline
Hexachlorobutadiene
2-Methylnaphthalene
Hexachlorocyclopentadiene
2-Chloronaphthalene
2-Nitroaniline
Dimethyl Phthalate
Acenaphthylene
3-Nitroaniline
Acenaphthene
Dibenzofuran
2,4-Dinitrotoluene
Acid Analyte
Phenol
2-Chlorophenol
Benzy Alcohol
2-Methylohenol
3- and 4-Methyphenole
2-Nitrophenol
2,4-Dimethylphenol
Benzoic Acid

Base Neutral Analyte N-Nitrosodimethylamine Bis(2-chloroethyl) Ether

3-Dichlorobenzen 1,4-Dichlorobenzene Bis(2-chloroisopropyl) Ether Hexachloroethene 1,2,4-Trichlorobenzene Hexachlorocyclopentadiene 2-Chloronaphthalene Dimethyl Phthalate Acenaphthylene 3-Nitroaniline

4-Dinitrotoluene

$\begin{array}{rc}\text { MRL } & \text { Result } \\ \mathbf{5} & \text { ND } \\ \mathbf{5} & \text { ND } \\ \mathbf{5} & \text { ND } \\ \mathbf{5} & \text { ND } \\ \mathbf{5} & \text { ND } \\ \mathbf{5} & \text { ND } \\ \mathbf{5} & \text { ND } \\ \mathbf{5} & \text { ND } \\ \mathbf{5} & \text { ND } \\ \mathbf{5} & \text { ND } \\ \mathbf{5} & \text { ND } \\ \mathbf{5} & \text { ND } \\ \mathbf{5} & \text { ND } \\ \mathbf{5} & \text { ND } \\ \mathbf{5} & \text { ND } \\ \mathbf{5} & \text { ND } \\ \mathbf{1 0} & \text { ND } \\ \mathbf{5} & \text { ND } \\ \mathbf{2 0} & \text { ND } \\ \mathbf{5} & \text { ND } \\ \mathbf{5} & \text { ND } \\ \mathbf{2 0} & \text { ND } \\ \mathbf{5} & \text { ND } \\ \mathbf{5} & \text { ND } \\ \mathbf{5} & \text { ND } \\ & \end{array}$

MRL Result

$\begin{array}{rr}5 & \text { ND } \\ 5 & \text { ND } \\ 10 & \text { ND } \\ 5 & \text { ND } \\ 5 & \text { ND } \\ 5 & \text { ND } \\ 5 & \text { ND } \\ 50 & \text { ND }\end{array}$

MRLMethod Reporting Limit ND None Detected at or above the method reporting limit - Quantified as 4-methylphenol.

\begin{tabular}{|c|c|c|}
\hline 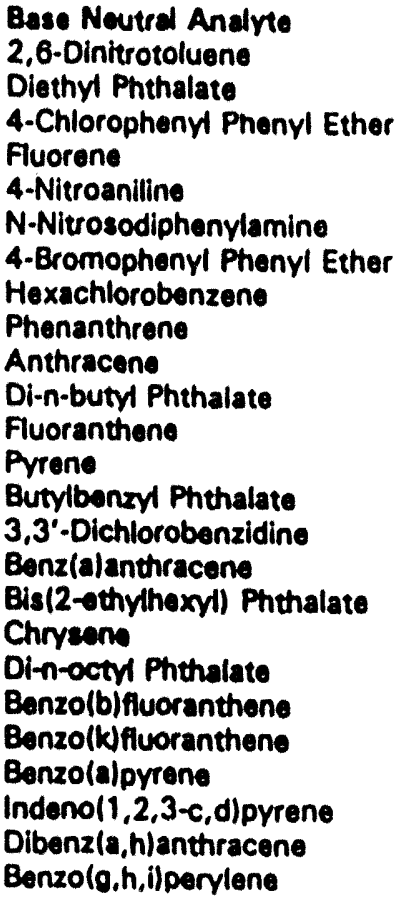 & $\begin{array}{r}\text { MRL } \\
\mathbf{5} \\
5 \\
5 \\
5 \\
20 \\
5 \\
5 \\
5 \\
5 \\
5 \\
5 \\
5 \\
5 \\
5 \\
20 \\
5 \\
5 \\
5 \\
5 \\
5 \\
5 \\
5 \\
5 \\
5 \\
5\end{array}$ & $\begin{array}{l}\text { Rosult } \\
\text { ND } \\
\text { ND } \\
\text { ND } \\
\text { ND } \\
\text { ND } \\
\text { ND } \\
\text { ND } \\
\text { ND } \\
\text { ND } \\
\text { ND } \\
\text { ND } \\
\text { ND } \\
\text { ND } \\
\text { ND } \\
\text { ND } \\
\text { ND } \\
\text { ND } \\
\text { ND } \\
\text { ND } \\
\text { ND } \\
\text { ND } \\
\text { ND } \\
\text { ND } \\
\text { ND } \\
\text { ND }\end{array}$ \\
\hline Acid Andyte & MRL. & Result \\
\hline $\begin{array}{l}\text { 2,4-Dichlorophenol } \\
\text { 4-Chlora-3-methylphenol } \\
\text { 2,4,6-Trichlorophenol } \\
\text { 2,4,6-Trichlorophenol } \\
\text { 2,4-Dinitrophenol } \\
\text { 4-Nitrophenol } \\
\text { 2-Methyi-4,6-dinitrophenol } \\
\text { Pentachlorophenol }\end{array}$ & $\begin{array}{r}5 \\
5 \\
5 \\
5 \\
50 \\
50 \\
20 \\
30\end{array}$ & $\begin{array}{l}\text { ND } \\
\text { ND } \\
\text { ND } \\
\text { ND } \\
\text { ND } \\
\text { ND } \\
\text { ND } \\
\text { ND }\end{array}$ \\
\hline
\end{tabular}


Client:

Peroxidation Systems Inc. S151 E. Broadway, Suite 600

Tucson, AZ 85711

Project Name: DOE

Project Number: 0345

P.O. Number: None
Sample I.D.: See Below

Laboratory I.D.: See Below

Sample Type: Waler

Date Sampled: $\quad 09 / 03 / 1993$

Date Received: 09/07/1993

Date Analyzed: $\quad 10 / 13 / 1993$

Analyzed By: See Comments

Surrogate Recovery Summary

Base Neutral/Acid Semivolatile Organic Compounds

EPA Methods 3510/8270

\begin{tabular}{|c|c|c|c|c|c|c|c|}
\hline Sample Name & Lab Code & $2 F P$ & $\underset{\text { PHL }}{\text { P }}$ & $r c \underset{T B P}{n} t$ & $A \cdot \underset{N B Z}{c} 0$ & $v \bullet \underset{F B P}{r y}$ & TPH \\
\hline $\begin{array}{l}\text { 090393-08 } \\
090793-22 \\
\text { 090793-26 } \\
\text { Method Blank }\end{array}$ & $\begin{array}{l}\text { LA3002-4 } \\
\text { LA3002-13 } \\
\text { LA3002-14 } \\
\text { LA3002-MB }\end{array}$ & $\begin{array}{l}54 \\
68 \\
53 \\
69\end{array}$ & $\begin{array}{l}25 \\
35 \\
25 \\
34\end{array}$ & $\begin{array}{l}48 \\
76 \\
64 \\
81\end{array}$ & $\begin{array}{r}93 \\
98 \\
91 \\
100\end{array}$ & $\begin{array}{l}80 \\
85 \\
79 \\
85\end{array}$ & $\begin{array}{l}86 \\
90 \\
93 \\
87\end{array}$ \\
\hline
\end{tabular}

EPA Acceptance Criteria

$21-100 \quad 10-94 \quad 10-123 \quad 35-114 \quad 43-116 \quad 33-141$

2FP 2-Fluorophenol

PHL Phenol-D,

TBP 2,4,6-Tribromophenol

NBZ Nitrobenzene-D.

FBP 2-Fluorobiphenyl

TPH Terphenyl-D 14 


\section{P5I Environmental Laboratories}

2595 E. Alrport Drive Tuceon. Arizana 65706 602.573.6565 FAx 602-573-6550 1-800-P5/-LABS ANALYTICAL REPORT

State of Arizona License w0009

Cllent:

Peroxidation Systems Inc.

5151 E. Broadway, Sulte 600

Tucson, AZ 85711

Project Name: DOE

Project Number: 0345

P.O. Number: None
Semple I.D.:
Laboratory I.D.:

Sample Type: Water

Date Sampled: $\quad 09 / 03 / 1993$

Date Received: $\quad 09 / 07 / 1993$

Date Analyzed: $\quad 10 / 13 / 1993$

Analyzed By: $\quad$ See Comments
Matrix Spike/Duplicate Matrix Spike Summary Base Neutral/Acid Semivolatile Organic Compounds

EPA Methods $3510 / 8270$

$\mu g \Omega(p p b)$
Analyte

Phenol

2-Chlorophenol

1,4-Dichlorobenzene

N-Nitrosodi-n-propylamine

1,2,4-Trichlorobenzene

4-Chloro-3-methylphenol

Acenaphthene

4-Nitrophenol

2,4-Dinitrotoluene

Pentachlorophenol

Pyrene
Percent $R \cdot c o v e r y$

\begin{tabular}{|c|c|c|c|c|c|c|c|c|}
\hline $\begin{array}{l}\text { Spike } \\
\text { MS }\end{array}$ & $\begin{array}{l}\text { Level } \\
\text { DMS }\end{array}$ & $\begin{array}{c}\text { Sample } \\
\text { Result }\end{array}$ & $\begin{array}{l}\text { Splke } \\
\text { MS }\end{array}$ & $\begin{array}{l}\text { Result } \\
\text { DMS }\end{array}$ & MS & DMS & $\begin{array}{c}\text { EPA } \\
\text { Acceptance } \\
\text { Criteria }\end{array}$ & $\begin{array}{c}\text { Relative } \\
\text { Percent } \\
\text { Difference }\end{array}$ \\
\hline $\begin{array}{l}10.0 \\
10.0 \\
10.0 \\
10.0 \\
10.0 \\
10.0 \\
10.0 \\
10.0 \\
10.0 \\
10.0 \\
10.0\end{array}$ & $\begin{array}{l}10.0 \\
10.0 \\
10.0 \\
10.0 \\
10.0 \\
10.0 \\
10.0 \\
10.0 \\
10.0 \\
10.0 \\
10.0\end{array}$ & $\begin{array}{l}\text { ND } \\
\text { ND } \\
\text { ND } \\
\text { ND } \\
\text { ND } \\
\text { ND } \\
\text { ND } \\
\text { ND } \\
\text { ND } \\
\text { ND } \\
\text { ND }\end{array}$ & $\begin{array}{c}4.50 \\
9.36 \\
6.64 \\
10.8 \\
6.59 \\
7.23 \\
9.67 \\
2.68 \\
8.62 \\
3.80 \\
9.48\end{array}$ & $\begin{array}{c}4.59 \\
9.71 \\
7.78 \\
11.6 \\
7.83 \\
7.56 \\
10.3 \\
2.60 \\
8.87 \\
4.03 \\
10.8\end{array}$ & $\begin{array}{r}45 \\
94 \\
66 \\
108 \\
66 \\
72 \\
97 \\
27 \\
86 \\
38 \\
95\end{array}$ & $\begin{array}{r}46 \\
97 \\
78 \\
116 \\
78 \\
76 \\
103 \\
26 \\
89 \\
40 \\
108\end{array}$ & $\begin{array}{l}12-89 \\
27-123 \\
36-97 \\
41-116 \\
39-98 \\
23-97 \\
46-118 \\
10-80 \\
24-96 \\
9-103 \\
26-127\end{array}$ & $\begin{array}{r}2 \\
4 \\
16 \\
7 \\
9 \\
3 \\
6 \\
2 \\
3 \\
6 \\
13\end{array}$ \\
\hline
\end{tabular}

ND None Detected at or above the method reporting limit

Comments: $\quad$ OC performed on a sample from another source.

Golden State/CAS Laboralories, Inc.

6925 Canoga Avenue

Canoga Park. CA 91303
Reviewed By:

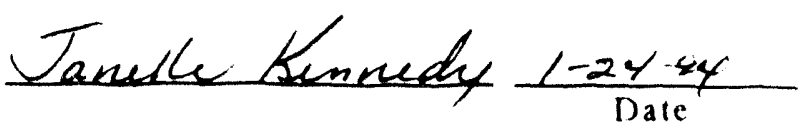


GOLDEN STATE/CAS

LABORATORIES, INC.
6925 CANOGA AVENUE, CANOGA PARK, CA 91304

8185875550 — FAX \# 8185875555
Chain of Custody Record Analytical Services Request

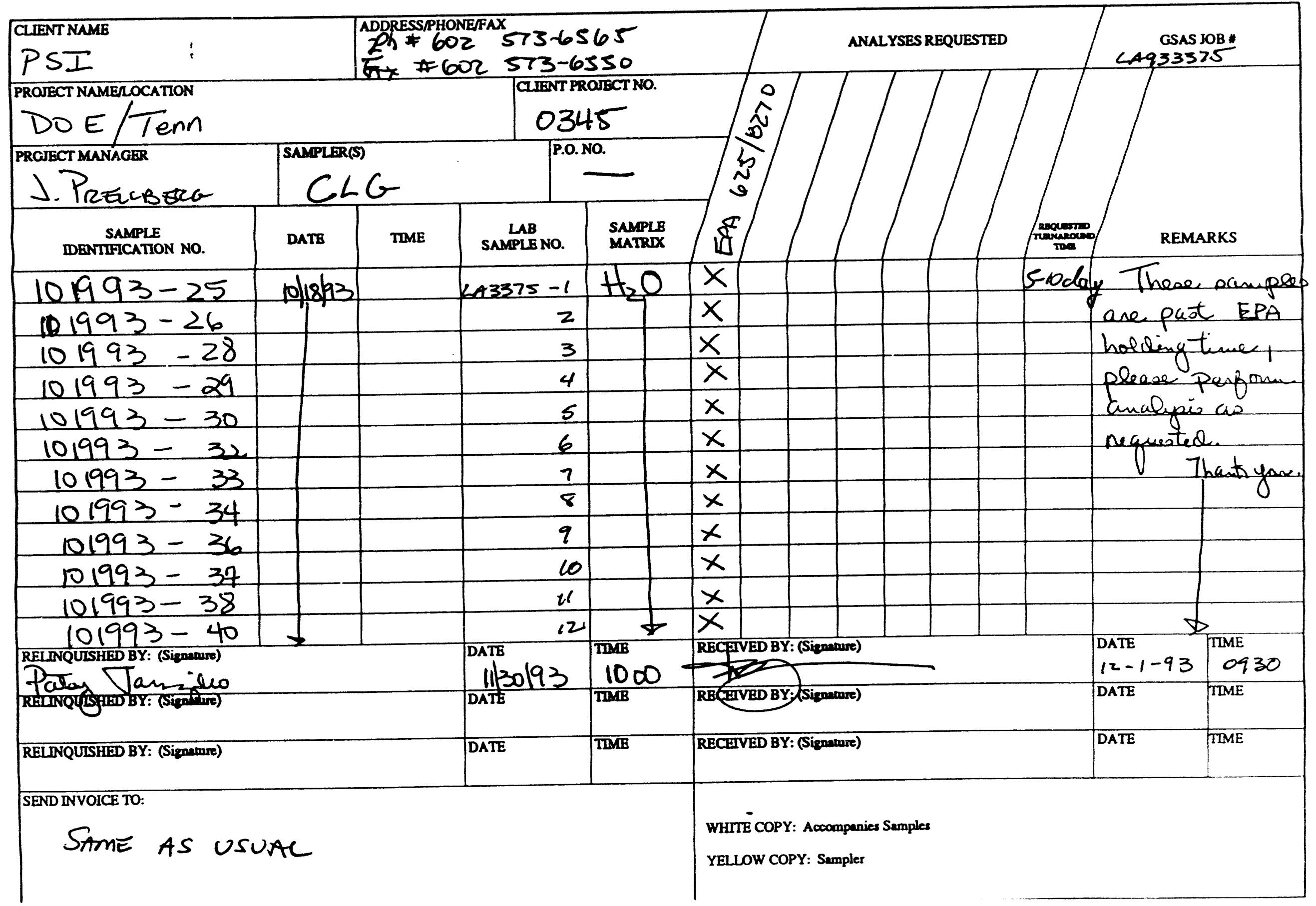


GOLDEN STATE/CAS

LABORATORIES, INC.
6925 CANOGA AVENUE, CANOGA PARK, CA 91304

$8185875550=$ FAX \# 8185875555
Chain of Custody Record $2 / 2$
Analytical Services Request

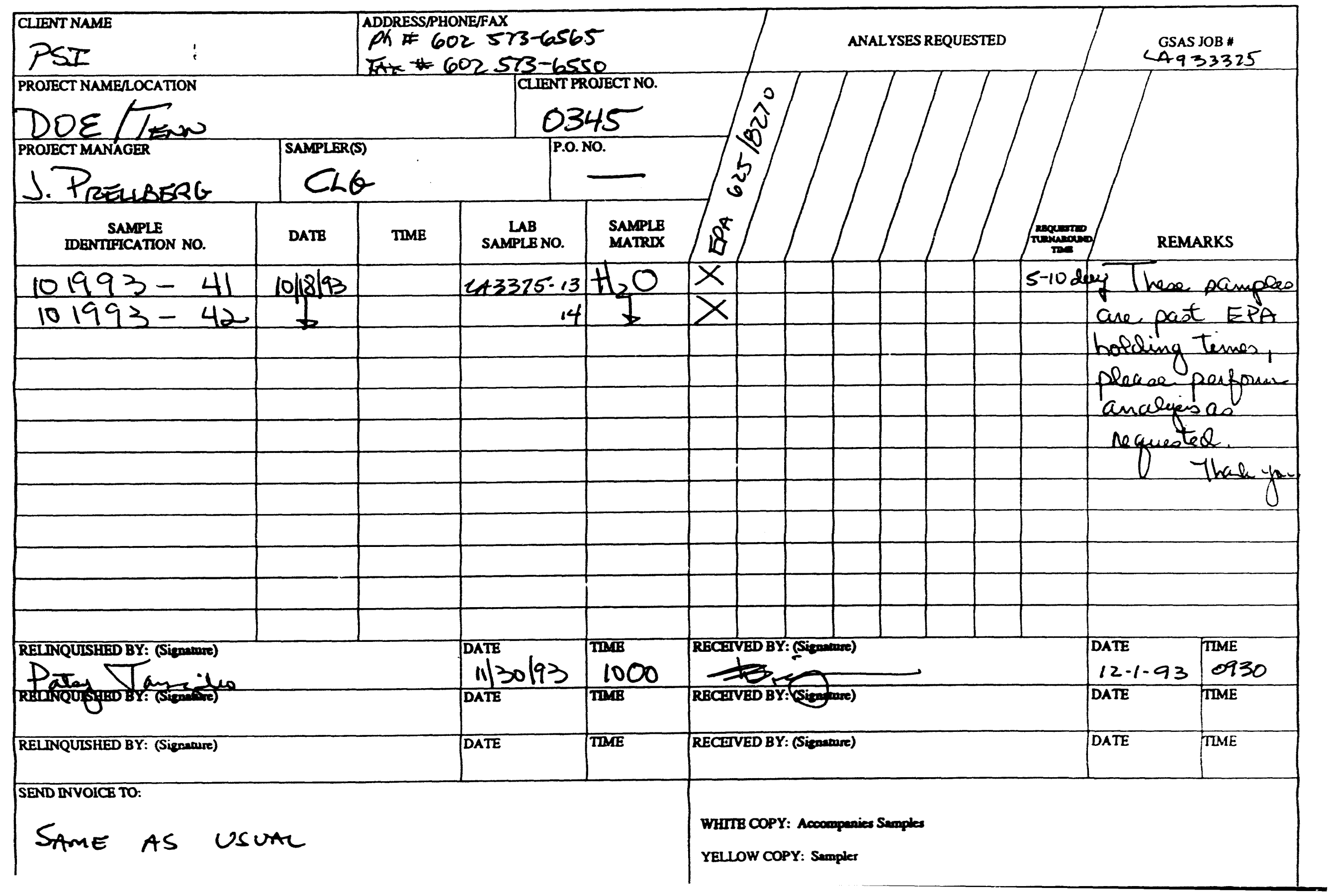




\section{ASI Environmental Laboratories}

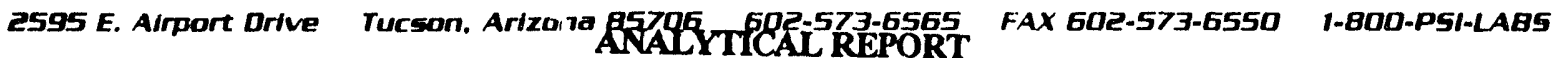
State of Arizona License $\$ 0009$

Client:

Peroxidation Systems Inc.

5151 E. Broadway, Suite 600

Tucson, AZ 85711

Project Name: DOE/Tenn

Project Number: 0345

P.O. Number: None
Sample I.D.: $\quad 16 \mathrm{~A}-0$

Laboratory I.D.: 101993-25

Sample Type: Water

Date Sampled: $\quad 10 / 18 / 1993$

Date Received: $\quad$ 10/19/1993

Date Analyzed: $\quad 12 / 10 / 1993$

Analyzed By: $\quad$ See Comments

Base Neutral/Acid Semivolatile Organic Compounds EPA Methods 3510/8270 $\mu \mathrm{g} / \mathrm{ppb})$

Base Neutral Analyte
N-Nitrosodimethylamine
Bis(2-chloroethyl) Ether
1,2-Dichlorobenzene
1,3-Dichlorobenzene
1,4-Dichlorobenzene
Bis(2-chloroisopropyl) Ether
N-Nitrosodi-n-propylamine
Hexachloroethane
Nitrobenzene
Isophorone
Bis(2-chloroethoxy) methane
1,2,4-Trichlorobenzene
Naphthalene
4-Chloroaniline
Hexachlorobutadiene
2-Methylnaphthalene
Hexachlorocyclopentadiene
2-Chloronaphthalene
2-Nitroaniline
Dimethyl Phthalate
Acenaphthylene
3-Nitroaniline
Acenaphthene
Dibenzofuran
2,4-Dinitrotoluene
A niline

Acid Analyte

Phenol

2-Chlorophenol

Benzyl Alcohol

2-Methylphenol

3- and 4-Methylphenol"

2-Nitrophenol

2,4-Dimethylphenol

Benzoic Acid

$\begin{array}{rc}\text { MRL } & \text { Result } \\ \mathbf{5} & \text { ND } \\ \mathbf{5} & \text { ND } \\ \mathbf{5} & \text { ND } \\ \mathbf{5} & \text { ND } \\ \mathbf{5} & \text { ND } \\ \mathbf{5} & \text { ND } \\ \mathbf{5} & \text { ND } \\ \mathbf{5} & \text { ND } \\ \mathbf{5} & \text { ND } \\ \mathbf{5} & \text { ND } \\ \mathbf{5} & \text { ND } \\ \mathbf{5} & \text { ND } \\ \mathbf{5} & \mathbf{8} \\ \mathbf{5} & \text { ND } \\ \mathbf{5} & \text { ND } \\ \mathbf{5} & \text { ND } \\ \mathbf{1 0} & \text { ND } \\ \mathbf{5} & \text { ND } \\ \mathbf{2 0} & \text { ND } \\ \mathbf{5} & \text { ND } \\ \mathbf{5} & \text { ND } \\ \mathbf{2 0} & \text { ND } \\ \mathbf{5} & \text { ND } \\ \mathbf{5} & \text { ND } \\ \mathbf{5} & \text { ND } \\ \mathbf{5} & \text { ND } \\ & \end{array}$

MRL

Result

$\begin{aligned} \mathbf{5} & \text { ND } \\ \mathbf{5} & \text { ND } \\ \mathbf{1 0} & \text { ND } \\ \mathbf{5} & \text { ND } \\ \mathbf{5} & \text { ND } \\ \mathbf{5} & \text { ND } \\ \mathbf{5} & \text { ND } \\ 50 & \text { ND }\end{aligned}$

MRLMethod Reporting Limit

NO None Detected at or above the method reporting limit

- Quantified as 4-methylphenol.

Comments:

Golden State/CAS Laboratories, Inc
Base Neutral Analyte

2,6-Dinitrotoluene

Diethyl Phthalate

4-Chlorophenyl Phenyl Ether

Fluorene

4-Nitroaniline

$\mathrm{N}$-Nitrosodiphenylamine

4-Bromophenyl Phenyl Ether

Hexachlorobenzene

Phenanthrene

Anthracene

Di-n-butyl Phthalate

riuoranthene

Pyrene

Butylbenzyl Phthalate

3,3'-Dichlorobenzidine

Benz(a)anthracene

Bis(2-ethylhexyl) Phthalate

Chrysene

Di-n-octyl Phthalate

Benzolb)fluoranthene

Benzo(k)fluoranthene

Benzola)pyrene

Indeno(1,2,3-c,d)pyrene

Dibenz (a,h)anthracene

Benzolg,h,ilperylene

Acid Analyte

Result

2,4-Dichlorophenol

4-Chloro-3-methylphenol

2,4,6-Trichlorophenol

2,4,5-Trichlorophenol

2,4-Dinitrophenol

4-Nitrophenol

2-Methyl-4,6-dinitrophenol

Pentachlorophenol
MRL

$\begin{array}{rr}\text { MRL } & \text { Result } \\ 5 & \text { ND } \\ \mathbf{5} & \text { ND } \\ 5 & \text { ND } \\ 5 & \text { ND } \\ 20 & \text { ND } \\ \mathbf{5} & \text { ND } \\ 5 & \text { ND } \\ \mathbf{5} & \text { ND } \\ \mathbf{5} & \text { ND } \\ \mathbf{5} & \text { ND } \\ \mathbf{5} & \text { ND } \\ \mathbf{5} & \text { ND } \\ \mathbf{5} & \text { ND } \\ \mathbf{5} & \text { ND } \\ \mathbf{2 0} & \text { ND } \\ \mathbf{5} & \text { ND } \\ \mathbf{5} & \text { ND } \\ \mathbf{5} & \text { ND } \\ \mathbf{5} & \text { ND } \\ \mathbf{5} & \text { ND } \\ \mathbf{5} & \text { ND } \\ \mathbf{5} & \text { ND } \\ \mathbf{5} & \text { ND } \\ \mathbf{5} & \text { ND } \\ \mathbf{5} & \text { ND }\end{array}$

5 ND

5 ND

ND

ND

ND

ND

ND

ND

Reviewed By:

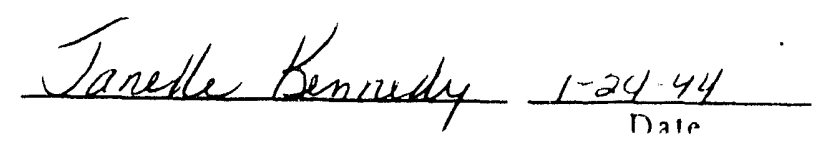




\section{PSI Environmental Laboratories}

2595 E. Airport Drive Tucson. Arizana 85706 602-573-6565 FAX 602-573-6550 1-800-P5I-LABS ANALYTICAL REPORT

State of Arizona License $\$ 0009$

Client:

Peroxidation Systems Inc.

5151 E. Broadway, Suite 600

Tucson, AZ 85711

Project Name: DOE/Tenn

Project Number: 0345

P.O. Number: None
Sample I.D.: 16A-3

Laboratory I.D.: 101993-26

Sample Type: Water

Date Sampled: $\quad 10 / 18 / 1993$

Date Received: $\quad 10 / 19 / 1993$

Date Analyzed: $\quad 12 / 10 / 1993$

Analyzed By: See Comments

Base Neutral/Acid Semivolatile Organic Compounds

EPA Methods $3510 / 8270$

$\mu \mathrm{g} \Omega$ (ppb)

Base Neutral Analyte

N-Nitrosodimethylamine

Bis(2-chloroethyl) Ether

1,2-Dichlorobenzene

1,3-Dichlorobenzene

1,4-Dichlorobenzene

Bis(2-chloroisopropyl) Ether

N-Nitrosodi-n-propylamine

Hexachloroethane

Nitrobenzene

Isophorone

Bis(2-chloroethoxy) methane

1,2,4-Trichlorobenzene

Naphthalene

4-Chloroaniline

Hexachlorobutadiene

2-Methylnaphthalene

Hexachlorocyclopentadiene

2-Chloronaphthalene

2-Nitroaniline

Dimethyl Phthalate

Acenaphthylene

3-Nitroaniline

Acenaphthene

Dibenzofuran

2,4-Dinitrotoluene

Aniline

Acid Analyte

Phenol

2-Chlorophenol

Benzyt Alcohol

2-Methylphenol

3- and 4-Methylphenol ${ }^{\circ}$

2-Nitrophenol

2,4-Dimethylphenol

Benzoic Acid

$\begin{array}{rc}\text { MRL } & \text { Result } \\ \mathbf{5} & \text { ND } \\ \mathbf{5} & \text { ND } \\ \mathbf{5} & \text { ND } \\ \mathbf{5} & \text { ND } \\ \mathbf{5} & \text { ND } \\ \mathbf{5} & \text { ND } \\ \mathbf{5} & \text { ND } \\ \mathbf{5} & \text { ND } \\ \mathbf{5} & \text { ND } \\ \mathbf{5} & \text { ND } \\ \mathbf{5} & \text { ND } \\ \mathbf{5} & \text { ND } \\ \mathbf{5} & \text { ND } \\ \mathbf{5} & \text { ND } \\ \mathbf{5} & \text { ND } \\ \mathbf{5} & \text { ND } \\ \mathbf{1 0} & \text { ND } \\ \mathbf{5} & \text { ND } \\ \mathbf{2 0} & \text { ND } \\ \mathbf{5} & \text { ND } \\ \mathbf{5} & \text { ND } \\ \mathbf{2 0} & \text { ND } \\ \mathbf{5} & \text { ND } \\ \mathbf{5} & \text { ND } \\ \mathbf{5} & \text { ND } \\ \mathbf{5} & \text { ND }\end{array}$

Base Neutral Analyte

2,6-Dinitrotoluene

Diethyl Phthalate

4-Chlorophenyl Phenyl Ether

Fluorene

4-Nitroaniline

N-Nitrosodiphenylamine

4-Bromophenyl Phenyl Ether

Hexachlorobenzene

Phenanthrene

Anthracene

Di-n-butyl Phthalate

Fluoranthene

Pyrene

Butylbenzyl Phthalate

3,3'-Dichlorobenzidine

Benz(a)anthracene

Bis(2-ethylhexyl) Phthalate

Chrysene

Di-n-octyl Phthalate

Benzolblfluoranthene

Benzo(k)fluoranthene

Benzolalpyrene

Indeno(1,2,3-c,d)pyrene

Dibenz(a,h)anthracene

Benzolg,h,i)perylene

Acid Analyte

2,4-Dichlorophenol

4-Chloro-3-methylphenol

2,4,6-Trichlorophenol

2,4,5-Trichlorophenol

2,4-Dinitrophenol

4-Nitrophenol

2-Methyl-4,6-dinitrophenol

Pentachlorophenol

$\begin{array}{cc}\text { MRL } & \text { Result } \\ \mathbf{5} & \text { ND } \\ \mathbf{5} & \text { ND } \\ \mathbf{5} & \text { ND } \\ \mathbf{5} & \text { ND } \\ 20 & \text { ND } \\ \mathbf{5} & \text { ND } \\ \mathbf{5} & \text { ND } \\ \mathbf{5} & \text { ND } \\ \mathbf{5} & \text { ND } \\ \mathbf{5} & \text { ND } \\ \mathbf{5} & \text { ND } \\ \mathbf{5} & \text { ND } \\ \mathbf{5} & \text { ND } \\ \mathbf{5} & \text { ND } \\ \mathbf{2 0} & \text { ND } \\ \mathbf{5} & \text { ND } \\ \mathbf{5} & \text { ND } \\ \mathbf{5} & \text { ND } \\ \mathbf{5} & \text { ND } \\ \mathbf{5} & \text { ND } \\ \mathbf{5} & \text { ND } \\ \mathbf{5} & \text { ND } \\ \mathbf{5} & \text { ND } \\ \mathbf{5} & \text { ND } \\ \mathbf{5} & \text { ND }\end{array}$

MRL Result

$5 \quad$ ND

5 ND

5 ND

5 ND

50 ND

50 ND

20 ND

$30 \quad$ ND

MRLMethod Reporting Limit

NO None Detected at or above the method reporting limit

- Quantified as 4-methylphenol. 


\section{PSI Environmental Laboratories}

2595 E. AJport Drive Tucson, Arizana 85706 602-573-6565 FAx 602-573-6550 1-800-P5I-LAB5

State of Arizona License $\$ 0009$

Client:

Peroxidation Systems Inc.

5151 E. Broadway, Suite 600

Tucson, AZ 85711

Sample I.D.: $\quad$ 16A-9

Laboratory I.D.: 101993-28

Sample Type: Water

Project Name: DOE/Tenn

Date Sampled: $\quad 10 / 18 / 1993$

Project Number: 0345

Date Received: $\quad 10 / 19 / 1993$

P.O. Number: None

Date Analyzed: $12 / 10 / 1993$

Analyzed By: See Comments

Base Neutral/Acid Semivolatile Organic Compounds

EPA Methods 3510/8270 $\mu q \Omega(p p b)$

Base Neutral Analyte

N-Nitrosodimethylamine

Bis(2-chloroethyl) Ether

1,2-Dichlorobenzene

1,3-Dichlorobenzene

1,4-Dichlorobenzene

Bis(2-chloroisopropyl) Ether

N-Nitrosodi-n-propylamine

Hexachloroethane

Nitrobenzene

Isophorone

Bis(2-chloroethoxy) methane

1,2,4-Trichlorobenzene

Naphthalene

4-Chloroaniline

Hexachlorobutadiene

2-Methylnaphthalene

Hexachlorocyclopentadiene

2-Chloronaphthalene

2-Nitroaniline

Dimethyl Phthalate

Acenaphthylene

3-Nitroaniline

Acenaphthene

Dibenzofuran

2,4-Dinitrotoluene

Aniline

Acid Analyte

Phenol

2-Chlorophenol

Benzyl Alcohol

2-Methylphenol

3- and 4-Methylphenol ${ }^{\circ}$

2-Nitrophenol

2,4-Dimethylphenol

Benzoic Acid

MRLMethod Reporting Limit

ND None Detected at or above the method reporting limit

- Quantified as 4-methylphenol.

Comments:

Golden State/CAS Laboratories, Inc.

6925 Canoga Avenue

Canoga Park, CA 91303
Base Neutral Analyte

2,6-Dinitrotoluene

Diethyl Phthalate

4-Chlorophenyl Phenyl Ether

Flúorene

4-Nitroaniline

N-Nitrosodiphenylamine

4-Bromophenyl Phenyl Ether

Hexachlorobenzene

Phenanthrene

Anthracene

Di-n-butyl Phthalate

Fluoranthene

Pyrene

Butylbenzyl Phthalate

3,3'-Dichlorobenzidine

Benz(a)anthracene

Bis(2-ethylhexyl) Phthalate

Chrysene

Di-n-octyl Phthalate

Benzo(b)fluoranthene

Benzo(k)fluoranthene

Benzo(a)pyrene

Indeno(1,2,3-c,d)pyrene

Dibenz(a,h)anthracene

Benzolg,h,i)perylene

Acid Analyte

2,4-Dichlorophenol

4-Chloro-3-methylphenol

2,4,6-Trichlorophenol

2,4,5-Trichlorophenol

2,4-Dinitrophenol

4-Nitrophenol

2-Methy-4,6-dinitrophenol

Pentachlorophenol
MRL Result

$\begin{array}{rc}\text { MRL } & \text { Result } \\ \mathbf{5} & \text { ND } \\ \mathbf{5} & \text { ND } \\ \mathbf{5} & \text { ND } \\ \mathbf{5} & \text { ND } \\ \mathbf{2 0} & \text { ND } \\ \mathbf{5} & \text { ND } \\ \mathbf{5} & \text { ND } \\ \mathbf{5} & \text { ND } \\ \mathbf{5} & \text { ND } \\ \mathbf{5} & \text { ND } \\ \mathbf{5} & \text { ND } \\ \mathbf{5} & \text { ND } \\ \mathbf{5} & \text { ND } \\ \mathbf{5} & \text { ND } \\ \mathbf{2 0} & \text { ND } \\ \mathbf{5} & \text { ND } \\ \mathbf{5} & \text { ND } \\ \mathbf{5} & \text { ND } \\ \mathbf{5} & \text { ND } \\ \mathbf{5} & \text { ND } \\ \mathbf{5} & \text { ND } \\ \mathbf{5} & \text { ND } \\ \mathbf{5} & \text { ND } \\ \mathbf{5} & \text { ND } \\ \mathbf{5} & \text { ND } \\ & \end{array}$

$\begin{array}{ll}\mathbf{5} & \text { ND } \\ \mathbf{5} & \text { ND } \\ \mathbf{5} & \text { ND } \\ \mathbf{5} & \text { ND } \\ \mathbf{5 0} & \text { ND } \\ \mathbf{5 0} & \text { ND } \\ \mathbf{2 0} & \text { ND } \\ \mathbf{3 0} & \text { ND }\end{array}$

Reviewed By:

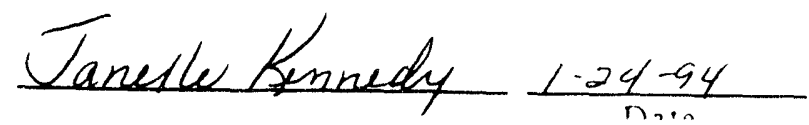




\section{PSI Environmental Laboratories}

2595 E. Airport Drive Tucson. Arizona 85706 602-573-6565 FAX 602-573-6550 1-800-P5/-LA85 ANALYTICAL REPORT

State of Arizona License $\$ 0009$

Client:

Peroxidation Systems Inc. 5151 E. Broadway, Suite 600

Tucson, AZ 85711

Project Name: DOE/Tenn

Project Number: 0345

P.O. Number: None
Sample I.D.: $\quad 17 A-0$

Laboratory I.D.: 101993-29

Sample Type: Water

Date Sampled: $\quad 10 / 18 / 1993$

Date Received: $\quad 10 / 19 / 1993$

Date Analyzed: $\quad 12 / 10 / 1993$

Analyzed By: See Comments

Base Neutral/Acid Semivolatile Organic Compounds EPA Methods $3510 / 8270$

$\mu \mathrm{g} / \mathrm{ppb}$ )

Base Neutral Analyte

N-Nitrosodimethylamine

Bis(2-chloroethyl) Ether

1,2-Dichlorobenzene

1,3-Dichlorobenzene

1,4-Dichlorobenzene

Bis(2-chloroisopropyl) Ether

N-Nitrosodi-n-propylamine

Hexachloroethane

Nitrobenzene

Isophorone

Bis(2-chloroethoxy) methane

1,2,4-Trichlorobenzene

Naphthalene

4-Chloroaniline

Hexachlorobutadiene

2-Methylnaphthalene

Hexachlorocyclopentadiene

2-Chloronaphthalene

2-Nitroaniline

Dimethyl Phthalate

Acenaphthylene

3-Nitroaniline

Acenaphthene

Dibenzofuran

2,4-Dinitrotoluene

Aniline

Acid Analyte

Phenol

2-Chlorophenol

Benzyl Alcohol

2-Methyiphenol

3- and 4-Methylphenol"

2-Nitrophenol

2,4-Dimethylphenol

Benzoic Acid

MRLMethod Reporting Limit

ND None Detected at or above the method reporting limit

- Quantified as 4-methylphenol.
Comments:

Golden State/CAS Laboratories, Inc
Base Neutral Analyte

2,6-Dinitrotoluene

Diethyl Phthalate

4-Chlorophenyl Phenyl Ether

Fluorene

4-Nitroaniline

N-Nitrosodiphenylamine

4-Bromophenyl Phenyl Ether

Hexachlorobenzene

Phenanthrene

Anthracene

Di-n-butyl Phthalate

Fluoranthene

Pyrene

Butylbenzyl Phthalate

3,3'-Dichlorobenzidine

Benz(a)anthracene

Bis(2-ethylhexyl) Phthalate

Chrysene

Di-n-octyl Phthalate

Benzo(b)fluoranthene

Benzo(k)fluoranthene

Benzola)pyrene

Indenol1,2,3-c,d)pyrene

Dibenz(a,h)anthracene

Benzolg, $h, i)$ perylene

Acid Analyte

2,4-Dichlorophenol

4-Chloro-3-methylphenol

2,4,6-Trichlorophenol

2,4,5-Trichlorophenol

2,4-Dinitrophenol

4-Nitrophenol

2-Methyl-4,6-dinitrophenol

Pentachlorophenol

$\begin{array}{rr}\text { MRL } & \text { Result } \\ \mathbf{5} & \text { ND } \\ \mathbf{5} & \text { ND } \\ \mathbf{5} & \text { ND } \\ \mathbf{5} & \text { ND } \\ 20 & \text { ND } \\ \mathbf{5} & \text { ND } \\ \mathbf{5} & \text { ND } \\ \mathbf{5} & \text { ND } \\ \mathbf{5} & \text { ND } \\ \mathbf{5} & \text { ND } \\ \mathbf{5} & \text { ND } \\ \mathbf{5} & \text { ND } \\ \mathbf{5} & \text { ND } \\ \mathbf{5} & \text { ND } \\ \mathbf{2 0} & \text { ND } \\ \mathbf{5} & \text { ND } \\ \mathbf{5} & \text { ND } \\ \mathbf{5} & \text { ND } \\ \mathbf{5} & \text { ND } \\ \mathbf{5} & \text { ND } \\ \mathbf{5} & \text { ND } \\ \mathbf{5} & \text { ND } \\ \mathbf{5} & \text { ND } \\ \mathbf{5} & \text { ND } \\ \mathbf{5} & \text { ND } \\ & \end{array}$

MRL Result

$\begin{array}{rr}\mathbf{5} & \text { ND } \\ \mathbf{5} & \text { ND } \\ \mathbf{5} & \text { ND } \\ \mathbf{5} & \text { ND } \\ \mathbf{5 0} & \text { ND } \\ 50 & \text { ND } \\ 20 & \text { ND } \\ 30 & \text { ND }\end{array}$

Reviewed By:

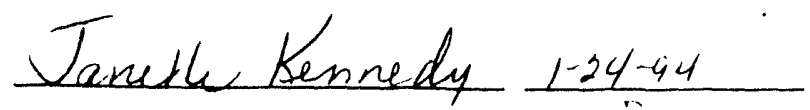


Client:

Peroxidation Systems lnc.

5151 E. Broadway, Suite 600

Tucson, AZ 85711

Project Name: DOE/Tenn

Project Number: 0345

P.O. Number: None

\author{
Sample I.D.: $\quad$ 17A-3 \\ Laboratory I.D.: 101993-30 \\ Sample Type: Water \\ Date Sampled: $\quad$ 10/18/1993 \\ Date Received: $\quad 10 / 19 / 1993$ \\ Date Analyzed: $\quad 12 / 10 / 1993$
}

Analyzed By: See Comments

Base Neutral/Acid Semivolatile Organic Compounds EPA Methods 3510/8270 $\mu \mathrm{g} \Omega(\mathrm{ppb})$

Base Neutral Analyte

N-Nitrosodimethylamine

Bis(2-chloroethyl) Ether

1.2-Dichlorobenzene

1,3-Dichlorobenzene

1,4-Dichlorobenzene

Bis(2-chloroisopropyl) Ether

$N$-Nitrosodi-n-propylamine

Hexachloroethane

Nitrobenzene

Isophorone

Bis(2-chloroethoxy) methane

1,2,4-Trichlorobenzene

Naphthalene

4-Chloroaniline

Hexachlorobutadiene

2-Methyinaphthalene

Hexachlorocyclopentadiene

2-Chloronaphthalene

2-Nitroaniline

Dimethyt Phthalate

Acenaphthylene

3-Nitroaniline

Acenaphthene

Dibenzofuran

2,4-Dinitrotoluene

Aniline

Acid Analyte

Phenol

2-Chlorophenol

Benzyl Alcohol

2-Methylphenol

3- and 4-Methylphenol*

2-Nitrophenol

2,4-Dimethylphenol

Benzoic Acid

Result
ND
ND
ND
ND
ND
ND
ND
ND
ND
ND
ND
ND
ND
ND
ND
ND
ND
ND
ND
ND
ND
ND
ND
ND
ND
ND

MRL

Result

ND

ND

ND

ND

ND

ND

ND

ND
Base Neutral Analyte

2,6-Dinitrotoluene

Diethyl Phthalate

4-Chlorophenyl Phenyl Ether Fluorene

4-Nitroaniline

N-Nitrosodiphenylamine

4-Bromophenyl Phenyl Ether

Hexachlorobenzene

Phenanthrene

Anthracene

Di-n-butyl Phthalate

Fuoranthene

Pyrene

Butylbenzyl Phthalate

3,3'-Dichlorobenzidine

Benz(a)anthracene

Bis(2-ethythexyl) Phthalate

Chrysene

Di-n-octyl Phthalate

Benzo(b)fiuoranthene

Benzolkffluoranthene

Benzolalpyrene

Indeno(1,2,3-c,d)pyrene

Dibenzla,hlanthracene

Benzo(o,h,i)peryiene

Acid Analyte

2,4-Dichlorophenol

4-Chloro-3-methylphenol

2,4,6-Trichlorophenol

2,4,5-Trichlorophenol

2,4-Dinitrophenol

4-Nitrophenol

2-Methyl-4,6-dinitrophenol

Pentachlorophenol

$\begin{array}{cc}\text { MRL } & \text { Result } \\ \mathbf{5} & \text { ND } \\ \mathbf{5} & \text { ND } \\ \mathbf{5} & \text { ND } \\ \mathbf{5} & \text { ND } \\ \mathbf{2 0} & \text { ND } \\ \mathbf{5} & \text { ND } \\ \mathbf{5} & \text { ND } \\ \mathbf{5} & \text { ND } \\ \mathbf{5} & \text { ND } \\ \mathbf{5} & \text { ND } \\ \mathbf{5} & \text { ND } \\ \mathbf{5} & \text { ND } \\ \mathbf{5} & \text { ND } \\ \mathbf{5} & \text { ND } \\ \mathbf{2 0} & \text { ND } \\ \mathbf{5} & \text { ND } \\ \mathbf{5} & \text { ND } \\ \mathbf{5} & \text { ND } \\ \mathbf{5} & \text { ND } \\ \mathbf{5} & \text { ND } \\ \mathbf{5} & \text { ND } \\ \mathbf{5} & \text { ND } \\ \mathbf{5} & \text { ND } \\ \mathbf{5} & \text { ND } \\ \mathbf{5} & \text { ND }\end{array}$

MKL Result

$\begin{array}{rr}\mathbf{5} & \text { ND } \\ \mathbf{5} & \text { ND } \\ \mathbf{5} & \text { ND } \\ \mathbf{5} & \text { ND } \\ \mathbf{5 0} & \text { ND } \\ \mathbf{5 0} & \text { ND } \\ 20 & \text { ND } \\ 30 & \text { ND }\end{array}$

MRLMethod Reporting Limit

ND None Detected at or above the method reporting limit

- Quantified as 4-methylphenol. 


\section{PSI Environmental Laboratories}

2595 E. Aimport Drtve Turwan, Arizona 85706 602-573-6565 FAX 602-573-6550 1-800-P5I-LAB5 ANALYTICAL REPORT

State of Arizona License $\$ 0009$

Client:

Peroxidation Systems Inc.

5151 E. Broadway, Suite 600

Tucson, AZ 85711

\author{
Sample I.D.: $\quad$ 17A-9 \\ Laboratory I.D.: 101993-32 \\ Sample Type: Water \\ Date Sampled: $\quad 10 / 18 / 1993$ \\ Date Received: $\quad 10 / 19 / 1993$ \\ Date Analyzed: 12/10/1993
}

Analyzed By: See Comments

P.O. Number: None Base Neutral/Acid Semivolatile Organic Compounds
EPA Methods $3510 / 8270$ $\mu \mathrm{g} \Omega(\mathrm{ppb})$

Base Neutral Analrte N-Nitrosodimethylamine Bis(2-chloroethyl) Ether

1,2-Dichlorobenzene

1,3-Dichlorobenzene

1,4-Dichlorobenzene

Bis(2-chloroisopropyl) Ether

$N$-Nitrosodi-n-propylamine

Hexachloroethane

Nitrobenzene

Isophorone

Bis (2-chloroethoxy) methane

1,2,4-Trichlorobenzene

Naphthalene

4-Chloroaniline

Hexachlorobutadiene

2-Methylnaphthalene

Hexachlorocyclopentadiene

2-Chloronaphthalene

2-Nitruaniline

Dimethyl Phthalate

Acenaphthylene

3-Nitroaniline

Acenaphthene

Dibenzofuran

2,4-Dinitrotoluene

Aniline

Acid Analyto

Phenol

2-Chlorophenol

Benzyl Alcohol

2-Methylphenol

3- and 4-Methylphenol*

2-Nitrophenol

2,4-Dimethylphenol

Benzoic Acid

$\begin{array}{rc}\text { MRL } & \text { Result } \\ \mathbf{5} & \text { ND } \\ \mathbf{5} & \text { ND } \\ \mathbf{5} & \text { ND } \\ \mathbf{5} & \text { ND } \\ \mathbf{5} & \text { ND } \\ \mathbf{5} & \text { ND } \\ \mathbf{5} & \text { ND } \\ \mathbf{5} & \text { ND } \\ \mathbf{5} & \text { ND } \\ \mathbf{5} & \text { ND } \\ \mathbf{5} & \text { ND } \\ \mathbf{5} & \text { ND } \\ \mathbf{5} & \text { ND } \\ \mathbf{5} & \text { ND } \\ \mathbf{5} & \text { ND } \\ \mathbf{5} & \text { ND } \\ \mathbf{1 0} & \text { ND } \\ \mathbf{5} & \text { ND } \\ \mathbf{2 0} & \text { ND } \\ \mathbf{5} & \text { ND } \\ \mathbf{5} & \text { ND } \\ \mathbf{2 0} & \text { ND } \\ \mathbf{5} & \text { ND } \\ \mathbf{5} & \text { ND } \\ \mathbf{5} & \text { ND } \\ \mathbf{5} & \text { ND } \\ & \end{array}$

MRL Result

$\begin{aligned} \mathbf{5} & \text { ND } \\ \mathbf{5} & \text { ND } \\ \mathbf{1 0} & \text { ND } \\ \mathbf{5} & \text { ND } \\ \mathbf{5} & \text { ND } \\ \mathbf{5} & \text { ND } \\ \mathbf{5} & \text { ND } \\ \mathbf{5 0} & \text { ND }\end{aligned}$

MRLMethod Reporting Limit

ND None Detected at or above the method reporting limit

- Quantified as 4-methylphenol.
Base Neutral Analyte

2,6-Dinitrotoluene

Diethyl Phthalate

4-Chlorophenyl Phenyl Ether

Fluorene

4-Nitroaniline

N-Nitrosodiphenylamine

4-Bromophenyl Phenyl Ether

Hexachlorobenzene

Phenanthrene

Anthracene

Di-n-butyl Phthalate

Fluoranthene

Pyrene

Butylbenzyl Phthalate

3,3'-Dichlorobenzidine

Benz(a)anthracene

Bis(2-othylhexy) Phthalate

Chrysene

Di-n-octyl Phthalate

Benzo(b)fluoranthene

Benzo(k)fluoranthene

Benzola)pyrene

Indeno(1,2,3-c,d)pyrene

Dibenz(a,h)anthracene

Benzolo, $h, i)$ perylene

Acid Analyte

2,4-Dichlorophenol

4-Chloro-3-methylphenol

2,4,6-Trichlorophenol

2,4,5-Trichlorophenol

2,4-Dinitrophenol

4-Nitrophenol

2-Methyl-4,6-dinitrophenol

Pentachlorophenol
Reviewed By:

$\begin{array}{rr}\text { MRL } & \text { Result } \\ \mathbf{5} & \text { ND } \\ \mathbf{5} & \text { ND } \\ \mathbf{5} & \text { ND } \\ \mathbf{5} & \text { ND } \\ \mathbf{2 0} & \text { ND } \\ \mathbf{5} & \text { ND } \\ \mathbf{5} & \text { ND } \\ \mathbf{5} & \text { ND } \\ \mathbf{5} & \text { ND } \\ \mathbf{5} & \text { ND } \\ \mathbf{5} & \text { ND } \\ \mathbf{5} & \text { ND } \\ \mathbf{5} & \text { ND } \\ \mathbf{5} & \text { ND } \\ \mathbf{2 0} & \text { ND } \\ \mathbf{5} & \text { ND } \\ \mathbf{5} & \text { ND } \\ \mathbf{5} & \text { ND } \\ \mathbf{5} & \text { ND } \\ \mathbf{5} & \text { ND } \\ \mathbf{5} & \text { ND } \\ \mathbf{5} & \text { ND } \\ \mathbf{5} & \text { ND } \\ \mathbf{5} & \text { ND } \\ \mathbf{5} & \text { ND }\end{array}$

MRL Result

$\begin{array}{rr}5 & \text { ND } \\ 5 & \text { ND } \\ 5 & \text { ND } \\ 5 & \text { ND } \\ 50 & \text { ND } \\ 50 & \text { ND } \\ 20 & \text { ND } \\ 30 & \text { ND }\end{array}$

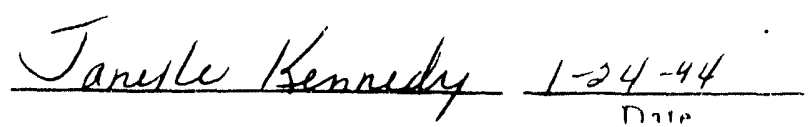




\section{PSI Environmental Laboratories}

\section{E. Almpart Orive Tucson. Arizona 85706 602.573-6565 FAX 602.573-6550 1-800-P5/-LA85}

ANALXTCAL REPORT

State of Arizona License $\$ 0009$

Client:

Peroxidation Systems Inc. 5151 E. Broadway, Suite 600

Tucson, AZ 85711

Project Name: LOE/Tenn

Project Number: 0345

P.O. Number: None

\author{
Sample I.D.: $\quad 18 \mathrm{~A}-0$ \\ Laboratory I.D.: 101993-33 \\ Sample Type: Water \\ Date Sampled: $\quad 10 / 18 / 1993$ \\ Date Received: $\quad 10 / 19 / 1993$ \\ Date Analyzed: $\quad 12 / 10 / 1993$
}

Analyzed By: $\quad$ See Comments

Base Neutral/Acid Semivolatile Organic Compounds EPA Methods $3510 / 8270$ $\mu \mathrm{g} \Omega(\mathrm{ppb})$

Base Neutral Analyte
N-Nitrosodimethylamine
Bis(2-chloroethyl) Ether
1,2-Dichlorobenzene
1,3-Dichlorobenzene
1,4-Dichlorobenzene
Bis(2-chloroisopropyl) Ether
N-Nitrosodi-n-propylamine
Hexachloroethane
Nitrobenzene
Isophorone
Bis(2-chloroethoxy) methane
1,2,4-Trichlorobenzene
Naphthalene
4-Chloroaniline
Hexachlorobutadiene
2-Methylnaphthalene
Hexachlorocyclopentadiene
2-Chloronaphthalene
2-Nitroaniline
Dimethyl Phthalate
Acenaphthylene
3-Nitroaniline
Acenaphthene
Dibenzofuran
2,4-Dinitrotoluene
Aniline

Acid Analyte

Phenol

2-Chlorophenol

Benzyl Alcohol

2-Methyiphenol

3- and 4-Methylphenol"

2-Nitrophenol

2,4-Dimethylphenol

Benzoic Acid

$\begin{array}{rr}\text { MRL } & \text { Result } \\ \mathbf{5} & \text { ND } \\ \mathbf{5} & \text { ND } \\ \mathbf{5} & \text { ND } \\ \mathbf{5} & \text { ND } \\ \mathbf{5} & \text { ND } \\ \mathbf{5} & \text { ND } \\ \mathbf{5} & \text { ND } \\ \mathbf{5} & \text { ND } \\ \mathbf{5} & \text { ND } \\ \mathbf{5} & \text { ND } \\ \mathbf{5} & \text { ND } \\ \mathbf{5} & \text { ND } \\ \mathbf{5} & 15 \\ \mathbf{5} & \text { ND } \\ \mathbf{5} & \text { ND } \\ \mathbf{5} & 8 \\ 10 & \text { ND } \\ \mathbf{5} & \text { ND } \\ \mathbf{2 0} & \text { ND } \\ \mathbf{5} & \text { ND } \\ \mathbf{5} & \text { ND } \\ \mathbf{2 0} & \text { ND } \\ \mathbf{5} & \text { ND } \\ \mathbf{5} & \text { ND } \\ \mathbf{5} & \text { ND } \\ \mathbf{5} & \text { ND }\end{array}$

MRL Result

$\begin{array}{rr}\mathbf{5} & \text { ND } \\ \mathbf{5} & \text { ND } \\ \mathbf{1 0} & \text { ND } \\ \mathbf{5} & \text { ND } \\ \mathbf{5} & \text { ND } \\ \mathbf{5} & \text { ND } \\ \mathbf{5} & \text { ND } \\ \mathbf{5 0} & \text { ND }\end{array}$

Base Neutral Analyte

2,6-Dinitrotoluene

Diethyl Phthalate

4-Chlorophenyl Phenyl Ether

Fiuorene

4-Nitroaniline

N-Nitrosodiphenylamine

4-Bromophenyl Phenyl Ether

Hexachlorobenzene

Phenanthrene

Anthracene

Di-n-butyl Phthalate

Fluoranthene

Pyrene

Butylbenzyl Phthalate

3,3'-Dichlorobenzidine

Benz(a)anthracene

Bis(2-ethythexyl) Phthalate

Chrysene

Di-n-octy Phthalate

Benzo(b)fluoranthene

Benzo(k)fluoranthene

Benzola)pyrene

Indeno(1,2,3-c,d)pyrene

Dibenz(a,h)anthracene

Benzolo,h,ilperylene

Acid Analrte

2,4-Dichlorophenol

4-Chloro-3-methylphenol

2,4,6-Trichlorophenol

2,4,5-Trichlorophenol

2,4-Dinitrophenol

4-Nitrophenol

2-Methyl-4,6-dinitrophenol

Pentachlorophenol $\begin{array}{rc}\text { MRL } & \text { Result } \\ \mathbf{5} & \text { ND } \\ \mathbf{5} & \text { ND } \\ \mathbf{5} & \text { ND } \\ \mathbf{5} & \text { ND } \\ \mathbf{2 0} & \text { ND } \\ \mathbf{5} & \text { ND } \\ \mathbf{5} & \text { ND } \\ \mathbf{5} & \text { ND } \\ \mathbf{5} & \text { ND } \\ \mathbf{5} & \text { ND } \\ \mathbf{5} & \text { ND } \\ \mathbf{5} & \text { ND } \\ \mathbf{5} & \text { ND } \\ \mathbf{5} & \text { ND } \\ \mathbf{2 0} & \text { ND } \\ \mathbf{5} & \text { ND } \\ \mathbf{5} & \text { ND } \\ \mathbf{5} & \text { ND } \\ \mathbf{5} & \text { ND } \\ \mathbf{5} & \text { ND } \\ \mathbf{5} & \text { ND } \\ \mathbf{5} & \text { ND } \\ \mathbf{5} & \text { ND } \\ \mathbf{5} & \text { ND } \\ \mathbf{5} & \text { ND }\end{array}$

MRL Result

$\begin{array}{rr}\mathbf{5} & \text { ND } \\ \mathbf{5} & \text { ND } \\ \mathbf{5} & \text { ND } \\ \mathbf{5} & \text { ND } \\ \mathbf{5 0} & \text { ND } \\ 50 & \text { ND } \\ 20 & \text { ND } \\ 30 & \text { ND }\end{array}$

MRLMethod Reporting Limit

ND None Detected at or above the method reporting limit

- Quantified as 4-methylphenol.

Comments:

Golden State/CAS Laboratories, Inc

6925 Canoga Avenue

Canoga Park. CA 91303
Reviewed By:

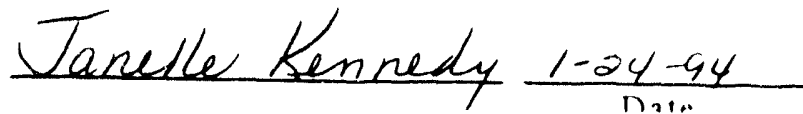


Client:

Peroxidation Systems Inc.

5151 E. Broadway, Suite 600

Tucson, AZ 85711

Project Name: DOE/Tenn

Project Number: 0345

P.O. Number: None
Sample I.D.:

18A-3

Laboratory I.D.: 101993-34

Sample Type: Water

Date Sampled: $\quad 10 / 18 / 1993$

Date Received: $\quad 10 / 19 / 1993$

Date Analyzed: $\quad 12 / 10 / 1993$

Analyzed By: See Comments

Base Neutral/Acid Semivolatile Organic Compounds

EPA Methods $3510 / 8270$

$\mu \mathrm{g} / \mathrm{ppb})$

Base Neutral Analyte
N-Nitrosodimethylamine
Bis(2-chloroethyl) Ether
1,2-Dichlorobenzene
1,3-Dichlorobenzene
1,4-Dichlorobenzene
Bis(2-chloroisopropyl) Ether
N-Nitrosodi-n-propylamine
Hexachloroethane
Nitrobenzene
Isophorone
Bis (2-chloroethoxy) methane
1,2,4-Trichlorobenzene
Naphthalene
4-Chloroaniline
Hexachlorobutadiene
2-Methylnaphthalene
Hexachlorocyclopentadiene
2-Chloronaphthalene
2-Nitroaniline
Dimethyl Phthalate
Acenaphthylene
3-Nitroaniline
Acenaphthene
Dibenzofuran
2,4-Dinitrotoluene
Aniline

Base Neutral Analyte

N-Nitrosodimethylamine

Bis(2-chloroethyl) Ether

1,3-Dichlorobenzene

1,4-Dichlorobenzene

Hexachloroethan

sophorone

Bis (2-chloroethoxy) methane

lorobenzene

Hexachlorobutadiene

2-Methylnaphthalene

2-Chloronaphthalene

2-Nitroaniline

Dimethyl Phthalate

Acenaphthene

2,4-Dinitrotoluene

Acid Analyte

Phenol

2-Chlorophenol

Benzyl Alcohol

2-Methyiphenol

3- and 4-Methylphenol*

2-Nitrophenol

2,4-Dimethylphenol

Benzoic Acid

MRLMethod Reporting Limit

ND None Detected at or above the method reporting limit

- Quantified as 4-methylphenol.

$\begin{array}{rr}\text { MRL } & \text { Result } \\ \mathbf{5} & \text { ND } \\ \mathbf{5} & \text { ND } \\ \mathbf{5} & \text { ND } \\ \mathbf{5} & \text { ND } \\ \mathbf{5} & \text { ND } \\ \mathbf{5} & \text { ND } \\ \mathbf{5} & \text { ND } \\ \mathbf{5} & \text { ND } \\ \mathbf{5} & \text { ND } \\ \mathbf{5} & \text { ND } \\ \mathbf{5} & \text { ND } \\ \mathbf{5} & \text { ND } \\ \mathbf{5} & \text { ND } \\ \mathbf{5} & \text { ND } \\ \mathbf{5} & \text { ND } \\ \mathbf{5} & \text { ND } \\ \mathbf{1 0} & \text { ND } \\ \mathbf{5} & \text { ND } \\ \mathbf{2 0} & \text { ND } \\ \mathbf{5} & \text { ND } \\ \mathbf{5} & \text { ND } \\ \mathbf{2 0} & \text { ND } \\ \mathbf{5} & \text { ND } \\ \mathbf{5} & \text { ND } \\ \mathbf{5} & \text { ND } \\ \mathbf{5} & \text { ND }\end{array}$

Base Neutral Analvte

2,6-Dinitrotoluene

Diethyl Phthalate

4-Chlorophenyl Phenyl Ether

Fluorene

4-Nitroaniline

N-Nitrosodiphenylamine

4-Bromophenyl Phenyl Ether

Hexachlorobenzene

Phenanthrene

Anthracene

Di-n-butyl Phthalate

Fluoranthene

Pyrene

Butylbenzyl Phthalate

3,3'-Dichlorobenzidine

Benz(a)anthracene

Bis(2-ethylhexyl) Phthalate

Chrysene

Di-n-octyl Phthalate

Benzo(b)fluoranthene

Benzo(k)fluoranthene

Benzolalpyrene

Indeno(1,2,3-c,d)pyrene

Dibenzla, h)anthracene

Benzolo, h,i)perylene

Acid Analyte

2,4-Dichlorophenol

4-Chloro-3-methylphenol

2,4,6-Trichlorophenol

2,4,5-Trichlorophenol

2,4-Dinitrophenol

4-Nitrophenol

2-Methyl-4,6-dinitrophenol

Pentachloropheno!

$\begin{array}{rc}\text { MRL } & \text { Result } \\ \mathbf{5} & \text { ND } \\ \mathbf{5} & \text { ND } \\ \mathbf{5} & \text { ND } \\ \mathbf{5} & \text { ND } \\ \mathbf{2 0} & \text { ND } \\ \mathbf{5} & \text { ND } \\ \mathbf{5} & \text { ND } \\ \mathbf{5} & \text { ND } \\ \mathbf{5} & \text { ND } \\ \mathbf{5} & \text { ND } \\ \mathbf{5} & \text { ND } \\ \mathbf{5} & \text { ND } \\ \mathbf{5} & \text { ND } \\ \mathbf{5} & \text { ND } \\ \mathbf{2 0} & \text { ND } \\ \mathbf{5} & \text { ND } \\ \mathbf{5} & \text { ND } \\ \mathbf{5} & \text { ND } \\ \mathbf{5} & \text { ND } \\ \mathbf{5} & \text { ND } \\ \mathbf{5} & \text { ND } \\ \mathbf{5} & \text { ND } \\ \mathbf{5} & \text { ND } \\ \mathbf{5} & \text { ND } \\ \mathbf{5} & \text { ND }\end{array}$

MRL Result

$\begin{array}{rr}5 & \text { ND } \\ 5 & \text { ND } \\ 5 & \text { ND } \\ 5 & \text { ND } \\ 50 & \text { ND } \\ 50 & \text { ND } \\ 20 & \text { ND } \\ 30 & \text { ND }\end{array}$

Comments:

Golden State/CAS Laboratories, Inc.

6925 Canoga Avenue

Canoga Park. CA 91303
Reviewed By:

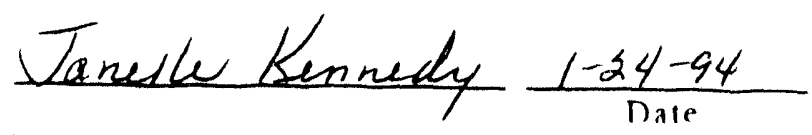


Client:

Peroxidation Systems Inc.

5151 E. Broadway, Suite 600

Tucson, AZ 85711
Sample I.D.: $\quad$ 18A-9

Laboratory I.D.: $101993-36$

Sample Type: Water

Date Sampled: $\quad$ 10/18/1993

Date Received: $\quad 10 / 19 / 1993$

Date Analyzed: $\quad 12 / 13 / 1993$

Analyzed By: See Commeats

P.O. Number: None

Base Neutral/Acid Semivolatile Organic Compounds EPA Methods 3510/8270

$\mathrm{LO} \Omega(\mathrm{ppb})$

Base Neutral Analyte

N-Nitrosodimethylamine

Bis(2-chloroethyl) Ether

1,2-Dichlorobenzene

1,3-Dichlorobenzene

1,4-Dichlorobenzene

Bis(2-chloroisopropyl) Ether

$\mathrm{N}$-Nitrosodi-n-propylamine

Hexachloroethane

Nitrobenzene

Isophorone

Bis (2-chloroethoxy) methane

1,2,4-Trichlorobenzene

Naphthalene

4-Chloroaniline

Hexachlorobutadiene

2-Methyinaphthalene

Hexachlorocyclopentadiene

2-Chloronaphthalene

2-Nitroaniline

Dimethyl Phthalate

Acenaphthylene

3-Nitroaniline

Acenaphthene

Dibenzofuran

2,4-Dinitrotoluene

Aniline

Acid Analvte

Phenol

2-Chlorophenol

Benzyl Alcohol

2-Methyiphenol

3- and 4-Methylphenol

2-Nitrophenol

2,4-Dimethylphenol

Benzoic Acid
Base Neutral Analvte

2,6-Dinitrotoluene

Diethyl Phthalate

4-Chlorophenyl Phenyl Ether

Fivorene

4-Nitroaniline

N-Nitrosodiphenylamine

4-Bromophenyl Phenyl Ether

Hexachlorobenzene

Phenanthrene

Anthracene

Di-n-butyl Phthalate

Fluoranthene

Pyrene

Butylbenzyl Phthalate

3,3'-Dichlorobenzidine

Benz(a)anthracene

Bis(2-othylhexyl) Phthalate

Chrysene

Din-octyl Phthalate

Benzo(b)fluoranthene

Benzo(k)fluoranthene

Benzola)pyrene

Indeno(1,2,3-c,d)pyrene

Dibenz (a,h)anthracene

Benzo(o,h,ilperyiene

Acid Analyte

2,4-Dichloropheriol

4-Chloro-3-methylphenol

2,4.6-Trichlorophenol

2,4,5-Trichlorophenol

2,4-Dinitrophenol

4-Nitrophenol

2-Methyt-4,6-dinitrophenol

Pentachlorophenol

$\begin{array}{cc}\text { MRL } & \text { Result } \\ \mathbf{5} & \text { ND } \\ \mathbf{5} & \text { ND } \\ \mathbf{5} & \text { ND } \\ \mathbf{5} & \text { ND } \\ \mathbf{2 0} & \text { ND } \\ \mathbf{5} & \text { ND } \\ \mathbf{5} & \text { ND } \\ \mathbf{5} & \text { ND } \\ \mathbf{5} & \text { ND } \\ \mathbf{5} & \text { ND } \\ \mathbf{5} & \text { ND } \\ \mathbf{5} & \text { ND } \\ \mathbf{5} & \text { ND } \\ \mathbf{5} & \text { ND } \\ \mathbf{2 0} & \text { ND } \\ \mathbf{5} & \text { ND } \\ \mathbf{5} & \text { ND } \\ \mathbf{5} & \text { ND } \\ \mathbf{5} & \text { ND } \\ \mathbf{5} & \text { ND } \\ \mathbf{5} & \text { ND } \\ \mathbf{5} & \text { ND } \\ \mathbf{5} & \text { ND } \\ \mathbf{5} & \text { ND } \\ \mathbf{5} & \text { ND }\end{array}$

MRL Result

5 ND

ND

ND

ND

ND

ND

ND

ND

MRLMethod Reporting Limit

ND None Detected at or above the method reporting limit

- Quantified as 4-methylphenol. 


\section{PSI Enviranmental Labarataries}

\section{E. Alrport Drive Tuceon. Arlzane 85706 602-573-6565 FAX 602.573-6550 1.800-p5/-LABS \\ ANALYTCAL REPORT \\ State of Arizona Lcense m0009}

Cllent:

\author{
Peroxidation Systems Inc. \\ 5151 E. Broadway, Suite 600 \\ Tucson, AZ 85711
}

Project Name: DOE/Tenn

Project Number: 0345

P.O. Number: None $\begin{array}{ll}\text { Sample I.D.: } & 19 A-0 \\ \text { Laboratory I.D.: } & 101993-37 \\ \text { Sample Type: } & \text { Water } \\ \text { Date Sampled: } & 10 / 18 / 1993\end{array}$

Date Received: 10/19/1993

Date Analyzed: $\quad 12 / 13 / 1993$

Analyzed By: See Comments

Base Neutral/Acid Semivolatile Organic Compounds EPA Methods $3510 / 8270$

$\mu \mathrm{p} / \mathrm{ppb}$

\author{
Base Neutral Analyte \\ N-Nitrosodimethylamine \\ Bis(2-chloroethyl) Ether \\ 1,2-Dichlorobenzene \\ 1,3-Dichlorobenzene \\ 1,4-Dichlorobenzene \\ Bis (2-chloroisopropyll Ether \\ N-Nitrosodi-n-propylamine \\ Hexachloroethane \\ Nitrobenzene \\ Isophorone \\ Bis(2-chloroethoxy) methane \\ 1,2,4-Trict orobenzene \\ Naphthalene \\ 4-Chloroaniline \\ Hexachlorobutadiene \\ 2-Methyinaphthalene \\ Hexachlorocyclopentadiene \\ 2-Chloronaphthalene \\ 2-Nitroaniline \\ Dimethyl Phthalate \\ Acenaphthylene \\ 3-Nitroaniline \\ Acenaphthene \\ Dibenzofuran \\ 2,4-Dinitrotoluene \\ Aniline
}

Acid Analyte

Phenol

2-Chlorophenol

Benzyl Alcohol

2-Methyiphenol

3. and 4-Methylphenol"

2-Nitrophenol

2,4-Dimethylphenol

Benzoic Acid

MRLMethod Reporting Limit

ND None Detected at or above the method reporting limit

- Quantified as 4-methylphenol.
Base Noutral Analyte

2,6-Oinitrotoluene

Diethyl Phthalate

4-Chlorophenyl Phenyl Ether

Fluorene

4-Nitroaniline

N-Nitrosodiphenylamine

4-Bromophenyl Phenyl Ether

Hexachlorobenzene

Phenanthrene

Anthracene

Di-n-buryl Phthalate

Fuoranthene

Pyreno

Butylbenzyl Phthalate

3,3'-Dichlorobenzidine

Benz(a)anthracene

Bis (2-thyhexyl) Phthalate

Chrreene

Di-n-octyl Phthalate

Benzolblfiluoranthene

Benzolkffluoranthene

Benzolalpyrene

Indeno(1,2,3-c,d)pyrene

Dibenz(a,h)anthracene

Benzolo,h,ilperylene

Acid Analrte

2.4-Dichlorophenol

4-Chloro-3-methylphenol

2,4,6-Trichlorophenol

2,4,5-Trichlorophenol

2,4-Dinitrophenol

4-Nitrophenol

2-Methyl-4,6-dinitrophenol

Pentachlorophenol

$\begin{array}{rr}\text { MRL } & \text { Result } \\ \mathbf{5} & \text { ND } \\ \mathbf{5} & \text { ND } \\ \mathbf{5} & \text { ND } \\ \mathbf{5} & \text { ND } \\ \mathbf{2 0} & \text { ND } \\ \mathbf{5} & \text { ND } \\ \mathbf{5} & \text { ND } \\ \mathbf{5} & \text { ND } \\ \mathbf{5} & \text { ND } \\ \mathbf{5} & \text { ND } \\ \mathbf{5} & \text { ND } \\ \mathbf{5} & \text { ND } \\ \mathbf{5} & \text { ND } \\ \mathbf{5} & \text { ND } \\ \mathbf{2 0} & \text { ND } \\ \mathbf{5} & \text { ND } \\ \mathbf{5} & \text { ND } \\ \mathbf{5} & \text { ND } \\ \mathbf{5} & \text { ND } \\ \mathbf{5} & \text { ND } \\ \mathbf{5} & \text { ND } \\ \mathbf{5} & \text { ND } \\ \mathbf{5} & \text { ND } \\ \mathbf{5} & \text { ND } \\ \mathbf{5} & \text { ND } \\ & \\ & \\ \text { MRL } & \text { Result } \\ & \\ \mathbf{5} & \text { ND } \\ \mathbf{5} & \text { ND } \\ \mathbf{5} & \text { ND } \\ \mathbf{5} & \text { ND } \\ \mathbf{5 0} & \text { ND } \\ \mathbf{5 0} & \text { ND } \\ \mathbf{2 0} & \text { ND } \\ \mathbf{3 0} & \text { ND } \\ & \end{array}$


Client:

Peroxidation Systems Inc.

5151 E. Broadway, Suile 600

Tucson, AZ 85711

Project Name: DOE/Tenn

Project Number: 0345

P.O. Number: None

\author{
Semple I.D.: $\quad$ 19A-3 \\ Laboratory I.D.: 101993-38 \\ Sample Type: Water \\ Date Sampled: $\quad 10 / 18 / 1993$ \\ Date Received: $\quad 10 / 19 / 1993$ \\ Date Analyzed: $\quad 12 / 13 / 1993$
}

Analyzed By: See Comments

Base Neutral/Acid Semivolatile Organic Compounds

EPA Mothods $3610 / 8270$ $\mu \mathrm{ph}$ (ppb)

\begin{tabular}{|c|c|c|c|c|c|}
\hline $\begin{array}{l}\text { Base Neutral Analyte } \\
\text { N-Nitrosodimathylamine } \\
\text { Bis(2-chloroethyll Ether } \\
\text { 1,2-Dichlorobenzene } \\
\text { 1,3-Dichlorobenzene } \\
\text { 1,4-Dichlorobenzene } \\
\text { Bis(2-chloroisopropyl) Ether } \\
\text { N-Nitrosodi-n-propylamine } \\
\text { Hexachlorosthane } \\
\text { Nitrobenzene } \\
\text { Isophorone }\end{array}$ & $\begin{array}{r}\text { MAL } \\
5 \\
5 \\
5 \\
5 \\
5 \\
5 \\
5 \\
5 \\
5 \\
5 \\
5 \\
5 \\
5 \\
5 \\
5 \\
5 \\
10 \\
5 \\
20 \\
5 \\
5 \\
20 \\
5 \\
5 \\
5 \\
5\end{array}$ & $\begin{array}{l}\text { Rosult } \\
\text { ND } \\
\text { ND } \\
\text { ND } \\
\text { ND } \\
\text { ND } \\
\text { ND } \\
\text { ND } \\
\text { ND } \\
\text { ND } \\
\text { ND } \\
\text { ND } \\
\text { ND } \\
\text { ND } \\
\text { ND } \\
\text { ND } \\
\text { ND } \\
\text { NO } \\
\text { NO } \\
\text { ND } \\
\text { ND } \\
\text { ND } \\
\text { ND } \\
\text { ND } \\
\text { ND } \\
\text { ND } \\
\text { ND }\end{array}$ & 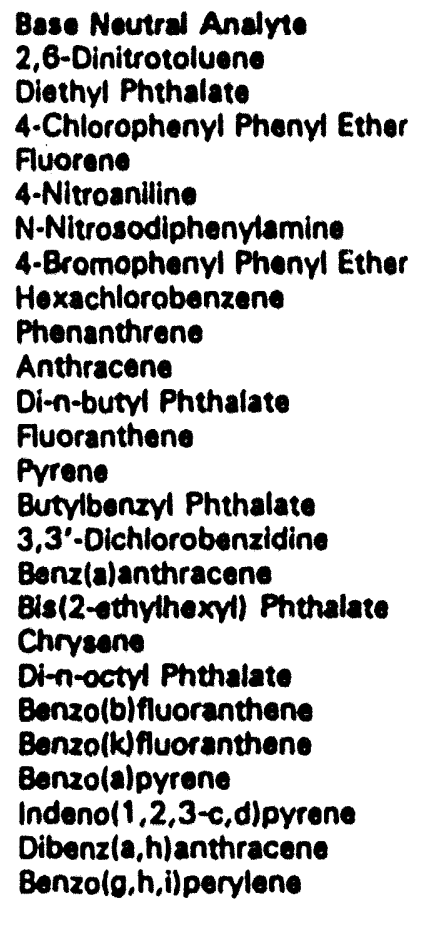 & $\begin{array}{r}\text { MRL } \\
5 \\
5 \\
5 \\
5 \\
20 \\
5 \\
5 \\
5 \\
5 \\
5 \\
5 \\
5 \\
5 \\
5 \\
20 \\
5 \\
5 \\
5 \\
5 \\
5 \\
5 \\
5 \\
5 \\
5 \\
5\end{array}$ & $\begin{array}{l}\text { Result } \\
\text { ND } \\
\text { ND } \\
\text { ND } \\
\text { ND } \\
\text { ND } \\
\text { ND } \\
\text { ND } \\
\text { ND } \\
\text { ND } \\
\text { ND } \\
\text { ND } \\
\text { ND } \\
\text { ND } \\
\text { ND } \\
\text { ND } \\
\text { ND } \\
\text { ND } \\
\text { ND } \\
\text { ND } \\
\text { ND } \\
\text { ND } \\
\text { ND } \\
\text { ND } \\
\text { ND } \\
\text { ND }\end{array}$ \\
\hline Acid Analyte & MRL & Result & Acid Analve & MRL & Result \\
\hline $\begin{array}{l}\text { Phenol } \\
\text { 2-Chlorophenol } \\
\text { Benzyl Alcohol } \\
\text { 2-Methylphenol } \\
\text { 3- and 4-Methylphenol" } \\
\text { 2-Nitrophenol } \\
\text { 2,4-Dimethylphenol } \\
\text { Benzoic Acid }\end{array}$ & $\begin{array}{r}5 \\
5 \\
10 \\
5 \\
5 \\
5 \\
5 \\
50\end{array}$ & $\begin{array}{l}\text { ND } \\
\text { ND } \\
\text { ND } \\
\text { ND } \\
\text { ND } \\
\text { ND } \\
\text { ND } \\
\text { ND }\end{array}$ & $\begin{array}{l}\text { 2,4-Dichlorophenol } \\
\text { 4-Chloro-3-methylphenol } \\
\text { 2,4,6-Trichlorophenol } \\
\text { 2,4,5-Trichlorophenol } \\
\text { 2,4-Dinitrophenol } \\
\text { 4-Nitrophenol } \\
\text { 2-Methit-4,6-dinitrophenol } \\
\text { Pentachlorophenol }\end{array}$ & $\begin{array}{r}5 \\
5 \\
5 \\
5 \\
50 \\
50 \\
20 \\
30\end{array}$ & $\begin{array}{l}\text { ND } \\
\text { ND } \\
\text { ND } \\
\text { ND } \\
\text { ND } \\
\text { ND } \\
\text { ND } \\
\text { ND }\end{array}$ \\
\hline
\end{tabular}

MRLMethod Reporting Limit ND None Detected at or above the method reporting limit - Quantified as 4-methylphenol. 


\section{PSI Enviranmental Laboratories}

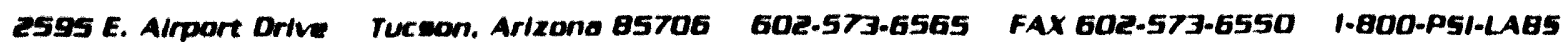

ANALYTICAL REPORT

State of Arizona I/cense 10009

Client:

Peroxidation Systems loc.

5151 E. Broadway, Suite 600

Tucson, AZ 85711

Semple I.D.: $\quad$ 19A-9

Laboratory I.D.: $101993-40$

Sample Type: Waler

Date Sampled: $\quad 10 / 18 / 1993$

Project Name: DOE/Tenn

Date Received: $\quad$ 10/19/1993

Project Number: 0345

Date Analyzed: $\quad 12 / 13 / 1993$

P.O. Number: None

Analyzed By: See Comments

Base Neutral/Acid Semivolatile Organic Compounds

EPA Methods $3510 / 8270$ $\mathrm{mon}$ (ppb)

Base Neutral Andrte

N-Nitrosodimethylamine

Bis(2-chloroethyl) Ether

1,2-Dichlorobenzene

1,3-Dichlorobenzene

1.4-Dichlorobenzene

Bis(2-chloroisopropyl) Ether

$N$-Nitrosodi-n-propyiamine

Hexachlorosthane

Nitrobenzene

Isophorone

Bis (2-chloroethoxy) mothane

1,2,4-Trichlorobenzene

Naphthalene

4-Chloroaniline

Hexachlorobutadiene

2-Methyinaphthalene

Hexachlorocyclopentadiene

2-Chloronaphthalene

2-Nitroanillne

Dimethyl Phthalate

Acenaphthylene

3-Nitroaniline

Acenaphthene

Dibenzofuran

2,4-Dinitrotoluene

Aniline

Acid Analrte

Phenol

2-Chlorophenol

Benzyl Alcohol

2-Methylphenol

3- and 4-Methrlphenol"

2-Nitrophenol

2,4-Dimethriphenol

Benzoic Acid

MRLMethod Reporting Limit

ND None Detected at or above the method reporting limit

- Quantified as 4-methriphenol.
MRL Result

$\begin{aligned} 5 & \text { ND } \\ 5 & \text { ND } \\ 10 & \text { ND } \\ 5 & \text { ND } \\ 5 & \text { ND } \\ 5 & \text { ND } \\ 5 & \text { ND } \\ 50 & \text { ND }\end{aligned}$

$\begin{array}{rr}\text { MRL } & \text { Result } \\ \mathbf{5} & \text { ND } \\ \mathbf{5} & \text { ND } \\ \mathbf{5} & \text { ND } \\ \mathbf{5} & \text { ND } \\ \mathbf{5} & \text { ND } \\ \mathbf{5} & \text { ND } \\ \mathbf{5} & \text { ND } \\ \mathbf{5} & \text { ND } \\ \mathbf{5} & \text { ND } \\ \mathbf{5} & \text { ND } \\ \mathbf{5} & \text { ND } \\ \mathbf{5} & \text { ND } \\ \mathbf{5} & \text { ND } \\ \mathbf{5} & \text { ND } \\ \mathbf{5} & \text { ND } \\ \mathbf{5} & \text { ND } \\ \mathbf{1 0} & \text { ND } \\ \mathbf{5} & \text { NO } \\ \mathbf{2 0} & \text { ND } \\ \mathbf{5} & \text { ND } \\ \mathbf{5} & \text { ND } \\ \mathbf{2 0} & \text { ND } \\ \mathbf{5} & \text { ND } \\ \mathbf{5} & \text { ND } \\ \mathbf{5} & \text { ND } \\ \mathbf{5} & \text { ND } \\ & \end{array}$

Base Neutral Analve

2,6-Dinitrotoluene

Diethyl Phthalate

4-Chlorophenyl Phenyl Ether

Fluorene

4-Nitroaniline

N-Nitrosodiphenviamine

4-Bromophenyl Phenyl Ether

Hexachlorobenzene

Phenanthrene

Anthracene

Di-n-butyl Phthalate

Fluoranthene

Pyrene

Butybenzy Phthalate

3,3'-Dichlorobenzidine

Benz(a)anthracene

Bis(2-othylhexyl) Phthalate

Chrysene

Din-octyl Phthalate

Benzo(b)filuoranthene

Benzo(kfluoranthene

Benzo(alpyrene

Indeno(1,2,3-c,d)pyrene

Dibenz(a, h)anthracene

Benzolg,h,ilperylene

Acid Andirte

2,4-Dichlorophenol

4-Chloro-3-methylphenol

2,4,6-Trichlornphenol

2,4,5-Trichlorophenol

2,4-Dinitrophenol

4-Nitrophenol

2-Methyt-4,6-dinitrophenol

Pentachlorophenol

$\begin{array}{rr}\text { MRL } & \text { Result } \\ \mathbf{5} & \text { ND } \\ \mathbf{5} & \text { ND } \\ \mathbf{5} & \text { ND } \\ \mathbf{5} & \text { ND } \\ \mathbf{2 0} & \text { ND } \\ \mathbf{5} & \text { ND } \\ \mathbf{5} & \text { ND } \\ \mathbf{5} & \text { ND } \\ \mathbf{5} & \text { ND } \\ \mathbf{5} & \text { ND } \\ \mathbf{5} & \text { ND } \\ \mathbf{5} & \text { ND } \\ \mathbf{5} & \text { ND } \\ \mathbf{5} & \text { ND } \\ \mathbf{2 0} & \text { ND } \\ \mathbf{5} & \text { ND } \\ \mathbf{5} & \text { ND } \\ \mathbf{5} & \text { ND } \\ \mathbf{5} & \text { ND } \\ \mathbf{5} & \text { ND } \\ \mathbf{5} & \text { ND } \\ \mathbf{5} & \text { ND } \\ \mathbf{5} & \text { ND } \\ \mathbf{5} & \text { ND } \\ \mathbf{5} & \text { ND }\end{array}$

MRL Result

$5 \quad$ ND

5 ND

ND

ND

ND

ND

ND

ND 
Client:

Peroxidation Systems Inc. 5151 E. Broadway, Suite 600 Tucson, AZ 85711

Project Name: DOE/Tenn

Project Number: 0345

P.O. Number: None
Sample I.D.: Field Blank

Laboratory I.D.: 101993-41

Sample Type: Water

Date Sampled: $\quad 10 / 18 / 1993$

Date Received: $\quad 10 / 19 / 1993$

Date Analyzed: $\quad 12 / 13 / 1993$

Analyzed By: Sec Comments

Base Neutral/Acid Semivolatile Organic Compounds EPA Methods $3510 / 8270$ $\mathrm{\mu ph}$ (ppb)

Base Neutral Analyte N-Nitrosodimethylamine Bis(2-chloroethyl) Ether 1,2-Dichlorobenzene 1,3-Dichlorobenzene 1,4-Dichlorobenzene Bis (2-chloroisopropyl) Ether N-Nitrosodi-n-propylamine Hexachloroethane Nitrobenzene Isophorone Bis(2-chloroethoxy) methane 1,2,4-Trichlorobenzene Naphthalene

4-Chloroaniline

Hexachlorobutadiene

2-Methyinaphthalene Hexachlorocyclopentadiene

2-Chloronaphthalene

2-Nitroaniline

Dimethyl Phthalate

Acenaphthylene

3-Nitroaniline

Acenaphthene

Dibenzofuran

2,4-Dinitrotoluene

Aniline

Acid Analyte

Phenol

2-Chlorophenol

Benzyl Alcohol

2-Methylphenol

3- and 4-Methylphenol

2-Nitrophenol

2,4-Dimethylphenol

Benzoic Acid

MRLMethod Reporting Limit

ND None Detected at or above the method reporting limit

- Quantified as 4-methylphenol.

$\begin{array}{rr}\text { MRL } & \text { Result } \\ \mathbf{5} & \text { ND } \\ \mathbf{5} & \text { ND } \\ \mathbf{5} & \text { ND } \\ \mathbf{5} & \text { ND } \\ \mathbf{5} & \text { ND } \\ \mathbf{5} & \text { ND } \\ \mathbf{5} & \text { ND } \\ \mathbf{5} & \text { ND } \\ \mathbf{5} & \text { ND } \\ \mathbf{5} & \text { ND } \\ \mathbf{5} & \text { ND } \\ \mathbf{5} & \text { ND } \\ \mathbf{5} & \text { ND } \\ \mathbf{5} & \text { ND } \\ \mathbf{5} & \text { ND } \\ \mathbf{5} & \text { ND } \\ \mathbf{1 0} & \text { ND } \\ \mathbf{5} & \text { ND } \\ \mathbf{2 0} & \text { ND } \\ \mathbf{5} & \text { ND } \\ \mathbf{5} & \text { ND } \\ \mathbf{2 0} & \text { ND } \\ \mathbf{5} & \text { ND } \\ \mathbf{5} & \text { ND } \\ \mathbf{5} & \text { ND } \\ \mathbf{5} & \text { ND } \\ & \end{array}$

MRL Result

$\begin{array}{rr}5 & \text { ND } \\ \mathbf{5} & \text { ND } \\ 10 & \text { ND } \\ 5 & \text { ND } \\ 5 & \text { ND } \\ 5 & \text { ND } \\ 5 & \text { ND } \\ 50 & \text { ND }\end{array}$

Base Neutral Analyte

2,6-Dinitrotoluene

Diethyl Phthalate

4-Chlorophenyl Phenyl Ether

Fluorerie

4-Nitroaniline

N-Nitrosodiphenylamine

4-Bromophenyl Phenyl Ether

Hexachlorobenzene

Phenanthrene

Anthracene

Di-n-butyl Phthalate

Fluoranthene

Pyrene

Butylbenzyl Phthalate

3,3'-Dichlorobenzidine

Benz(a)anthracene

Bis(2-ethylhexyl) Phthalate

Chrysene

Di-n-octyl Phthalate

Benzo(b)fluoranthene

Benzo(k)fluoranthene

Benzolalpyrene

Indeno(1,2,3-c,dlpyrene

Dibenz(a,h)anthracene

Benzolg, $h, i)$ perylene

Acid Analyte

2,4-Dichlorophenol

4-Chloro-3-methylphenol

2,4,6-Trichlorophenol

2,4,5-Trichlorophenol

2,4-Dinitrophenol

4-Nitrophenol

2-Methyl-4,6-dinitrophenol

Pentachlorophenol

$\begin{array}{cc}\text { MRL } & \text { Result } \\ \mathbf{5} & \text { ND } \\ \mathbf{5} & \text { ND } \\ \mathbf{5} & \text { ND } \\ \mathbf{5} & \text { ND } \\ \mathbf{2 0} & \text { ND } \\ \mathbf{5} & \text { ND } \\ \mathbf{5} & \text { ND } \\ \mathbf{5} & \text { ND } \\ \mathbf{5} & \text { ND } \\ \mathbf{5} & \text { ND } \\ \mathbf{5} & \text { ND } \\ \mathbf{5} & \text { ND } \\ \mathbf{5} & \text { ND } \\ \mathbf{5} & \text { ND } \\ \mathbf{2 0} & \text { ND } \\ \mathbf{5} & \text { ND } \\ \mathbf{5} & \text { ND } \\ \mathbf{5} & \text { ND } \\ \mathbf{5} & \text { ND } \\ \mathbf{5} & \text { ND } \\ \mathbf{5} & \text { ND } \\ \mathbf{5} & \text { ND } \\ \mathbf{5} & \text { ND } \\ \mathbf{5} & \text { ND } \\ \mathbf{5} & \text { ND }\end{array}$

MRL Result

5 ND

5 ND

ND

ND

ND

ND

ND

ND 


\section{PSI Environmental Laboratories}

2595 E. Airport Drlve

Tucson. Arizona 85706 602.573-6565

FAX 602.573-6550

1-800.PSI-LABS

ANALYTICAL REPOR'T

State of Arizona License $\$ 0009$

Client:

Peroxidation Systems Inc.

5151 E. Broadway, Suite 600

Tucson, AZ 85711

Sample I.D.: Trip Blank

Laboratory I.D.: 101993.42

Sample Type: Water

Date Sampled: $\quad 10 / 18 / 1993$

Project Name: DOE/Tenn

Date Received: $\quad 10 / 19 / 1993$

Project Number: 0345

Date Analyzed: $\quad 12 / 13 / 1993$

P.O. Number: None

Analyzed By: See Comments

Base Neutral/Acid Semivolatile Organic Compounds

EPA Methods $3510 / 8270$ $\mu \mathrm{g} / \mathrm{p}(\mathrm{ppb})$

Base Neutral Analyte.

N-Nitrosodimethylamine

Bis(2-chloroethyl) Ether

1.2-Dichlorobenzene

1,3-Dichlorobenzene

1,4-Dichlorobenzene

Bis(2-chloroisopropyl) Ether

N-Nitrosodi-n-propylamine

Hexachloroethane

Nitrobenzene

Isophorone

Bis(2-chloroethoxy) methane

1,2,4-Trichlorobenzene

Naphthalene

4-Chloroaniline

Hexachlorobutadiene

2-Methynaphthalene

Hexachlorocyclopentadiene

2-Chloronaphthalene

2-Nitroaniline

Dimethyl Phthalate

Acenaphthylene

3-Nitroaniline

Acenaphthene

Dibenzofuran

2,4-Dinitrotoluene

Aniline

Acid Analrte

Phenol

2-Chlorophenol

Benzyl Alcohol

2-Methyiphenol

3- and 4-Methylphenol*

2-Nitrophenol

2,4-Dimethylphenol

Benzoic Acid

$\begin{array}{rc}\text { MRL } & \text { Result } \\ \mathbf{5} & \text { ND } \\ \mathbf{5} & \text { ND } \\ \mathbf{5} & \text { ND } \\ \mathbf{5} & \text { ND } \\ \mathbf{5} & \text { ND } \\ \mathbf{5} & \text { ND } \\ \mathbf{5} & \text { ND } \\ \mathbf{5} & \text { ND } \\ \mathbf{5} & \text { ND } \\ \mathbf{5} & \text { ND } \\ \mathbf{5} & \text { ND } \\ \mathbf{5} & \text { ND } \\ \mathbf{5} & \text { ND } \\ \mathbf{5} & \text { ND } \\ \mathbf{5} & \text { ND } \\ \mathbf{5} & \text { ND } \\ \mathbf{1 0} & \text { ND } \\ \mathbf{5} & \text { ND } \\ \mathbf{2 0} & \text { ND } \\ \mathbf{5} & \text { ND } \\ \mathbf{5} & \text { ND } \\ \mathbf{2 0} & \text { ND } \\ \mathbf{5} & \text { ND } \\ \mathbf{5} & \text { ND } \\ \mathbf{5} & \text { ND } \\ \mathbf{5} & \text { ND }\end{array}$

MRL

Result

$5 \quad$ ND

ND

ND

ND

ND

ND

ND

ND
Base Neutral Analyte

2,6-Dinitrotoluene

Diethyl Phthalate

4-Chlorophenyl Phenyl Ether

Fluorene

4-Nitroaniline

N-Nitrosodiphenylamine

4-Bromophenyl Phenyl Ether

Hexachlorobenzene

Phenanthrene

Anthracene

Di-n-butyl Phthalate

Fluoranthene

Pyrene

Butylbenzyl Phthalate

3.3'-Dichlorobenzidine

Benz(a) anthracene

Bis(2-ethylhexyl) Phthalate

Chrysene

Di-n-octyl Phthalate

Benzo(b)fluoranthene

Benzo(k)fluoranthene

Benzolalpyrene

Indeno(1,2,3-c,d)pyrene

Dibenz $(a, h)$ anthracene

Benzolo,h,ilperylene

Acid Analrte

2,4-Dichlorophenol

4-Chloro-3-methylphenol

2,4,6-Trichlorophenol

2,4,5-Trichlorophenol

2,4-Dinitrophenol

4-Nitrophenol

2-Methyl-4,6-dinitrophenol

Pentachlorophenol

$\begin{array}{rr}\text { MRL } & \text { Result } \\ \mathbf{5} & \text { ND } \\ \mathbf{5} & \text { ND } \\ \mathbf{5} & \text { ND } \\ \mathbf{5} & \text { ND } \\ \mathbf{2 0} & \text { ND } \\ \mathbf{5} & \text { ND } \\ \mathbf{5} & \text { ND } \\ \mathbf{5} & \text { ND } \\ \mathbf{5} & \text { ND } \\ \mathbf{5} & \text { ND } \\ \mathbf{5} & \text { ND } \\ \mathbf{5} & \text { ND } \\ \mathbf{5} & \text { ND } \\ \mathbf{5} & \text { ND } \\ \mathbf{2 0} & \text { ND } \\ \mathbf{5} & \text { ND } \\ \mathbf{5} & \text { ND } \\ \mathbf{5} & \text { ND } \\ \mathbf{5} & \text { ND } \\ \mathbf{5} & \text { ND } \\ \mathbf{5} & \text { ND } \\ \mathbf{5} & \text { ND } \\ \mathbf{5} & \text { ND } \\ \mathbf{5} & \text { ND } \\ \mathbf{5} & \text { ND }\end{array}$

MRL Result

5 ND

5 ND

ND

ND

ND

ND

ND

ND

MRLMethod Reporting Limit

ND None Detected at or above the method reporting limit

- Quantified as 4-methylphenol 
Client:

Peroxidation Systems Inc.

5151 E. Broadway, Suite 600

Tucson, AZ 85711

Sample I.D.: Method Blank

Laboratory I.D.: Method Blank

Sample Type: Water

Date Sampled: $\quad 10 / 18 / 1993$

Project Name: DOE/Tenn

Date Received: $\quad 10 / 19 / 1993$

Project Number: 0345

Date Analyzed: $\quad 12 / 10 / 1993$

P.O. Number: None

Analyzed By: See Comments

Base Neutral/Acid Semivolatile Organic Compounds EPA Methods 3510/8270 $\mu \mathrm{g} / \mathrm{p}(\mathrm{ppb})$

Base Neutral Analyte N-Nitrosodimethylamine Bis(2-chloroethyl) Ether

1,2-Dichlorobenzene

1,3-Dichlorobenzene

1,4-Dichlorobenzene

Bis(2-chloroisopropyl) Ether

$\mathrm{N}$-Nitrosodi-n-propylamine

Hexachloroethane

Nitrobenzene

Isophorone

Bis(2-chloroethoxy) methane

1,2,4-Trichlorobenzene

Naphthalene

4-Chloroaniline

Hexachlorobutadiene

2-Methylnaphthalene

Hexachlorocyclopentadiene

2-Chloronaphthalene

2-Nitroaniline

Dimethyl Phthalate

Acenaphthylene

3-Nitroaniline

Acenaphthene

Dibenzofuran

2,4-Dinitrotoluene

Aniline

Acid Analrte

Phenol

2-Chlorophenol

Benzyl Alcohol

2-Methyiphenol

3- and 4-Methylphenol

2-Nitrophenol

2,4-Dimethylphenol

Benzoic Acid

ND None Detected at or above the method reporting limit

- Quantified as 4-methylphenol.

\begin{tabular}{|c|c|c|}
\hline $\begin{array}{l}\text { Base Neutral Analyte } \\
\text { 2,6-Dinitrotoluene } \\
\text { Diethyl Phthalate } \\
\text { 4-Chlorophenyl Phenyl Ether } \\
\text { Fluorene } \\
\text { 4-Nitroaniline } \\
\text { N-Nitrosodiphenylamine } \\
\text { 4-Bromophenyl Phenyl Ether } \\
\text { Hexachlorobenzene } \\
\text { Phenanthrene } \\
\text { Anthracene } \\
\text { Di-n-butyl Phthalate } \\
\text { Fluoranthene } \\
\text { Pyrene } \\
\text { Butylbenzyl Phthalate } \\
\text { 3,3'-Dichlorobenzidine } \\
\text { Benz(a)anthracene } \\
\text { Bis(2-ethylhexyl) Phthalate } \\
\text { Chrysene } \\
\text { Di-n-octyl Phthalate } \\
\text { Benzo(b)fluoranthene } \\
\text { Benzo(k)fluoranthene } \\
\text { Benzo(a)pyrene } \\
\text { Indeno(1,2,3-c,dlpyrene } \\
\text { Dibenz(a,h)anthracene } \\
\text { Benzolg,h,i)perylene }\end{array}$ & $\begin{array}{r}\text { MRL } \\
5 \\
5 \\
5 \\
5 \\
20 \\
5 \\
5 \\
5 \\
5 \\
5 \\
5 \\
5 \\
5 \\
5 \\
20 \\
5 \\
5 \\
5 \\
5 \\
5 \\
5 \\
5 \\
5 \\
5 \\
5\end{array}$ & $\begin{array}{l}\text { Result } \\
\text { ND } \\
\text { ND } \\
\text { ND } \\
\text { ND } \\
\text { ND } \\
\text { ND } \\
\text { ND } \\
\text { ND } \\
\text { ND } \\
\text { ND } \\
\text { ND } \\
\text { ND } \\
\text { ND } \\
\text { ND } \\
\text { ND } \\
\text { ND } \\
\text { ND } \\
\text { ND } \\
\text { ND } \\
\text { ND } \\
\text { ND } \\
\text { ND } \\
\text { ND } \\
\text { ND } \\
\text { ND }\end{array}$ \\
\hline Acid Analyte & MRL & Result \\
\hline $\begin{array}{l}\text { 2,4-Dichlorophenol } \\
\text { 4-Chloro-3-methyiphenol } \\
\text { 2,4,6-Trichlorophenol } \\
\text { 2,4,5-Trichlorophenol } \\
\text { 2,4-Dinitrophenol } \\
\text { 4-Nitrophenol } \\
\text { 2-Methyl-4,6-dinitrophenol } \\
\text { Pentachlorophenol }\end{array}$ & $\begin{array}{r}5 \\
5 \\
5 \\
5 \\
50 \\
50 \\
20 \\
30\end{array}$ & $\begin{array}{l}\text { ND } \\
\text { ND } \\
\text { ND } \\
\text { ND } \\
\text { ND } \\
\text { ND } \\
\text { ND } \\
\text { ND }\end{array}$ \\
\hline
\end{tabular}

Reviewed By:

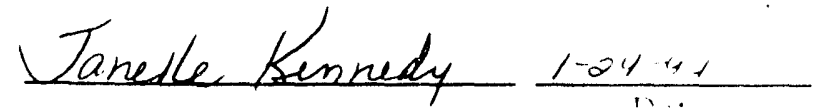




\section{PSI Environmental Laboratories}

\section{ANALYTICAL REPORT}

State of Arizona License $\$ 0009$

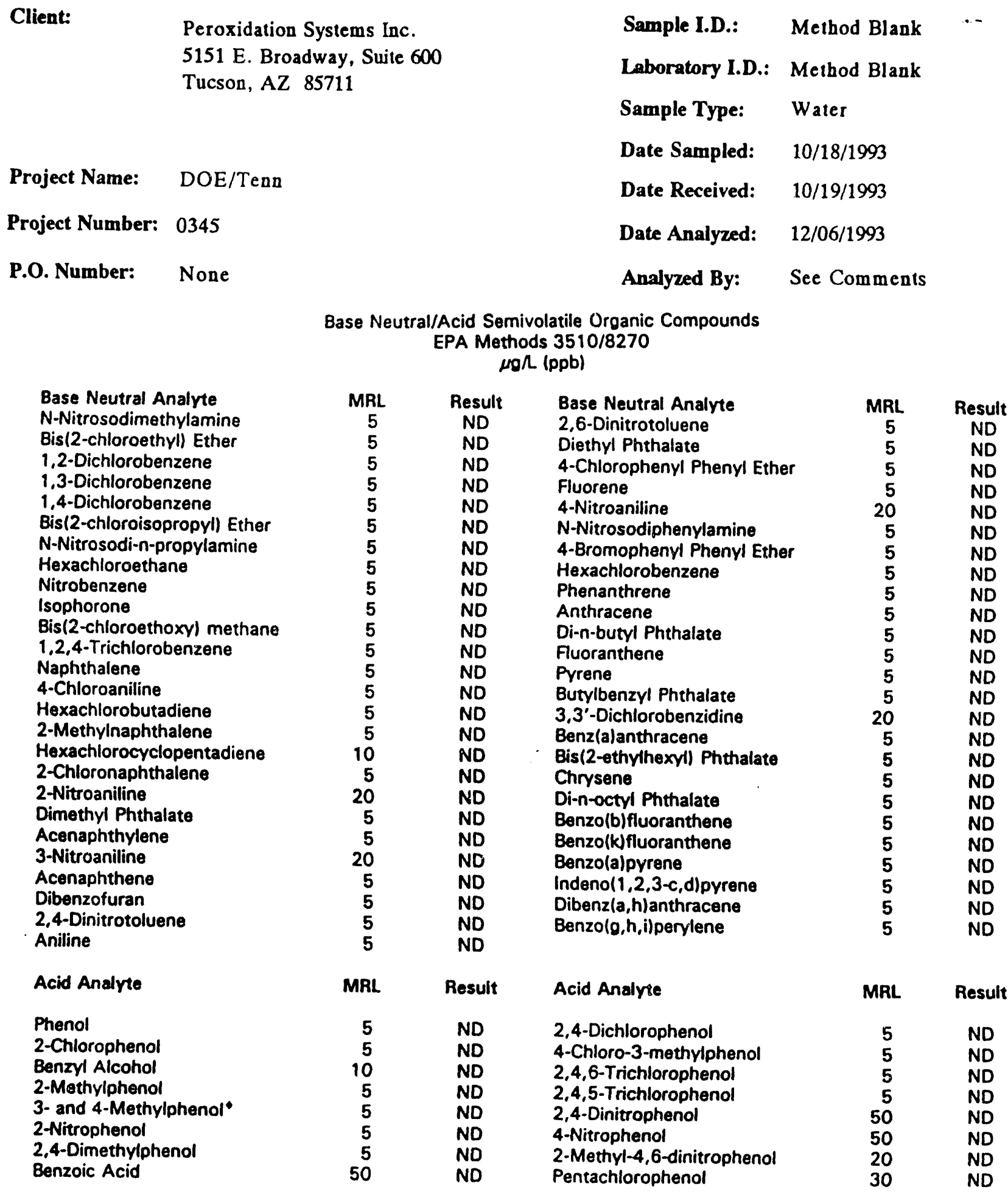

MRLMethod Reporting Limit

ND None Detected at or above the method reporting limit

- Quantified as 4-methylphenol. 


\section{A PSI Enviranmental Laboratories}

2595 E. Aimport Drive Tucson. Arizona 85706 602-573-6565 FAX 602-573-6550 I-800-p5I-LABS ANALYTICAL REPORT

State of Arizona License $\$ 0009$

Client:

Peroxidation Systems Inc.

5151 E. Broadway, Suite 600

Tucson, AZ 85711

Project Name: $\quad$ DOE/Tenn

Project Number: 0345

P.O. Number: None
Sample I.D.: $\quad 16$ A.9 $\quad \cdots$

Laboratory I.D.: 101993-28

Sample Type: Water

Date Sampled: $\quad 10 / 18 / 1993$

Date Received: 10/19/1993

Date Analyzed: $\quad 12 / 10 / 1993$

Analyzed By: See Comments

Matrix Spike/Duplicate Matrix Spike Summary Base Neutral/Acid Semivolatile Organic Compounds

EPA Methods 3510/8270

$\mu g \Omega(p p b)$

Analyte

Phenol

2-Chlorophenol

1,4-Dichlorobenzene

$\mathrm{N}$-Nitrosodi-n-propylamine

1,2,4-Trichlorobenzene

4-Chloro-3-methylphenol

Acenaphthene

4-Nitrophenol

2,4-Dinitrotoluene

Pentachlorophenol

Pyrene

Percent Recovery

Spike Level Sample Spike Result

50.0

50.0

50.0

50.0

50.0

50.0

50.0

50.0

50.0

50.0

50.0
DMS

Result

50.0

50.0

50.0

50.0

50.0

50.0

50.0

50.0

50.0

50.0

50.0

ND
ND
ND
ND
ND
ND
ND
ND
ND
ND
ND

ND
DMS

18.2

38.0

41.2

40.2

41.7

40.5

47.7

14.2

49.4

38.0

46.5
16.8

35.6

37.0

36.4

38.3

38.0

45.4

13.4

45.2

34.2

41.4
EPA Acceptance

MS DMS

Criteria

12-89

27-123

36-97

41-116

39-98

23-97

46-118

10-80

24-96

9-103

26-127

Relative

Percent

Difference

$\begin{array}{lll}76 & 68 & 9-103 \\ 93 & 83 & 26-127\end{array}$

8

7

ND None Detected at or above the method reporting limit Spike recoveries were outside acceptance limits due to matrix effects. Laboratory control spike was acceptable, therefore, data was approved.

Reviewed By:

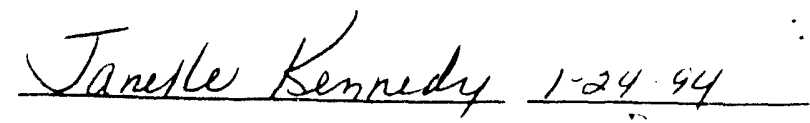


Client:

Peroxidation Systems Inc. 5151 E. Broadway, Suite 600

Tucson, AZ 85711

Project Name: DOE/Tenn

Project Number: 0345

P.O. Number: None
Sample I.D.: See Below $\quad$ - -

Laboratory I.D.: See Below

Sample Type: Water

Date Sampled: $\quad 10 / 18 / 1993$

Date Received: $\quad 10 / 19 / 1993$

Date Analyzed: $\quad 12 / 06-12 / 13 / 1993$

Analyzed By: See Comments

Surrogate Recovery Summary

Base Neutral/Acid Semivolatile Organic Compounds EPA Methods $3510 / 8270$

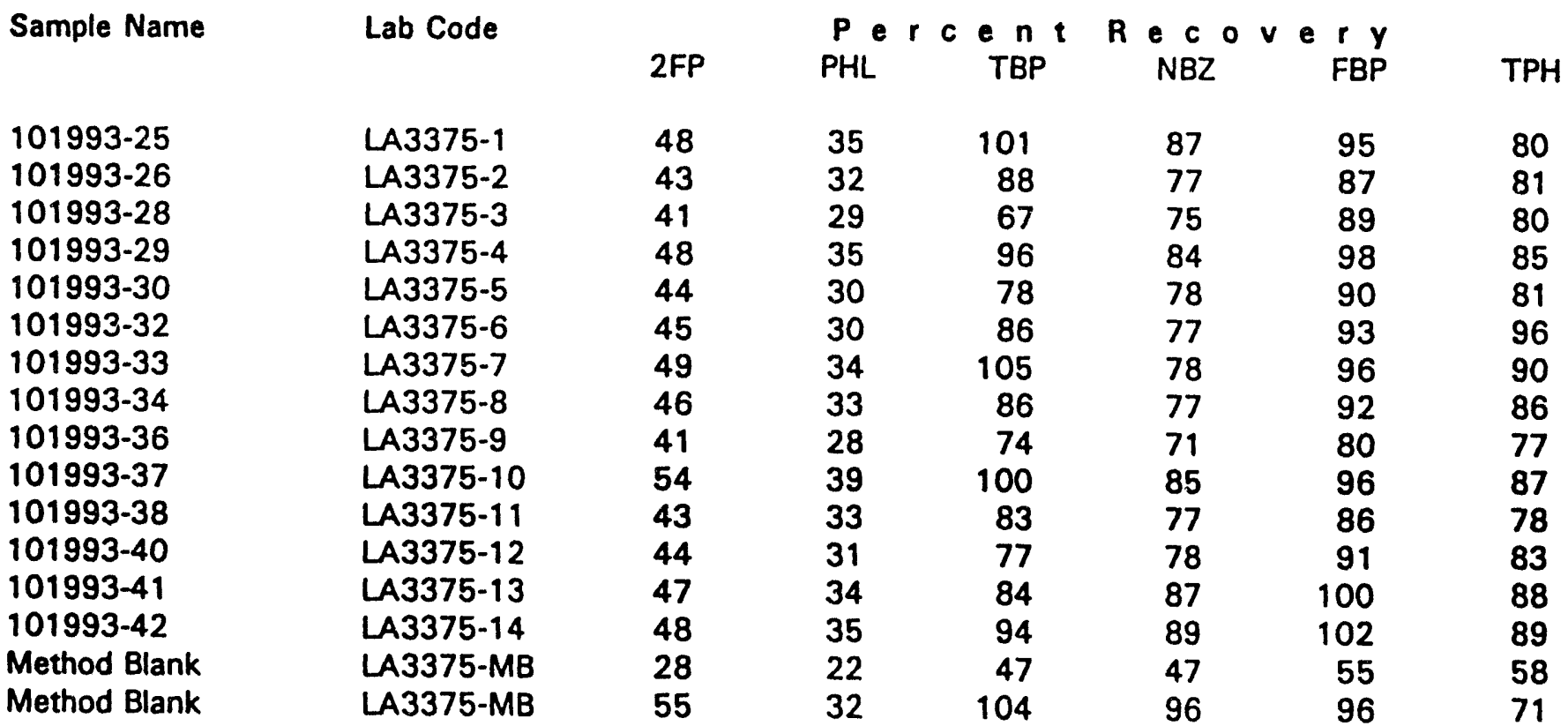

EPA Acceptance Criteria

$\begin{array}{llllll}21-100 & 10-94 & 10-123 & 35-114 & 43-116 & 33-141\end{array}$

$\begin{array}{ll}\text { 2FP } & \text { 2-Fluorophenol } \\ \text { PHL } & \text { Phenol-D。 } \\ \text { TBP } & 2,4,6-T \text { Tribromophenol } \\ \text { NBZ } & \text { Nitrobenzene- } D_{5} \\ \text { FBP } & 2-\text { Fluorobiphenyl } \\ \text { TPH } & \text { Terphenyl- } D_{14}\end{array}$

Comments:

Golden State/CAS Laboratories, Inc.

6925 Canoga Avenue

Canoga Park, CA 91303
Reviewed By:

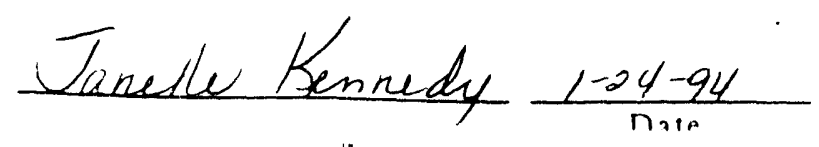



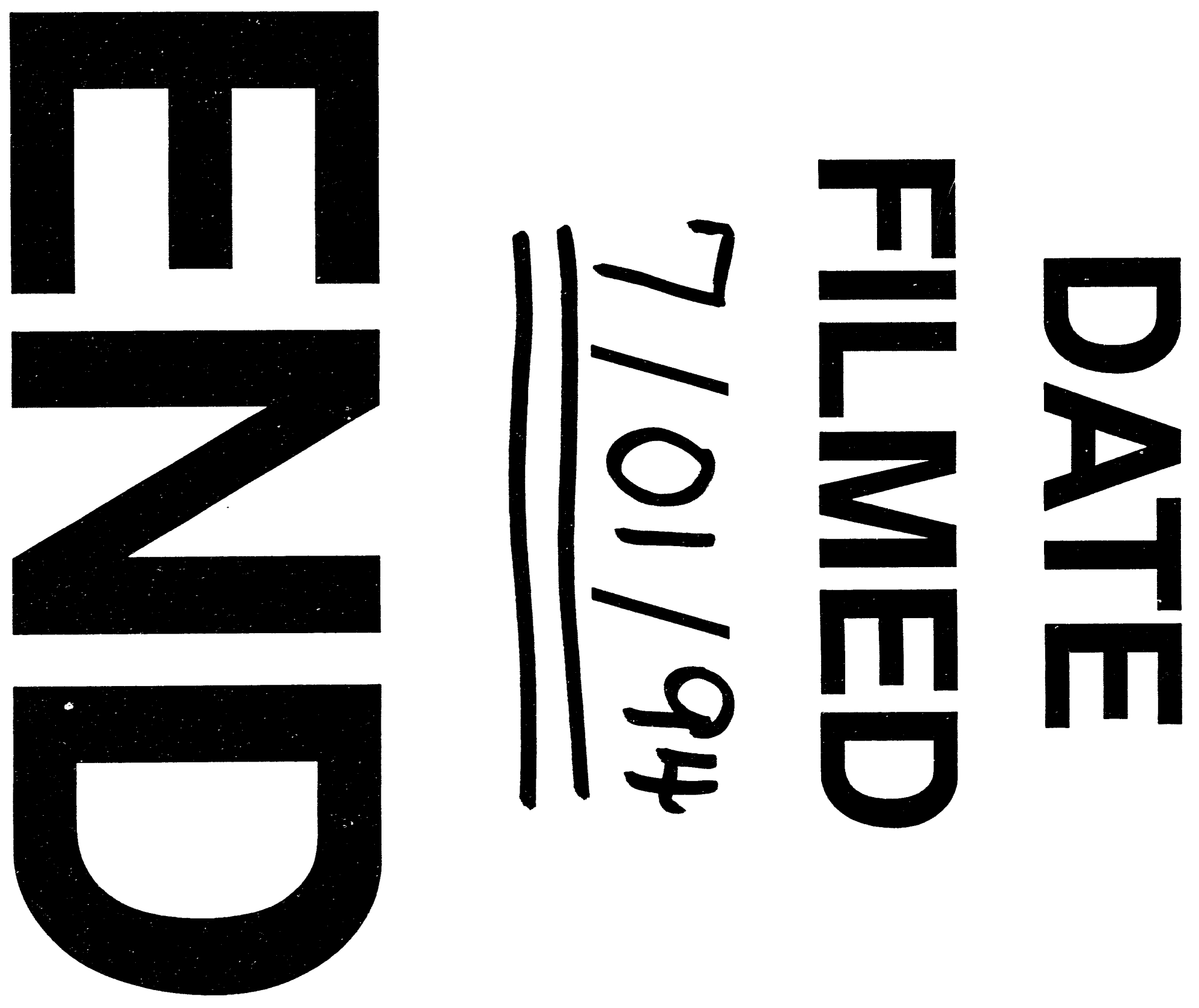
$$
\text { }
$$

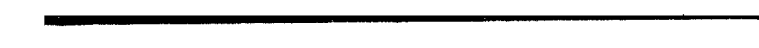

